\title{
PRECIPITATION, ATMOSPHERIC DEPOSITION, STREAMFLOW, AND WATER-QUALITY DATA FROM SELECTED SITES IN THE CITY OF CHARLOTTE AND MECKLENBURG COUNTY, NORTH CAROLINA, 1997-98
}

By K.M. Sarver, W.F. Hazell, and J.B. Robinson

U.S. GEOLOGICAL SURVEY

Open-File Report 99-273

Prepared in cooperation with the City of Charlotte and Mecklenburg County, North Carolina

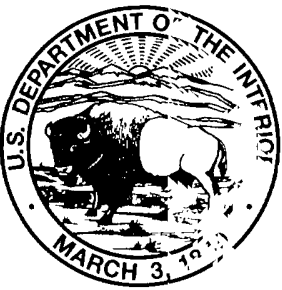




\section{U.S. DEPARTMENT OF THE INTERIOR \\ BRUCE BABBITT, Secretary}

U.S. GEOLOGICAL SURVEY

Charles G. Groat, Director

The use of firm, trade, and brand names in this report is for identification purposes only and does not constitute endorsement by the U.S. Geological Survey.

For additional information write to:

District Chief

U.S. Geological Survey

3916 Sunset Ridge Road

Raleigh, North Carolina 27607
Copies of this report can be purchased from:

U.S. Geological Survey

Information Services

Box 25286, Federal Center

Denver, CO 80225 


\section{CONTENTS}

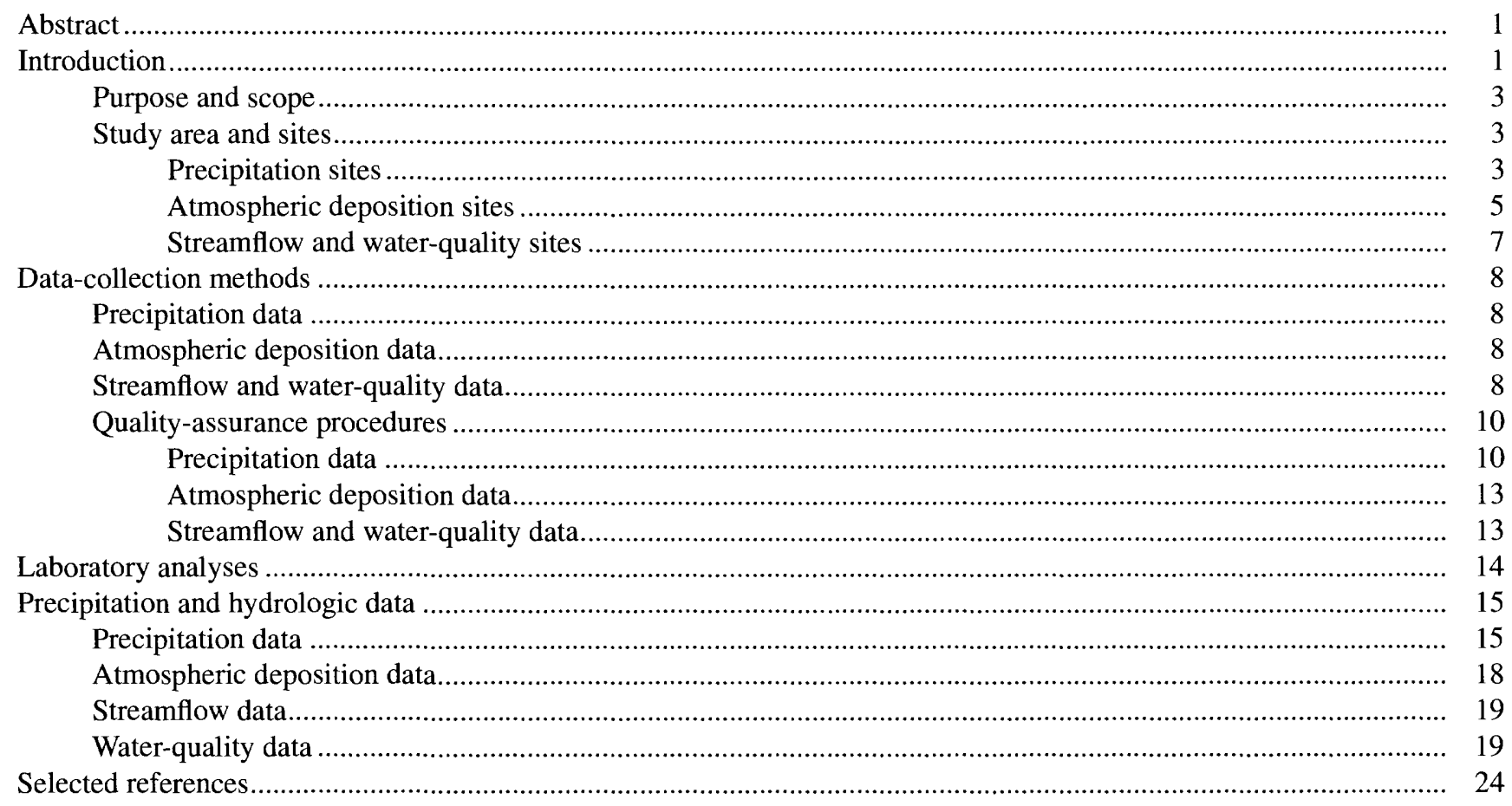

\section{FIGURES}

1. Map showing city of Charlotte and Mecklenburg County data-collection network, 1993-98.......................... 2

2. Flow chart showing raingage locations, by basin, in Charlotte and Mecklenburg County ............................... 5

3. Statistical summary of monthly rainfall totals at site 21 (CRN11) .............................................................. 12

4-6. Maps showing:

4. Annual rainfall distribution in Charlotte and Mecklenburg County for October 1, 1997, through September 30, 1998

5. Rainfall distribution in Charlotte and Mecklenburg County for July 22-24, 1997.

6. Rainfall recurrence intervals for peak 24-hour durations in Charlotte and Mecklenburg County for July $22-24,1997$.

7. Distribution of concentrations of selected constituents measured in equipment, churn, and ambient blank samples associated with stream environmental samples.

\section{TABLES}

1. Precipitation network sites located in Mecklenburg County, October 1988 through September 1998.

2. Types of data collected at streamflow and water-quality sites, December 1993 through September 1998........... 6

3. Land-use distribution, in percent, for study site drainage areas .

4. Accuracy of discharge records at streamflow and water-quality sites.

5. Containers, container treatment, and preservation procedures required for samples collected at the streamflow and water-quality study sites and analyzed by the Mecklenburg County Department of Environmental Protection Laboratory, July 1997 through September 1998. 
6. Summary of atmospheric deposition blank sample results compared to minimum and median concentrations for atmospheric deposition environmental samples, March 1997 through March 1998.

7. Statistical summary of inorganic constituents in blank samples associated with water-quality samples collected from streams, May 1994 through September 1998

8. Blank 95th-percentile values compared to minimum and median concentrations for stream environmental samples, May 1994 through September 1998

9. Analytical procedures and method detection limits for chemical constituents in water analyzed by the Mecklenburg County Department of Environmental Protection Laboratory, July 1997 through

September 1998

10-55. Daily accumulated rainfall totals, July 1997 through September 1998, at:

10. Site 3 (CRN10)

11. Site 5 (CRN06)

12. Site 6 (CRN18)

13. Site 13 (CRN01)

14. Site 14 (CRNO2)

15. Site 15 (CRN03)

16. Site 16 (CRN04)

17. Site 17 (CRN05)

18. Site 18 (CRN07)

19. Site 19 (CRN08)

20. Site 20 (CRN09)

21. Site 21 (CRN11)

22. Site 22 (CRN12)

23. Site 23 (CRN13)

24. Site 24 (CRN14)

25. Site 25 (CRN15)

26. Site 26 (CRN16)

27. Site 27 (CRN17)

28. Site 28 (CRN19)

29. Site 29 (CRN20)

30. Site 30 (CRN21)

31. Site 31 (CRN22)

32. Site 32 (CRN23)

33. Site 33 (CRN25)

34. Site 34 (CRN24)

35. Site 35 (CRN26)

36. Site 36 (CRN27)

37. Site 37 (CRN28)

38. Site 44 (CRN41)

39. Site 45 (CRN29)

40. Site 46 (CRN30)

41. Site 47 (CRN31)

42. Site 48 (CRN32)

43. Site 49 (CRN33)

44. Site 50 (CRN34)

45. Site 51 (CRN35)

46. Site 52 (CRN36)

47. Site 53 (CRN37)

48. Site 54 (CRN38)

49. Site 55 (CRN39)

50. Site 56 (CRN40)

51. Site 57 (CRN42)

52. Site 58 (CRN43) 
53. Site 59 (CRN44)

54. Site $60(\mathrm{CRN} 45)$

55. Site 61 (CRN46)

56-58. Statistical summary of atmospheric deposition water-quality data, March 1997 through March 1998, at:

56. Site 37 (CSW06)

57. Site 42 (CSW04)

58. Site 43 (CSW07)

59-61. Atmospheric deposition water-quality data, March 1997 through March 1998, at:

59. Site 37 (CSW06)

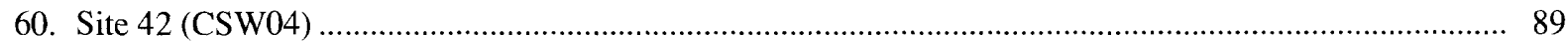

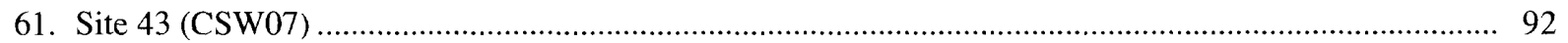

62. Streamflow statistics at the streamflow and water-quality study sites, December 1993 through

September 1998

63-68. Daily mean discharge values, July 1997 through September 1998, at:

63. Site 37 (CSW06)

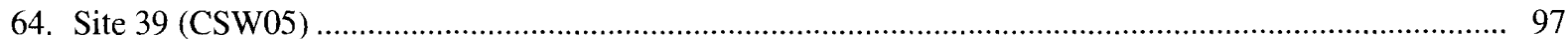

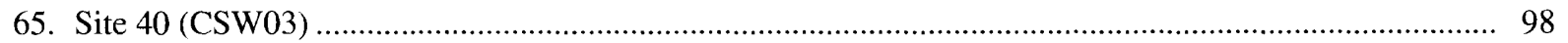

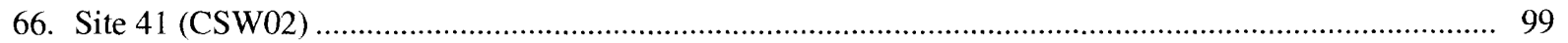

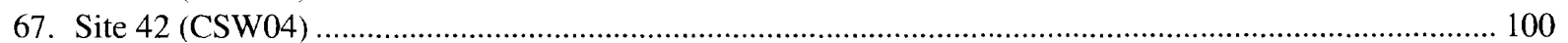

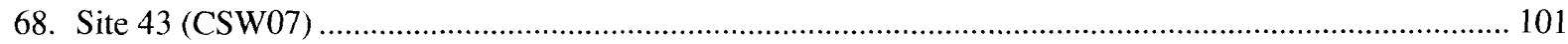

69. Maximum and minimum specific conductance and water temperature recorded by monitors at the streamflow and water-quality study sites, October 1994 through September 1998 …............................... 102

70-75. Statistical summary of water-quality data at:

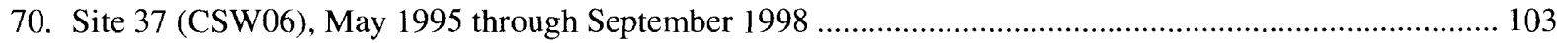

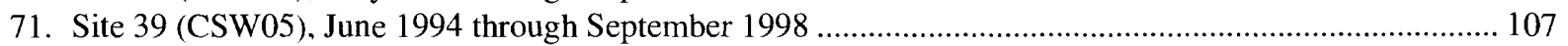

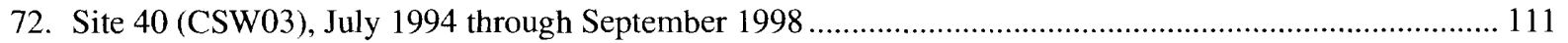

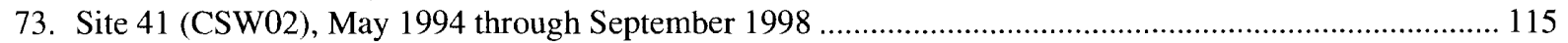

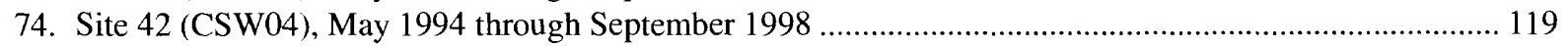

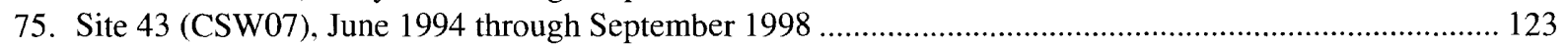

76-81. Water-quality data, July 1997 through September 1998, at:

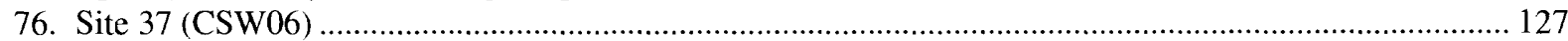

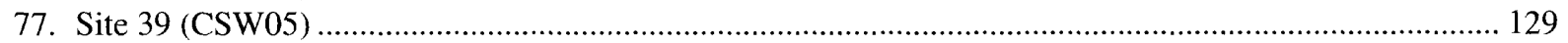

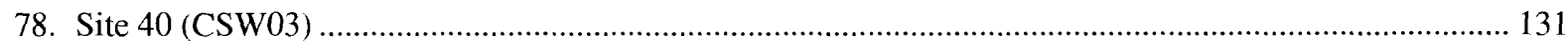

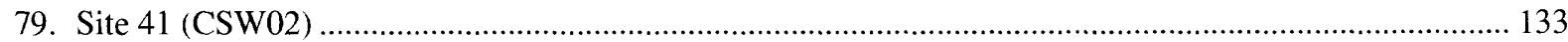

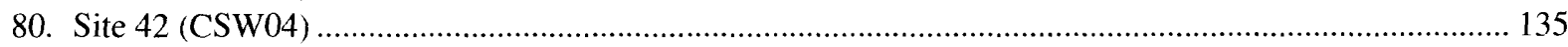

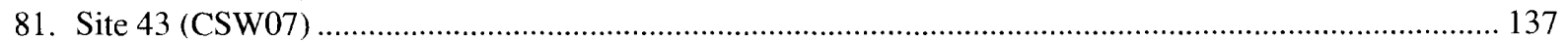

82-87. Rainfall and streamflow characteristics, July 1997 through September 1998, for the monitored storms at:

82. Site 37 (CSW06)

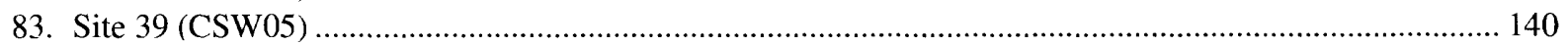

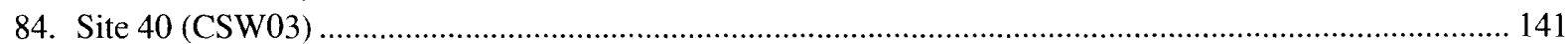

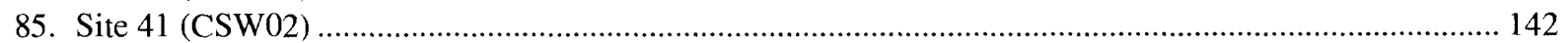

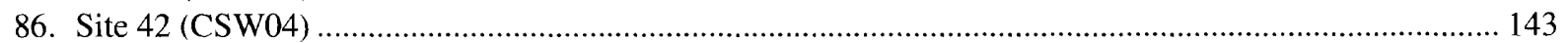

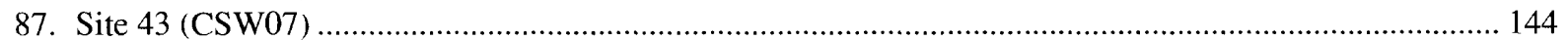


CONVERSION FACTORS, VERTICAL DATUM, SPECIFIC CONDUCTANCE, AND TEMPERATURE

\begin{tabular}{rll}
\hline Multiply & By & To obtain \\
\hline inch (in.) & 25.4 & millimeter \\
foot $(\mathrm{ft})$ & 0.3048 & meter \\
square mile $\left(\mathrm{mi}^{2}\right)$ & 2.59 & square kilometer \\
pint $(\mathrm{pt})$ & 0.4732 & liter \\
inch per year (in/yr) & 25.4 & millimeter per year \\
\hline
\end{tabular}

Sea level: In this report "sea level" refers to the National Geodetic Vertical Datum of 1929 (NGVD of 1929)-A geodetic datum derived from a general adjustment of the first-order level nets of both the United States and Canada, formerly called Sea Level Datum of 1929.

Specific conductance is given in microsiemens per centimeter at 25 dgrees Celsius $\left(\mu \mathrm{S} / \mathrm{cm}\right.$ at $\left.25^{\circ} \mathrm{C}\right)$.

Temperature conversions for degrees Celsius $\left({ }^{\circ} \mathrm{C}\right)$ and degrees Fahrenheit $\left({ }^{\circ} \mathrm{F}\right)$ :

$$
\begin{aligned}
& { }^{\circ} \mathrm{C}=5 / 9\left({ }^{\circ} \mathrm{F}-32\right) \\
& { }^{\circ} \mathrm{F}=1.8\left({ }^{\circ} \mathrm{C}\right)+32
\end{aligned}
$$




\title{
Precipitation, Atmospheric Deposition, Streamflow, and Water-Quality Data from Selected Sites in the City of Charlotte and Mecklenburg County, North Carolina, 1997-98
}

\author{
By K.M. Sarver, W.F. Hazell, and J.B. Robinson
}

\section{ABSTRACT}

Precipitation data were collected at 46 precipitation sites and 3 atmospheric deposition sites, and hydrologic data were collected at 6 stream sites in the vicinity of Charlotte and Mecklenburg County, North Carolina, from July 1997 through September 1998. Data were collected to identify the type, concentration, and amount of nonpoint-source stormwater runoff in the study area. The data collected include measurements of precipitation; streamflow; physical characteristics, such as water temperature, $\mathrm{pH}$, specific conductance, biochemical oxygen demand, oil and grease, and suspendedsediment concentrations; and concentrations of nutrients, metals and minor constituents, and organic compounds. These data will provide information needed for (1) planned watershed simulation models, (2) estimates of nonpointsource constituent loadings to the Catawba River, and (3) characterization of water quality in relation to basin conditions. Streamflow and rainfall data have been used to provide early warnings of possible flooding.

\section{INTRODUCTION}

In October 1993, the U.S. Geological Survey (USGS), in cooperation with the City of Charlotte, Mecklenburg County, and Charlotte-Mecklenburg Utility Department (CMU), began a water-quality study in the Catawba River Basin near Charlotte, North Carolina (fig. 1). Study efforts for the City of Charlotte, which are described in this report, focused on characterizing stormwater quantity and quality from selected land uses, collecting information on nonpointsource loadings to the Catawba River, and installing and operating a precipitation network. Study efforts for CMU and Mecklenburg County focused on Mountain Island Lake and included inflow sampling from two basins, outflow sampling, and reservoir monitoring (Sarver and Steiner, 1998).

An earlier study was conducted during 1992-94 by the USGS, in cooperation with the Western Piedmont Council of Governments, to investigate water quality in the upper Catawba River Basin (Jaynes, 1994). The objectives of the study were to collect and interpret water-quality data from streams and reservoirs in the region and to develop circulation and transport models for two reservoirs in the Catawba River Basin-Rhodhiss Lake and Lake Hickorynorthwest of the study area described in this report (Giorgino and Bales, 1997; Bales and Giorgino, 1998).

In addition, the USGS is conducting an investigation of water quality in the Catawba River Basin downstream from Lake Wylie to evaluate the potential effects of increased point-source inputs on water quality in the river. The Catawba River Basin also is part of the USGS National Water-Quality Assessment (NAWQA) Program's Santee-Coastal Basin Study Unit (Hughes, 1994). Together, these studies are providing consistent methods of data collection, interpretation, and modeling techniques for a large portion of the Catawba River Basin. 


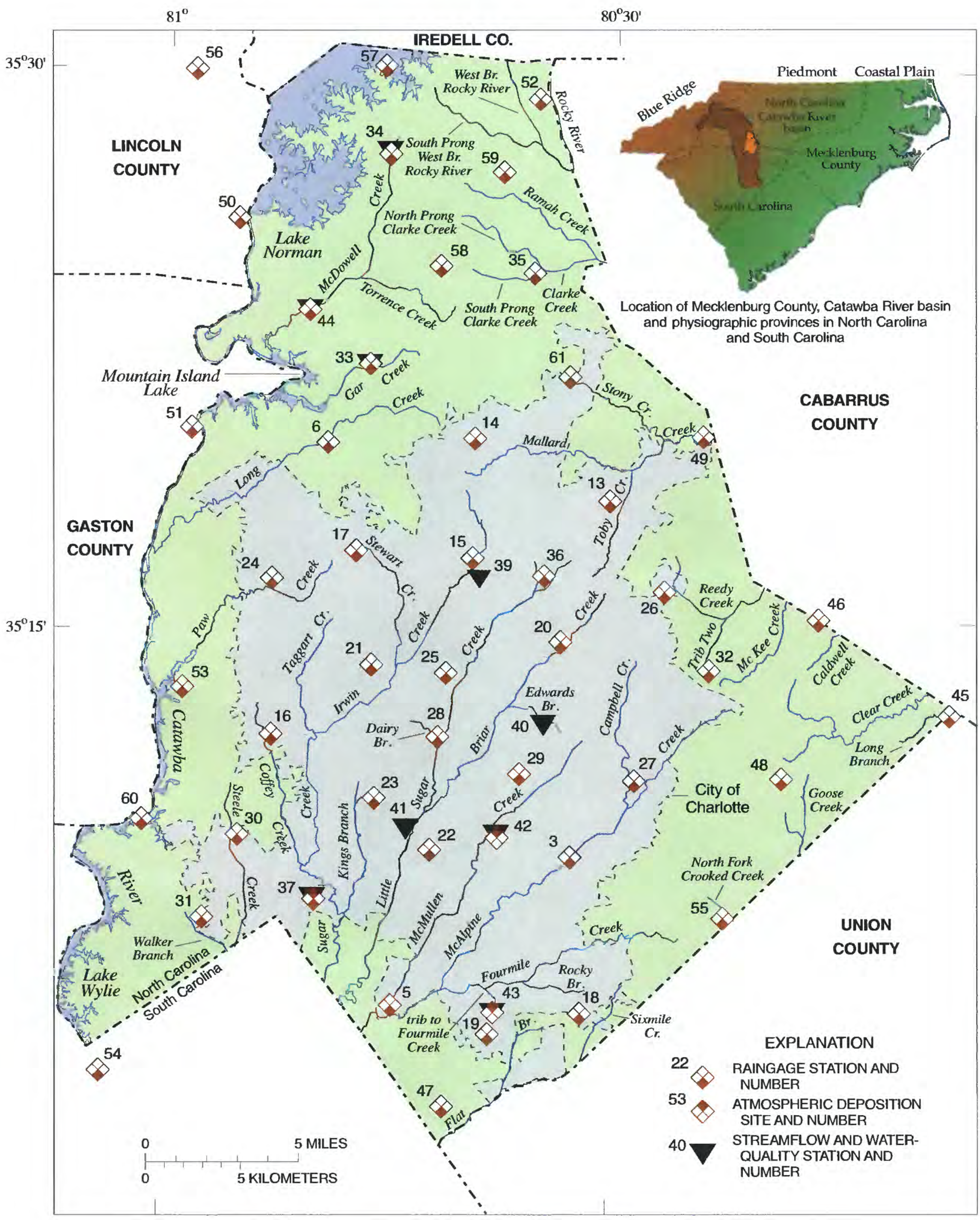

Figure 1. City of Charlotte and Mecklenburg County data-collection network, 1993-98. 


\section{Purpose and Scope}

The purpose of this report is to summarize the precipitation and hydrologic data collected in Charlotte and Mecklenburg County from July 1997 through September 1998. Summary statistics are presented for the entire period of record (May 1994 through September 1998). The data collected include measurements of precipitation; atmospheric deposition; streamflow; physical characteristics, such as water temperature, $\mathrm{pH}$, specific conductance, biochemical oxygen demand, oil and grease, and suspended-sediment concentrations; and concentrations of nutrients, metals and minor constituents, and organic compounds. This report also describes the field and laboratory methods that were used to collect and analyze these data. Earlier reports documented data that were collected during October 1993 through June 1995 (Robinson and others, 1996) and July 1995 through June 1997 (Robinson, Hazell, and Garrett, 1998).

The data-collection network that was initiated in October 1993 with the City of Charlotte, Mecklenburg County, and CMU consisted of 46 precipitation sites, 3 atmospheric deposition sites, and 9 stream sites (fig. 1), which were needed to determine the effects of land development on water quality and to evaluate the effectiveness of control measures. Six of the sites defined runoff characteristics from streams with differing land-use characteristics within the city; these sites were discontinued in September 1998. Three of the sites defined runoff characteristics from streams located within the county; these sites were discontinued in September 1997. These data will provide information needed for stormwater management, estimates of nonpoint-source constituent loadings to the Catawba River, and information needed to calibrate watershed models used for evaluating stormwater management options.

\section{Study Area and Sites}

Mecklenburg County is located in south-central North Carolina in the southern Piedmont Province (fig. 1). Prior to July 1998, the county encompassed an area of 528 square miles $\left(\mathrm{mi}^{2}\right)$. County boundaries were changed slightly in the northwest corner on July 1, 1998. As a result, Mecklenburg County currently (1999) encompasses about $524 \mathrm{mi}^{2}$. The county is bounded on the west by the Catawba River and its reservoirs-Lake Norman, Mountain Island Lake, and Lake Wylie (fig. 1). The Catawba River drains approximately 75 percent of the county. The remaining 25 percent of the county is draine 1 by the Rocky River and its tributaries in the Pee Dee River Basin (McCachren, 1980). Lake Norman is the major water-supply reservoir for several municipalities in northern Mecklenburg County. Mountain Island Lake supplies Charlotte and several other municipalities in Mecklenburg and surrounding counties.

Charlotte is the principal municipality in Mecklenburg County and the largest city in North Carolina. The metropolitan area had a 1997 fopulation of 513,000 - an increase of approximately 55,000 people since 1994. An additional 97,000 peonle live in Mecklenburg County outside the city limits of Charlotte (Steve Patterson, City of Charlotte Planning Office, oral commun., 1997). The city area encompasses $234 \mathrm{mi}^{2}$, or approximately 45 percent of the county. Most of the city's urban area is drained by four large creeks-Irwin, Little Sugar, McAlpine, and Briar (fig. 1). Irwin, Little Sugar, and McAlpine Creeks receive effluent from Charlotte wastewater-treatment plants, as well as effluent from smaller dischargers.

The climate of the study area is characterized by hot, humid summers, moderate but short winters, and long growing seasons. The mean monthly temperature ranges from about $41^{\circ} \mathrm{F}$ in January to about $79^{\circ} \mathrm{F}$ in July. Precipitation in the study area averages about 43 inches per year (in/yr) (McCachren, 1980). The topography of the area is characterized by brcad, gently rolling interstream areas and by steeper slop's along the drainageways. The elevation of the study area has a range of 520 feet (ft) above mean sea level at the State line south of Pineville, N.C., to about $830 \mathrm{ft}$ in the extreme northern portion of the county (McCachren, 1980). The area is predominately underlain by granite with some slate in the southeast (LeGrand and Mundorff, 1952). The soils in the study area are described as well-drained sandy loams with a clayey subsoil (McCachren, 1980).

\section{Precipitation Sites}

Since 1963, the USGS has collected frecipitation data at various locations throughout Charlotte and Mecklenburg County. Forty-six raingages provided precipitation data for this report (fig. 1; table 1). These raingage sites previously have been referred to by using Charlotte rainfall network (CRN) numbers, which are included in this report for reference. 
Table 1. Precipitation network sites located in Mecklenburg County, October 1988 through Septernber 1998 [Shaded rows indicate collection-well sites. All others are tipping bucket sites. CMU, Charlotte-Mecklenburg Utility Department; SR, Secondary Road; WWTP, wastewater-treatment plant]

\begin{tabular}{|c|c|c|c|c|c|}
\hline $\begin{array}{c}\text { Siteno. } \\
\text { (fig.1) }\end{array}$ & Station no. ${ }^{a}$ & Latitude & Longitude & Location & $\begin{array}{l}\text { Friod of } \\
\text { record }\end{array}$ \\
\hline 3 & 02146600 & $35^{\circ} 08^{\prime} 14^{\prime \prime}$ & $80^{\circ} 46^{\prime} 05^{\prime \prime}$ & CRN10, McAlpine Creek at Sardis Rd. nr Charlotte, N.C. & $11 / 92-9 / 98$ \\
\hline 5 & 02146750 & $35^{\circ} 03^{\prime} 59^{\prime \prime}$ & $80^{\circ} 52^{\prime} 12^{\prime \prime}$ & CRN06, McAlpine Creek below McMullen Creek nr Pineville, N.C. & $5 / 93-9 / 98$ \\
\hline 6 & 02142900 & $35^{\circ} 19^{\prime} 42^{\prime \prime}$ & $80^{\circ} 54^{\prime} 35^{\prime \prime}$ & CRN18, Long Creek nr Paw Creek, N.C., at Oakdale Rd. & $3 / 93-9 / 98$ \\
\hline 13 & 351812080445545 & $35^{\circ} 18^{\prime} 12^{\prime \prime}$ & $80^{\circ} 44^{\prime} 55^{\prime \prime}$ & CRN01, Fire Station 27, 111 Ken Hoffman Dr. & $10 / 92-9 / 98$ \\
\hline 14 & 351954080493445 & $35^{\circ} 19^{\prime} 54^{\prime \prime}$ & $80^{\circ} 49^{\prime} 34^{\prime \prime}$ & CRN02, Fire Station 28,8013 Old Statesville Rd. & $10 / 92-9 / 98$ \\
\hline 15 & 0214620760 & $35^{\circ} 16^{\prime} 32^{\prime \prime}$ & $80^{\circ} 49^{\prime} 35^{\prime \prime}$ & CRN03, Irwin Creek at Starita Rd. at Charlotte, N.C. & $10 / 92-9 / 98$ \\
\hline 16 & 351132080562345 & $35^{\circ} 11^{\prime} 32^{\prime \prime}$ & $80^{\circ} 56^{\prime} 23^{\prime \prime}$ & CRN04, Fire Station 30, 4707 Belle Oaks Rd. & $10 / 92-9 / 98$ \\
\hline 17 & 351642080533445 & $35^{\circ} 16^{\prime} 42^{\prime \prime}$ & $80^{\circ} 53^{\prime} 34^{\prime \prime}$ & CRN05, CMU, Administration Building, 5100 Brookshire Blvd. & $10 / 92-9 / 98$ \\
\hline 18 & 350351080454145 & $35^{\circ} 03^{\prime} 51^{\prime \prime}$ & $80^{\circ} 45^{\prime} 41^{\prime \prime}$ & CRN07, Fire Station 9, 4529 McKee Rd. & $10 / 92-9 / 98$ \\
\hline 19 & 350314080484945 & $35^{\circ} 03^{\prime} 14^{\prime \prime}$ & $80^{\circ} 48^{\prime} 49^{\prime \prime}$ & CRN08, 11515 Elm Lane at intersection of Providence Rd. West ${ }^{c}$ & $10 / 92-9 / 98$ \\
\hline 20 & 351414080463245 & $35^{\circ} 14^{\prime} 14^{\prime \prime}$ & $80^{\circ} 46^{\prime} 32^{\prime \prime}$ & CRN09, Fire Station 15, 3617 Frontenac Ave. & $11 / 92-9 / 98$ \\
\hline 21 & 351331080525945 & $35^{\circ} 13^{\prime} 31^{\prime \prime}$ & $80^{\circ} 52^{\prime} 59^{\prime \prime}$ & CRN11, Fire Station 10, 2135 Remount Rd. & $11 / 92-9 / 98$ \\
\hline 22 & 350823080505345 & $35^{\circ} 08^{\prime} 23^{\prime \prime}$ & $80^{\circ} 50^{\prime} 53^{\prime \prime}$ & CRN12, Fire Station 16, 6623 Park South Dr. & $3 / 93-9 / 98$ \\
\hline 23 & 350947080524945 & $35^{\circ} 09^{\prime} 47^{\prime \prime}$ & $80^{\circ} 52^{\prime} 49^{\prime \prime}$ & CRN13, USGS Office, 810 Tyvola Rd. & $3 / 93-9 / 98$ \\
\hline 24 & 351553080562645 & $35^{\circ} 15^{\prime} 53^{\prime \prime}$ & $80^{\circ} 56^{\prime} 26^{\prime \prime}$ & CRN14, Fire Station 21, 1023 Little Rock Rd. & $3 / 93-9 / 98$ \\
\hline 25 & 351320080502645 & $35^{\circ} 1320^{\prime \prime}$ & $80^{\circ} 50^{\prime} 26^{\prime \prime}$ & CRN15, Charlotte-Mecklenburg Govt. Center, 600 E. Fourth St. & $3 / 93-9 / 98$ \\
\hline 26 & 351540080430045 & $35^{\circ} 15^{\prime} 40^{\prime \prime}$ & $80^{\circ} 43^{\prime} 00^{\prime \prime}$ & CRN16, Reedy Creek Environmental Center, 2900 Rocky River Rd. & $3 / 93-9 / 98$ \\
\hline 27 & 351023080435745 & $35^{\circ} 10^{\prime} 23^{\prime \prime}$ & $80^{\circ} 43^{\prime} 57^{\prime \prime}$ & CRN17, Piney Grove Elementary School, 8801 Eaglewind Dr. & $3 / 93-9 / 98$ \\
\hline 28 & 351132080504145 & $35^{\circ} 11^{\prime} 32^{\prime \prime}$ & $80^{\circ} 50^{\prime} 41^{\prime \prime}$ & CRN19, Freedom Park, Cumberland Dr. & 9/93-9/98 \\
\hline 29 & 351032080475245 & $35^{\circ} 10^{\prime} 32^{\prime \prime}$ & $80^{\circ} 47^{\prime} 52^{\prime \prime}$ & CRN20, Fire Station 14, 114 N. Sharon Amity Rd. & 9/93-9/98 \\
\hline 30 & 350842080572801 & $35^{\circ} 08^{\prime} 42^{\prime \prime}$ & $80^{\circ} 57^{\prime} 28^{\prime \prime}$ & CRN21, Kennedy Middle School, 4000 Gallant Lane & $9 / 90-9 / 98$ \\
\hline 31 & 350623080583801 & $35^{\circ} 06^{\prime} 23^{\prime \prime}$ & $80^{\circ} 58^{\prime} 38^{\prime \prime}$ & CRN22, Walker Branch Basin, Choate Cir. & $9 / 90-9 / 98$ \\
\hline 32 & 351302080412701 & $35^{\circ} 1302^{\prime \prime}$ & $80^{\circ} 41^{\prime} 27^{\prime \prime}$ & CRN23, Harrisburg Rd. Landfill, 7817 Harrisburg Rd. & $10 / 88-9 / 98$ \\
\hline 33 & 0214266075 & $35^{\circ} 21^{\prime} 55^{\prime \prime}$ & $80^{\circ} 53^{\prime} 12^{\prime \prime}$ & CRN25, Gar Creek at SR 2120 (McCoy Rd.) nr Oakdale, N.C. & $4 / 9.4-9 / 98$ \\
\hline 34 & 02142651 & $35^{\circ} 27^{\prime} 49^{\prime \prime}$ & $80^{\circ} 52^{\prime} 36^{\prime \prime}$ & CRN24, McDowell Creek at Westmoreland Rd. nr Cornelius, N.C. & $5 / 9.4-9 / 98$ \\
\hline 35 & 352432080473745 & $35^{\circ} 24^{\prime} 32^{\prime \prime}$ & $80^{\circ} 47^{\prime} 37^{\prime \prime}$ & CRN26, Bradford Airfield, Huntersville-Concord Rd. & $6 / 94-9 / 98$ \\
\hline 36 & 351604080470845 & $35^{\circ} 16^{\prime} 04^{\prime \prime}$ & $80^{\circ} 47^{\prime} 08^{\prime \prime}$ & CRN27, Hidden Valley Elementary School, 5100 Snow White Lane & $10 / 94-9 / 98$ \\
\hline 37 & 0214635212 & $35^{\circ} 06^{\prime} 57^{\prime \prime}$ & $80^{\circ} 54^{\prime} 49^{\prime \prime}$ & CRN28, Unnamed tributary to Sugar Creek at Crompton St. & $4 / 95-9 / 98$ \\
\hline 44 & 0214266000 & $35^{\circ} 23^{\prime} 22^{\prime \prime}$ & $80^{\circ} 55^{\prime} 16^{\prime \prime}$ & CRN41, McDowell Creek nr Charlotte & $11 / 96-9 / 98$ \\
\hline 45 & 351218080331345 & $35^{\circ} 12^{\prime} 18^{\prime \prime}$ & $80^{\circ} 33^{\prime} 13^{\prime \prime}$ & CRN29, Clear Creek Boy Scout Camp, 9408 Belt Rd. & 2/96-9/98 \\
\hline 46 & 351455080374445 & $35^{\circ} 14^{\prime} 55^{\prime \prime}$ & $80^{\circ} 37^{\prime} 44^{\prime \prime}$ & CRN30, Rhyne Farm, 3600 Peach Orchard Rd. & 2/96-9/98 \\
\hline 47 & 350110080502045 & $35^{\circ} 01^{\prime} 10^{\prime \prime}$ & $80^{\circ} 50^{\prime} 20^{\prime \prime}$ & CRN31, Elon Homes, 11401 Ardrey-Kell Rd. & 2/96-9/98 \\
\hline 48 & 351028080385545 & $35^{\circ} 10^{\prime} 28^{\prime \prime}$ & $80^{\circ} 38^{\prime} 55^{\prime \prime}$ & CRN32, Bain Elementary School, 11524 Bain School Rd. & 2/96-9/98 \\
\hline 49 & 352000080414645 & $35^{\circ} 20^{\prime} 00^{\prime \prime}$ & $80^{\circ} 41^{\prime} 46^{\prime \prime}$ & CRN33, Mallard Creek WWTP, 12400 Hwy. 29 North & $12 / 95-9 / 98$ \\
\hline 50 & 352555080574445 & $35^{\circ} 25^{\prime} 55^{\prime \prime}$ & $80^{\circ} 57^{\prime} 44^{\prime \prime}$ & CRN34, Cowans Ford Dam area, 257 Duke Lane & $2 / 96-9 / 98$ \\
\hline 51 & 0214267600 & $35^{\circ} 200^{\prime \prime}$ & $80^{\circ} 59^{\prime} 12^{\prime \prime}$ & CRN35, Catawba River at Mountain Island Dam & $1 / 96-9 / 98$ \\
\hline 52 & 352921080473245 & $35^{\circ} 29^{\prime} 21^{\prime \prime}$ & $80^{\circ} 47^{\prime} 32^{\prime \prime}$ & CRN36, West Fork substation, 20801 Shearer Rd. & $2 / 96-9 / 98$ \\
\hline 53 & 351247080592745 & $35^{\circ} 12^{\prime} 47^{\prime \prime}$ & $80^{\circ} 59^{\prime} 27^{\prime \prime}$ & CRN37, Berryhill Elementary School, 10501 Walkers Ferry Rd. & $2 / 96-9 / 98$ \\
\hline 54 & 350200081020345 & $35^{\circ} 02^{\prime} 00^{\prime \prime}$ & $81^{\circ} 02^{\prime} 03^{\prime \prime}$ & CRN38, Tega Cay city offices, 7000 Tega Cay Dr. & $2 / 96-9 / 98$ \\
\hline 55 & 350634080405245 & $35^{\circ} 06^{\prime} 34^{\prime \prime}$ & $80^{\circ} 40^{\prime} 52^{\prime \prime}$ & CRN39, Phillips Farm, 2248 Mount Harmony Church Rd. & $2 / 96-9 / 98$ \\
\hline 56 & 353003080591745 & $35^{\circ} 30^{\prime} 03^{\prime \prime}$ & $80^{\circ} 59^{\prime} 17^{\prime \prime}$ & CRN40, Westport Golf Course ${ }^{\mathrm{d}}$ & $2 / 96-9 / 98$ \\
\hline 57 & 353014080524945 & $35^{\circ} 30^{\prime} 14^{\prime \prime}$ & $80^{\circ} 52^{\prime} 49^{\prime \prime}$ & CRN42, Horton pool house, 21509 Norman Shores Dr. & $1 / 97-9 / 98$ \\
\hline 58 & 352440080505045 & $35^{\circ} 24^{\prime} 40^{\prime \prime}$ & $80^{\circ} 50^{\prime} 50^{\prime \prime}$ & CRN43, Huntersville Elementary School. 200 Gilead Rd. & $1 / 97-9 / 98$ \\
\hline 59 & 352718080484345 & $35^{\circ} 27^{\prime} 18^{\prime \prime}$ & $80^{\circ} 48^{\prime} 43^{\prime \prime}$ & CRN44, Knox Farm, 13516 Mayes Rd. & $1 / 97-9 / 98$ \\
\hline 60 & 350903081004545 & $35^{\circ} 09^{\prime} 03^{\prime \prime}$ & $81^{\circ} 00^{\prime} 45^{\prime \prime}$ & CRN45, 12700 Withers Cove Rd. & $1 / 97-9 / 98$ \\
\hline 61 & 352135080462045 & $35^{\circ} 21^{\prime} 35^{\prime \prime}$ & $80^{\circ} 46^{\prime} 20^{\prime \prime}$ & CRN46, Oehler Farm, 3491 Johnston-Oehler Rd. & $1 / 97-9 / 98$ \\
\hline
\end{tabular}

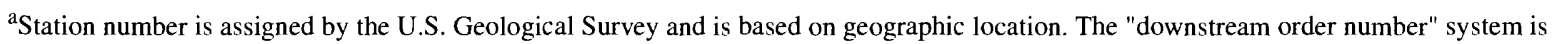
used for streamflow sites, and the "latitude-longitude" system is used for well sites.

${ }^{b}$ Precipitation data collection currently (1999) is ongoing.

${ }^{c}$ Prior to August 4, 1994, located at McAlpine Creek Elementary School, 9100 Carswell Lane, station number 350458080493245.

${ }^{\mathrm{d}}$ Prior to June 4, 1996, located at Lake Norman Volunteer Fire Department, 1206 Brawley School Road, station number $35340208 \mathrm{C} 543145$. 
The primary criterion for site selection of raingage locations was to provide good areal coverage of Charlotte and Mecklenburg County. Consideration also was given to providing optimum precipitation data for water-quality sampling events and to combining installations with existing stream-gaging locations. Four raingages were installed with streamflow and water-quality sites-sites $33,34,37$, and 44 . Three raingages were installed at existing USGS streamgaging stations-sites 3, 5, and 6 (fig. 1; table 1). Data collection currently (1999) is ongoing at all 46 raingages.

Forty-one named stream basins are covered by the raingage locations, including all major stream basins in Charlotte and Mecklenburg County (fig. 1). Figure 2 is a generalized chart that identifies the raingages and the specific stream subbasins in which they are located. Four raingages-sites $14,18,46$, and 59-are located on basin divides and, therefore, represent rainfall coverage in multiple headwater basins.

\section{Atmospheric Deposition Sites}

Atmospheric deposition sites were located in basins with existing streamflow and water-quality datacollection sites. Atmospheric deposition data were collected at sites 37, 42, and 43, which represent different land uses in Charlotte (fig. 1; table 2). A detailed description of these sites is given in the following section. Atmospheric deposition samples were collected weekly from March 1997 through March 1998.
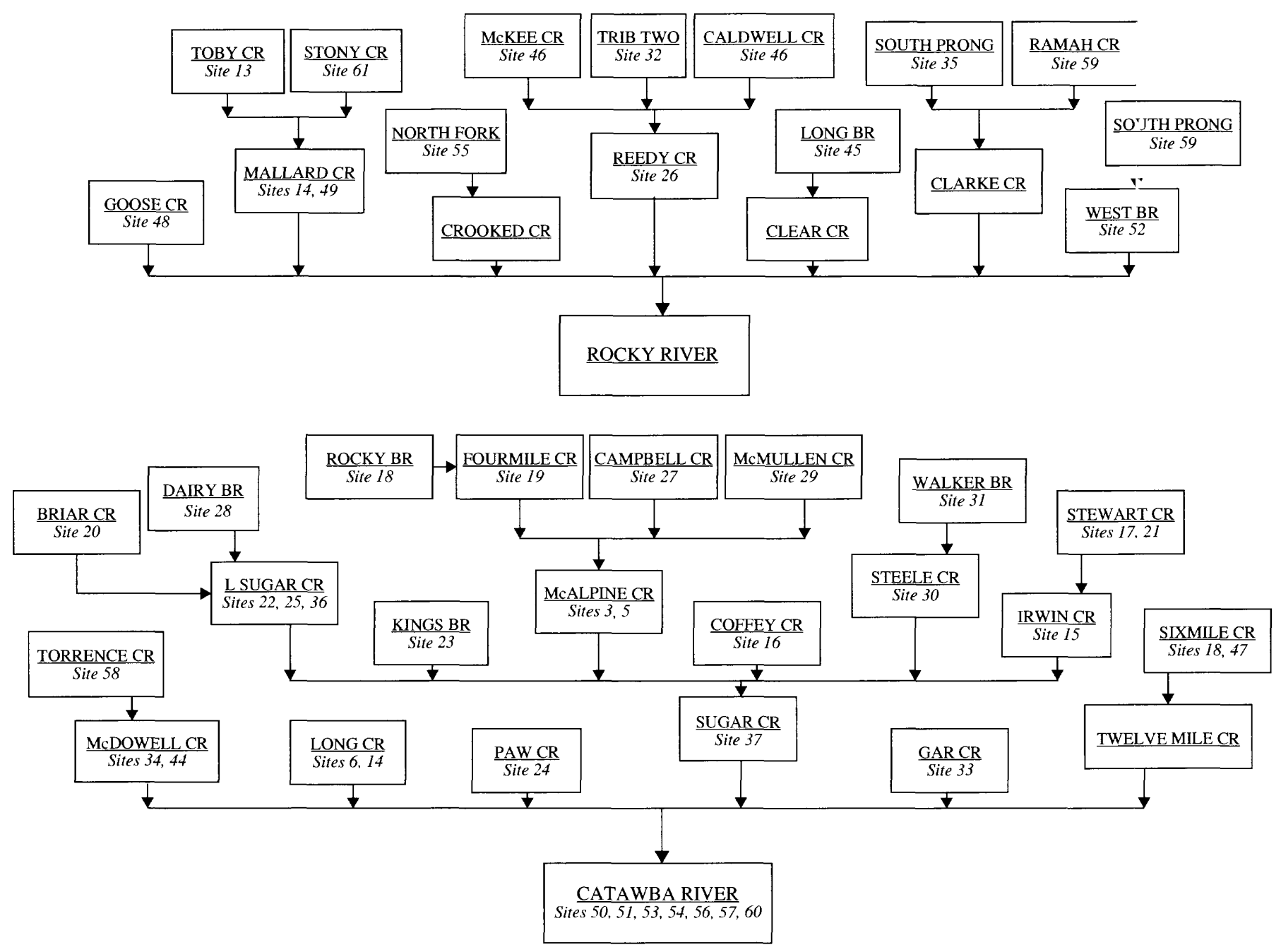

Figure 2. Raingage locations, by basin, in Charlotte and Mecklenburg County. 


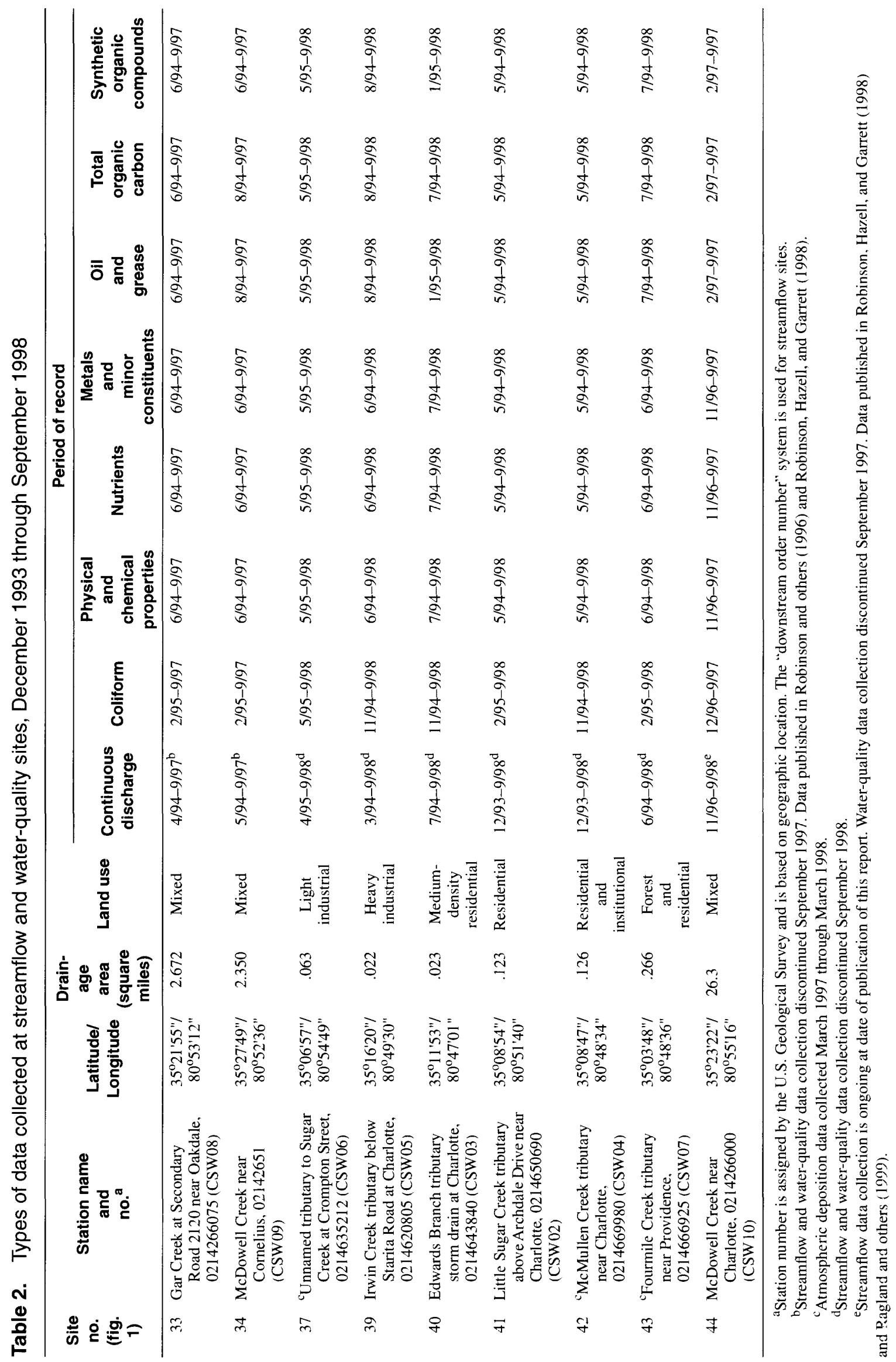




\section{Streamflow and Water-Quality Sites}

Streamflow and water-quality site selection was based on the size of the drainage areas and the type of land use. The land-use information presented in this report was obtained from the City of Charlotte and is based on data classified from 1990 aerial photographs and reconnaissance conducted by USGS personnel. Basin land-use maps previously have been published (Robinson and others, 1996) except for site 44. Six sites were chosen within the city limits of Charlottesites 37, 39, 40, 41, 42, and 43; and three sites were chosen in the northern part of the county-sites 33, 34 . and 44 (fig. 1; table 2). These sites have previously been referred to using Charlotte stormwater (CSW) numbers; those CSW numbers are included in this report for reference.

Each site within the city drained into one of the four major streams carrying runoff from the metropolitan area. Sites 33, 34, and 44 all drained directly into Mountain Island Lake water-supply reservoir. All sites had continuous records of stage and discharge, water temperature, and specific conductance. Waterquality samples were collected once per season when possible, during runoff events.

Streamflow and water-quality data collection was discontinued at sites 33 and 34 in September 1997. Collection of water-quality data was discontinued at site 44 in September 1997; however, streamflow data collection currently (1999) is ongoing (Ragland and others, 1999). Streamflow and water-quality data collected through September 1997 at sites ?3, 34, and 44 are presented in Robinson, Hazell, and Garrett (1998); thus, these sites are not discussed further in this report. Streamflow and water-quality data collection at sites $37,39,40,41,42$, and 43 was discontinued in September 1998.

Site 37 is located on a tributary to Sugar Creek (fig. 1). The drainage area encompasses $0.0<3 \mathrm{mi}^{2}$ and consists of light industrial, light commercial, and some woods or brush (table 3). A small portion of an active railroad also is within the basin.

Site 39 is located on a tributary to Irwin Creek (fig. 1). Land use is almost entirely heavy industrial with a drainage area of $0.022 \mathrm{mi}^{2}$ (table 3 ).

Site 40 is located in a storm drain to a tributary of Edwards Branch, which flows into Briar Creek (fig. 1). Land use is almost entirely medium-density residential (table 3) with a drainage area of $0.023 \mathrm{mi}^{2}$. A very small portion of the basin includes some light industry as well as an elementary school.

Site 41 is located on a tributary to Little Sugar Creek (fig. 1) and has a multi-use drainage area of $0.123 \mathrm{mi}^{2}$. Residential housing is the primary land use. The basin also includes a portion of a large chemical

Table 3. Land-use distribution, in percent, for study site drainage areas

[Values are in percent. - , no land-use data for this category]

\begin{tabular}{|c|c|c|c|c|c|c|c|c|c|c|}
\hline \multirow[b]{2}{*}{$\begin{array}{l}\text { Site no. } \\
\text { (fig. 1) }\end{array}$} & \multirow[b]{2}{*}{$\begin{array}{l}\text { Woods/ } \\
\text { Brush }\end{array}$} & \multicolumn{4}{|c|}{ Residential } & \multirow[b]{2}{*}{$\begin{array}{c}\text { Institu- } \\
\text { tional }\end{array}$} & \multicolumn{2}{|c|}{ Industrial } & \multicolumn{2}{|c|}{ Commercia' } \\
\hline & & $\begin{array}{c}\text { Greater } \\
\text { than } \\
2 \text { acres }\end{array}$ & $\begin{array}{c}\text { Greater } \\
\text { than } \\
1 / 2 \text { to } \\
2 \text { acres }\end{array}$ & $\begin{array}{c}\text { Greater } \\
\text { than } \\
1 / 4 \text { to } \\
1 / 2 \text { acre }\end{array}$ & $\begin{array}{l}\text { Less } \\
\text { than or } \\
\text { equal to } \\
1 / 4 \text { acre }\end{array}$ & & Light $^{a}$ & Heavy ${ }^{b}$ & Light $^{\mathrm{a}}$ & Heas $\cdots b$ \\
\hline 37 [CSW06] & 10.3 & 0.1 & - & - & - & - & 63.5 & - & 26.1 & - \\
\hline 39 [CSW05] & .1 & - & - & - & - & 一 & - & 99.8 & .1 & 一 \\
\hline 40 [CSW03] & - & - & 2.1 & 96.8 & - & - & 1.1 & - & - & - \\
\hline 41 [CSW02] & 1.7 & - & - & 57.7 & - & 5.8 & - & 22.9 & 11.9 & - \\
\hline 42 [CSW04] & - & - & 7.9 & 19.4 & 31.3 & 40.6 & - & - & .8 & - \\
\hline 43 [CSW07] & 17.2 & - & 2.5 & 33.0 & - & 3.2 & - & - & 44.1 & - \\
\hline
\end{tabular}

a Light is defined as less than 44 percent impervious.

${ }^{\mathrm{b}} \mathrm{Heavy}$ is defined as greater than 56 percent impervious. 
research laboratory, an elementary school, and some light commercial activity (table 3 ).

Site 42 is located on a tributary to McMullen Creek (fig. 1) and has a drainage area of $0.126 \mathrm{mi}^{2}$. Land use within the basin is residential and institutional (a private school). Some light commercial activity also is present (table 3 ).

Site 43 is located on a tributary to Fourmile Creek (fig. 1). At the time of site selection, existing land use was predominately light commercial, singlefamily residential, and woods/brush (table 3 ). Future development for the basin was planned, and much of the drainage area is now residential (single and multifamily) and light commercial, with ongoing new construction. A large church, a rest home, and some woods/brush also are present. The drainage area is $0.266 \mathrm{mi}^{2}$.

\section{DATA-COLLECTION METHODS}

All sites were equipped with electronic dataloggers for instrument operation and data collection. Storage modules with independent, internal batteries and nonvolatile memory also stored programs and data for backup. Modems at the sites allowed remote communication and interaction with the dataloggers. Software was developed to automatically retrieve and process data daily. Remote interaction also allowed users to monitor, test, and activate peripheral devices from any offsite location.

\section{Precipitation Data}

Two types of raingages were installed in the study area-tipping bucket raingages and 3-inch (in.) diameter collection wells with water-level sensors. The type of rainfall measuring equipment installed was determined on a site-by-site basis. Thirteen sites initially were installed with collection-well pipes in less secure areas or where tipping buckets were not feasible. Site 17 was converted to a tipping bucket site on Dec. 29, 1994. As of September 1998, there were 34 tipping bucket sites and 12 collection-well sites (table 1).

All sites recorded rainfall amounts at 5-minute intervals. The raingages located at water-quality sampling sites also recorded rainfall at 1-minute intervals when rainfall was detected. During periods of equipment problems when incremental resolution was lost, total rainfall still may have been measured.
Although neither type of raingage was designed to measure frozen precipitation, rainfall equivalent totals may have been recorded as the frozen precipitation in the catchment was melted by warming temperatures. When possible, daily or monthly totals were computed and published.

\section{Atmospheric Deposition Data}

Atmospheric deposition data collection began in March 1997 and continued through March 1998 at sites 37, 42, and 43 (fig. 1; table 2). Hydrologic data included the quantity of wet deposition and analysis of wet-deposition samples for specific conductance, $\mathrm{pH}$, nutrients, selected metals, chloride, and sulfate. No collection of dry deposition (dust particles) was conducted during this investigation.

Wet-deposition samples were collected using an automatic wet/dry sampler equipped with a plastic sample-collection container and powered by a 12-volt battery. This device had a motorized protective lid that kept the sample-collection container covere 1 during periods of no precipitation. When the moist rre sensor detected precipitation, the lid mechanically moved to allow wet deposition to be collected in the sample container. When the precipitation stopped, the lid mechanically returned to the protective position. Samples typically were retrieved on Monday of each week.

Samples were weighed in the USGS Charlotte Field Office using an analytical balance. Orce weighed, the precipitation amount was computed in inches equivalent. Samples were then decanted directly from the collection container into the appronriate subsample containers, preserved, and delivered to the laboratory for analysis.

\section{Streamflow and Water-Quality Data}

Data collection began in December 1993 at sites 41 and 42, in March 1994 at site.39, in June 1994 at site 43, in July 1994 at site 40, and in April 199: at site 37. Hydrologic data included measurements of streamflow, fecal coliform bacteria, physical and chemical properties, nutrients, concentrations of metals and minor constituents, oil and grease, organic compounds in water (table 2), and suspended sediment. Streamflow and water-quality data collection were discontinued September 1998 . 
Instrumentation at each site included sensors for the collection of water level, water temperature, and specific conductance data. Water-quality samples were collected by using an automatic refrigerated sampler. All equipment was housed in a walk-in shelter with alternating current. Site 37 had a tipping bucket raingage for the collection of precipitation data.

Continuous-record gages were established at each site. Because of the rapid response of streamflow to precipitation in small urban basins, instantaneous water levels (stage) were recorded every 5 minutes. Water levels were recorded every minute when stream stage rose above a predetermined threshold and during water-quality sampling events. At the beginning of the study (December 1993), the recording interval at sites 41 and 42 was 15 minutes.

Where conditions allowed, streamflow (discharge) was measured by following procedures outlined by Rantz and others (1982). Stage-discharge relations, or ratings, were subsequently developed and used to quantify streamflow at each recorded 5-minute interval. In the absence of suitable measuring conditions, concrete weirs, V-notches, and indirect methods of flow computations were used to define the stage-discharge relations. The point control and, therefore, the stage-discharge relations remained stable at all sites throughout the study period.

The accuracy of the discharge records depends primarily on (1) the stability of the stage-discharge relation and (2) the accuracy of the measurement of stage. Accuracy is attributed to the discharge records as follows: "Excellent" means that about 95 percent of the daily discharges are within 5 percent of the true value; "good," within 10 percent; and "fair," within 15 percent. Records that do not meet these criteria are rated "poor." Rated accuracy of records for each site is shown in table 4.

Water temperature and specific conductance were measured every 5 minutes with an in situ probe. Initially, these data also were collected at 1-minute intervals when flow was above the predetermined threshold and during water-quality sampling events.

Review of these data indicated that water temperature and specific conductance did not vary significantly at 1-minute intervals. Thus, the collection interval was increased to 5 minutes in June 1995. Specific conductance measurements also were made with grab samples collected during an event.

Measurements of $\mathrm{pH}$ were made during waterquality sampling events. Prior to January 1996, pH was
Table 4. Accuracy of discharge records at streamflow and water-quality sites

$\left[\mathrm{ft}^{3} / \mathrm{s}\right.$, cubic foot per second]

\begin{tabular}{|c|c|c|}
\hline $\begin{array}{l}\text { Site no. } \\
\text { (fig. 1) }\end{array}$ & $\begin{array}{c}\text { Flow range } \\
\left(\mathrm{ft}^{3} / \mathrm{s}\right)\end{array}$ & Ratinn \\
\hline 37 (CSW06) & $\begin{array}{l}0.00 \text { to }<2.20 \\
\geq 2.20\end{array}$ & $\begin{array}{l}\text { good } \\
\text { fair }\end{array}$ \\
\hline 39 (CSW05) & $\begin{array}{l}0.00 \text { to }<1.50 \\
1.50 \text { to }<2.50 \\
\geq 2.50\end{array}$ & $\begin{array}{l}\text { good } \\
\text { fair } \\
\text { poor }\end{array}$ \\
\hline 40 (CSW03) & $\begin{array}{l}0.00 \text { to }<1.00 \\
1.00 \text { to }<3.00 \\
\geq 3.00\end{array}$ & $\begin{array}{l}\text { good } \\
\text { fair } \\
\text { poor }\end{array}$ \\
\hline 41 (CSW02) & $\begin{array}{l}0.00 \text { to }<1.50 \\
1.50 \text { to }<3.00 \\
\geq 3.00\end{array}$ & $\begin{array}{l}\text { good } \\
\text { fair } \\
\text { poor }\end{array}$ \\
\hline 42 (CSW04) & $\begin{array}{l}0.00 \text { to }<0.80 \\
0.80 \text { to }<2.00 \\
\geq 2.00\end{array}$ & $\begin{array}{l}\text { good } \\
\text { fair } \\
\text { poor }\end{array}$ \\
\hline 43 (CSW07) & all & poor \\
\hline
\end{tabular}

measured by using a probe that was mounted in a flowthrough cell connected to a water pump. Collection of $\mathrm{pH}$ data was initiated simultaneously with waterquality sample collection and continued for a period of 10 minutes. After January 1996, raw water samples collected by the automatic samplers, as well as grab samples collected during an event, were used for $\mathrm{pH}$ determinations.

Water samples were collected at each study site during runoff events, once per season when possible. The criteria, provided by the cooperators, that were used to determine if the sampled event met the requirements of the project were (1) the minimum period between sampled events was at least 21 days, (2) the rainfall duration was between 3 and 13 hours, (3) the rainfall amount was between 0.2 and 0.8 in., and (4) less than 0.1 in. of rainfall had occurred in the 72 hours prior to the sampled event. The rainfall amount could exceed 0.8 in. and(or) the duration could be longer than 13 hours as long as the total rainfall amount during the first 3 ho' $x$ rs was less than 0.8 in. Every effort was made to adhere to these criteria, but there were times when all criteria were not met.

Generally, three discrete samples were collected during increasing, near peak, and receding streamflows associated with the runoff event. The specific conductance and $\mathrm{pH}$ of each sample were measured as the sample was processed. Samples were analyzed for a broad range of constituents. 
Water samples for inorganic analysis were collected using an automatic refrigerated sampler. Each discrete sample consisted of two raw water samples collected in 1.9-liter glass bottles. The two bottles were composited in a polycarbonate churn splitter, processed and preserved as described by Horowitz and others (1994), and analyzed by the USGS National Water Quality Laboratory. Beginning in September 1995, the Mecklenburg County Department of Environmental Protection Laboratory began analyzing the samples that were collected for inorganic constituents. These samples were preserved as required by the Mecklenburg County laboratory (table 5). Total organic carbon (TOC) samples were taken from the discrete samples prior to placement in the churn splitter. Samples for the analysis of dissolved constituents were filtered through a 0.45 -micron poresize capsule filter by using a peristaltic pump.

Samples for most organic analyses were collected using an automatic refrigerated sampler with methanol-cleaned Teflon tubing or by hand as a grab sample. Pesticide samples were collected during the spring and were decanted directly from the glass collection bottles to the appropriate sample containers.

Grab samples included oil and grease and volatile organic compounds (VOC's), which were collected during the first 20-30 minutes of the runoff event. Bacteria samples were collected manually during increasing, near peak, and receding streamflows. Because of possible contamination from methanol-cleaned tubing used in the automatic sampler during the spring, TOC was collected as a grab sample during spring sampling events.

\section{Quality-Assurance Procedures}

Quality-assurance procedures for precipitation, atmospheric deposition, streamflow, and water-quality data collection and processing are presented in the following sections. All procedures followed standard USGS guidelines as documented in each section. Detailed quality-assurance procedures were prepared and are documented in a USGS administrative report (U.S. Geological Survey, written commun., 1997).

\section{Precipitation Data}

Tipping bucket raingages were delivered from the factory with documented calibration. Factory calibration consisted of pouring a known amount of water into the bucket at a fixed rate and comparing the recorded amount with the known rainfall equivalency. Collection-well raingages were designed an 1 constructed according to generally accepted standards.

All sites were field calibrated in July and August 1998. Tipping buckets were calibrated using a technique similar to that applied in the factcry. At collection-well sites, catchment dimensions were measured and a surface area was computed. A known amount of water was poured into the catchment, and the rainfall total recorded was compared to the rainfall equivalent of the known volume. Measured precipitation for 32 raingages was within 5 percent of the actual amount, and all of the raingages recorded precipitation within 12 percent of the actual amount. Where errors greater than 5 percent were computed, the equipment was adjusted and recalibrated. No corrections were applied to the data.

Sites were visited on an average of once every 6 to 8 weeks. Initial readings of time and rainfall were recorded. Catchments, funnels, and tubing vere inspected for blockage, and conditions were noted. Catchments and funnels were wiped clean and rinsed free of debris. Tubing was reamed, rinsed, and brushed clean. Battery voltage was measured with an external volt meter, and the reading was compared to that of the datalogger. Freshly charged batteries were installed as needed. The installation and phone lines were inspected for vandalism or tampering.

Tipping bucket pivots were oiled, and buckets were inspected for freedom of movement and assurance of interaction with the datalogger. After draining a collection well, a small amount of water was returned to the well. Inspections included visibly watching the float wheel turn and physically checking the response of the float wheel.

Final readings of time and rainfall were recorded before leaving the site. After completion of t ${ }^{\circ}$ site visit but before leaving the area of the site, contact was made with the datalogger by using a cellular phono to assure that all phone connections were working properly.

Data were automatically retrieved daily by modem and phone line. Daily summary printouts available for inspection include: daily rainfall total, accumulated rainfall total since last service, and battery voltage. A location map of the raingages with corresponding rainfall totals for the previous day also is available. This allows for early identification and correction of problems.

Data were inspected for signs of drifting float wheels. This drift is easily spotted, and any 
Table 5. Containers, container treatment, and preservation procedures required for samples collected at the streamflow and water-quality study sites and analyzed by the Mecklenburg County Department of Environmental Protection Laboratory, July 1997 through September 1998

$\left[{ }^{\circ} \mathrm{C}\right.$, degrees Celsius; $\mathrm{mL}$, milliliter]

\begin{tabular}{|c|c|c|c|}
\hline $\begin{array}{l}\text { Compounds, elements, } \\
\text { or } \\
\text { properties analyzed }\end{array}$ & $\begin{array}{l}\text { Container } \\
\text { size }\end{array}$ & Container type & $\begin{array}{l}\text { Container treatment } \\
\quad \text { and } \\
\text { sample preservation }\end{array}$ \\
\hline \multicolumn{4}{|c|}{ Physical and chemical properties } \\
\hline Dissolved solids, residue at $180^{\circ} \mathrm{C}$ & $500 \mathrm{~mL}$ & $\begin{array}{l}\text { Polyethylene, red cap, } \\
\text { disposable }\end{array}$ & $\begin{array}{l}\text { Filter through a disposable capsule filter with } 0.45 \text {-micron pore } \\
\text { size; use filtered sample to rinse containers. }\end{array}$ \\
\hline $\begin{array}{l}\mathrm{pH} \text {, specific conductance, alkalinity, } \\
\text { chloride, sulfate }\end{array}$ & $1,000 \mathrm{~mL}$ & $\begin{array}{l}\text { Polyethylene, blue cap, } \\
\text { disposable }\end{array}$ & Unfiltered; use unfiltered sample to rinse containers. \\
\hline $\begin{array}{l}\text { Volatile suspended solids, total } \\
\text { suspended solids }\end{array}$ & $1,000 \mathrm{~mL}$ & $\begin{array}{l}\text { Polyethylene, blue cap, } \\
\text { disposable }\end{array}$ & Unfiltered; use unfiltered sample to rinse containers. \\
\hline Chemical oxygen demand & $250 \mathrm{~mL}$ & $\begin{array}{l}\text { Polyethylene, orange cap, } \\
\text { disposable }\end{array}$ & $\begin{array}{l}\text { Acidify collected sample with } 1.0 \mathrm{~mL} \mathrm{H}_{2} \mathrm{SO}_{4} \text {; chill and maintain } \\
\text { sample at } 4{ }^{\circ} \mathrm{C} \text {. }\end{array}$ \\
\hline Biochemical oxygen demand ${ }^{a}$ & $1,000 \mathrm{~mL}$ & $\begin{array}{l}\text { Polyethylene, blue cap, } \\
\text { disposable }\end{array}$ & Unfiltered, chill and maintain sample at $4{ }^{\circ} \mathrm{C}$. \\
\hline Coliform ${ }^{\mathrm{a}}$ & $200 \mathrm{~mL}$ & Glass & Sterile, chill and maintain sample at $4{ }^{\circ} \mathrm{C}$. \\
\hline \multicolumn{4}{|c|}{ Nutrients } \\
\hline Dissolved nutrients & $250 \mathrm{~mL}$ & $\begin{array}{l}\text { Polyethylene, green cap, } \\
\text { disposable }\end{array}$ & $\begin{array}{l}\text { Filter through a disposable capsule filter with } 0.45 \text {-micron pore } \\
\text { size; use filtered sample to rinse containers. Add } 1.0 \mathrm{~mL} \\
\mathrm{H}_{2} \mathrm{SO}_{4} \text {; chill and maintain sample at } 4^{\circ} \mathrm{C} \text {. }\end{array}$ \\
\hline Total nutrients & $250 \mathrm{~mL}$ & $\begin{array}{l}\text { Polyethylene, orange cap. } \\
\text { disposable }\end{array}$ & $\begin{array}{l}\text { Unfiltered; use unfiltered sample to rinse containers. Add } 1.0 \mathrm{~mL} \\
\mathrm{H}_{2} \mathrm{SO}_{4} \text {; chill and maintain sample at } 4{ }^{\circ} \mathrm{C} \text {. }\end{array}$ \\
\hline \multicolumn{4}{|c|}{ Metals and minor constituents } \\
\hline $\begin{array}{l}\text { As, } \mathrm{Se}, \mathrm{Hg}, \mathrm{Sb}, \mathrm{Be}, \mathrm{Cr}, \mathrm{Cu}, \mathrm{Cd}, \mathrm{Pb} \text {, } \\
\mathrm{Ni}, \mathrm{Ag}, \mathrm{Zn}\end{array}$ & $500 \mathrm{~mL}$ & $\begin{array}{l}\text { Polyethylene, acid rinsed, } \\
\text { white cap, disposable }\end{array}$ & $\begin{array}{l}\text { Unfiltered; use unfiltered sample to rinse containers. Add } \\
1.25 \mathrm{~mL} \text { of } \mathrm{HNO}_{3} \text {. }\end{array}$ \\
\hline \multicolumn{4}{|c|}{ Organic compounds } \\
\hline Oil and grease & $2,500 \mathrm{~mL}$ & Glass & Surface skim, unfiltered. Add $5.0 \mathrm{~mL} \mathrm{HCl}$. \\
\hline Pesticides and herbicides ${ }^{b}$ & $1 \mathrm{~L}$ & Glass, amber & $\begin{array}{l}\text { Bottle baked at } 450{ }^{\circ} \mathrm{C} \text {. Do not rinse container in field. Chill and } \\
\text { maintain sample at } 4{ }^{\circ} \mathrm{C} \text {. }\end{array}$ \\
\hline Total organic carbon ${ }^{b}$ & $125 \mathrm{~mL}$ & Glass, amber & $\begin{array}{l}\text { Bottle baked at } 450^{\circ} \mathrm{C} \text {. Do not rinse container in field. Chill and } \\
\text { maintain sample at } 4{ }^{\circ} \mathrm{C} \text {. }\end{array}$ \\
\hline Volatile organic compounds ${ }^{b}$ & $40 \mathrm{~mL}$ & Glass septum vial, amber & $\begin{array}{l}\text { Do not rinse container in field. Exclude all air bubble in sample } \\
\text { by completely filling vial. Acidify sample with } \mathrm{HCl} \text { to } \mathrm{pH}<2 \text {. } \\
\text { Protect sample from sunlight. Chill and maintain sample at } \\
4^{\circ} \mathrm{C} \text {. }\end{array}$ \\
\hline \multicolumn{4}{|c|}{ Sediment } \\
\hline Suspended sediment ${ }^{\mathrm{C}}$ & 1 pint & Glass & None. \\
\hline
\end{tabular}

${ }^{\text {a }}$ Analyses performed by the Mecklenburg County Department of Environmental Protection, May 1994 through September 1998.

${ }^{b}$ Analyses performed by the U.S. Geological Survey National Water-Quality Laboratory, May 1994 through September 1998.

${ }^{c}$ Analyses performed by U.S. Geological Survey Sediment Laboratories, May 1994 through September 1998. 
accumulated rainfall amounts resulting from the drift were removed from the database. Rainfall data during and after site visits were inspected and compared to field notes to assure proper readings. Daily totals were compared with data from surrounding sites to check for reasonable agreement.

During periods of sub-freezing air temperature or suspected frozen precipitation, data were inspected for signs of improper recording of precipitation. Incremental data for periods of apparent frozen precipitation were deleted from the database. When possible, daily or monthly totals were estimated on the basis of readings recorded as the snow and ice melted.

The mean and standard deviation of monthly rainfall totals for all of the gages in the network were computed. These statistics were used to identify stations that reported rainfall amounts significantly different from most of the other stations in the network. Monthly statistics for the period October 1993 to September 1998 were evaluated to determine if individual sites consistently reported rainfall amounts that were at least two standard deviations less than or greater than the monthly mean. For example, monthly totals available for the period of record at site 21 (CRN11) indicate that all of the monthly totals fell within two standard deviations of the mean for the respective month (fig. 3).

From July 1997 through September 1998, there were 690 station months of record. There were seven occurrences of monthly rainfall amounts at least two

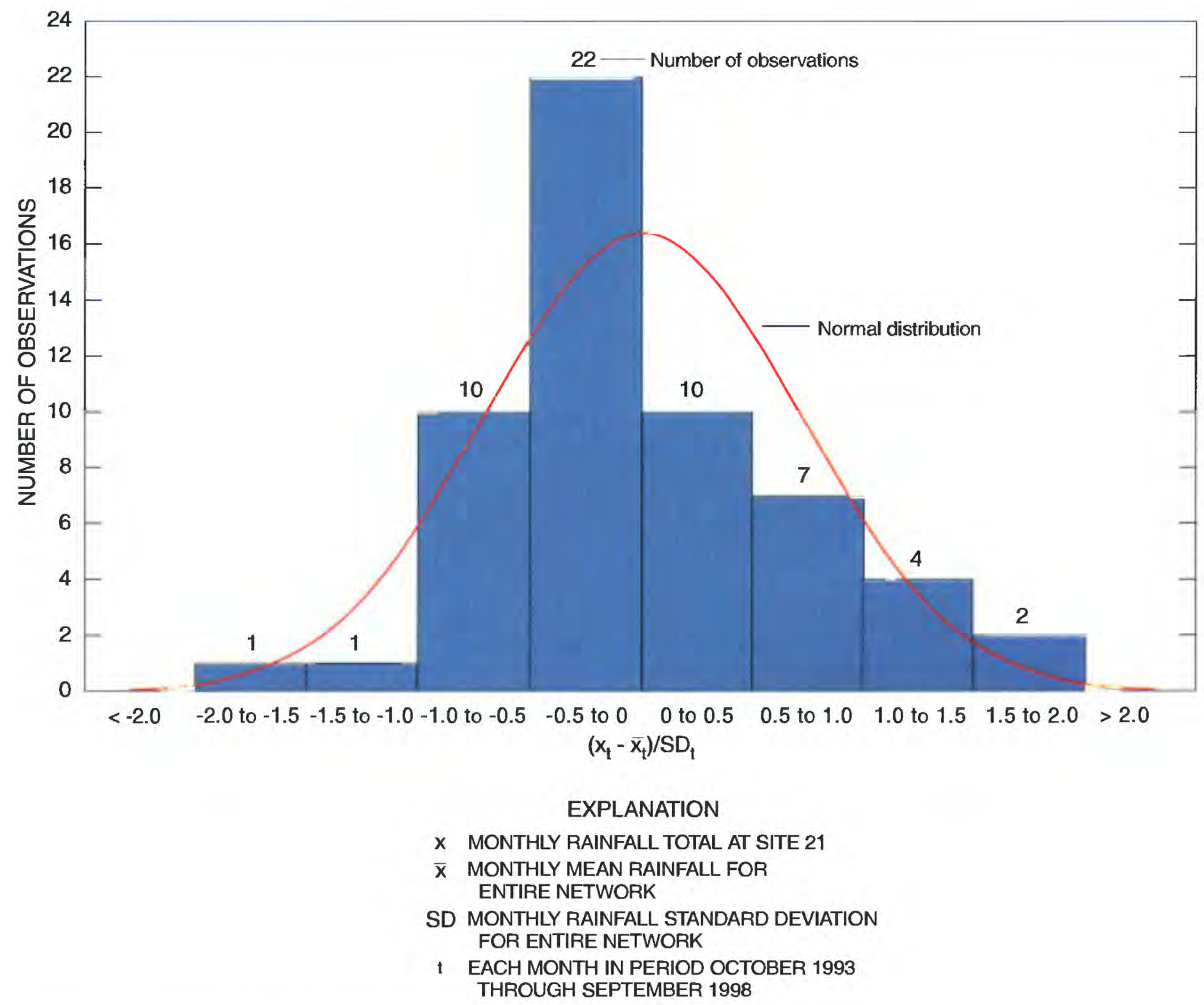

Figure 3. Statistical summary of monthly rainfall totals at site 21 (CRN11). 
standard deviations less than the monthly mean and 16 occurrences of monthly rainfall amounts at least two standard deviations greater than the monthly mean. In general, there did not appear to be a consistent over or under reporting of rainfall at any of the stations.

\section{Atmospheric Deposition Data}

Installation and operation of the automatic wet/dry samplers were in accordance with protocols established by the National Atmospheric Deposition Program (NADP) (Bigelow, 1984; Bigelow and Dossett, 1988), except samples were retrieved on Mondays rather than Tuesdays. Samplers were equipped with polycarbonate protective lids and Teflon-coated arms to prevent metal contamination of samples collected for metals and minor constituents.

Plastic sample-collection containers were prepared by washing with a nonphosphate detergent and soaking in a 5-percent hydrochloric acid solution as described by Horowitz and others (1994).

Equipment was assigned to each site to prevent possible cross-contamination between sites.

Quality-assurance samples composed approximately 20 percent of the samples analyzed. Equipment blanks using inorganic blank water provided by the USGS laboratory were prepared and analyzed for nutrients and metals and minor constituents to validate the cleaning procedures and to ensure that no contaminants were leaching from the sample-collection container. The quality-assurance blanks for nutrients were analyzed using the low-level automated-segment flow (ASF) method (Fishman, 1993), and the metals and minor constituents were analyzed using the Inductively Coupled Plasma-Mass Spectrometry (ICP-MS) method (Faires, 1993; Fishman, 1993; Struzeski and others, 1996). In addition, split samples were analyzed periodically for each site when sample volume allowed.

\section{Streamflow and Water-Quality Data}

Installation and operation of the continuousrecord gages were in accordance with USGS standards described in the Techniques of Water-Resources Investigations (TWRI) series of manuals published by the USGS. Streamflow was measured, and discharge record from stage was computed according to TWRI specifications.

Discharge measurements were made as needed at each site to develop stage-discharge relation curves.
Periodic check measurements of the rating were made when warranted by extreme or unstable confitions. Variable stage-discharge shifts were generally applied for periods when the absolute difference between the measured discharge and the expected discharge from the rating curve exceeded 5 percent.

Site visits routinely were conducted once every 4 to 6 weeks. Corrections to gage height record were made when the absolute difference between the reference gage observations and the water-level sensor exceeded $0.015 \mathrm{ft}$.

All sensors that were used for measuring water temperature and specific conductance were tested prior to being placed in the field. Thereafter, sens ors were routinely calibrated every 4 to 6 weeks. This procedure began with an initial check of the probe in its current state. The probes were then thoroughly cleaned and calibrated by using several standards. Adjustments to the sensor readings were applied over time and range, as needed, on the basis of calibration record`. Sensors were calibrated as soon as possible following sampling events to minimize any potential problems vith drift.

All data were automatically retrieved daily using a modem and phone line. Plots of stage and specific conductance for the 4 previous days were generated and reviewed daily. This allowed quick detertion and reconciliation of potential problems caused by instrumentation malfunctions.

The water temperature sensor was calibrated by using either an American Bureau of Standards mercury thermometer or an electronic thermistor that previously had been calibrated. The thermometer or thermistor was placed in the stream and allowed to equilibrate prior to disturbing the temperature sensor. All readings were recorded on the calibration sheet. The temperature sensor then was removed, cleaned, returned to the stream, and allowed to equilibrate. All readings were recorded a second time. As needed, adjustments to the data were time corrected on the basis of observed versus actual readings.

The specific conductance probe was calibrated by using five standards ranging from 20 to 500 microsiemens per centimeter at 25 degrees Celsius $\left(\mu \mathrm{S} / \mathrm{cm}\right.$ at $\left.25^{\circ} \mathrm{C}\right)$. The three standards that bast bracketed the typically observed specific conductance readings were used to apply any needed adjustments to the data. The probe was rinsed with deionized water, sequentially immersed in each standard, and allowed to equilibrate. Readings were recorded on the calibration sheet with the actual standard value. The probe then 
was cleaned thoroughly by using a special scrub brush and deionized water to remove any accumulation of dirt and algae. The probe readings then were checked once more using the same procedure. This allowed for adjustments to the data with time and range in the event of probe degradation.

All equipment that was used to collect waterquality samples was prepared by washing with a nonphosphate detergent and soaking in a 5-percent hydrochloric acid solution as described by Horowitz and others (1994). Equipment was assigned to each site to prevent cross-contamination between sites. Blanks were run on each piece of sampling equipment at each site on a yearly basis and analyzed for nutrients and metals and minor constituents by using inorganic blank water prepared by the USGS laboratory.

The Teflon-lined tubing on all automatic samplers was replaced with new tubing yearly. Between sampling events, this tubing was field-cleaned by using the above procedure. In addition, the tubing was rinsed with methanol, and the sample-collection bottles were baked at $450{ }^{\circ} \mathrm{C}$ prior to the spring collection of organic constituents. An equipment blank for the analysis of pesticides and herbicides was performed yearly at one randomly chosen site by using organic-free water purchased from a scientific supply company. Sample-collection volume was checked and calibrated at least yearly or when problems were suspected.

Sample-processing equipment assigned to each site was prepared with the cleaning procedures described above. Samples for the analysis of organic constituents were decanted directly from the glass collection bottles into the appropriate glass sample containers, then set aside and chilled. The remaining water was placed in a polycarbonate churn splitter to remove homogenous subsamples for inorganic and sediment analyses. Samples for the analysis of dissolved constituents were filtered using silicone tubing prepared with the previously described cleaning procedure and a disposable 0.45 -micron pore-size capsule filter (table 5). Prior to September 1995, samples collected for inorganic analyses were preserved by using USGS protocols as described by Horowitz and others (1994). Subsequently, samples collected for inorganic analyses were analyzed by the Mecklenburg County Department of Environmental Protection Laboratory and were preserved according to their requirements (table 5).
Churn splitters were field-cleaned with deionized water and 5-percent hydrochloric acid solution between each discrete sample collected at each site during an event. Blanks were processed on these field-cleaned churns for the analysis of nutrients and metals with a frequency of one blank per site per event to verify that field cleaning procedures were adequate. One ambient blank per event was collected and analyzed to check for contamination resulting from atmospheric deposition in the USGS Charlote Field Office sample processing area.

In addition to churn blanks and ambient blanks, other quality-assurance samples that were collected include split, duplicate, and blank samples for all constituents analyzed. The quality-assurance blanks for nutrients were analyzed using the low-level ASF method (Fishman, 1993), and the metals and minor constituents were analyzed using the ICP-MS method (Faires, 1993; Fishman, 1993; Struzeski and others 1996).

\section{LABORATORY ANALYSES}

Samples collected during May 1994 through August 1995 were analyzed by the USGS National Water Quality Laboratory (NWQL) in Denver, Colo. The analytical methods that were used by the NWQL are documented in Wershaw and others (1987), Britton and Greeson (1989), Fishman and Friedman (1989), Fishman (1993), Rose and Schroeder (1995), Zaugg and others (1995), and Werner and others (1996). Beginning in September 1995, samples collected for inorganic constituents were analyzed by the Mecklenburg County Department of Environmental Protection Laboratory. Analytical procedure: and method detection limits for the Mecklenburg County Department of Environmental Protection Laboratory are listed in table 9 (p. 27-36). The NWQL continued to analyze samples for organic constituents. All quality-assurance blanks were analyzed by the NWQL. Suspended-sediment concentrations were determined during the study period by the USGS sediment laboratories in Raleigh, N.C., Baton Rouge, La., and Louisville, Ky., by using methods and proce tures documented by Guy (1969).

Method detection limits (MDL's) for a particular compound and analytical method are determined statistically from laboratory method performance tests. MDL's for the 88 dissolved pesticide organic compounds (table 9) were revised by the NWQL on April 15, 1996, on the basis of detailed meth od 
performance tests. MDL's generally were lowered by one-half to an order of magnitude from values previously published in Robinson and others (1996). The USGS water-quality database was updated in late 1996. Hence, concentrations of dissolved organic compounds reported in statistical summary tables may be different from previously published values.

\section{PRECIPITATION AND HYDROLOGIC DATA}

Precipitation and hydrologic data collected from 46 precipitation sites and 6 stream sites during July 1997 through September 1998 are discussed in the following sections. Rainfall and streamflow

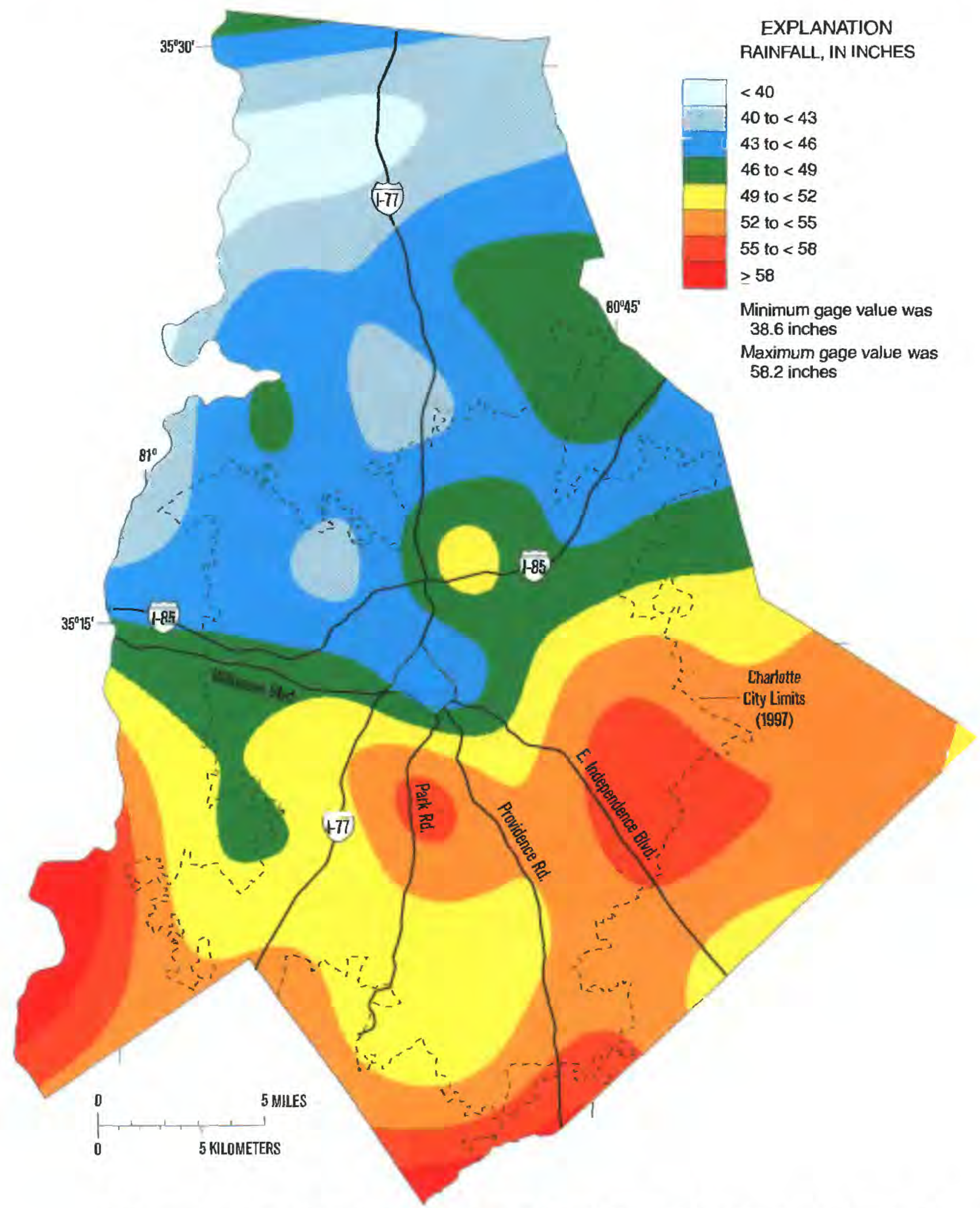

Figure 4. Annual rainfall distribution in Charlotte and Mecklenburg County for October 1, 1997, through September 30, 1998. characteristics for monitored storms at the stream sites are summarized, and atmospheric deposition data collected during March 1997 through March 1998 are presented.

\section{Precipitation Data}

Daily and monthly rainfall totals at the 46 rainfall sites (fig. 1) are presented in tables 10-55 (p. 37-82). The distribution of annual rainfall in Mecklenburg County, based on data from the 46 rainfall sites for October 1, 1997, through September 30,1998 , ranged from approximately $39 \mathrm{in}$. to $58 \mathrm{in}$. (fig. 4). For the storm of July 22-24, 1997, rainfall 
totals in Mecklenburg County ranged from approximately 4 in. to 13 in. (fig. 5), and the recurrence intervals for a 24-hour rainfall duration ranged from less than 1 year to greater than 100 years on the basis of data from 45 raingages (fig. 6).
These recurrence intervals were derived from Hershfield (1961) and are published in two USGS Fact Sheets-FS-052-97 (Hazell and Bales, 1997) and FS-036-98 (Robinson, Hazell, and Young, 1998).

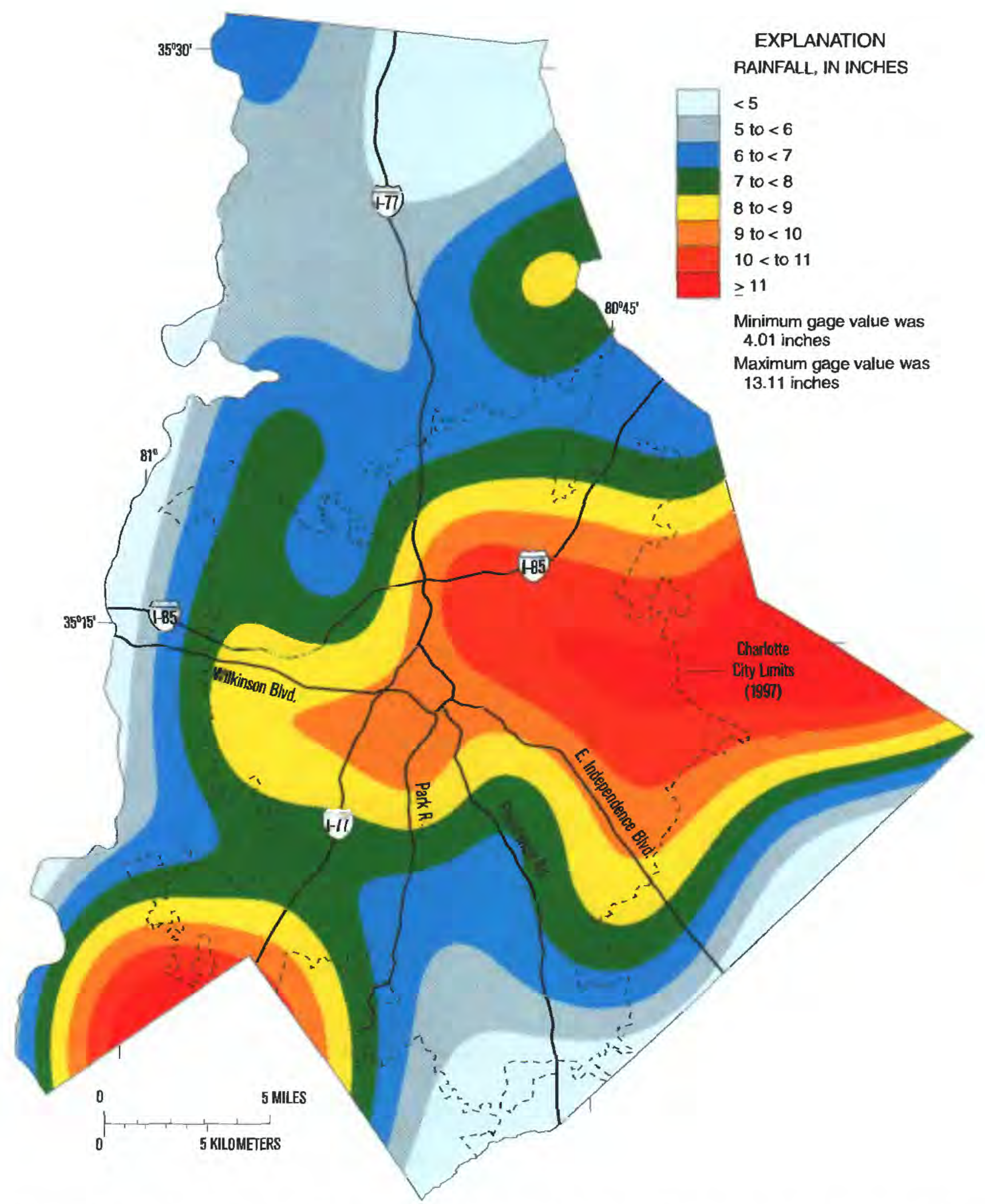

Figure 5. Rainfall distribution in Charlotte and Mecklenburg County for July 22-24, 1997 (from Robinson, Hazell, and Young, 1998). 


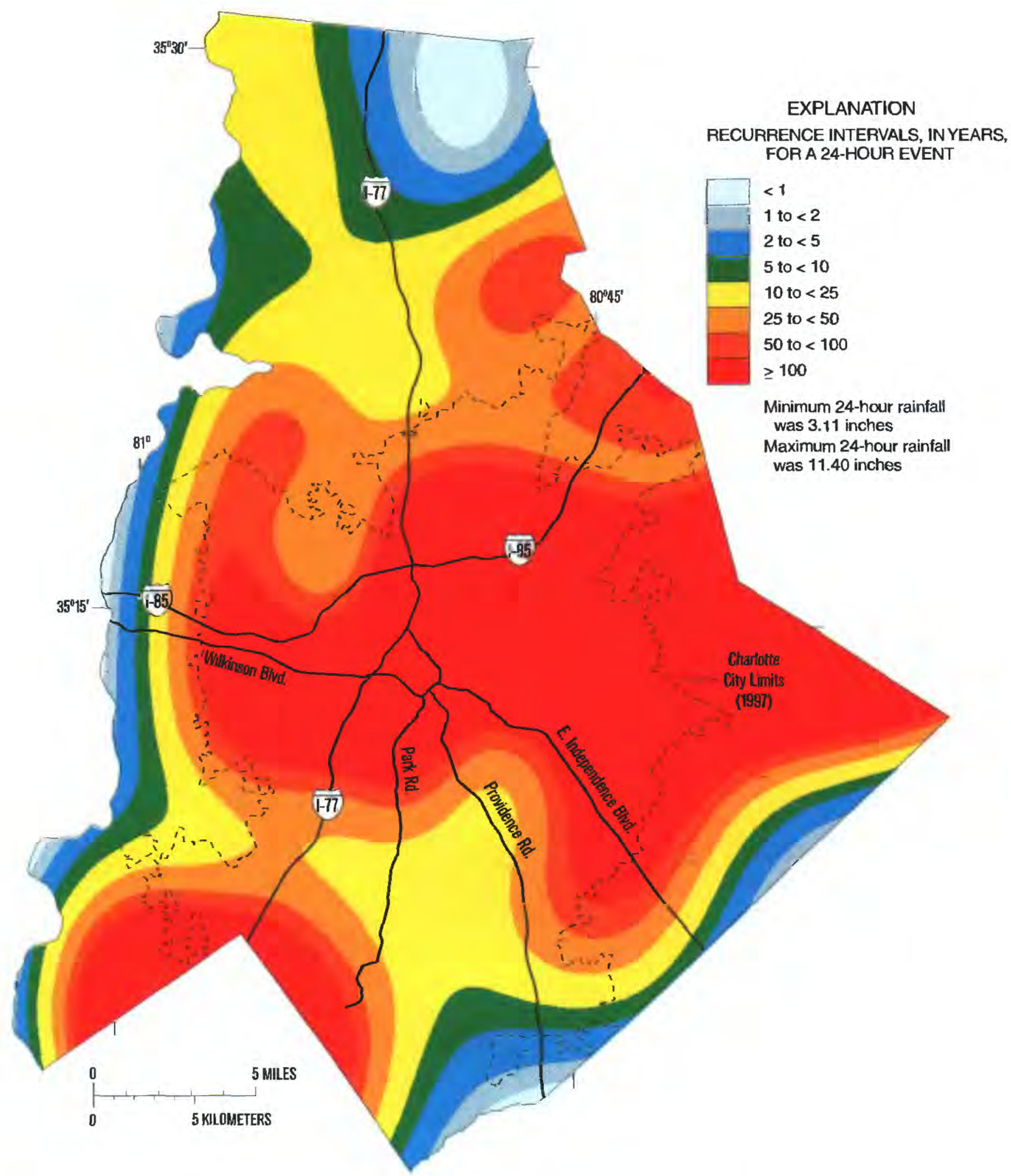

Figure 6. Rainfall recurrence intervals for peak 24-hour durations in Charlotte and Mecklenburg County for July 22-24, 1997 (from Robinson, Hazell, and Young, 1998). 


\section{Atmospheric Deposition Data}

Statistical summary tables of approximately 25 chemical constituents include measurements of precipitation, physical and chemical properties, chloride and sulfate, nutrients, and concentrations of total metals (tables 56-58, p. 83-85). Data for individual atmospheric deposition samples collected during March 1997 through March 1998 are provided in tables 59-61 (p. 86-94). Weekly rainfall amounts measured in each atmospheric deposition sampler were compared to rainfall amounts recorded at nearby raingages. The raingage at site 37 was $600 \mathrm{ft}$ from the sampler; at site 42 , the raingage was $1 \mathrm{mi}$ from the sampler; and at site 43 , the sampler and raingage were collocated. For each sample collected, two values are shown for the precipitation total-(1) parameter code 00045 is the total weekly precipitation recorded by the nearest recording raingage and (2) parameter code
00046 is the total weekly precipitation, in inches equivalent, collected in the wet/dry sampler.

Statistical summaries were prepared by using programs developed by the USGS (Maddy and others, 1992). If the total number of observations above and below the method detection limit is greater than 1 but less than or equal to 5 , only the maximum and minimum values are reported in the tables. If only one observation is available, the value is reported as the maximum value. Percentiles are not shown if sample sizes were small or if a large proportion of the data was censored (less than laboratory reporting level). Statistical summaries are for the period of record, March 1997 through March 1998.

Analytical results of blank samples were evaluated to ensure that atmospheric deposition samples were not being contaminated by the samplecollection process. Statistical summaries of atmospheric deposition blank data (table 6) indicate that 8 of the 12 constituents of interest were not

Table 6. Summany of atmospheric deposition blank sample results compared to minimum and median concentrations for atmospheric deposition environmental samples, March 1997 through March 1998

\begin{tabular}{|c|c|c|c|c|c|c|c|c|c|c|}
\hline \multirow{3}{*}{ Constituent } & \multirow{3}{*}{$\begin{array}{l}\text { Biank } \\
\text { detection } \\
\text { limit }\end{array}$} & \multicolumn{3}{|c|}{$\begin{array}{l}\text { Atmospheric deposition sampler } \\
\text { blanks }\end{array}$} & \multicolumn{3}{|c|}{$\begin{array}{l}\text { Atmospheric deposition bucket } \\
\text { bianks }\end{array}$} & \multirow{2}{*}{\multicolumn{3}{|c|}{$\begin{array}{c}\begin{array}{c}\text { Atmospheric deposition } \\
\text { environmentai sampies }\end{array} \\
\begin{array}{c}\text { Minimum (median) } \\
\text { concentration }\end{array}\end{array}$}} \\
\hline & & \multirow{2}{*}{$\begin{array}{l}\text { No. } \\
\text { of } \\
\text { bianks }\end{array}$} & \multirow{2}{*}{$\begin{array}{c}\text { No. } \\
\text { of } \\
\text { detections }\end{array}$} & \multirow{2}{*}{$\begin{array}{l}\text { Maximum } \\
\text { concen- } \\
\text { tration }\end{array}$} & \multirow{2}{*}{$\begin{array}{l}\text { No. } \\
\text { of } \\
\text { blanks }\end{array}$} & \multirow{2}{*}{$\begin{array}{c}\text { No. } \\
\text { of } \\
\text { detections }\end{array}$} & \multirow{2}{*}{$\begin{array}{l}\text { Maximum } \\
\text { concen- } \\
\text { tration }\end{array}$} & & & \\
\hline & & & & & & & & CSW04 & CSW06 & csw07 \\
\hline Ammonia (mg/L as $\mathrm{N})$ & 0.002 & 7 & 1 & 0.003 & 2 & 1 & 0.006 & $\begin{array}{l}<0.015 \\
(0.070)\end{array}$ & $\begin{array}{l}0.015 \\
(0.085)\end{array}$ & $\begin{array}{l}<0.015 \\
(0.070)\end{array}$ \\
\hline $\mathrm{NO}_{2}+\mathrm{NO}_{3}(\mathrm{mg} / \mathrm{L}$ as $\mathrm{N})$ & .005 & 7 & 0 & $<.005$ & 2 & 0 & $<.005$ & $\begin{array}{l}0.070 \\
(0.170)\end{array}$ & $\begin{array}{l}0.060 \\
(0.190)\end{array}$ & $\begin{array}{l}0.050 \\
(0.175)\end{array}$ \\
\hline Orthophosphorus (mg/L as P) & .001 & 7 & 3 & .002 & 2 & 2 & .002 & $\begin{array}{l}<0.010 \\
(<0.010)\end{array}$ & $\begin{array}{l}<0.010 \\
(<0.010)\end{array}$ & $\begin{array}{l}<0.010 \\
(<0.010)\end{array}$ \\
\hline Beryllium $(\mu \mathrm{g} / \mathrm{L})$ & .20 & 7 & 0 & $<.20$ & 2 & 0 & $<.20$ & $\begin{array}{l}<10 \\
(<10)\end{array}$ & $\begin{array}{l}<10 \\
(<10)\end{array}$ & $\begin{array}{l}<10 \\
(<10)\end{array}$ \\
\hline Cadmium $(\mu \mathrm{g} / \mathrm{L})$ & .30 & 7 & 0 & $<.30$ & 2 & 0 & $<.30$ & $\begin{array}{l}<1.0 \\
(<1.0)\end{array}$ & $\begin{array}{l}<1.0 \\
(<1.0)\end{array}$ & $\begin{array}{l}<1.0 \\
(<1.0)\end{array}$ \\
\hline Chromium $(\mu \mathrm{g} / \mathrm{L})$ & .20 & 7 & 0 & $<20$ & 2 & 0 & $<.20$ & $\begin{array}{l}<1.0 \\
(<1.0)\end{array}$ & $\begin{array}{l}<1.0 \\
(<1.0)\end{array}$ & $\begin{array}{l}<1.0 \\
(<1.0)\end{array}$ \\
\hline Copper $(\mu \mathrm{g} / \mathrm{L})$ & .20 & 7 & 2 & .37 & 2 & 0 & $<.20$ & $\begin{array}{l}<1.0 \\
(<1.0)\end{array}$ & $\begin{array}{l}<1.0 \\
(<1.0)\end{array}$ & $\begin{array}{l}<1.0 \\
(<1.0)\end{array}$ \\
\hline Lead $(\mu \mathrm{g} / \mathrm{L})$ & .30 & 7 & 0 & $<.30$ & 2 & 0 & $<.30$ & $\begin{array}{l}<1.0 \\
(<1.0)\end{array}$ & $\begin{array}{l}<1.0 \\
(<1.0)\end{array}$ & $\begin{array}{l}<1.0 \\
(<1.0)\end{array}$ \\
\hline Nickel $(\mu \mathrm{g} / \mathrm{L})$ & .50 & 7 & 0 & $<.50$ & 2 & 0 & $<.50$ & $\begin{array}{l}<1.0 \\
(<1.0)\end{array}$ & $\begin{array}{l}<1.0 \\
(<1.0)\end{array}$ & $\begin{array}{l}<1.0 \\
(<1.0)\end{array}$ \\
\hline Silver $(\mu \mathrm{g} / \mathrm{L})$ & .20 & 7 & 0 & $<.20$ & 2 & 0 & $<.20$ & $\begin{array}{l}<1.0 \\
(<1.0)\end{array}$ & $\begin{array}{l}<1.0 \\
(<1.0)\end{array}$ & $\begin{array}{l}<1.0 \\
(<1.0)\end{array}$ \\
\hline Zinc $(\mu \mathrm{g} / \mathrm{L})$ & .50 & 7 & 2 & 3.1 & 2 & 1 & 4.4 & $\begin{array}{l}<10 \\
(<10)\end{array}$ & $\begin{array}{l}<10 \\
(10)\end{array}$ & $\begin{array}{l}<10 \\
(<10)\end{array}$ \\
\hline Antimony $(\mu \mathrm{g} / \mathrm{L})$ & .20 & 7 & 0 & $<.20$ & 2 & 0 & $<.20$ & $\begin{array}{l}<1.0 \\
(<1.0)\end{array}$ & $\begin{array}{l}<1.0 \\
(<1.0)\end{array}$ & $\begin{array}{l}<1.0 \\
(<1.0)\end{array}$ \\
\hline
\end{tabular}


detected in any of the blank samples. These results suggest no contribution of these compounds to atmospheric deposition samples from the samplecollection process. Maximum concentrations of compounds detected in at least one blank sample (ammonia, orthophosphorus, copper, and zinc) were from one-half to 12 times less than the minimum concentration detected in any atmospheric deposition sample. These blank results suggest that the potential for contamination of the atmospheric deposition samples from the sample-collection process was negligible and will not affect interpretation of atmospheric deposition sample data.

\section{Streamflow Data}

Streamflow statistics for December 1993 through September 1998 are presented in table 62 (p. 95). Daily mean discharge data at the six streamflow sites (fig. 1) are presented in tables 63-68 (p. 96-101). During periods of missing record, a daily mean discharge was estimated on the basis of rainfall and computed streamflow for other gages in the area.

\section{Water-Quality Data}

Continuous specific conductance and water temperature data were collected at 5-minute intervals at the streamflow sites. These data are available from the USGS District Office in Raleigh, N.C. Continuous specific conductance and water temperature statistics for the six streamflow sites are presented in table 69 (p. 102). Statistical summary tables of approximately 250 chemical constituents include measurements of fecal coliform bacteria, physical and chemical properties, nutrients, concentrations of metals and minor constituents, oil and grease, suspended sediment, and organic compounds in water (tables 70-75, p. 103-126). Statistical summaries are for the period of record, May 1994 through September 1998, unless otherwise noted.

The instantaneous discharges reported in the statistical summary tables and discrete sample tables are associated with individual water-quality samplecollection dates. The sampling period for collection of discrete water-quality samples was July 1997 through September 1998 (tables 76-81, p. 127-138). These data include measurements of fecal coliform bacteria, physical and chemical properties, nutrients, metals and minor constituents, oil and grease, total organic carbon, and suspended sediment. The organic compounds were not included in these tables because of the small number of samples with concentrations greater than the MDL and the large number of organic constituents.

Samples were collected on May 20, 1938, at four sites (sites 41,42, 43, and 39) during a period of no rainfall runoff to determine base-flow levels of selected constituents. These data are included in the statistical summaries. Samples were not collected at sites 37 or 40 on May 20, 1998. because of zero flow conditions.

Rainfall and streamflow characteristics for the monitored storms at the stream sites are presented in tables 82-87 (p. 139-144). Total accumulated rainfall is reported as the total rainfall for the duration of each storm event.

Analytical results of blank samp'es were evaluated throughout the project to ensire that stream environmental samples were not being contaminated by the overall sample-collection process. Field equipment blanks were subjected to all aspects of sample collection, field processing, preservation, transportation. and laboratory handling, the same as an environmental sample. Churn blank samples were mixed and separated using field-cleane $\mathrm{d}$ churns in the same manner as was used for environmental samples. Churn blank samples were collected to verify that procedures that were used to field-clean the churns were adequate. Ambient blanks were prepared with the same type of bottle that was used for environmental samples, leaving the blanks exposed to ambient conditions during sample processing. The blank samples were analyzed for nutrients and trace metals by using low-level analytical methods (Faires, 1993; Fishman, 1993; Struzeski and others, 1996), with detection limits up to an order of magnitude less than detection limits for stream environmen ${ }^{+}$al samples.

Statistical summaries of inorganic blank data (table 7) indicated that four of the metals analyzed (beryllium, cadmium, silver, and antimony) were not detected in any of the 230 blank samples. These results suggest no contribution of these compcunds to stream environmental samples from the data-collection process. The 95 th-percentile blank con entration of lead was less than the blank detection I'mit. Median stream environmental sample concentrations were 3 to 310 times greater than the 95 th-percentile inorganic blank concentrations (table 8 ), depending on the constituent. In addition, the 95th-percentile values for all blank samples were less than the minimum 


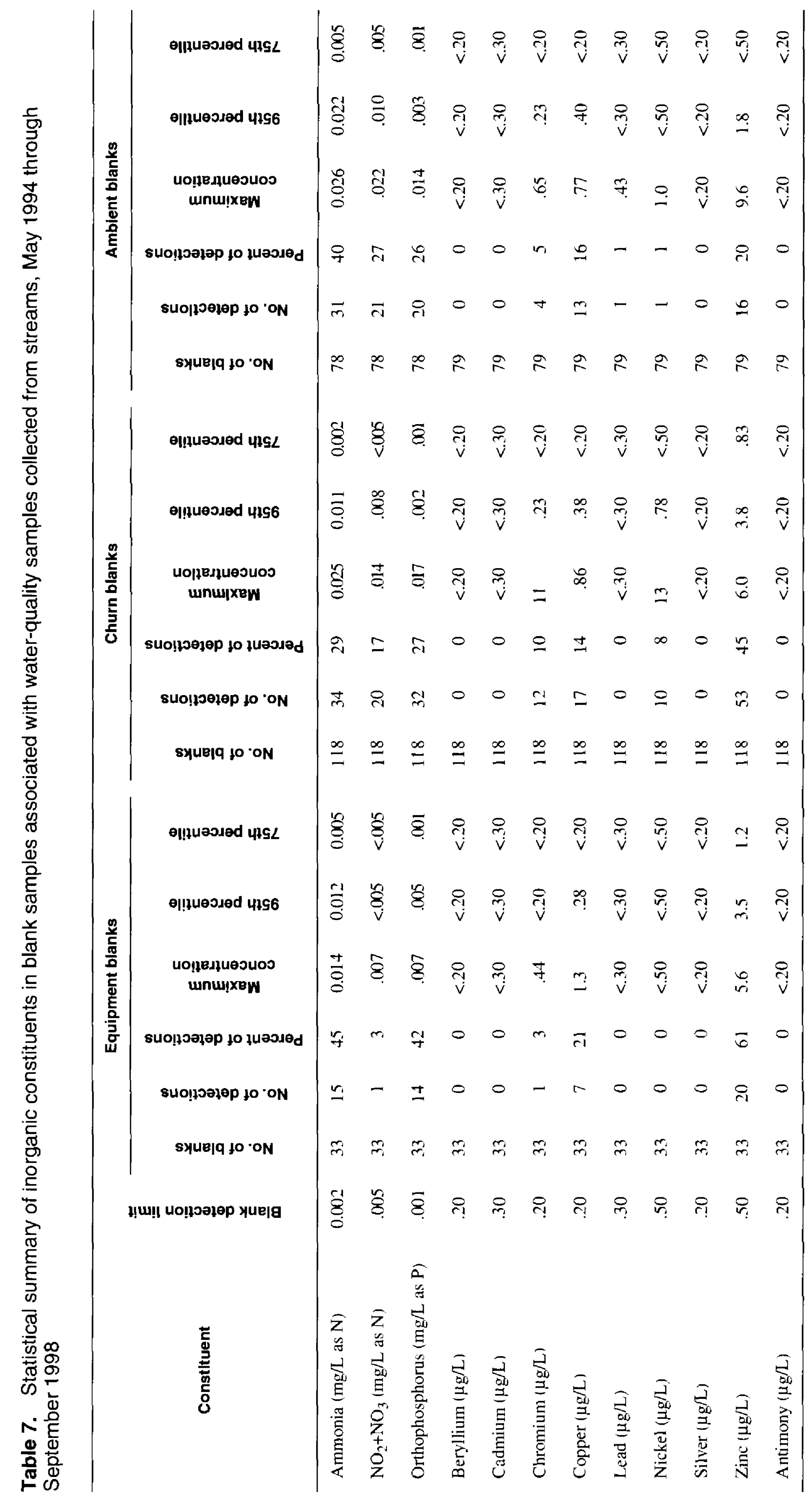


concentrations detected in any stream environmental sample for all constituents except ammonia (table 8). Blank sample concentrations greater than the 95th percentile indicate very low levels of sample contamination (fig. 7). Careful comparison of the blank data with associated stream environmental samples indicated minimal impact of contamination on the stream sample results.

Sample collection procedures were modified, as necessary, based on blank sample results. For example, from May 1994 through September 1998, the maximum ammonia concentration in ambient blanks was $0.026 \mathrm{mg} / \mathrm{L}$, with a 95 th-percentile concentration of $0.022 \mathrm{mg} / \mathrm{L}$ (table 7; fig. 7). Prior to July 1995 , water-quality samples were processed (split and preserved) at each sampling site. Subsequently, waterquality samples were processed in the USGS Charlotte
Field Office. Following this procedural change, ammonia concentrations detected in ambient blanks decreased considerably (maximum $0.008 \mathrm{mg} / \mathrm{L}$; 95 th percentile $0.005 \mathrm{mg} / \mathrm{L}$ ). The change in processing location, based on evaluation of ambient blank data, dramatically lowered the potential for introduction of ammonia to the environmental samples from ambient conditions.

In summary, blank sample results suggest that the potential for nutrient and metals contamination of the stream environmental samples from the overall sample-collection process was negligible and will not affect interpretation of the environmental sample data. Evaluation of equipment blank organic constituent results indicated no contamination of stream environmental samples from the sample-collection process.

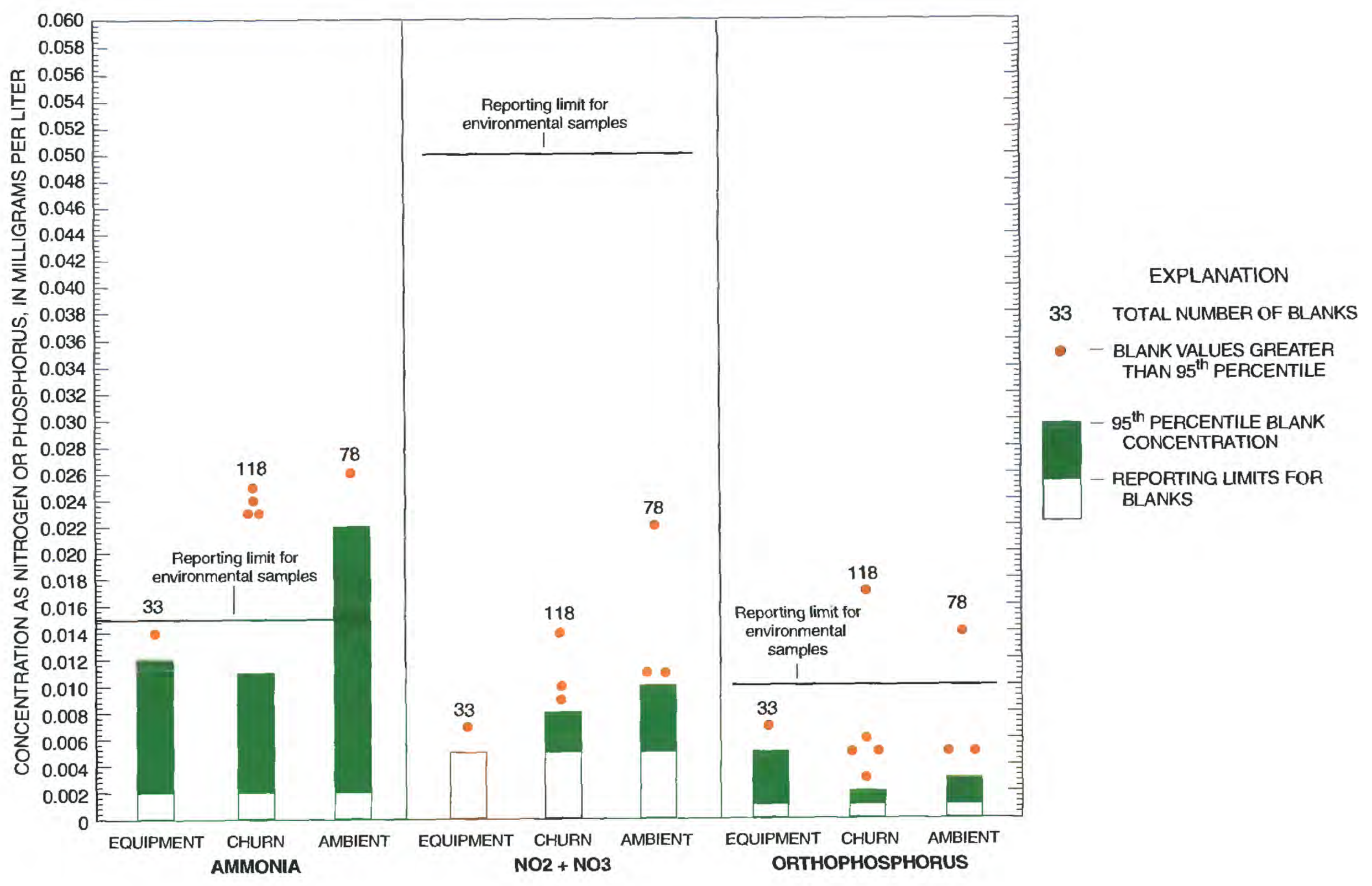

Figure 7. Distribution of concentrations of selected constituents measured in equipment, churn, and ambient blank samples associated with stream environmental samples. 


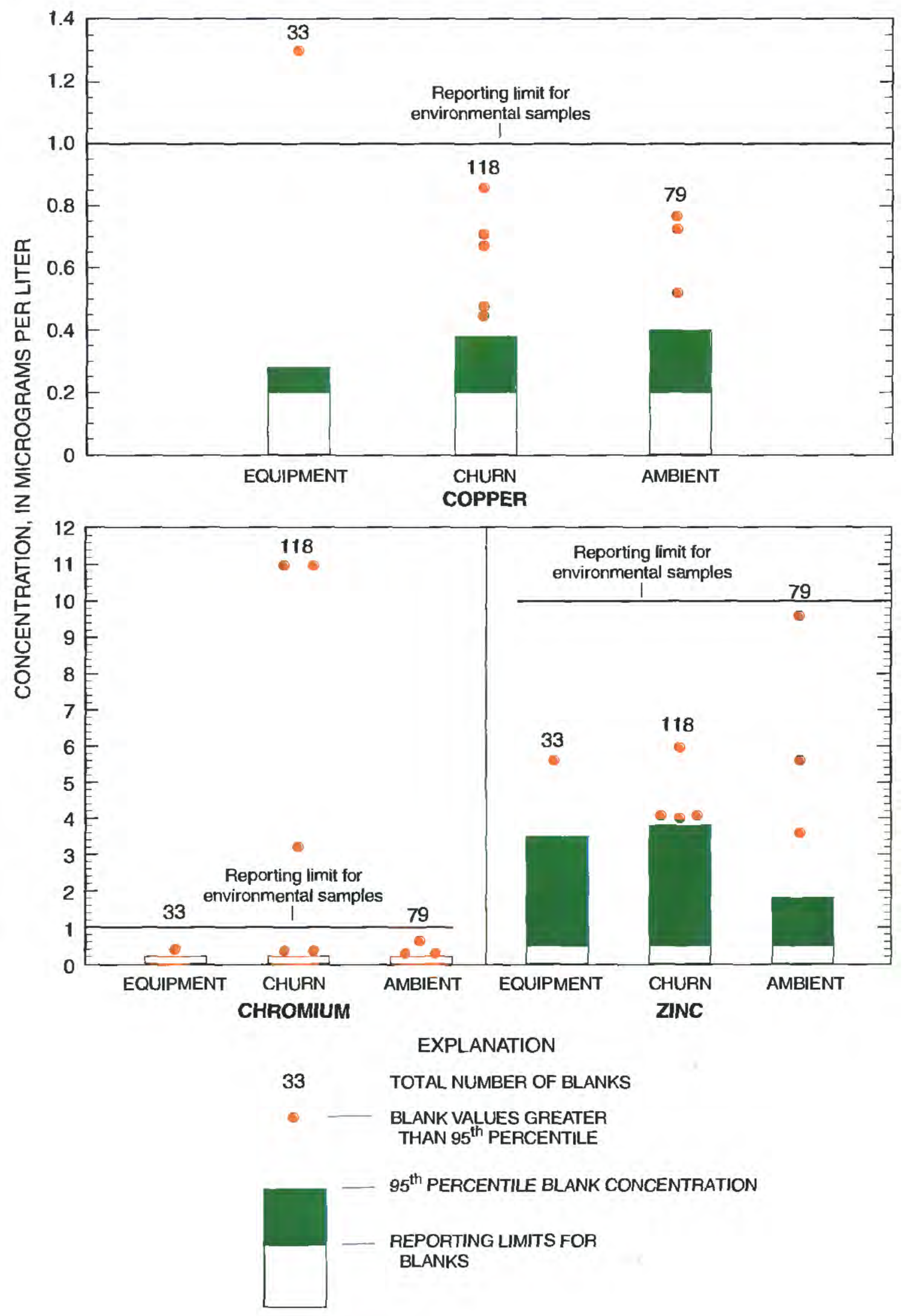

Figure 7. (Continued) Distribution of concentrations of selected constituents measured in equipment, churn, and ambient blank samples associated with stream environmental samples. 


\section{SELECTED REFERENCES}

Bales, J.D., and Giorgino, M.J., 1998, Lake Hickory, North Carolina-Analysis of ambient conditions and simulation of hydrodynamics, constituent transport, and water-quality characteristics, 1993-94: U.S. Geological Survey Water-Resources Investigations Report 98-4149, 62 p.

Bigelow, D.S., 1984, Instruction manual, NADP/NTN site selection and installation: National Atmospheric Deposition Program, 23 p.

Bigelow, D.S., and Dossett, S.R., 1988, Instruction manual, NADP/NTN site operation: National Atmospheric Deposition Program, $40 \mathrm{p}$.

Britton, L.J., and Greeson, P.E., eds., 1989, Methods for collection and analyses of aquatic biological and microbiological samples: U.S. Geological Survey Techniques of Water-Resources Investigations, book 5 . chap. A4, 363 p.

Edwards, T.K., and Glysson, G.D., 1988, Field methods for measurements of fluvial sediment: U.S. Geological Survey Open-File Report 86-531, 118 p.

Faires, L.M., 1993, Methods of analysis by the U.S. Geological Survey National Water Quality Laboratory-Determination of metals in water by inductively coupled plasma-mass spectrometry: U.S. Geological Survey Open-File Report 92-634, 28 p.

Fishman, M.J., ed., 1993, Methods of analysis by the U.S. Geological Survey National Water Quality Laboratory-Determination of inorganic and organic constituents in water and fluvial sediments: U.S. Geological Survey Open-File Report 93-125, 217 p.

Fishman, M.J., and Friedman, L.C., eds.. 1989, Methods for determination of inorganic substances in water and fluvial sediments: U.S. Geological Survey Techniques of Water-Resources Investigations, book 5, chap. A1, $545 \mathrm{p}$.

Giorgino, M.J., and Bales, J.D., 1997, Rhodhiss Lake, North Carolina-Analysis of ambient conditions and simulation of hydrodynamics, constituent transport, and water-quality characteristics, 1993-94: U.S. Geological Survey Water-Resources Investigations Report 97-4131, 62 p.

Guy, H.P., 1969, Laboratory theory and methods for sediment analyses: U.S. Geological Survey Techniques of Water-Resources Investigations, book 5, chap. C1, $58 \mathrm{p}$.

Hazell, W.F., and Bales, J.D., 1997, Real-time rainfall measurement in the city of Charlotte and Mecklenburg County, North Carolina: U.S. Geological Survey Fact Sheet FS-052-97, 4 p.

Hershfield. D.M., 1961, Rainfall frequency atlas of the United States: Washington, D.C., Department of Commerce, Weather Bureau, Technical Paper No. 40, $114 \mathrm{p}$.
Horowitz, A.J., Demas, C.R., Fitzgerald, K.K., Miller, T.L., and Rickert, D.A., 1994, U.S. Geological Survey protocol for the collections and processing of surfacewater samples for the subsequent determination of inorganic constituents in filtered water: U.S. Geological Survey Open-File Report 94-539, 57 p.

Hughes, W.B., 1994, National Water-Qua'ity Assessment Program-The Santee River Basin and coastal drainages, N.C. and S.C.: U.S. Geolrgical Survey Fact Sheet 94-010, $2 \mathrm{p}$.

Jaynes, M.L., 1994, Hydrologic, water-quality, and meteorologic data from selected sites in the upper Catawba River Basin, North Carolina, January 1993 through March 1994: U.S. Geological Survey OpenFile Report 94-509, 76 p.

Legrand, H.E., and Mundorff, M.J., 1952, Geology and groundwater in the Charlotte area, North Carolina: North Carolina Department of Conservation and Development, Bulletin 63, 88 p.

Maddy, D.V., Lopp, L.E., Jackson, D.L., Coupe, R.H., Schertz, T.L., and Garcia, K.T., 1992, National water information systenı user's manual, v. 2, chap. 2, waterquality system, p. 5-14 - 5-17.

McCachren, C.M., 1980, Soil survey of Mecklenburg County, North Carolina: U.S. Department of Agriculture, Soil Conservation Servise, 97 p.

Outlaw, G.S., Hoos, A.B., and Pankey, J.T., 1994, Rainfall, streamflow, and water-quality data for five small watersheds, Nashville, Tenn., 1990-92: U.S. Geological Survey Open-File Report 94-68, 43 p.

Ragland, B.C., Smith, D.G., Barker, R.G. and Robinson, J.B., 1999, Water resources data, Noth Carolina. water year 1998-Volume 1. Surface-water records: U.S. Geological Survey Water-Data Report NC-98-1, $708 \mathrm{p}$.

Rantz, S.E., and others, 1982, Measurements and computation of streamflow-Volume 1, Measurement of stage and discharge, and Volume 2, Computation of discharge: U.S. Geological Survey Water-Supply Paper $2175,631 \mathrm{p}$.

Robinson, J.B., Hazell, W.F., and Garrett, R.G., 1996, Precipitation, streamflow, and water-quality data from selected sites in the city of Charlotte and Mecklenburg County, North Carolina, 1993-95: U.S. Geological Survey Open-File Report 96-150, 135 p.

1998, Precipitation, streamflow, and water-quality data from selected sites in the city of Charlotte and Mecklenburg County, North Carolina, 1995-97: U.S. Geological Survey Open-File Report 98-67, $220 \mathrm{p}$.

Robinson, J.B., Hazell, W.F., and Young, W.S., 1998, Effects of August 1995 and July 1997 storm in the city of Charlotte and Mecklenburg County, North Carolina: U.S. Geological Survey Fact Sheet FS-036-98, 6 p. 
Rose, D.L., and Schroeder, M.P., 1995, Methods of analysis by the U.S. Geological Survey National Water Quality Laboratory-Determination of volatile organic compounds in water by purge and trap capillary gas chromatography/mass spectrometry: U.S. Geological Survey Open-File Report 94-708, 26 p.

Sarver, K.M., and Steiner, B.C., 1998, Hydrologic and water-quality data from Mountain Island Lake, North Carolina, 1994-97: U.S. Geological Survey Open-File Report 98-549, 165 p.

Stanley, D.W., 1994, An evaluation of pollutant removal by a demonstration of an urban stormwater detention pond: Institute for Coastal and Marine Resources, and Department of Biology, East Carolina University, $112 \mathrm{p}$.

Struzeski, T.M., DeGiacomo, W.J., and Zayhowski, E.J., 1996, Methods of analysis by the U.S. Geological Survey National Water Quality LaboratoryDetermination of dissolved aluminum and boron in water by inductively coupled plasma-atomic emission spectrometry: U.S. Geological Survey Open-File Report 96-149, 17 p.

Timme, P.J., 1995, National Water Quality Laboratory 1995 services catalog: U.S. Geological Survey Open-File Report 95-352, $120 \mathrm{p}$.
U.S. Environmental Protection Agency, 1990, National pollutant regulations for storm water discharges: U.S. Federal Register, v. 55, no. 222, p. 47990 - 48091.

Ward, J.R., and Harr, C.A., eds., 1990, Methods for collection and processing of surface-water ard bedmaterial samples for physical and chemical analyses: U.S. Geological Survey Open-File Report 90-140, 71 p. Werner, S.L., Burkhardt, M.R., and DeRusseau, S.N., 1996, Methods of analysis by the U.S. Geological Survey National Water Quality Laboratory-Detern ination of pesticides in water by Carbopak-B solid-phase extraction and high-performance liquid chromatography: U.S. Geological Survey Open-File Report 96-216, $42 \mathrm{p}$.

Wershaw, R.L., Fishman, M.J., Grabbe, R.R., and Lowe, L.E., 1987, Methods for the determination of organic substances in water and fluvial sediments: U.S. Geological Survey Techniques of Water-Resources Investigations, book 5, chap. A3, $80 \mathrm{p}$.

Zaugg, S.D., Sandstrom, M.W., Smith, S.G., and Fehlberg, K.M., 1995, Methods of analysis by the U.S. Geological Survey National Water Quality Laboratory-Determination of pesticides in water by C-18 solid-phase extraction and capillary-column gas chromatography/mass spectrometry with selected-ion monitoring: U.S. Geological Survey Open-File Report 95-181, $60 \mathrm{p}$. 


\section{ABBREVIATIONS USED IN DATA TABLES 9-87}

ACCUM accumulation

COLS. PER 100 ML, COLS./100 ML colonies per 100 milliliters

CFSM cubic feet per second per square mile

$\mathrm{ft}^{3} / \mathrm{s} \quad$ cubic feet per second

${ }^{\circ} \mathrm{C}$, DEG. C degrees Celsius

IN., IN inch

in./wk, IN./WK inch per week

INST. instantaneous

MAX maximum

$\mu \mathrm{g} / \mathrm{L}, \mathrm{UG} / \mathrm{L} \quad$ micrograms per liter

$\mu \mathrm{S} / \mathrm{cm}, \mathrm{US} / \mathrm{CM}$ microsiemens per centimeter

$\mathrm{mg} / \mathrm{L}, \mathrm{MG} / \mathrm{L}$ milligrams per liter

$\mathrm{mi}^{2} \quad$ square mile

$\mathrm{mL}$ milliliter

MIN minimum

RECOV. recoverable

$>$ greater than

$\geq \quad$ greater than or equal to

$<$ less than

$\leq \quad$ less than or equal to 


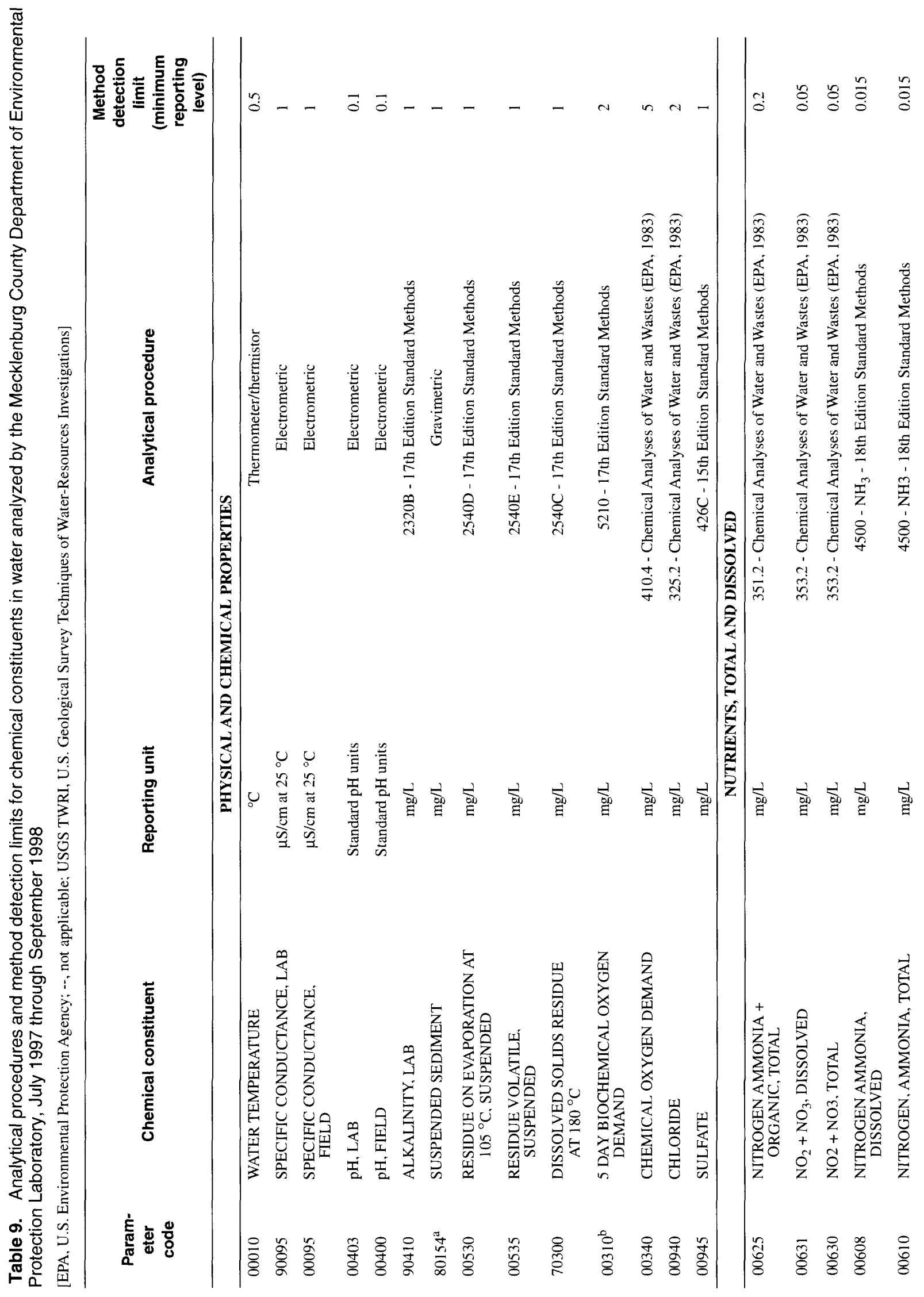




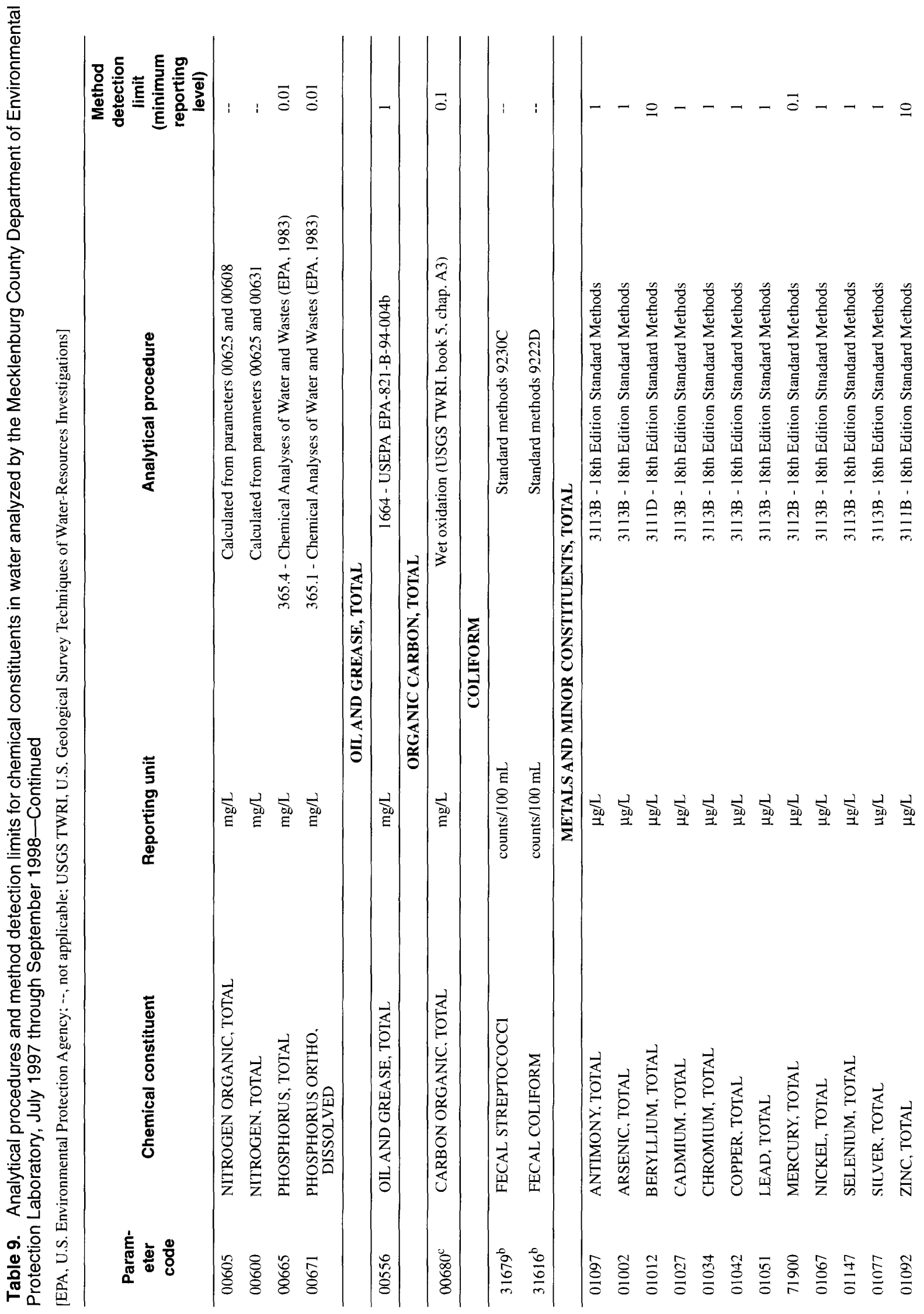




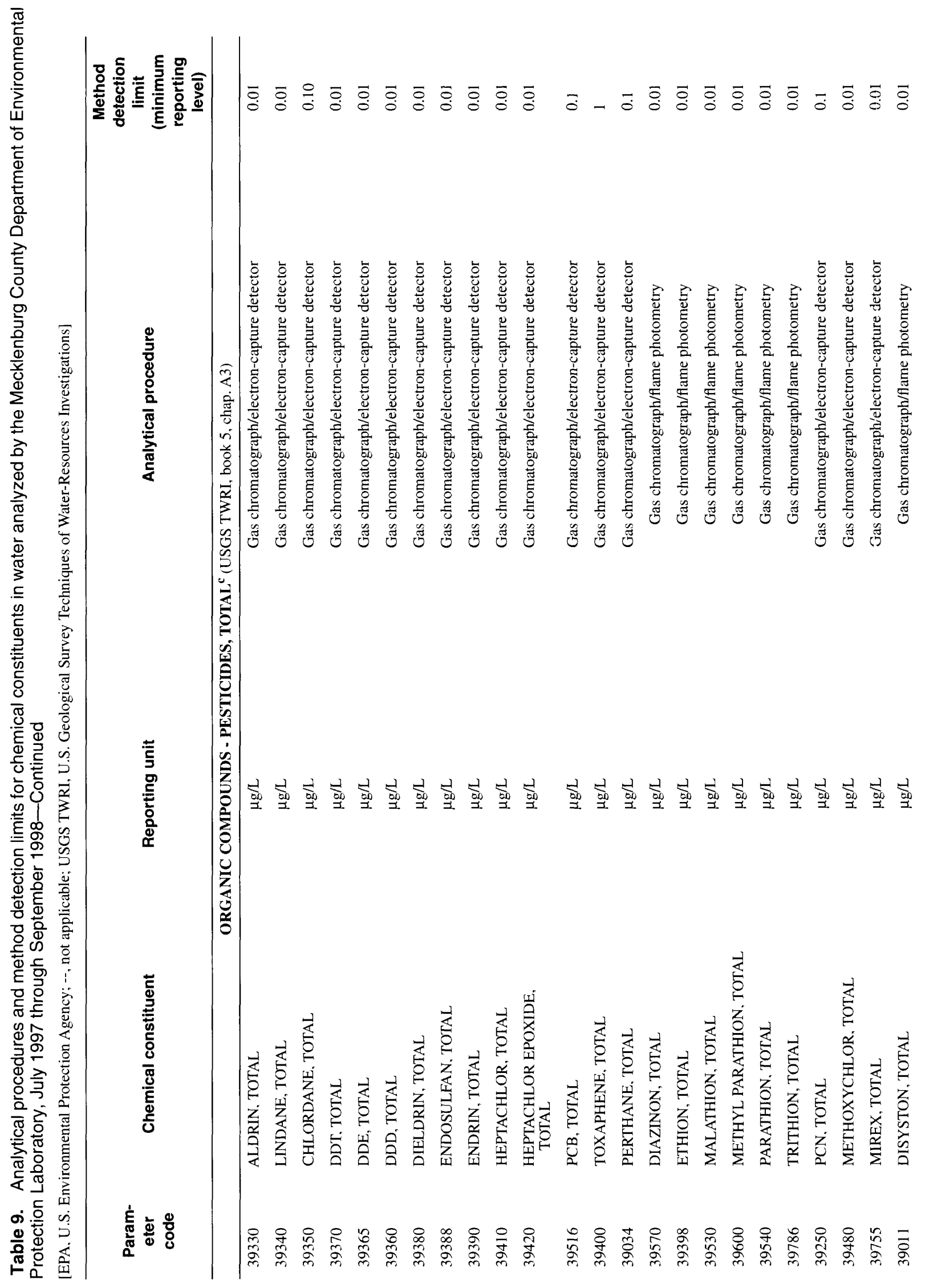




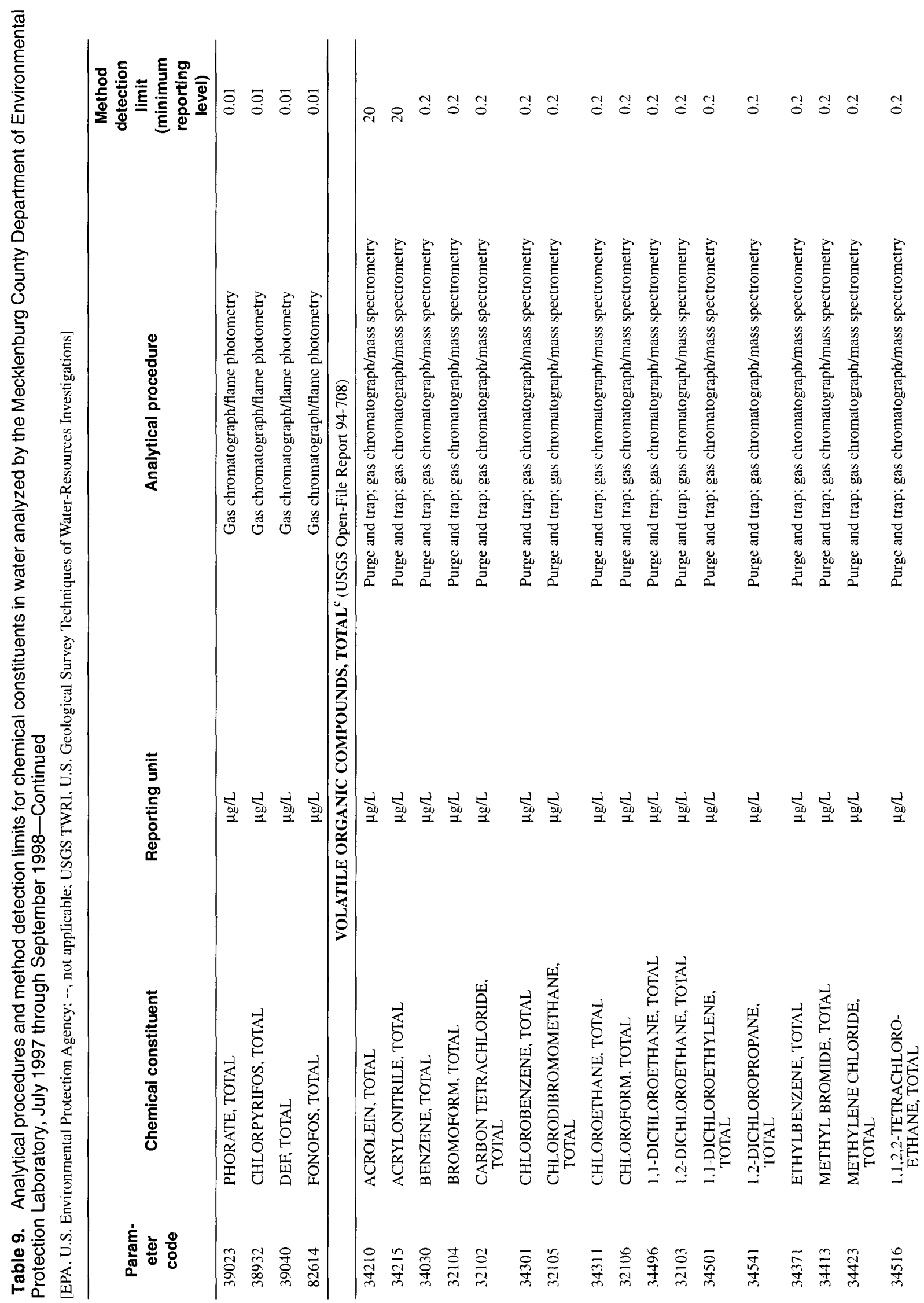




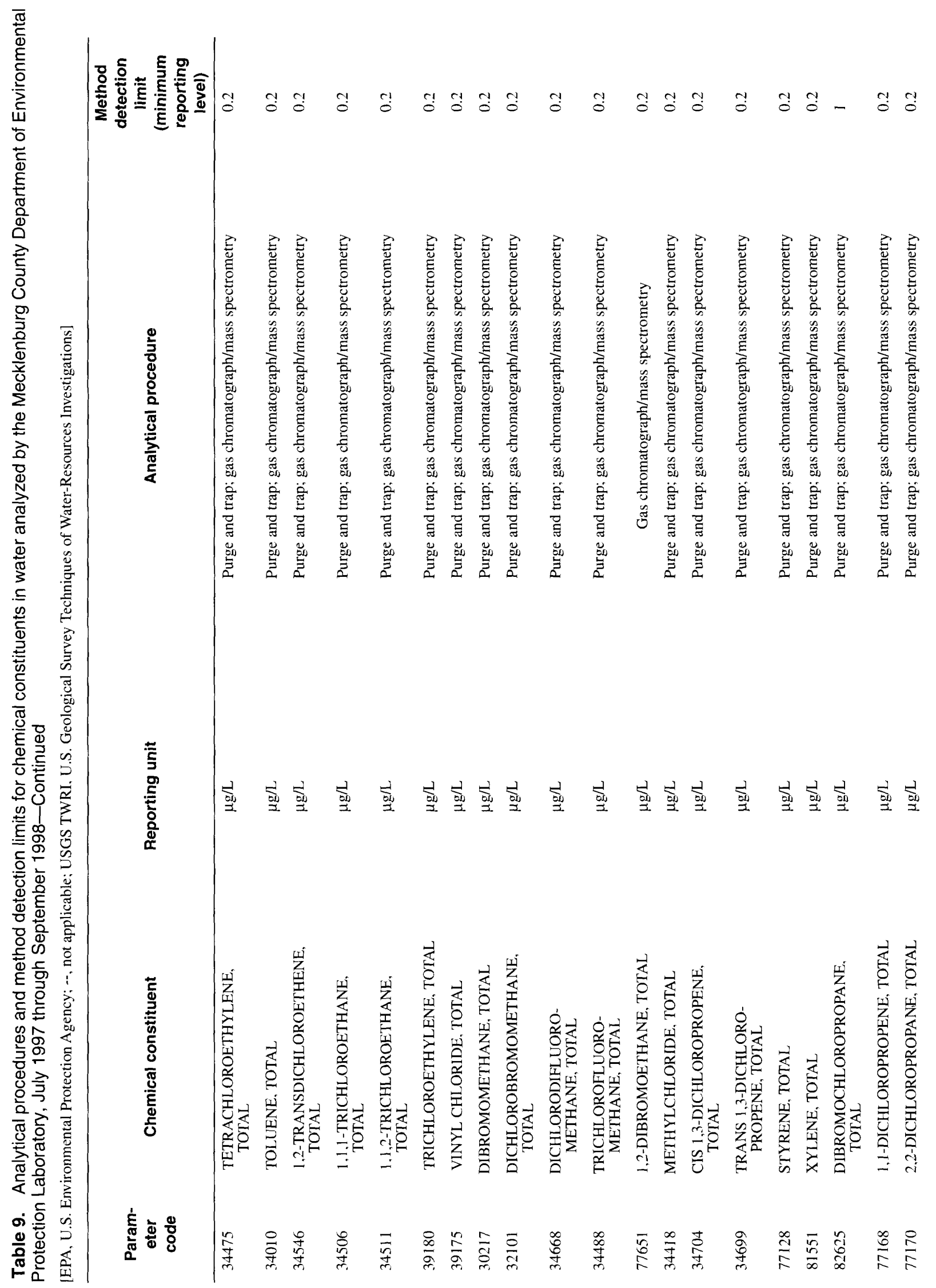




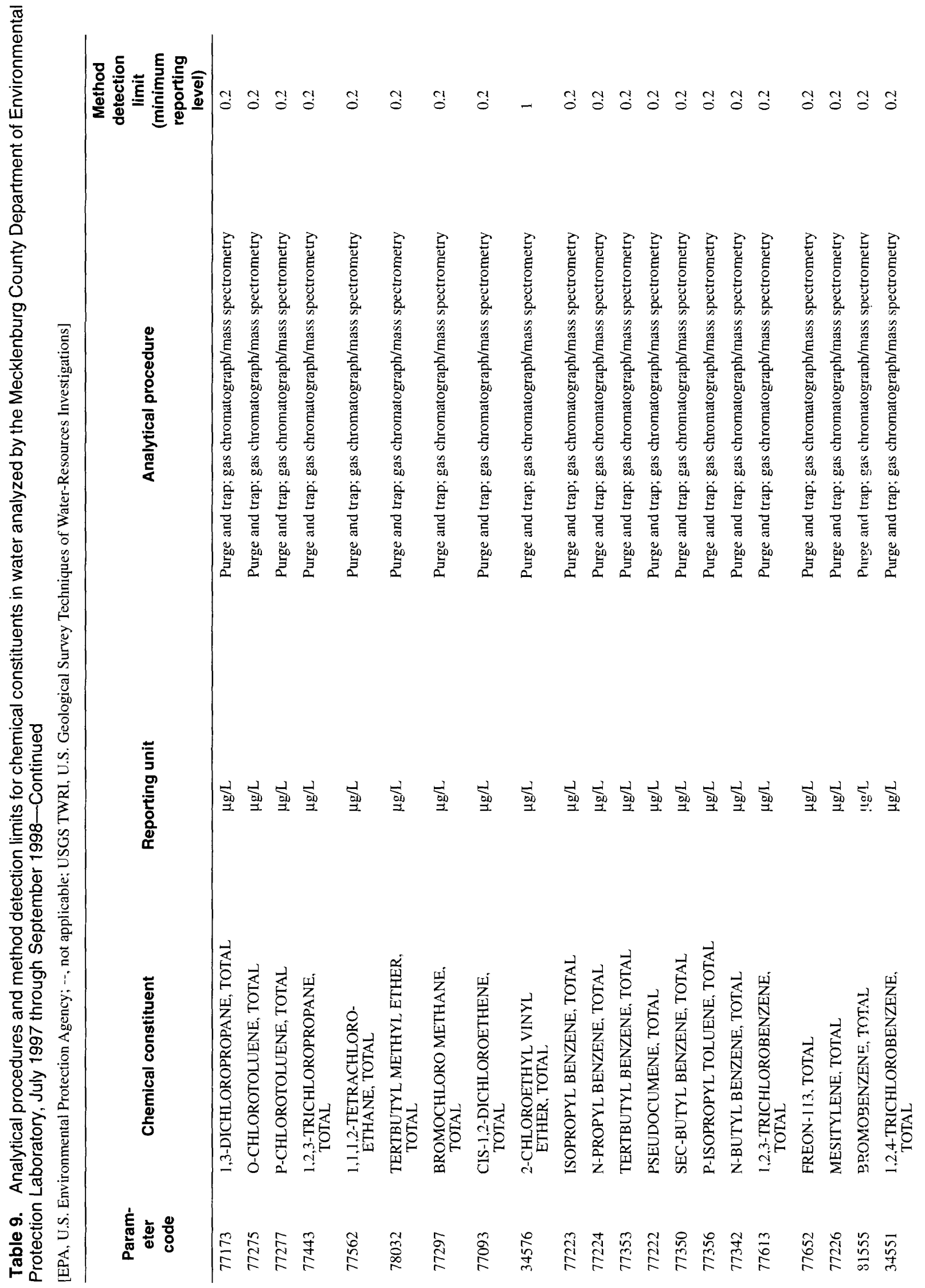




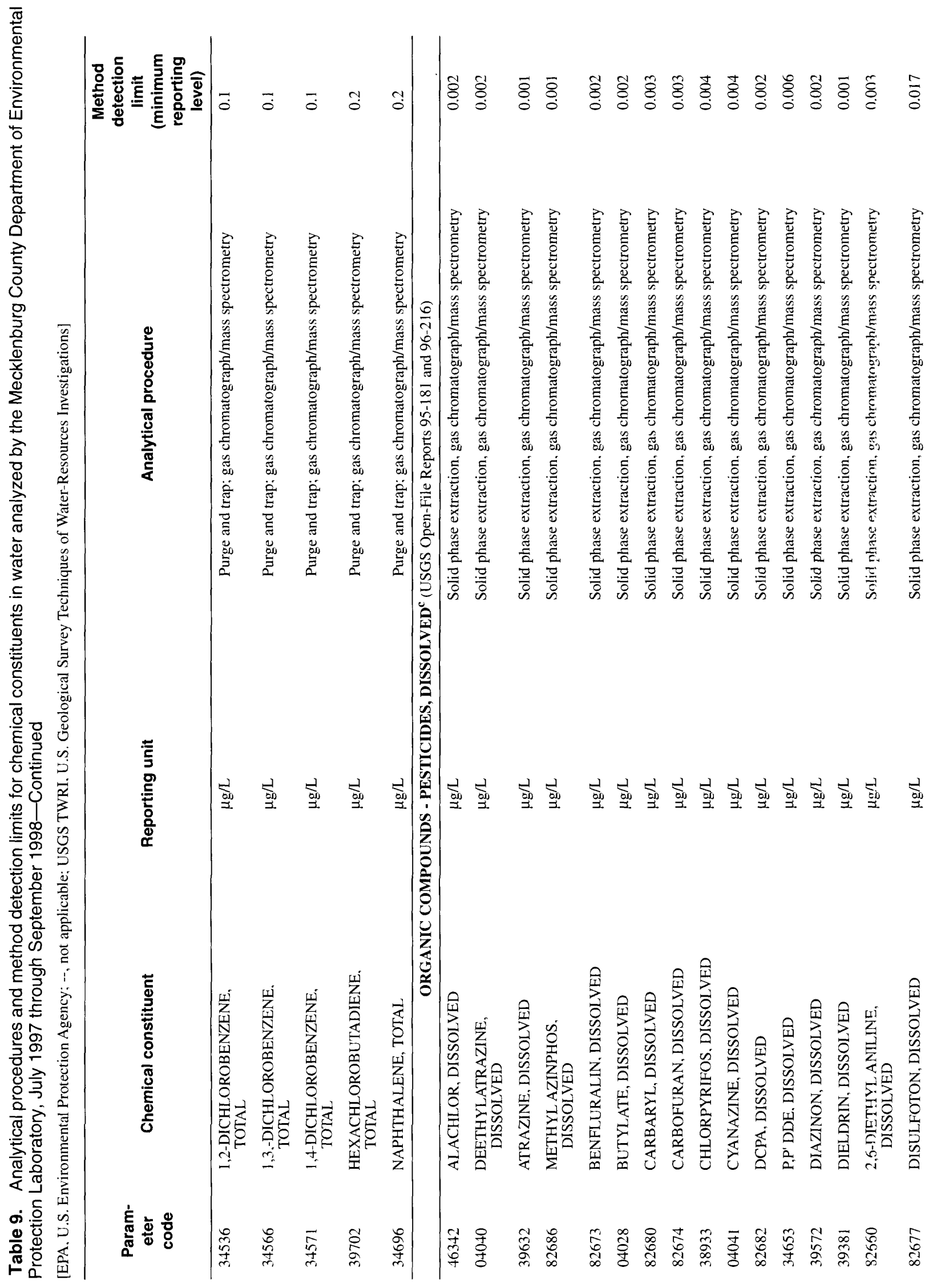




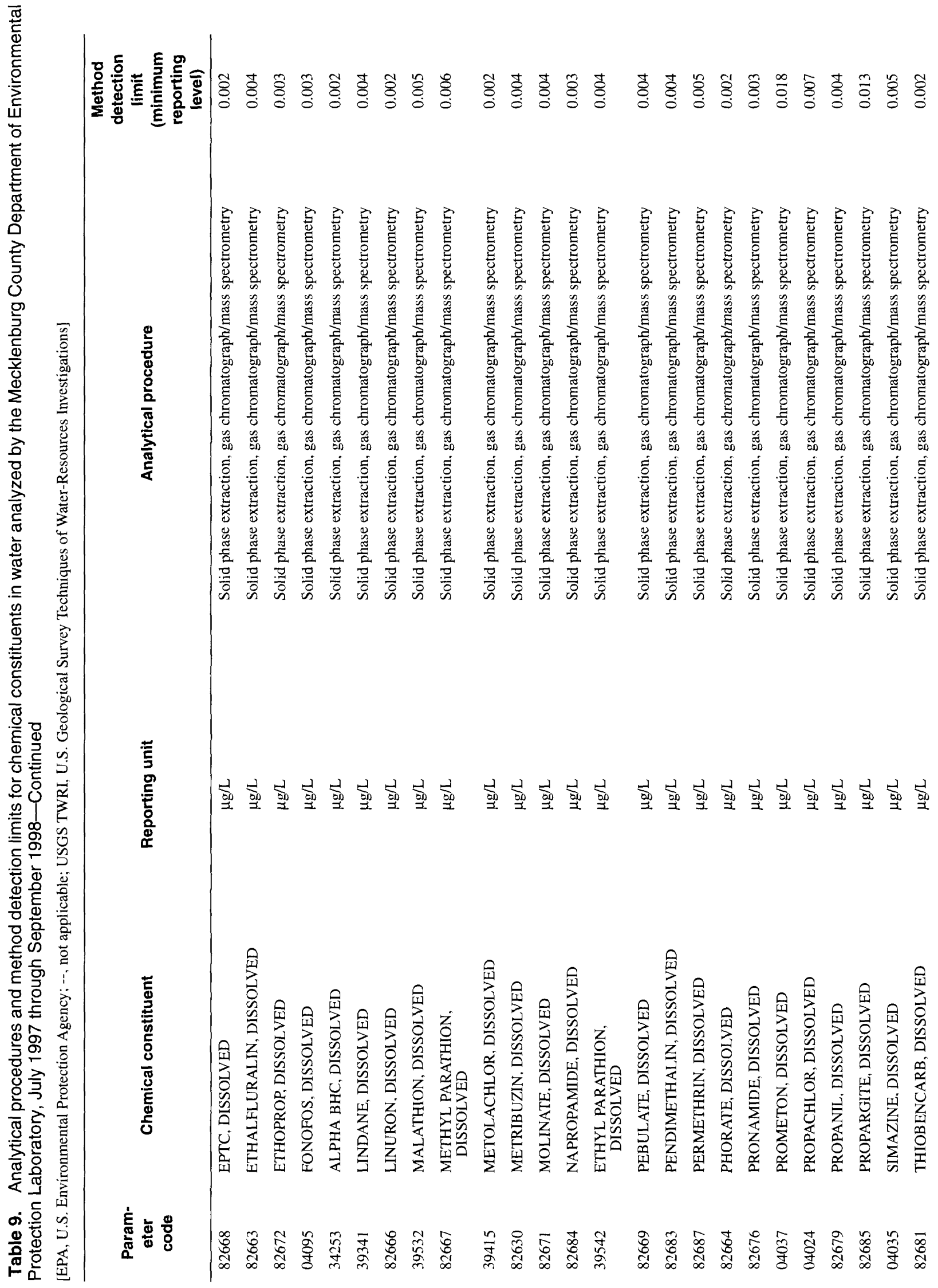




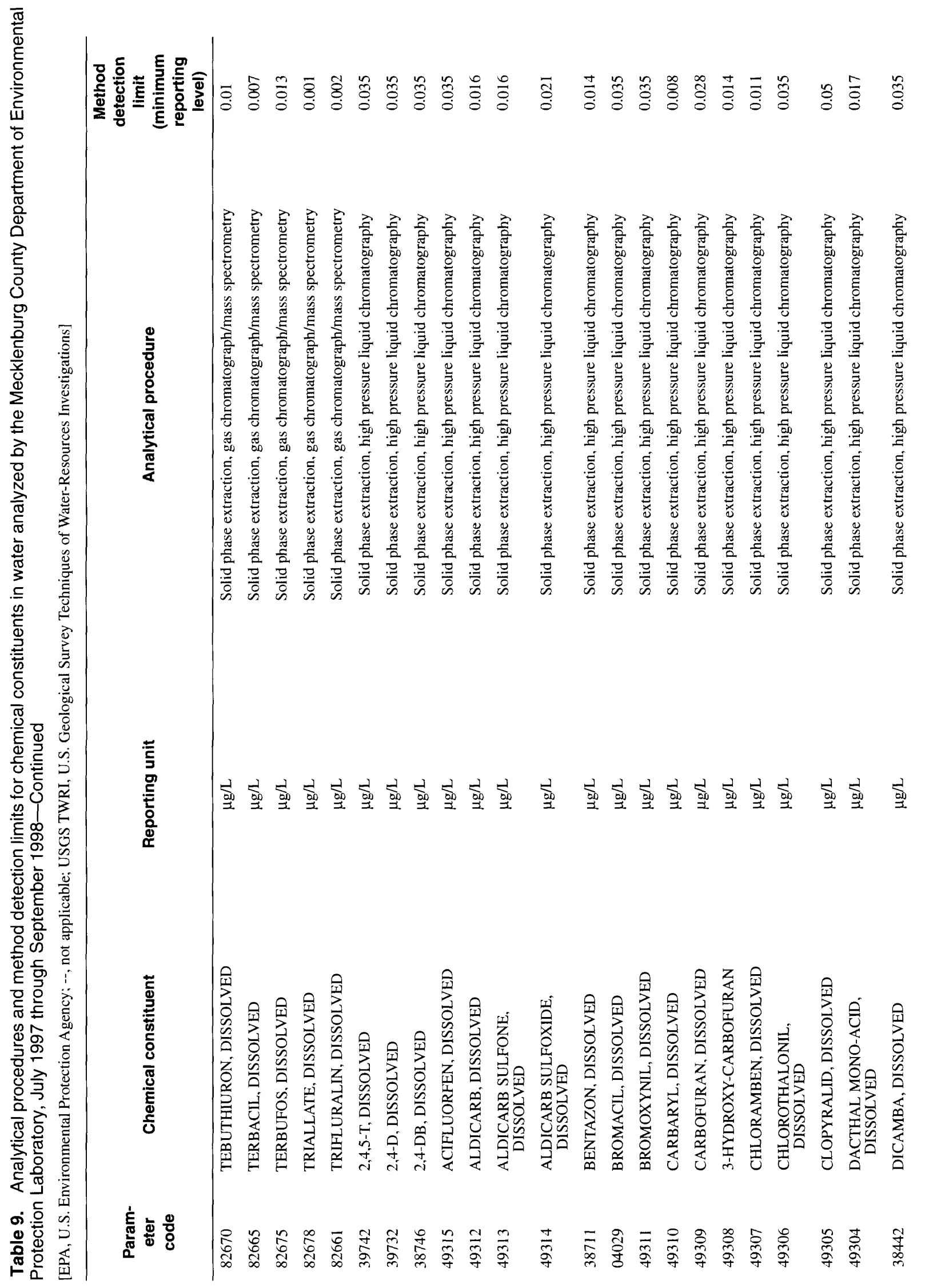




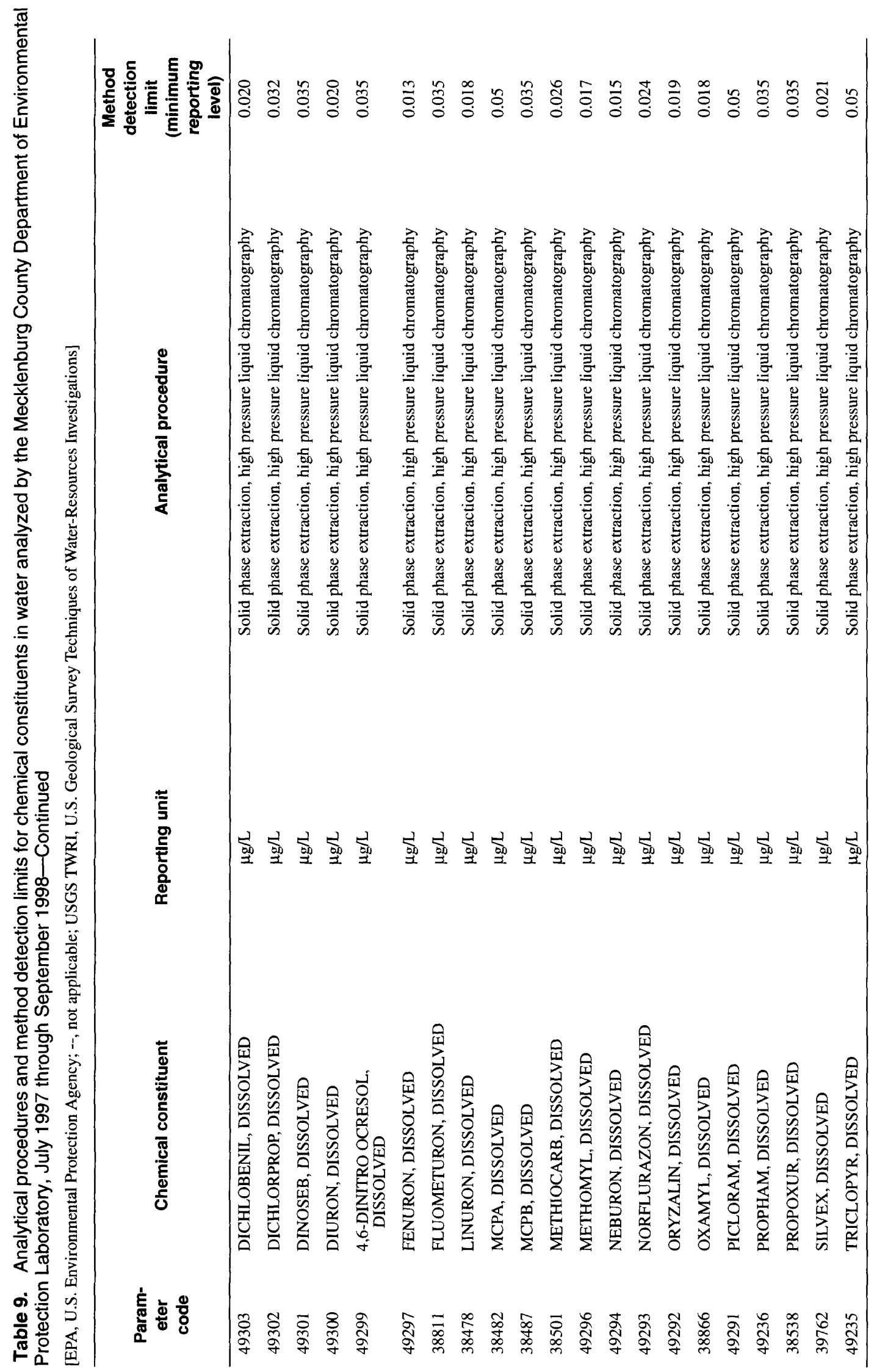




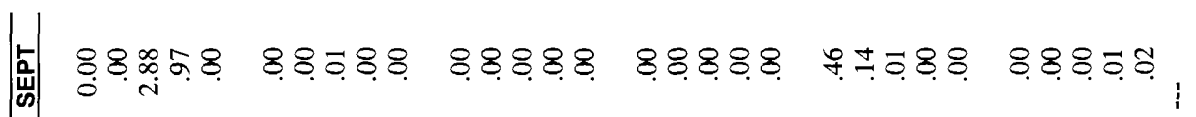

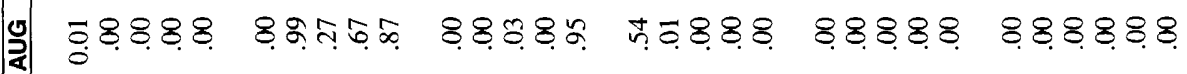

ว

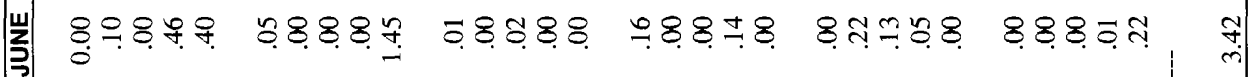

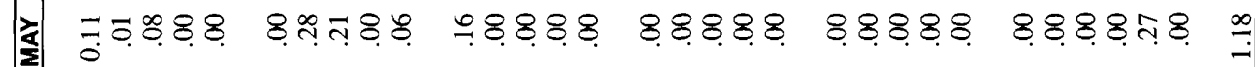

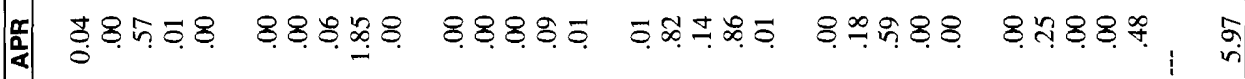

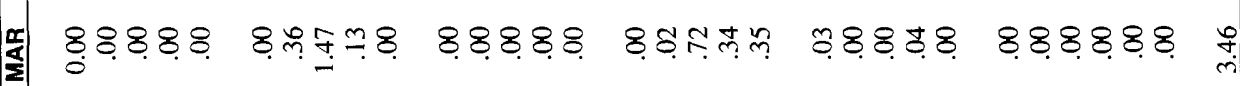

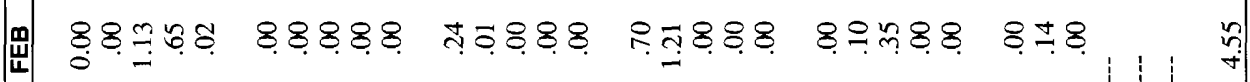

خे

$\sum_{\substack{0 \\ 0}}^{\underline{0}}$

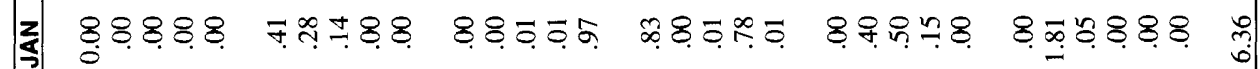

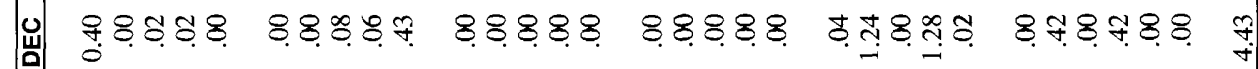

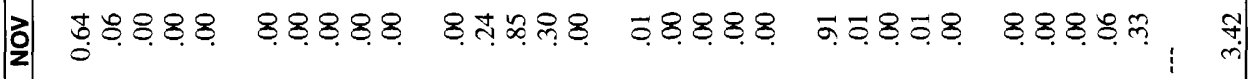

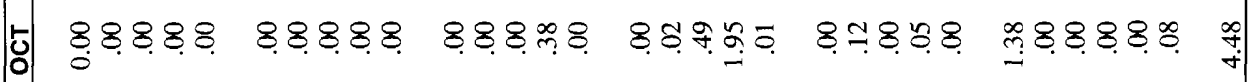

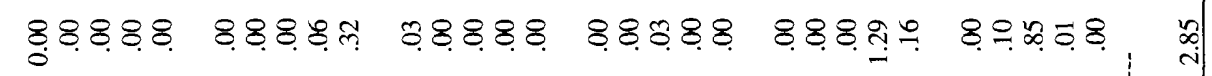

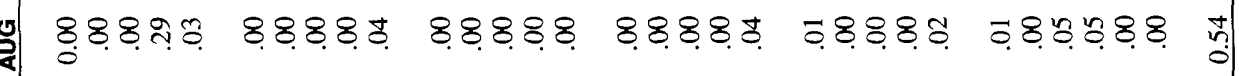

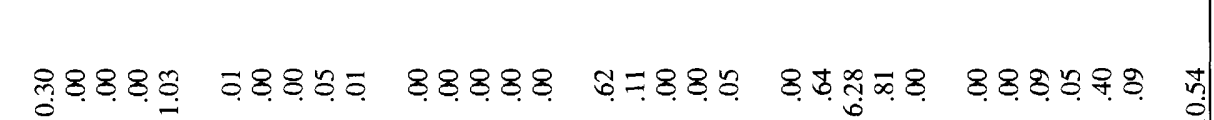

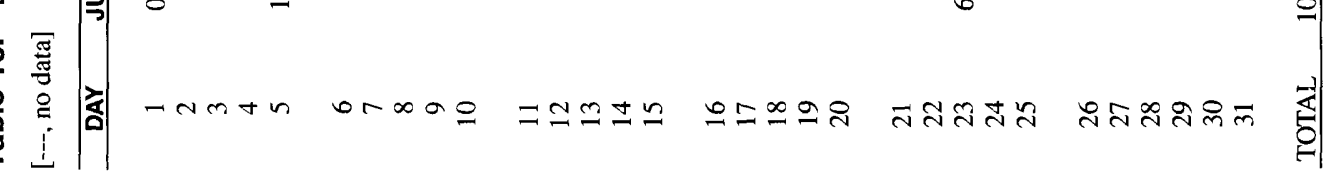




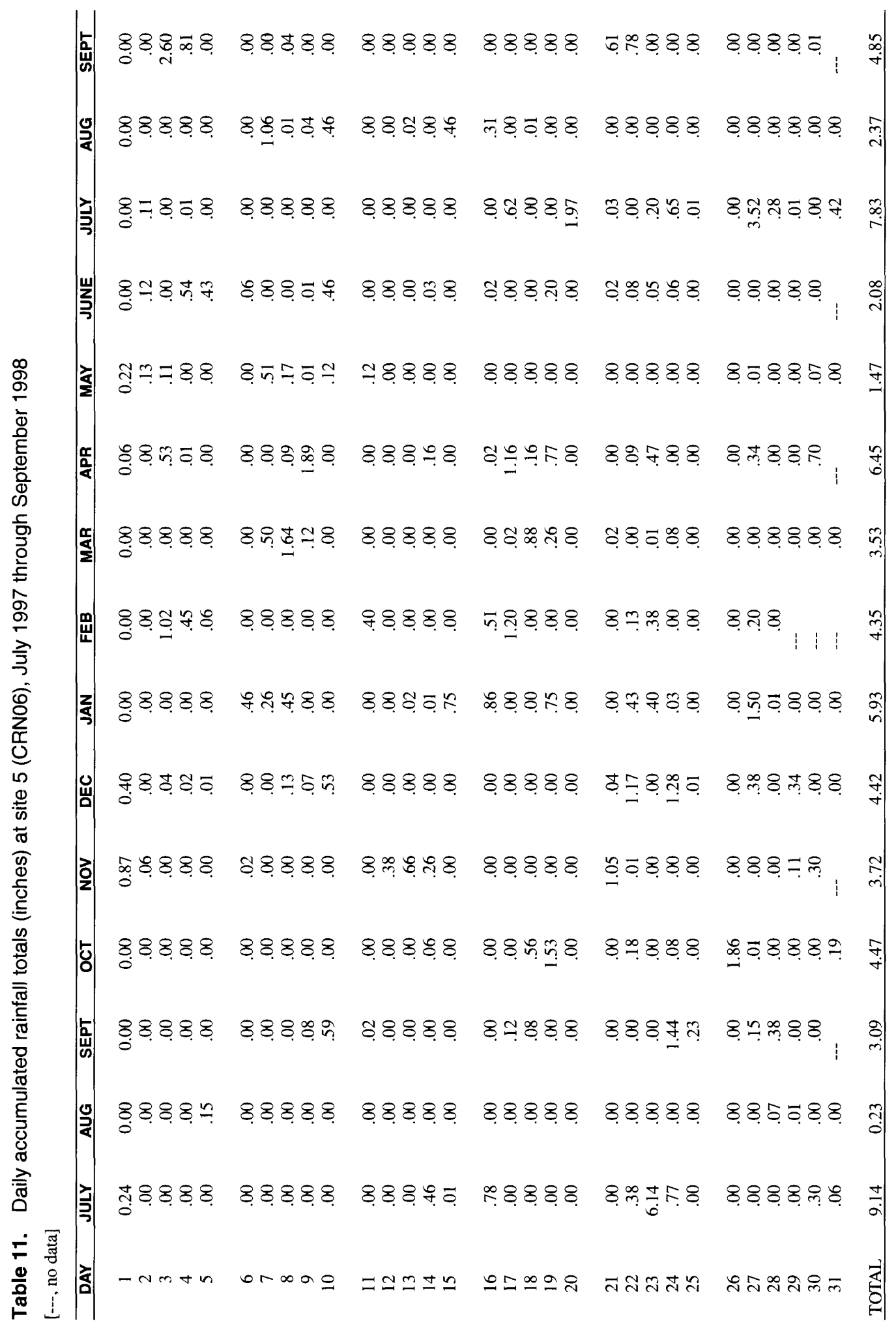




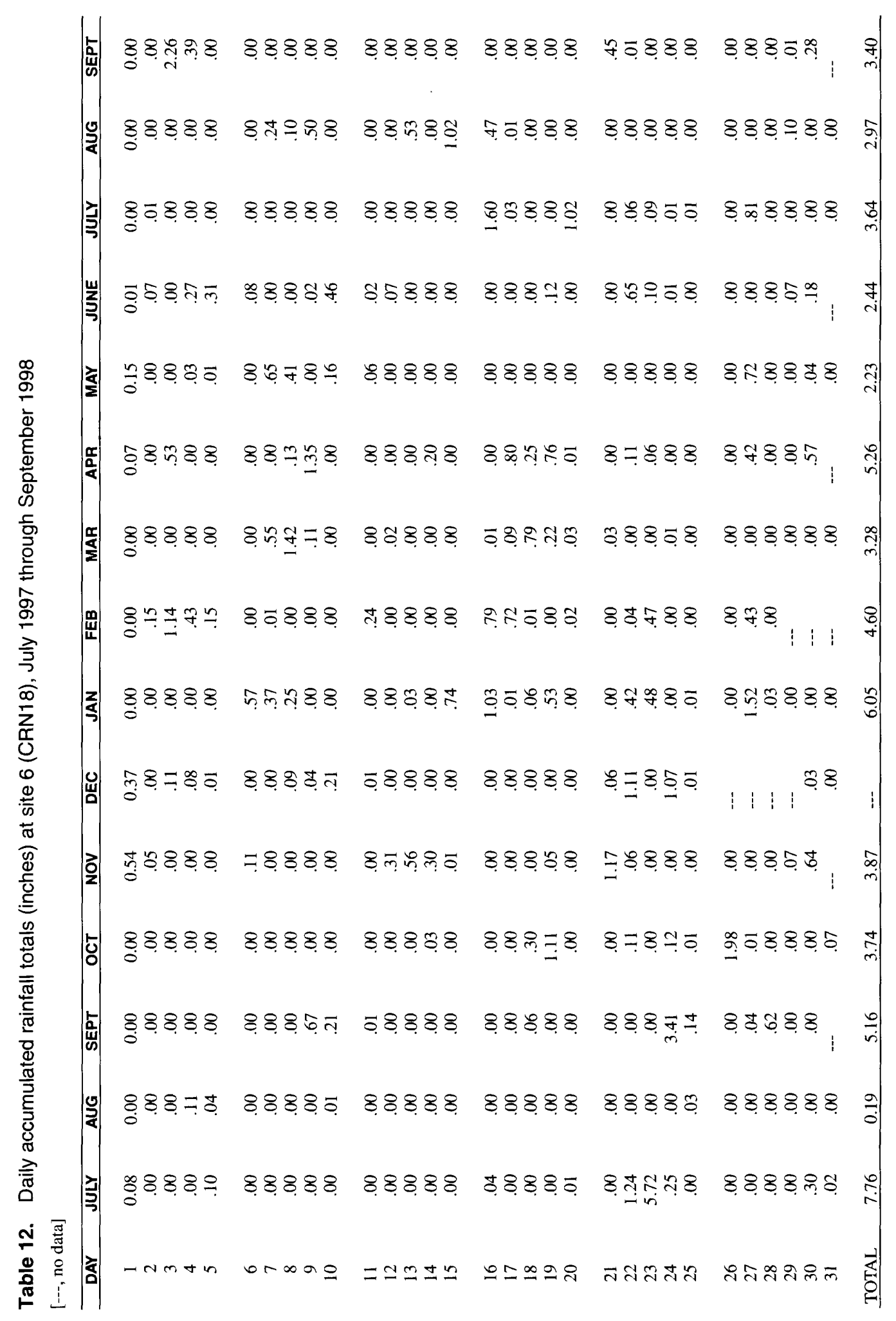




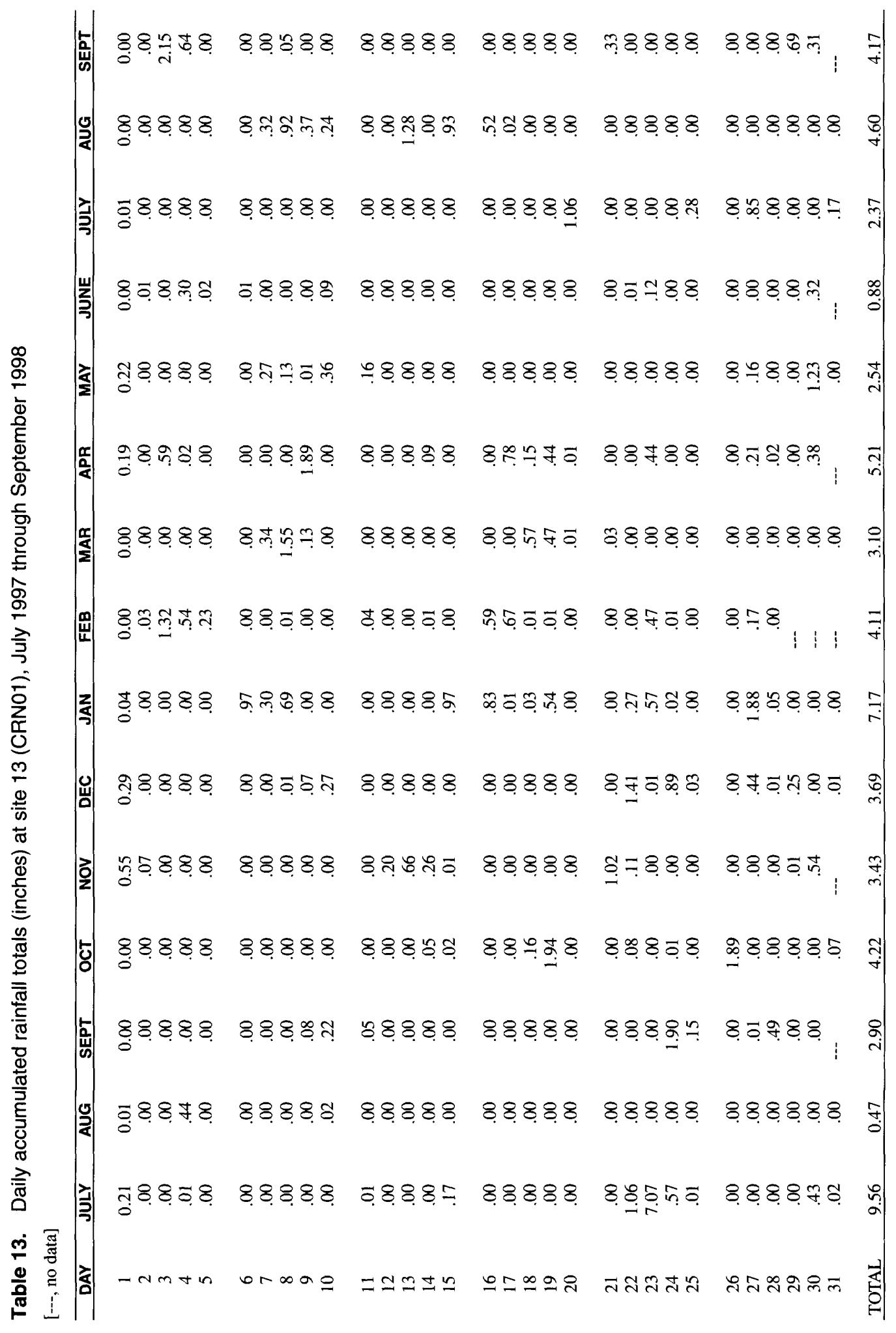




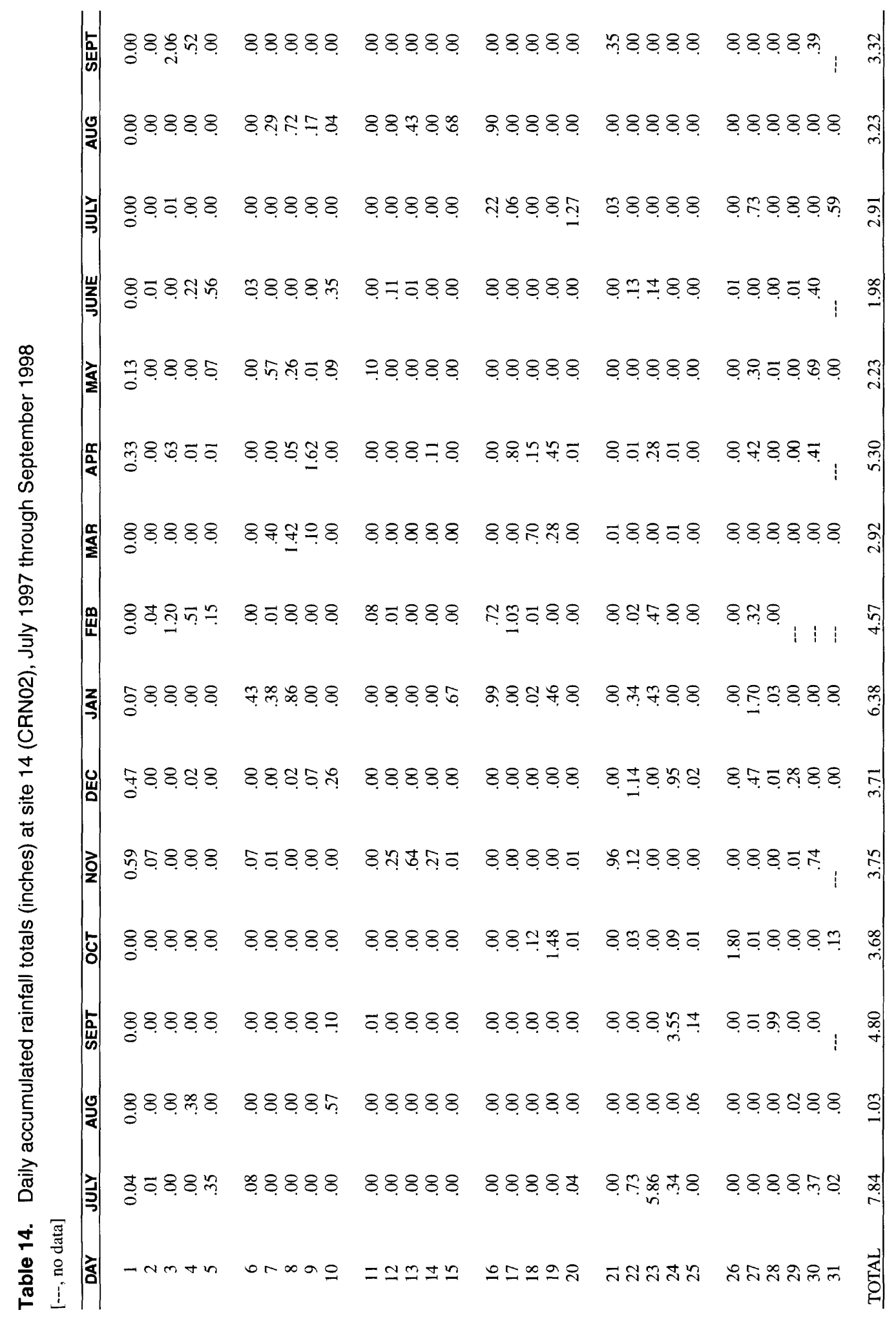




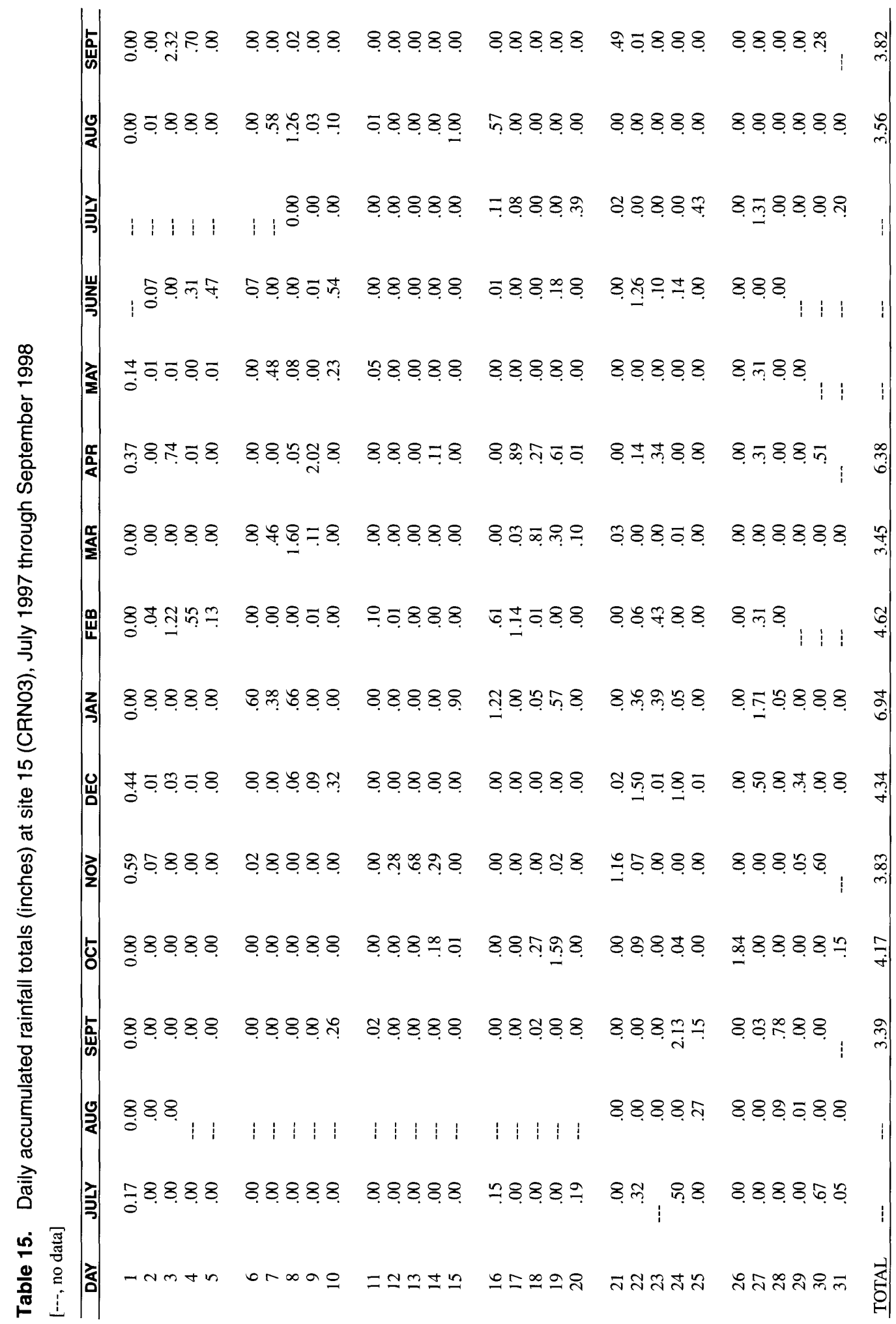




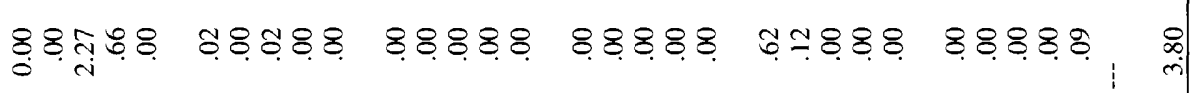

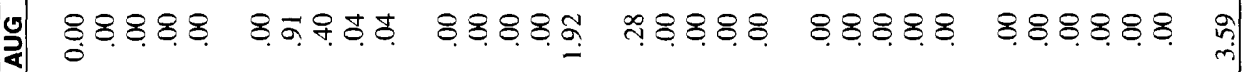

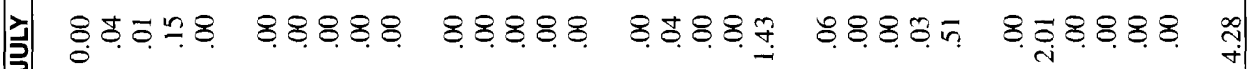

य

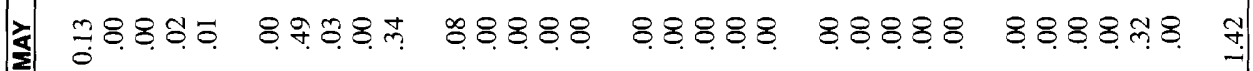

শ

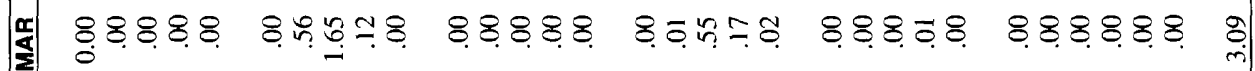

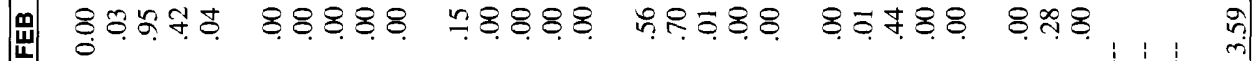

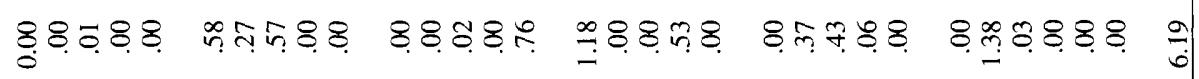

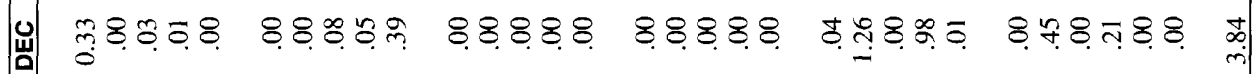

\$

H.

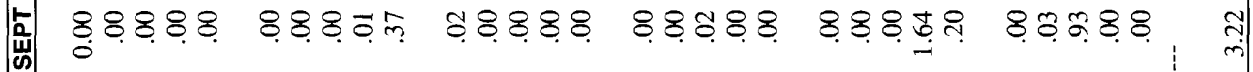

4 8.8.8\%

خ

至 


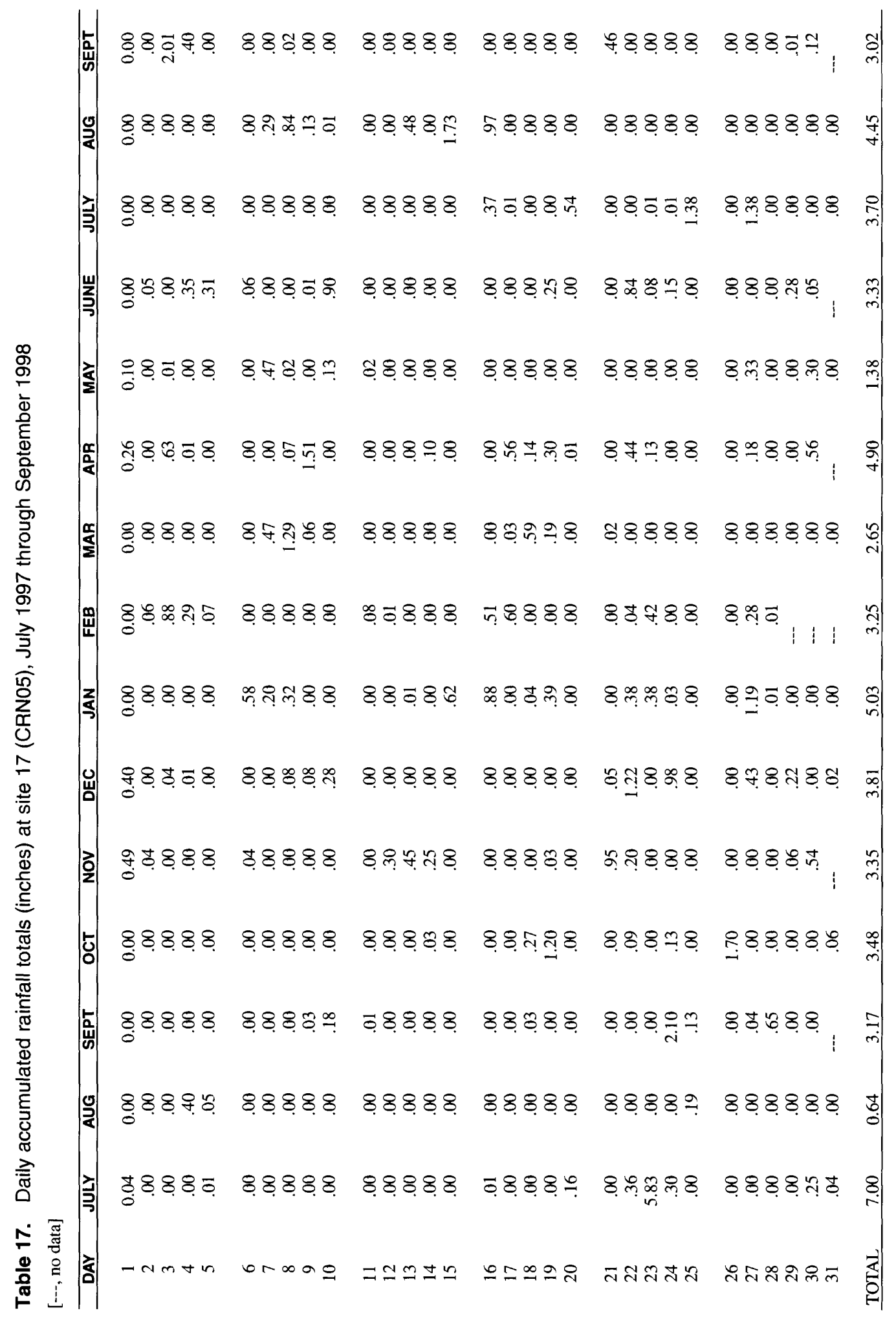




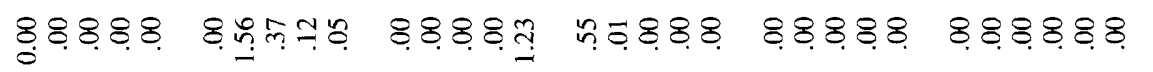

官 8.

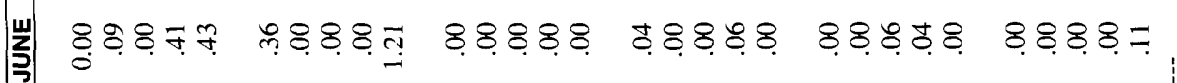

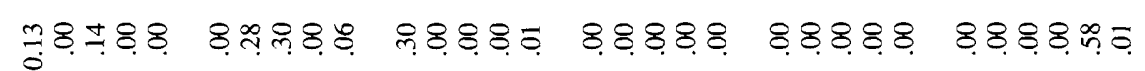

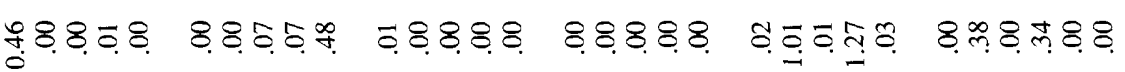

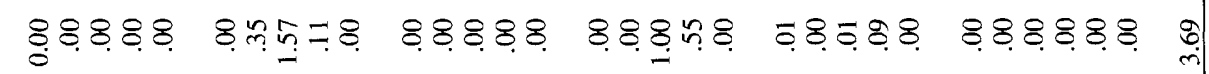

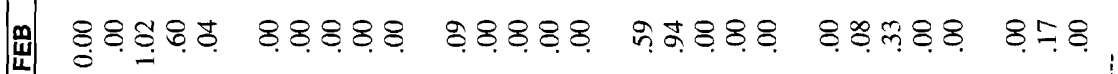

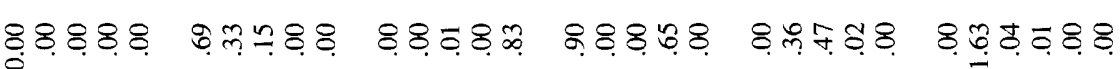

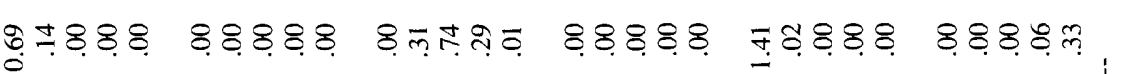

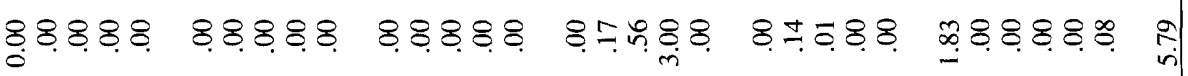

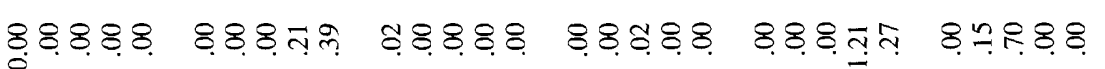

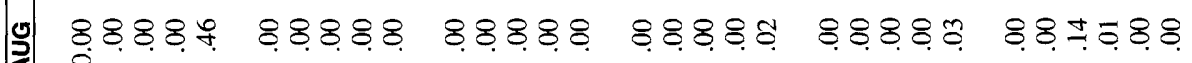

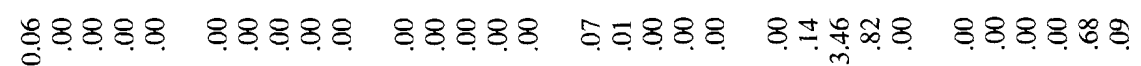

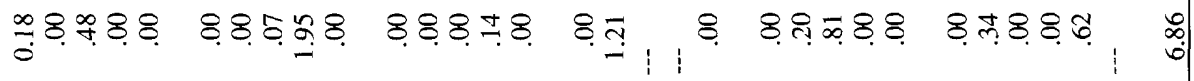




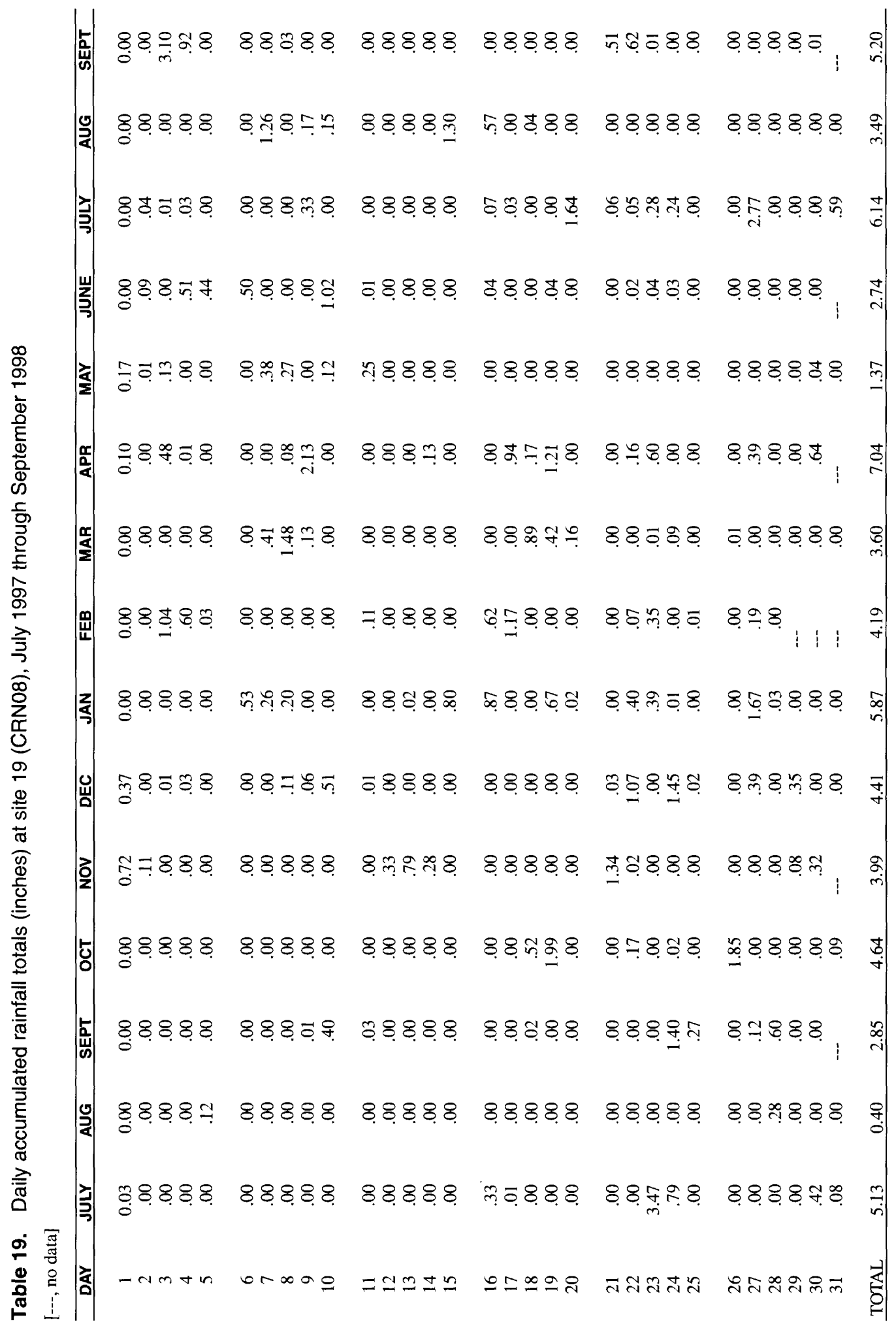




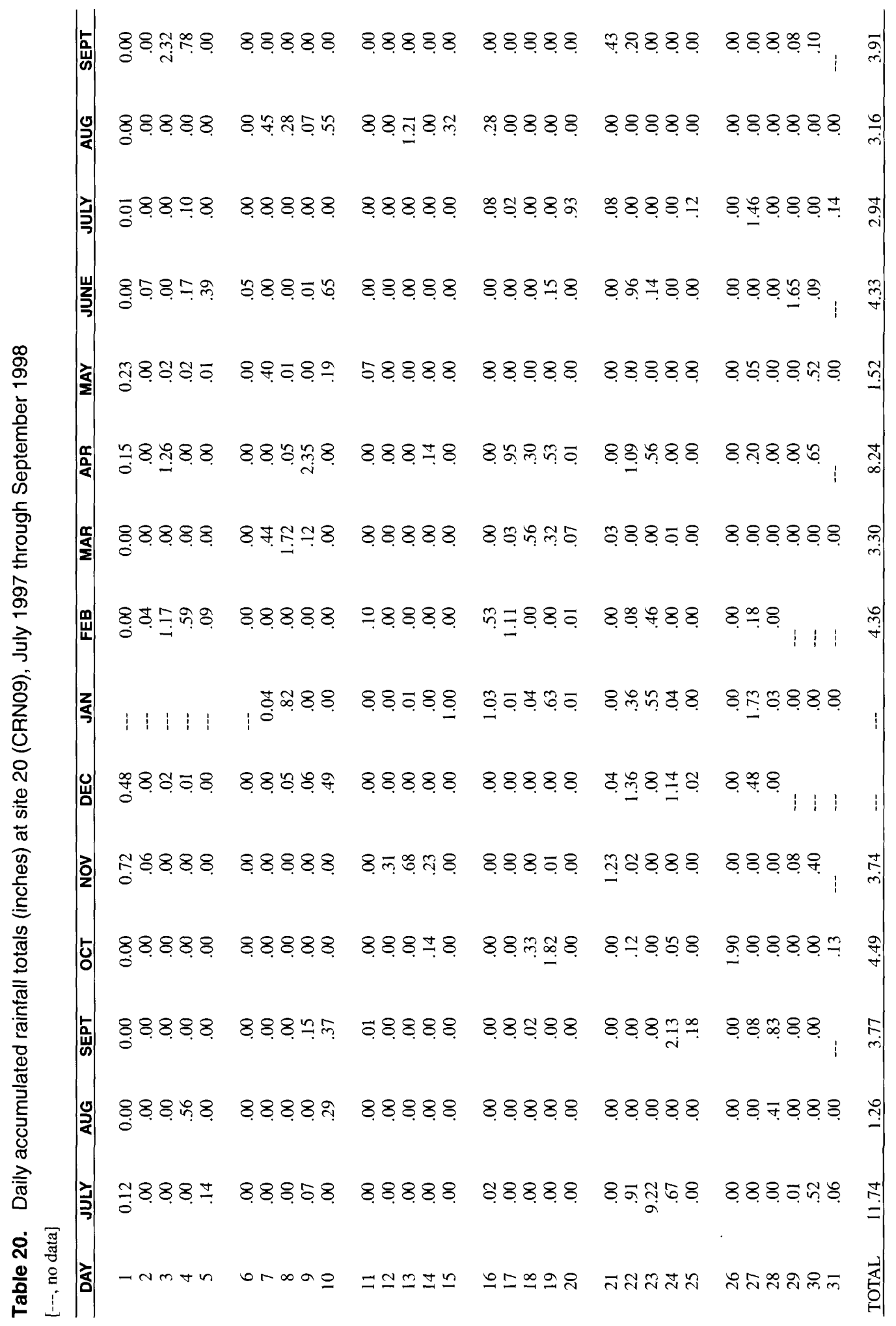




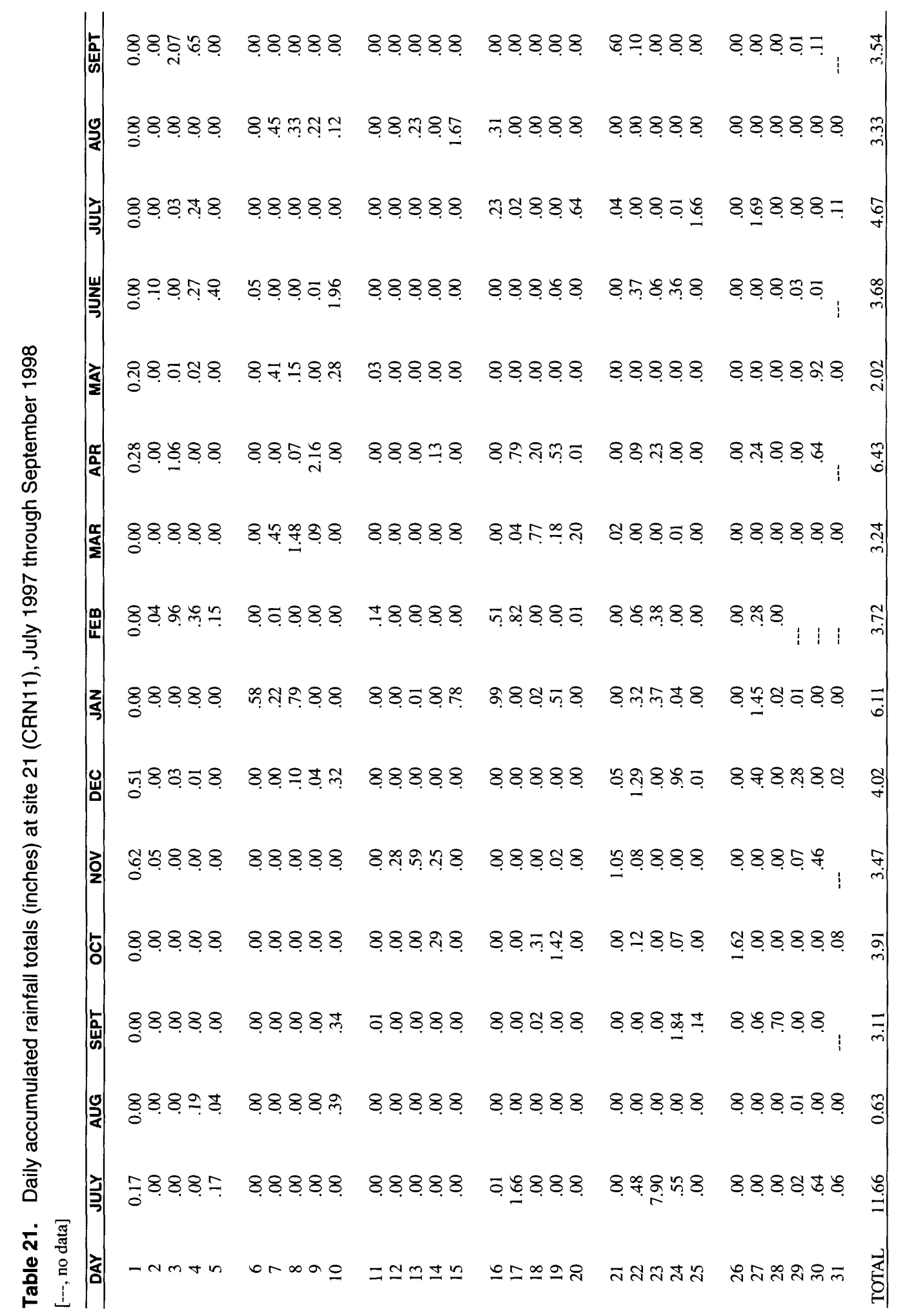




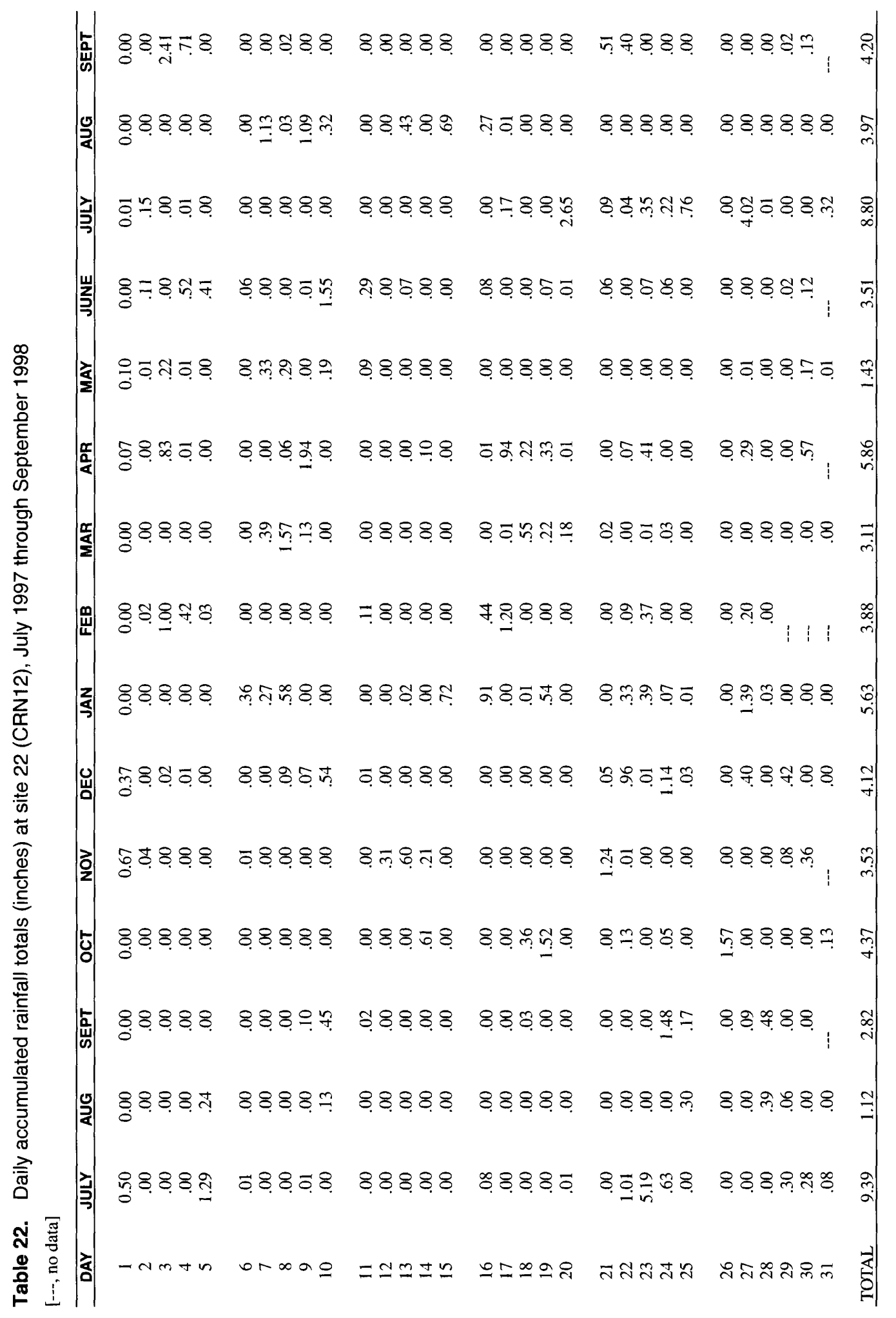




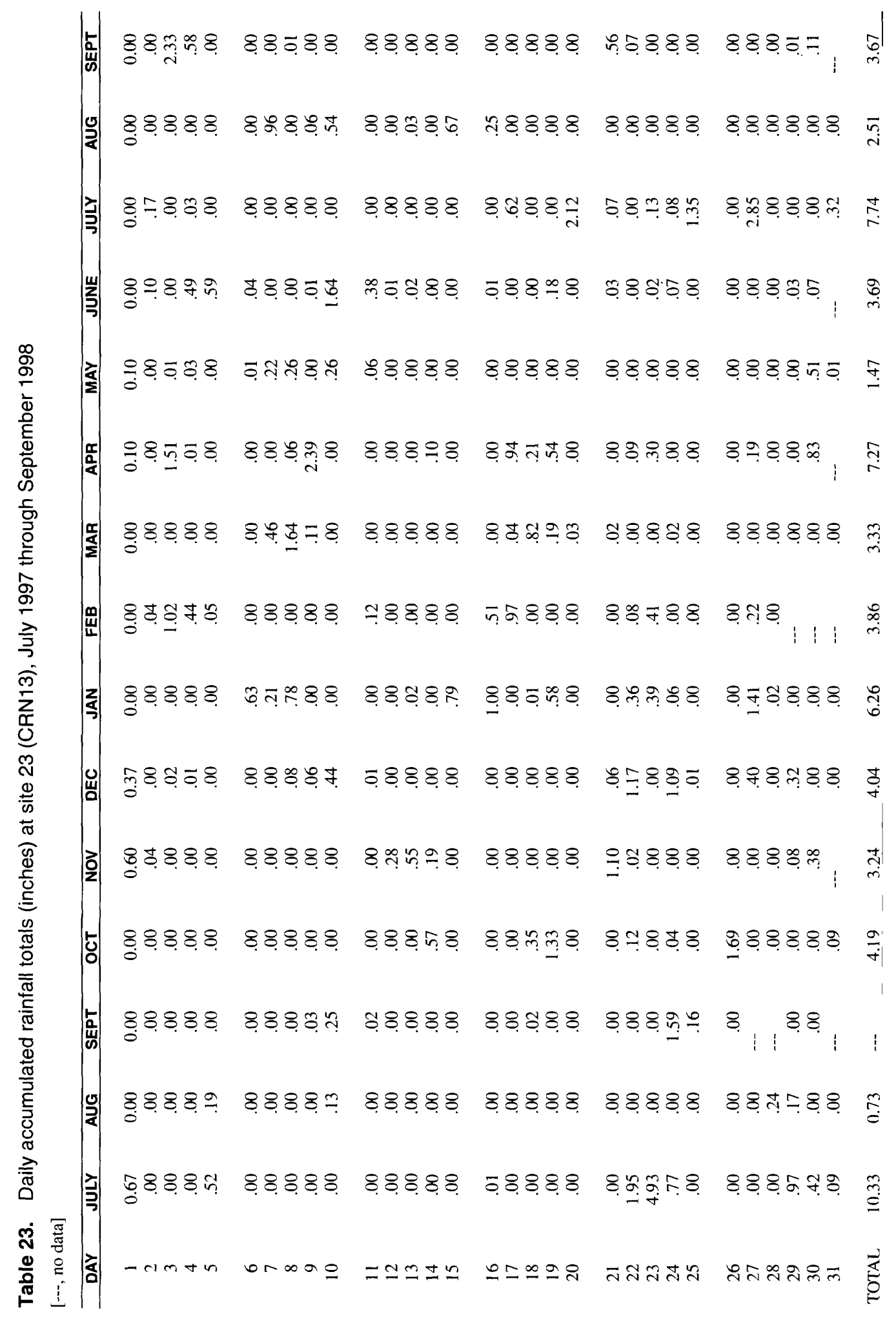




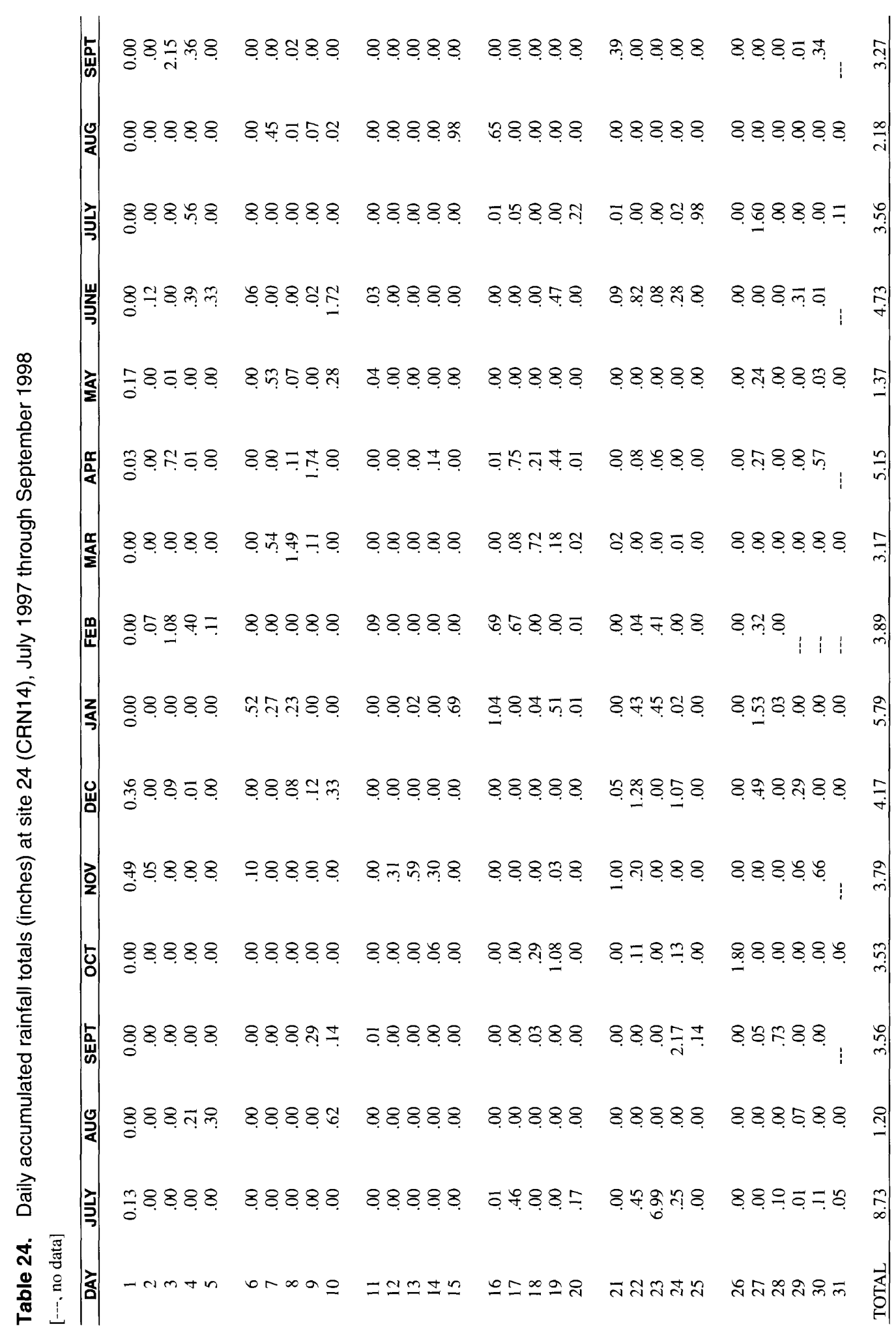




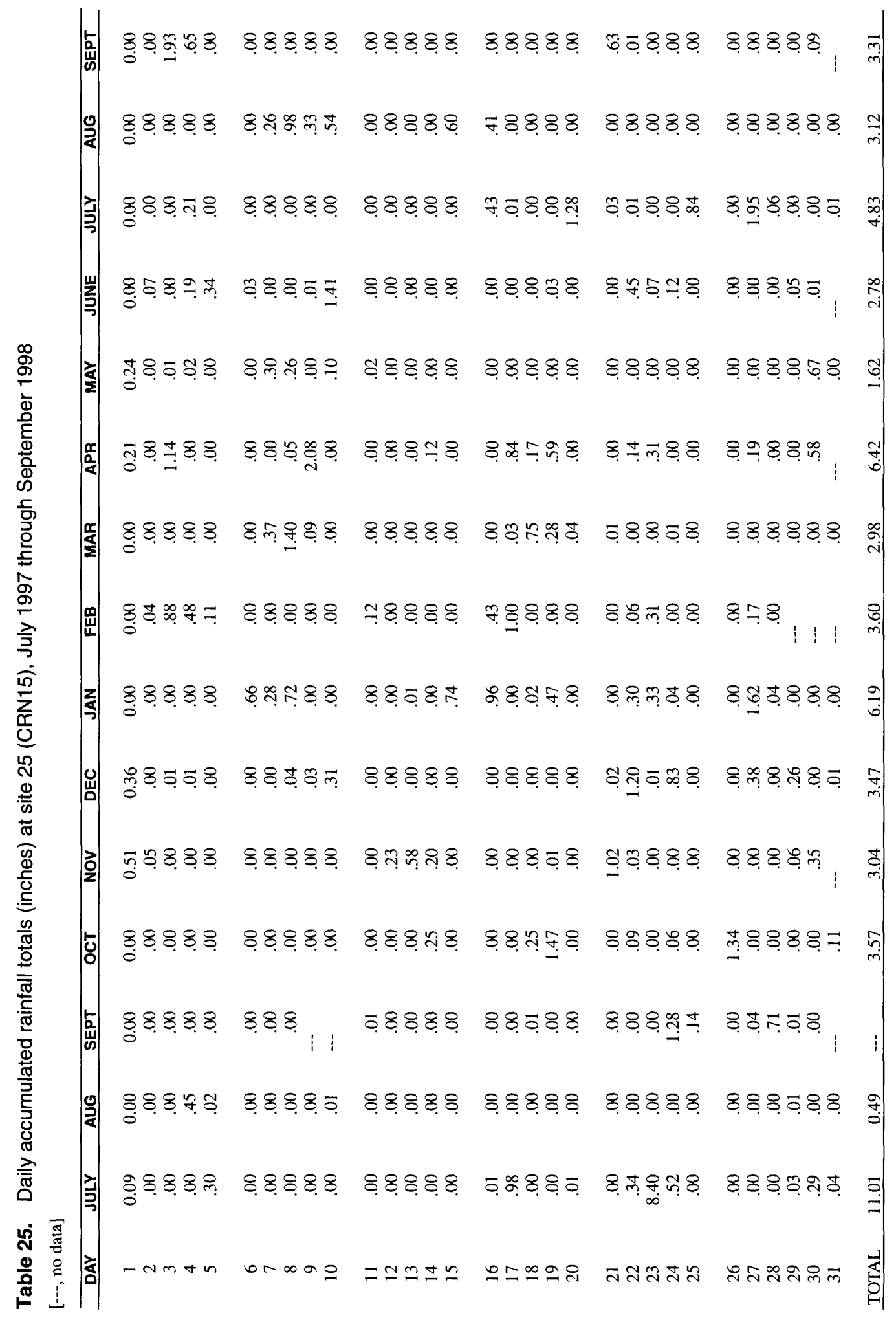




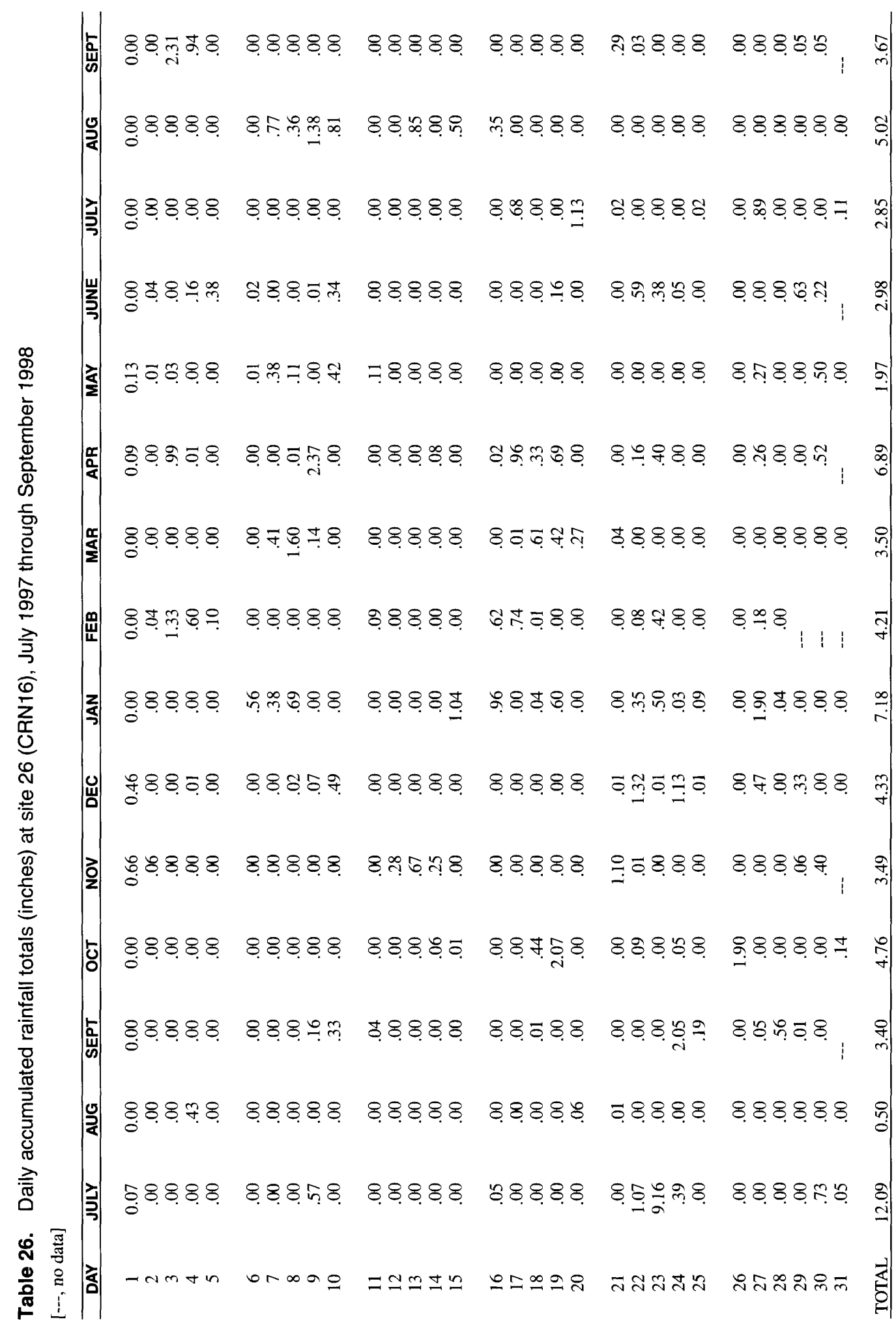




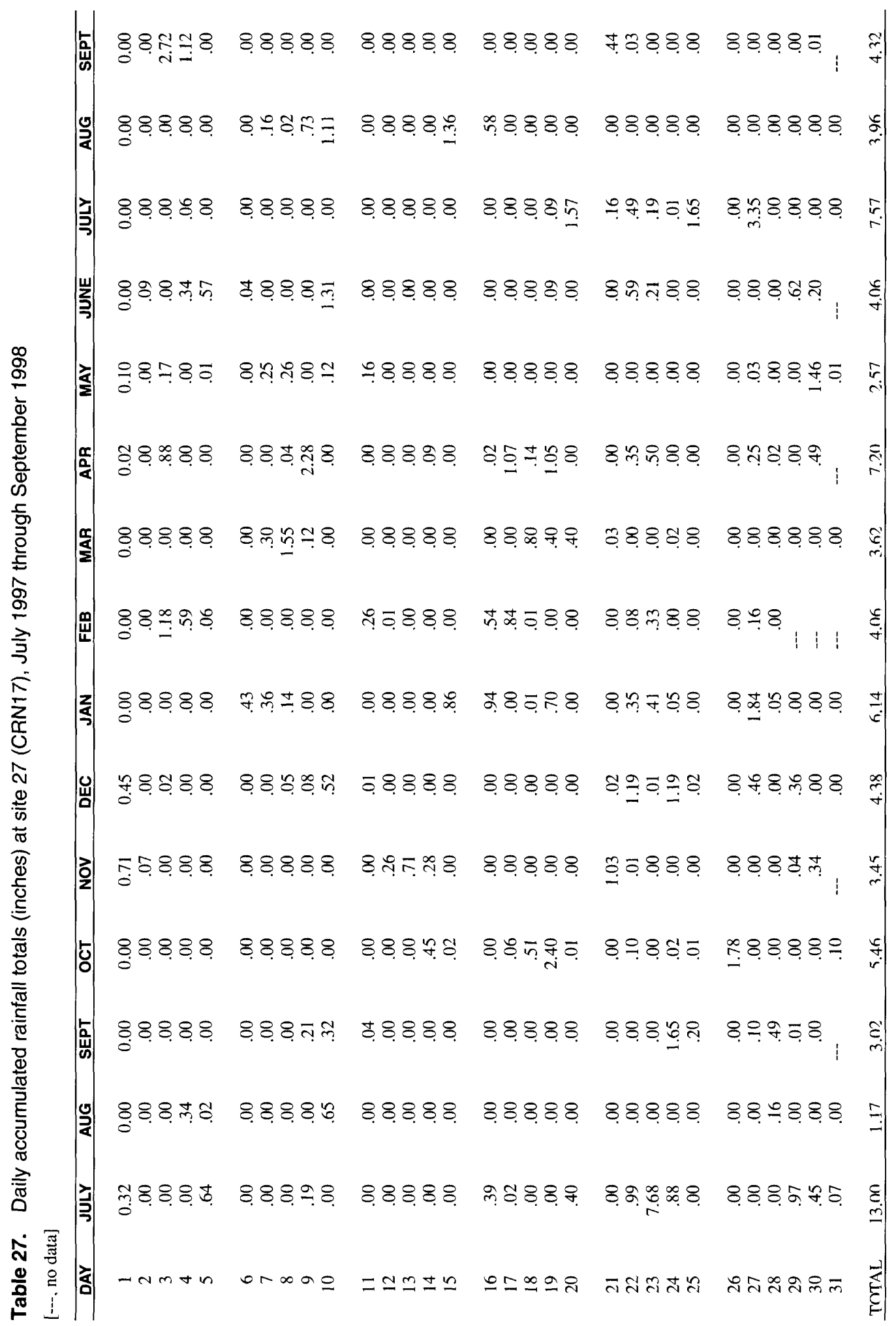




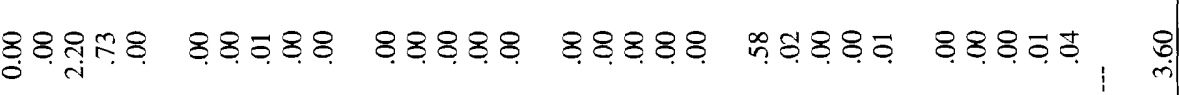

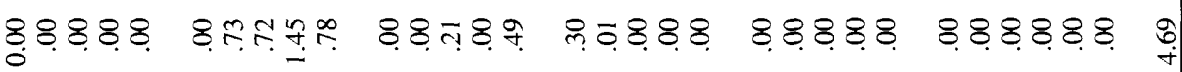

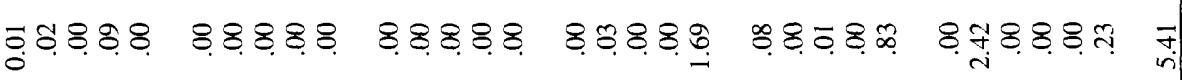

w

₹

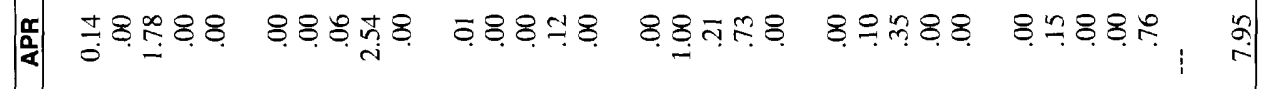

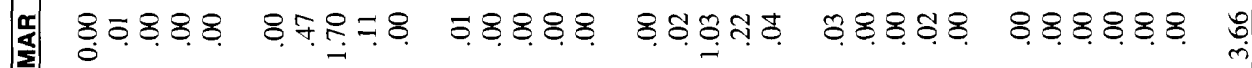

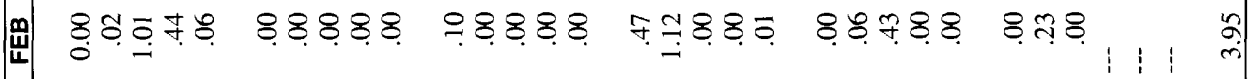

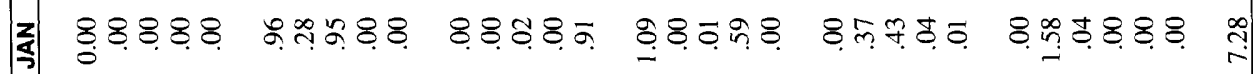

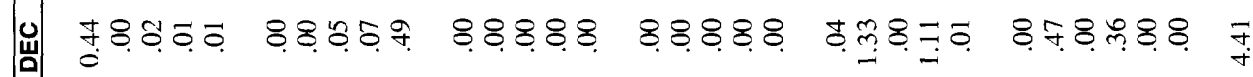

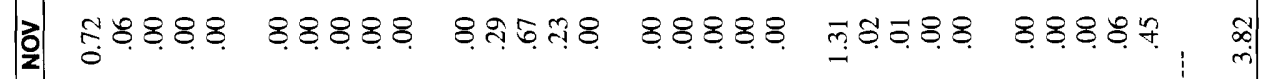

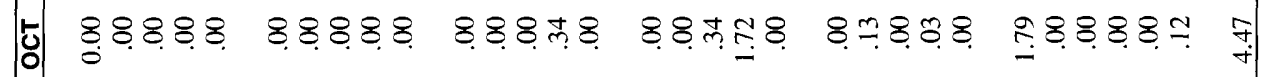

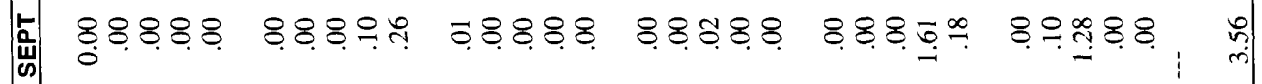

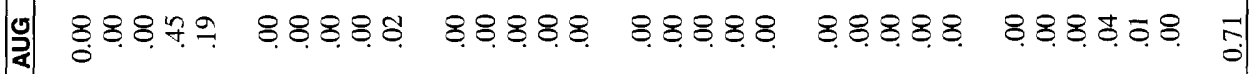
औ ल

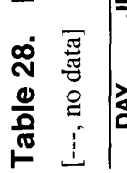
- 


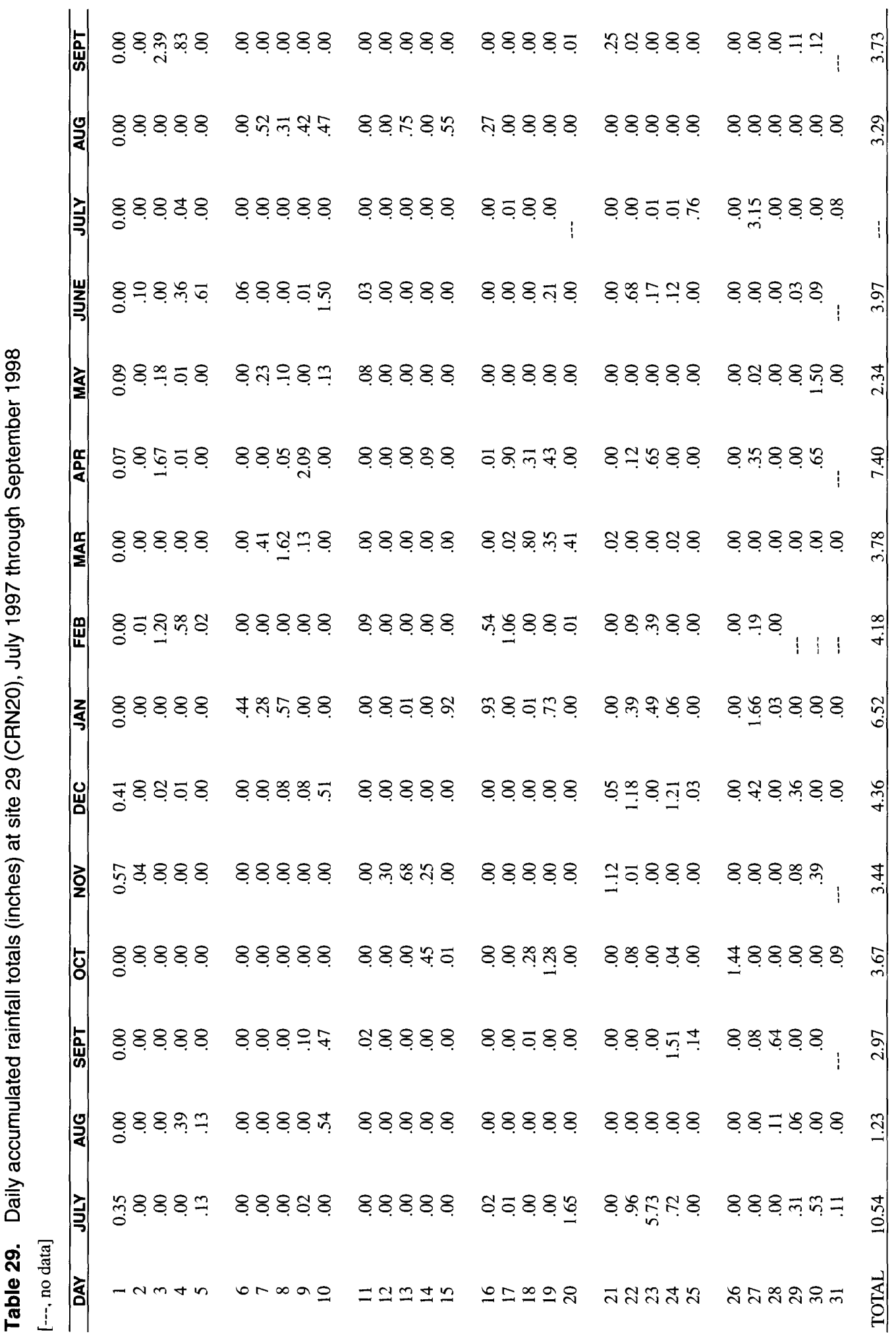




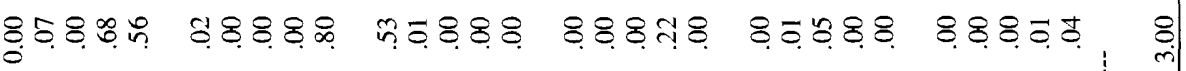

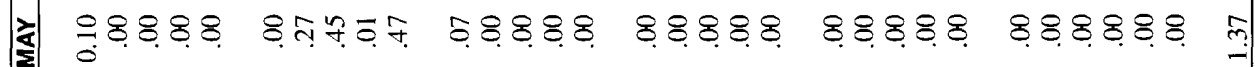

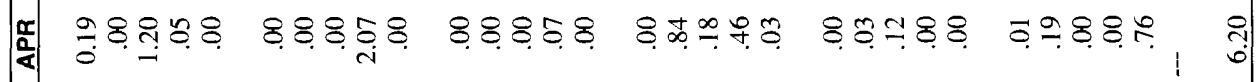

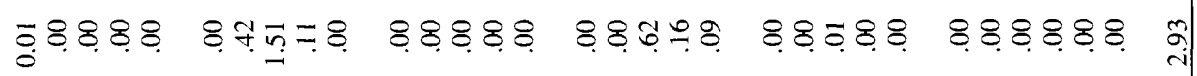

س

य

요

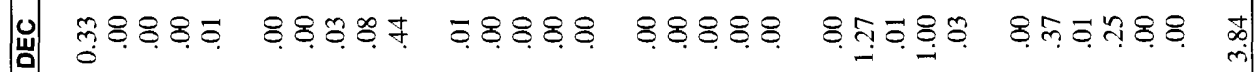

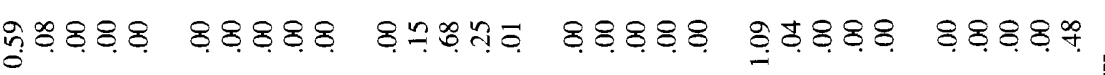

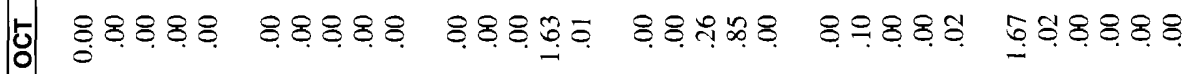

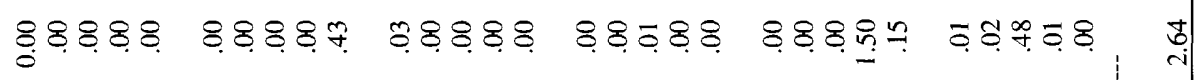

\% 8.80\% ठ

营

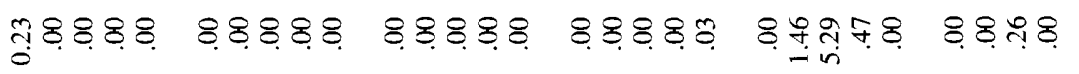




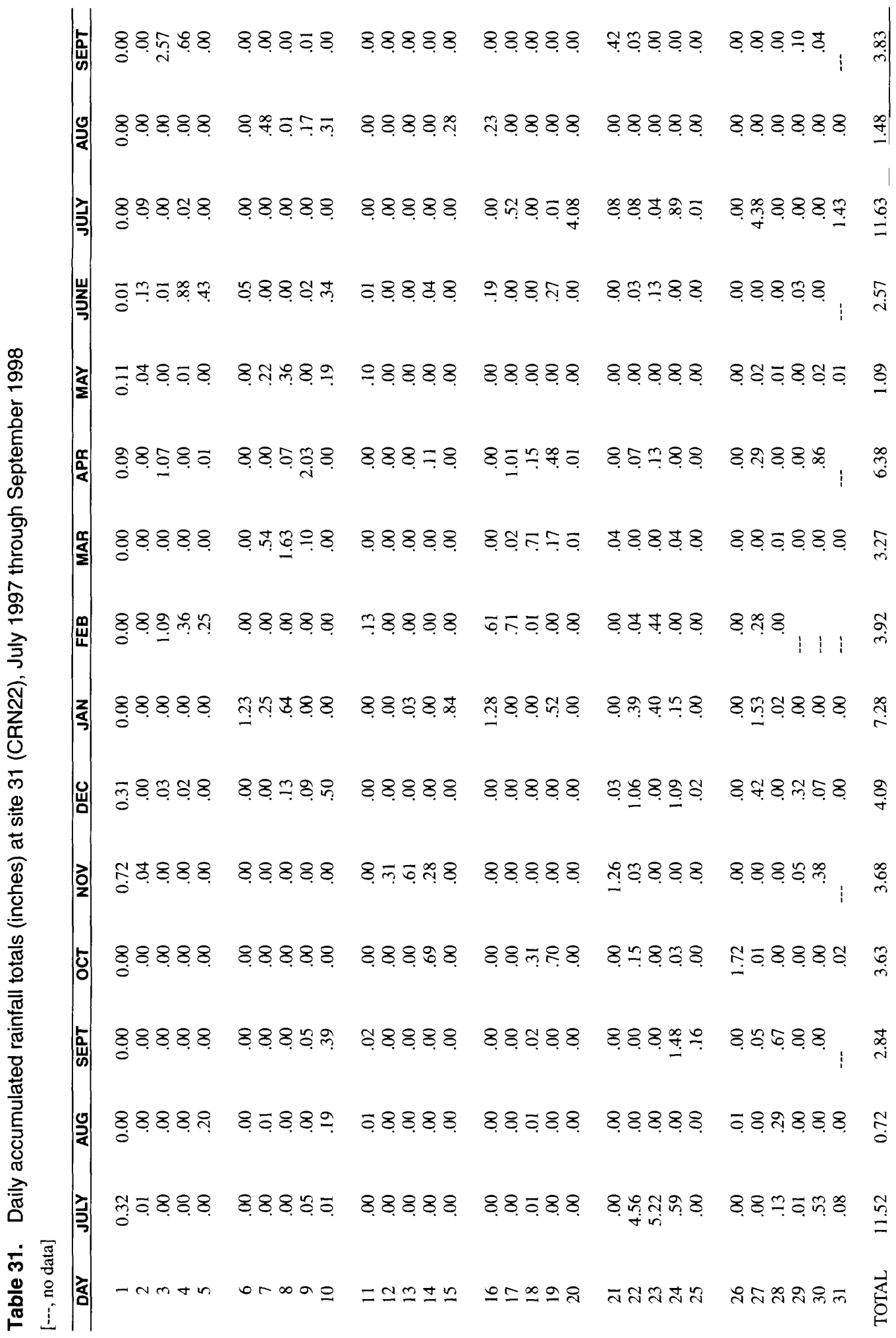




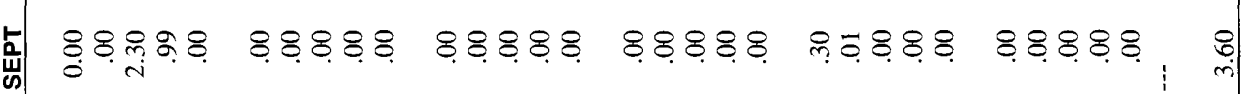

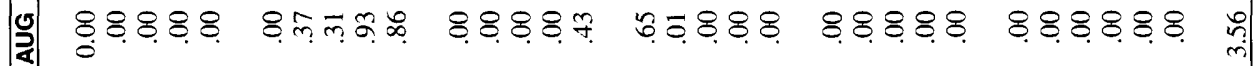

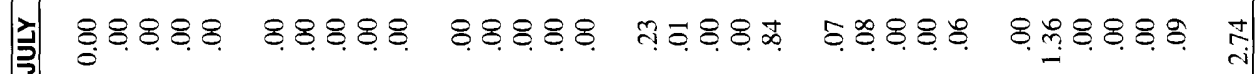

س

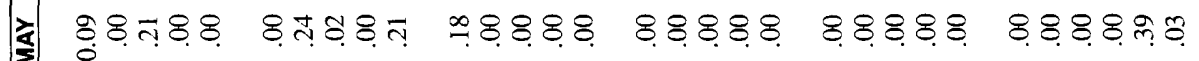

䋮

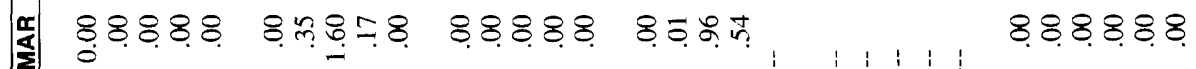

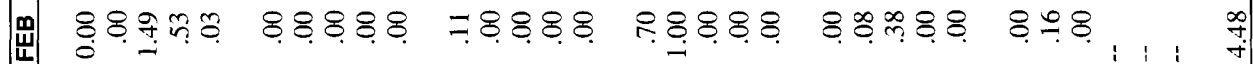

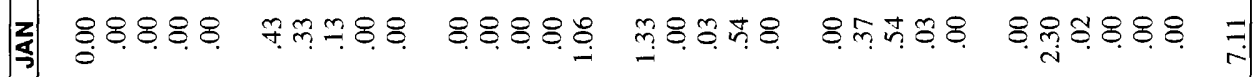

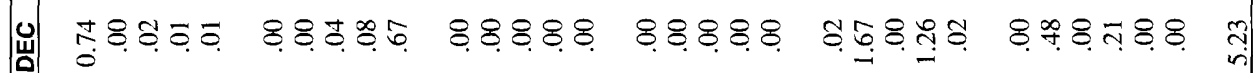

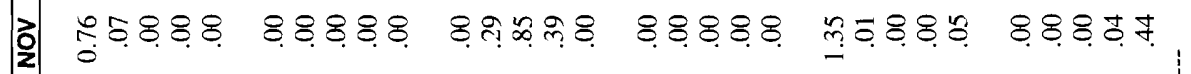

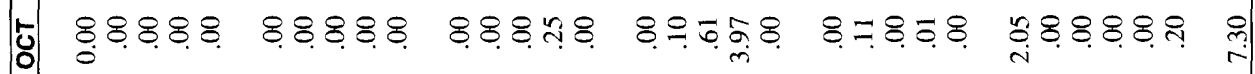

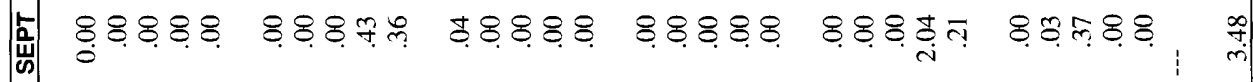

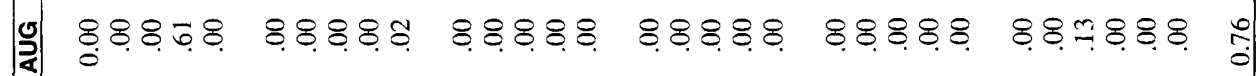

خ

กู่ 藏

- 


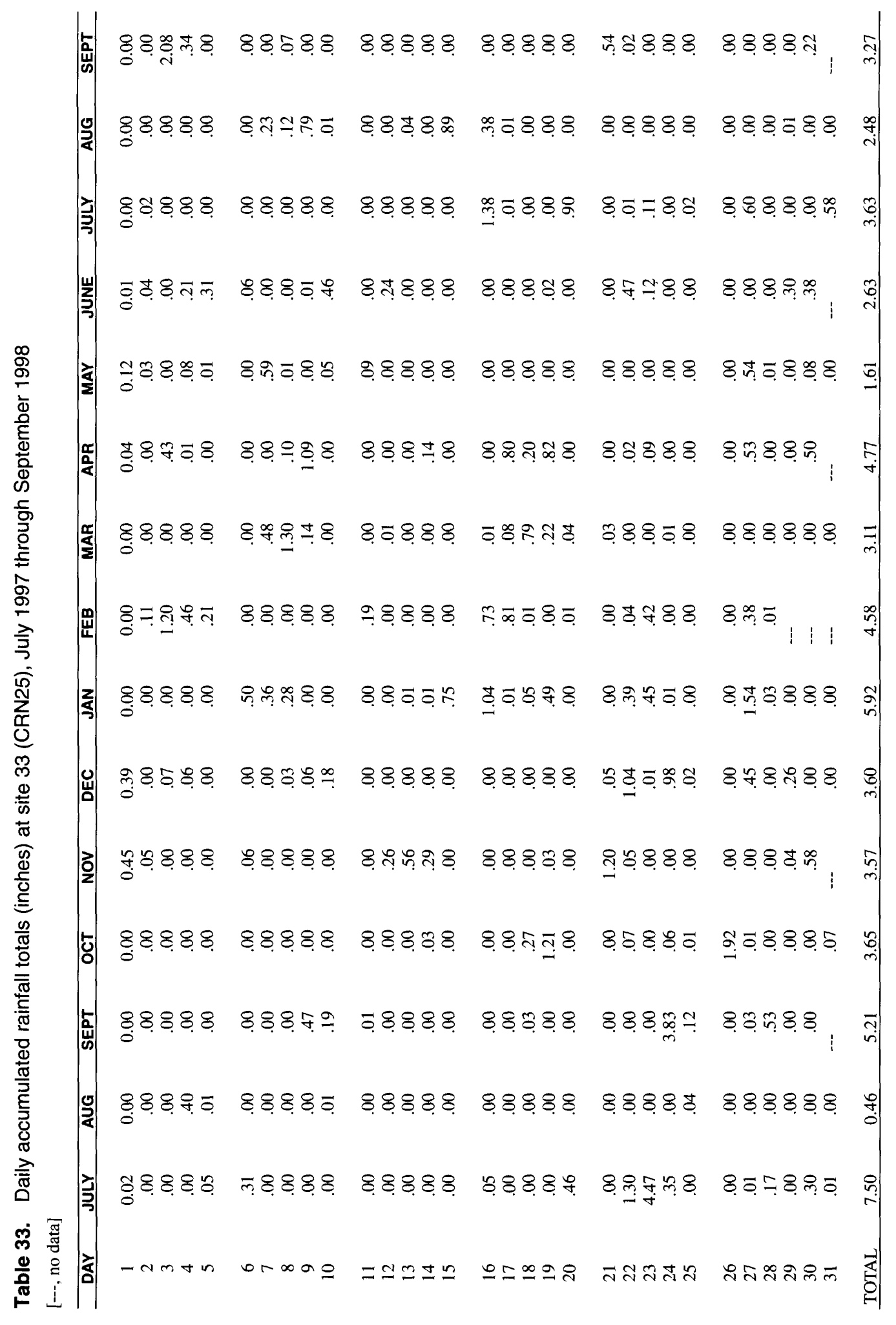


|

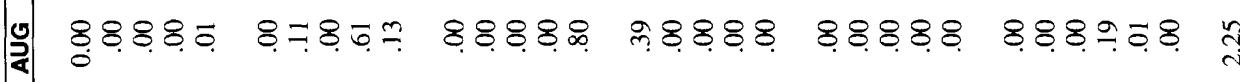

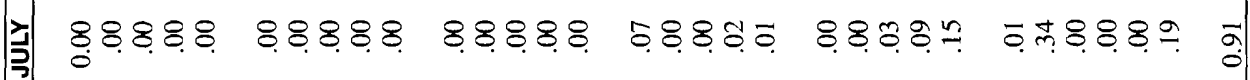

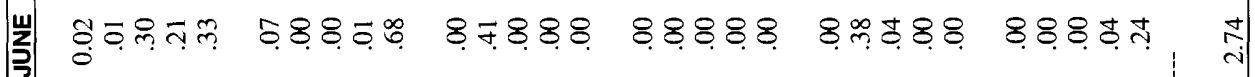

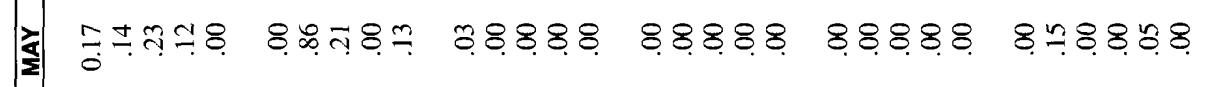

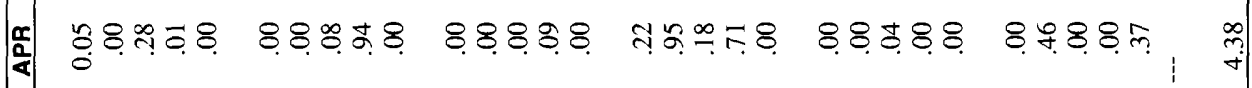

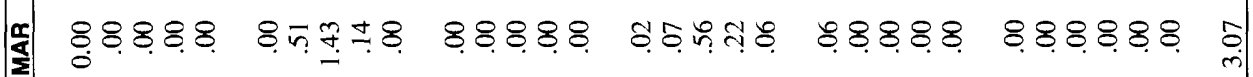

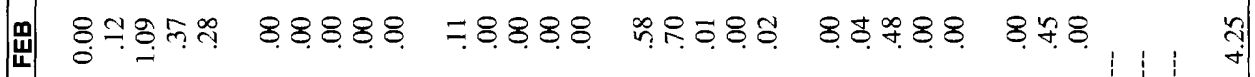

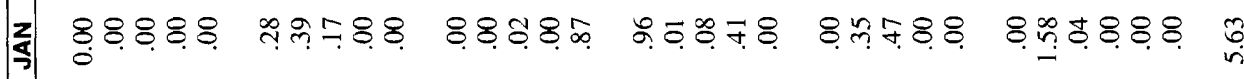

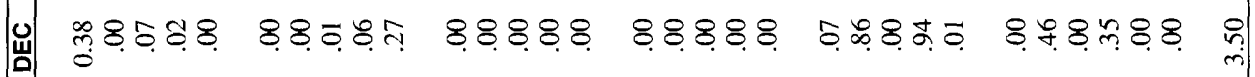

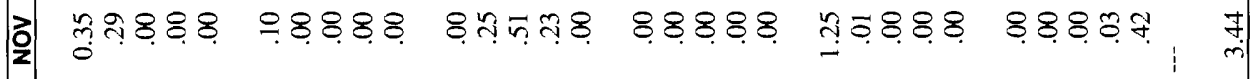

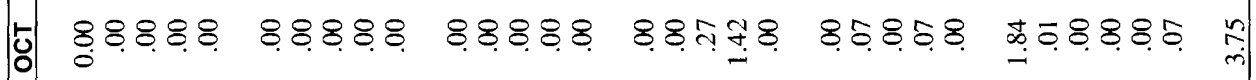

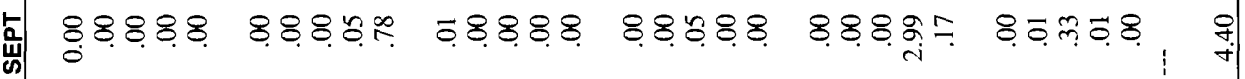

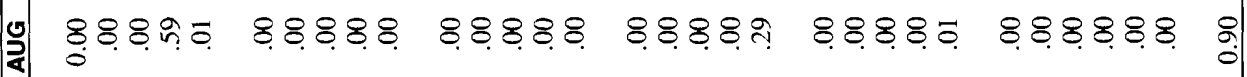

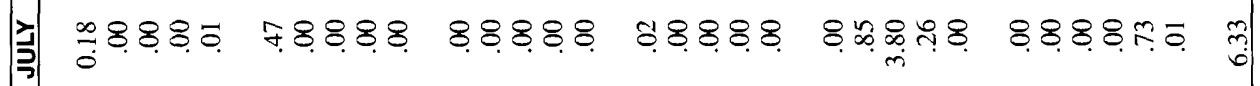

矢 


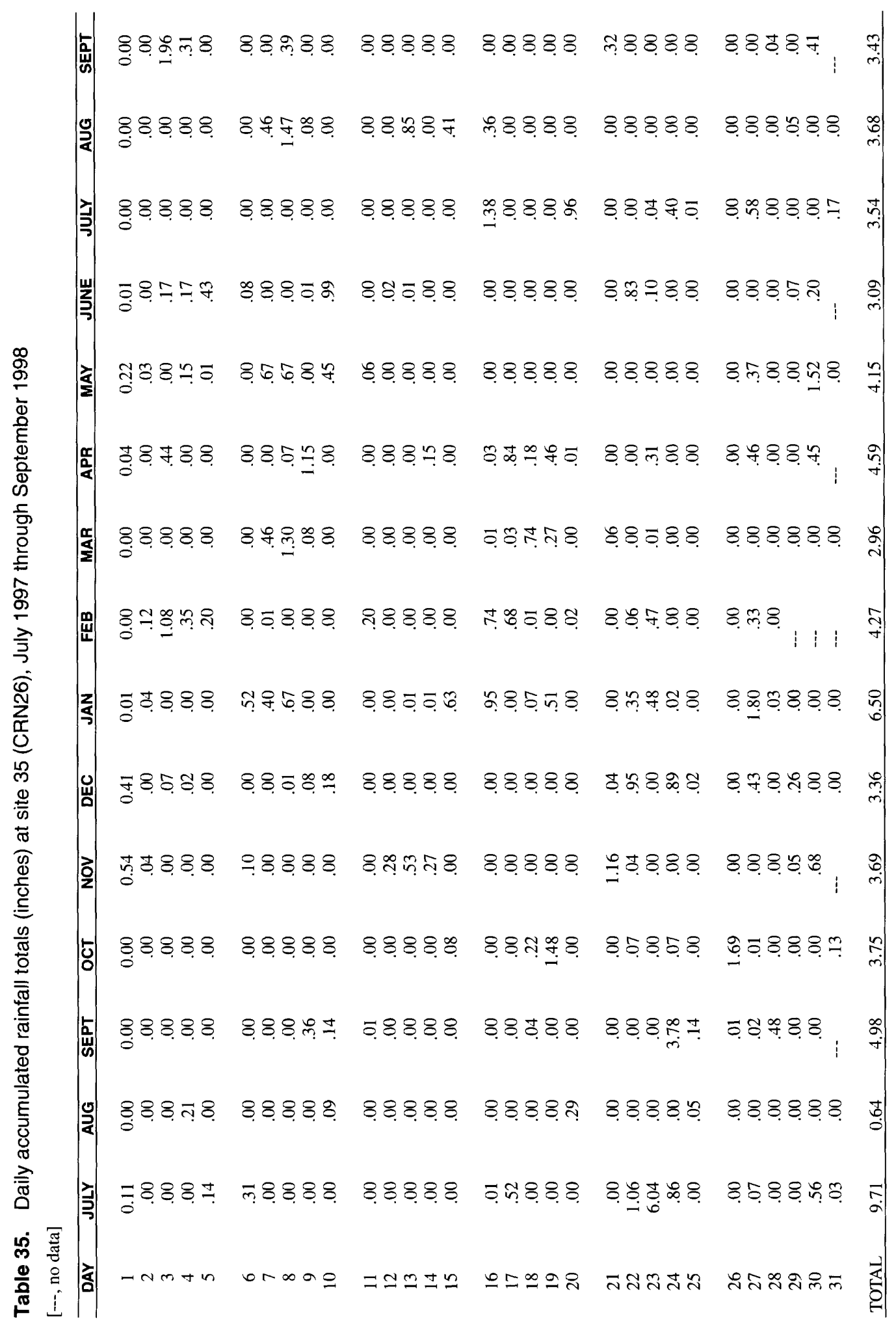


8.8.

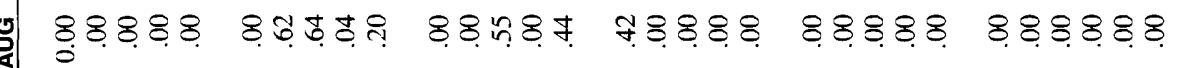

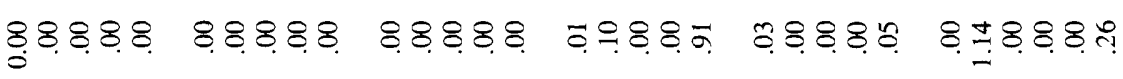

แ

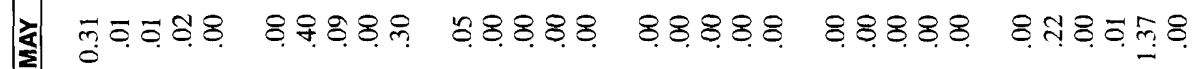

这

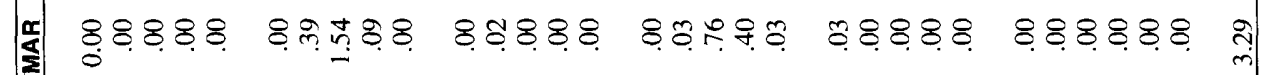

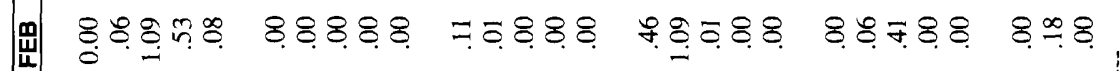

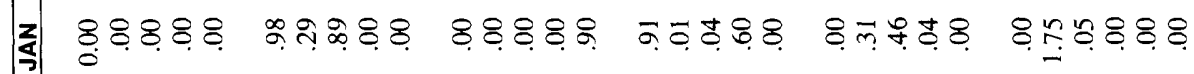

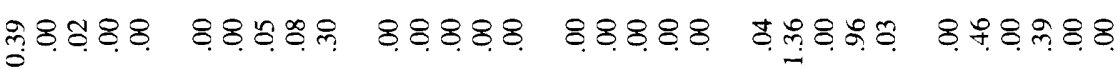

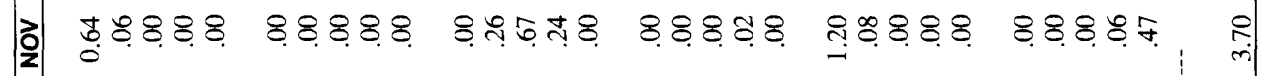

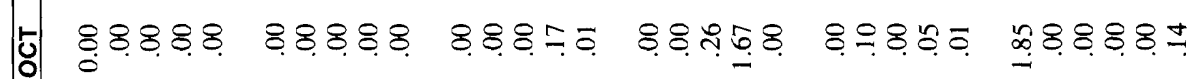

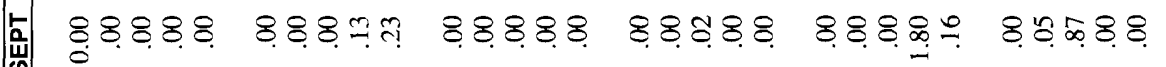

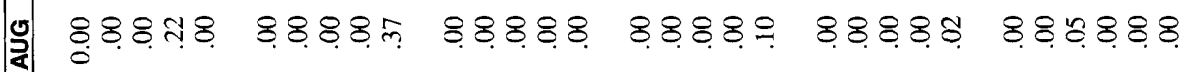

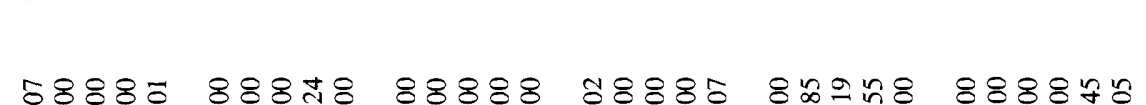

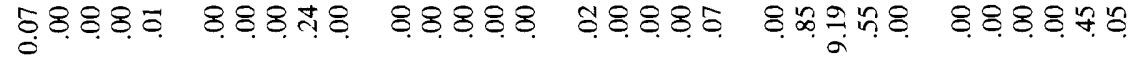

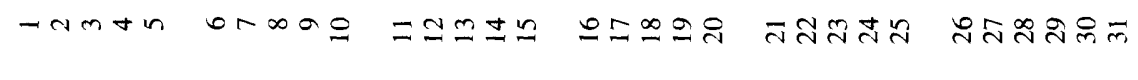




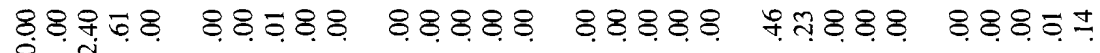

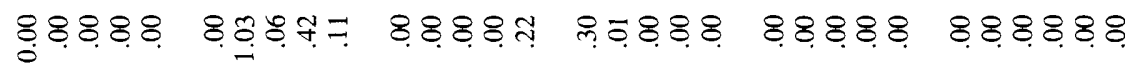

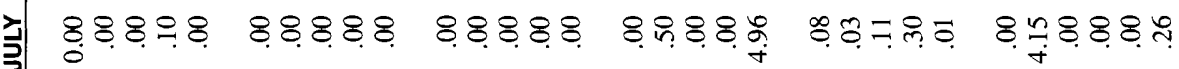

แ

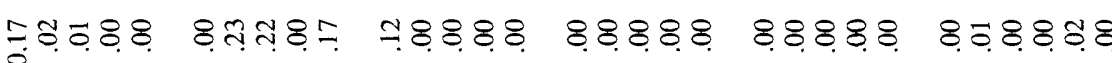

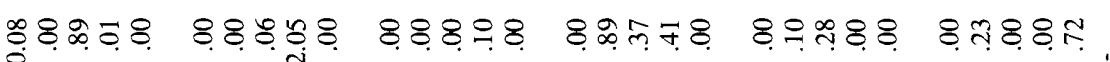

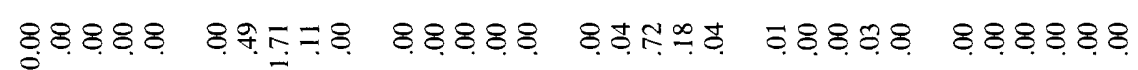

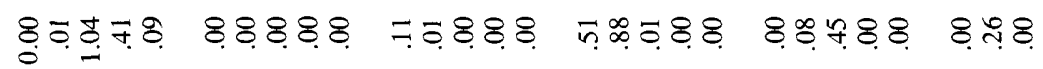

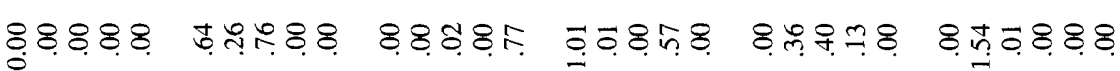

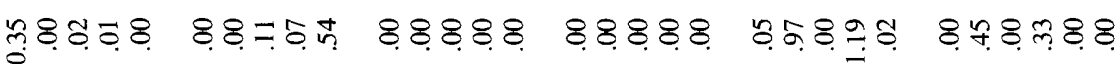

วิ

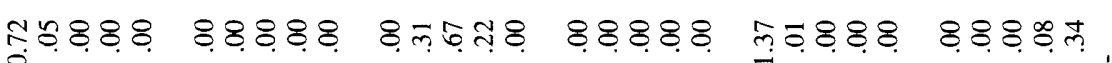

เ.

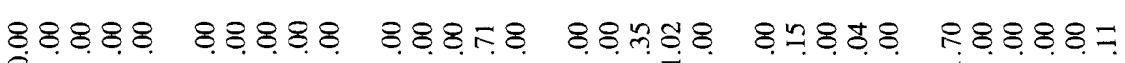

8.8.8.8. 8.8.8.

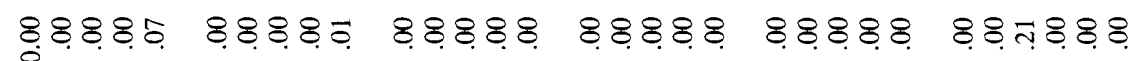

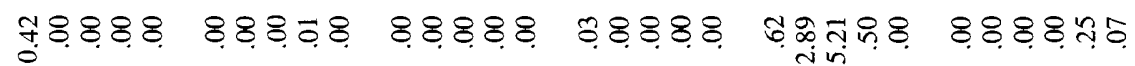

ผ่ 覀

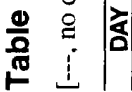

$-4 m+n$

or $\infty$ o

$=\simeq 9 \pm n$

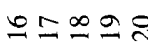

ปปูป

유싱్ㅀㅇㅠ 


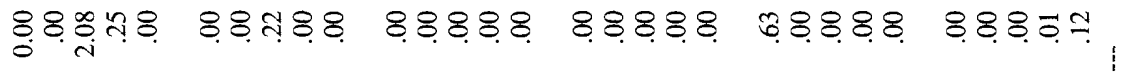

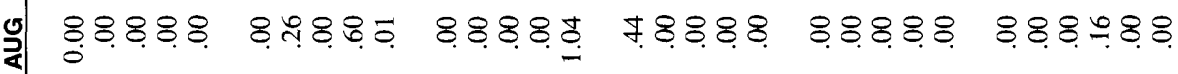

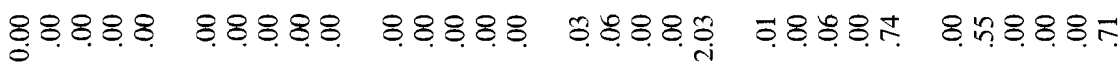

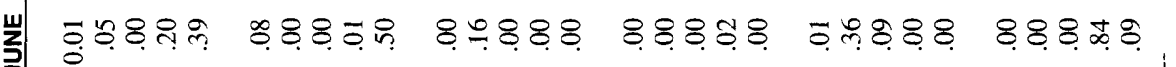

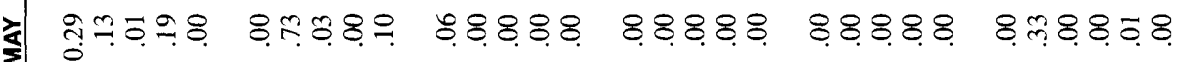

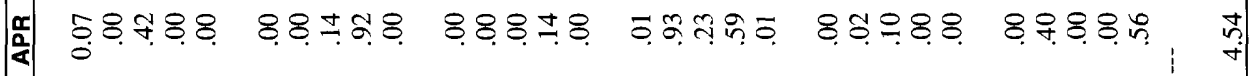

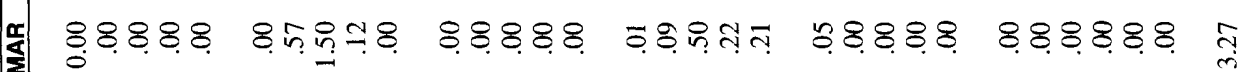

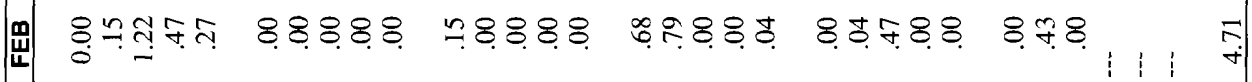

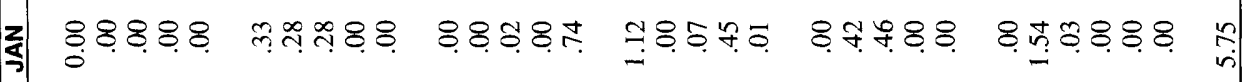

u

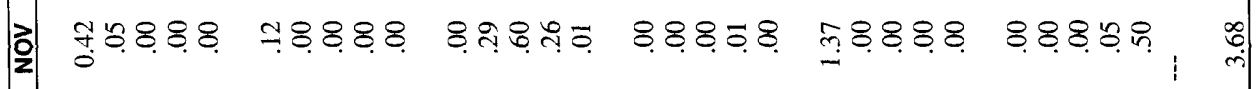

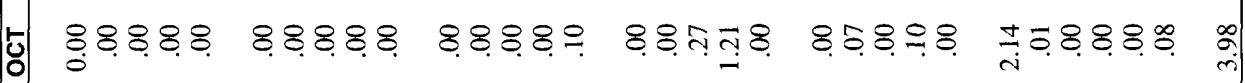

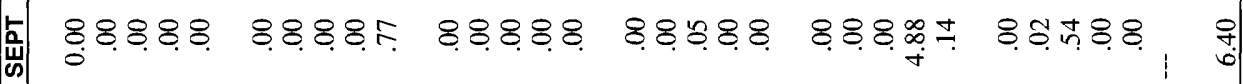

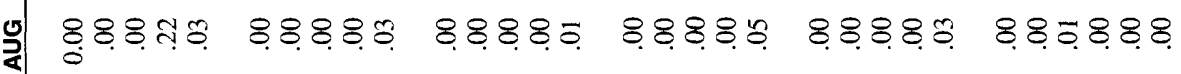

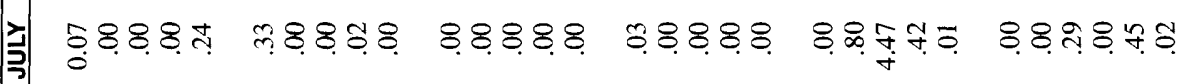

高 


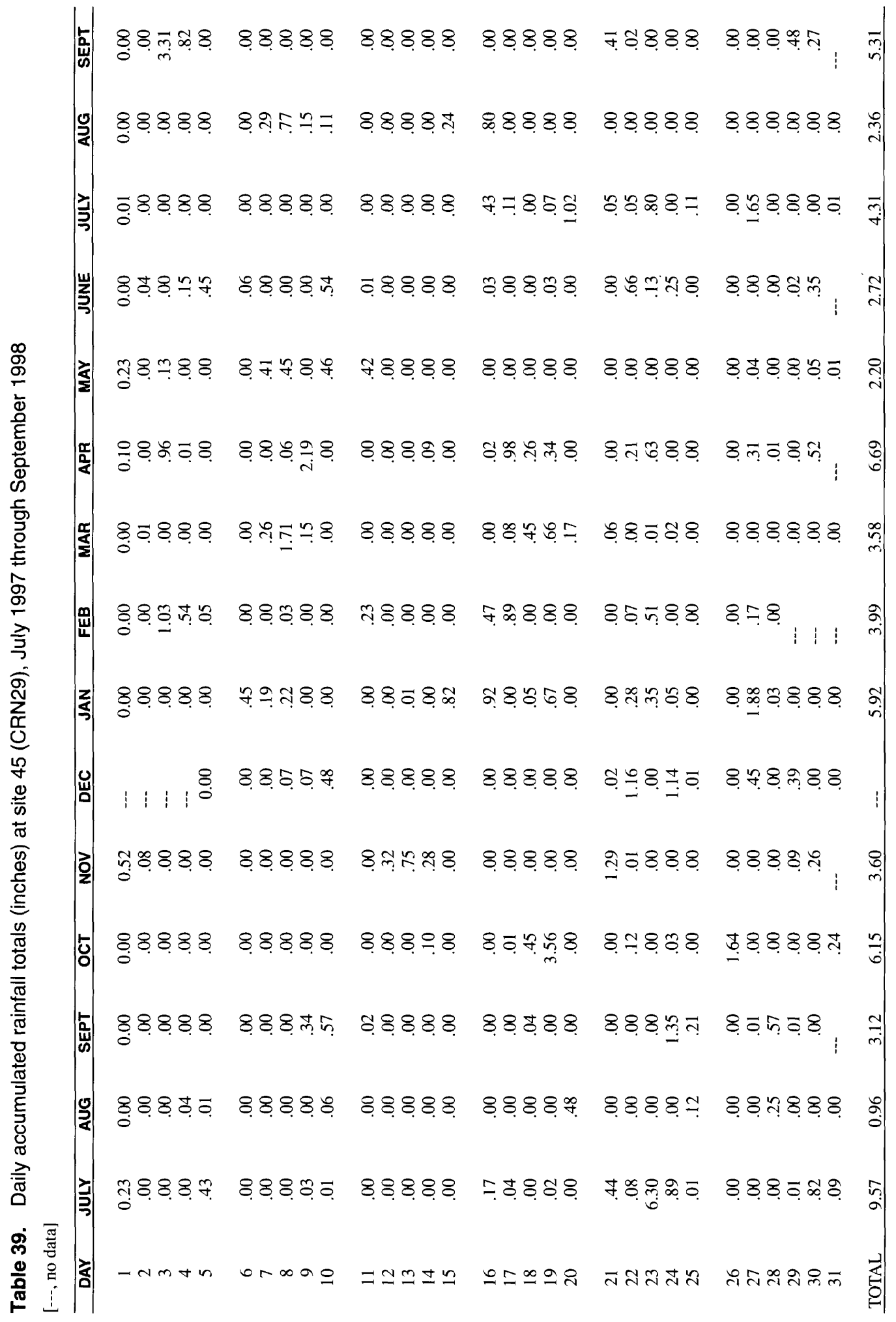




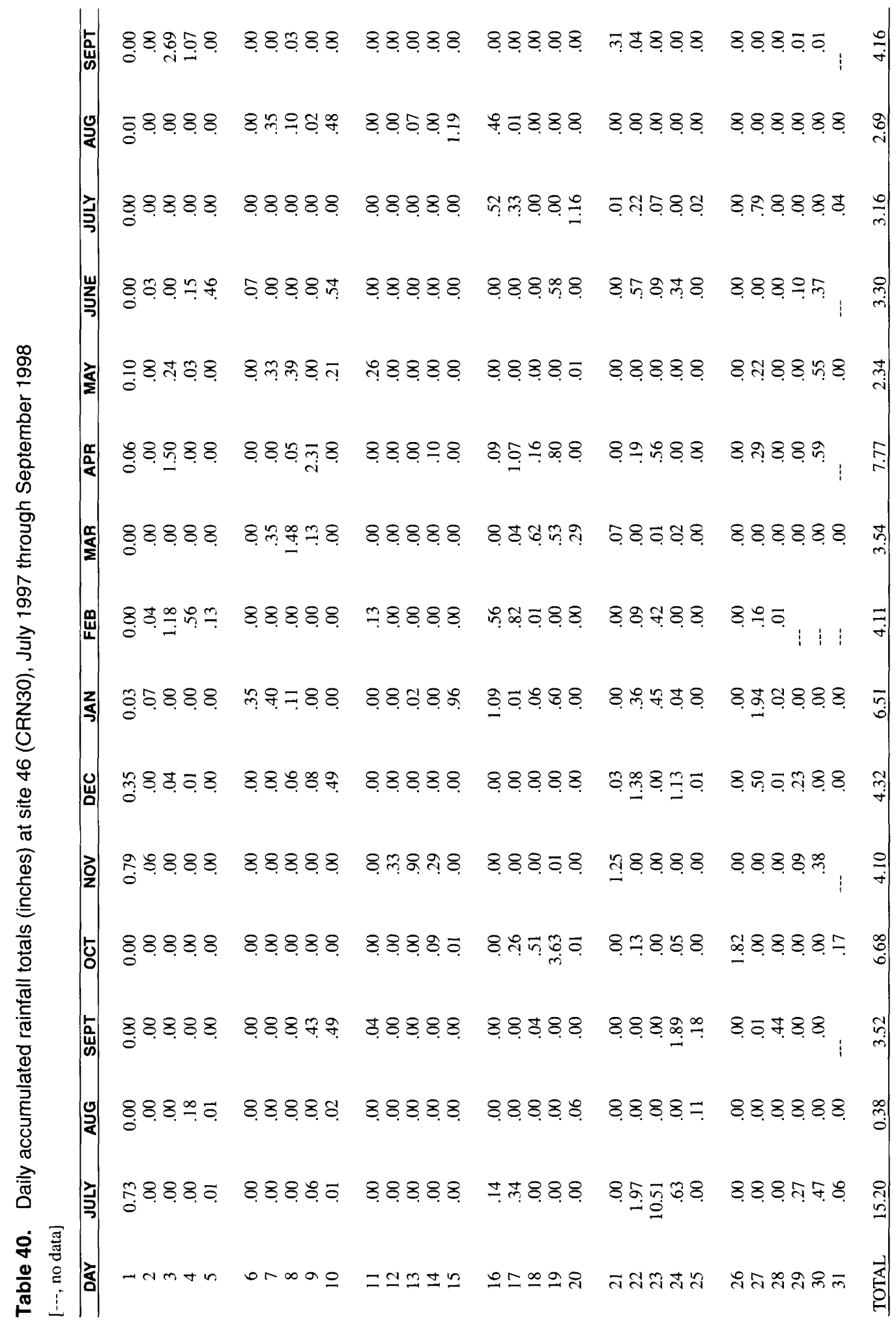




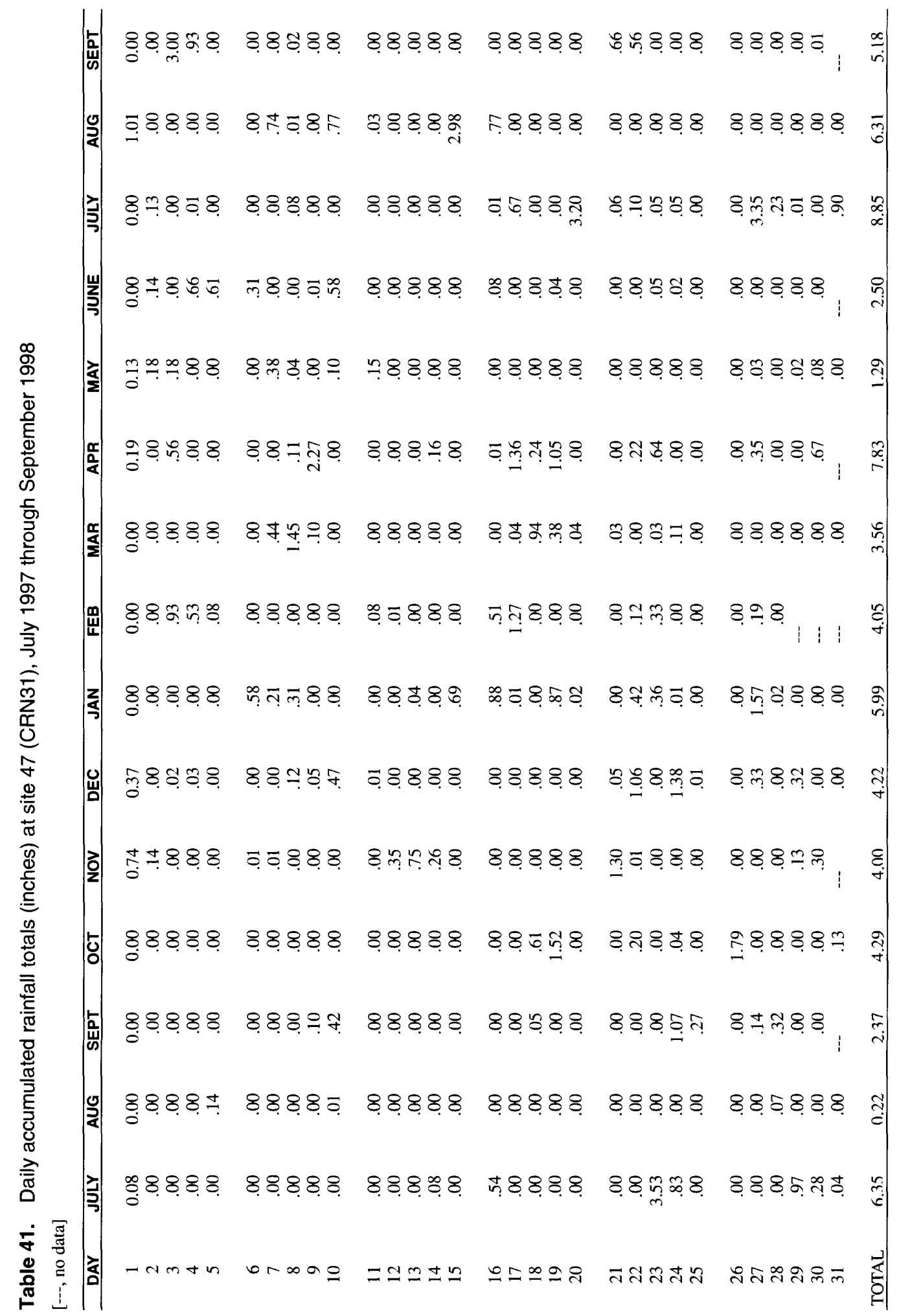




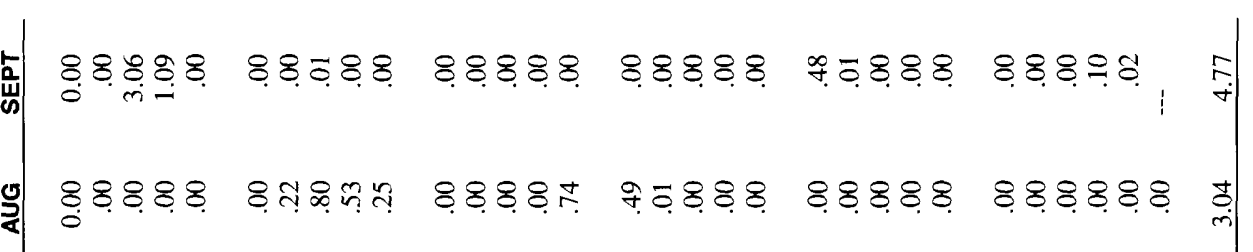

$\Rightarrow$ 8.8.

س

年

ฟ

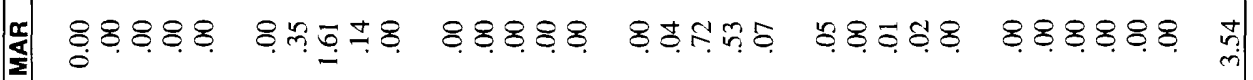

巡

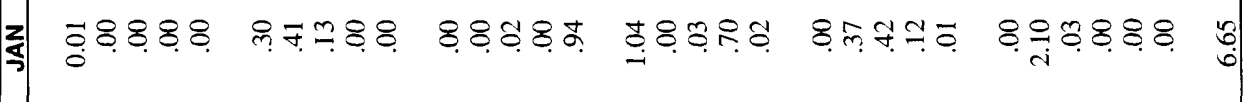

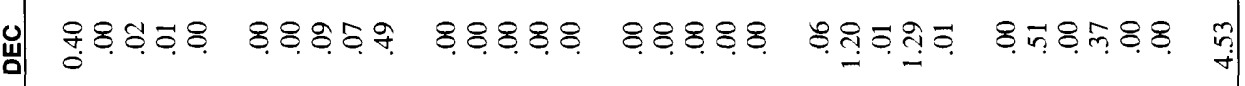

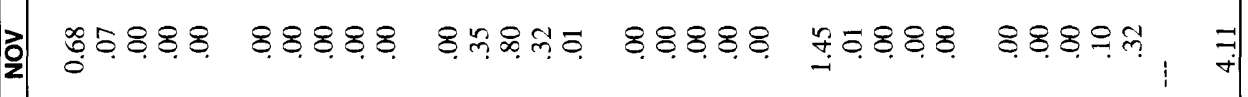

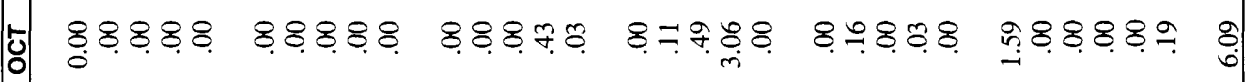

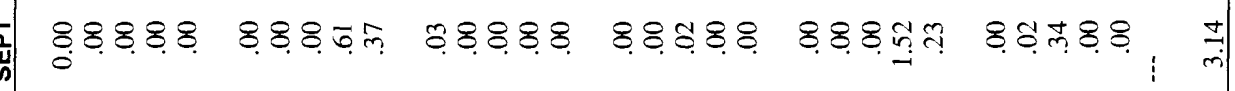

4 \$.8\%

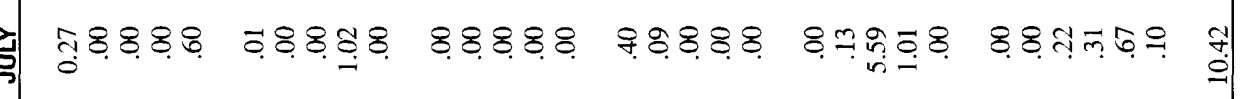




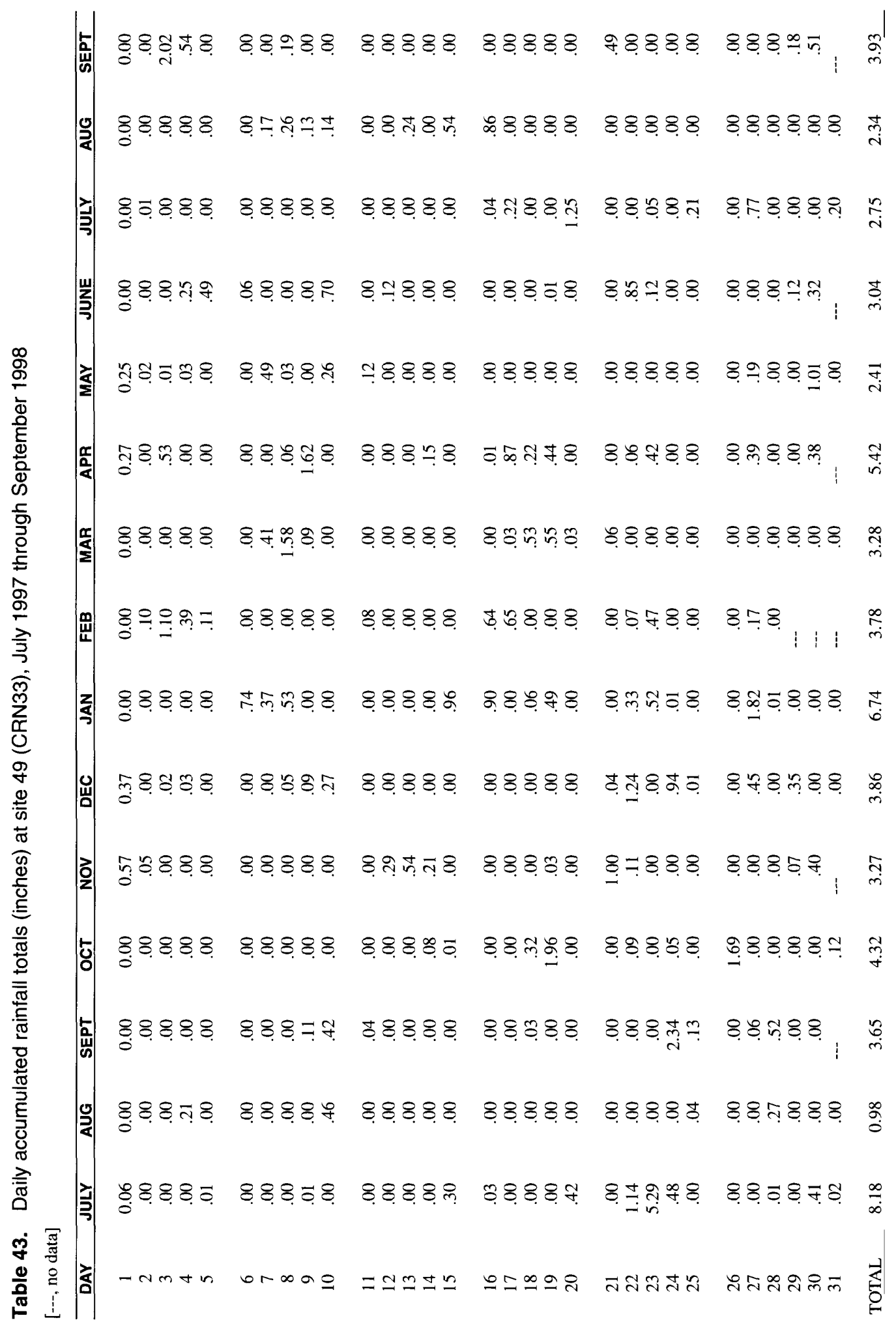




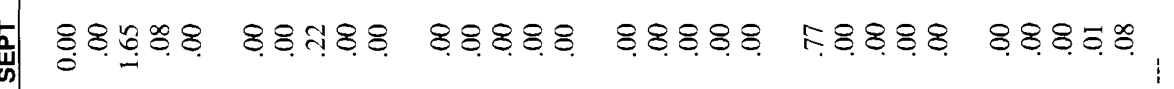

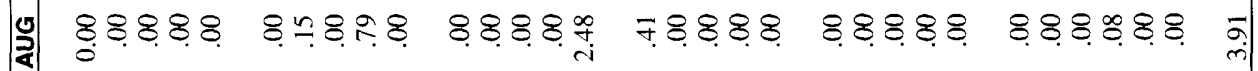

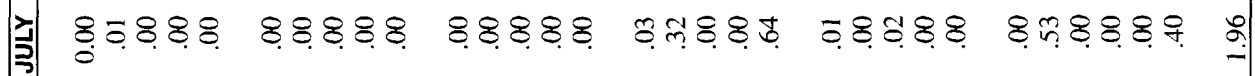

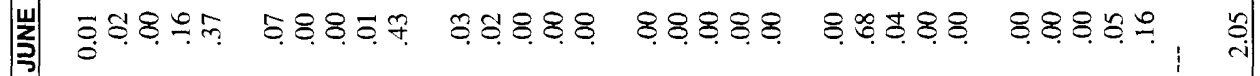

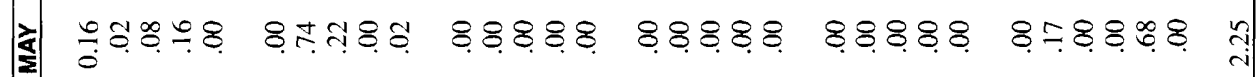

商

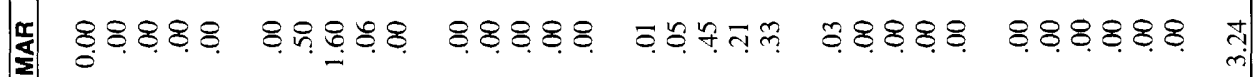

भ

z

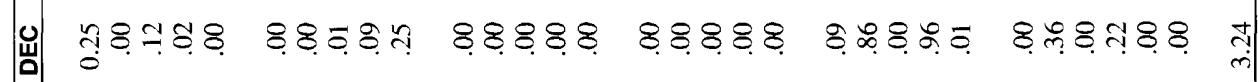

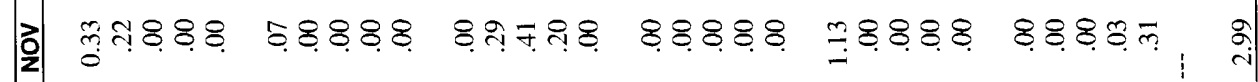

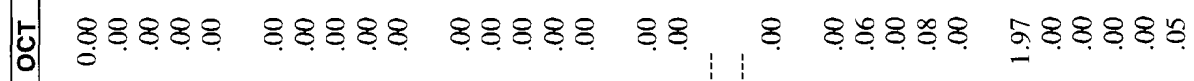

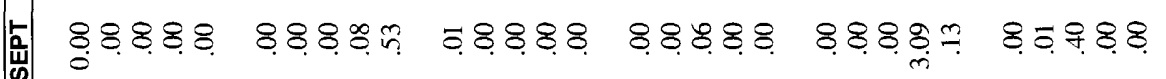

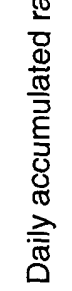

प 8.8\%

$\Rightarrow$ ะ.8.5

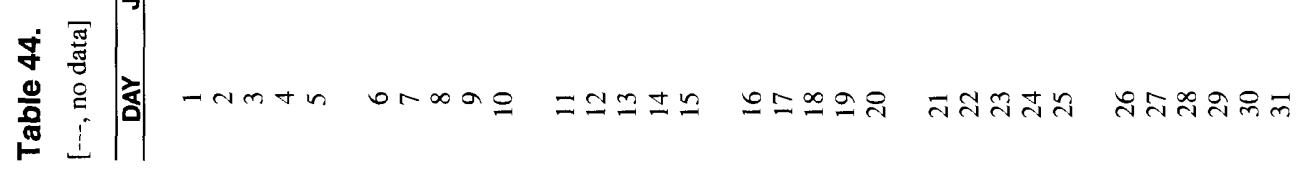




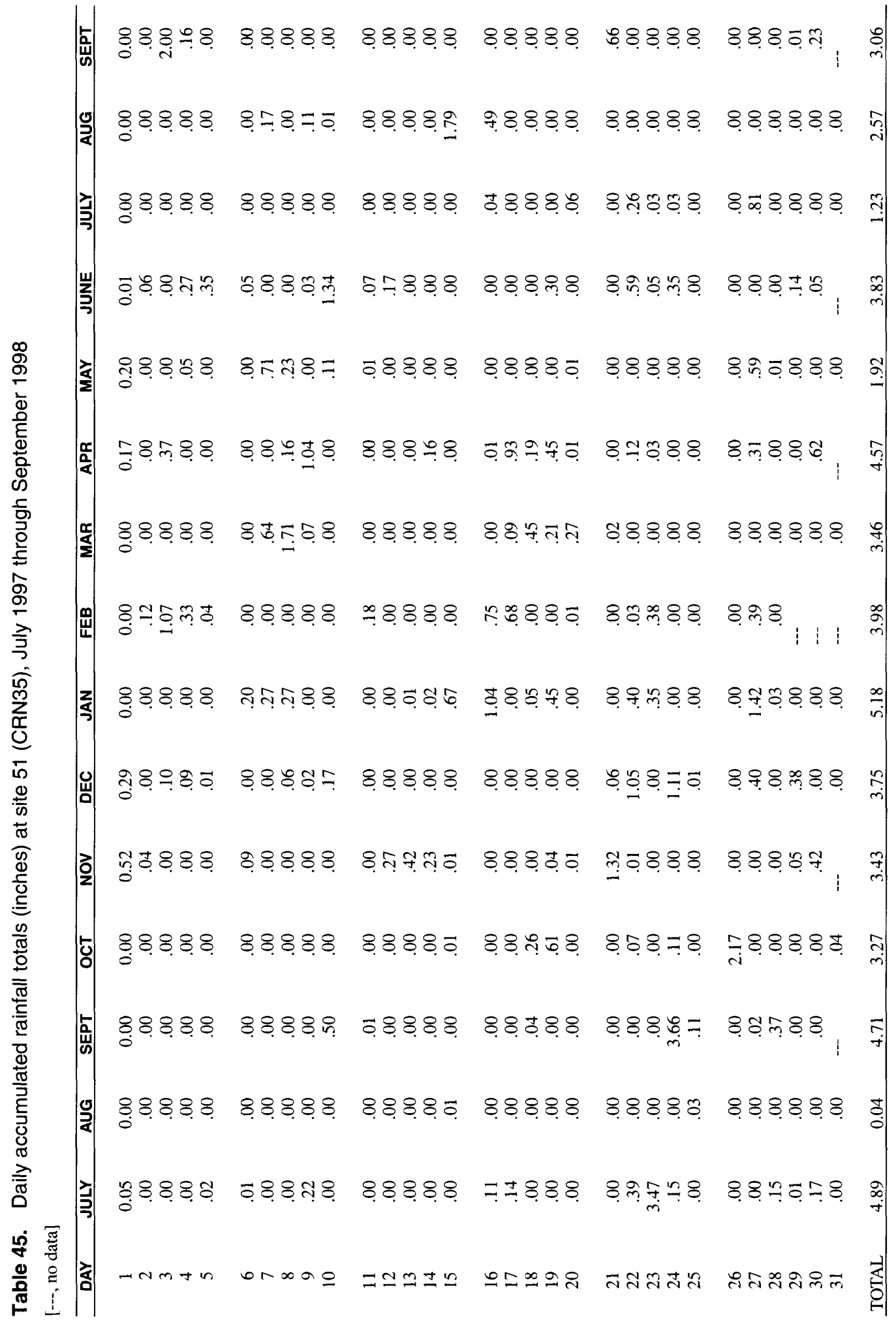




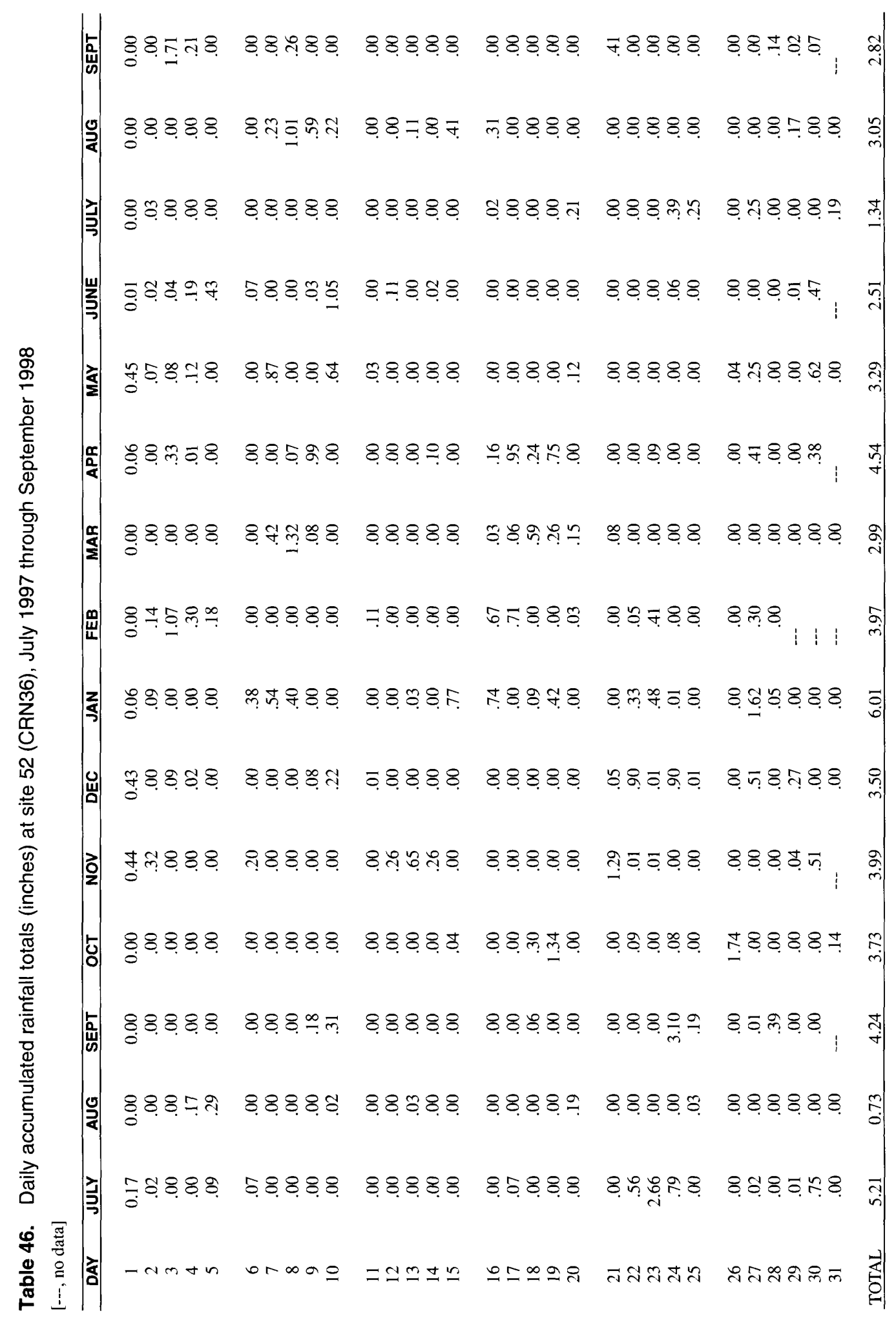




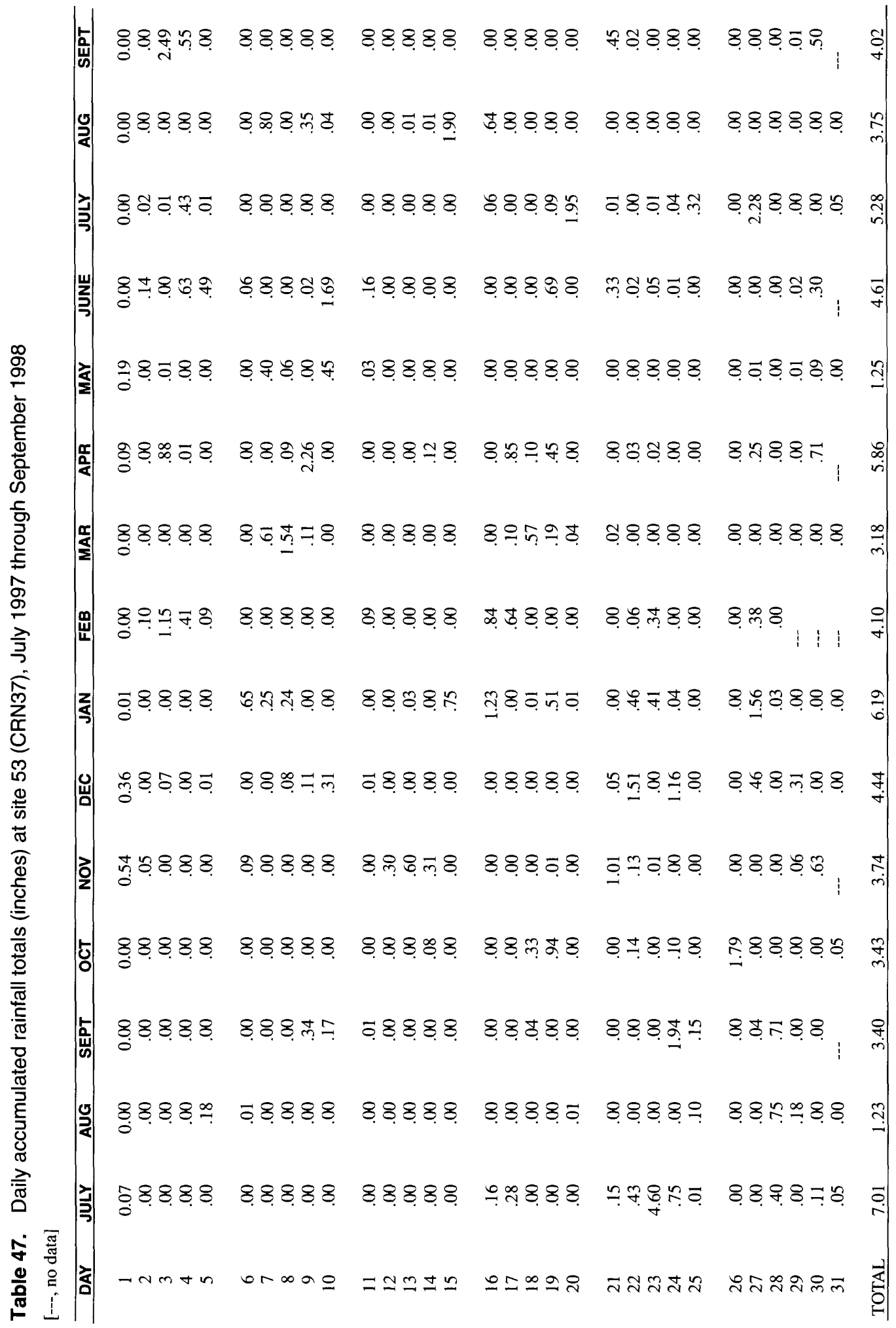


点

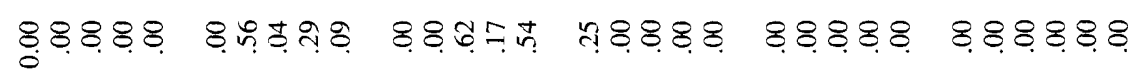

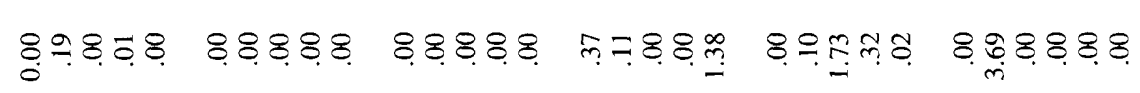

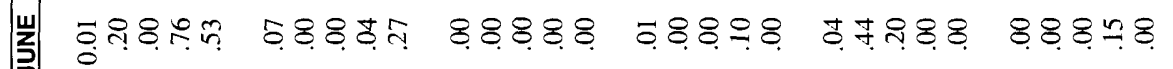

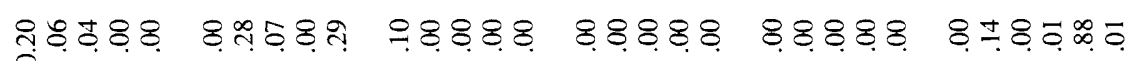

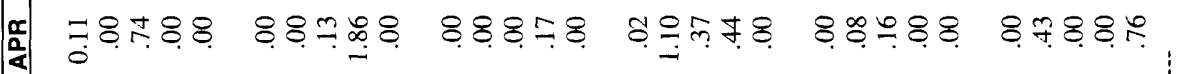

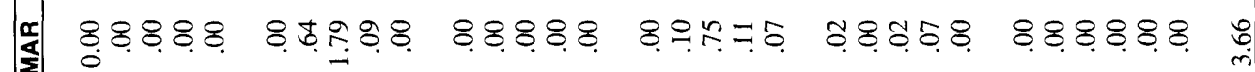

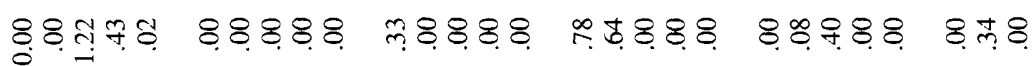

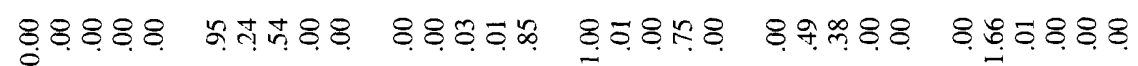

ஸे

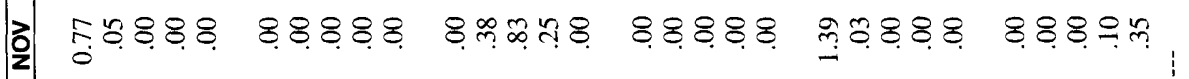

용

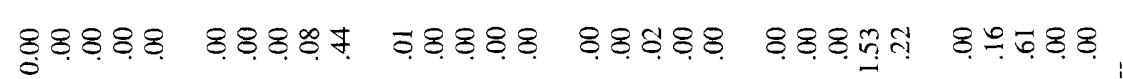

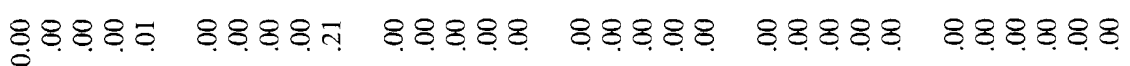

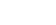

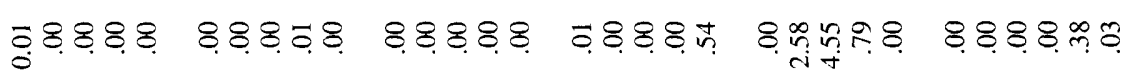

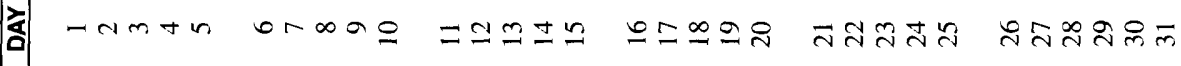




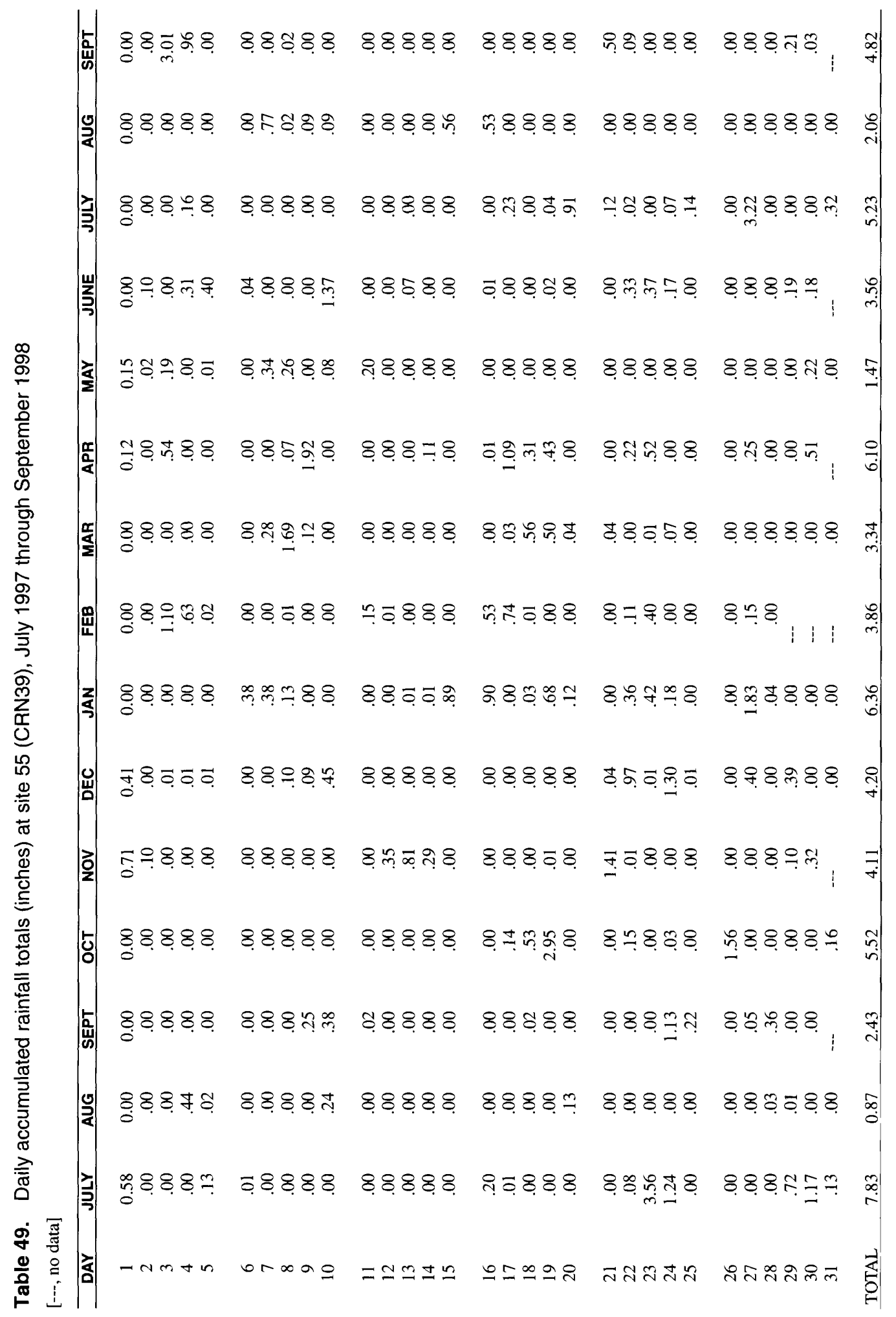




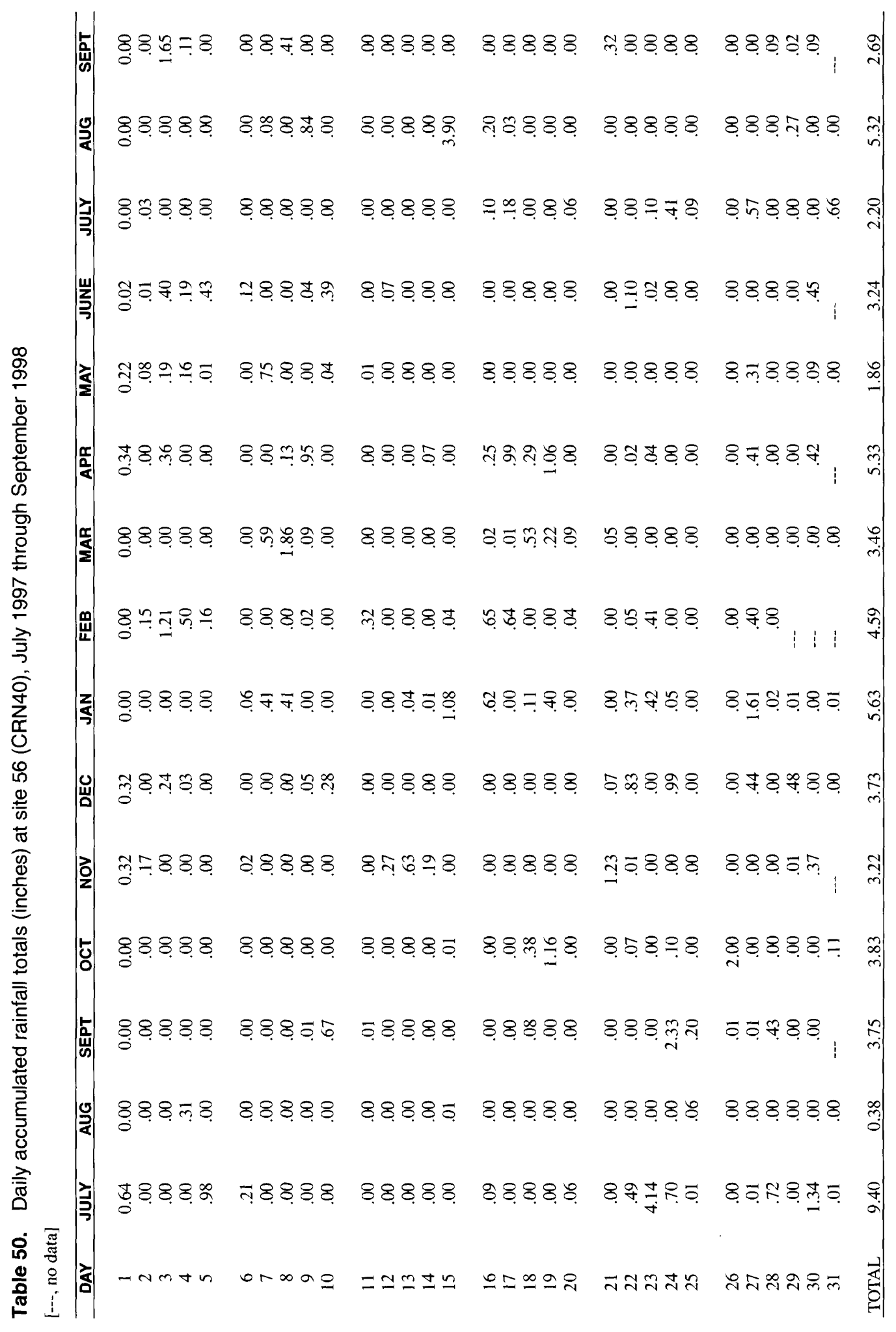




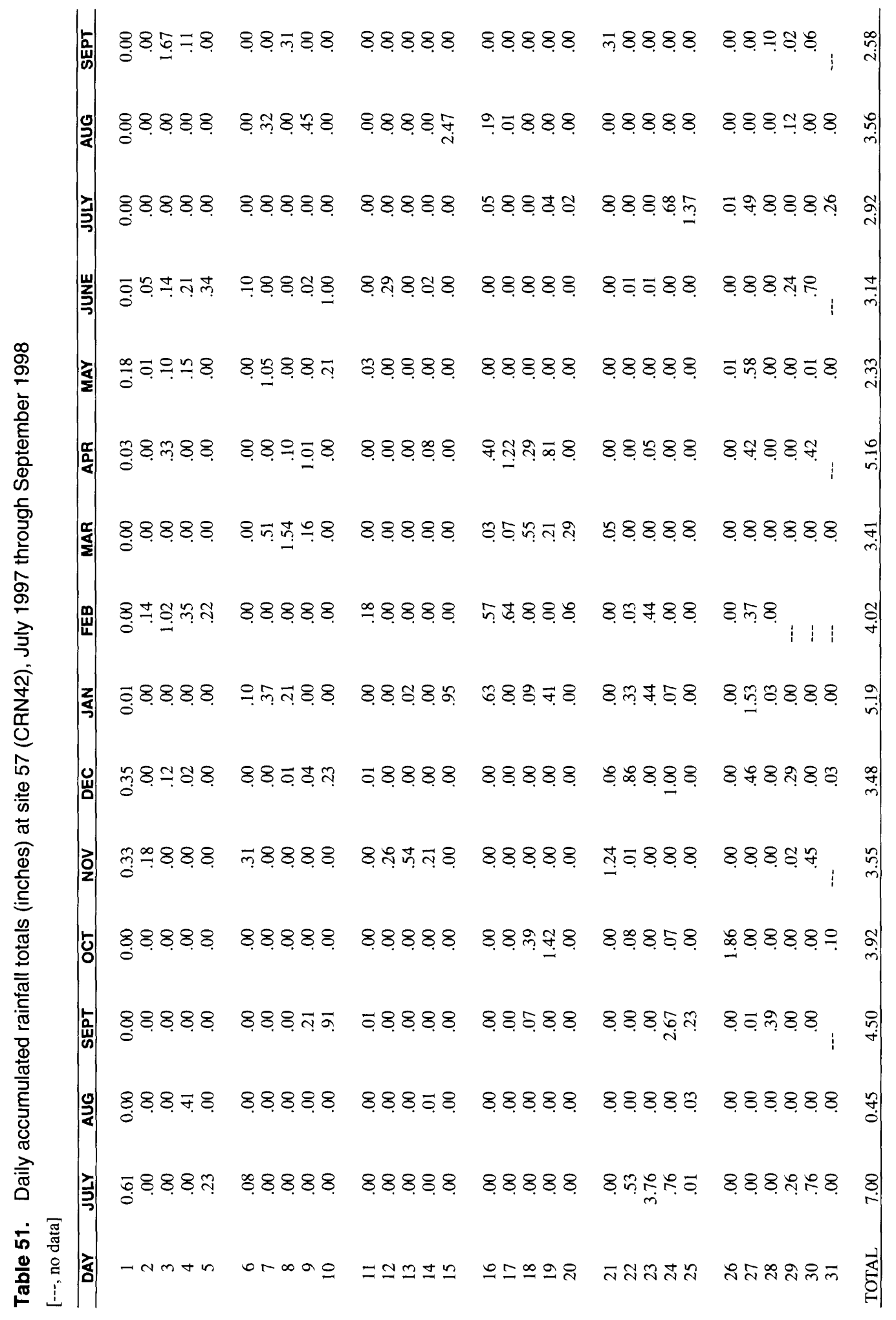




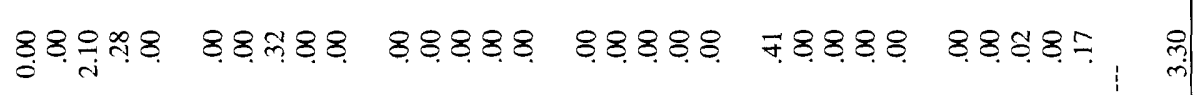

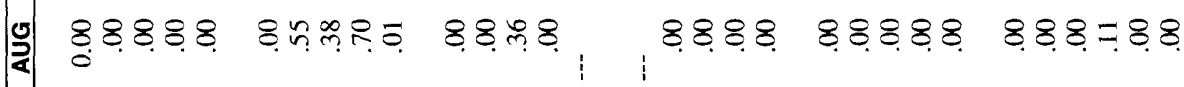

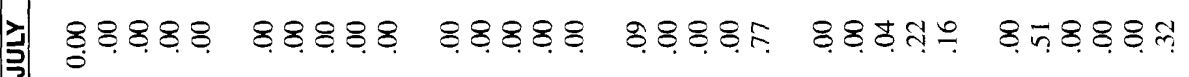

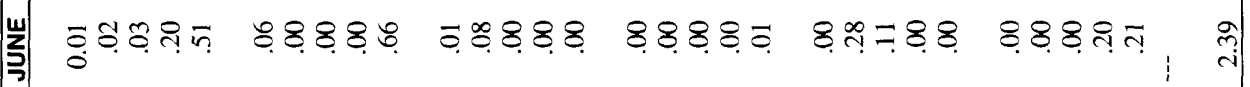

$\overrightarrow{\mathbf{a}}$

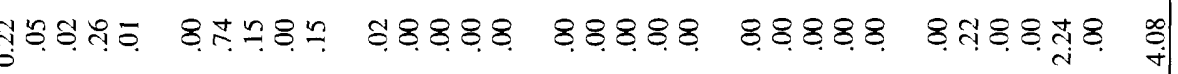

产

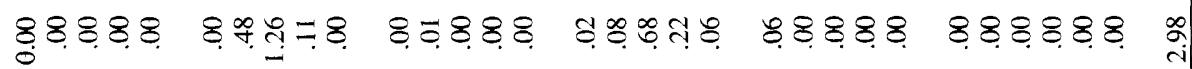

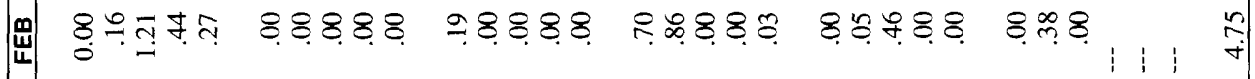

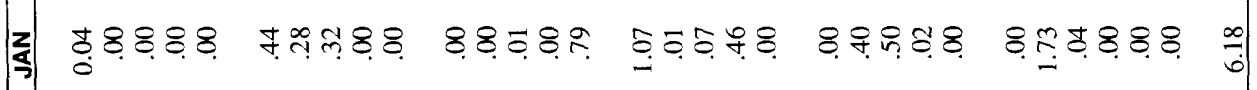

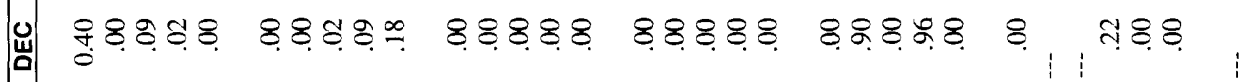

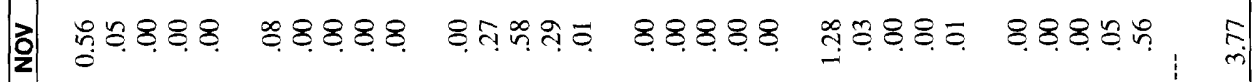

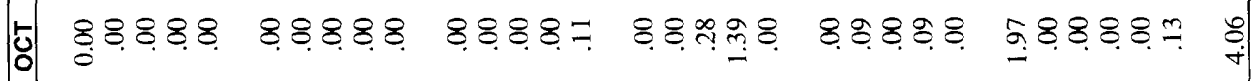

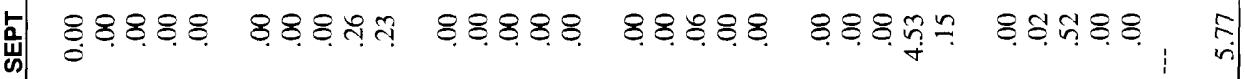

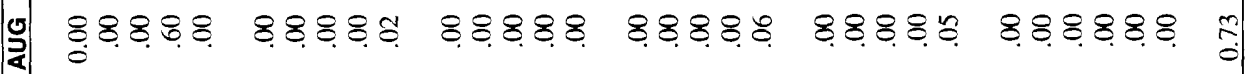

ว

ஸิ่

堐 吾 


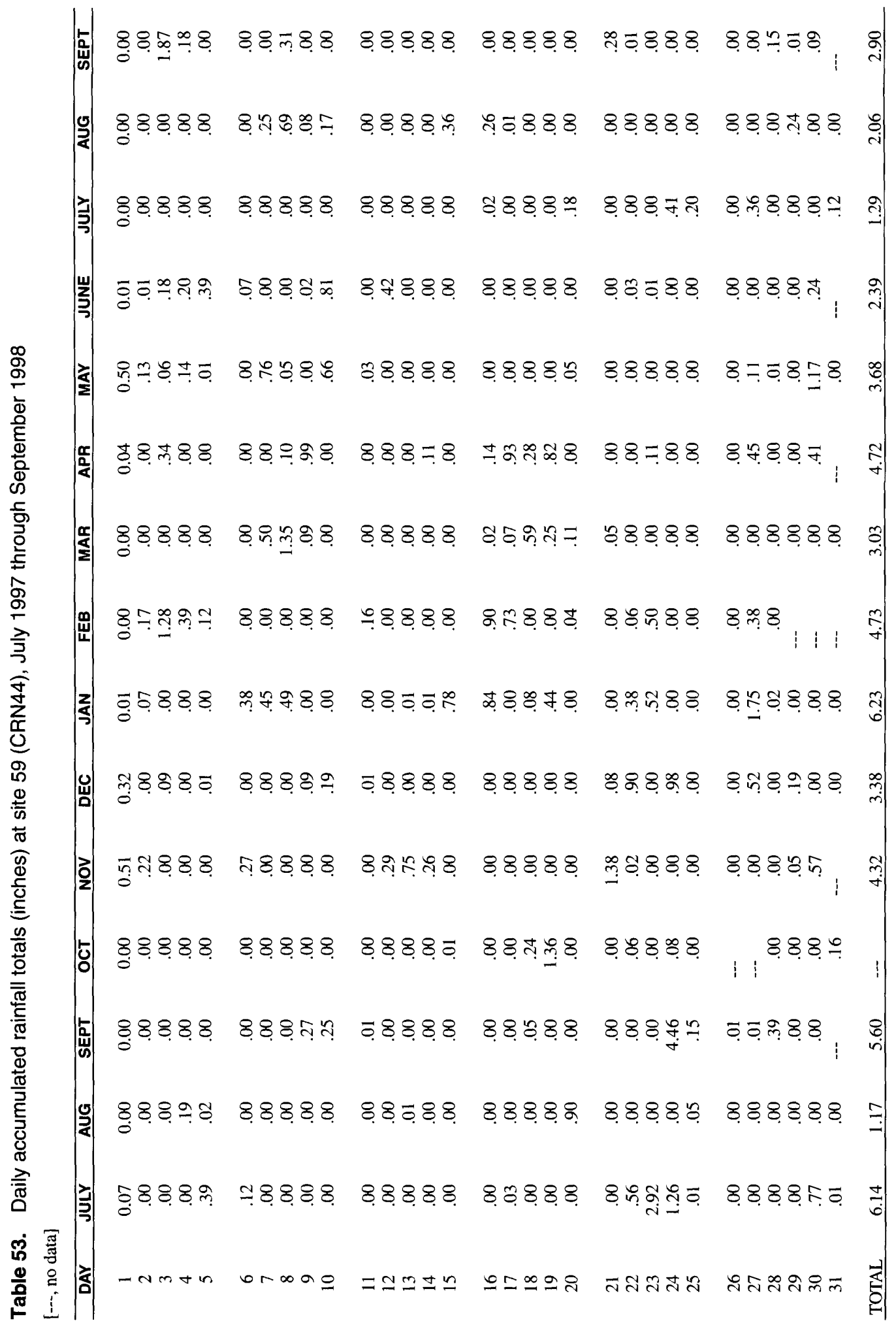




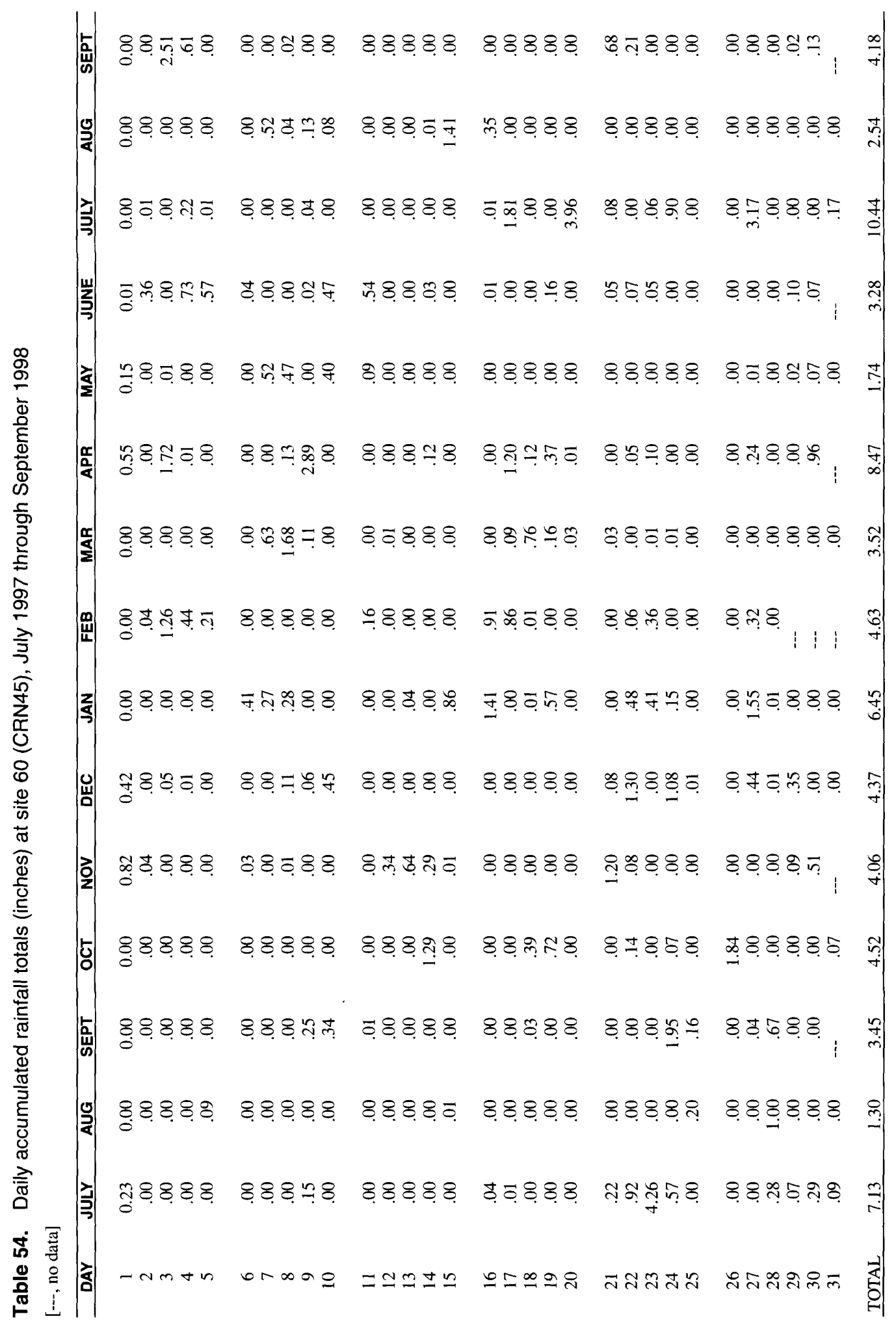




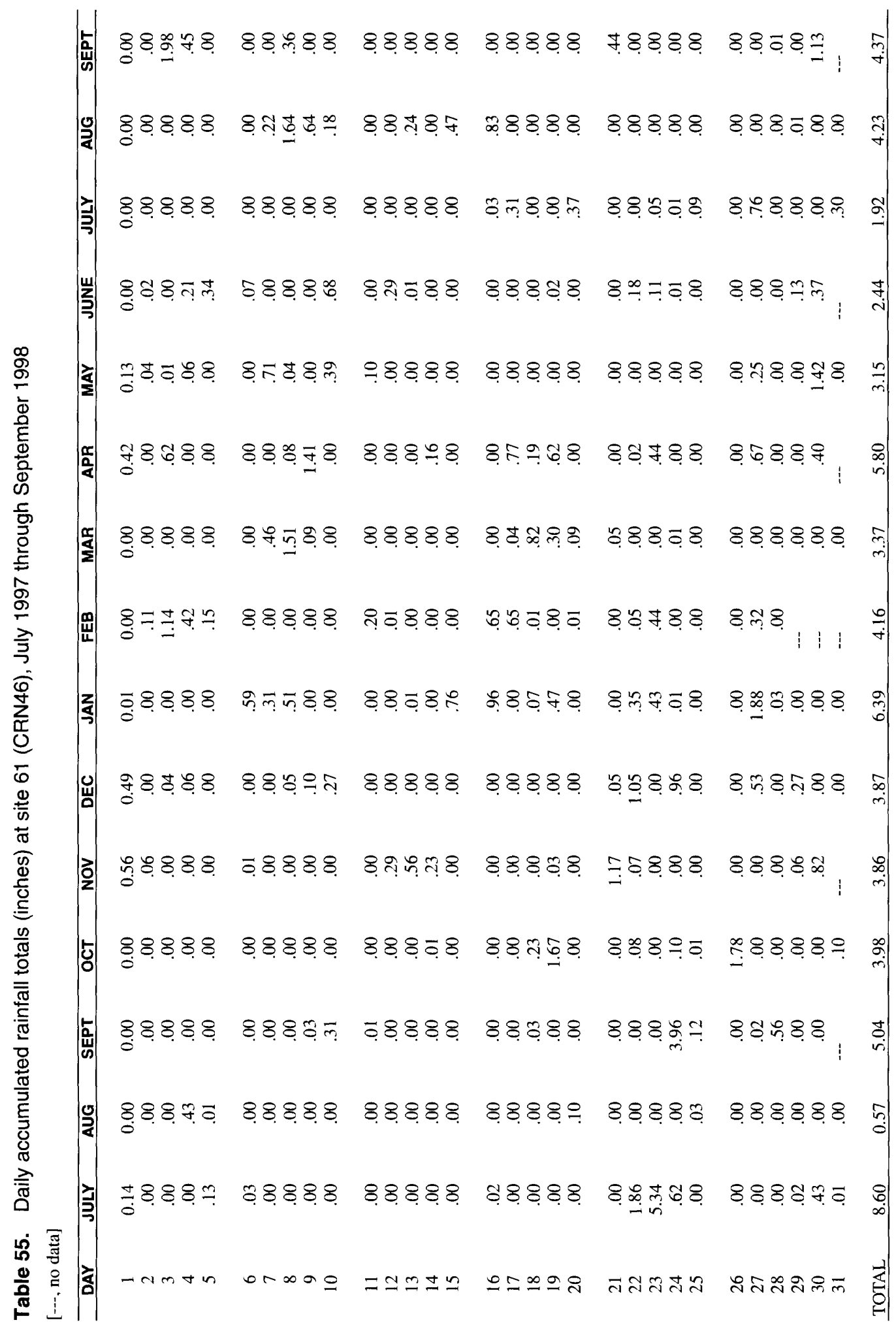


Table 56. Statistical summary of atmospheric deposition water-quality data at site 37 (CSWO6), March 1997 thrcugh March 1998

\begin{tabular}{|c|c|c|c|c|c|c|c|c|c|c|}
\hline \multirow{2}{*}{$\begin{array}{c}\text { PARAM- } \\
\text { ETER } \\
\text { CODE }\end{array}$} & \multirow{2}{*}{ PROPERTY OR CONSTITUENT } & \multicolumn{4}{|c|}{ DESCRIPTIVE STATISTICS } & \multicolumn{5}{|c|}{$\begin{array}{c}\text { PERCENT OF SAMPLES IN WHICH VALUES WERE LESS } \\
\text { THAN OR EQUAL TO THOSE SHOWN }\end{array}$} \\
\hline & & $\begin{array}{l}\text { SAMPLE } \\
\text { SIZE }\end{array}$ & MAXIMUM & MINIMUM & MEAN & $95 \%$ & $75 \%$ & $\begin{array}{c}50 \% \\
\text { (median) }\end{array}$ & $25 \%$ & $5 \%$ \\
\hline \multicolumn{11}{|c|}{ PHYSICAL AND CHEMICAL PROPERTIES } \\
\hline 00045 & RAINFALL (in./wk) & 52 & 9.220 & 0.000 & 0.964 & 2.820 & 1.525 & 0.555 & 0.027 & 0.000 \\
\hline 00046 & RAINFALL, TOTAL (in./wk) & 53 & 8.020 & 0.000 & 0.944 & 2.857 & 1.460 & 0.440 & 0.027 & 0.000 \\
\hline 90095 & $\begin{array}{l}\text { SPECIFIC CONDUCTANCE, } \\
\mathrm{LAB}\left(\mu \mathrm{S} / \mathrm{cm} \text { at } 25^{\circ} \mathrm{C}\right)\end{array}$ & 34 & 78.000 & 6.000 & 25.529 & 78.000 & 32.750 & 19.000 & 13.759 & 6.750 \\
\hline 00403 & $\begin{array}{l}\text { pH, LAB (STANDARD pH } \\
\text { UNITS) }\end{array}$ & 39 & 5.500 & 3.200 & 4.467 & 5.400 & 4.700 & 4.500 & 4.107 & 3.600 \\
\hline 00940 & $\begin{array}{l}\text { CHLORIDE, DISSOLVED } \\
(\mathrm{mg} / \mathrm{L} \text { as } \mathrm{Cl})\end{array}$ & 35 & 32.000 & $<2.000$ & - & 3.400 & $<2.000$ & $<2.000$ & $<2.00$ ? & $<2.000$ \\
\hline 00945 & $\begin{array}{l}\text { SULFATE, DISSOLVED } \\
\left(\mathrm{mg} / \mathrm{L} \text { as } \mathrm{SO}_{4}\right)\end{array}$ & 33 & 6.900 & $<1.000$ & $1.699 *$ & 6.000 & 2.000 & 1.400 & $<1.00$ ) & $<1.000$ \\
\hline \multicolumn{11}{|c|}{ NUTRIENTS, TOTAL } \\
\hline 00630 & $\mathrm{NO}_{2}+\mathrm{NO}_{3}$, TOTAL $(\mathrm{mg} / \mathrm{L}$ as $\mathrm{N})$ & 26 & 1.090 & 0.060 & 0.230 & 0.862 & 0.260 & 0.190 & 0.127 & 0.064 \\
\hline 00610 & $\begin{array}{l}\text { NITROGEN AMMONIA, TOTAL } \\
(\mathrm{mg} / \mathrm{L} \text { as } \mathrm{N})\end{array}$ & 26 & 0.680 & 0.015 & 0.120 & 0.540 & 0.142 & 0.085 & $0.05 ?$ & 0.024 \\
\hline 00605 & $\begin{array}{l}\text { NITROGEN ORGANIC, TOTAL } \\
(\mathrm{mg} / \mathrm{L} \text { as } \mathrm{N})\end{array}$ & 26 & 1.300 & $<0.020$ & $0.237^{*}$ & 0.620 & 0.220 & 0.160 & 0.127 & $<0.040$ \\
\hline 00625 & $\begin{array}{l}\text { NITROGEN AMMONIA + } \\
\text { ORGANIC, TOTAL }(\mathrm{mg} / \mathrm{L} \text { as N)}\end{array}$ & 26 & 1.400 & $<0.150$ & $0.337^{*}$ & 1.300 & 0.370 & 0.250 & 0.187 & $<0.150$ \\
\hline 00600 & NITROGEN, TOTAL (mg/L as N) & 26 & 2.400 & 0.060 & 0.550 & 2.085 & 0.615 & 0.470 & 0.287 & 0.064 \\
\hline 00665 & $\begin{array}{l}\text { PHOSPHORUS, TOTAL } \\
(\mathrm{mg} / \mathrm{L} \text { as } \mathrm{P})\end{array}$ & 26 & 0.090 & $<0.010$ & $0.015^{*}$ & 0.070 & 0.011 & $<0.010$ & $<0.010$ & $<0.010$ \\
\hline 70507 & $\begin{array}{l}\text { PHOSPHORUS ORTHO, TOTAL } \\
(\mathrm{mg} / \mathrm{L} \text { as } \mathrm{P})\end{array}$ & 26 & 0.038 & $<0.010$ & -- & $<0.010$ & $<0.010$ & $<0.010$ & $<0.010$ & $<0.010$ \\
\hline \multicolumn{11}{|c|}{ METALS AND MINOR CONSTITUENTS, TOTAL } \\
\hline 01002 & ARSENIC, TOTAL $(\mu \mathrm{g} / \mathrm{L}$ as As) & 31 & 1.000 & $<1.000$ & -- & $<1.000$ & $<1.000$ & $<1.000$ & $<1.007$ & $<1.000$ \\
\hline 01027 & CADMIUM, TOTAL $(\mu \mathrm{g} / \mathrm{L}$ as $\mathrm{Cd})$ & 31 & $<1.000$ & $<1.000$ & -- & -- & -- & -- & -- & -- \\
\hline 01034 & $\begin{array}{l}\text { CHROMIUM, TOTAL } \\
(\mu \mathrm{g} / \mathrm{L} \text { as } \mathrm{Cr})\end{array}$ & 31 & 3.000 & $<1.000$ & -- & 1.000 & $<1.000$ & $<1.000$ & $<1.007$ & $<1.000$ \\
\hline 01042 & COPPER, TOTAL $(\mu \mathrm{g} / \mathrm{L}$ as $\mathrm{Cu})$ & 31 & 3.000 & $<1.000$ & -- & $<1.000$ & $<1.000$ & $<1.000$ & $<1.007$ & $<1.000$ \\
\hline 01051 & LEAD, TOTAL $(\mu \mathrm{g} / \mathrm{L}$ as $\mathrm{Pb})$ & 31 & 2.000 & $<1.000$ & -- & 1.000 & $<1.000$ & $<1.000$ & $<1.007$ & $<1.000$ \\
\hline 71900 & MERCURY, TOTAL ( $\mu \mathrm{g} / \mathrm{L}$ as $\mathrm{Hg}$ ) & 31 & 0.500 & $<0.100$ & -- & $<0.100$ & $<0.100$ & $<0.100$ & $<0.107$ & $<0.100$ \\
\hline 01067 & NICKEL. TOTAL $(\mu \mathrm{g} / \mathrm{L}$ as $\mathrm{Ni})$ & 31 & $<1.000$ & $<1.000$ & -- & -- & -- & -- & -- & -- \\
\hline 01092 & ZINC, TOTAL ( $\mu \mathrm{g} / \mathrm{L}$ as $\mathrm{Zn})$ & 31 & 40.000 & $<10.000$ & $13.007^{*}$ & 30.000 & 20.000 & 10.000 & $<10.007$ & $<10.000$ \\
\hline 01012 & $\begin{array}{l}\text { BERYLLIUM, TOTAL } \\
(\mu \mathrm{g} / \mathrm{L} \text { as } \mathrm{Be})\end{array}$ & 27 & $<10.000$ & $<10.000$ & -- & -- & -- & -- & -- & -- \\
\hline 01097 & ANTIMONY, TOTAL ( $\mu \mathrm{g} / \mathrm{L}$ as $\mathrm{Sb})$ & 27 & $<1.000$ & $<1.000$ & -- & -- & -- & -- & -- & -- \\
\hline 01147 & SELENIUM, TOTAL $(\mu \mathrm{g} / \mathrm{L}$ as $\mathrm{Se})$ & 27 & $<1.000$ & $<1.000$ & -- & -- & - & -- & -- & -- \\
\hline 01077 & SILVER, TOTAL $(\mu \mathrm{g} / \mathrm{L}$ as $\mathrm{Ag})$ & 27 & $<1.000$ & $<1.000$ & - & - & -- & -- & -- & -- \\
\hline
\end{tabular}

NOTE: Multiple detection limits during the period of record may result in different values flagged with a " $<$."

* Value is estimated by using a log-probability regression to predict the values of data below the detection limit. 
Table 57. Statistical summary of atmospheric deposition water-quality data at site 42 (CSW04), March 1997 through March 1998

\begin{tabular}{|c|c|c|c|c|c|c|c|c|c|c|}
\hline \multirow{2}{*}{$\begin{array}{l}\text { PARAM- } \\
\text { ETER } \\
\text { CODE }\end{array}$} & \multirow{2}{*}{ PROPERTY OR CONSTITUENT } & \multicolumn{4}{|c|}{ DESCRIPTIVE STATISTICS } & \multicolumn{5}{|c|}{$\begin{array}{c}\text { PERCENT OF SAMPLES IN WHICH VALIIES WERE LESS } \\
\text { THAN OR EQUAL TO THOSE S" NWN }\end{array}$} \\
\hline & & $\begin{array}{l}\text { SAMPLE } \\
\text { SIZE }\end{array}$ & MAXIMUM & MINIMUM & MEAN & $95 \%$ & $75 \%$ & $\begin{array}{c}50 \% \\
\text { (median) }\end{array}$ & $25 \%$ & $5 \%$ \\
\hline \multicolumn{11}{|c|}{ PHYSICAL AND CHEMICAL PROPERTIES } \\
\hline 00045 & RAINFALL (in./wk) & 53 & 6.830 & 0.000 & 0.927 & 2.598 & 1.430 & 0.750 & 0.025 & 0.000 \\
\hline 00046 & RAINFALL, TOTAL (in./wk) & 53 & 7.540 & 0.000 & 1.025 & 2.688 & 1.695 & 0.760 & 0.030 & 0.000 \\
\hline 90095 & $\begin{array}{l}\text { SPECIFIC CONDUCTANCE, } \\
\mathrm{LAB}\left(\mu \mathrm{S} / \mathrm{cm} \text { at } 25^{\circ} \mathrm{C}\right)\end{array}$ & 38 & 101.000 & 6.000 & 27.105 & 93.400 & 28.000 & 17.000 & 12.000 & 6.950 \\
\hline 00403 & $\begin{array}{l}\text { pH, LAB (STANDARD pH } \\
\text { UNITS) }\end{array}$ & 42 & 5.500 & 3.700 & 4.493 & 5.370 & 4.800 & 4.600 & 4.100 & 3.715 \\
\hline 00940 & $\begin{array}{l}\text { CHLORIDE, DISSOLVED } \\
(\mathrm{mg} / \mathrm{L} \text { as } \mathrm{Cl})\end{array}$ & 39 & 3.900 & $<2.000$ & -- & $<2.000$ & $<2.000$ & $<2.000$ & $<2.000$ & $<2.000$ \\
\hline 00945 & $\begin{array}{l}\text { SULFATE, DISSOLVED } \\
\left(\mathrm{mg} / \mathrm{L} \text { as } \mathrm{SO}_{4}\right)\end{array}$ & 36 & 10.000 & $<1.000$ & $2.043 *$ & 9.000 & 2.000 & $<1.000$ & $<1.000$ & $<1.000$ \\
\hline \multicolumn{11}{|c|}{ NUTRIENTS, TOTAL } \\
\hline 00630 & $\mathrm{NO}_{2}+\mathrm{NO}_{3}$, TOTAL $(\mathrm{mg} / \mathrm{L}$ as $\mathrm{N})$ & 30 & 0.650 & 0.070 & 0.230 & 0.639 & 0.303 & 0.170 & 0.127 & 0.075 \\
\hline 00610 & $\begin{array}{l}\text { NITROGEN AMMONIA, TOTAL } \\
(\mathrm{mg} / \mathrm{L} \text { as } \mathrm{N})\end{array}$ & 30 & 0.340 & $<0.015$ & $0.110^{*}$ & 0.260 & 0.150 & 0.070 & 0.050 & $<0.015$ \\
\hline 00605 & $\begin{array}{l}\text { NITROGEN ORGANIC, TOTAL } \\
(\mathrm{mg} / \mathrm{L} \text { as } \mathrm{N})\end{array}$ & 30 & 0.470 & $<0.080$ & $0.203 *$ & 0.460 & 0.260 & 0.150 & 0.100 & $<0.090$ \\
\hline 00625 & $\begin{array}{l}\text { NITROGEN AMMONIA + } \\
\text { ORGANIC, TOTAL }(\mathrm{mg} / \mathrm{L} \text { as N)}\end{array}$ & 30 & 0.800 & $<0.150$ & $0.303 *$ & 0.650 & 0.390 & 0.250 & 0.170 & $<0.150$ \\
\hline 00600 & NITROGEN, TOTAL (mg/L as N) & 30 & 1.400 & 0.070 & 0.503 & 1.235 & 0.700 & 0.430 & 0.270 & 0.075 \\
\hline 00665 & $\begin{array}{l}\text { PHOSPHORUS, TOTAL } \\
(\mathrm{mg} / \mathrm{L} \text { as } \mathrm{P})\end{array}$ & 29 & 0.090 & $<0.010$ & $0.009 *$ & 0.025 & $<0.010$ & $<0.010$ & $<0.010$ & $<0.010$ \\
\hline 70507 & $\begin{array}{l}\text { PHOSPHORUS ORTHO, TOTAL } \\
(\mathrm{mg} / \mathrm{L} \text { as } \mathrm{P})\end{array}$ & 29 & $<0.100$ & $<0.010$ & -- & -- & -- & -- & -- & -- \\
\hline \multicolumn{11}{|c|}{ METALS AND MINOR CONSTITUENTS, TOTAL } \\
\hline 01002 & ARSENIC, TOTAL $(\mu \mathrm{g} / \mathrm{L}$ as $\mathrm{As})$ & 34 & 1.000 & $<1.000$ & -- & $<1.000$ & $<1.000$ & $<1.000$ & $<1.000$ & $<1.000$ \\
\hline 01027 & CADMIUM, TOTAL ( $\mu \mathrm{g} / \mathrm{L}$ as $\mathrm{Cd})$ & 34 & $<1.000$ & $<1.000$ & -- & -- & - & -- & -- & -- \\
\hline 01034 & $\begin{array}{l}\text { CHROMIUM, TOTAL } \\
(\mu \mathrm{g} / \mathrm{L} \text { as } \mathrm{Cr})\end{array}$ & 34 & 3.000 & $<1.000$ & -- & 1.000 & $<1.000$ & $<1.000$ & $<1.000$ & $<1.000$ \\
\hline 01042 & COPPER, TOTAL ( $\mu \mathrm{g} / \mathrm{L}$ as $\mathrm{Cu})$ & 34 & 6.000 & $<1.000$ & -- & 1.000 & $<1.000$ & $<1.000$ & $<1.000$ & $<1.000$ \\
\hline 01051 & LEAD, TOTAL ( $\mu \mathrm{g} / \mathrm{L}$ as $\mathrm{Pb})$ & 34 & 1.000 & $<1.000$ & -- & $<1.000$ & $<1.000$ & $<1.000$ & $<1.000$ & $<1.000$ \\
\hline 71900 & MERCURY, TOTAL $(\mu \mathrm{g} / \mathrm{L}$ as $\mathrm{Hg})$ & 33 & 0.100 & $<0.100$ & -- & $<0.100$ & $<0.100$ & $<0.100$ & $<0.100$ & $<0.100$ \\
\hline 01067 & NICKEL, TOTAL ( $\mu \mathrm{g} / \mathrm{L}$ as Ni) & 34 & $<1.000$ & $<1.000$ & -- & -- & -- & -- & -- & -- \\
\hline 01092 & ZINC, TOTAL ( $\mu \mathrm{g} / \mathrm{L}$ as $\mathrm{Zn})$ & 34 & 30.000 & $<10.000$ & $9.922 *$ & 30.000 & 10.000 & $<10.000$ & $<10.000$ & $<10.000$ \\
\hline 01012 & $\begin{array}{l}\text { BERYLLIUM, TOTAL } \\
(\mu \mathrm{g} / \mathrm{L} \text { as } \mathrm{Be})\end{array}$ & 32 & $<10.000$ & $<10.000$ & -- & -- & -- & -- & -- & -- \\
\hline 01097 & ANTIMONY, TOTAL $(\mu \mathrm{g} / \mathrm{L}$ as $\mathrm{Sb})$ & 31 & $<1.000$ & $<1.000$ & -- & -- & -- & -- & -- & -- \\
\hline 01147 & SELENIUM, TOTAL $(\mu \mathrm{g} / \mathrm{L}$ as $\mathrm{Se})$ & 31 & $<1.000$ & $<1.000$ & -- & -- & -- & -- & -- & -- \\
\hline 01077 & SILVER, TOTAL $(\mu \mathrm{g} / \mathrm{L}$ as $\mathrm{Ag})$ & 32 & $<1.000$ & $<1.000$ & -- & - & -- & -- & -- & -. \\
\hline
\end{tabular}

NOTE: Multiple detection limits during the period of record may result in different values flagged with a " $<$."

* Value is estimated by using a log-probability regression to predict the values of data below the detection limit. 
Table 58. Statistical summary of atmospheric deposition water-quality data at site 43 (CSW07), March 1997 throuxh March 1998

\begin{tabular}{|c|c|c|c|c|c|c|c|c|c|c|}
\hline \multirow{2}{*}{$\begin{array}{c}\text { PARAM- } \\
\text { ETER } \\
\text { CODE }\end{array}$} & \multirow{2}{*}{ PROPERTY OR CONSTITUENT } & \multicolumn{4}{|c|}{ DESCRIPTIVE STATISTICS } & \multicolumn{5}{|c|}{$\begin{array}{l}\text { PERCENT OF SAMPLES IN WHICH VALUES WERE LESS } \\
\text { THAN OR EQUAL TO THOSE SHOI'N }\end{array}$} \\
\hline & & $\begin{array}{l}\text { SAMPLE } \\
\text { SIZE }\end{array}$ & MAXIMUM & MINIMUM & MEAN & $95 \%$ & $75 \%$ & $\begin{array}{c}50 \% \\
\text { (median) }\end{array}$ & $25 \%$ & $5 \%$ \\
\hline \multicolumn{11}{|c|}{ PHYSICAL AND CHEMICAL PROPERTIES } \\
\hline 00045 & RAINFALL (in./wk) & 53 & 4.260 & 0.000 & 0.871 & 2.977 & 1.570 & 0.500 & 0.010 & 0.000 \\
\hline 00046 & RAINFALL. TOTAL (in./wk) & 53 & 4.120 & 0.000 & 0.868 & 2.965 & 1.540 & 0.500 & 0.045 & 0.000 \\
\hline 90095 & $\begin{array}{l}\text { SPECIFIC CONDUCTANCE, } \\
\text { LAB }\left(\mu \mathrm{S} / \mathrm{cm} \text { at } 25^{\circ} \mathrm{C}\right)\end{array}$ & 38 & 74.000 & 4.000 & 22.947 & 70.200 & 31.000 & 17.500 & $\mathrm{I} 2.750$ & 5.900 \\
\hline 00403 & $\begin{array}{l}\text { pH, LAB (STANDARD pH } \\
\text { UNITS) }\end{array}$ & 41 & 5.400 & 3.800 & 4.590 & 5.290 & 4.850 & 4.600 & 4.200 & 3.910 \\
\hline 00940 & $\begin{array}{l}\text { CHLORIDE, DISSOLVED } \\
(\mathrm{mg} / \mathrm{L} \text { as } \mathrm{Cl})\end{array}$ & 39 & 3.100 & $<2.000$ & -- & $<2.000$ & $<2.000$ & $<2.000$ & $<2.000$ & $<2.000$ \\
\hline 00945 & $\begin{array}{l}\text { SULFATE, DISSOLVED } \\
\left(\mathrm{mg} / \mathrm{L} \text { as } \mathrm{SO}_{4}\right)\end{array}$ & 34 & 7.000 & $<1.000$ & $1.612^{*}$ & 6.500 & 2.000 & $<1.000$ & $<1.000$ & $<1.000$ \\
\hline \multicolumn{11}{|c|}{ NUTRIENTS, TOTAL } \\
\hline 00630 & $\mathrm{NO}_{2}+\mathrm{NO}_{3}$. TOTAL $(\mathrm{mg} / \mathrm{L}$ as $\mathrm{N})$ & 28 & 0.830 & 0.050 & 0.217 & 0.672 & 0.248 & 0.175 & 0.093 & 0.059 \\
\hline 00610 & $\begin{array}{l}\text { NITROGEN AMMONIA, TOTAL } \\
(\mathrm{mg} / \mathrm{L} \text { as N)}\end{array}$ & 28 & 0.390 & $<0.015$ & $0.107^{*}$ & 0.210 & 0.150 & 0.070 & 0.050 & $<0.015$ \\
\hline 00605 & $\begin{array}{l}\text { NITROGEN ORGANIC. TOTAL } \\
(\mathrm{mg} / \mathrm{L} \text { as } \mathrm{N})\end{array}$ & 28 & 2.000 & $<0.090$ & $0.258^{*}$ & 0.670 & 0.210 & 0.140 & 0.110 & $<0.100$ \\
\hline 00625 & $\begin{array}{l}\text { NITROGEN AMMONIA + } \\
\text { ORGANIC, TOTAL }(\mathrm{mg} / \mathrm{L} \text { as } \mathrm{N})\end{array}$ & 28 & 2.200 & $<0.150$ & $0.356^{*}$ & 0.880 & 0.300 & 0.210 & 0.180 & $<0.150$ \\
\hline 00600 & NITROGEN, TOTAL (mg/L as N) & 28 & 2.300 & 0.070 & 0.560 & 1.940 & 0.665 & 0.395 & 0.292 & 0.079 \\
\hline 00665 & $\begin{array}{l}\text { PHOSPHORUS, TOTAL } \\
(\mathrm{mg} / \mathrm{L} \text { as } \mathrm{P})\end{array}$ & 27 & 0.190 & $<0.010$ & $0.017 *$ & 0.090 & $<0.010$ & $<0.010$ & $<0.010$ & $<0.010$ \\
\hline 70507 & $\begin{array}{l}\text { PHOSPHORUS ORTHO, TOTAL } \\
(\mathrm{mg} / \mathrm{L} \text { as } \mathrm{P})\end{array}$ & 28 & 0.210 & $<0.010$ & -- & 0.039 & $<0.010$ & $<0.010$ & $<0.010$ & $<0.010$ \\
\hline \multicolumn{11}{|c|}{ METALS AND MINOR CONSTITUENTS, TOTAL } \\
\hline 01002 & ARSENIC, TOTAL ( $\mu \mathrm{g} / \mathrm{L}$ as As) & 31 & 16.000 & $<1.000$ & -- & $<1.000$ & $<\mathrm{I} .000$ & $<1.000$ & $<1.000$ & $<1.000$ \\
\hline 01027 & CADMIUM, TOTAL $(\mu \mathrm{g} / \mathrm{L}$ as $\mathrm{Cd})$ & 31 & $<1.000$ & $<1.000$ & -- & -- & -- & -- & -- & -- \\
\hline 01034 & $\begin{array}{l}\text { CHROMIUM, TOTAL } \\
(\mu \mathrm{g} / \mathrm{L} \text { as } \mathrm{Cr})\end{array}$ & 31 & 1.000 & $<1.000$ & -- & 1.000 & $<\mathrm{l} .000$ & $<1.000$ & $<1.000$ & $<1.000$ \\
\hline 01042 & COPPER, TOTAL $(\mu \mathrm{g} / \mathrm{L}$ as $\mathrm{Cu})$ & 31 & 3.000 & $<1.000$ & -- & 1.000 & $<\mathrm{l} .000$ & $<1.000$ & $<1.000$ & $<1.000$ \\
\hline $0105 \mathrm{I}$ & LEAD, TOTAL $(\mu \mathrm{g} / \mathrm{L}$ as $\mathrm{Pb})$ & $3 I$ & 2.000 & $<1.000$ & -- & 2.000 & $<\mathrm{I} .000$ & $<1.000$ & $<1.000$ & $<1.000$ \\
\hline 71900 & MERCURY, TOTAL $(\mu \mathrm{g} / \mathrm{L}$ as $\mathrm{Hg})$ & 31 & $<0.100$ & $<0.100$ & -- & -- & -- & -- & -- & -- \\
\hline 01067 & NICKEL, TOTAL $(\mu \mathrm{g} / \mathrm{L}$ as Ni) & 31 & $<1.000$ & $<1.000$ & -- & -- & -- & -- & -- & -- \\
\hline 01092 & ZINC, TOTAL $(\mu \mathrm{g} / \mathrm{L}$ as $\mathrm{Zn})$ & 31 & 100.000 & $<10.000$ & $10.993 *$ & 30.000 & 10.000 & $<10.000$ & $<10.000$ & $<\mathrm{I} 0.000$ \\
\hline 01012 & $\begin{array}{l}\text { BERYLLIUM, TOTAL } \\
(\mu \mathrm{g} / \mathrm{L} \text { as } \mathrm{Be})\end{array}$ & 29 & $<10.000$ & $<10.000$ & -- & -- & -- & -- & -- & - \\
\hline 01097 & ANTIMONY, TOTAL ( $\mu \mathrm{g} / \mathrm{L}$ as $\mathrm{Sb})$ & 29 & $<1.000$ & $<1.000$ & -- & -- & -- & -- & -- & -- \\
\hline 01147 & SELENIUM, TOTAL ( $\mu \mathrm{g} / \mathrm{L}$ as $\mathrm{Se})$ & 29 & $<1.000$ & $<1.000$ & - & -- & -- & -- & -- & -- \\
\hline 01077 & SILVER, TOTAL $(\mu \mathrm{g} / \mathrm{L}$ as $\mathrm{Ag})$ & 29 & $<1.000$ & $<1.000$ & -- & -- & -- & -- & -- & -- \\
\hline
\end{tabular}

NOTE: Multiple detection limits during the period of record may result in different values flagged with a " $<$."

* Value is estimated by using a log-probability regression to predict the values of data below the detection limit. 
Table 59. Atmospheric deposition water-quality data at site 37 (CSWO6), March 1997 through March 1998

\begin{tabular}{|c|c|c|c|c|c|c|c|c|}
\hline DATE & $\begin{array}{c}\text { RAINFALL } \\
\text { (IN./WK) } \\
(\text { (00045) }\end{array}$ & $\begin{array}{c}\text { RAINFALL, } \\
\text { TOTAL } \\
\text { (IN.WKK) } \\
\text { (00046) } \\
\end{array}$ & $\begin{array}{l}\text { SPECIFIC } \\
\text { CONDUC- } \\
\text { TANCE } \\
\text { LAB } \\
(\text { US/CM) } \\
(90095) \\
\end{array}$ & $\begin{array}{c}\text { PH WATER } \\
\text { RAW } \\
\text { LAB } \\
\text { (STANDARD } \\
\text { UNITS) } \\
(00403) \\
\end{array}$ & $\begin{array}{c}\text { CHLORIDE, } \\
\text { DISSOLVED } \\
\text { (MG/L } \\
\text { AS CL) } \\
(00940) \\
\end{array}$ & $\begin{array}{c}\text { SULFATE, } \\
\text { DISSOLVED } \\
\text { (MG/L AS } \\
\text { SO4) } \\
(00945) \\
\end{array}$ & $\begin{array}{l}\text { NITROGEN, } \\
\text { NO2+NO3, } \\
\text { TOTAL } \\
\text { (MG/L } \\
\text { AS N) } \\
(00630) \\
\end{array}$ & $\begin{array}{l}\text { NITROGEN, } \\
\text { AMMTNIA, } \\
\text { TOTAL } \\
\text { (M V/L } \\
\text { AS N) } \\
(00 \div 10) \\
\end{array}$ \\
\hline MAR 24-31, 1997 & 1.02 & 1.06 & 28 & 4.5 & $<2.0$ & 2.5 & 0.380 & 0.280 \\
\hline MAR 31-APR 7 & .26 & .29 & 25 & 4.5 & $<2.0$ & 1.5 & -- & -- \\
\hline APR 7-14 & .87 & .92 & 6 & 5.1 & $<2.0$ & $<1.0$ & .070 & .110 \\
\hline APR 14-21 & .00 & .00 & -- & -- & -- & -- & -- & -- \\
\hline APR 2I-28 & 3.34 & 3.48 & 16 & 4.6 & $<2.0$ & $<1.0$ & .220 & .180 \\
\hline APR 28-MAY 5 & 1.45 & 1.46 & 16 & 4.8 & $<2.0$ & $<1.0$ & .240 & .160 \\
\hline MAY 5-12 & .38 & .42 & 78 & 4.0 & $<2.0$ & 6.9 & 1.09 & 680 \\
\hline MAY I2-I9 & .00 & .00 & -- & -- & -- & -- & -- & -- \\
\hline MAY 19-27 & .11 & .13 & 78 & 3.6 & -- & 1.0 & -- & -- \\
\hline MAY 27-JUNE 2 & .02 & $.0 \mathrm{I}$ & -- & -- & - & $-\cdot$ & -- & -- \\
\hline JUNE 2-9 & 1.00 & .59 & 13 & 4.6 & $<2.0$ & 1.5 & .190 & .080 \\
\hline JUNE 9-I6 & 2.54 & 2.59 & 20 & 4.6 & $<2.0$ & 1.5 & .260 & .140 \\
\hline JUNE 16-23 & .00 & .00 & -- & -- & -- & -- & -- & -- \\
\hline JUNE 23-30 & I.14 & 1.22 & 20 & 4.5 & $<2.0$ & 2.8 & .330 & .140 \\
\hline JUNE 30-JULY 7 & .42 & .44 & 43 & 4.0 & $<2.0$ & 5.0 & .340 & .070 \\
\hline JULY 7-14 & .01 & .00 & -- & -- & -- & -- & -- & -- \\
\hline JULY 14-2I & .03 & .04 & -- & 3.7 & $<2.0$ & -- & -- & -- \\
\hline JULY 2I-28 & 9.22 & 8.02 & 8 & 5.4 & $<2.0$ & $<1.0$ & .060 & $<.015$ \\
\hline JULY 28-AUG 4 & .32 & .35 & 48 & 4.0 & $<2.0$ & 2.0 & -- & -- \\
\hline AUG 4-II & .08 & .11 & 56 & 4.0 & $<2.0$ & -- & -- & -- \\
\hline AUG II-18 & .00 & .00 & -- & -- & -- & -- & -- & -- \\
\hline AUG $18-25$ & .00 & .00 & - & -- & -- & -- & -- & -- \\
\hline AUG 25-SEPT 2 & .21 & $.2 \mathrm{I}$ & 46 & 4.1 & $<2.0$ & 6.0 & -- & -- \\
\hline SEPT 2-8 & .00 & .00 & -- & -- & -- & -- & -- & -- \\
\hline SEPT 8-15 & -- & .27 & 46 & 4.1 & $<2.0$ & 3.2 & -- & -- \\
\hline SEPT 15-22 & .02 & .02 & -- & 3.2 & -- & -- & -- & - \\
\hline SEPT $22-29$ & 2.12 & 2.15 & 35 & 4. I & $<2.0$ & 2.5 & .440 & .120 \\
\hline SEPT 29-OCT 6 & .00 & .00 & -- & -- & -- & -- & -- & -- \\
\hline ОСТ 6-I4 & .00 & .00 & -- & -- & -- & -- & -- & -- \\
\hline ОСТ 14-20 & 2.08 & 2.06 & 18 & 4.7 & $<2.0$ & 1.5 & .190 & .090 \\
\hline ОСТ 20-27 & 1.89 & 1.88 & 7 & 5.5 & $<2.0$ & $<1.0$ & .080 & .060 \\
\hline OCT 27-NOV 3 & .88 & .95 & 15 & 4.7 & $<2.0$ & 1.0 & .170 & .060 \\
\hline NOV 3-10 & .00 & .00 & -- & -- & -- & -- & -- & -- \\
\hline NOV $10-17$ & 1.20 & 1.19 & 17 & 4.5 & $<2.0$ & 1.2 & .210 & .050 \\
\hline NOV 17-24 & 1.38 & 1.46 & 23 & 4.4 & $<2.0$ & 2.2 & .220 & .110 \\
\hline NOV 24-DEC 1 & .69 & $.8 \mathrm{I}$ & 13 & 5.1 & $<2.0$ & $<\mathrm{l} .0$ & .100 & .060 \\
\hline DEC 1-8 & .03 & .04 & -- & 3.8 & 3.4 & -- & -- & -- \\
\hline DEC 8-15 & .72 & .75 & 16 & 4.6 & $<2.0$ & 1.5 & .140 & .050 \\
\hline DEC $15-22$ & 1.00 & 1.10 & 22 & 4.4 & $<2.0$ & $<1.0$ & .260 & .050 \\
\hline DEC 22-29 & $2.0 \mathrm{I}$ & 2.05 & 16 & 5.4 & $<2.0$ & $<1.0$ & .150 & .150 \\
\hline $\begin{array}{r}\text { DEC } 29,1997- \\
\text { JAN } 5,1998\end{array}$ & .00 & .02 & - & -- & -- & -- & -- & -- \\
\hline JAN 5-12 & I.66 & 1.75 & 8 & 5.2 & $<2.0$ & $<\mathrm{I} .0$ & .070 & .050 \\
\hline JAN $12-20$ & 2.38 & 2.56 & -- & -- & -- & -- & -- & -- \\
\hline JAN 20-26 & .89 & .94 & $\cdots$ & - & -- & -- & -- & -- \\
\hline JAN 26-FEB 2 & 1.55 & 1.55 & 14 & 4.6 & $<2.0$ & $<1.0$ & .120 & .050 \\
\hline FEB 2-9 & 1.55 & 1.40 & 17 & 4.4 & 32 & $<1.0$ & .190 & .050 \\
\hline FEB 9-16 & .28 & .33 & 32 & 4.7 & $<2.0$ & 1.8 & -- & -- \\
\hline FEB 16-23 & 1.74 & $\mathrm{I} .78$ & 9 & 5.1 & 2.3 & $<\mathrm{I} .0$ & .120 & .150 \\
\hline FEB 23-MAR 2 & .29 & .28 & 22 & 4.6 & $<2.0$ & 2.0 & -- & -- \\
\hline MAR 2-9 & 2.31 & 2.32 & 12 & 4.8 & $<2.0$ & $<1.0$ & .160 & .130 \\
\hline MAR 9-16 & .00 & .02 & -- & 4.1 & -- & -- & -- & -- \\
\hline MAR 16-23 & .99 & $1.0 \mathrm{I}$ & 25 & 4.4 & $<2.0$ & 1.4 & .180 & .040 \\
\hline MAR 23-30 & .03 & .02 & -- & 3.8 & -- & -- & -- & -- \\
\hline
\end{tabular}


Table 59. Atmospheric deposition water-quality data at site 37 (CSW06), March 1997 through March 1998-Continued

\begin{tabular}{|c|c|c|c|c|c|c|c|c|}
\hline DATE & $\begin{array}{l}\text { NITROGEN, } \\
\text { ORGANIC } \\
\text { TOTAL } \\
\text { (MG/L } \\
\text { AS N) } \\
(00605) \\
\end{array}$ & $\begin{array}{c}\text { NITROGEN, } \\
\text { AMMONIA+ } \\
\text { ORGANIC } \\
\text { TOTAL } \\
\text { (MG/L } \\
\text { AS N) } \\
(00625) \\
\end{array}$ & $\begin{array}{c}\text { NITROGEN, } \\
\text { TOTAL } \\
\text { (MG/L } \\
\text { AS N) } \\
(00600)\end{array}$ & $\begin{array}{l}\text { PHOS- } \\
\text { PHORUS, } \\
\text { TOTAL } \\
\text { (MG/L } \\
\text { AS P) } \\
(00665) \\
\end{array}$ & $\begin{array}{c}\text { PHOS- } \\
\text { PHORUS, } \\
\text { ORTHO, } \\
\text { TOTAL } \\
\text { (MG/L } \\
\text { AS P) } \\
(70507) \\
\end{array}$ & $\begin{array}{c}\text { ARSENIC, } \\
\text { TOTAL } \\
\text { (UG/L } \\
\text { AS AS) } \\
(01002) \\
\end{array}$ & $\begin{array}{l}\text { CADMIUM } \\
\text { WATER } \\
\text { UNFIL- } \\
\text { TERED, } \\
\text { TOTAL } \\
\text { (UG/L } \\
\text { AS CD) } \\
\text { (01027) } \\
\end{array}$ & $\begin{array}{c}\text { CHRO- } \\
\text { MIUM, } \\
\text { TOTAL } \\
\text { RECOVE?- } \\
\text { ABLE } \\
\text { (UG/L } \\
\text { AS CR) } \\
(01034) \\
\end{array}$ \\
\hline MAR $24-31,1997$ & 0.16 & 0.44 & 0.82 & $<0.010$ & $<0.010$ & $<1$ & $<1$ & 3 \\
\hline MAR 31-APR 7 & -- & - & -- & -- & -- & $<1$ & $<1$ & $<1$ \\
\hline APR $7-14$ & $<.04$ & $<.15$ & .07 & $<.010$ & $<.010$ & $<1$ & $<1$ & $<1$ \\
\hline APR $14-21$ & -. & $\ldots$ & -- & -- & -- & -- & -- & - \\
\hline APR $21-28$ & .17 & .35 & .57 & $<.010$ & $<.010$ & $<1$ & $<1$ & 1 \\
\hline APR 28-MAY 5 & .32 & .48 & .72 & $<.010$ & $<.010$ & $<1$ & $<1$ & $<1$ \\
\hline MAY 5-12 & .62 & 1.3 & 2.4 & .016 & $<.010$ & $<1$ & $<1$ & $<1$ \\
\hline MAY $12-19$ & -- &.- & -- & $\ldots$ & -- & -- & -- & -- \\
\hline MAY 19-27 & -- & -- & -- & -- & -- & -- & -- & -- \\
\hline MAY 27-JUNE 2 & -- & - & -- & -- & -- & -- & -- & -- \\
\hline JUNE 2-9 & .17 & .25 & .44 & .070 & $<.010$ & $<1$ & $<1$ & $<1$ \\
\hline JUNE 9-16 & .29 & .43 & .69 & $<.010$ & $<.010$ & $<1$ & $<1$ & $<1$ \\
\hline JUNE $16-23$ & -- & -- & -- & -- & -- & -- & -- & -- \\
\hline JUNE $23-30$ & .12 & .26 & .59 & $<.010$ & $<.010$ & $<1$ & $<1$ & 1 \\
\hline JUNE 30-JULY 7 & .11 & .18 & .52 & $<.010$ & $<.010$ & 1 & $<1$ & $<1$ \\
\hline JULY 7-14 & -- & - & -- & -- & - & -. & -- & -- \\
\hline JULY 14-21 & -- & -- & -- & -- & -- & -- & -- & -- \\
\hline JULY $21-28$ & $<.14$ & $<.15$ & .06 & $<.010$ & $<.010$ & $<1$ & $<1$ & $<1$ \\
\hline JULY 28-AUG 4 & -- & -- & -- & -- & - & $<1$ & $<1$ & $<1$ \\
\hline AUG 4-11 & -- & -- & -- & -- & - & -- & -- & - \\
\hline AUG 11-18 & -- & -- & -- & -- & -- & -- & -- & -- \\
\hline AUG 18-25 & -- & -- & -- & -- & -- & -- & -- & -- \\
\hline AUG 25-SEPT 2 & -- & -- & -- & -- & -- & -- & -- & -- \\
\hline SEPT 2-8 & -- & -- & -- & -- & -- & -- & -- & -- \\
\hline SEPT 8-15 & - & -- & -- & -- & -- & $<1$ & $<1$ & $<1$ \\
\hline SEPT 15-22 & -- & -- & -- & -- & -- & -- & -- & -- \\
\hline SEPT $22-29$ & .29 & .41 & .85 & $<.010$ & $<.010$ & $<1$ & $<1$ & $<1$ \\
\hline SEPT 29-OCT 6 & -- & - & -- & -- & -- & -- & -- & -- \\
\hline OCT 6-14 & -- & -. & - & -. & -- & -. & -- & -- \\
\hline OCT $14-20$ & .19 & .28 & .47 & .090 & $<.010$ & $<1$ & $<1$ & $<1$ \\
\hline OCT 20-27 & 1.3 & 1.4 & 1.5 & .060 & .038 & $<1$ & $<1$ & $<1$ \\
\hline OCT $27-N O V 3$ & .22 & .28 & .45 & $<.010$ & $<.010$ & $<1$ & $<1$ & $<1$ \\
\hline NOV $3-10$ & -- & -- & -- & -- & - & -- & - & - \\
\hline NOV $10-17$ & .12 & .17 & .38 & $<.010$ & $<.010$ & $<1$ & $<1$ & $<1$ \\
\hline NOV $17-24$ & .14 & .25 & .47 & $<.010$ & $<.010$ & $<1$ & $<1$ & $<1$ \\
\hline NOV $24-D E C ~ 1$ & .13 & .19 & .29 & $<.010$ & $<.010$ & $<1$ & $<1$ & $<1$ \\
\hline DEC $1-8$ & -- & -- & -- & -- & -- & -- & -- & -- \\
\hline DEC $8-15$ & .16 & .21 & .35 & .050 & $<.010$ & $<1$ & $<1$ & $<1$ \\
\hline DEC 15-22 & .16 & .21 & .47 & .036 & $<.010$ & $<1$ & $<1$ & $<1$ \\
\hline DEC 22-29 & .22 & .37 & .52 & .011 & $<.010$ & $<1$ & $<1$ & $<1$ \\
\hline $\begin{array}{r}\text { DEC } 29,1997- \\
\text { JAN } 5,1998\end{array}$ & -- & -- & -- & -- & -- & -- & -- & -- \\
\hline JAN 5-12 & .16 & .21 & .28 & $<.010$ & $<.010$ & $<1$ & $<1$ & $<1$ \\
\hline JAN 12-20 & -- & -- & -- & -. & -- & -- & -- & -- \\
\hline JAN 20-26 & -- & -- & -- & -- & -- & -- & -- & -- \\
\hline JAN 26-FEB 2 & $<.10$ & $<.15$ & .12 & $<.010$ & $<.010$ & $<1$ & $<1$ & $<1$ \\
\hline FEB 2-9 & $<.10$ & $<.15$ & .19 & $<.010$ & $<.010$ & $<1$ & $<1$ & $<1$ \\
\hline FEB 9-16 & -- & -- & -- & -- & -- & $<1$ & $<1$ & $<1$ \\
\hline FEB 16-23 & .14 & .29 & .41 & .010 & $<.010$ & $<1$ & $<1$ & $<1$ \\
\hline FEB 23-MAR 2 & -- & -- & -- & -- & -- & $<1$ & $<1$ & $<1$ \\
\hline MAR 2-9 & $<.02$ & $<.15$ & .16 & $<.010$ & $<.010$ & $<1$ & $<1$ & $<1$ \\
\hline MAR 9-16 & -- & -- & -- & -- & -- & -- & -- & -- \\
\hline MAR 16-23 & .29 & .33 & .51 & $<.010$ & $<.010$ & $<1$ & $<1$ & $<1$ \\
\hline MAR 23-30 & -- & -- & -- & -- & -- & -- & -- & -- \\
\hline
\end{tabular}


Table 59. Atmospheric deposition water-quality data at site 37 (CSW06), March 1997 through March 1998--Continued

\begin{tabular}{|c|c|c|c|c|c|c|c|c|c|}
\hline DATE & $\begin{array}{l}\text { COPPER, } \\
\text { TOTAL } \\
\text { RECOVER- } \\
\text { ABLE } \\
\text { (UG/L } \\
\text { AS CU) } \\
(01042) \\
\end{array}$ & $\begin{array}{l}\text { LEAD, } \\
\text { TOTAL } \\
\text { RECOVER- } \\
\text { ABLE } \\
\text { (UG/L } \\
\text { AS PB) } \\
\text { (01051) }\end{array}$ & $\begin{array}{l}\text { MERCURY, } \\
\text { TOTAL } \\
\text { RECOVER- } \\
\text { ABLE } \\
\text { (UG/L } \\
\text { AS HG) } \\
\text { (71900) }\end{array}$ & $\begin{array}{c}\text { NICKEL, } \\
\text { TOTAL } \\
\text { RECOVER- } \\
\text { ABLE } \\
\text { (UG/L } \\
\text { AS NI) } \\
(01067) \\
\end{array}$ & $\begin{array}{c}\text { ZINC, } \\
\text { TOTAL } \\
\text { RECOVER- } \\
\text { ABLE } \\
\text { (UG/L } \\
\text { AS ZN) } \\
(01092) \\
\end{array}$ & $\begin{array}{c}\text { BERYL- } \\
\text { LIUM, } \\
\text { TOTAL, } \\
\text { RECOVER- } \\
\text { ABLE } \\
\text { (UG/L } \\
\text { AS BE) } \\
\text { (01012) } \\
\end{array}$ & $\begin{array}{l}\text { ANTIMONY, } \\
\text { TOTAL } \\
\text { (UG/L } \\
\text { AS SB) } \\
\text { (01097) }\end{array}$ & $\begin{array}{c}\text { SELENIUM, } \\
\text { TOTAL } \\
\text { (UG/L } \\
\text { AS SE) } \\
\text { (01147) }\end{array}$ & $\begin{array}{c}\text { SILVER, } \\
\text { TOTAL } \\
\text { RECOVER- } \\
\text { ABLE } \\
\text { (UG/L AS } \\
\text { AG) } \\
\text { (01077) }\end{array}$ \\
\hline MAR 24-31, 1997 & 3 & $<1$ & $<0.10$ & $<1$ & 30 & $<10$ & $<1$ & $<1$ & $<1$ \\
\hline MAR 31-APR 7 & $<1$ & $<1$ & $<.10$ & $<1$ & $<10$ & -- & -- & -- & -- \\
\hline APR 7-14 & $<1$ & $<1$ & $<.10$ & $<1$ & $<10$ & $<10$ & $<1$ & $<1$ & $<1$ \\
\hline APR $14-21$ & -- & -- & - & -- & -- & -. & -- & -- & -- \\
\hline APR 21-28 & $<1$ & $<1$ & $<.10$ & $<1$ & $<10$ & $<10$ & $<1$ & $<1$ & $<1$ \\
\hline APR 28-MAY 5 & $<1$ & 2 & $<.10$ & $<1$ & 20 & $<10$ & $<1$ & $<1$ & $<1$ \\
\hline MAY 5-12 & $<1$ & 1 & $<.10$ & $<1$ & $<10$ & - & - & - & -. \\
\hline MAY $12-19$ & -- & - & -- & -- & -- & -- & -- & -- & -- \\
\hline MAY 19-27 & -- & -- & -- & -- & -- & -- & -- & -- & -- \\
\hline MAY 27-JUNE 2 & -- & -- & -- & -- & -- & -- & - & -- & -- \\
\hline JUNE 2-9 & $<1$ & $<1$ & $<.10$ & $<1$ & 10 & $<10$ & $<1$ & $<1$ & $<1$ \\
\hline JUNE 9-16 & $<1$ & $<1$ & $<.10$ & $<1$ & 10 & $<10$ & $<1$ & $<1$ & $<1$ \\
\hline JUNE $16-23$ & -- & - & -- & -- & -- & -- & -- & -- & -- \\
\hline JUNE $23-30$ & $<1$ & $<1$ & $<.10$ & $<1$ & $<10$ & $<10$ & $<1$ & $<1$ & $<1$ \\
\hline JUNE 30-JULY 7 & $<1$ & $<1$ & $<.10$ & $<1$ & $<10$ & $<10$ & $<1$ & $<1$ & $<1$ \\
\hline JULY 7-14 & -- & - & -- & -- & -- & -- & -- & -- & -- \\
\hline JULY $14-21$ & -- & -- & -- & -- & -- & -- & -- & -- & -- \\
\hline JULY 21-28 & $<1$ & $<1$ & $<.10$ & $<1$ & 10 & $<10$ & $<1$ & $<1$ & $<1$ \\
\hline JULY 28-AUG 4 & $<1$ & 1 & $<.10$ & $<1$ & $<10$ & $<10$ & $<1$ & $<1$ & $<1$ \\
\hline AUG 4-11 & -- & - & -- & -- & - & -- & -- & -- & -- \\
\hline AUG 11-18 & - & -- & -- & -- & -- & -- & -- & -- & -- \\
\hline AUG $18-25$ & -- & -- & -- & -- & -- & -- & -- & -- & -- \\
\hline AUG 25-SEPT 2 & - & - & - & - & - & - & - & - & - \\
\hline SEPT 2-8 & -- & -- & -- & - & -- & -- & -- & -- & -- \\
\hline SEPT 8-15 & $<1$ & $<1$ & .50 & $<1$ & 20 & -- & -- & -- & -- \\
\hline SEPT $15-22$ & -- & -- & -- & -- & -- & -- & -- & -- & -- \\
\hline SEPT 22-29 & $<1$ & $<1$ & $<.10$ & $<1$ & 20 & $<10$ & $<1$ & $<1$ & $<1$ \\
\hline SEPT 29-OCT 6 & -. & -. & -- & -- & -- & -- & -- & -- & -- \\
\hline OCT $6-14$ & -- & -- & -- & -- & -- & -- & -- & -- & -- \\
\hline OCT $14-20$ & $<1$ & $<1$ & $<.10$ & $<1$ & 10 & $<10$ & $<1$ & $<1$ & $<1$ \\
\hline OCT $20-27$ & $<1$ & $<1$ & $<.10$ & $<1$ & 20 & $<10$ & $<1$ & $<1$ & $<1$ \\
\hline OCT $27-$ NOV 3 & $<1$ & $<1$ & $<.10$ & $<1$ & 40 & $<10$ & $<1$ & $<1$ & $<1$ \\
\hline NOV $3-10$ & - & - & -- & - & - & - & - & -- & - \\
\hline NOV $10-17$ & $<1$ & $<1$ & $<.10$ & $<1$ & 20 & $<10$ & $<1$ & $<1$ & $<1$ \\
\hline NOV $17-24$ & $<1$ & $<1$ & $<.10$ & $<1$ & $<10$ & $<10$ & $<1$ & $<1$ & $<1$ \\
\hline NOV 24-DEC 1 & $<1$ & $<1$ & $<.10$ & $<1$ & 20 & $<10$ & $<1$ & $<1$ & $<1$ \\
\hline DEC 1-8 & -- & -- & -- & -- & -- & -- & -- & -- & -- \\
\hline DEC $8-15$ & $<1$ & $<1$ & $<.10$ & $<1$ & 20 & $<10$ & $<1$ & $<1$ & $<1$ \\
\hline DEC $15-22$ & $<1$ & $<1$ & $<.10$ & $<1$ & 30 & $<10$ & $<1$ & $<1$ & $<1$ \\
\hline DEC 22-29 & $<\mathrm{I}$ & $<1$ & $<.10$ & $<1$ & 10 & $<10$ & $<1$ & $<1$ & $<1$ \\
\hline $\begin{array}{r}\text { DEC } 29,1997- \\
\text { JAN } 5,1998\end{array}$ & - & - & - & - & - & - & - & - & - \\
\hline JAN 5-12 & $<1$ & $<1$ & $<.10$ & $<1$ & $<10$ & $<10$ & $<1$ & $<1$ & $<1$ \\
\hline JAN $12-20$ & - & - & -- & - & - & - & - & - & -. \\
\hline JAN 20-26 & -- & -- & -- & -- & -- & -- & -- & -- & -- \\
\hline JAN 26-FEB 2 & $<1$ & $<1$ & $<.10$ & $<1$ & $<10$ & $<10$ & $<1$ & $<1$ & $<1$ \\
\hline FEB 2-9 & $<1$ & $<1$ & $<.10$ & $<1$ & $<10$ & $<10$ & $<1$ & $<1$ & $<1$ \\
\hline FEB 9-16 & $<1$ & $<1$ & $<.10$ & $<1$ & $<10$ & $<10$ & $<1$ & $<1$ & $<1$ \\
\hline FEB 16-23 & $<1$ & $<1$ & $<.10$ & $<1$ & 20 & $<10$ & $<1$ & $<1$ & $<1$ \\
\hline FEB 23-MAR 2 & $<1$ & $<1$ & $<.10$ & $<1$ & 10 & - & - & -- & - \\
\hline MAR 2-9 & $<1$ & $<1$ & $<.10$ & $<1$ & 10 & $<10$ & $<1$ & $<1$ & $<1$ \\
\hline MAR 9-16 & -- & -- & -- & -- & -- & -- & - & -- & - \\
\hline MAR $16-23$ & $<1$ & $<1$ & $<.10$ & $<1$ & $<10$ & $<10$ & $<1$ & $<1$ & $<1$ \\
\hline MAR 23-30 & $\ldots$ & - & -. & -. & - & - & - & - & -- \\
\hline
\end{tabular}


Table 60. Atmospheric deposition water-quality data at site 42 (CSW04), March 1997 through March 1979

\begin{tabular}{|c|c|c|c|c|c|c|c|c|}
\hline DATE & $\begin{array}{l}\text { RAINFALL } \\
\text { (IN_NKK) } \\
(00045)\end{array}$ & $\begin{array}{c}\text { RAINFALL, } \\
\text { TOTAL } \\
\text { (IN./WK) } \\
\text { (00046) }\end{array}$ & $\begin{array}{l}\text { SPECIFIC } \\
\text { CONDUC- } \\
\text { TANCE } \\
\text { LAB } \\
\text { (US/CM) } \\
\text { (90095) } \\
\end{array}$ & $\begin{array}{c}\text { PH WATER } \\
\text { RAW } \\
\text { LAB } \\
\text { (STANDARD } \\
\text { UNITS) } \\
\text { (00403) } \\
\end{array}$ & $\begin{array}{c}\text { CHLORIDE, } \\
\text { DISSOLVED } \\
\text { (MG/L } \\
\text { AS CL) } \\
(00940) \\
\end{array}$ & $\begin{array}{c}\text { SULFATE, } \\
\text { DISSOLVED } \\
\text { (MG/ AS } \\
\text { SO4) } \\
(00945) \\
\end{array}$ & $\begin{array}{l}\text { NITROGEN, } \\
\text { NO2+NO3, } \\
\text { TOTAL } \\
\text { (MG/L } \\
\text { AS N) } \\
(00630) \\
\end{array}$ & $\begin{array}{l}\text { NITROGEN, } \\
\text { AMMON'A, } \\
\text { TOTAL. } \\
\text { (MG/L } \\
\text { AS N) } \\
\left(00610^{\circ}\right.\end{array}$ \\
\hline MAR 25-31, 1997 & 0.91 & 0.97 & 27 & 4.6 & $<2.0$ & 2.5 & 0.370 & 0.260 \\
\hline MAR 31-APR 7 & .20 & .23 & 27 & 4.4 & $<2.0$ & -- & -- & -- \\
\hline APR 7-14 & .80 & .76 & 6 & 5.2 & $<2.0$ & $<1.0$ & .070 & .060 \\
\hline APR 14-21 & .00 & .00 & -- & -- & -- & -- & -- & -- \\
\hline APR $21-28$ & 2.85 & 2.94 & 16 & 4.7 & $<2.0$ & $<1.0$ & .250 & .170 \\
\hline APR 28-MAY 5 & 1.44 & 1.51 & 16 & 4.8 & $<2.0$ & $<1.0$ & .240 & .230 \\
\hline MAY 5-12 & .37 & .33 & 77 & 4.0 & $<2.0$ & 6.5 & -- & -- \\
\hline MAY 12-19 & .02 & .00 & -- & -- & -- & -- & -- & -- \\
\hline MAY 19-27 & .18 & .18 & 31 & 4.0 & -- & -- & -- & -- \\
\hline MAY 27-JUNE 2 & .00 & .00 & -- & -- & -- & -- & -- & -- \\
\hline JUNE 2-9 & 1.02 & 1.04 & 10 & 4.7 & $<2.0$ & $<1.0$ & .160 & .070 \\
\hline JUNE 9-16 & 2.35 & 2.27 & 24 & 4.6 & $<2.0$ & 2.0 & .330 & .150 \\
\hline JUNE 16-23 & .02 & .10 & 62 & 3.8 & $<2.0$ & 4.3 & -- & -- \\
\hline JUNE 23-30 & .98 & 1.70 & 18 & 4.5 & $<2.0$ & 1.5 & .240 & .090 \\
\hline JUNE 30-JULY 7 & 1.80 & 2.04 & 24 & 4.4 & $<2.0$ & 2.0 & .310 & .190 \\
\hline JULY 7-14 & .01 & .02 & -- & 3.9 & -- & -- & -- & -- \\
\hline JULY 14-21 & .09 & .15 & 93 & 3.7 & $<2.0$ & 10 & -- & -- \\
\hline JULY 21-28 & 6.83 & 7.54 & 7 & 5.1 & $<2.0$ & $<1.0$ & .080 & $<.015$ \\
\hline JULY 28-AUG 4 & .66 & 1.12 & 43 & 4.1 & $<2.0$ & 4.0 & .650 & .250 \\
\hline AUG 4-11 & .37 & .58 & 85 & 3.8 & $<2.0$ & 9.0 & .630 & .340 \\
\hline AUG 11-18 & .00 & .00 & -- & -- & -- & -- & -- & -- \\
\hline AUG $18-25$ & .00 & .00 & -- & -- & -- & -- & -- & -- \\
\hline AUG 25-SEPT 2 & .75 & .16 & I0I & 3.7 & $<2.0$ & 8.5 & -- & -- \\
\hline SEPT 2-8 & .00 & .00 & -- & -- & -- & -- & -- & -- \\
\hline SEPT 8-15 & .57 & .68 & 38 & 4.1 & $<2.0$ & 3.0 & .470 & .180 \\
\hline SEPT 15-22 & .03 & .02 & -- & 4.2 & -- & -- & -- & -- \\
\hline SEPT 22-29 & 2.22 & 2.44 & 31 & 4.3 & $<2.0$ & 1.8 & .390 & .090 \\
\hline SEPT 29-OCT 6 & .00 & .00 & -- & -- & - & -- & -- & -- \\
\hline OCT 6-I4 & .00 & .00 & -- & -- & -- & -- & -- & -- \\
\hline OCT $14-20$ & 2.49 & 2.50 & 10 & 4.8 & $<2.0$ & $<1.0$ & .110 & .040 \\
\hline OCT $20-27$ & 1.75 & 1.86 & 9 & 4.9 & $<2.0$ & $<1.0$ & .080 & .050 \\
\hline OCT 27-NOV 3 & .84 & .93 & 13 & 4.7 & $<2.0$ & $<1.0$ & .160 & .060 \\
\hline NOV 3-10 & .01 & .01 & -- & -- & -- & -- & -- & -- \\
\hline NOV $10-17$ & $\mathrm{I} .12$ & 1.27 & 16 & 4.6 & $<2.0$ & 1.2 & .160 & .070 \\
\hline NOV 17-24 & 1.25 & 1.34 & 20 & -- & $<2.0$ & 2.0 & .250 & .130 \\
\hline NOV 24-DEC 1 & .81 & .98 & 12 & 4.8 & $<2.0$ & $<\mathrm{I} .0$ & .130 & .070 \\
\hline DEC 1-8 & .03 & .04 & -- & 3.8 & 3.9 & -- & -- & -- \\
\hline DEC 8-15 & .71 & .73 & 15 & 4.8 & $<2.0$ & 1.5 & .150 & .040 \\
\hline DEC 15-22 & .98 & 1.14 & 24 & 4.4 & $<2.0$ & 1.7 & .270 & .040 \\
\hline DEC $22-29$ & 2.03 & 2.07 & 14 & 5.4 & $<2.0$ & $<\mathrm{I} .0$ & .150 & .150 \\
\hline $\begin{array}{r}\text { DEC } 29,1997- \\
\text { JAN } 5,1998\end{array}$ & .00 & .00 & -- & -- & -- & -- & -- & -- \\
\hline JAN 5-12 & 1.21 & 1.11 & 10 & 5.1 & $<2.0$ & $<1.0$ & .080 & .070 \\
\hline JAN $12-20$ & 2.20 & 2.58 & 16 & 4.6 & $<2.0$ & $<\mathrm{I} .0$ & .180 & .060 \\
\hline JAN 20-26 & .80 & .97 & 25 & 4.6 & $<2.0$ & $<1.0$ & .300 & .080 \\
\hline JAN 26-FEB 2 & 1.42 & 1.69 & 15 & 4.7 & $<2.0$ & $<1.0$ & .120 & .050 \\
\hline FEB 2-9 & 1.47 & 1.66 & 12 & 4.6 & $<2.0$ & $<\mathrm{I} .0$ & .140 & .031 \\
\hline FEB 9-16 & .20 & .40 & 25 & 4.7 & $<2.0$ & 1.8 & -- & -- \\
\hline FEB 16-23 & 1.99 & 1.98 & 7 & 5.5 & $<2.0$ & $<\mathrm{I} .0$ & .100 & .110 \\
\hline FEB 23-MAR 2 & .22 & .20 & 25 & 4.7 & $<2.0$ & 1.5 & -- & -- \\
\hline MAR 2-9 & 2.09 & 2.14 & 10 & 4.9 & $<2.0$ & $<1.0$ & .170 & .140 \\
\hline MAR 9-16 & .00 & .03 & -- & 4.2 & $<2.0$ & -- & -- & -- \\
\hline MAR 16-23 & .98 & 1.86 & 16 & 4.5 & $<2.0$ & $<\mathrm{I} .0$ & .170 & $<.015$ \\
\hline MAR 23-30 & .04 & .03 & -- & 3.8 & -- & -- & -- & - \\
\hline
\end{tabular}


Table 60. Atmospheric deposition water-quality data at site 42 (CSW04), March 1997 through March 1998-Continued

\begin{tabular}{|c|c|c|c|c|c|c|c|c|}
\hline DATE & $\begin{array}{l}\text { NITROGEN, } \\
\text { ORGANIC } \\
\text { TOTAL } \\
\text { (MG/L } \\
\text { AS N) } \\
\text { (00605) } \\
\end{array}$ & $\begin{array}{l}\text { NITROGEN, } \\
\text { AMMONIA+ } \\
\text { ORGANIC } \\
\text { TOTAL } \\
\text { (MG/L } \\
\text { AS N) } \\
(00625) \\
\end{array}$ & $\begin{array}{l}\text { NITROGEN, } \\
\text { TOTAL } \\
\text { (MG/L } \\
\text { AS N) } \\
(00600) \\
\end{array}$ & $\begin{array}{l}\text { PHOS- } \\
\text { PHORUS, } \\
\text { TOTAL } \\
\text { (MG/L } \\
\text { AS P) } \\
\text { (00665) }\end{array}$ & $\begin{array}{c}\text { PHOS- } \\
\text { PHORUS, } \\
\text { ORTHO, } \\
\text { TOTAL } \\
\text { (MG/L } \\
\text { AS P) } \\
(70507)\end{array}$ & $\begin{array}{l}\text { ARSENIC, } \\
\text { TOTAL } \\
\text { (UGlL } \\
\text { AS AS) } \\
\text { (01002) } \\
\end{array}$ & $\begin{array}{l}\text { CADMIUM } \\
\text { WATER } \\
\text { UNFIL- } \\
\text { TERED, } \\
\text { TOTAL } \\
\text { (UG/L } \\
\text { AS CD) } \\
\text { (01027) } \\
\end{array}$ & $\begin{array}{c}\text { CHR?- } \\
\text { MIUM, } \\
\text { TOTAL } \\
\text { RECOI'SR- } \\
\text { ABLE } \\
\text { (UG'l. } \\
\text { AS CR) } \\
\text { (01034) } \\
\end{array}$ \\
\hline MAR 25-31, 1997 & 0.27 & 0.53 & 0.90 & $<0.010$ & $<0.010$ & $<1$ & $<\mathrm{I}$ & 3 \\
\hline MAR 31-APR 7 & -- & -- & -- & -- & -- & $<1$ & $<1$ & $<1$ \\
\hline APR 7-14 & $<.09$ & $<.15$ & .07 & $<.010$ & $<.010$ & $<1$ & $<1$ & $<1$ \\
\hline APR 14-21 & -- & -- & -- & -- & -- & -- & -- & -- \\
\hline APR 21-28 & .26 & .43 & .68 & .010 & $<.010$ & $<1$ & $<1$ & 1 \\
\hline APR 28-MAY 5 & .12 & .35 & .59 & $<.010$ & $<.010$ & $<1$ & $<\mathrm{I}$ & $<1$ \\
\hline MAY 5-12 & -- & -- & -- & -- & -- & $<1$ & $<\mathrm{I}$ & $<1$ \\
\hline MAY 12-19 & -- & -- & -- & -- & -- & -- & -- & -- \\
\hline MAY 19-27 & -- & -- & -- & -- & -- & $<1$ & $<1$ & $<1$ \\
\hline MAY 27-JUNE 2 & -- & -- & -- & -- & -- & -- & -- & -- \\
\hline JUNE 2-9 & .18 & .25 & .41 & .021 & $<.010$ & $<1$ & $<1$ & $<1$ \\
\hline JUNE 9-16 & .31 & .46 & .79 & .013 & $<.010$ & $<1$ & $<1$ & $<1$ \\
\hline JUNE 16-23 & -- & -- & -- & - & -- & -- & -- & -- \\
\hline JUNE 23-30 & .37 & .46 & .70 & $<.010$ & $<.010$ & $<1$ & $<1$ & 1 \\
\hline JUNE 30-JULY 7 & .20 & .39 & .70 & $<.010$ & $<.010$ & 1 & $<1$ & $<1$ \\
\hline JULY 7-I4 & -- & -- & -- & -- & -- & -- & -- & -- \\
\hline JULY 14-21 & -- & -- & -- & -- & -- & -- & -- & -- \\
\hline JULY 21-28 & $<.14$ & $<.15$ & .08 & $<.010$ & $<.010$ & $<1$ & $<\mathrm{I}$ & $<1$ \\
\hline JULY 28-AUG 4 & .10 & .35 & 1.0 & $<.010$ & $<.010$ & $<\mathrm{I}$ & $<1$ & $<1$ \\
\hline AUG 4-11 & .46 & .80 & 1.4 & .017 & $<.100$ & $<1$ & $<1$ & $<1$ \\
\hline AUG 11-18 & -- & -- & -- & -- & -- & -- & -- & -- \\
\hline AUG $18-25$ & -- & -- & -- & -- & -- & -- & -- & -- \\
\hline AUG 25-SEPT 2 & -- & -- & -- & -- & -- & -- & -- & -- \\
\hline SEPT $2-8$ & -- & -- & -. & -- & -- & -- & -- & -- \\
\hline SEPT 8-15 & .47 & .65 & 1.1 & $<.010$ & $<.010$ & $<1$ & $<1$ & $<1$ \\
\hline SEPT 15-22 & -- & -- & -- & -. & -- & -- & -- & -- \\
\hline SEPT 22-29 & .26 & .35 & .74 & $<.010$ & $<.010$ & $<1$ & $<1$ & $<1$ \\
\hline SEPT 29-OCT 6 & -- & -- & -- & -- & -- & -- & -- & -- \\
\hline OCT 6-14 & -- & -- & -- & -- & -- & -- & -- & -- \\
\hline OCT $14-20$ & $<.11$ & $<.15$ & .11 & .090 & $<.010$ & $<1$ & $<1$ & $<1$ \\
\hline OCT $20-27$ & $<.10$ & $<.15$ & .08 & $<.010$ & $<.010$ & $<1$ & $<1$ & $<1$ \\
\hline OCT 27-NOV 3 & .19 & .25 & .41 & $<.010$ & $<.010$ & $<1$ & $<1$ & $<1$ \\
\hline NOV 3-10 & -- & -- & -- & -- & -- & -- & -- & -- \\
\hline NOV $10-17$ & .15 & .22 & .38 & $<.010$ & $<.010$ & $<1$ & $<1$ & $<1$ \\
\hline NOV 17-24 & .10 & .23 & .48 & $<.010$ & -- & $<1$ & $<1$ & $<1$ \\
\hline NOV 24-DEC 1 & $<.08$ & $<.15$ & .13 & -- & $<.010$ & $<1$ & $<1$ & $<1$ \\
\hline DEC 1-8 & -- & -- & -- & -- & -- & -- & -- & - \\
\hline DEC 8-15 & $<.11$ & $<.15$ & .15 & .025 & $<.010$ & $<1$ & $<1$ & $<1$ \\
\hline DEC $15-22$ & .13 & .17 & .44 & .025 & $<.010$ & $<1$ & $<1$ & $<1$ \\
\hline DEC 22-29 & .11 & .26 & .41 & $<.010$ & $<.010$ & $<1$ & $<1$ & $<1$ \\
\hline $\begin{array}{r}\text { DEC } 29,1997- \\
\text { JAN } 5,1998\end{array}$ & -- & -- & -- & -- & -- & -- & -- & -- \\
\hline JAN 5-12 & .16 & .23 & .31 & $<.010$ & $<.010$ & $<1$ & $<1$ & $<1$ \\
\hline JAN 12-20 & .13 & .19 & .37 & $<.010$ & $<.010$ & $<1$ & $<1$ & $<1$ \\
\hline JAN 20-26 & .23 & .31 & .61 & $<.010$ & $<.010$ & $<1$ & $<1$ & $<1$ \\
\hline JAN 26-FEB 2 & $<.10$ & $<.15$ & .12 & $<.010$ & $<.010$ & $<1$ & $<1$ & $<1$ \\
\hline FEB 2-9 & .16 & .19 & .33 & $<.010$ & $<.010$ & $<1$ & $<1$ & $<1$ \\
\hline FEB 9-16 & -- & -- & -- & -- & -- & $<1$ & $<1$ & $<1$ \\
\hline FEB 16-23 & .43 & .54 & .64 & $<.010$ & $<.010$ & $<1$ & $<1$ & $<1$ \\
\hline FEB 23-MAR 2 & -- & -- & -- & -- & -- & -- & -- & -- \\
\hline MAR 2-9 & .11 & .25 & .42 & $<.010$ & $<.010$ & $<1$ & $<1$ & $<\mathrm{I}$ \\
\hline MAR 9-16 & -- & -- & -- & -- & -- & -- & -- & -- \\
\hline MAR 16-23 & .36 & .36 & .53 & $<.010$ & $<.010$ & $<1$ & $<1$ & $<1$ \\
\hline MAR 23-30 & -- & -- & -- & -- & -- & -- & -- & -- \\
\hline
\end{tabular}


Table 60. Atmospheric deposition water-quality data at site 42 (CSW04), March 1997 through March 1998—Continued

\begin{tabular}{|c|c|c|c|c|c|c|c|c|c|}
\hline DATE & $\begin{array}{l}\text { COPPER, } \\
\text { TOTAL } \\
\text { RECOVER- } \\
\text { ABLE } \\
\text { (UG/ } \\
\text { AS CU) } \\
(01042) \\
\end{array}$ & $\begin{array}{c}\text { LEAD, } \\
\text { TOTAL } \\
\text { RECOVER- } \\
\text { ABLE } \\
\text { (UG/L } \\
\text { AS PB) } \\
(01051) \\
\end{array}$ & $\begin{array}{c}\text { MERCURY, } \\
\text { TOTAL } \\
\text { RECOVER- } \\
\text { ABLE } \\
\text { (UG/L } \\
\text { AS HG) } \\
\text { (71900) } \\
\end{array}$ & $\begin{array}{c}\text { NICKEL, } \\
\text { TOTAL } \\
\text { RECOVER- } \\
\text { ABLE } \\
\text { (UG/L } \\
\text { AS NI) } \\
(01067) \\
\end{array}$ & $\begin{array}{c}\text { ZINC, } \\
\text { TOTAL } \\
\text { RECOVER- } \\
\text { ABLE } \\
\text { (UG/ } \\
\text { AS ZN) } \\
(01092) \\
\end{array}$ & $\begin{array}{l}\text { BERYL- } \\
\text { LIUM, } \\
\text { TOTAL, } \\
\text { RECOVER- } \\
\text { ABLE } \\
\text { (UG/L } \\
\text { AS BE) } \\
(01012) \\
\end{array}$ & $\begin{array}{c}\text { ANTIMONY, } \\
\text { TOTAL } \\
\text { (UG/L } \\
\text { AS SB) } \\
(01097)\end{array}$ & $\begin{array}{c}\text { SELENIUM, } \\
\text { TOTAL } \\
\text { (UG/L } \\
\text { AS SE) } \\
\text { (01147) }\end{array}$ & $\begin{array}{c}\text { SILVER, } \\
\text { TOTAL } \\
\text { FFCOVER- } \\
\text { ABLE } \\
\text { (UG/L AS } \\
\text { AG) } \\
(01077)\end{array}$ \\
\hline MAR 25-31, 1997 & 6 & $<1$ & $<0.10$ & $<1$ & 20 & $<10$ & $<1$ & $<1$ & $<1$ \\
\hline MAR 31-APR 7 & $<1$ & $<1$ & $<.10$ & $<1$ & 10 & -- & -- & -- & -- \\
\hline APR 7-14 & $<1$ & $<1$ & $<.10$ & $<1$ & $<10$ & $<10$ & $<1$ & $<1$ & $<1$ \\
\hline APR $14-21$ & -. & -- & -- & -- & - & -- & -- & -- & - \\
\hline APR $21-28$ & $<1$ & $<1$ & $<.10$ & $<1$ & $<10$ & $<10$ & $<1$ & $<1$ & $<1$ \\
\hline APR 28-MAY 5 & $<1$ & $<1$ & $<.10$ & $<1$ & $<10$ & $<10$ & $<1$ & $<1$ & $<1$ \\
\hline MAY 5-12 & $<1$ & 1 & $<.10$ & $<1$ & $<10$ & $<10$ & $<1$ & $<1$ & $<1$ \\
\hline MAY $12-19$ & -- & -- & -- & -- & -- & -- & .. & -. & -- \\
\hline MAY $19-27$ & $<1$ & $<1$ & .10 & $<1$ & $<10$ & -- & .- & -- & -- \\
\hline MAY 27-JUNE 2 & -- & -- & -- & -- & - & -- & -- & -- & -- \\
\hline JUNE 2-9 & 1 & $<1$ & $<.10$ & $<1$ & $<10$ & $<10$ & $<1$ & $<1$ & $<1$ \\
\hline JUNE 9-16 & $<1$ & $<1$ & $<.10$ & $<1$ & $<10$ & $<10$ & $<1$ & $<1$ & $<1$ \\
\hline JUNE 16-23 & -- & -- & -- & -- & -- & -- & -- & -- & -- \\
\hline JUNE 23-30 & $<1$ & $<1$ & $<.10$ & $<1$ & $<10$ & $<10$ & $<1$ & $<1$ & $<1$ \\
\hline JUNE 30-JULY 7 & $<1$ & $<1$ & $<.10$ & $<1$ & $<10$ & $<10$ & $<1$ & $<1$ & $<1$ \\
\hline JULY 7-14 & -- & -- & -- & - & -- & - & -- & -- & -- \\
\hline JULY $14-21$ & -- & -- & - & -. & - & -. & -- & -- & -- \\
\hline JULY 21-28 & $<1$ & $<1$ & $<.10$ & $<1$ & $<10$ & $<10$ & $<1$ & $<1$ & $<1$ \\
\hline JULY 28 -AUG 4 & $<1$ & $<1$ & $<.10$ & $<1$ & $<10$ & $<10$ & $<1$ & $<1$ & $<1$ \\
\hline AUG 4-11 & I & $<1$ & $<.10$ & $<1$ & $<10$ & $<10$ & $<1$ & $<1$ & $<1$ \\
\hline AUG $11-18$ & -- & -- & -- & -- & -- & -- & -- & -- & -- \\
\hline AUG 18-25 & - & -- & -- & -- & -- & -- & -- & - & -- \\
\hline AUG 25-SEPT 2 & -. & -- & -. & -- & -- & -- & -- & -- & -- \\
\hline SEPT 2-8 & -- & -- & -- & -- & -- & -- & -- & -- & -- \\
\hline SEPT 8-15 & $<1$ & $<1$ & $<.10$ & $<1$ & 20 & $<10$ & $<1$ & $<1$ & $<1$ \\
\hline SEPT $15-22$ & -- & -- & -- & -- & -- & -- & -- & -- & -- \\
\hline SEPT $22-29$ & $<1$ & $<1$ & $<.10$ & $<1$ & 20 & $<10$ & $<1$ & $<1$ & $<1$ \\
\hline SEPT 29-OCT 6 & -- & -- & -- & -- & -- & -- & -- & -- & -- \\
\hline OCT 6-14 & -- & -- & -- & -- & -- & -- & -- & -- & -- \\
\hline OCT $14-20$ & $<1$ & $<1$ & $<.10$ & $<1$ & 10 & $<10$ & $<1$ & $<1$ & $<1$ \\
\hline OCT $20-27$ & $<1$ & $<1$ & $<.10$ & $<1$ & 20 & $<10$ & $<1$ & $<1$ & $<\mathrm{I}$ \\
\hline OCT 27-NOV 3 & $<1$ & $<1$ & $<.10$ & $<1$ & 10 & $<10$ & $<1$ & $<1$ & $<1$ \\
\hline NOV $3-10$ & -- & -- & -- & -- & -- & -. & -- & -- & -- \\
\hline NOV $10-17$ & $<1$ & $<1$ & $<.10$ & $<1$ & 30 & $<10$ & $<1$ & $<1$ & $<1$ \\
\hline NOV 17-24 & $<1$ & $<1$ & $<.10$ & $<1$ & $<10$ & $<10$ & $<1$ & $<1$ & $<1$ \\
\hline NOV 24-DEC 1 & $<1$ & $<1$ & $<.10$ & $<1$ & $<10$ & $<10$ & $<1$ & $<1$ & $<1$ \\
\hline DEC 1-8 & -- & -- & -- & -. & -- & -- & -- & -- & -- \\
\hline DEC 8-15 & $<1$ & $<1$ & $<.10$ & $<1$ & 30 & $<10$ & $<1$ & $<1$ & $<1$ \\
\hline DEC $15-22$ & $<1$ & $<1$ & $<.10$ & $<1$ & 10 & $<10$ & $<1$ & $<1$ & $<1$ \\
\hline DEC 22-29 & $<1$ & $<1$ & $<.10$ & $<1$ & $<10$ & $<10$ & $<1$ & $<1$ & $<1$ \\
\hline $\begin{array}{r}\text { DEC } 29,1997- \\
\text { JAN } 5,1998\end{array}$ & -- & -- & -- & -- & -- & -- & -- & -- & -- \\
\hline JAN 5-12 & $<1$ & $<1$ & -- & $<1$ & $<10$ & $<10$ & -- & -- & $<1$ \\
\hline JAN 12-20 & $<1$ & $<1$ & $<.10$ & $<1$ & $<10$ & $<10$ & $<1$ & $<1$ & $<1$ \\
\hline JAN 20-26 & $<1$ & $<1$ & $<.10$ & $<1$ & $<10$ & $<10$ & $<1$ & $<1$ & $<1$ \\
\hline JAN 26-FEB 2 & $<1$ & $<1$ & $<.10$ & $<1$ & $<10$ & $<10$ & $<1$ & $<1$ & $<1$ \\
\hline FEB 2-9 & $<1$ & $<1$ & $<.10$ & $<1$ & $<10$ & $<10$ & $<1$ & $<1$ & $<1$ \\
\hline FEB 9-16 & $<1$ & $<1$ & $<.10$ & $<1$ & $<10$ & $<10$ & $<1$ & $<1$ & $<1$ \\
\hline FEB 16-23 & $<1$ & $<1$ & $<.10$ & $<1$ & 10 & $<10$ & $<1$ & $<1$ & $<1$ \\
\hline FEB 23-MAR 2 & -- & -- & -- & -- & -- & -- & -- & -- & -- \\
\hline MAR 2-9 & $<1$ & $<1$ & $<.10$ & $<1$ & 20 & $<10$ & $<1$ & $<1$ & $<1$ \\
\hline MAR 9-16 & -- & -- & -- & -- & -. & -- & -. & -- & -- \\
\hline MAR $16-23$ & $<1$ & $<1$ & $<.10$ & $<1$ & 20 & $<10$ & $<1$ & $<1$ & $<1$ \\
\hline MAR 23-30 & -- & - & -- & -- & -- & -- & -- & -- & -- \\
\hline
\end{tabular}


Table 61. Atmospheric deposition water-quality data at site 43 (CSW07), March 1997 through March 1998

\begin{tabular}{|c|c|c|c|c|c|c|c|c|}
\hline DATE & $\begin{array}{l}\text { RAINFALL } \\
\text { (IN.NKK) } \\
\text { (00045) }\end{array}$ & $\begin{array}{c}\text { RAINFALL, } \\
\text { TOTAL } \\
\text { (IN } / W K \text { ) } \\
\text { (00046) }\end{array}$ & $\begin{array}{l}\text { SPECIFIC } \\
\text { CONDUC- } \\
\text { TANCE } \\
\text { LAB } \\
\text { (US/CM) } \\
(90095) \\
\end{array}$ & $\begin{array}{c}\text { PH WATER } \\
\text { RAW } \\
\text { LAB } \\
\text { (STANDARD } \\
\text { UNITS) } \\
\text { (00403) } \\
\end{array}$ & $\begin{array}{c}\text { CHLORIDE, } \\
\text { DISSOLVED } \\
\text { (MG/L } \\
\text { AS CL) } \\
(00940) \\
\end{array}$ & $\begin{array}{c}\text { SULFATE, } \\
\text { DISSOLVED } \\
\text { (MG/L AS } \\
\text { S04) } \\
(00945) \\
\end{array}$ & $\begin{array}{c}\text { NITROGEN, } \\
\text { NO2+NO3, } \\
\text { TOTAL } \\
\text { (MG/L } \\
\text { AS N) } \\
(00630) \\
\end{array}$ & $\begin{array}{c}\text { NITROGEN, } \\
\text { AMMIONIA, } \\
\text { TO-AL } \\
\text { (MG/L } \\
\text { AS N) } \\
(00610) \\
\end{array}$ \\
\hline MAR 24-31, 1997 & 0.55 & 0.70 & 22 & 4.8 & $<2.0$ & 1.5 & 0.390 & .120 \\
\hline MAR 31-APR 7 & .04 & .07 & 39 & 4.3 & -- & -- & -- & -- \\
\hline APR 7-14 & .67 & .70 & 7 & 5.4 & $<2.0$ & $<1.0$ & .070 & .060 \\
\hline APR 14-21 & .00 & .02 & -- & 3.8 & -- & -- & -. & -- \\
\hline APR 21-28 & 3.18 & 3.14 & 13 & 4.7 & $<2.0$ & $<1.0$ & .200 & .140 \\
\hline APR 28-MAY 5 & 1.91 & 1.94 & 14 & 4.8 & $<2.0$ & $<1.0$ & .210 & .190 \\
\hline MAY 5-12 & .31 & .36 & 70 & 4.1 & $<2.0$ & 6.4 & -- & -. \\
\hline MAY $12-19$ & .00 & .00 & -- & -- & -- & -- & -- & -- \\
\hline MAY 19-27 & .17 & .25 & 34 & 4.0 & -. & -- & $\ldots$ & -- \\
\hline MAY 27-JUNE 2 & .00 & .00 & -- & -- & -- & -- & -- & - \\
\hline JUNE 2-9 & .71 & .75 & 4 & 5.2 & $<2.0$ & $<1.0$ & .070 & $<.015$ \\
\hline JUNE 9-16 & 2.89 & 2.89 & 17 & 4.8 & $<2.0$ & $<1.0$ & .240 & .150 \\
\hline JUNE 16-23 & .00 & .02 & -. & 3.9 & -- & -- & -- & -- \\
\hline JUNE 23-30 & .61 & .65 & 22 & -- & $<2.0$ & 2.8 & -- & -- \\
\hline JUNE 30-JUL Y7 & .03 & .07 & 32 & 4.2 & -- & - & -- & -- \\
\hline JULY 7-14 & .00 & .00 & -- & -- & -- & - & -- & -- \\
\hline JULY 14-21 & .34 & .37 & 52 & 4.0 & $<2.0$ & 6.5 & -- & -- \\
\hline JULY 21-28 & 4.26 & 4.12 & 8 & 5.1 & $<2.0$ & $<1.0$ & $<.070$ & $<.015$ \\
\hline JULY 28-AUG 4 & .50 & .50 & 27 & 5.2 & $<2.0$ & 5.5 & .830 & .390 \\
\hline AUG 4-11 & .12 & .16 & 74 & 4.3 & $<2.0$ & 2.0 & -- & -- \\
\hline AUG 11-18 & .00 & .00 & -- & -- & -- & -- & -- & -- \\
\hline AUG $18-25$ & .00 & .04 & -- & 4.2 & $<2.0$ & -- & -- & -- \\
\hline AUG 25-SEPT 2 & .28 & .31 & 54 & 4.0 & $<2.0$ & 7.0 & -- & -- \\
\hline SEPT 2-8 & .00 & .00 & -- & -- & -. & - & -- & -- \\
\hline SEPT 8-15 & .44 & .48 & 41 & 4.1 & $<2.0$ & 2.0 & .480 & .160 \\
\hline SEPT 15-22 & .02 & .05 & -- & -- & 3.1 & -- & -- & -. \\
\hline SEPT $22-29$ & 2.39 & 2.26 & 31 & 4.3 & $<2.0$ & 2.2 & .450 & .210 \\
\hline SEPT 29-OCT 6 & .00 & .00 & -- & $\ldots$ & -- &.- & -- & - \\
\hline OCT 6-14 & .00 & .00 & -- & -- & -- & -- & -- & -- \\
\hline OCT $14-20$ & 2.51 & 2.37 & 6 & 5.3 & $<2.0$ & $<1.0$ & .090 & $<. C^{15}$ \\
\hline OCT $20-27$ & 2.04 & 1.98 & 7 & 5.1 & $<2.0$ & $<1.0$ & .050 & $<. C 15$ \\
\hline OCT $27-N O V 3$ & .92 & .93 & 14 & 4.7 & $<2.0$ & 1.2 & .180 & .060 \\
\hline NOV 3-10 & .00 & .00 & -. & -- & .- & -- & -- & -- \\
\hline NOV $10-17$ & 1.40 & 1.34 & 14 & 4.8 & $<2.0$ & $<1.0$ & .150 & .040 \\
\hline NOV $17-24$ & 1.36 & 1.32 & 19 & 4.6 & $<2.0$ & 1.8 & .210 & .120 \\
\hline NOV 24-DEC 1 & .77 & .75 & 13 & 4.8 & $<2.0$ & 1.0 & .120 &.$c 50$ \\
\hline DEC 1-8 & .04 & .06 & -- & 4.0 & $<2.0$ & -- & -- & -- \\
\hline DEC 8-15 & .69 & .66 & 16 & 4.6 & $<2.0$ & 2.0 & .170 & .040 \\
\hline DEC 15-22 & 1.09 & 1.07 & 22 & 4.4 & $<2.0$ & 1.2 & .250 & .060 \\
\hline DEC 22-29 & 2.22 & 2.08 & 12 & 4.7 & $<2.0$ & $<1.0$ & .130 & .120 \\
\hline $\begin{array}{r}\text { DEC } 29,1997- \\
\text { JAN } 5,1998\end{array}$ & .00 & .02 & -- & -- & -- & -- & -- & -- \\
\hline JAN 5-12 & .99 & .95 & 7 & 5.1 & $<2.0$ & $<1.0$ & .080 & .070 \\
\hline JAN $12-20$ & 2.38 & 2.45 & 15 & 4.6 & $<2.0$ & $<1.0$ & .160 & .060 \\
\hline JAN 20-26 & .80 & .78 & 30 & 4.4 & $<2.0$ & $<1.0$ & .370 & .100 \\
\hline JAN 26-FEB 2 & 1.70 & 1.65 & 13 & 4.7 & $<2.0$ & $<1.0$ & .090 & .050 \\
\hline FEB 2-9 & 1.67 & 1.58 & 19 & 4.5 & $<2.0$ & $<1.0$ & .220 & .070 \\
\hline FEB 9-16 & .22 & .29 & 27 & 4.8 & $<2.0$ & 1.3 & .370 & .190 \\
\hline FEB 16-23 & 2.09 & 2.00 & 6 & 5.1 & $<2.0$ & $<1.0$ & .100 & .110 \\
\hline FEB 23-MAR 2 & .23 & .24 & 18 & 4.6 & $<2.0$ & 1.5 & -- & -- \\
\hline MAR 2-9 & 2.02 & 1.97 & 9 & 4.9 & $<2.0$ & $<1.0$ & .180 & .170 \\
\hline MAR 9-16 & .00 & .04 & -- & -- & $<2.0$ & -- & -- & -- \\
\hline MAR $16-23$ & 1.47 & 1.50 & 13 & 5.2 & $<2.0$ & 1.5 & .150 & .150 \\
\hline MAR 23-30 & .11 & .11 & 31 & 4.1 & $<2.0$ & -- & -- & -- \\
\hline
\end{tabular}


Table 61. Atmospheric deposition water-quality data at site 43 (CSW07), March 1997 through March 1998-Continued

\begin{tabular}{|c|c|c|c|c|c|c|c|c|}
\hline DATE & $\begin{array}{l}\text { NITROGEN, } \\
\text { ORGANIC } \\
\text { TOTAL } \\
\text { (MG/L } \\
\text { AS N) } \\
(00605)\end{array}$ & $\begin{array}{l}\text { NITROGEN, } \\
\text { AMMONIA + } \\
\text { ORGANIC } \\
\text { TOTAL } \\
\text { (MG/L } \\
\text { AS N) } \\
(00625)\end{array}$ & $\begin{array}{l}\text { NITROGEN, } \\
\text { TOTAL } \\
\text { (MG/L } \\
\text { AS N) } \\
(00600)\end{array}$ & $\begin{array}{c}\text { PHOS- } \\
\text { PHORUS, } \\
\text { TOTAL } \\
\text { (MG/L } \\
\text { AS P) } \\
\text { (00665) }\end{array}$ & $\begin{array}{l}\text { PHOS- } \\
\text { PHORUS, } \\
\text { ORTHO, } \\
\text { TOTAL } \\
\text { (MG/L } \\
\text { AS P) } \\
\text { (70507) }\end{array}$ & $\begin{array}{l}\text { ARSENIC, } \\
\text { TOTAL } \\
\text { (UG/L } \\
\text { AS AS) } \\
(01002)\end{array}$ & $\begin{array}{l}\text { CADMIUM } \\
\text { WATER } \\
\text { UNFIL- } \\
\text { TERED, } \\
\text { TOTAL } \\
\text { (UG/L } \\
\text { AS CD) } \\
\text { (01027) }\end{array}$ & $\begin{array}{l}\text { CHA?- } \\
\text { MIU } \%, \\
\text { TOT'", } \\
\text { RECO'"ER- } \\
\text { ABI E } \\
\text { (UG". } \\
\text { AS C.R) } \\
\text { (01034) }\end{array}$ \\
\hline MAR 24-31, 1997 & 0.52 & 0.64 & 1.0 & 0.080 & 0.019 & $<1$ & $<\mathrm{I}$ & 1 \\
\hline MAR 3I-APR 7 & -- & -- & -- & -- & -- & -- & -- & -- \\
\hline APR 7-14 & $<.09$ & $<.15$ & .07 & $<.010$ & $<.010$ & $<1$ & $<1$ & $<1$ \\
\hline APR 14-21 & -- & -- & -- & -- & -- & -- & -- & -- \\
\hline APR 21-28 & .13 & .27 & .47 & $<.010$ & $<.010$ & $<1$ & $<1$ & 1 \\
\hline APR 28-MAY 5 & .31 & .50 & .71 & $<.010$ & $<.010$ & $<1$ & $<1$ & $<1$ \\
\hline MAY 5-12 & -- & -- & -- & -- & -- & $<1$ & $<1$ & $<1$ \\
\hline MAY $12-19$ & -- & -- & -- & -- & -- & -- & -- & -- \\
\hline MAY 19-27 & -- & -- & -- & -- & -- & $<1$ & $<1$ & $<1$ \\
\hline MAY 27-JUNE 2 & -- & -- & -- & -- & -- & -- & - & -- \\
\hline JUNE 2-9 & .14 & .14 & .21 & $<.010$ & $<.010$ & $<1$ & $<1$ & $<1$ \\
\hline JUNE 9-16 & .22 & .37 & $.6 \mathrm{I}$ & $<.010$ & $<.010$ & $<\mathrm{l}$ & $<1$ & $<1$ \\
\hline JUNE 16-23 & -- &.- & .. & -- &.- & -- & -- & -- \\
\hline JUNE $23-30$ & -- & -- & -- & -- & -- & $<1$ & $<1$ & 1 \\
\hline JUNE 30-JULY 7 & -- & -- & -- & -- & -- & -- & -- & -- \\
\hline JULY 7-I4 & -- & -- & -- & -- & -- & -- & -- & -- \\
\hline JULY 14-21 & -- & -- & -- & -- & -- & -- & -- & -- \\
\hline JULY 2I-28 & $<.14$ & $<.15$ & $<.22$ & $<.010$ & $<.010$ & $<1$ & $<\mathrm{I}$ & $<\mathrm{I}$ \\
\hline JULY 28-AUG 4 & .32 & .71 & 1.5 & $<.010$ & $<.010$ & $<1$ & $<1$ & $<1$ \\
\hline AUG 4-11 & -- & -- & -- & -- & -- & -- & -- & -- \\
\hline AUG I1-I8 & -- & -- & -- & -- & -- & -- & -- & -- \\
\hline AUG 18-25 & -- & -- & -- & -- & -- & -- & -- & -- \\
\hline AUG 25-SEPT 2 & -- & -- & - & $\cdots$ & -- & $<1$ & $<1$ & $<1$ \\
\hline SEPT 2-8 & -- & -- & -- & -- & -- & -- & -- & -- \\
\hline SEPT 8-15 & .48 & .64 & I.I & $<.010$ & $<.010$ & $<1$ & $<1$ & $<\mathrm{I}$ \\
\hline SEPT $15-22$ & -- & -- & -- & -- & -- & -- & -- & -- \\
\hline SEPT $22-29$ & .67 & .88 & 1.3 & .039 & .039 & I6 & $<\mathrm{l}$ & $<\mathrm{I}$ \\
\hline SEPT 29 -OCT 6 & -- & -- & -- & -- & -- & -- & -- & -- \\
\hline OCT 6-14 & -- & -- & - & - & -- & -- & -- & -- \\
\hline OCT $14-20$ & $<.14$ & $<.15$ & .09 & .090 & $<.010$ & $<1$ & $<1$ & $<\mathrm{I}$ \\
\hline ОСТ $20-27$ & .21 & $.2 \mathrm{I}$ & .26 & $<.010$ & $<.010$ & $<\mathrm{I}$ & $<\mathrm{l}$ & $<\mathrm{l}$ \\
\hline OCT 27-NOV 3 & .18 & .24 & .42 & $<.010$ & $<.010$ & $<1$ & $<1$ & $<1$ \\
\hline NOV 3-10 & -- & -- & -- & -- & -- & -- & -- & -- \\
\hline NOV $10-17$ & .11 & .15 & .30 & -- & $<.010$ & $<\mathrm{I}$ & $<1$ & $<1$ \\
\hline NOV 17-24 & .12 & .24 & .45 & $<.010$ & $<.010$ & $<\mathrm{I}$ & $<\mathrm{l}$ & $<\mathrm{I}$ \\
\hline NOV 24-DEC I & .14 & .20 & .32 & $<.010$ & $<.010$ & $<\mathrm{I}$ & $<\mathrm{I}$ & $<\mathrm{I}$ \\
\hline DEC $1-8$ & -- & -- & -- & -- & -- & -- & -- & -- \\
\hline DEC $8-15$ & .14 & .18 & .35 & $<.010$ & $<.010$ & $<1$ & $<\mathrm{I}$ & $<1$ \\
\hline DEC $15-22$ & .13 & .19 & .44 & $<.010$ & $<.010$ & $<\mathrm{I}$ & $<\mathrm{I}$ & $<1$ \\
\hline DEC $22-29$ & .11 & .23 & .36 & .015 & $<.010$ & $<1$ & $<1$ & $<\mathrm{l}$ \\
\hline $\begin{array}{r}\text { DEC 29, } 1997- \\
\text { JAN 5, } 1998\end{array}$ & -- & -- & -- & -- & -- & -- & -- & -- \\
\hline JAN 5-12 & .14 & .21 & .29 & $<.010$ & $<.010$ & $<1$ & $<1$ & $<\mathrm{I}$ \\
\hline JAN I2-20 & .13 & .19 & .35 & $<.010$ & $<.010$ & $<\mathrm{I}$ & $<1$ & $<\mathrm{I}$ \\
\hline JAN 20-26 & .18 & .28 & .65 & $<.010$ & $<.010$ & $<1$ & $<1$ & $<\mathrm{l}$ \\
\hline JAN 26-FEB 2 & $<.10$ & $<.15$ & .09 & $<.010$ & $<.010$ & $<\mathrm{I}$ & $<1$ & $<\mathrm{I}$ \\
\hline FEB 2-9 & .12 & .19 & $.4 \mathrm{I}$ & $<.010$ & $<.010$ & $<\mathrm{I}$ & $<1$ & $<1$ \\
\hline FEB 9-16 & .11 & .30 & .67 & .016 & $<.010$ & -- & -- & -- \\
\hline FEB 16-23 & .16 & .27 & .37 & $<.010$ & $<.010$ & $<\mathrm{I}$ & $<\mathrm{I}$ & $<1$ \\
\hline FEB 23-MAR 2 & -- & -- & -- & -- & -- & -- & -- & -- \\
\hline MAR 2-9 & .03 & .20 & .38 & $<.010$ & $<.010$ & $<\mathrm{I}$ & $<1$ & $<1$ \\
\hline MAR 9-16 & -- & -- & -- & -- & -- & -- & -- & -- \\
\hline MAR I6-23 & 2.0 & 2.2 & 2.3 & .190 & .210 & $<1$ & $<1$ & $<\mathrm{I}$ \\
\hline MAR 23-30 & -- & -- & -- & -- & -- & -- & -- & -- \\
\hline
\end{tabular}


Table 61. Atmospheric deposition water-quality data at site 43 (CSW07), March 1997 through March 1998-Continued

\begin{tabular}{|c|c|c|c|c|c|c|c|c|c|}
\hline DATE & $\begin{array}{c}\text { COPPER, } \\
\text { TOTAL } \\
\text { RECOVER- } \\
\text { ABLE } \\
\text { (UG/L } \\
\text { AS CU) } \\
\text { (01042) }\end{array}$ & $\begin{array}{c}\text { LEAD, } \\
\text { TOTAL } \\
\text { RECOVER- } \\
\text { ABLE } \\
\text { (UG/L } \\
\text { AS PB) } \\
(01051)\end{array}$ & $\begin{array}{c}\text { MERCURY, } \\
\text { TOTAL } \\
\text { RECOVER- } \\
\text { ABLE } \\
\text { (UG/L } \\
\text { AS HG) } \\
(71900) \\
\end{array}$ & $\begin{array}{c}\text { NICKEL, } \\
\text { TOTAL } \\
\text { RECOVER- } \\
\text { ABLE } \\
\text { (UG/L } \\
\text { AS NI) } \\
\text { (01067) }\end{array}$ & $\begin{array}{c}\text { ZINC, } \\
\text { TOTAL } \\
\text { RECOVER- } \\
\text { ABLE } \\
\text { (UG/L } \\
\text { AS ZN) } \\
(01092) \\
\end{array}$ & $\begin{array}{l}\text { BERYL- } \\
\text { LIUM, } \\
\text { TOTAL, } \\
\text { RECOVER- } \\
\text { ABLE } \\
\text { (UG/L } \\
\text { AS BE) } \\
\text { (01012) } \\
\end{array}$ & $\begin{array}{c}\text { ANTIMONY, } \\
\text { TOTAL } \\
\text { (UG/L } \\
\text { AS SB) } \\
(01097) \\
\end{array}$ & $\begin{array}{c}\text { SELENIUM, } \\
\text { TOTAL } \\
\text { (UG/L } \\
\text { AS SE) } \\
\text { (01147) } \\
\end{array}$ & $\begin{array}{c}\text { SILVER, } \\
\text { TOTAL } \\
\text { RECOVER- } \\
\text { ABLE } \\
\text { (UG/L AS } \\
\text { AG) } \\
\text { (01077) }\end{array}$ \\
\hline MAR 24-31, 1997 & 3 & $<1$ & $<0.10$ & $<1$ & 20 & $<10$ & $<1$ & $<1$ & $<1$ \\
\hline MAR 31-APR 7 & -- & - & - & -- & -- & -- & -- & -- & -- \\
\hline APR 7-14 & $<1$ & $<1$ & $<.10$ & $<1$ & $<10$ & $<10$ & $<1$ & $<1$ & $<1$ \\
\hline APR 14-21 & -- & - & - & -- & -. & -- & -- & -- & -- \\
\hline APR 21-28 & $<1$ & $<1$ & $<.10$ & $<1$ & $<10$ & $<10$ & $<1$ & $<1$ & $<1$ \\
\hline APR 28-MAY 5 & $<1$ & 1 & $<.10$ & $<1$ & $<10$ & $<10$ & $<1$ & $<1$ & $<1$ \\
\hline MAY 5-12 & 1 & 2 & $<.10$ & $<1$ & $<10$ & $<10$ & $<1$ & $<1$ & $<1$ \\
\hline MAY $12-19$ & -- & - & -- & -- & -- & -- & -- & -- & -. \\
\hline MAY $19-27$ & 1 & 2 & $<.10$ & $<1$ & $<10$ & -- & -- & -- & -- \\
\hline MAY 27-JUNE 2 & -- & -- & -- & -- & -- & -- & -- & -- & -- \\
\hline JUNE 2-9 & $<1$ & $<1$ & $<.10$ & $<1$ & 10 & $<10$ & $<1$ & $<1$ & $<1$ \\
\hline JUNE 9-16 & $<1$ & $<1$ & $<.10$ & $<1$ & $<10$ & $<10$ & $<1$ & $<1$ & $<1$ \\
\hline JUNE 16-23 & -- & -- & -- & -- & -- & -- & - & - & -- \\
\hline JUNE 23-30 & $<1$ & $<1$ & $<.10$ & $<1$ & $<10$ & $<10$ & $<1$ & $<1$ & $<1$ \\
\hline JUNE 30-JULY 7 & -- & -- & -- & -- & -- & - & -- & - & - \\
\hline JULY 7-14 & -- & -- & -- & -- & -- & - & - & -- & -- \\
\hline JULY 14-21 & -- & -- & -- & -- & -- & -- & -- & -- & -- \\
\hline JULY $21-28$ & $<1$ & $<1$ & $<.10$ & $<1$ & $<10$ & $<10$ & $<1$ & $<1$ & $<1$ \\
\hline JULY 28-AUG 4 & $<1$ & 2 & $<.10$ & $<1$ & 100 & $<10$ & $<1$ & $<1$ & $<1$ \\
\hline AUG 4-11 & -- & -- & -- & -- & -- & - & -- & -- & -- \\
\hline AUG 11-18 & -- & -- & -- & -- & -- & -- & - & -- & -- \\
\hline AUG $18-25$ & -- & -- & -- & -- & -. & -. & - & -- & - \\
\hline AUG 25-SEPT 2 & $<1$ & $<1$ & $<.10$ & $<1$ & 10 & - & -- & -- & -- \\
\hline SEPT 2-8 & -- & -- & -- & -- & -- & -- & -- & -- & -- \\
\hline SEPT $8-15$ & $<1$ & $<1$ & $<.10$ & $<1$ & 20 & $<10$ & $<1$ & $<1$ & $<1$ \\
\hline SEPT $15-22$ & -- & -- & -- & -- & -- & -- & -- & -- & -- \\
\hline SEPT $22-29$ & $<1$ & $<1$ & $<.10$ & $<1$ & $<10$ & $<10$ & $<1$ & $<1$ & $<1$ \\
\hline SEPT 29-OCT 6 & -- & -- & -- & -- & -. & -- & -- & -- & -- \\
\hline OCT $6-14$ & -- & -- & -- & -- & -- & -- & -- & -- & -. \\
\hline OCT $14-20$ & $<1$ & $<1$ & $<.10$ & $<1$ & $<10$ & $<10$ & $<1$ & $<1$ & $<1$ \\
\hline OCT $20-27$ & $<1$ & $<1$ & $<.10$ & $<1$ & 10 & $<10$ & $<1$ & $<1$ & $<1$ \\
\hline OCT $27-N O V 3$ & $<1$ & $<1$ & $<.10$ & $<1$ & 20 & $<10$ & $<1$ & $<1$ & $<1$ \\
\hline NOV $3-10$ & -- & - & -- & -- & -- & -- & -- & -- & -- \\
\hline NOV $10-17$ & $<1$ & $<1$ & $<.10$ & $<1$ & 30 & $<10$ & $<1$ & $<1$ & $<1$ \\
\hline NOV $17-24$ & $<1$ & $<1$ & $<.10$ & $<1$ & 10 & $<10$ & $<1$ & $<1$ & $<1$ \\
\hline NOV 24-DEC 1 & $<1$ & $<1$ & $<.10$ & $<1$ & $<10$ & $<10$ & $<1$ & $<1$ & $<1$ \\
\hline DEC $1-8$ & -- & -- & -- & -- & -- & - & -- & -- & -- \\
\hline DEC 8-15 & $<1$ & $<1$ & $<.10$ & $<1$ & 20 & $<10$ & $<1$ & $<1$ & $<1$ \\
\hline DEC $15-22$ & $<1$ & $<1$ & $<.10$ & $<1$ & $<10$ & $<10$ & $<1$ & $<1$ & $<1$ \\
\hline DEC 22-29 & $<1$ & $<1$ & $<.10$ & $<1$ & 10 & $<10$ & $<1$ & $<1$ & $<1$ \\
\hline $\begin{array}{r}\text { DEC } 29,1997- \\
\text { JAN } 5,1998\end{array}$ & -- & - & -- & -- & -- & -- & -- & -- & -- \\
\hline JAN 5-12 & $<1$ & $<1$ & $<.10$ & $<1$ & $<10$ & $<10$ & $<1$ & $<1$ & $<1$ \\
\hline JAN $12-20$ & $<1$ & $<1$ & $<.10$ & $<1$ & $<10$ & $<10$ & $<1$ & $<1$ & $<1$ \\
\hline JAN 20-26 & $<1$ & $<1$ & $<.10$ & $<1$ & $<10$ & $<10$ & $<1$ & $<1$ & $<1$ \\
\hline JAN 26-FEB 2 & $<1$ & $<1$ & $<.10$ & $<1$ & $<10$ & $<10$ & $<1$ & $<1$ & $<1$ \\
\hline FEB 2-9 & $<1$ & $<1$ & $<.10$ & $<1$ & 10 & $<10$ & $<1$ & $<1$ & $<1$ \\
\hline FEB 9-16 & -- & - & $-\cdot$ & -- & -- & -- & -- & -- & -- \\
\hline FEB 16-23 & $<1$ & $<1$ & $<.10$ & $<1$ & 10 & $<10$ & $<1$ & $<1$ & $<1$ \\
\hline FEB 23-MAR 2 & -- & -- & -- & -- & -- & -- & -- & -- & -- \\
\hline MAR 2-9 & $<1$ & $<1$ & $<.10$ & $<1$ & 10 & $<10$ & $<1$ & $<1$ & $<1$ \\
\hline MAR 9-16 & -- & -- & -- & -- & -- & -- & -- & -- & -- \\
\hline MAR 16-23 & $<1$ & $<1$ & $<.10$ & $<1$ & $<10$ & $<10$ & $<1$ & $<1$ & $<1$ \\
\hline MAR 23-30 & -- & - & -- & -- & -- & -- & - & - & - \\
\hline
\end{tabular}


Table 62. Streamflow statistics at the streamflow and water-quality study sites, December 1993 through September 1998 $\left[\mathrm{mi}^{2}\right.$, square mile; $\mathrm{ft}^{3} / \mathrm{s}$, cubic foot per second]

\begin{tabular}{|c|c|c|c|c|c|}
\hline $\begin{array}{l}\text { Site no. } \\
\text { (fig. 1) }\end{array}$ & $\begin{array}{l}\text { Drainage } \\
\text { area } \\
\left(\mathbf{m i}^{2}\right)\end{array}$ & $\begin{array}{l}\text { Period of } \\
\text { record }\end{array}$ & $\begin{array}{l}\text { Daily mean } \\
\text { discharge for } \\
\text { period of record } \\
\left(\mathrm{ft}^{3} / \mathrm{s}\right)\end{array}$ & $\begin{array}{c}\text { Maximum } \\
\text { instantaneous } \\
\text { discharge recorded } \\
\left(\mathrm{ft}^{3} / \mathrm{s}\right)\end{array}$ & $\begin{array}{c}\text { Minimum } \\
\text { instantaneous } \\
\text { discharge recorde } \mathrm{f} \\
\left(\mathrm{ft}^{3} / \mathrm{s}\right) \\
\text { and number of days of oc ?urrence }\end{array}$ \\
\hline $\begin{array}{c}37 \\
(\mathrm{CSW} 06)\end{array}$ & 0.063 & $4 / 95-9 / 98$ & 0.088 & Not determined & $\begin{array}{l}0 \\
\text { (482 days during period of record: } \\
38 \% \text { of days during period of record) }\end{array}$ \\
\hline $\begin{array}{c}39 \\
\text { (CSW05) }\end{array}$ & .022 & $3 / 94-9 / 98$ & 0.037 & $\begin{array}{c}27 \\
(5 / 29 / 96) \\
(7 / 23 / 97)\end{array}$ & $\begin{array}{c}0 \\
\text { (192 days during period of record; } \\
14 \% \text { of days during perio } d \text { of record) }\end{array}$ \\
\hline $\begin{array}{c}40 \\
\text { (CSW03) }\end{array}$ & .023 & $7 / 94-9 / 98$ & 0.009 & $\begin{array}{c}\geq 27^{\mathrm{a}} \\
(8 / 27 / 95)\end{array}$ & $\begin{array}{c}0 \\
\text { (1380 days during period of record; } \\
89 \% \text { of days during periot of record) }\end{array}$ \\
\hline $\begin{array}{c}41 \\
(\mathrm{CSW} 02)\end{array}$ & .123 & $12 / 93-9 / 98$ & 0.17 & $\begin{array}{c}334 \\
(8 / 27 / 95)\end{array}$ & $\begin{array}{c}0.010 \\
(7 \text { days during period of record; } \\
<1 \% \text { of days during pericd of record })\end{array}$ \\
\hline $\begin{array}{c}42 \\
\text { (CSW04) }\end{array}$ & .126 & $12 / 93-9 / 98$ & 0.32 & $\begin{array}{c}305 \\
(7 / 23 / 97)\end{array}$ & $\begin{array}{c}0 \\
\text { (12 days during period of record; } \\
1 \% \text { of days during periot of record) }\end{array}$ \\
\hline $\begin{array}{c}43 \\
\text { (CSW07) }\end{array}$ & .266 & $6 / 94-9 / 98$ & 0.46 & $\begin{array}{c}371 \\
(7 / 3 / 95)\end{array}$ & $\begin{array}{c}0 \\
\text { (18 days during period of record; } \\
1 \% \text { of days during period of record) }\end{array}$ \\
\hline
\end{tabular}

${ }^{\mathrm{a}}$ Instantaneous discharge during storm of July 23, 1997, may have exceeded $27 \mathrm{ft}^{3} / \mathrm{s}$. 


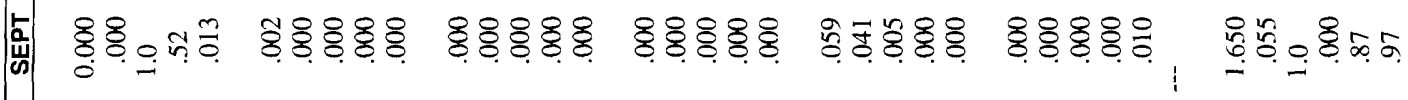

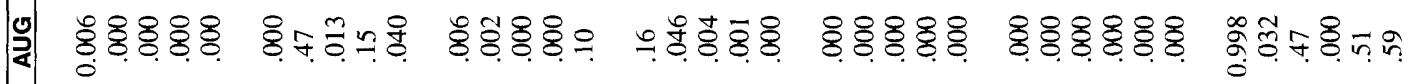

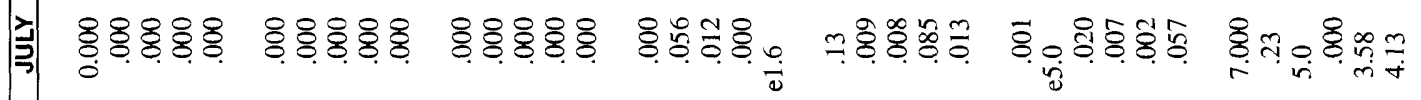

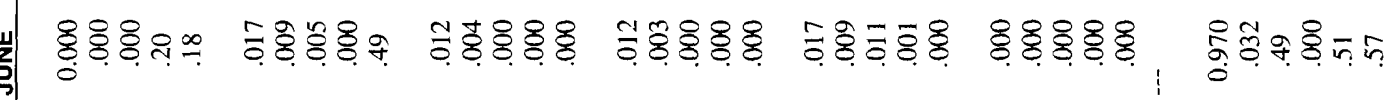

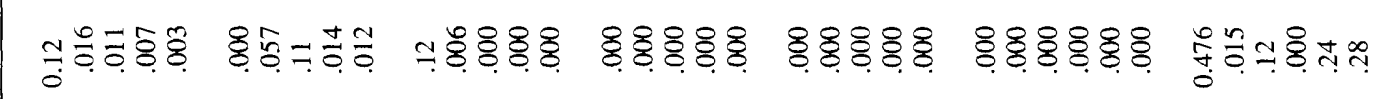

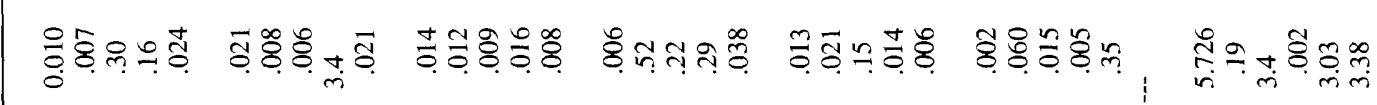

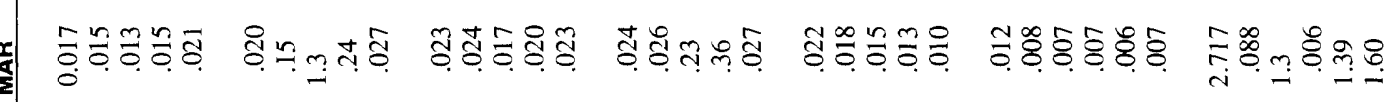

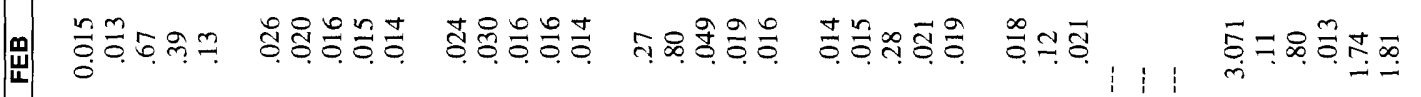
₹

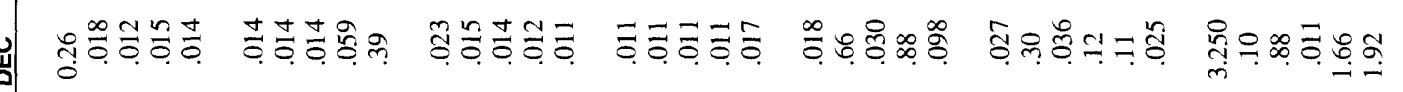

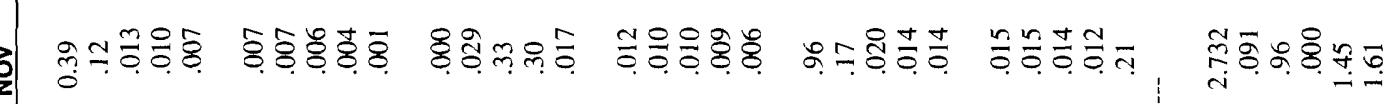
$\frac{0}{3}$ . (1)

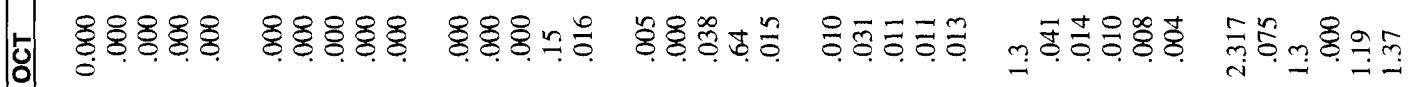

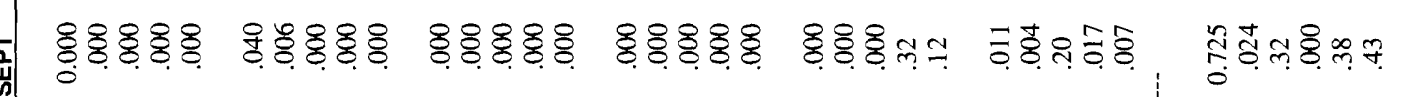

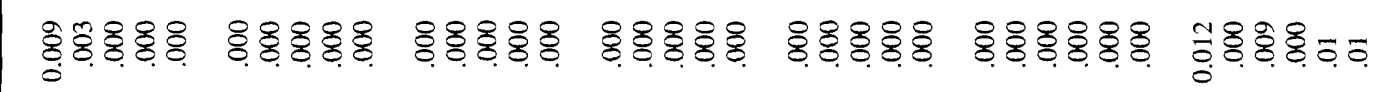
ฮั

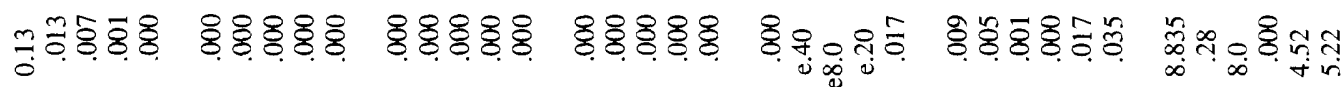

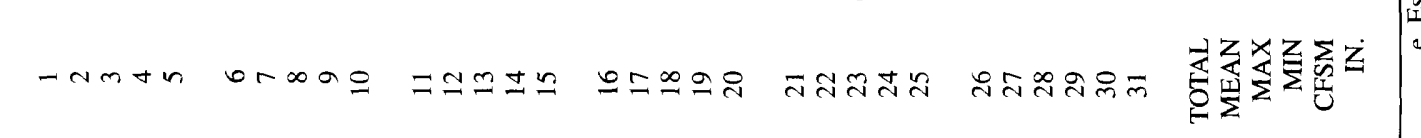




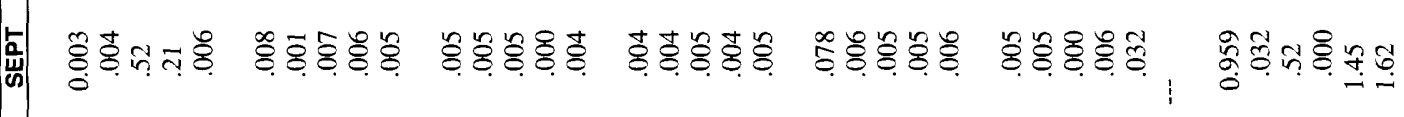

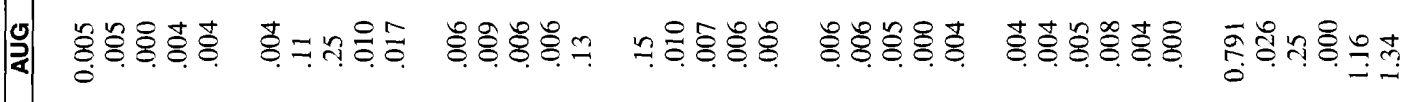

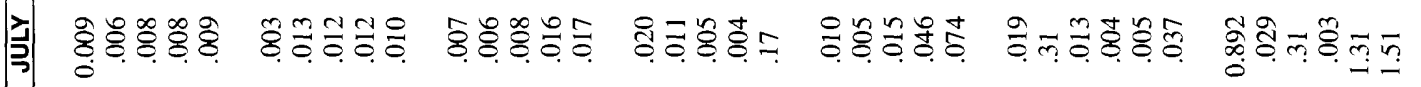

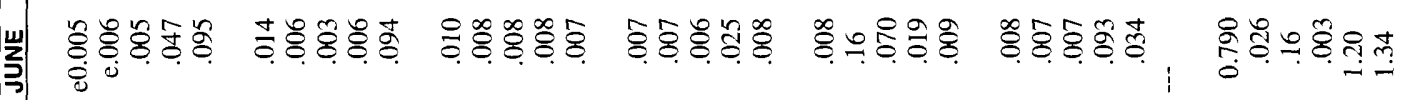

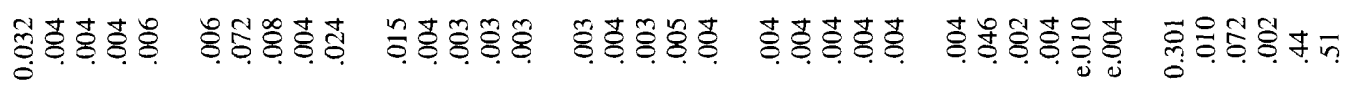

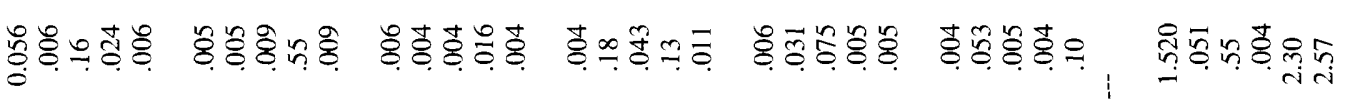

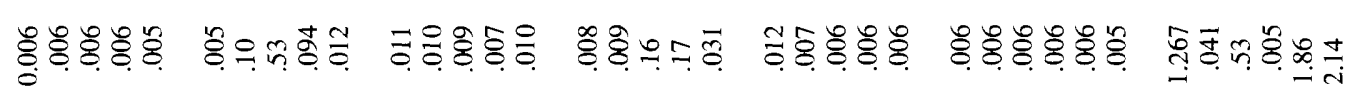

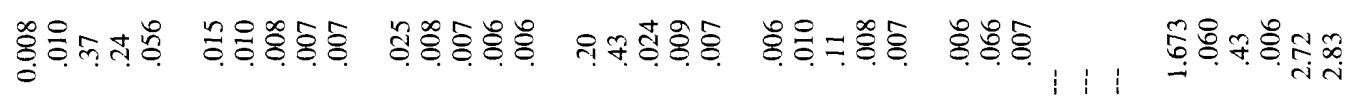

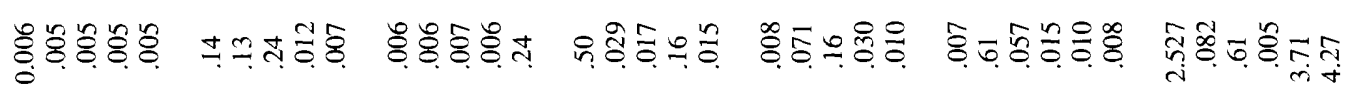

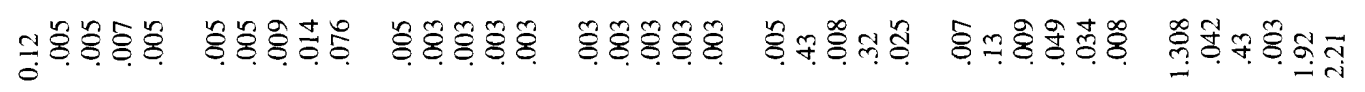

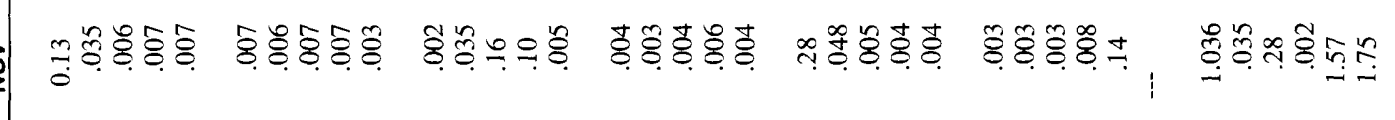

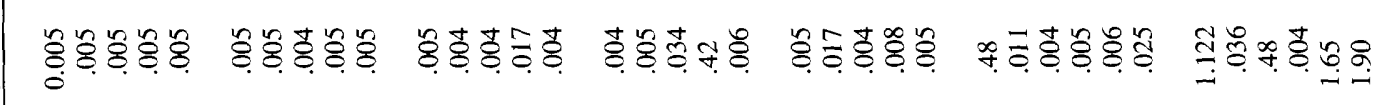

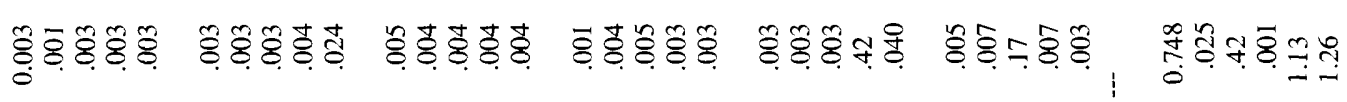

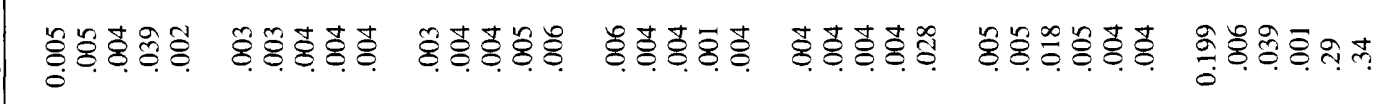
胥

츰

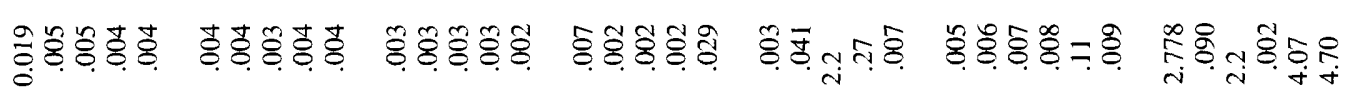




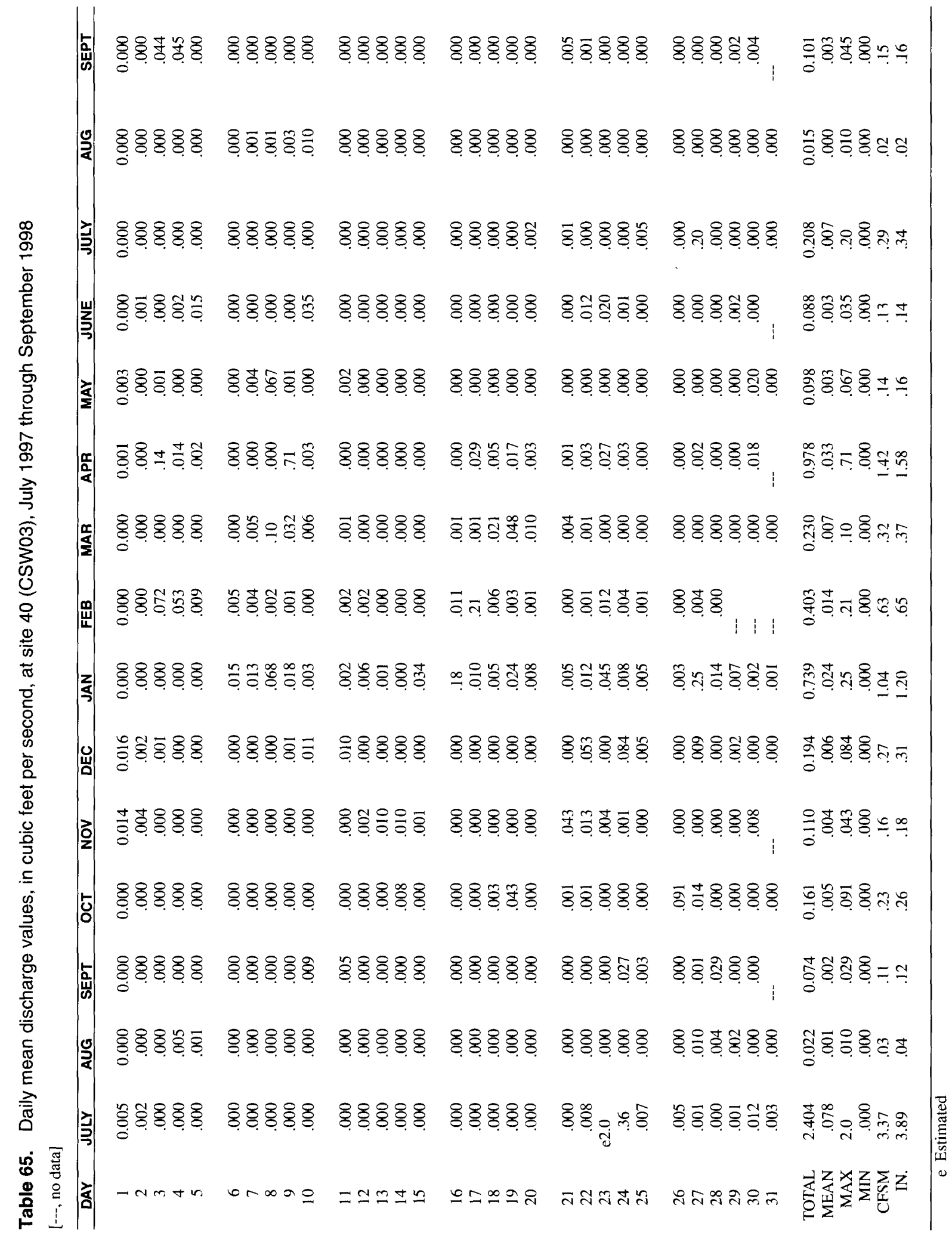

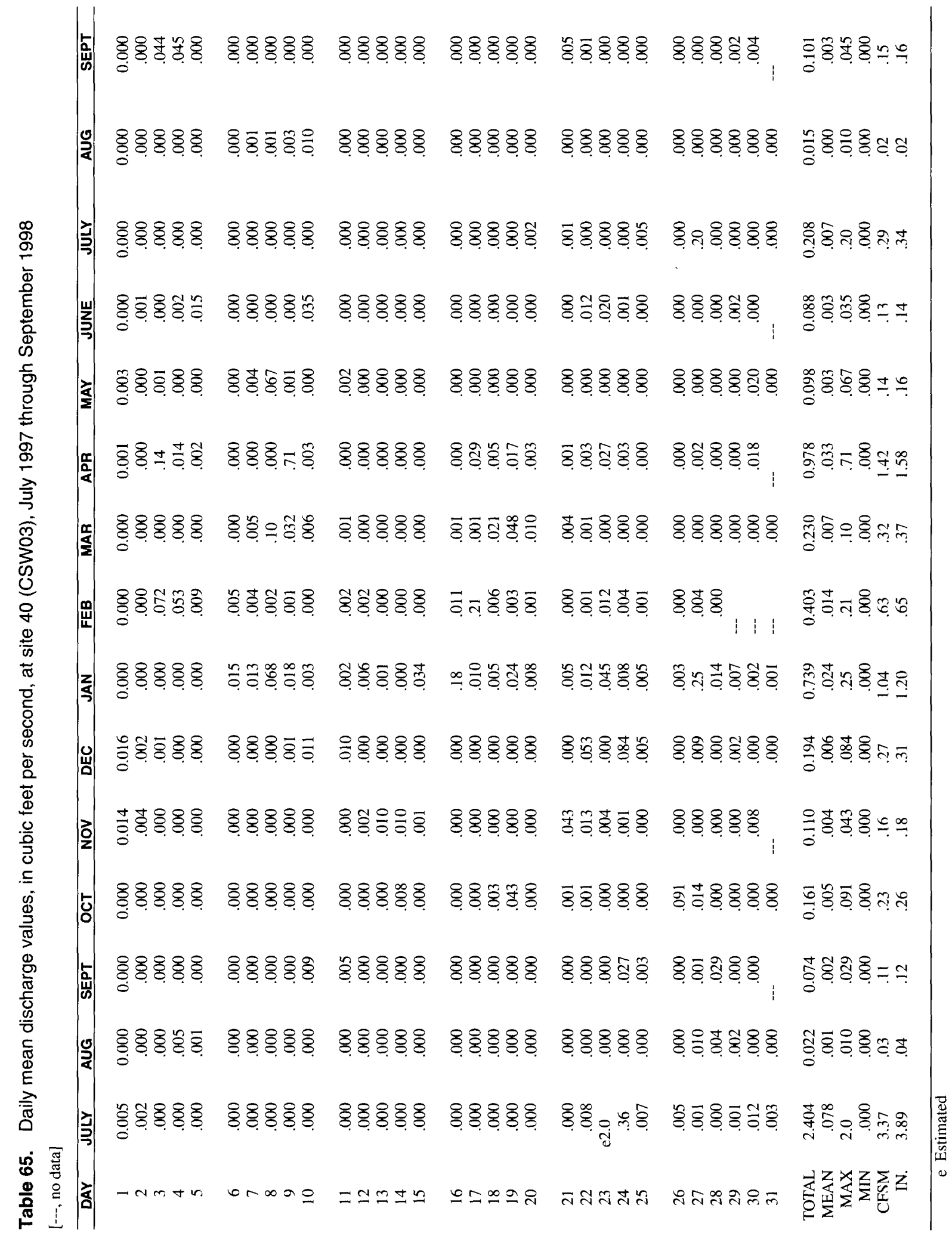

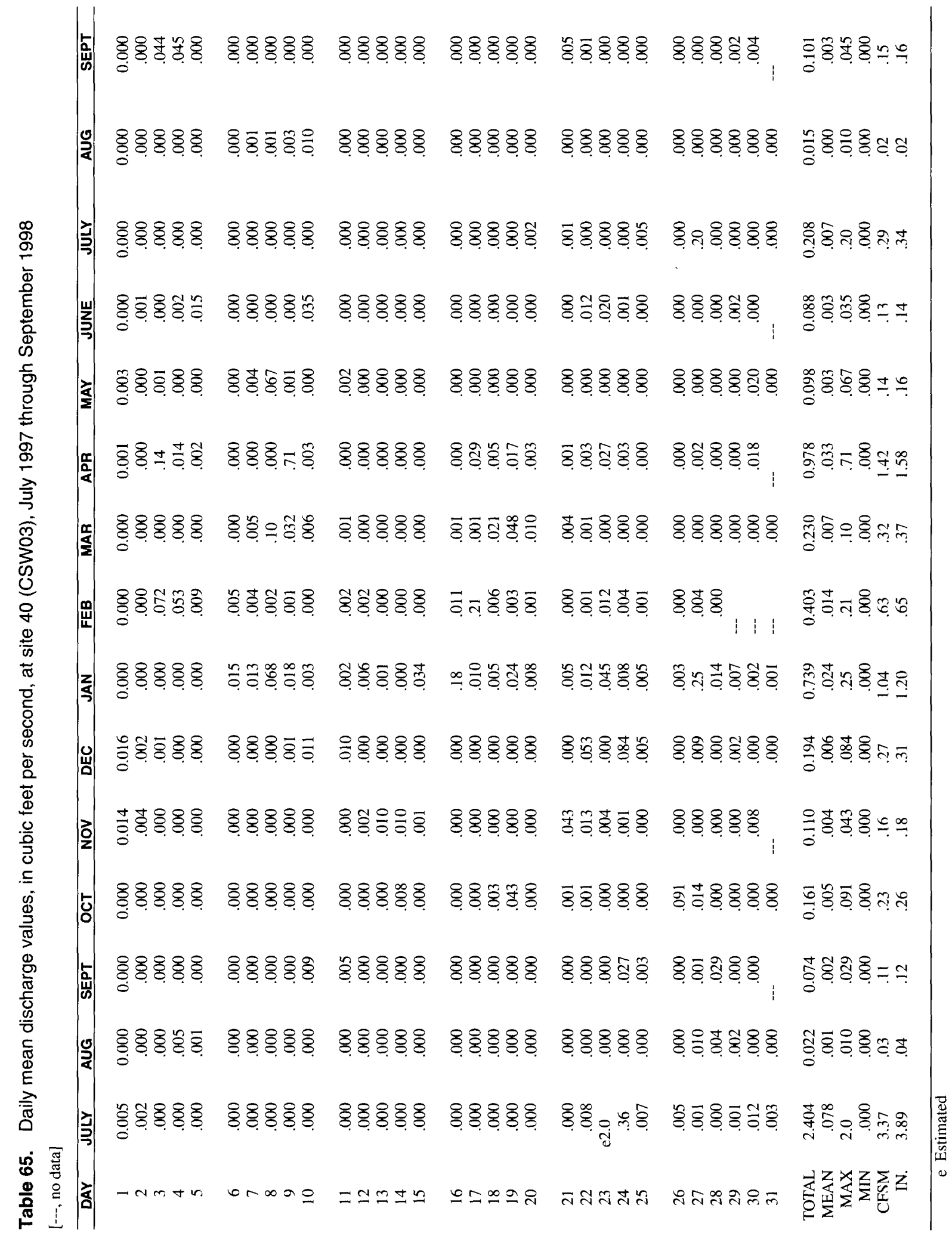

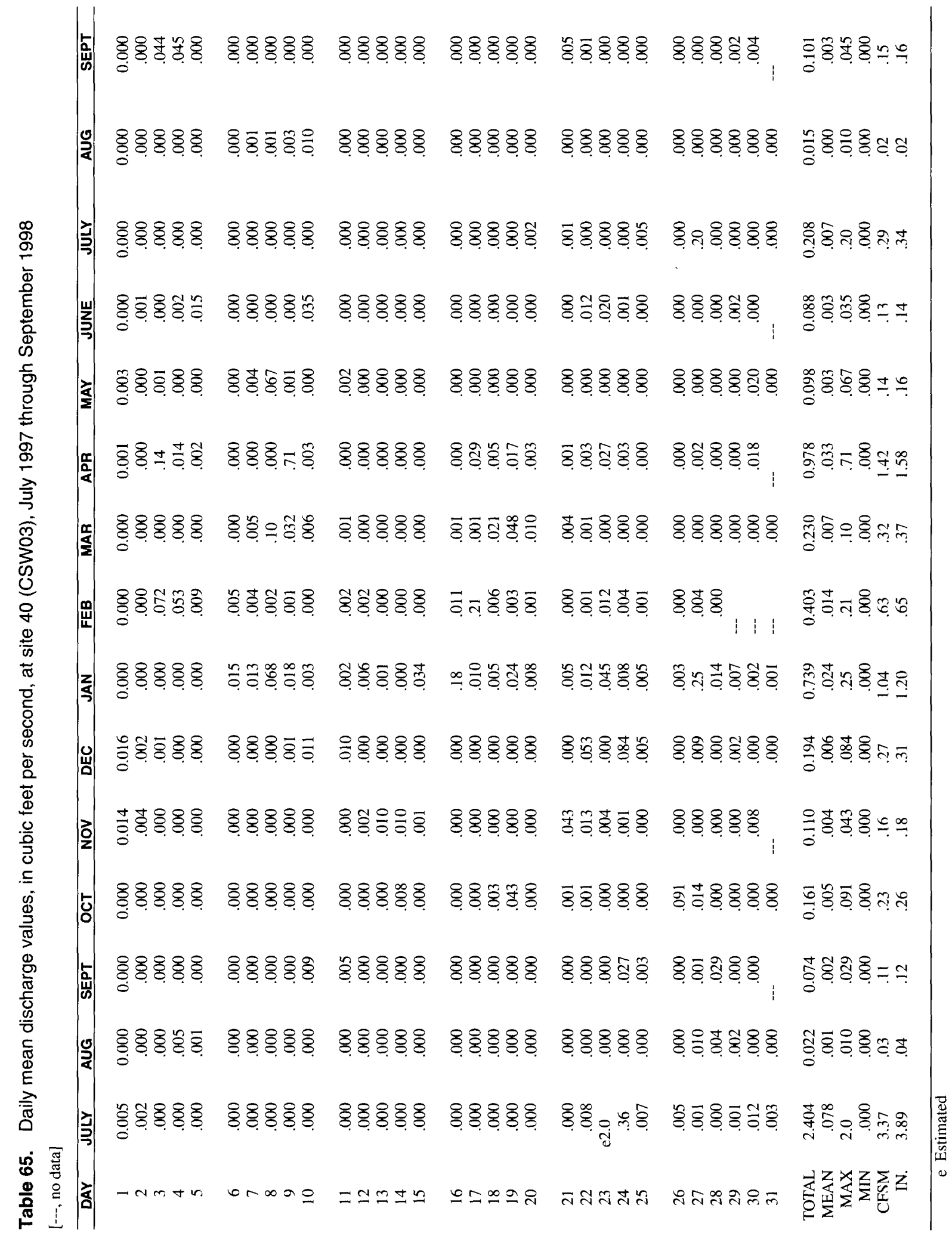

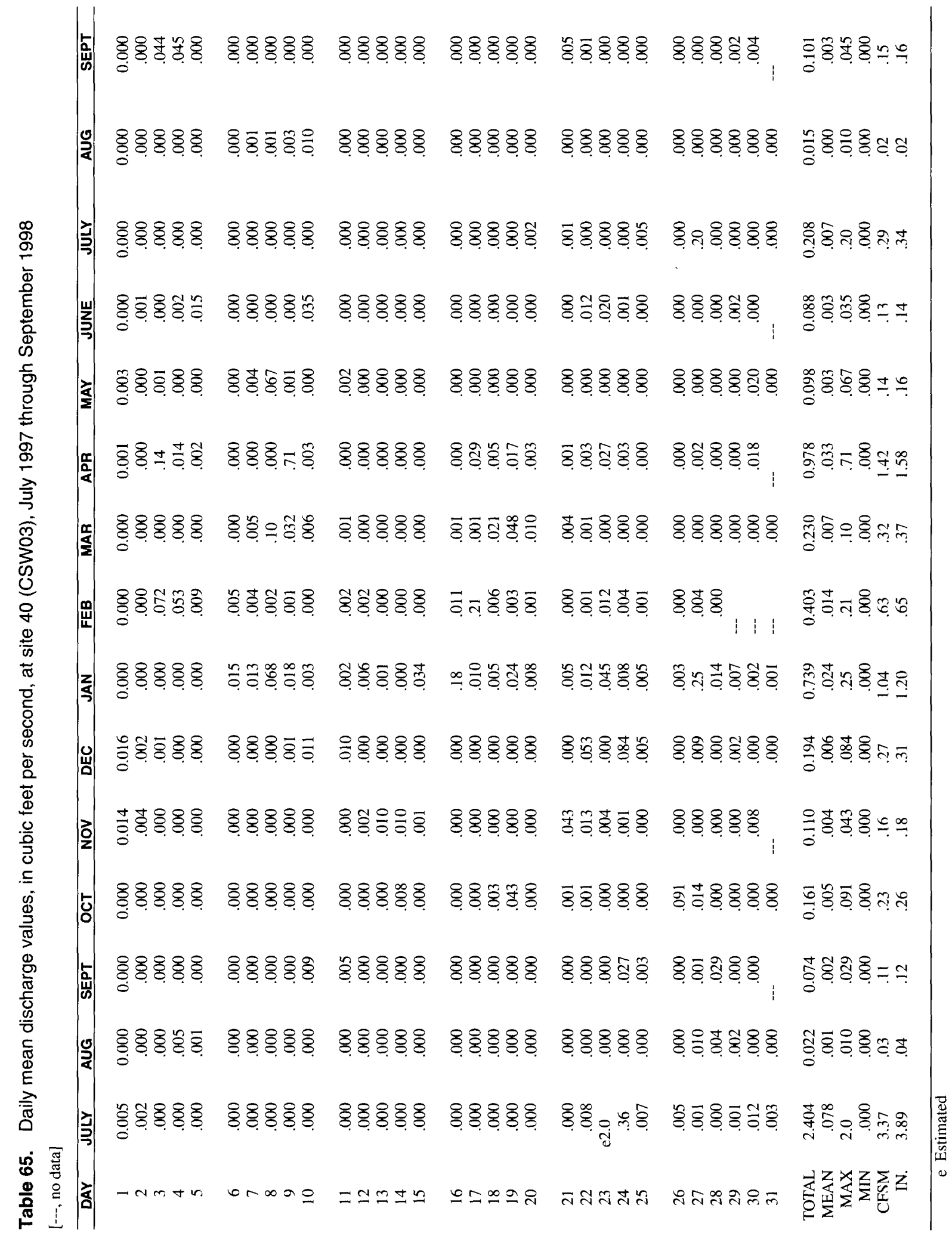

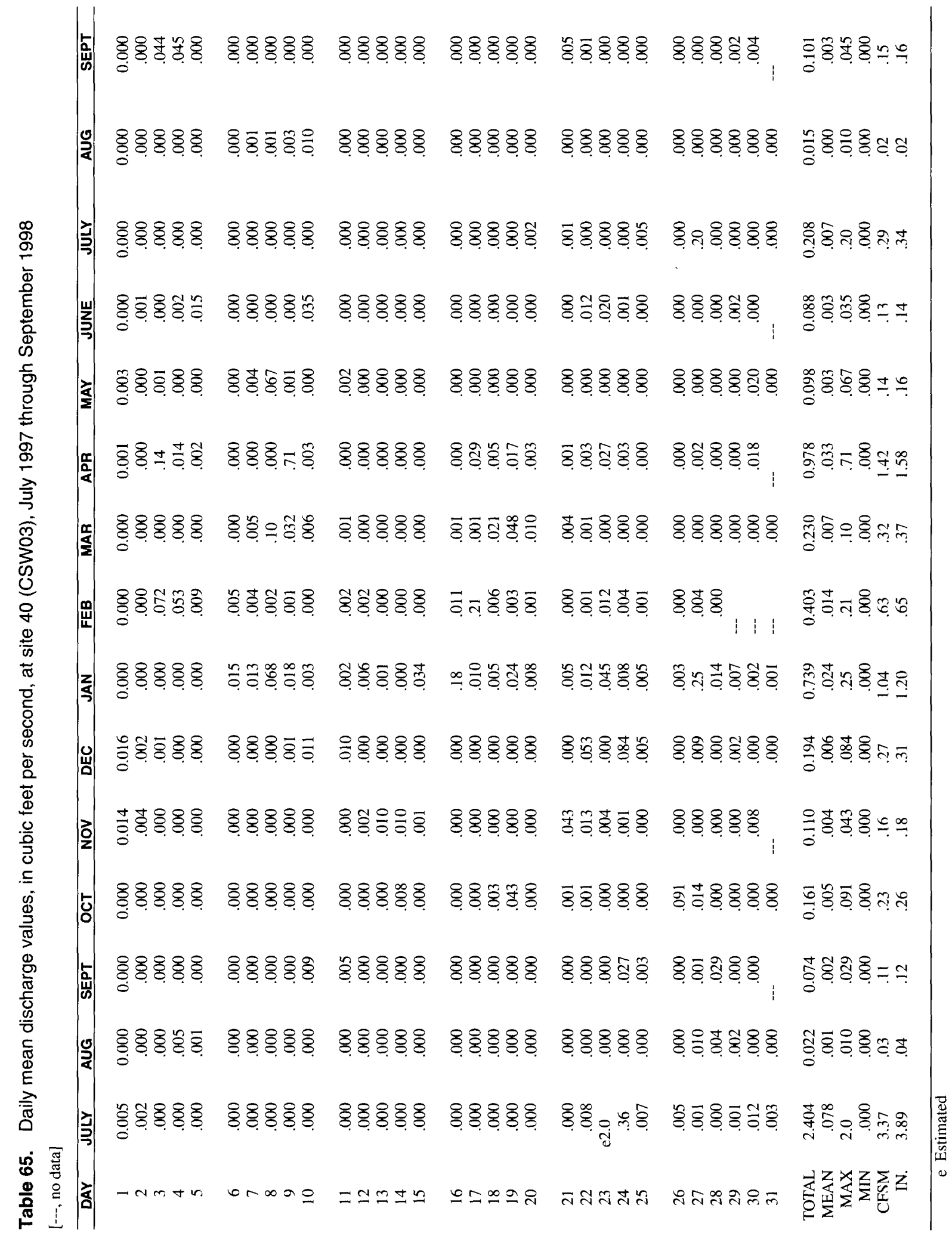

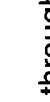

$$
\text { 各 }
$$

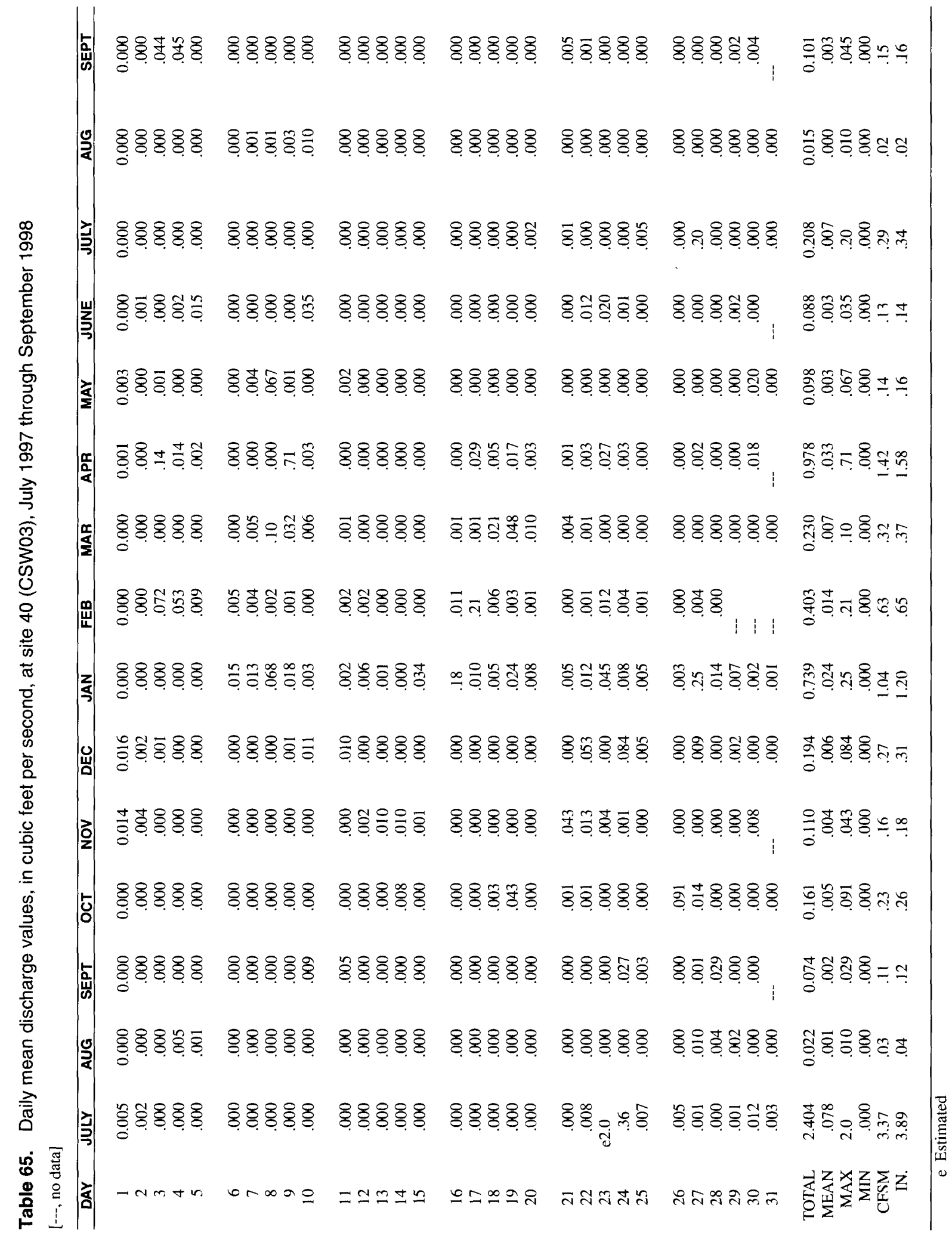

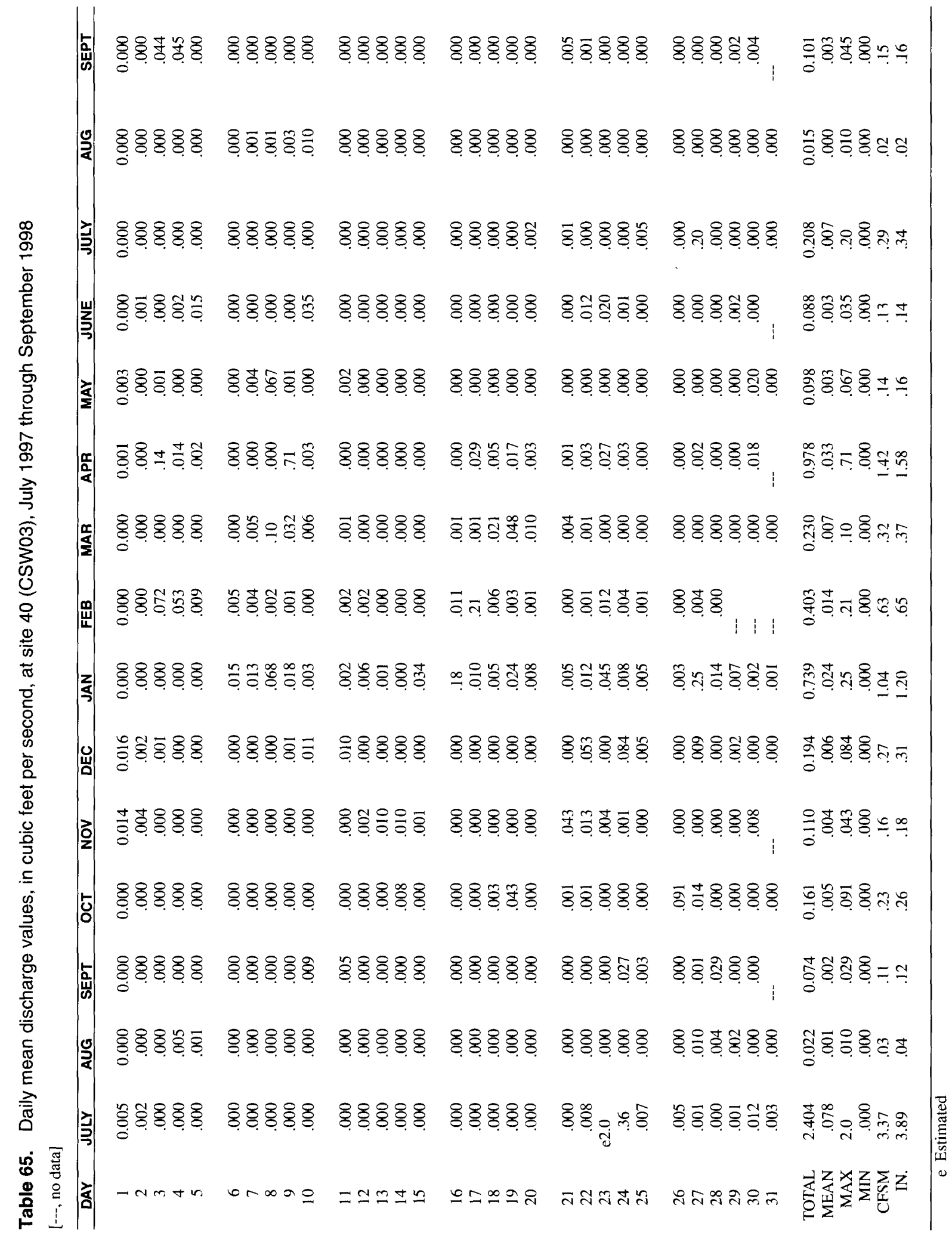

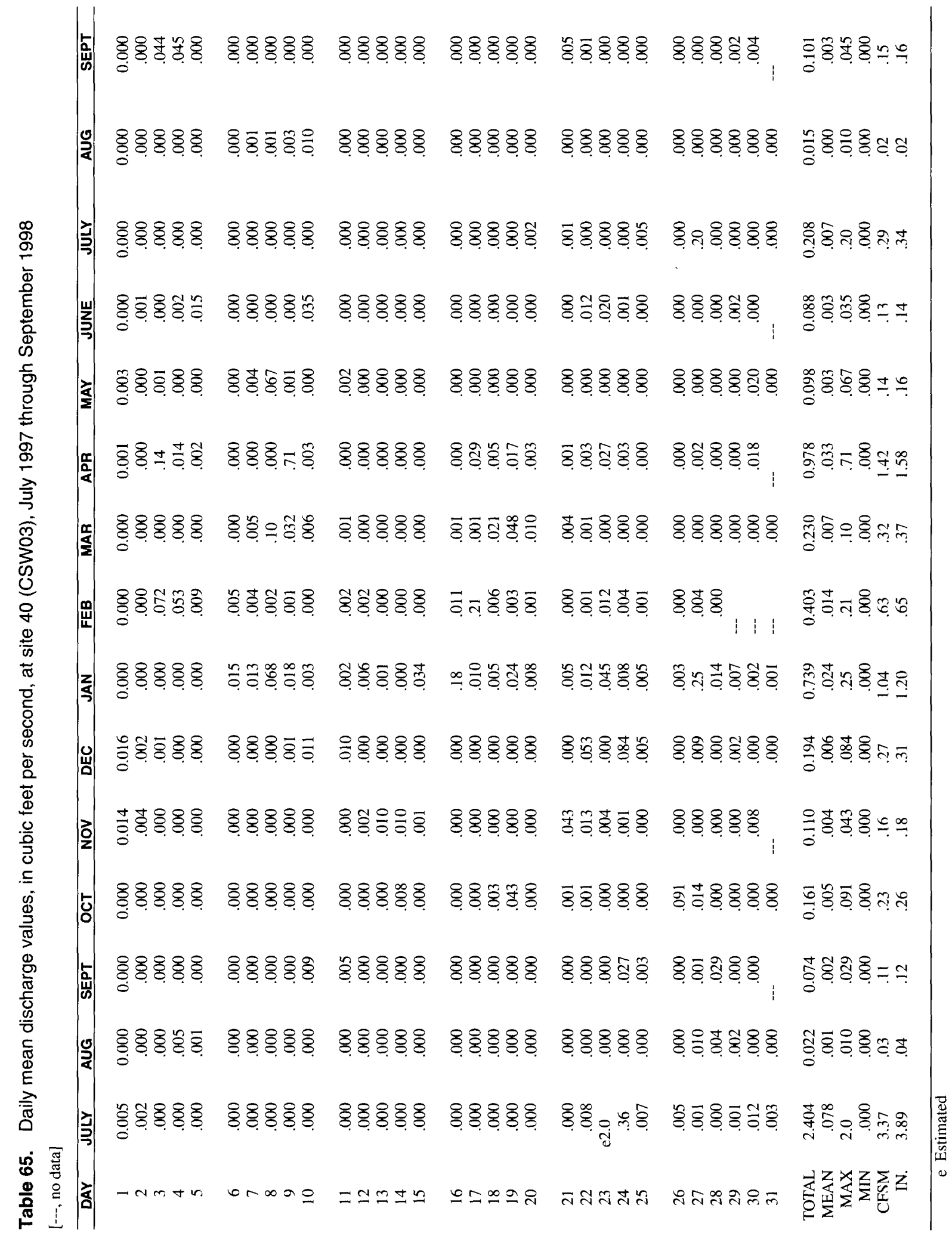

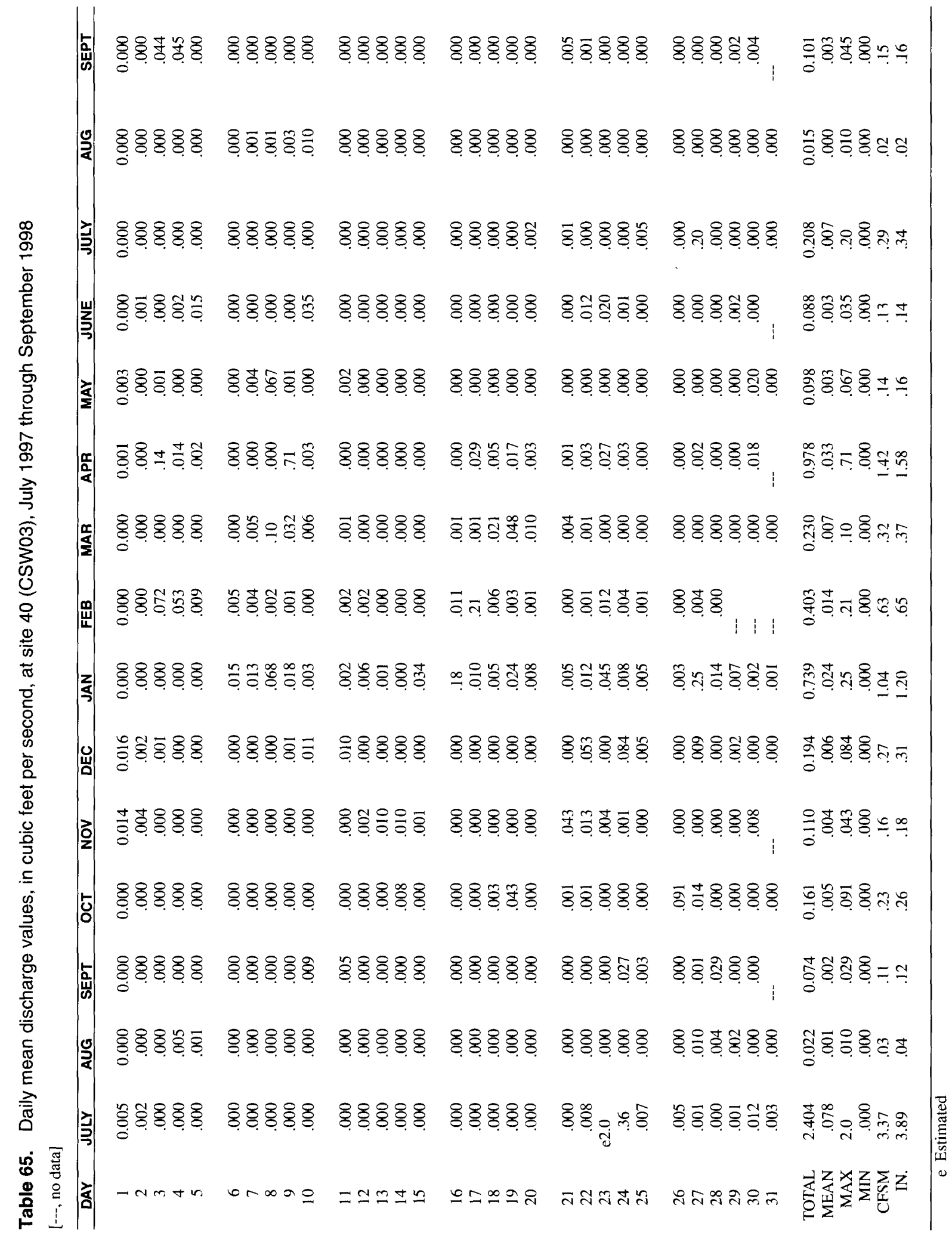

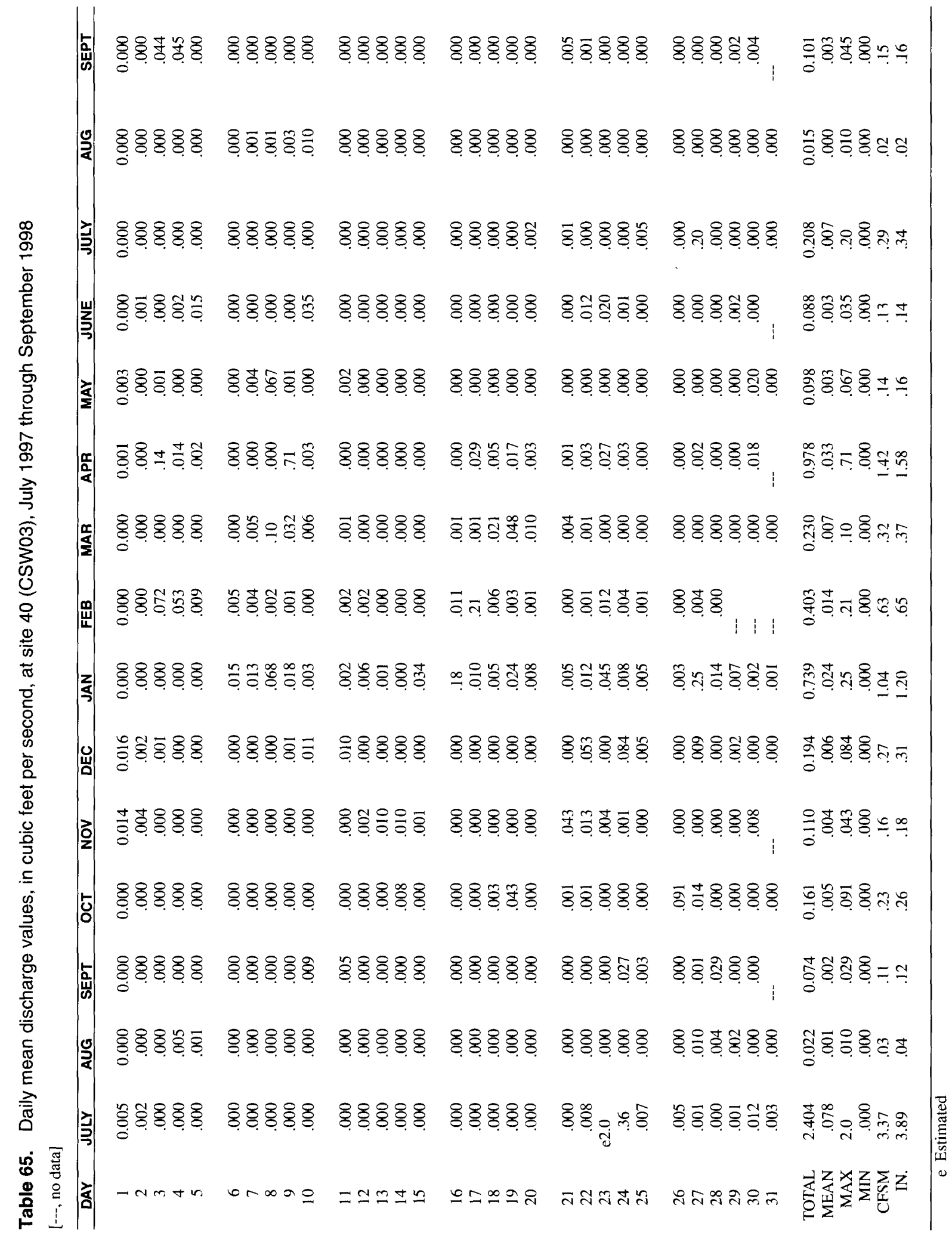

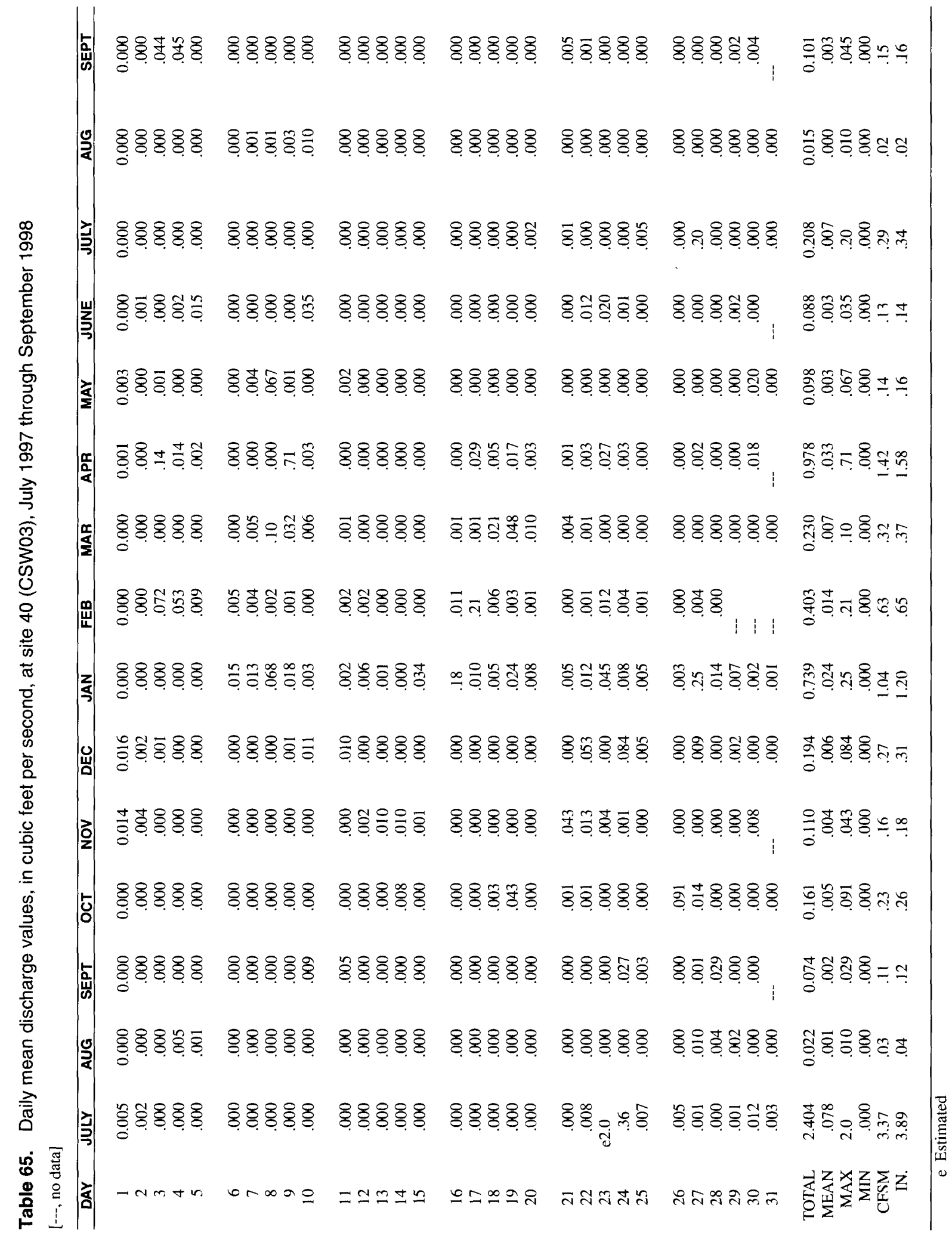

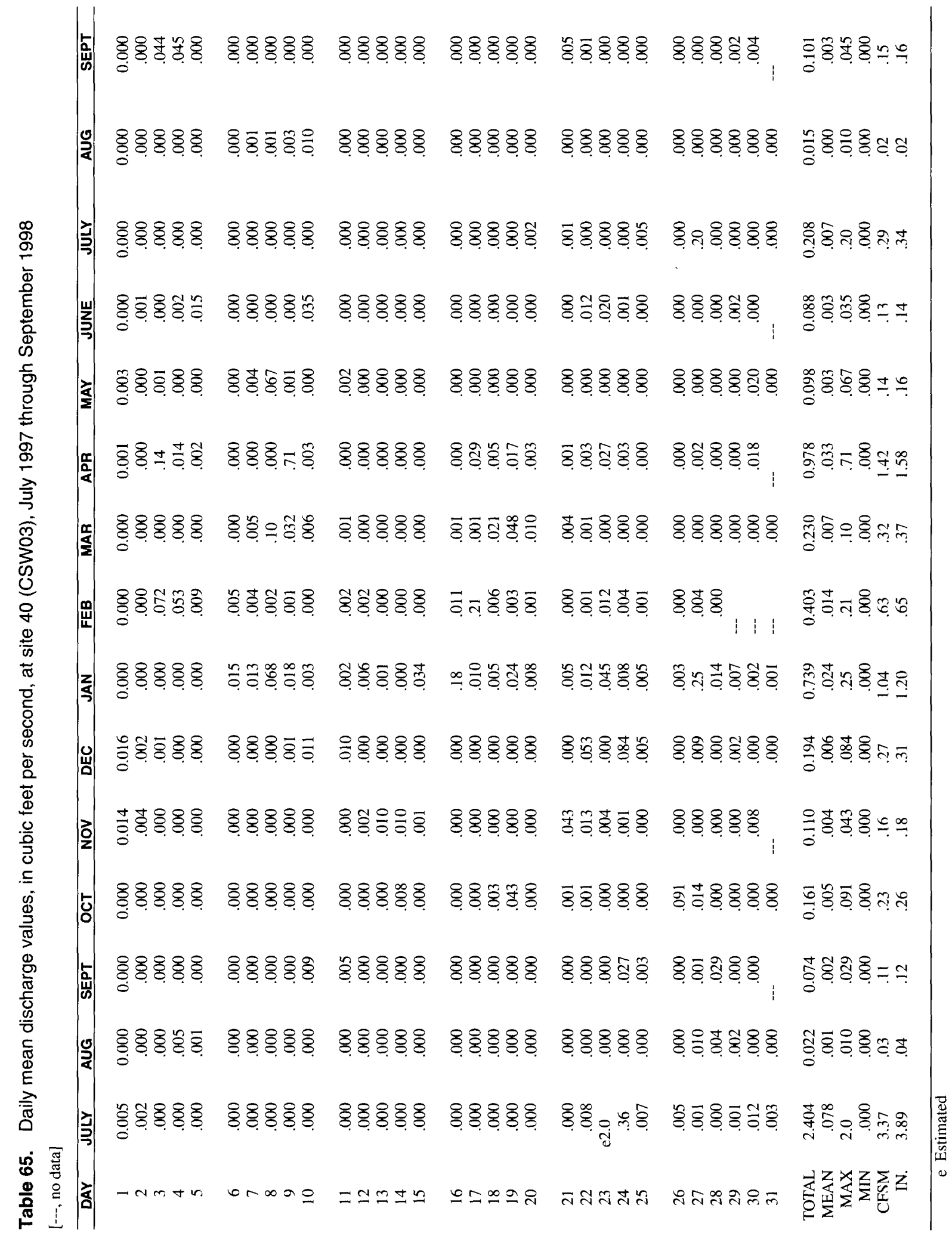

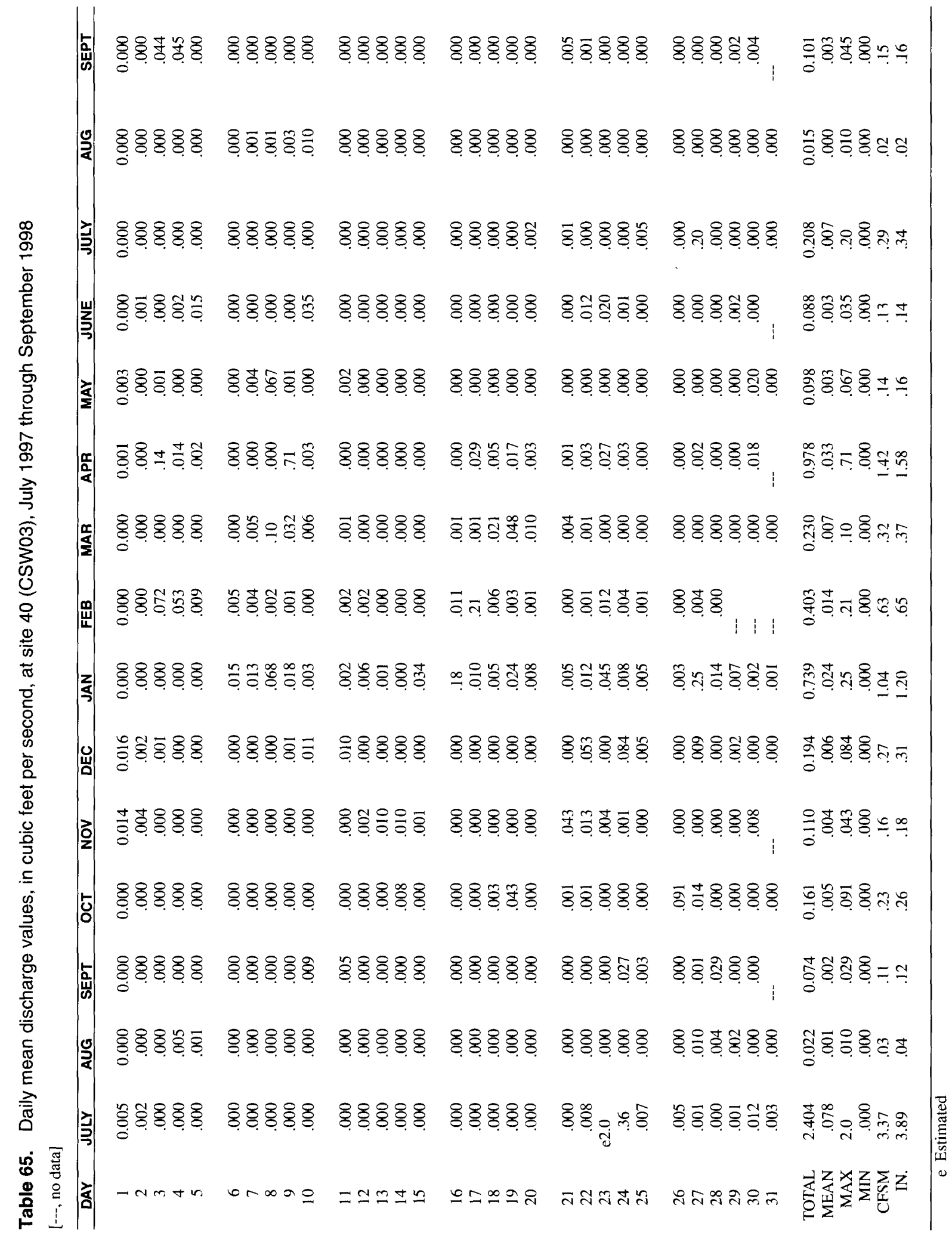

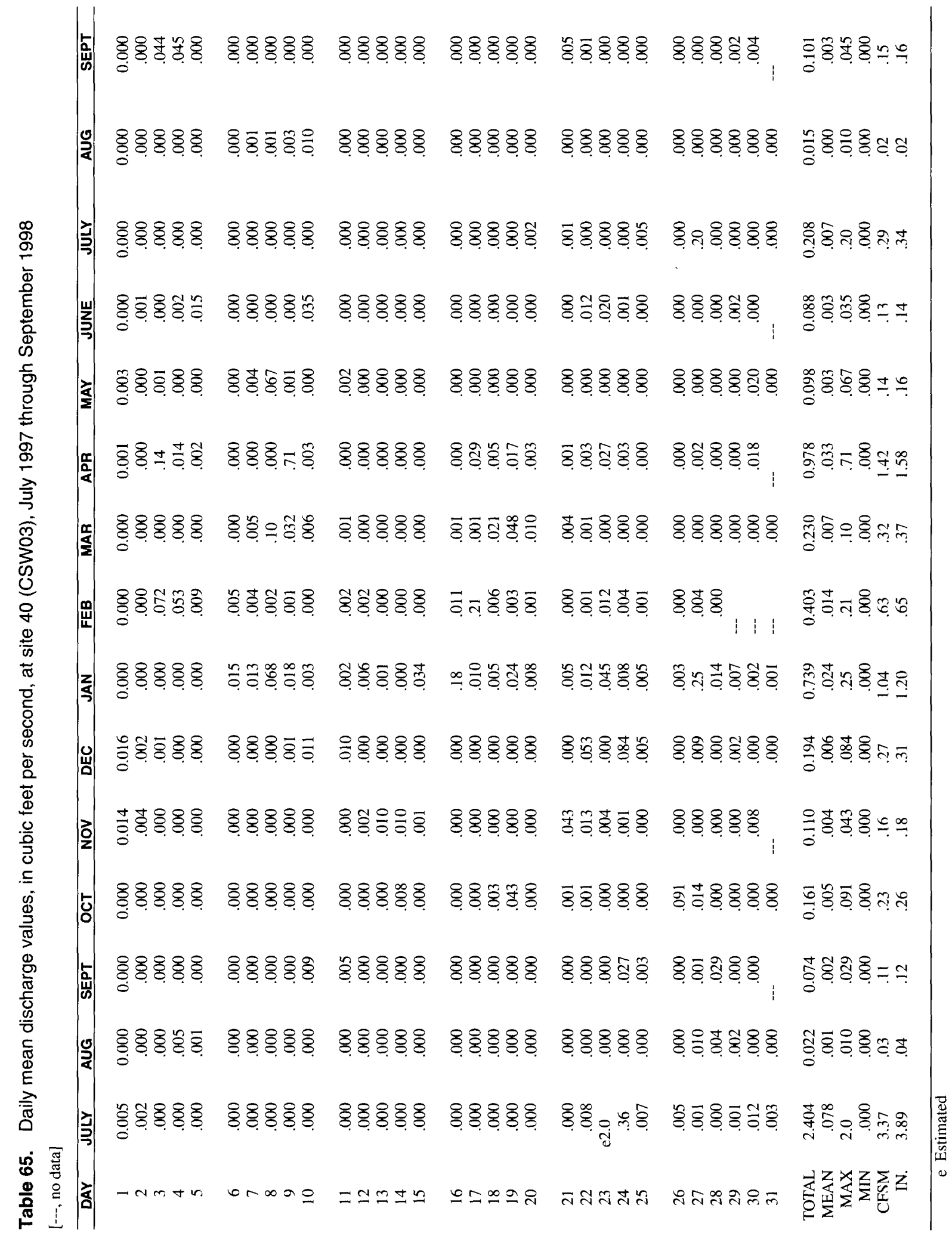

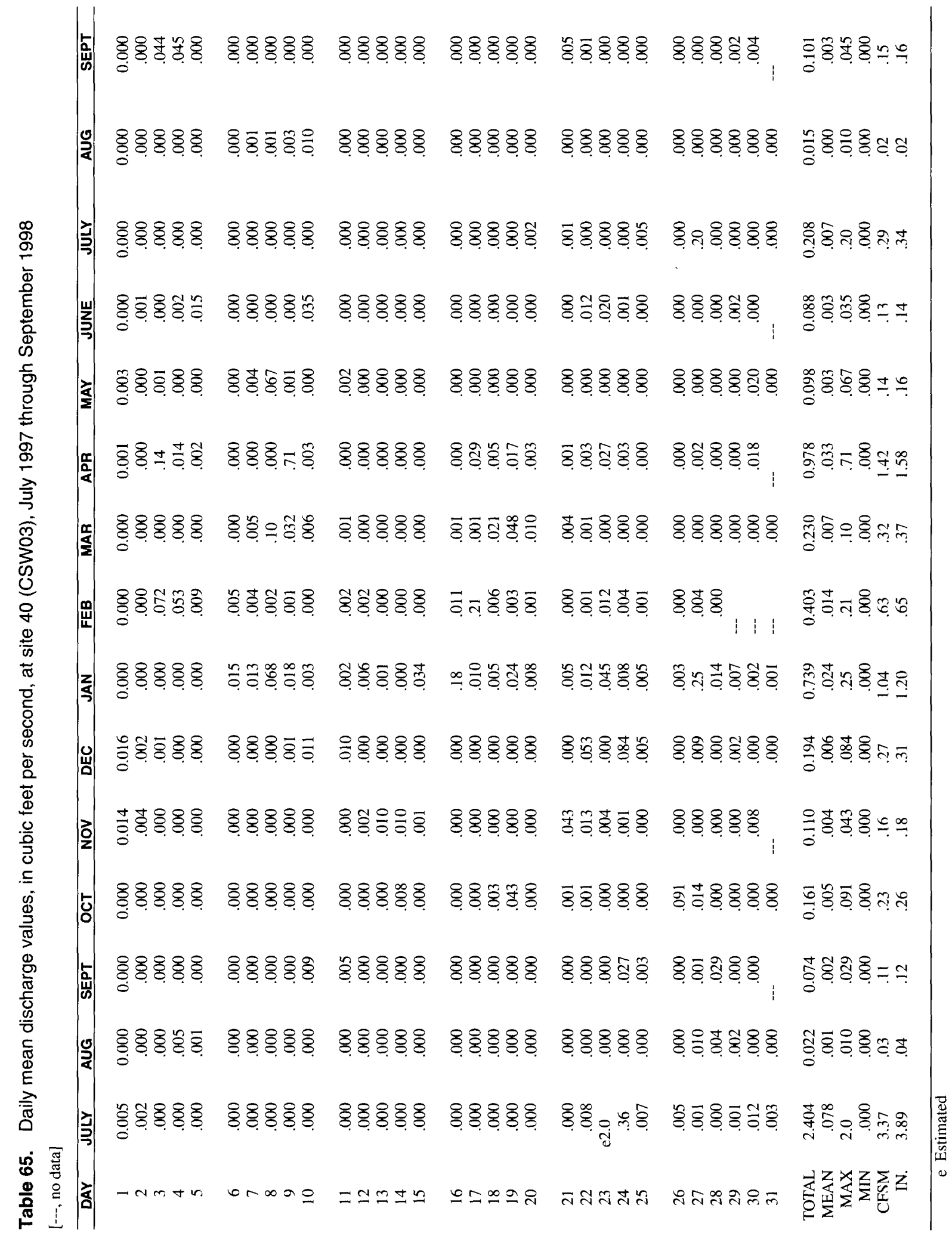

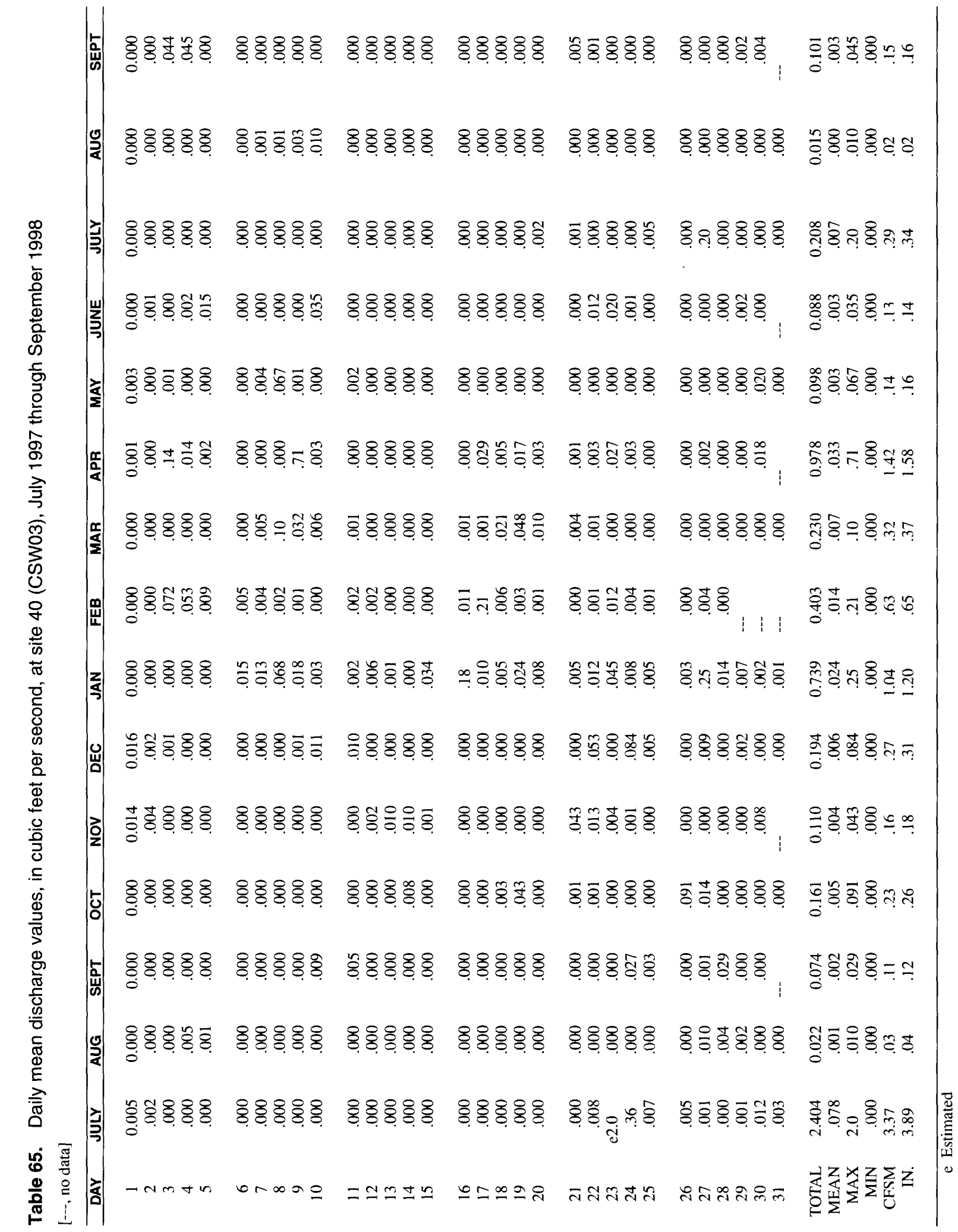




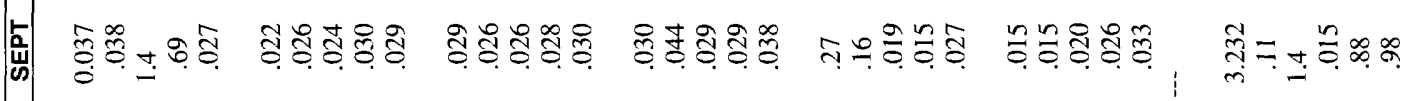

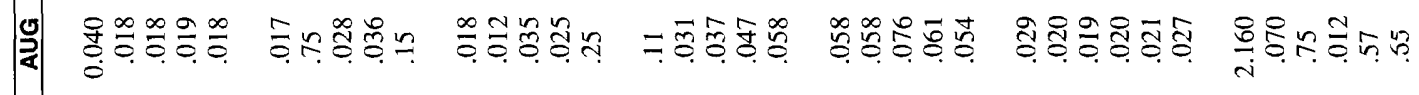

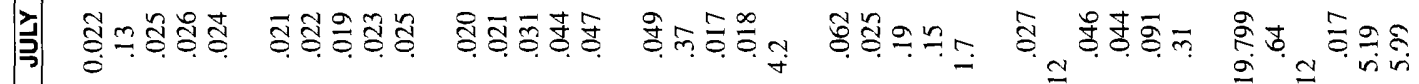

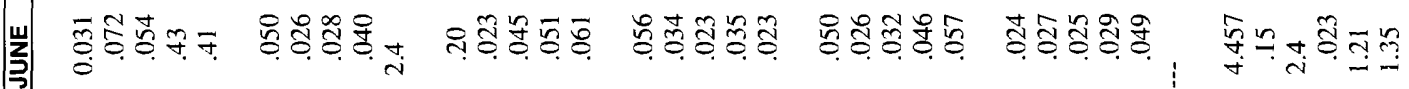

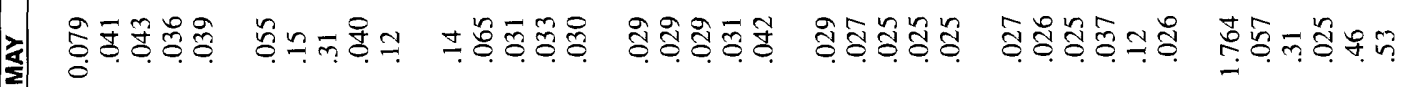

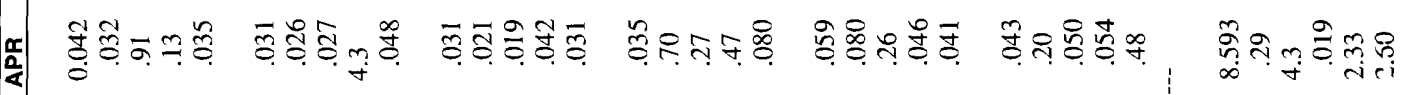

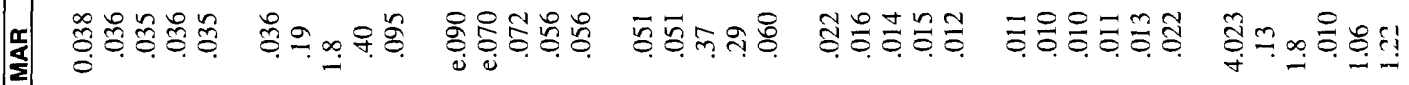

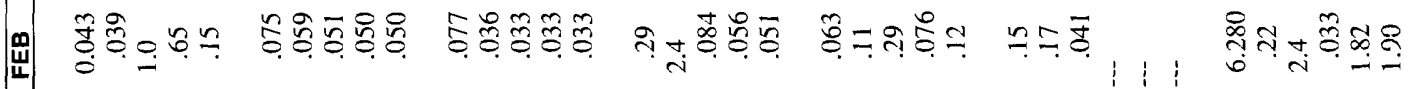

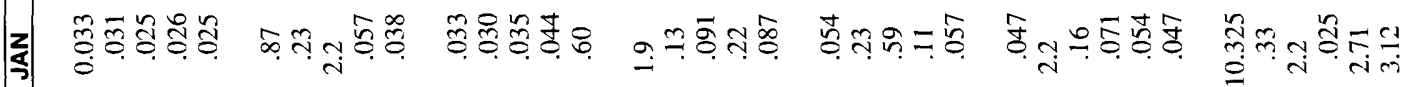

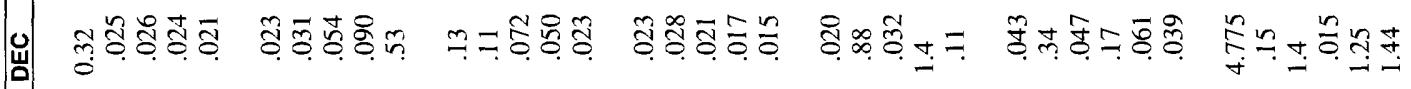

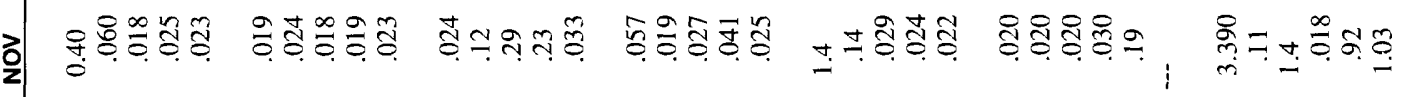

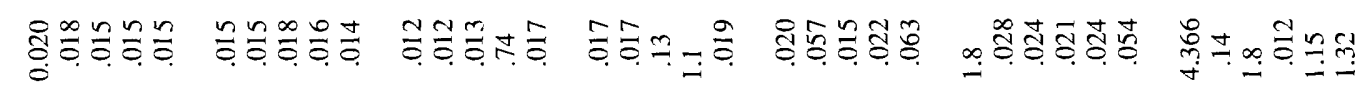
兽

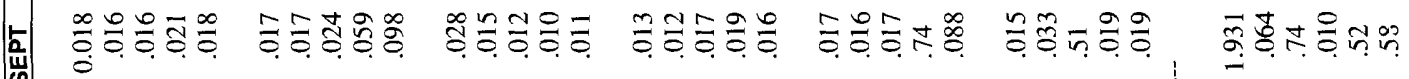

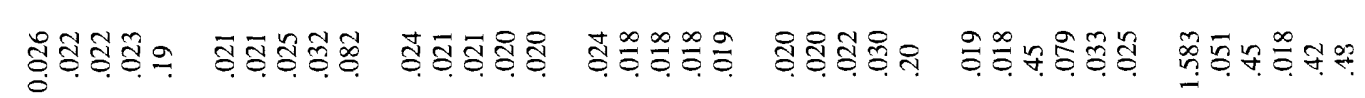

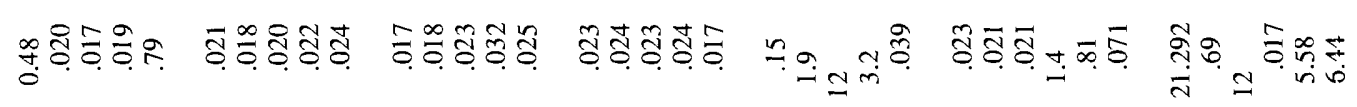




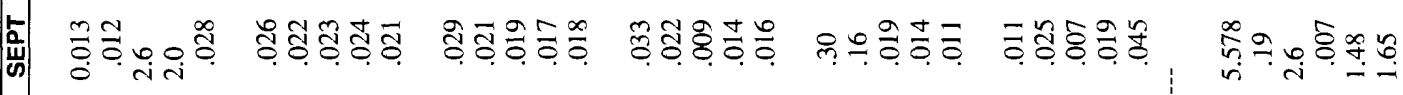

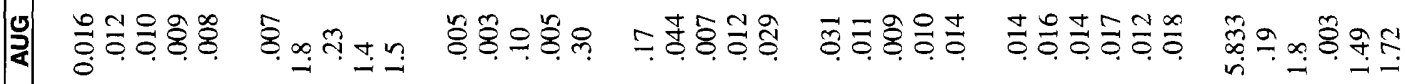

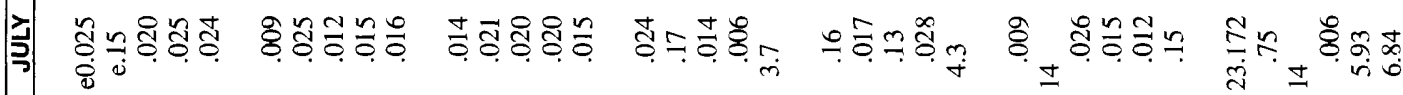

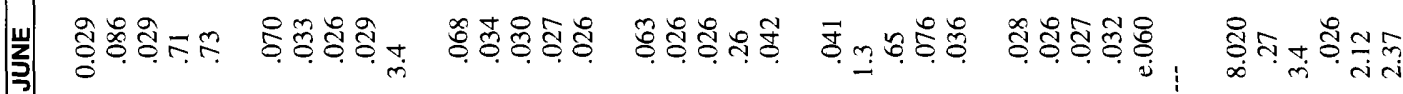

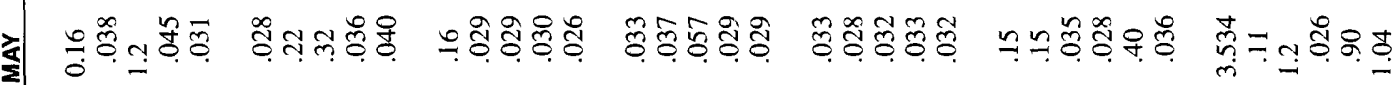

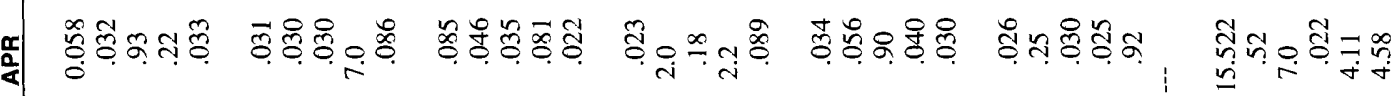

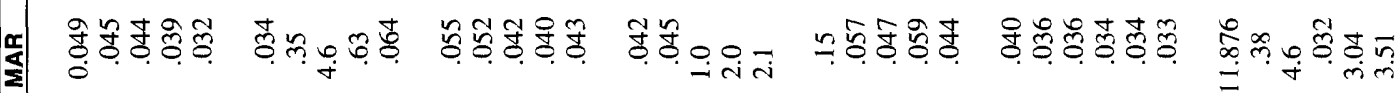

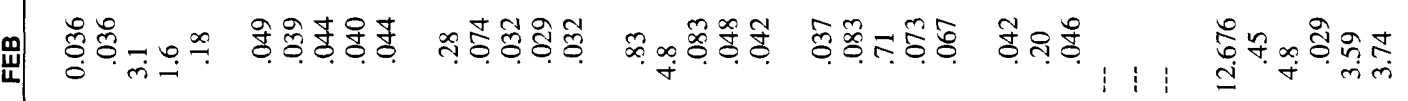

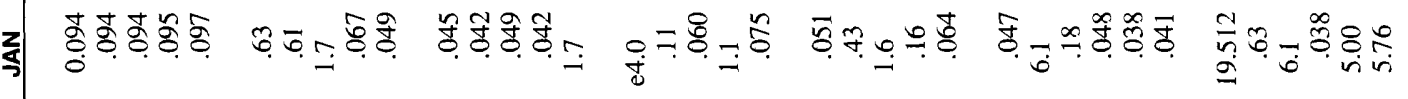

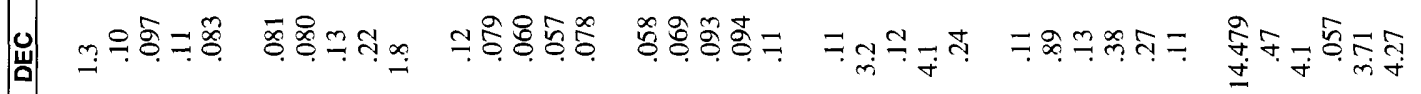

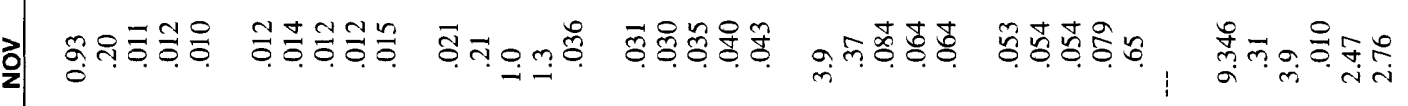

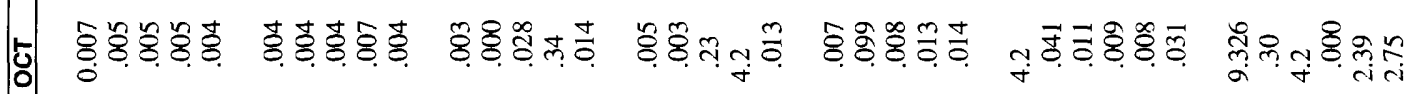

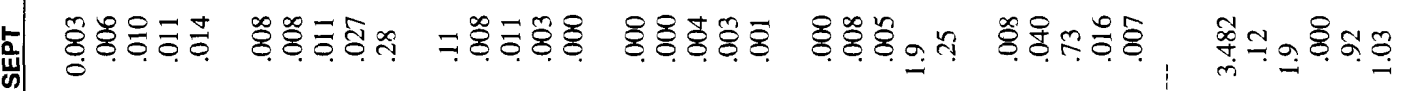

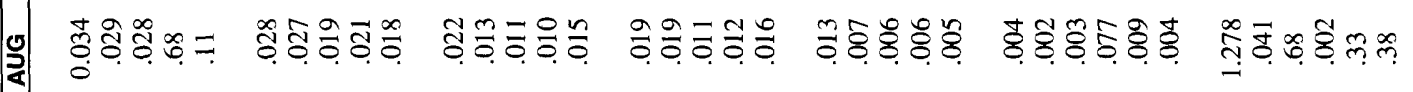

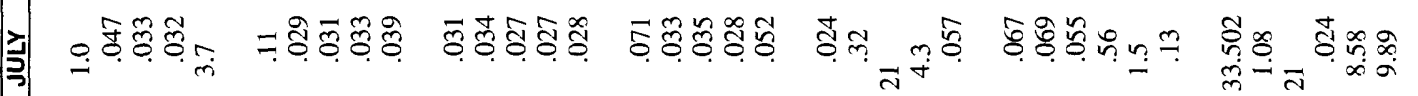

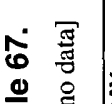

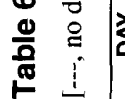

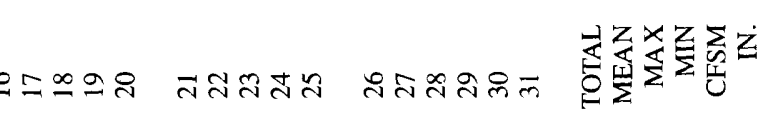




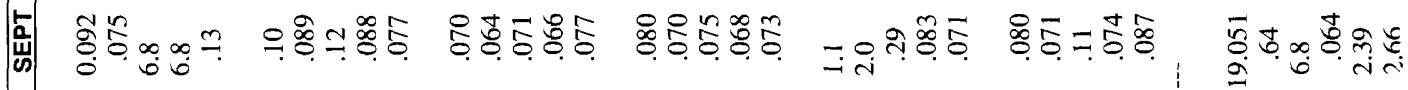

ำ

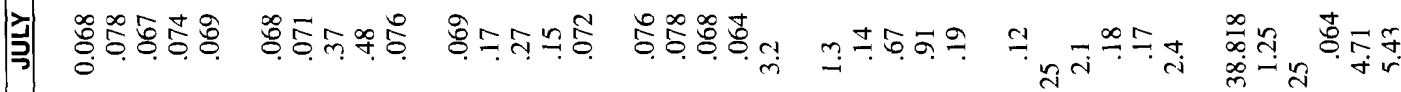

แ

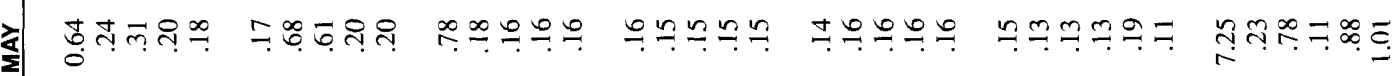

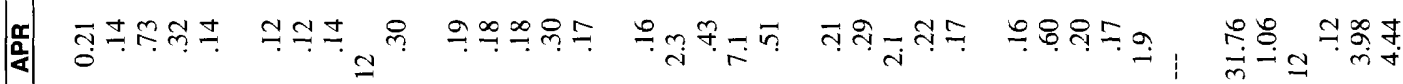

๓人

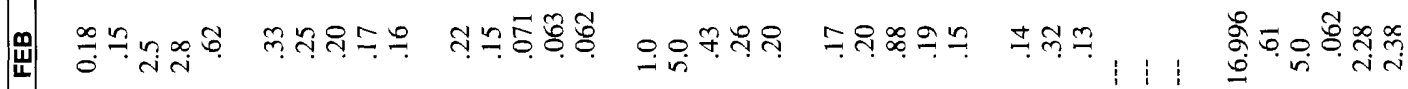

ร)

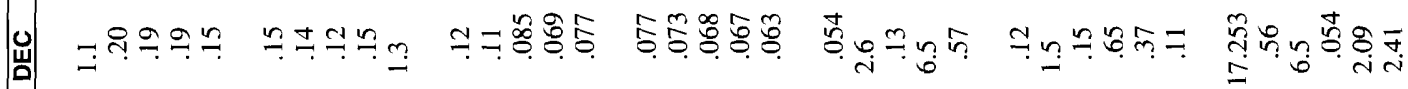

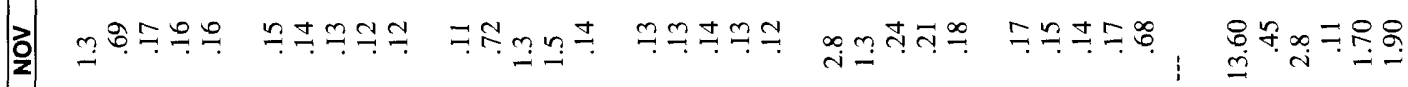

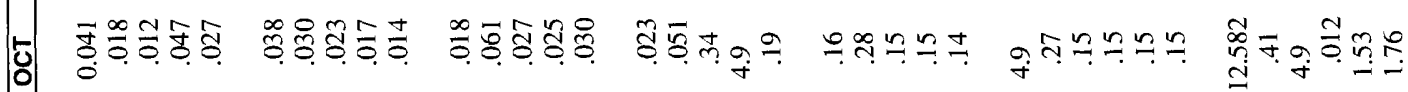

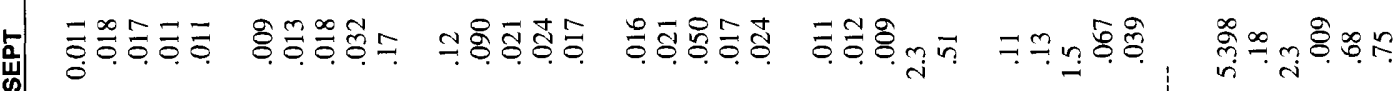

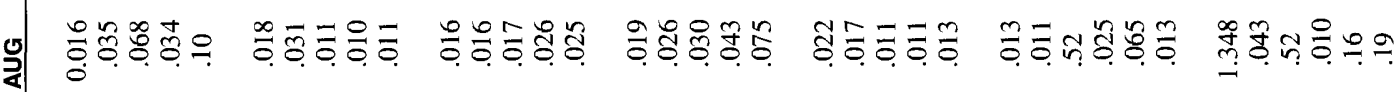

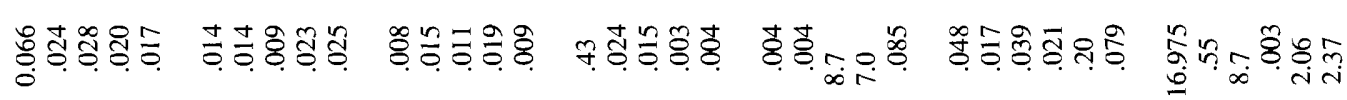


Table 69. Maximum and minimum specific conductance and water temperature recorded by monitors at the streamflow and water-quality study sites, October 1994 through September 1998

$\left[\mu \mathrm{S} / \mathrm{cm}\right.$; microsiemens per centimeter; ${ }^{\circ} \mathrm{C}$, degrees Celsius]

\begin{tabular}{|c|c|c|c|c|c|}
\hline \multirow{2}{*}{$\begin{array}{l}\text { Site no. } \\
\text { (fig. 1) }\end{array}$} & \multirow{2}{*}{ Period of record } & \multicolumn{2}{|c|}{$\begin{array}{c}\text { Specific conductance } \\
\left(\mu \mathrm{S} / \mathrm{cm} \text { at } 25^{\circ} \mathrm{C}\right)\end{array}$} & \multicolumn{2}{|c|}{$\begin{array}{c}\text { Water temperature } \\
\left({ }^{\circ} \mathrm{C}\right)\end{array}$} \\
\hline & & $\begin{array}{l}\text { Maximum } \\
\text { (Date) }\end{array}$ & $\begin{array}{l}\text { Minimum } \\
\text { (Date) }\end{array}$ & $\begin{array}{l}\text { Maximum } \\
\text { (Date) }\end{array}$ & $\underset{\text { (Date) }}{\text { Minimum }}$ \\
\hline $\begin{array}{c}37 \\
\text { [CSW06] }\end{array}$ & $5 / 95-9 / 98$ & $\begin{array}{c}960 \\
(12 / 29 / 97)\end{array}$ & $\begin{array}{c}22 \\
(4 / 9 / 98)\end{array}$ & $\begin{array}{c}49.3 \\
(7 / 25 / 98)\end{array}$ & $\begin{array}{c}0.3 \\
(1 / 7 / 96 ; 11 / 29 / 97)\end{array}$ \\
\hline $\begin{array}{c}39 \\
\text { [CSW05] }\end{array}$ & $10 / 94-9 / 98$ & $\begin{array}{c}7,060 \\
(1 / 9 / 96)\end{array}$ & $\begin{array}{c}10 \\
(1 / 13 / 95)\end{array}$ & $\begin{array}{c}34.7 \\
(7 / 31 / 95)\end{array}$ & $\begin{array}{c}0.7 \\
(2 / 13 / 97)\end{array}$ \\
\hline $\begin{array}{c}40 \\
{[\mathrm{CSW} 03]}\end{array}$ & $10 / 94-9 / 98$ & $\begin{array}{c}3,000 \\
(1 / 12 / 96)\end{array}$ & $\begin{array}{c}8 \\
(11 / 1 / 97)\end{array}$ & $\begin{array}{c}31.0 \\
(8 / 15 / 95)\end{array}$ & $\begin{array}{c}0.4 \\
(2 / 5 / 96 ; 2 / 6 / 96)\end{array}$ \\
\hline $\begin{array}{c}41 \\
\text { [CSW02] }\end{array}$ & $10 / 94-9 / 98$ & $\begin{array}{c}4,480 \\
(2 / 4 / 96)\end{array}$ & $\begin{array}{c}12 \\
(6 / 19 / 95)\end{array}$ & $\begin{array}{c}31.6 \\
(8 / 15 / 95 \\
7 / 23 / 98)\end{array}$ & $\begin{array}{c}2.0 \\
(2 / 13 / 97)\end{array}$ \\
\hline $\begin{array}{c}42 \\
\text { [CSW04] }\end{array}$ & $10 / 94-9 / 98$ & $\begin{array}{c}13,900 \\
(5 / 22 / 96)\end{array}$ & $\begin{array}{c}10 \\
(1 / 5 / 96)\end{array}$ & $\begin{array}{c}32.9 \\
(7 / 23 / 98)\end{array}$ & $\begin{array}{c}0.1 \\
(1 / 7 / 96)\end{array}$ \\
\hline $\begin{array}{c}43 \\
\text { [CSW07] }\end{array}$ & $10 / 94-9 / 98$ & $\begin{array}{c}1320 \\
(1 / 22 / 98)\end{array}$ & $\begin{array}{c}27 \\
(1 / 7 / 96)\end{array}$ & $\begin{array}{c}33.0 \\
(6 / 24 / 97)\end{array}$ & $\begin{array}{c}0.0 \\
(1 / 6,2 / 7,9,12 / 25-30 / 95 \\
1 / 7,12,2 / 4-6 / 96 ; 1 / 18 / 97)\end{array}$ \\
\hline
\end{tabular}


Table 70. Statistical summary of water-quality data at site 37 (CSW06), May 1995 through September 1998

\begin{tabular}{|c|c|c|c|c|c|c|c|c|c|c|}
\hline \multirow{2}{*}{$\begin{array}{l}\text { PARAM- } \\
\text { ETER } \\
\text { CODE }\end{array}$} & \multirow{2}{*}{ PROPERTY OR CONSTITUENT } & \multicolumn{4}{|c|}{ DESCRIPTIVE STATISTICS } & \multicolumn{5}{|c|}{$\begin{array}{c}\text { PERCENT OF SAMPLES IN WHICH VALUES WERE LESS THAN OF } \\
\text { EQUAL TO THOSE SHOI'N }\end{array}$} \\
\hline & & $\begin{array}{l}\text { SAMPLE } \\
\text { SIZE }\end{array}$ & MAXIMUM & MINIMUM & MEAN & $95 \%$ & $75 \%$ & $\begin{array}{c}50 \% \\
\text { (medlan) }\end{array}$ & $25 \%$ & $\mathbf{5} \%$ \\
\hline \multicolumn{11}{|c|}{ PHYSICAL AND CHEMICAL PROPERTIES } \\
\hline 00061 & INSTANTANEOUS DISCHARGE $\left(\mathrm{ft}^{3} / \mathrm{s}\right)$ & 75 & 40.000 & 0.010 & 3.572 & 20.000 & 4.100 & 1.200 & 0.530 & 0.068 \\
\hline 00010 & WATER TEMPERATURE $\left({ }^{\circ} \mathrm{C}\right)$ & 68 & 29.000 & 5.000 & 17.069 & 27.500 & 22.000 & 17.750 & 9.625 & 5.725 \\
\hline 90095 & SPECIFIC CONDUCTANCE, LAB ( $\mu \mathrm{S} / \mathrm{cm}$ at $25^{\circ} \mathrm{C}$ ) & 45 & 164.000 & 22.000 & 63.400 & 141.500 & 80.000 & 53.000 & 45.000 & 27.400 \\
\hline 00095 & $\begin{array}{l}\text { SPECIFIC CONDUCTANCE, FIELD }(\mu \mathrm{S} / \mathrm{cm} \text { at } 25 \\
\left.{ }^{\circ} \mathrm{C}\right)\end{array}$ & 62 & 152.000 & 20.000 & 63.806 & 143.950 & 79.250 & 53.500 & 43.750 & 27.300 \\
\hline 00403 & pH, LAB (STANDARD pH UNITS) & 45 & 8.900 & 6.200 & 6.798 & 7.340 & 7.000 & 6.800 & 6.500 & 6.300 \\
\hline 00400 & $\mathrm{pH}$, FIELD (STANDARD $\mathrm{pH}$ UNITS) & 62 & 7.900 & 6.000 & 6.661 & 7.200 & 6.800 & 6.600 & 6.500 & 6.215 \\
\hline 90410 & ALKALINITY, LAB (mg/L as $\left.\mathrm{CaCO}_{3}\right)$ & 45 & 52.000 & 5.600 & 11.982 & 25.700 & 14.000 & 8.900 & 7.000 & 5.930 \\
\hline 80154 & SUSPENDED SEDIMENT (mg/L) & 45 & 364.000 & 10.000 & 69.667 & 228.700 & 81.000 & 45.000 & 27.500 & 11.000 \\
\hline 00530 & $\begin{array}{l}\text { RESIDUE ON EVAPORATION AT } 105^{\circ} \mathrm{C} \text {, SUSPENDED } \\
(\mathrm{mg} / \mathrm{L})\end{array}$ & 30 & 140.000 & 4.000 & 40.267 & 133.400 & 57.750 & 22.500 & 8.750 & 4.550 \\
\hline 00535 & RESIDUE VOLATILE， SUSPENDED (mg/L) & 40 & 54.000 & $<1.000$ & $10.301 *$ & 36.000 & 11.000 & 5.000 & 2.000 & $<1.000$ \\
\hline 70300 & DISSOLVED SOLIDS, RESIDUE AT $180^{\circ} \mathrm{C}(\mathrm{mg} / \mathrm{L})$ & 45 & 190.000 & 19.000 & 51.511 & 116.100 & 54.000 & 43.000 & 35.000 & 22.000 \\
\hline 00310 & 5 DAY BIOCHEMICAL OXYGEN DEMAND (mg/L) & 42 & 23.000 & $<2.000$ & $6.595 *$ & 13.000 & 7.600 & 5.400 & 4.300 & $<2.000$ \\
\hline 00340 & CHEMICAL OXYGEN DEMAND $(\mathrm{mg} / \mathrm{L})$ & 45 & 190.000 & 5.000 & 31.333 & 86.400 & 34.500 & 26.000 & 18.000 & 10.300 \\
\hline \multicolumn{11}{|c|}{ NUTRIENTS, TOTAL AND DISSOLVED } \\
\hline 00625 & $\begin{array}{l}\text { NITROGEN AMMONI } \bar{A}+\text { ORGANIC, } \\
\text { TOTAL (mg/L as N) }\end{array}$ & 45 & 3.200 & 0.360 & 0.887 & $2 . \overline{360}$ & 1.000 & 0.710 & 0.560 & 0.372 \\
\hline 00631 & $\mathrm{NO}_{2}+\mathrm{NO}_{3}$, DISSOLVED $(\mathrm{mg} / \mathrm{L}$ as $\mathrm{N})$ & 45 & 0.850 & 0.050 & 0.334 & 0.694 & 0.445 & 0.340 & 0.190 & 0.093 \\
\hline 00608 & NITROGEN AMMONIA, DISSOLVED (mg/L as N) & 45 & 0.430 & $<0.015$ & $0.097 *$ & 0.330 & 0.120 & 0.060 & 0.030 & $<0.015$ \\
\hline 00605 & NITROGEN ORGANIC，TOTAL (mg/L as N) & 45 & 2.800 & 0.310 & 0.794 & 2.330 & 0.840 & 0.660 & 0.515 & 0.353 \\
\hline 00600 & NITROGEN, TOTAL (mg/L as N) & 45 & 3.700 & 0.360 & 1.220 & 2.730 & 1.500 & 1.100 & 0.865 & 0.517 \\
\hline 00665 & PHOSPHORUS, TOTAL (mg/L as P) & 45 & 1.900 & 0.028 & 0.294 & 1.193 & 0.330 & 0.200 & 0.135 & 0.080 \\
\hline 00671 & PHOSPHORUS ORTHO, DISSOLVED (mg/L as P) & 45 & 1.180 & 0.010 & 0.115 & 0.261 & 0.130 & 0.080 & 0.040 & 0.012 \\
\hline & & & OIL AND & REASE, TOT & $\mathbf{A L}$ & & & & & \\
\hline 00556 & OIL AND GREASE, TOTAL (mg/L) & 12 & 3.000 & $<1.000$ & $1.739^{\star}$ & 3.000 & 3.000 & 1.000 & $<1.000$ & $<1.000$ \\
\hline & & & ORGANIC & ARBON, TON & TAL & & & & & \\
\hline 00680 & CARBON ORGANIC, TOTAL (mg/L) & 27 & 32.000 & 5.700 & 11.778 & 27.200 & 14.000 & 10.000 & 8.500 & 5.780 \\
\hline & & & & IFORM & & & & & & \\
\hline 31679 & FECAL STREPTOCOCCI (Colonies per $100 \mathrm{~mL}$ ) & 35 & 140000.000 & 100.000 & 43991.145 & 124000.016 & 73000.000 & 45000.000 & 3000.000 & 380.000 \\
\hline 31616 & FECAL COLIFORM (Colonies per $100 \mathrm{~mL}$ ) & 35 & 480000.000 & 90.000 & 68390.570 & 392000.094 & 66000.000 & 27000.000 & 6300.000 & 90.000 \\
\hline & & ORG & AN1C COMPOI & DS-PEST1C & IDES, TOTAL & & & & & \\
\hline 39330 & ALDRIN, TOTAL $(\mu \mathrm{g} / \mathrm{L})$ & 4 & $<0.010$ & $<0.010$ & -- & -- & -- & -- & -- & -- \\
\hline 39340 & LINDANE, TOTAL $(\mu \mathrm{g} / \mathrm{L})$ & 4 & $<0.010$ & $<0.010$ & -- & -- & -- & -- & -- & $\ldots$ \\
\hline 39350 & CHLORDANE, TOTAL $(\mu \mathrm{g} / \mathrm{L})$ & 4 & $<0.100$ & $<0.100$ & -- & -- & -- & -- & -- & -- \\
\hline 39370 & DDT, TOTAL $(\mu \mathrm{g} / \mathrm{L})$ & 4 & $<0.010$ & $<0.010$ & -- & -- & -- & -- & -- & -- \\
\hline 39365 & DDE, TOTAL $(\mu \mathrm{g} / \mathrm{L})$ & 4 & $<0.010$ & $<0.010$ & -- & -- & -- & -- & -- & -- \\
\hline 39360 & DDD, TOTAL $(\mu \mathrm{g} / \mathrm{L})$ & 4 & $<0.010$ & $<0.010$ & -- & -- & -- & -- & -- & - \\
\hline 39380 & DIELDRIN, TOTAL $(\mu \mathrm{g} / \mathrm{L})$ & 4 & $<0.010$ & $<0.010$ & -- & -- & -- & -- & -- & -- \\
\hline 39388 & ENDOSULFAN, TOTAL $(\mu \mathrm{g} / \mathrm{L})$ & 4 & $<0.010$ & $<0.010$ & -. & -- & -- & -- & -- & -- \\
\hline 39390 & ENDRIN， TOTAL $(\mu \mathrm{g} / \mathrm{L})$ & 4 & $<0.010$ & $<0.010$ & -- & -- & -- & -- & -- & -- \\
\hline 39410 & HEPTACHLOR, TOTAL $(\mu \mathrm{g} / \mathrm{L})$ & 4 & $<0.010$ & $<0.010$ & -- & -- & -- & -- & -- & -- \\
\hline 39420 & HEPTACHLOR EPOXIDE, TOTAL $(\mu \mathrm{g} / \mathrm{L})$ & 4 & $<0.010$ & $<0.010$ & -- & -- & -- & -- & -- & -. \\
\hline 39516 & PCB, TOTAL $(\mu \mathrm{g} / \mathrm{L})$ & 4 & 0.130 & $<0.100$ & -- & -- & -- & -- & -- & -- \\
\hline 39400 & TOXAPHENE, TOTAL $(\mu \mathrm{g} / \mathrm{L})$ & 4 & $<1.000$ & $<1.000$ & -- & -- & -- & -- & -- & -- \\
\hline 39034 & PERTHANE, TOTAL $(\mu \mathrm{g} / \mathrm{L})$ & 4 & $<0.100$ & $<0.100$ & -- & -- & -- & -- & -- & -- \\
\hline 39570 & DIAZINON, TOTAL $(\mu \mathrm{g} / \mathrm{L})$ & 4 & 0.010 & $<0.010$ & -- & -- & -- & -- & -- & -- \\
\hline 39398 & ETHION, TOTAL $(\mu \mathrm{g} / \mathrm{L})$ & 4 & $<0.010$ & $<0.010$ & -- & $\cdots$ & - & -- & -- & -- \\
\hline 39530 & MALATHION, TOTAL $(\mu \mathrm{g} / \mathrm{L})$ & 4 & 0.010 & $<0.010$ & -- & -- & -- & -- & - & -- \\
\hline 39600 & METHYL PARATHION, TOTAL $(\mu \mathrm{g} / \mathrm{L})$ & 4 & 0.010 & $<0.010$ & -- & -- & -- & -- & -- & -- \\
\hline 39540 & PARATHION, TOTAL $(\mu \mathrm{g} / \mathrm{L})$ & 4 & $<0.010$ & $<0.010$ & -- & -- & -- & -- & -- & -- \\
\hline 39786 & TRITHION, TOTAL $(\mu \mathrm{g} / \mathrm{L})$ & 4 & $<0.010$ & $<0.010$ & -- & -- & -- & -- & -- & - \\
\hline 39250 & PCN, TOTAL $(\mu \mathrm{g} / \mathrm{L})$ & 4 & $<0.100$ & $<0.100$ & -- & -- & -- & -- & -- & -- \\
\hline 39480 & METHOXYCHLOR, TOTAL $(\mu \mathrm{g} / \mathrm{L})$ & 4 & $<0.010$ & $<0.010$ & -- & -- & -- & -- & -- & - \\
\hline 39755 & MIREX, TOTAL $(\mu \mathrm{g} / \mathrm{L})$ & 4 & $<0.010$ & $<0.010$ & $\rightarrow$ & - & -- & -- & -- & -- \\
\hline 39011 & DISYSTON, TOTAL $(\mu \mathrm{g} / \mathrm{L})$ & 4 & $<0.010$ & $<0.010$ & -- & - & - & -- & -- & -- \\
\hline 39023 & PHORATE, TOTAL $(\mu \mathrm{g} / \mathrm{L})$ & 4 & $<0.010$ & $<0.010$ & -- & -- & -- & -- & -- & -- \\
\hline 38932 & CHLORPYRIFOS, TOTAL $(\mu \mathrm{g} / \mathrm{L})$ & 4 & $<0.010$ & $<0.010$ & -- & -- & -- & -- & -- & - \\
\hline 39040 & DEF, TOTAL $(\mu \mathrm{g} / \mathrm{L})$ & 4 & $<0.010$ & $<0.010$ & -- & -- & -- & -- & -- & - \\
\hline 82614 & FONOFOS, TOTAL $(\mu \mathrm{g} / \mathrm{L})$ & 4 & $<0.010$ & $<0.010$ & - & - & -- & -- & - & - \\
\hline & & & LATILE ORGA & COMPOUN & NDS, TOTAL & & & & & \\
\hline 34215 & ACRYLONITRILE, TOTAL $(\mu \mathrm{g} / \mathrm{L})$ & 1 & $<5.000$ & -- & $\ldots$ & -- & -- & -- & -- & -- \\
\hline 34030 & BENZENE，TOTAL $(\mu \mathrm{g} / \mathrm{L})$ & 9 & $<2.000$ & $<0.200$ & -- & -. & -- & -- & -- & $\ldots$ \\
\hline 32104 & BROMOFORM, TOTAL $(\mu \mathrm{g} / \mathrm{L})$ & 9 & $<2.000$ & $<0.200$ & $\ldots$ & -- & -- & -- & -- & -- \\
\hline 32102 & CARBON TETRACHLORIDE， TOTAL $(\mu \mathrm{g} / \mathrm{L})$ & 9 & $<2.000$ & $<0.200$ & -- & -- & -- & -- & -- & -- \\
\hline 34301 & CHLOROBENZENE, TOTAL $(\mu \mathrm{g} / \mathrm{L})$ & 9 & $<2.000$ & $<0.200$ & -- & - & -- & -- & -- & - \\
\hline 32105 & CHLORODIBROMOMETHANE, TOTAL $(\mu \mathrm{g} / \mathrm{L})$ & 9 & $<2.000$ & $<0.200$ & -- & -- & -- & -- & -- & -- \\
\hline 34311 & CHLOROETHANE, TOTAL $(\mu \mathrm{g} / \mathrm{L})$ & 9 & $<2.000$ & $<0.200$ & -- & -- & -- & -- & -- & -- \\
\hline 32106 & CHLOROFORM, TOTAL $(\mu \mathrm{g} / \mathrm{L})$ & 9 & $<2.000$ & $<0.200$ & -- & -- & -- & -- & -- & $\cdots$ \\
\hline 34496 & 1,1-DICHLOROETHANE, TOTAL $(\mu \mathrm{g} / \mathrm{L})$ & 9 & $<2.000$ & $<0.200$ & -- & -. & -- & -- & -- & -- \\
\hline 32103 & 1,2 -DICHLOROETHANE, TOTAL $(\mu \mathrm{g} / \mathrm{L})$ & 9 & $<2.000$ & $<0.200$ & -- & -- & -- & -- & -- & -- \\
\hline 34501 & 1,1 -DICHLOROETHYLENE, TOTAL $(\mu \mathrm{g} / \mathrm{L})$ & 9 & $<2.000$ & $<0.200$ & -- & -- & -- & -- & -- & -- \\
\hline 34541 & 1,2 -DICHLOROPROPANE, TOTAL $(\mu \mathrm{g} / \mathrm{L})$ & 9 & $<2.000$ & $<0.200$ & -- & -- & -- & -- & -- & -- \\
\hline 34371 & ETHYLBENZENE, TOTAL $(\mu \mathrm{g} / \mathrm{L})$ & 9 & $<2.000$ & $<0.200$ & -- & -- & -- & -- & -- & - \\
\hline 34413 & METHYL BROMIDE, TOTAL $(\mu \mathrm{g} / \mathrm{L})$ & 9 & $<2.000$ & $<0.200$ & -- & -- & -- & -- & -- & - \\
\hline
\end{tabular}

NOTE: Multiple detection limits during the period of record may result in different values flagged with a "<."

* Value is estimated by using a log-probability regression to predict the values of data below the detection limit. 
Table 70. Statistical summary of water-quality data at site 37 (CSW06), May 1995 through September 1998-Conti 7 ued

\begin{tabular}{|c|c|c|c|c|c|c|c|c|c|c|}
\hline \multirow{2}{*}{$\begin{array}{c}\text { PARAM- } \\
\text { ETER } \\
\text { CODE }\end{array}$} & \multirow{2}{*}{ PROPERTY OR CONSTITUENT } & & DESCRIPTI & STATISTICS & & PERCENT O & $\begin{array}{r}\text { SAMPLES I } \\
\text { EQUA }\end{array}$ & $\begin{array}{l}\text { WHICH VALI } \\
\text { TO THOSE S }\end{array}$ & $\begin{array}{l}\text { SWERE LE } \\
\text { WN }\end{array}$ & THAN OR \\
\hline & & $\begin{array}{c}\text { SAMPLE } \\
\text { SIZE }\end{array}$ & MAXIMUM & MINIMUM & MEAN & $95 \%$ & $75 \%$ & $\begin{array}{c}50 \% \\
\text { (median) }\end{array}$ & $25 \%$ & $5 \%$ \\
\hline 34423 & METHYLENE CHLORIDE, TOTAL $(\mu \mathrm{g} / \mathrm{L})$ & 9 & $<2.0 \overline{00}$ & $<0.200$ & -- & -- & -- & -- & -- & - \\
\hline 34516 & $1,1,2,2$-TETRACHLOROETHANE, TOTAL $(\mu \mathrm{g} / \mathrm{L})$ & 9 & $<2.000$ & $<0.200$ & -- & - & - & -- & - & -- \\
\hline 34475 & TETRACHLOROETHYLENE， TOTAL $(\mu \mathrm{g} / \mathrm{L})$ & 9 & $<2.000$ & $<0.200$ & -- & -- & -- & -- & - & -- \\
\hline 34010 & TOLUENE, TOTAL $(\mu \mathrm{g} / \mathrm{L})$ & 9 & 0.300 & $<0.200$ & -- & 0.300 & $<0.800$ & $<0.800$ & $<0.400$ & $<0.400$ \\
\hline 34546 & 1,2-TRANSDICHLOROETHENE, TOTAL $(\mu \mathrm{g} / \mathrm{L})$ & 9 & $<2.000$ & $<0.200$ & -- & -- & -- & -- & -- & - \\
\hline 34506 & $1,1,1$-TRICHLOROETHANE, TOTAL $(\mu \mathrm{g} / \mathrm{L})$ & 9 & 1.300 & $<0.200$ & -- & 1.300 & $<0.800$ & $<0.400$ & $<0.400$ & $<0.400$ \\
\hline 34511 & $1,1,2$-TRICHLOROETHANE, TOTAL $(\mu \mathrm{g} / \mathrm{L})$ & 9 & $<2.000$ & $<0.200$ & -- & -- & -- & -- & -- & -- \\
\hline 39180 & TRICHLOROETHYLENE, TOTAL $(\mu \mathrm{g} / \mathrm{L})$ & 9 & $<2.000$ & $<0.200$ & -- & -- & -- & -- & -- & -- \\
\hline 39175 & VINYL CHLORIDE， TOTAL $(\mu \mathrm{g} / \mathrm{L})$ & 9 & $<2.000$ & $<0.200$ & -- & -- & -- & -- & -+ & -- \\
\hline 30217 & DIBROMOMETHANE, TOTAL $(\mu \mathrm{g} / \mathrm{L})$ & 9 & $<2.000$ & $<0.200$ & -- & -- & -- & -- & -- & -- \\
\hline 32101 & DICHLOROBROMOMETHANE, TOTAL $(\mu \mathrm{g} / \mathrm{L})$ & 9 & $<2.000$ & $<0.200$ & -- & -- & -- & -- & -- & -- \\
\hline 34668 & DICHLORODIELUOROMETHANE, TOTAL $(\mu \mathrm{g} / \mathrm{L})$ & 9 & $<2.000$ & $<0.200$ & -- & -- & -- & -- & -- & -- \\
\hline 34488 & TRICHLOROFLUOROMETHANE, TOTAL $(\mu \mathrm{g} / \mathrm{L})$ & 9 & $<2.000$ & $<0.200$ & -- & -- & -- & -- & -- & -- \\
\hline 77651 & 1,2 -DIBROMOETHANE, TOTAL $(\mu \mathrm{g} / \mathrm{L})$ & 9 & $<2.000$ & $<0.200$ & -- & -- & -- & -- & -+ & -- \\
\hline 34418 & METHYLCHLORIDE, TOTAL $(\mu \mathrm{g} / \mathrm{L})$ & 9 & $<2.000$ & $<0.200$ & -- & -- & -- & -- & -- & -- \\
\hline 34704 & CIS 1,3 -DICHLOROPROPENE, TOTAL $(\mu \mathrm{g} / \mathrm{L})$ & 9 & $<2.000$ & $<0.200$ & -- & -- & -- & -- & -- & -- \\
\hline 34699 & TRANS 1,3 -DICHLOROPROPENE, TOTAL $(\mu \mathrm{g} / \mathrm{L})$ & 9 & $<2.000$ & $<0.200$ & -- & -- & -- & -- & -- & -- \\
\hline 77128 & STYRENE, TOTAL $(\mu \mathrm{g} / \mathrm{L})$ & 9 & $<2.000$ & $<0.200$ & -- & -- & -- & -- & -- & -- \\
\hline 81551 & XYLENE，TOTAL $(\mu \mathrm{g} / L)$ & 9 & $<2.000$ & $<0.200$ & -- & -- & -- & -- & -- & -- \\
\hline 82625 & DIBROMOCHLOROPROPANE， TOTAL $(\mu \mathrm{g} / \mathrm{L})$ & 9 & $<10.000$ & $<1.000$ & -- & -- & -- & -- & -- & -- \\
\hline 77168 & 1,1 -DICHLOROPROPENE, TOTAL $(\mu \mathrm{g} / \mathrm{L})$ & 9 & $<2.000$ & $<0.200$ & -- & -- & -- & -- & -- & -- \\
\hline 77170 & 2,2 -DICHLOROPROPANE，TOTAL $(\mu \mathrm{g} / \mathrm{L})$ & 9 & $<2.000$ & $<0.200$ & -- & $\cdots$ & -- & -- & -- & -- \\
\hline 77173 & 1,3 -DICHLOROPROPANE，TOTAL $(\mu \mathrm{g} / \mathrm{L})$ & 9 & $<2.000$ & $<0.200$ & -- & -- & -- & -- & -- & -- \\
\hline 77275 & O-CHLOROTOLUENE, TOTAL $(\mu \mathrm{g} / \mathrm{L})$ & 9 & $<2.000$ & $<0.200$ & -- & -- & -- & -- & -- & -- \\
\hline 77277 & P-CHLOROTOLUENE, TOTAL $(\mu \mathrm{g} / \mathrm{L})$ & 9 & $<2.000$ & $<0.200$ & -- & -- & -- & -- & -- & -- \\
\hline 77443 & 123-TRICHLOROPROPANE，TOTAL $(\mu \mathrm{g} / \mathrm{L})$ & 9 & $<2.000$ & $<0.200$ & -- & -- & -- & -- & -- & - \\
\hline 77562 & 1112-TETRACHLOROETHANE, TOTAL $(\mu \mathrm{g} / \mathrm{L})$ & 9 & $<2.000$ & $<0.200$ & -- & -- & - & -- & -- & -- \\
\hline 78032 & TERTBUTYL METHYL ETHER, TOTAL $(\mu \mathrm{g} / \mathrm{L})$ & 9 & $<2.000$ & $<0.200$ & -- & -- & -- & -- & -- & -- \\
\hline 77297 & BROMOCHLORO METHANE, TOTAL $(\mu \mathrm{g} / \mathrm{L})$ & 9 & $<2.000$ & $<0.200$ & -- & -- & - & -- & -- & -- \\
\hline 77093 & CIS- 1,2 -DICHLOROETHENE, TOTAL $(\mu \mathrm{g} / \mathrm{L})$ & 9 & $<2.000$ & $<0.200$ & -- & -- & -- & -- & -- & -- \\
\hline 34576 & 2-CHLOROETHYL VINYL ETHER, TOTAL $(\mu \mathrm{g} / \mathrm{L})$ & 5 & $<10.000$ & $<1.000$ & -- & -- & -- & -- & -- & -- \\
\hline 77223 & ISOPROPYL BENZENE，TOTAL $(\mu \mathrm{g} / \mathrm{L})$ & 9 & $<2.000$ & $<0.200$ & -- & -- & -- & -- & -- & -- \\
\hline 77224 & N-PROPYL BENZENE, TOTAL $(\mu \mathrm{g} / \mathrm{L})$ & 9 & $<2.000$ & $<0.200$ & -- & -- & - & -- & -- & -- \\
\hline 77353 & TERTBUTYL BENZENE, TOTAL $(\mu \mathrm{g} / \mathrm{L})$ & 9 & $<2.000$ & $<0.200$ & -- & -- & -- & -- & -- & -- \\
\hline 77222 & PSEUDOCUMENE, TOTAL $(\mu \mathrm{g} / \mathrm{L})$ & 9 & $<2.000$ & $<0.200$ & -- & -- & -- & -- & -- & -- \\
\hline 77350 & SEC-BUTYL BENZENE, TOTAL $(\mu \mathrm{g} / \mathrm{L})$ & 9 & $<2.000$ & $<0.200$ & -- & -- & - & -- & -- & -- \\
\hline 77356 & P-ISOPROPYL TOLUENE, TOTAL $(\mu \mathrm{g} / \mathrm{L})$ & 9 & $<2.000$ & $<0.200$ & -- & - & -- & -- & -- & -- \\
\hline 77342 & N-BUTYL BENZENE, TOTAL $(\mu \mathrm{g} / \mathrm{L})$ & 9 & $<2.000$ & $<0.200$ & -- & -- & -- & -- & -- & -- \\
\hline 77613 & $1,2,3$-TRICHLOROBENZENE，TOTAL $(\mu \mathrm{g} / \mathrm{L})$ & 9 & $<2.000$ & $<0.200$ & -- & -- & -- & -- & -- & -- \\
\hline 77652 & FREON-113, TOTAL $(\mu \mathrm{g} / \mathrm{L})$ & 9 & $<2.000$ & $<0.200$ & -- & -- & - & -- & -- & -- \\
\hline 77226 & MESITYLENE, TOTAL $(\mu \mathrm{g} / \mathrm{L})$ & 9 & $<2.000$ & $<0.200$ & -- & -- & - & -- & -- & - \\
\hline 81555 & BROMOBENZENE, TOTAL $(\mu \mathrm{g} / \mathrm{L})$ & 9 & $<2.000$ & $<0.200$ & -- & -- & -- & -- & -- & -- \\
\hline 34551 & $1,2,4$-TRICHLOROBENZENE, TOTAL $(\mu \mathrm{g} / \mathrm{L})$ & 9 & $<2.000$ & $<0.200$ & -- & -- & -- & -- & -- & -- \\
\hline 34536 & 1,2 -DICHLOROBENZENE，TOTAL $(\mu \mathrm{g} / \mathrm{L})$ & 9 & $<2.000$ & $<0.200$ & -- & -- & -- & -- & -- & -- \\
\hline 34566 & 1, 3-DICHLOROBENZENE, TOTAL $(\mu \mathrm{g} / \mathrm{L})$ & 9 & $<2.000$ & $<0.200$ & -- & -- & -- & -- & -- & -- \\
\hline 34571 & 1,4 -DICHLOROBENZENE, TOTAL $(\mu \mathrm{g} / \mathrm{L})$ & 9 & $<2.000$ & $<0.200$ & -- & -- & -- & -- & -- & -- \\
\hline 39702 & HEXACHLOROBUTADIENE, TOTAL $(\mu \mathrm{g} / \mathrm{L})$ & 9 & $<2.000$ & $<0.200$ & -- & -- & -- & -- & -- & -- \\
\hline 34696 & NAPHTHALENE, TOTAL $(\mu \mathrm{g} / \mathrm{L})$ & 9 & $<2.000$ & $\leq 0.200$ & -- & -- & -- & -- & -- & - \\
\hline & & MET & LS AND MIN & CONSTITUI & TS, TOTAL & & & & & \\
\hline 01097 & ANTIMONY, TOTAL $(\mu \mathrm{g} / \mathrm{L}$ as $\mathrm{Sb})$ & 32 & $1 . \overline{000}$ & $<1.000$ & -- & 1.000 & $<1.000$ & $<1.000$ & $<1.000$ & $<1.000$ \\
\hline 01002 & ARSENIC, TOTAL $(\mu \mathrm{g} / \mathrm{L}$ as As $)$ & 32 & 5.000 & $<1.000$ & -- & 3.000 & $<1.000$ & $<1.000$ & $<1.000$ & $<1.000$ \\
\hline 01012 & BERYLLIUM, TOTAL $(\mu \mathrm{g} / \mathrm{L}$ as $\mathrm{Be})$ & 19 & $<10.000$ & $<10.000$ & - & $<10.000$ & $<10.000$ & $<10.000$ & $<10.000$ & $<10.000$ \\
\hline 01027 & CADMIUM, TOTAL $(\mu \mathrm{g} / \mathrm{L}$ as $\mathrm{Cd})$ & 19 & $<1.000$ & $<1.000$ & -- & $<1.000$ & $<1.000$ & $<1.000$ & $<1.000$ & $<1.000$ \\
\hline 01034 & CHROMIUM, TOTAL $(\mu \mathrm{g} / \mathrm{L}$ as $\mathrm{Cr})$ & 32 & 32.000 & $<1.000$ & $5.796^{\star}$ & 15.000 & 7.000 & 3.000 & 2.000 & $<1.000$ \\
\hline 01042 & COPPER, TOTAL $(\mu \mathrm{g} / \mathrm{L}$ as $\mathrm{Cu})$ & 32 & 44.000 & 5.000 & 15.250 & 33.600 & 18.500 & 14.000 & 11.000 & 5.000 \\
\hline 01051 & LEAD, TOTAL $(\mu \mathrm{g} / \mathrm{L}$ as $\mathrm{Pb})$ & 32 & 41.000 & $<1.000$ & $6.626^{*}$ & 25.000 & 8.000 & 3.000 & 2.000 & $<1.000$ \\
\hline 71900 & MERCURY, TOTAL $(\mu \mathrm{g} / \mathrm{L}$ as $\mathrm{Hg})$ & 32 & 0.200 & $<0.100$ & -- & 0.200 & $<0.100$ & $<0.100$ & $<0.100$ & $<0.100$ \\
\hline 01067 & NICKEL, TOTAL $(\mu \mathrm{g} / \mathrm{L}$ as Ni $)$ & 32 & 41.000 & 3.000 & 10.406 & 35.150 & 10.750 & 8.000 & 6.000 & 3.000 \\
\hline 01147 & SELENIUM, TOTAL ( $\mu \mathrm{g} / \mathrm{L}$ as $\mathrm{Se})$ & 19 & $<1.000$ & $<1.000$ & -- & $<1.000$ & $<1.000$ & $<1.000$ & $<1.000$ & $<1.000$ \\
\hline 01077 & SILVER, TOTAL $(\mu \mathrm{g} / \mathrm{L}$ as $\mathrm{Ag})$ & 19 & 2.000 & $<1.000$ & -- & 2.000 & $<1.000$ & $<1.000$ & $<1.000$ & $<1.000$ \\
\hline 01092 & $\mathrm{ZINC}, \operatorname{TOTAL}(\mu \mathrm{g} / \mathrm{L}$ as $\mathrm{zn})$ & 32 & 240.000 & 40.000 & 90.938 & 207.500 & 117.500 & 85.000 & 60.000 & 40.000 \\
\hline 00720 & CYANIDE, TOTAL (mg/L as $\mathrm{Cn}$ ) & 15 & $<0.010$ & $<0.010$ & -- & $<0.010$ & $<0.010$ & $<0.010$ & $<0.010$ & $<0.010$ \\
\hline & & ORGAN & IC COMPOUN & -PESTICID & DISSOLVE & & & & & \\
\hline 49260 & ACETOCHLOR， DISSOLVED $(\mu \mathrm{g} / \mathrm{L})$ & 3 & $<0.002$ & $<0.002$ & -+ & $=$ & -- & -- & -- & -- \\
\hline 46342 & ALACHLOR, DISSOLVED $(\mu \mathrm{g} / \mathrm{L})$ & 3 & 0.010 & $<0.002$ & -- & -- & -- & -- & -- & -- \\
\hline 04040 & DEETHYL ATRAZINE, DISSOLVED $\{\mu \mathrm{g} / \mathrm{L}\rangle$ & 3 & 0.008 & $<0.002$ & -- & -- & -- & -- & -- & -- \\
\hline 39632 & ATRAZINE, DISSOLVED $(\mu \mathrm{g} / \mathrm{L})$ & 3 & 0.084 & 0.011 & -- & -- & -- & -- & -- & -- \\
\hline 82686 & METHYL AZINPHOS, DISSOLVED $(\mu \mathrm{g} / \mathrm{L})$ & 3 & $<0.001$ & $<0.001$ & -- & -- & -- & -- & -- & -- \\
\hline 82673 & BENFLURALIN， DISSOLVED $(\mu \mathrm{g} / \mathrm{L})$ & 3 & $<0.002$ & $<0.002$ & -- & -- & - & -- & -- & -- \\
\hline 04028 & BUTYLATE, DISSOLVED $(\mu \mathrm{g} / \mathrm{L})$ & 3 & $<0.002$ & $<0.002$ & -- & - & -- & -- & -- & -- \\
\hline 82680 & CARBARYL， DISSOLVED $(\mu \mathrm{g} / \mathrm{L})$ & 3 & 0.048 & 0.014 & -- & -- & -- & -- & -- & -- \\
\hline 82674 & CARBOFURAN， DISSOLVED $(\mu \mathrm{g} / \mathrm{L})$ & 3 & 0.007 & $<0.003$ & -- & -- & -- & -- & -- & -- \\
\hline 38933 & CHLORPYRIFOS, DISSOLVED $(\mu \mathrm{g} / \mathrm{L})$ & 3 & $<0.004$ & $<0.004$ & -- & -- & -- & -- & -- & -- \\
\hline 04041 & CYANAZINE, DISSOLVED $(\mu \mathrm{g} / \mathrm{L})$ & 3 & 0.034 & $<0.004$ & -- & -- & -- & -- & -- & -- \\
\hline 82682 & DCPA, DISSOLVED $(\mu \mathrm{g} / \mathrm{L})$ & 3 & $<0.002$ & $<0.002$ & -- & -- & -- & -- & -- & -- \\
\hline 34653 & $P, P^{\prime}$ DDE, DISSOLVED $(\mu \mathrm{g} / \mathrm{L})$ & 3 & $<0.006$ & $<0.006$ & -- & -- & -- & -- & -- & -- \\
\hline 39572 & DIAZINON, DISSOLVED $(\mu \mathrm{g} / \mathrm{L})$ & 3 & $<0.002$ & $<0.002$ & -- & -- & - & -- & $\ldots$ & -- \\
\hline 39381 & DIELDRIN， DISSOLVED $(\mu \mathrm{g} / \mathrm{L})$ & 3 & $<0.001$ & $<0.001$ & -- & -- & -- & -- & -- & -- \\
\hline 82660 & 2,6 -DIETHYL ANILINE, DISSOLVED $(\mu \mathrm{g} / \mathrm{L})$ & 3 & $<0.003$ & $<0.003$ & -- & -- & -- & -- & -- & -- \\
\hline 82677 & DISULFOTON, DISSOLVED $(\mu \mathrm{g} / \mathrm{L})$ & 3 & $<0.017$ & $<0.017$ & -- & -- & -- & -- & -- & -- \\
\hline 82668 & EPTC， DISSOLVED $(\mu \mathrm{g} / \mathrm{L})$ & 3 & $<0.002$ & $<0.002$ & -- & -- & -- & -- & -- & -- \\
\hline
\end{tabular}

NOTE: Multiple detection limits during the period of record may result in different values flagged with a "<."

* Value is estimated by using a log-probability regression to predict the values of data below the detection limit. 
Table 70. Statistical summary of water-quality data at site 37 (CSW06), May 1995 through September 1998-Continued

\begin{tabular}{|c|c|c|c|c|c|c|c|c|c|c|}
\hline \multirow{2}{*}{$\begin{array}{c}\text { PARAM- } \\
\text { ETER } \\
\text { CODE }\end{array}$} & \multirow{2}{*}{ PROPERTY OR CONSTITUENT } & & DESCRIPTII & STATISTICS & & PERCEN & $\begin{array}{r}\text { AMPLE } \\
\text { EQ }\end{array}$ & $\begin{array}{l}\text { WHICH VAL } \\
\text { TO THOSE }\end{array}$ & $\begin{array}{l}\text { WEAE } \\
\text { NN }\end{array}$ & HAN OR \\
\hline & & $\begin{array}{c}\text { SAMPLE } \\
\text { SIZE }\end{array}$ & MAXIMUM & MINIMUM & MEAN & $95 \%$ & $75 \%$ & $\begin{array}{c}50 \% \\
\text { (median) }\end{array}$ & $25 \%$ & $5 \%$ \\
\hline 82663 & ETHALFLURALIN， DISSOLVED $(\mu \mathrm{g} / \mathrm{L})$ & 3 & $<0.004$ & $<0.004$ & -- & -- & -- & - & -- & $-\cdots$ \\
\hline 82672 & ETHOPROP, DISSOLVED $(\mu \mathrm{g} / \mathrm{L})$ & 3 & $<0.003$ & $<0.003$ & -- & -- & -- & -- & -- & -- \\
\hline 04095 & FONOFOS, DISSOLVED $(\mu \mathrm{g} / \mathrm{L})$ & 3 & $<0.003$ & $<0.003$ & -- & -- & -- & -- & -- & -- \\
\hline 34253 & ALPHA BHC, DISSOLVED $(\mu \mathrm{g} / \mathrm{L})$ & 3 & $<0.002$ & $<0.002$ & -- & - & -. & -- & -- & -- \\
\hline 39341 & LINDANE， DISSOLVED $(\mu \mathrm{g} / \mathrm{L})$ & 3 & $<0.004$ & $<0.004$ & -- & -- & -- & -- & -- & -- \\
\hline 82666 & LINURON, DISSOLVED $(\mu \mathrm{g} / \mathrm{L})$ & 3 & $<0.002$ & $<0.002$ & -- & -- & -- & -- & -- & -- \\
\hline 39532 & MALATHION，DISSOLVED $\left(\mu \mathrm{g} / \mathrm{L}_{\mathrm{f}}\right)$ & 3 & 0.018 & $<0.005$ & -- & -. & -- & -- & -- & -- \\
\hline 82667 & METHYL PARATHION, DISSOLVED $(\mu \mathrm{g} / \mathrm{L})$ & 3 & 0.031 & $<0.006$ & -- & -- & -- & -- & -- & -- \\
\hline 39415 & METOLACHLOR, DISSOLVED $(\mu \mathrm{g} / \mathrm{L})$ & 3 & 0.034 & 0.008 & -- & - & -- & -- & -- & -- \\
\hline 82630 & METRIBUZIN, DISSOLVED $(\mu \mathrm{g} / \mathrm{L})$ & 3 & $<0.004$ & $<0.004$ & -- & -- & -- & -- & $\cdots$ & -- \\
\hline 82671 & MOLINATE, DISSOLVED $(\mu \mathrm{g} / \mathrm{L})$ & 3 & $<0.004$ & $<0.004$ & -- & -- & -- & -- & -- & -- \\
\hline 82684 & NAPROPAMIDE, DISSOLVED $(\mu \mathrm{g} / \mathrm{L})$ & 3 & $<0.003$ & $<0.003$ & -- & -- & -- & -- & -- & -- \\
\hline 39542 & PARATHION, DISSOLVED $(\mu \mathrm{g} / \mathrm{L})$ & 3 & $<0.004$ & $<0.004$ & -- & -- & -- & - & -- & -- \\
\hline 82669 & PEBULATE, DISSOLVED $(\mu \mathrm{g} / \mathrm{L})$ & 3 & $<0.004$ & $<0.004$ & -. & -- & -- & -. & -- & -- \\
\hline 82683 & PENDIMETHALIN， DISSOLVED $(\mu \mathrm{g} / \mathrm{L})$ & 3 & $<0.004$ & $<0.004$ & -- & -- & -- & -- & -- & -- \\
\hline 82687 & PERMETHRIN, DISSOLVED $(\mu \mathrm{g} / \mathrm{L})$ & 3 & $<0.005$ & $<0.005$ & - & -- & -- & -- & - & -- \\
\hline 82664 & PHORATE, DISSOLVED $(\mu \mathrm{g} / \mathrm{L})$ & 3 & $<0.002$ & $<0.002$ & -. & -- & -- & -. & -- & -- \\
\hline 82676 & PRONAMIDE, DISSOLVED $(\mu \mathrm{g} / \mathrm{L})$ & 3 & 0.010 & $<0.003$ & -- & -- & -- & -- & -- & -- \\
\hline 04037 & PROMETON, DISSOLVED $(\mu \mathrm{g} / \mathrm{L})$ & 3 & 0.148 & $<0.018$ & -- & -- & -- & -- & - & -- \\
\hline 04024 & PROPACHLOR, DISSOLVED $(\mu \mathrm{g} / \mathrm{L})$ & 3 & $<0.007$ & $<0.007$ & -- & -- & -- & -. & -- & -- \\
\hline 82679 & PROPANIL, DISSOLVED $(\mu \mathrm{g} / \mathrm{L})$ & 3 & 0.009 & $<0.004$ & -- & -- & -- & -- & -- & -- \\
\hline 82685 & PROPARGITE, DISSOLVED $(\mu \mathrm{g} / \mathrm{L})$ & 3 & $<0.013$ & $<0.013$ & -- & -- & -- & -- & -- & -- \\
\hline 04035 & SIMAZINE, DISSOLVED $(\mu \mathrm{g} / \mathrm{L})$ & 3 & $<0.009$ & $<0.005$ & -- & -- & -- & -- & -- & -- \\
\hline 82681 & THIOBENCARB, DISSOLVED $(\mu \mathrm{g} / \mathrm{L})$ & 3 & $<0.002$ & $<0.002$ & -- & -- & -- & -- & -- & -- \\
\hline 82670 & TEBUTHIURON, DISSOLVED $(\mu \mathrm{g} / \mathrm{L})$ & 3 & 0.508 & $<0.010$ & -- & -- & -- & -- & - & -- \\
\hline 82665 & TERBACIL, DISSOLVED $(\mu \mathrm{g} / \mathrm{L})$ & 3 & $<0.025$ & $<0.007$ & -- & -- & -- & -- & -- & -- \\
\hline 82675 & TERBUFOS, DISSOLVED $(\mu \mathrm{g} / \mathrm{L})$ & 3 & $<0.013$ & $<0.013$ & -- & -- & - & -- & -- & -- \\
\hline 82678 & TRIALLATE, DISSOLVED $(\mu \mathrm{g} / \mathrm{L})$ & 3 & $<0.001$ & $<0.001$ & -- & -- & -. & -- & -- & -- \\
\hline 82661 & TRIFLURALIN, DISSOLVED $(\mu \mathrm{g} / \mathrm{L})$ & 3 & $<0.002$ & $<0.002$ & $-\cdots$ & -- & -- & -- & -- & -- \\
\hline 39742 & $2,4,5-\mathrm{T}$, DISSOLVED $(\mu \mathrm{g} / \mathrm{L})$ & 3 & $<0.035$ & $<0.035$ & -- & -- & -- & -- & -- & -- \\
\hline 39732 & $2,4-D$, DISSOLVED $(\mu \mathrm{g} / \mathrm{L})$ & 3 & $<0.150$ & $<0.035$ & -- & -- & -- & -- & -- & -- \\
\hline 38746 & 2,4 -DB, DISSOLVED $(\mu \mathrm{g} / \mathrm{L})$ & 3 & $<0.240$ & $<0.035$ & -- & -- & -- & -- & -- & -- \\
\hline 49315 & ACIFLUORFEN, DISSOLVED $(\mu \mathrm{g} / \mathrm{L})$ & 3 & $<0.035$ & $<0.035$ & -- & -- & -- & -- & -- & -- \\
\hline 49312 & ALDICARB， DISSOLVED $(\mu \mathrm{g} / \mathrm{L})$ & 3 & $<0.550$ & $<0.016$ & -- & -- & -- & -- & -- & -- \\
\hline 49313 & ALDICARB SULFONE, DISSOLVED $(\mu \mathrm{g} / \mathrm{L})$ & 3 & $<0.100$ & $<0.016$ & -- & -- & -- & -- & -- & -- \\
\hline 49314 & ALDICARB SULFOXIDE, DISSOLVED $(\mu \mathrm{g} / \mathrm{L})$ & 3 & $<0.021$ & $<0.021$ & -- & -- & - & -- & -- & -- \\
\hline 38711 & BENTAZON, DISSOLVED $(\mu \mathrm{g} / \mathrm{L})$ & 3 & $<0.014$ & $<0.014$ & -- & -- & -- & -- & -- & -- \\
\hline 04029 & BROMACIL, DISSOLVED $(\mu \mathrm{g} / \mathrm{L})$ & 3 & $<0.035$ & $<0.035$ & -- & -- & -- & -- & -- & -- \\
\hline 49311 & BROMOXYNIL, DISSOLVED $(\mu \mathrm{g} / \mathrm{L})$ & 3 & $<0.035$ & $<0.035$ & -- & -- & -- & -- & -- & -- \\
\hline 49310 & CARBARYL, DISSOLVED $(\mu \mathrm{g} / \mathrm{L})$ & 3 & $<0.008$ & $<0.008$ & -- & -- & -- & -- & -- & -- \\
\hline 49309 & CARBOFURAN, DISSOLVED $(\mu \mathrm{g} / \mathrm{L})$ & 3 & $<0.120$ & $<0.028$ & -- & -- & -- & -- & -- & -- \\
\hline 49308 & 3-HYDROXY-CARBOFURAN $(\mu \mathrm{g} / \mathrm{L})$ & 3 & $<0.014$ & $<0.014$ & -- & -- & $\cdots$ & -- & -- & -- \\
\hline 49307 & AMIBEN, DISSOLVED $(\mu \mathrm{g} / \mathrm{L})$ & 3 & $<0.420$ & $<0.011$ & -- & -- & - & -- & -- & -- \\
\hline 49306 & CHLOROTHALONIL， DISSOLVED $(\mu \mathrm{g} / \mathrm{L})$ & 3 & $<0.480$ & $<0.035$ & -- & -- & -- & -- & -- & -- \\
\hline 49305 & CLOPYRALID, DISSOLVED $(\mu \mathrm{g} / \mathrm{L})$ & 3 & $<0.230$ & $<0.050$ & -- & -- & - & -- & -- & -- \\
\hline 49304 & DACTHALMONO-ACID, DISSOLVED $(\mu \mathrm{g} / \mathrm{L})$ & 3 & $<0.017$ & $<0.017$ & -- & -- & -- & -- & -- & -- \\
\hline 38442 & DICAMBA，DISSOLVED $(\mu \mathrm{g} / \mathrm{L})$ & 3 & $<0.035$ & $<0.035$ & -- & -- & -- & -- & -- & -- \\
\hline 49303 & DICHLOBENIL，DISSOLVED $(\mu \mathrm{g} / \mathrm{L})$ & 2 & $<1.200$ & $<0.020$ & -- & -- & -- & -- & -- & -- \\
\hline 49302 & DICHLORPROP，DISSOLVED $(\mu \mathrm{g} / \mathrm{L})$ & 3 & $<0.032$ & $<0.032$ & -- & -- & -- & -- & -- & -- \\
\hline 49301 & DINOSEB, DISSOLVED $(\mu \mathrm{g} / \mathrm{L})$ & 3 & $<0.035$ & $<0.035$ & -- & -- & -- & -- & -- & -- \\
\hline 49300 & DIURON, DISSOLVED $(\mu \mathrm{g} / \mathrm{L})$ & 3 & 12.300 & $<0.020$ & -- & -- & -- & -- & -- & -- \\
\hline 49299 & 4,6-DINITRO OCRESOL, DISSOLVED $(\mu \mathrm{g} / \mathrm{L})$ & 3 & $<0.420$ & $<0.035$ & - & -- & -- & -- & -- & -- \\
\hline 49298 & ESFENVALERATE, DISSOLVED $(\mu \mathrm{g} / \mathrm{L})$ & 2 & $<0.019$ & $<0.019$ & -- & - & -- & -- & -- & -- \\
\hline 49297 & FENURON， DISSOLVED $(\mu \mathrm{g} / \mathrm{L})$ & 3 & $<0.013$ & $<0.013$ & -- & -- & -- & -- & -- & -- \\
\hline 38811 & FLUOMETURON, DISSOLVED $(\mu \mathrm{g} / \mathrm{L})$ & 3 & $<0.035$ & $<0.035$ & -- & -- & -- & -- & -- & -- \\
\hline 38478 & LINURON, DISSOLVED $(\mu \mathrm{g} / \mathrm{L})$ & 3 & $<0.018$ & $<0.018$ & -- & -- & -- & -- & -- & -- \\
\hline 38482 & MCPA, DISSOLVED $(\mu \mathrm{g} / \mathrm{L})$ & 3 & $<0.170$ & $<0.050$ & -- & -- & -- & -- & -- & -- \\
\hline 38487 & MCPB, DISSOLVED $(\mu \mathrm{g} / \mathrm{L})$ & 3 & $<0.140$ & $<0.035$ & -- & -- & -- & -- & -- & -- \\
\hline 38501 & METHIOCARB, DISSOLVED $(\mu \mathrm{g} / \mathrm{L})$ & 3 & $<0.026$ & $<0.026$ & -- & -- & -- & $\ldots$ & -- & -- \\
\hline 49296 & METHOMYL， DISSOLVED $(\mu \mathrm{g} / \mathrm{L})$ & 3 & $<0.017$ & $<0.017$ & -- & -- & -- & - & -- & -- \\
\hline 49295 & 1-NAPHTHOL, DISSOLVED $(\mu \mathrm{g} / \mathrm{L})$ & 2 & $<0.007$ & $<0.007$ & -- & -- & -- & -- & -- & -- \\
\hline 49294 & NEBURON， DISSOLVED $(\mu \mathrm{g} / \mathrm{L})$ & 3 & $<0.015$ & $<0.015$ & -- & -. & -- & -- & -- & -- \\
\hline 49293 & NORFLURAZON, DISSOLVED $(\mu \mathrm{g} / \mathrm{L})$ & 3 & $<0.024$ & $<0.024$ & -- & -- & -- & -- & -- & -- \\
\hline 49292 & ORYZALIN, DISSOLVED $(\mu \mathrm{g} / \mathrm{L})$ & 3 & $<0.310$ & $<0.019$ & -- & -- & -- & -- & -- & -- \\
\hline 38866 & OXAMYL, DISSOLVED $(\mu \mathrm{g} / \mathrm{L})$ & 3 & $<0.018$ & $<0.018$ & -- & -- & -- & -- & -- & -- \\
\hline 49291 & PICLORAM, DISSOLVED $(\mu \mathrm{g} / \mathrm{L})$ & 3 & $<0.050$ & $<0.050$ & -- & -- & -- & -- & - & -- \\
\hline 49236 & PROPHAM, DISSOLVED $(\mu \mathrm{g} / \mathrm{L})$ & 3 & $<0.035$ & $<0.035$ & -- & -- & -- & -- & -- & -- \\
\hline 38538 & PROPOXUR, DISSOLVED $(\mu \mathrm{g} / \mathrm{L})$ & 3 & $<0.035$ & $<0.035$ & -- & -- & -- & -- & -- & -- \\
\hline 39762 & SILVEX, DISSOLVED $(\mu \mathrm{g} / \mathrm{L})$ & 3 & $<0.021$ & $<0.021$ & -- & -- & -- & -- & -- & -- \\
\hline 49235 & TRICLOPYR，DISSOLVED $(\mu \mathrm{g} / \mathrm{L})$ & 3 & 1.460 & $<0.050$ & -- & -- & -- & -- & -- & -- \\
\hline & & ORGANI & COMPOUND & ORGANONI & OGEN, T & TAL & & & & \\
\hline 39057 & PROMETRYNE, TOTAL $(\mu \mathrm{g} / \mathrm{L})$ & 1 & $<0.100$ & -- & $\overline{--}$ & $-\overline{--}$ & $=-$ & -- & -- & -- \\
\hline 39056 & PROMETONE, TOTAL $(\mu \mathrm{g} / \mathrm{L})$ & 1 & $<0.200$ & $\sim$ & -- & -- & -- & -- & -- & -- \\
\hline 39054 & SIMETRYNE, TOTAL $(\mu g / L)$ & 1 & $<0.100$ & -- & -- & -- & -- & -- & -- & -- \\
\hline 81757 & CYANAZINE, TOTAL $(\mu \mathrm{g} / \mathrm{L})$ & 1 & $<0.200$ & -- & -- & -- & -- & - & -- & -- \\
\hline 77825 & ALACHLOR, TOTAL $(\mu \mathrm{g} / \mathrm{L})$ & 1 & $<0.100$ & -- & -- & -- & -- & -- & -- & -- \\
\hline 82611 & METRIBUZIN, TOTAL $(\mu \mathrm{g} / \mathrm{L})$ & 1 & $<0.100$ & -- & -- & -- & -- & -- & -- & - \\
\hline 30311 & TERBACIL, TOTAL $(\mu \mathrm{g} / \mathrm{L})$ & 1 & $<0.200$ & -- & -- & -- & -- & -- & -- & -- \\
\hline 30245 & CARBOXIN, TOTAL $(\mu \mathrm{g} / \mathrm{L})$ & 1 & $<0.200$ & -- & -- & -- & -- & -- & -- & -- \\
\hline 30264 & HEXAZINONE, TOTAL $(\mu \mathrm{g} / \mathrm{L})$ & 1 & $<0.200$ & -- & -- & -- & -- & -- & -- & -- \\
\hline 30235 & BUTACHLOR, TOTAL $(\mu \mathrm{g} / \mathrm{L})$ & 1 & $<0.100$ & -- & -- & -- & -- & -- & -- & -- \\
\hline 30236 & BUTYLATE, TOTAL $(\mu \mathrm{g} / \mathrm{L})$ & 1 & $<0.100$ & -- & -- & -- & -- & -- & -- & -- \\
\hline
\end{tabular}

NOTE: Multiple detection limits during the period of record may result in different values flagged with a "<."

* Value is estimated by using a log-probability regression to predict the values of data below the detection limit. 
Table 70. Statistical summary of water-quality data at site 37 (CSW06), May 1995 through September 1998—Continued

\begin{tabular}{|c|c|c|c|c|c|c|c|c|c|c|}
\hline \multirow{2}{*}{$\begin{array}{l}\text { PARAM- } \\
\text { ETER } \\
\text { CODE }\end{array}$} & \multirow{2}{*}{ PROPERTY OR CONSTITUENT } & \multicolumn{4}{|c|}{ DESCRIPTIVE STATISTICS } & \multicolumn{5}{|c|}{$\begin{array}{c}\text { PERCENT OF SAMPLES IN WHICH VALUES WERE LESS THAN OP } \\
\text { EQUAL TO THOSE SHOWN }\end{array}$} \\
\hline & & $\begin{array}{l}\text { SAMPLE } \\
\text { SIZE }\end{array}$ & MAXIMUM & MINIMUM & MEAN & $95 \%$ & $75 \%$ & $\begin{array}{c}50 \% \\
\text { (medlan) }\end{array}$ & $\mathbf{2 5} \%$ & $5 \%$ \\
\hline 75981 & DE-ETHYLATRAZINE, TOTAL $(\mu \mathrm{g} / \mathrm{L})$ & 1 & $<0.200$ & -- & -- & -- & -- & -- & -- & -- \\
\hline 39630 & ATRAZINE, TOTAL $(\mu \mathrm{g} / \mathrm{L})$ & 1 & 0.100 & -- & -- & -- & -- & -- & -- & - \\
\hline 39055 & SIMAZINE, TOTAL $(\mu \mathrm{g} / \mathrm{L})$ & 1 & $<0.100$ & -- & -- & -- & -- & $\cdots$ & -- & -- \\
\hline 39024 & PROPAZINE, TOTAL $(\mu \mathrm{g} / \mathrm{L})$ & 1 & $<0.100$ & -- & -- & -- & -- & -- & -- & -- \\
\hline 82184 & AMETRYNE, TOTAL $(\mu \mathrm{g} / \mathrm{L})$ & 1 & $<0.100$ & -- & -- & -- & -- & -- & -- & -- \\
\hline 39030 & TRIFLURALIN, TOTAL $(\mu \mathrm{g} / \mathrm{L})$ & 1 & $<0.100$ & -- & -- & -- & -- & -- & -- & -- \\
\hline 82612 & METOLACHLOR, TOTAL $(\mu \mathrm{g} / \mathrm{L})$ & 1 & $<0.200$ & -- & -- & -- & -- & -- & -- & -- \\
\hline 30234 & BROMACIL, TOTAL $(\mu \mathrm{g} / \mathrm{L})$ & 1 & $<0.200$ & -- & -- & -- & -- & -- & -- & -- \\
\hline 30255 & DIPHENAMID, TOTAL $(\mu \mathrm{g} / \mathrm{L})$ & 1 & $<0.100$ & -- & -- & -- & -- & -- & -- & -- \\
\hline 30324 & VERNOLATE， TOTAL $(\mu \mathrm{g} / \mathrm{L})$ & 1 & $<0.100$ & -- & -- & -- & -- & -- & -- & - \\
\hline 30254 & CYCLOATE, TOTAL $(\mu \mathrm{g} / \mathrm{L})$ & 1 & $<0.100$ & -- & -- & -- & -- & -- & -- & -- \\
\hline 30295 & PROPACHLOR, TOTAL $(\mu \mathrm{g} / \mathrm{L})$ & 1 & $<0.100$ & -- & -- & -- & -- & -- & -- & -- \\
\hline 75980 & DE-ISOPROPYLATRAZIN, TOTAL $(\mu \mathrm{g} / \mathrm{L})$ & 1 & $<0.200$ & -- & -- & -- & -- & -- & -- & -- \\
\hline \multicolumn{11}{|c|}{ ORGANIC COMPOUNDS-HERBICIDES, TOTAL } \\
\hline 39730 & $2,4-D$, TOTAL $(\mu \mathrm{g} / \mathrm{L})$ & 1 & $<0.010$ & -- & -- & -- & -- & - & -- & -- \\
\hline 39760 & SILVEX, TOTAL $(\mu \mathrm{g} / \mathrm{L})$ & 1 & $<0.010$ & -- & -- & -- & -- & -- & -- & -- \\
\hline 39720 & PICLORAM, TOTAL $(\mu \mathrm{g} / \mathrm{L})$ & 1 & $<0.010$ & -- & -- & -- & -- & -- & -- & -- \\
\hline 39740 & $2,4,5-\mathrm{T}$, TOTAL $(\mu \mathrm{g} / \mathrm{L})$ & 1 & $<0.010$ & -- & -- & -- & -- & -- & -- & -- \\
\hline 82183 & 2,4-DP, TOTAL $(\mu \mathrm{g} / \mathrm{L})$ & 1 & $<0.010$ & -- & - & -- & -- & -- & -- & -- \\
\hline 82052 & DICAMBA， TOTAL $(\mu \mathrm{g} / \mathrm{L})$ & 1 & $<0.010$ & -- & $\cdots$ & -- & -- & -- & -- & -- \\
\hline \multicolumn{11}{|c|}{ ORGANIC COMPOUNDS - CARBAMATE PESTICIDES, TOTAL } \\
\hline 39750 & SEVIN, TOTAL $(\mu \mathrm{g} / \mathrm{L})$ & 1 & $<0.500$ & -- & $\overline{--}$ & - & $\cdots$ & -- & - & - \\
\hline 39051 & METHOMYL, TOTAL $(\mu \mathrm{g} / \mathrm{L})$ & 1 & $<0.500$ & -- & -- & -- & -- & -- & $-\infty$ & -- \\
\hline 82619 & ALDICARD, TOTAL $(\mu \mathrm{g} / L)$ & 1 & $<0.500$ & -- & -- & -- & -- & -- & -- & -- \\
\hline 30296 & PROPOXUR, TOTAL $(\mu \mathrm{g} / \mathrm{L})$ & 1 & $<0.500$ & -- & 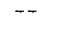 & -- & -- & -- & -- & -- \\
\hline 39052 & PROPHAM，TOTAL $(\mu \mathrm{g} / \mathrm{L})$ & 1 & $<0.500$ & -- & -- & -- & -- & -- & -- & -- \\
\hline 82615 & CARBOFURAN, TOTAL $(\mu \mathrm{g} / \mathrm{L})$ & 1 & $<0.500$ & -- & - & - & -- & -- & -- & -- \\
\hline 77441 & 1-NAPHTHOL, TOTAL $(\mu \mathrm{g} / \mathrm{L})$ & 1 & $<0.500$ & -- & -- & -- & -- & -- & -- & -- \\
\hline 30282 & METHIOCARB， TOTAL $(\mu \mathrm{g} / \mathrm{L})$ & 1 & $<0.500$ & -- & -- & -- & -- & -- & - & -- \\
\hline
\end{tabular}

NOTE: Multiple detection limits during the period of record may result in different values flagged with a "<."

* Value is estimated by using a log-probability regression to predict the values of data below the detection limit. 
Table 71. Statistical summary of water-quality data at site 39 (CSW05), June 1994 through September 1998

\begin{tabular}{|c|c|c|c|c|c|c|c|c|c|c|}
\hline \multirow{2}{*}{$\begin{array}{c}\text { PARAM- } \\
\text { ETER } \\
\text { CODE }\end{array}$} & \multirow{2}{*}{ PROPERTY OR CONSTITUENT } & \multicolumn{4}{|c|}{ DESCRIPTIVE STATISTICS } & \multicolumn{5}{|c|}{$\begin{array}{c}\text { PERCENT OF SAMPLES IN WHICH VALUES WERE LESS THAN OR } \\
\text { EQUAL TO THOSE SHOWN }\end{array}$} \\
\hline & & $\begin{array}{l}\text { SAMPLE } \\
\text { SIZE }\end{array}$ & MAXIMUM & MINIMUM & MEAN & $95 \%$ & $75 \%$ & $\begin{array}{c}50 \% \\
\text { (median) }\end{array}$ & $25 \%$ & $5 \%$ \\
\hline \multicolumn{11}{|c|}{ PHYSICAL AND CHEMICAL PROPERTIES } \\
\hline 00061 & INSTANTANEOUS DISCHARGE $\left(\mathrm{ft}^{3} / \mathrm{s}\right)$ & 94 & 21.000 & 0.000 & 1.192 & 4.850 & 1.125 & 0.420 & 0.140 & 0.010 \\
\hline 00010 & WATER TEMPERATURE $\left({ }^{\circ} \mathrm{C}\right)$ & 94 & 31.000 & 1.700 & 17.063 & 27.575 & 21.925 & 18.000 & 11.500 & 4.200 \\
\hline 90095 & SPECIFIC CONDUCTANCE, LAB $\left(\mu \mathrm{S} / \mathrm{cm}\right.$ at $\left.25{ }^{\circ} \mathrm{C}\right)$ & 54 & 456.000 & 21.000 & 92.111 & 380.000 & 108.500 & 63.500 & 42.000 & 25.750 \\
\hline 00095 & $\begin{array}{l}\text { SPECIFIC CONDUCTANCE, FIELD ( } \mu \text { S/Cm at } 25 \\
\left.{ }^{\circ} \mathrm{C}\right)\end{array}$ & 73 & 429.000 & 11.000 & 78.479 & 239.400 & 98.500 & 49.000 & 36.500 & 20.700 \\
\hline 00400 & pH, FIELD (STANDARD pH UNITS) & 71 & 7.900 & 5.200 & 6.732 & 7.300 & 6.900 & 6.800 & 6.600 & 5.680 \\
\hline 90410 & ALKALINITY, LAB (mg/L as $\mathrm{CaCO}_{3}$ ) & 57 & 150.000 & 2.000 & 19.216 & 117.300 & 16.500 & 10.000 & 6.250 & 2.950 \\
\hline 80154 & SUSPENDED SEDIMENT $(\mathrm{mg} / \mathrm{L})$ & 60 & 650.000 & 7.000 & 118.550 & 420.600 & 182.500 & 64.500 & 41.000 & 18.050 \\
\hline 00530 & $\begin{array}{l}\text { RESIDUE ON EVAPORATION AT } 105^{\circ} \mathrm{C} \text {, SUSPENDED } \\
(\mathrm{mg} / \mathrm{L})\end{array}$ & 32 & 232.000 & 1.000 & 66.094 & 228.100 & 132.500 & 28.000 & 14.000 & 2.950 \\
\hline 00535 & RESIDUE VOLATILE, SUSPENDED $(\mathrm{mg} / \mathrm{L})$ & 51 & 157.000 & $<1.000$ & $23.695^{*}$ & 64.000 & 44.000 & 10.000 & 4.000 & $<1.000$ \\
\hline 70300 & DISSOLVED SOLIDS, RESIDUE AT $180^{\circ} \mathrm{C}(\mathrm{mg} / \mathrm{L})$ & 57 & 297.000 & 1.000 & 68.982 & 234.300 & 84.000 & 48.000 & 28.500 & 9.200 \\
\hline 00310 & 5 DAY BIOCHEMICAL OXYGEN DEMAND (mg/L) & 44 & 25.000 & $<2.000$ & $8.638^{*}$ & 23.000 & 14.000 & 6.600 & 3.700 & $<2.000$ \\
\hline 00340 & CHEMICAL OXYGEN DEMAND $(\mathrm{mg} / \mathrm{L})$ & 59 & 580.000 & 5.000 & 78.407 & 300.000 & 100.000 & 41.000 & 22.000 & 7.000 \\
\hline \multicolumn{11}{|c|}{ NUTRIENTS, TOTAL AND DISSOLVED } \\
\hline 00631 & $\mathrm{NO}_{2}+\mathrm{NO}_{3}$, DISSOLVED (mg/L as $\mathrm{N}$ ) & 60 & 2.800 & 0.150 & 0.658 & 1.553 & 0.858 & 0.565 & 0.313 & 0.171 \\
\hline 00608 & NITROGEN AMMONIA, DISSOLVED ( $\mathrm{mg} / \mathrm{L}$ as N) & 60 & 2.300 & $<0.0 .15$ & 0.349 * & 1.200 & 0.420 & 0.130 & 0.080 & $<0.015$ \\
\hline 00605 & NITROGEN ORGANIC, TOTAL (mg/L as N) & 60 & 7.500 & 0.000 & 1.486 & 6.110 & 1.900 & 0.865 & 0.545 & 0.311 \\
\hline 00600 & NITROGEN, TOTAL (mg/L as N) & 60 & 11.000 & 0.600 & 2.494 & 8.375 & 3.100 & 1.600 & 1.100 & 0.692 \\
\hline 00665 & PHOSPHORUS, TOTAL (mg/L as P) & 60 & 1.500 & 0.020 & 0.416 & 1.199 & 0.605 & 0.265 & 0.165 & 0.091 \\
\hline 00671 & PHOSPHORUS ORTHO, DISSOLVED (mg/L as P) & 60 & 1.000 & 0.010 & 0.209 & 0.938 & 0.218 & 0.130 & 0.072 & 0.027 \\
\hline \multicolumn{11}{|c|}{ OIL AND GREASE, TOTAL } \\
\hline 00556 & OIL AND GREASE, TOTAL (mg/L) & 17 & 19.000 & $<1.000$ & $5.550^{\star}$ & 19.000 & 8.000 & 4.000 & 3.000 & $<1.000$ \\
\hline \multicolumn{11}{|c|}{ ORGANIC CARBON, TOTAL } \\
\hline 00680 & CARBON ORGANIC, TOTAL $(\mathrm{mg} / \mathrm{L})$ & 37 & 120.000 & 4.200 & 25.924 & 95.700 & 31.000 & 14.000 & 9.500 & 5.190 \\
\hline & & & & IFORM & & & & & & \\
\hline 31679 & FECĀL STREPTOCOCCI (CO1Onles per $100 \mathrm{~mL}$ ) & 30 & $65 \overline{000.000}$ & 72.000 & 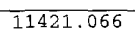 & $50 \overline{150.020}$ & $\overline{15500 . \overline{000}}$ & $5 \overline{800.000}$ & 1900.000 & 279.900 \\
\hline 31616 & FECAL COLIFORM (Colonies per $100 \mathrm{~mL}$ ) & 30 & 310000.000 & 60.000 & 33298.332 & 200000.156 & 37250.000 & 6750.000 & 1072.500 & 82.000 \\
\hline & & ORC & ANIC COMPOI & DS-PESTIC & CIDES, TOTAL & & & & & \\
\hline 39330 & ALDRIN, TOTAL $(\mu \mathrm{g} / \mathrm{L})$ & 5 & $<0.010$ & $<0.010$ & -- & -- & -- & -- & -- & -- \\
\hline 39340 & LINDANE, TOTAL $(\mu \mathrm{g} / \mathrm{L})$ & 5 & $<0.010$ & $<0.010$ & -- & -- & -- & -- & -- & -- \\
\hline 39360 & DDD, TOTAL $(\mu \mathrm{g} / \mathrm{L})$ & 5 & $<0.010$ & $<0.010$ & -- & -- & -- & -- & -- & -- \\
\hline 39380 & DIELDRIN, TOTAL $(\mu \mathrm{g} / \mathrm{L})$ & 5 & $<0.010$ & $<0.010$ & -- & -- & -- & -- & -- & -- \\
\hline 39388 & ENDOSULFAN, TOTAL $(\mu \mathrm{g} / \mathrm{L})$ & 5 & $<0.010$ & $<0.010$ & -- & - & -- & -- & -- & - \\
\hline 39390 & ENDRIN, TOTAL $(\mu \mathrm{g} / \mathrm{L})$ & 5 & $<0.010$ & $<0.010$ & -- & - & -- & -- & -- & -- \\
\hline 39410 & HEPTACHLOR, TOTAL $(\mu \mathrm{g} / \mathrm{L})$ & 5 & $<0.010$ & $<0.010$ & -- & -- & -- & -- & -- & -- \\
\hline 39420 & HEPTACHLOR EPOXIDE, TOTAL $(\mu \mathrm{g} / \mathrm{L})$ & 5 & $<0.010$ & $<0.010$ & -- & -- & -- & -. & - & -- \\
\hline 39516 & $\mathrm{PCB}, \operatorname{TOTAL}(\mu \mathrm{g} / \mathrm{L})$ & 5 & 0.100 & $<0.100$ & -- & -- & -- & -- & -- & -- \\
\hline 39400 & TOXAPHENE, TOTAL $(\mu \mathrm{g} / \mathrm{L})$ & 5 & $<1.000$ & $<1.000$ & $\ldots$ & -- & -- & -- & -- & - \\
\hline 39034 & PERTHANE, TOTAL $(\mu \mathrm{g} / \mathrm{L})$ & 5 & $<0.100$ & $<0.100$ & -- & -- & -- & -- & -- & -- \\
\hline 39570 & DIAZINON, TOTAL $(\mu \mathrm{g} / \mathrm{L})$ & 5 & 0.020 & $<0.010$ & -- & -- & -- & -- & - & - \\
\hline 39398 & ETHION, TOTAL $(\mu \mathrm{g} / \mathrm{L})$ & 5 & $<0.010$ & $<0.010$ & -- & -- & -- & - & - & -- \\
\hline 39530 & MALATHION, TOTAL $(\mu \mathrm{g} / \mathrm{L})$ & 5 & 0.060 & $<0.010$ & -- & -- & -- & -- & -- & -. \\
\hline 39600 & METHYL PARATHION, TOTAL $(\mu \mathrm{g} / \mathrm{L})$ & 5 & 0.010 & $<0.010$ & -- & -- & -- & -- & -- & $-\cdots$ \\
\hline 39540 & PARATHION, TOTAL $(\mu \mathrm{g} / \mathrm{L})$ & 5 & $<0.010$ & $<0.010$ & -- & -- & -- & -- & -- & -- \\
\hline 39786 & TRITHION, TOTAL $(\mu \mathrm{g} / \mathrm{L})$ & 5 & 0.020 & $<0.010$ & -- & - & -- & - & -- & - \\
\hline 39250 & PCN， TOTAL $(\mu \mathrm{g} / \mathrm{L})$ & 5 & $<0.100$ & $<0.100$ & - & -- & -- & -- & -- & - \\
\hline 39480 & METHOXYCHLOR, TOTAL $(\mu \mathrm{g} / \mathrm{L})$ & 5 & 0.020 & $<0.010$ & -- & -- & -- & -- & -- & -- \\
\hline 39755 & MIREX, TOTAL $(\mu \mathrm{g} / \mathrm{L})$ & 5 & $<0.010$ & $<0.010$ & -- & -- & -- & -- & -- & -- \\
\hline 39011 & DISYSTON, TOTAL $(\mu \mathrm{g} / \mathrm{L})$ & 3 & $<0.010$ & $<0.010$ & -- & -- & -- & -- & -- & -- \\
\hline 39023 & PHORATE, TOTAL $(\mu \mathrm{g} / \mathrm{L})$ & 5 & $<0.010$ & $<0.010$ & - & -- & -- & -- & -- & -- \\
\hline 38932 & CHLORPYRIFOS, TOTAL $(\mu g / L)$ & 5 & 0.010 & $<0.010$ & -- & - & -- & -- & -- & - \\
\hline 39040 & DEF, TOTAL $(\mu \mathrm{g} / \mathrm{L})$ & 5 & $<0.010$ & $<0.010$ & -- & -- & -- & -- & -- & -- \\
\hline 82614 & FONOFOS, TOTAL $(\mu \mathrm{g} / \mathrm{L})$ & 5 & $<0.010$ & $<0.010$ & -- & - & -- & -- & - & -- \\
\hline & & & LATILE ORGA & C COMPOU & NDS, TOTAL & & & & & \\
\hline 34210 & ACROLEIN, TOTAL $\overline{(\mu \mathrm{g} / \mathrm{L})}$ & 1 & $<20.000$ & - & -- & -- & -- & -- & -- & -- \\
\hline 34215 & ACRYLONITRILE, TOTAL $(\mu \mathrm{g} / \mathrm{L})$ & 2 & $<20.000$ & $<5.000$ & -- & - & -- & -- & -- & - \\
\hline 34030 & BENZENE, TOTAL $(\mu \mathrm{g} / \mathrm{L})$ & 11 & $<2.000$ & $<0.200$ & -- & -- & -- & -- & - & -- \\
\hline 32104 & BROMOFORM, TOTAL $(\mu \mathrm{g} / \mathrm{L})$ & 11 & $<2.000$ & $<0.200$ & -- & -- & -- & -- & - & -- \\
\hline 32102 & CARBON TETRACHLORIDE, TOTAL $(\mu \mathrm{g} / \mathrm{L})$ & 11 & $<2.000$ & $<0.200$ & -- & -- & -- & -- & -- & - \\
\hline 34301 & CHLOROBENZENE, TOTAL $(\mu \mathrm{g} / \mathrm{L})$ & 11 & $<2.000$ & $<0.200$ & -- & - & -- & -- & -- & -- \\
\hline 32105 & CHLORODIBROMOMETHANE, TOTAL $(\mu \mathrm{g} / \mathrm{L})$ & $1 I$ & $<2.000$ & $<0.200$ & -- & - & -- & -- & -- & - \\
\hline 34311 & CHLOROETHANE, TOTAL $(\mu \mathrm{g} / \mathrm{L})$ & 11 & $<2.000$ & $<0.200$ & -- & -- & -- & -. & - & -- \\
\hline 32106 & CHLOROFORM， TOTAL $(\mu \mathrm{g} / \mathrm{L})$ & 11 & $<2.000$ & $<0.200$ & -- & -- & -- & -. & -- & -- \\
\hline 34496 & 1,1 -DICHLOROETHANE, TOTAL $(\mu \mathrm{g} / \mathrm{L})$ & 11 & $<2.000$ & $<0.200$ & -- & -- & - & -- & -- & -- \\
\hline 32103 & 1,2 -DICHLOROETHANE, TOTAL $(\mu \mathrm{g} / \mathrm{L})$ & 11 & $<2.000$ & $<0.200$ & -- & -- & -- & -- & -- & -- \\
\hline
\end{tabular}

NOTE: Multiple detection Jimits during the period of record may result in different values flagged with a "<."

* Value is estimated by using a log-probability regression to predict the values of data below the detection limit. 
Table 71. Statistical summary of water-quality data at site 39 (CSW05), June 1994 through September 1998-Continued

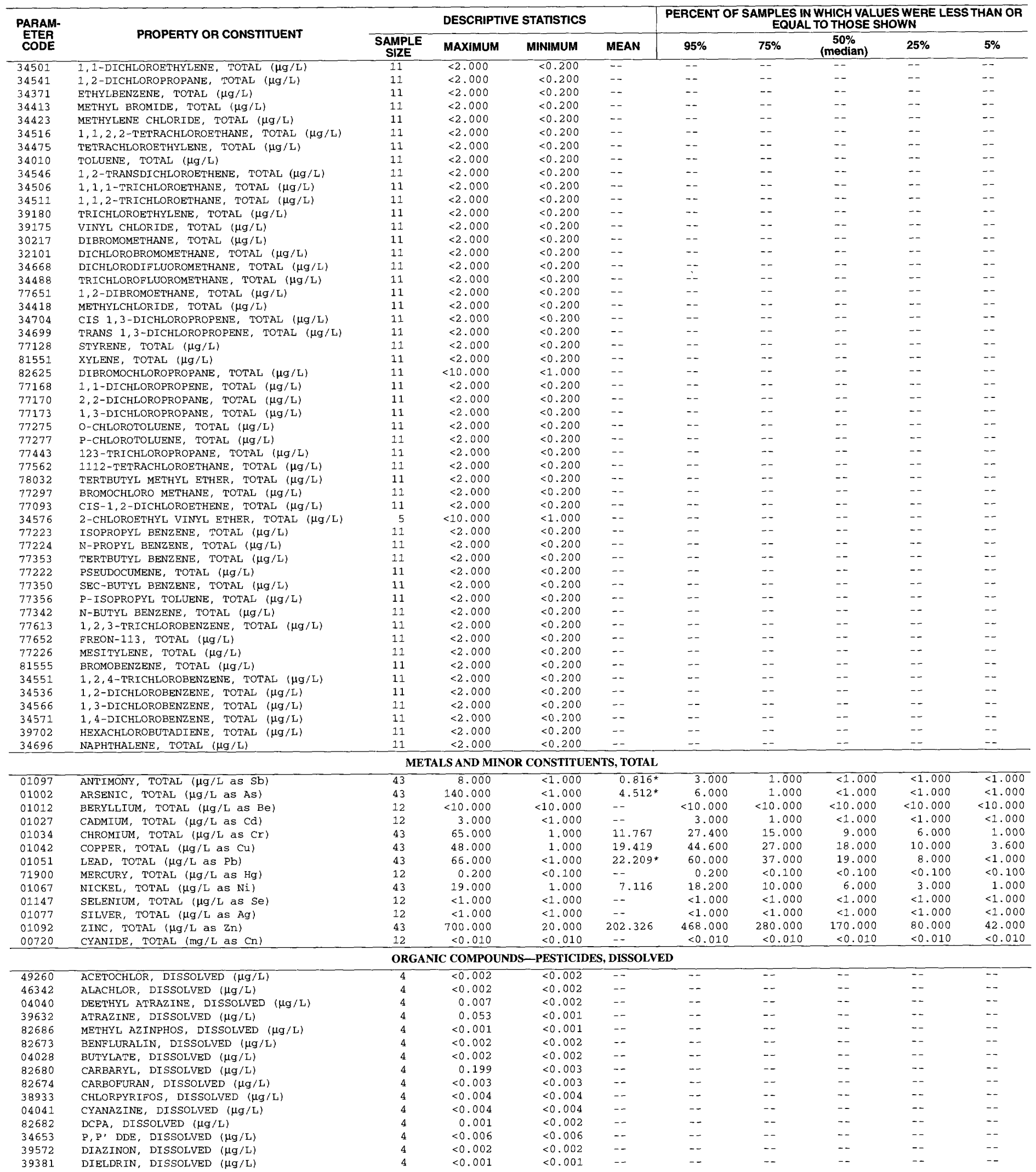

NOTE: Multiple detection limits during the period of record may result in different values flagged with a "<."

* Value is estimated by using a log-probability regression to predict the values of data below the detection limit. 
Table 71. Statistical summary of water-quality data at site 39 (CSW05), June 1994 through September 1998-Continued

\begin{tabular}{|c|c|c|c|c|c|c|c|c|c|c|}
\hline \multirow{2}{*}{$\begin{array}{c}\text { PARAM- } \\
\text { ETER } \\
\text { CODE }\end{array}$} & \multirow{2}{*}{ PROPERTY OR CONSTITUENT } & & DESCRIPTI & STATISTICS & & PERCEN & $\begin{array}{l}\text { AMPLE } \\
\text { EC }\end{array}$ & $\begin{array}{l}\text { WHICH VAL } \\
\text { TO THOSE }\end{array}$ & WERE & HAN OA \\
\hline & & $\begin{array}{l}\text { SAMPLE } \\
\text { SIZE }\end{array}$ & MAXIMUM & MINIMUM & MEAN & $95 \%$ & $75 \%$ & $\begin{array}{c}50 \% \\
\text { (medlan) }\end{array}$ & $25 \%$ & $5 \%$ \\
\hline $826 \overline{60}$ & 2,6 -DIETHYL ANILINE, DISSOLVED $\overline{(\mu \mathrm{g} / \bar{L})}$ & 4 & $<0.003$ & $<0.003$ & -- & -- & -- & -- & -- & -- \\
\hline 82677 & DISULFOTON，DISSOLVED $(\mu \mathrm{g} / \mathrm{L})$ & 4 & $<0.017$ & $<0.017$ & - & -- & -- & -- & -- & -- \\
\hline 82568 & EPTC, DISSOLVED $(\mu \mathrm{g} / \mathrm{L})$ & 4 & $<0.002$ & $<0.002$ & -- & -- & -- & -- & -- & -- \\
\hline 82563 & ETHALFLURALIN，DISSOLVED $(\mu \mathrm{g} / \mathrm{L})$ & 4 & $<0.004$ & $<0.004$ & -- & -- & - & -- & -- & -- \\
\hline 82672 & ETHOPROP, DISSOLVED $(\mu \mathrm{g} / \mathrm{L})$ & 4 & $<0.003$ & $<0.003$ & -- & -- & - & -- & -- & -- \\
\hline 04095 & FONOFOS, DISSOLVED $(\mu \mathrm{g} / \mathrm{L})$ & 4 & $<0.003$ & $<0.003$ & -- & -- & - & -- & -- & -- \\
\hline 34253 & ALPHA BHC, DISSOLVED $(\mu \mathrm{g} / L)$ & 4 & $<0.002$ & $<0.002$ & $\ldots$ & -- & -- & -- & $\rightarrow$ & -- \\
\hline 39341 & LINDANE， DISSOLVED $(\mu \mathrm{g} / \mathrm{L})$ & 4 & $<0.004$ & $<0.004$ & -- & -- & - & -. & -- & -- \\
\hline 82666 & LINURON， DISSOLVED $(\mu \mathrm{g} / \mathrm{L})$ & 4 & $<0.002$ & $<0.002$ & -- & -- & - & -- & -- & -- \\
\hline 39532 & MALATHION, DISSOLVED $(\mu \mathrm{g} / \mathrm{L})$ & 4 & 0.132 & $<0.005$ & -- & -- & $\ldots$ & -- & -- & -- \\
\hline 82667 & METHYL PARATHION, DISSOLVED $(\mu \mathrm{g} / \mathrm{L})$ & 4 & $<0.006$ & $<0.006$ & - & -- & - & $-\rightarrow$ & -- & -- \\
\hline 39415 & METOLACHLOR, DISSOLVED $(\mu \mathrm{g} / \mathrm{L})$ & 3 & 0.039 & $<0.002$ & -- & -- & -- & -- & -- & -- \\
\hline 82630 & METRIBUZIN， DISSOLVED $(\mu \mathrm{g} / L)$ & 4 & $<0.004$ & $<0.004$ & -- & -- & - & -- & -- & -- \\
\hline 82671 & MOLINATE, DISSOLVED $(\mu \mathrm{g} / \mathrm{L})$ & 4 & $<0.004$ & $<0.004$ & -- & -- & -- & -. & -. & -- \\
\hline 82684 & NAPROPAMIDE, DISSOLVED $(\mu \mathrm{g} / L)$ & 4 & $<0.003$ & $<0.003$ & -- & -- & -- & -- & -- & -- \\
\hline 39542 & PARATHION， DISSOLVED $(\mu \mathrm{g} / L)$ & 4 & $<0.004$ & $<0.004$ & -- & -- & -- & -- & -- & -- \\
\hline 82669 & PEBULATE, DISSOLVED $(\mu \mathrm{g} / \mathrm{L})$ & 4 & $<0.004$ & $<0.004$ & -- & -- & -- & -- & -- & -- \\
\hline 82683 & PENDIMETHALIN， DISSOLVED $(\mu \mathrm{g} / \mathrm{L})$ & 4 & $<0.004$ & $<0.004$ & -- & -- & - & -- & -- & -- \\
\hline 82687 & PERMETHRIN， DISSOLVED $(\mu \mathrm{g} / \mathrm{L})$ & 4 & $<0.005$ & $<0.005$ & -- & -- & - & - & -- & -- \\
\hline 82664 & PHORATE, DISSOLVED $(\mu \mathrm{g} / \mathrm{L})$ & 4 & $<0.002$ & $<0.002$ & -- & - & -- & -- & -- & -- \\
\hline 82676 & PRONAMIDE, DISSOLVED $(\mu \mathrm{g} / \mathrm{L})$ & 4 & $<0.003$ & $<0.003$ & -- & -- & -- & -- & -- & -- \\
\hline 04037 & PROMETON， DISSOLVED $(\mu \mathrm{g} / \mathrm{L})$ & 4 & 0.053 & $<0.018$ & -- & -- & -- & - & -- & -- \\
\hline 04024 & PROPACHLOR， DISSOLVED $(\mu \mathrm{g} / \mathrm{L})$ & 4 & $<0.007$ & $<0.007$ & -- & -- & -- & $-\infty$ & -- & -- \\
\hline 82679 & PROPANIL, DISSOLVED $(\mu \mathrm{g} / \mathrm{L})$ & 4 & $<0.004$ & $<0.004$ & -- & -- & -- & $-\infty$ & -- & -- \\
\hline 82685 & PROPARGITE，DISSOLVED $(\mu \mathrm{g} / \mathrm{L})$ & 4 & $<0.013$ & $<0.013$ & -- & -. & -- & -- & -- & -- \\
\hline 04035 & SIMAZINE, DISSOLVED $(\mu \mathrm{g} / \mathrm{L})$ & 4 & $<0.005$ & $<0.005$ & -- & -- & -- & $\cdots$ & -- & -- \\
\hline 82681 & THIOBENCARB， DISSOLVED $(\mu \mathrm{g} / L)$ & 4 & $<0.002$ & $<0.002$ & -- & -- & -- & -- & -- & -- \\
\hline 82670 & TEBUTHIURON， DISSOLVED ( $\mu \mathrm{g} / \mathrm{L})$ & 4 & $<0.010$ & $<0.010$ & -- & -. & -- & -- & -- & -- \\
\hline 82665 & TERBACIL，DISSOLVED $(\mu \mathrm{g} / \mathrm{L})$ & 4 & 0.019 & $<0.007$ & -- & -- & -- & -- & -- & -- \\
\hline 82675 & TERBUFOS, DISSOLVED $(\mu \mathrm{g} / \mathrm{L})$ & 4 & $<0.013$ & $<0.013$ & -- & -- & -- & -- & -- & -- \\
\hline 82678 & TRIALLATE, DISSOLVED $(\mu \mathrm{g} / \mathrm{L})$ & 4 & $<0.001$ & $<0.001$ & -- & -- & -- & - & -- & -- \\
\hline 82661 & TRIFLURALIN， DISSOLVED $(\mu \mathrm{g} / \mathrm{L})$ & 4 & $<0.002$ & $<0.002$ & -- & -- & -- & -- & -- & -- \\
\hline 39742 & $2,4,5-T$, DISSOLVED $(\mu \mathrm{g} / \mathrm{L})$ & 4 & $<0.035$ & $<0.035$ & -- & -- & -- & -- & -- & -- \\
\hline 39732 & $2,4-\mathrm{D}$, DISSOLVED $(\mu \mathrm{g} / \mathrm{L})$ & 4 & $<0.150$ & $<0.035$ & -- & -- & -- & - & -- & -- \\
\hline 38746 & $2,4-\mathrm{DB}, \quad$ DISSOLVED $(\mu \mathrm{g} / \mathrm{L})$ & 4 & $<0.240$ & $<0.035$ & -- & -- & -- & -. & -- & - - \\
\hline 49315 & ACIFLUORFEN， DISSOLVED $(\mu \mathrm{g} / L)$ & 4 & $<0.035$ & $<0.035$ & -- & -- & -- & -- & -- & -- \\
\hline 49312 & ALDICARB, DISSOLVED $(\mu \mathrm{g} / \mathrm{L})$ & 4 & $<0.550$ & $<0.016$ & -- & -- & -- & -- & -- & -- \\
\hline 49313 & ALDICARB SULFONE, DISSOLVED $\left(\mu_{\mathrm{g}} / \mathrm{L}\right)$ & 4 & $<0.100$ & $<0.016$ & -- & -- & -- & -- & -- & -- \\
\hline 49314 & ALDICARB SULFOXIDE, DISSOLVED $(\mu \mathrm{g} / \mathrm{L})$ & 4 & $<0.021$ & $<0.021$ & -- & -- & -- & -- & -- & - - \\
\hline 38711 & BENTAZON, DISSOLVED $(\mu \mathrm{g} / \mathrm{L})$ & 4 & $<0.014$ & $<0.014$ & -- & -- & -- & -- & -- & -- \\
\hline 04029 & BROMACIL，DISSOLVED $(\mu \mathrm{g} / \mathrm{L})$ & 4 & $<0.035$ & $<0.035$ & -- & -- & -- & $-\ldots$ & -- & - \\
\hline 49311 & BROMOXYNIL, DISSOLVED $(\mu \mathrm{g} / \mathrm{L})$ & 4 & $<0.035$ & $<0.035$ & -- & -- & -- & -- & -- & -- \\
\hline 49310 & CARBARYL， DISSOLVED $(\mu \mathrm{g} / \mathrm{L})$ & 4 & $<0.008$ & $<0.008$ & -- & -- & -- & -- & -- & -- \\
\hline 49309 & CARBOFURAN， DISSOLVED $(\mu \mathrm{g} / \mathrm{L})$ & 4 & $<0.120$ & $<0.028$ & -- & -- & .- & -- & -- & -- \\
\hline 49308 & 3 -HYDROXY-CARBOFURAN $(\mu \mathrm{g} / \mathrm{L})$ & 4 & $<0.014$ & $<0.014$ & -- & - & -- & -- & -- & -- \\
\hline 49307 & AMIBEN, DISSOLVED $(\mu \mathrm{g} / \mathrm{L})$ & 4 & $<0.420$ & $<0.011$ & -- & -- & -- & -- & -- & -- \\
\hline 49306 & CHLOROTHALONIL, DISSOLVED $(\mu \mathrm{g} / \mathrm{L})$ & 4 & $<0.480$ & $<0.035$ & -- & - - & -- & - - & -- & - - \\
\hline 49305 & CLOPYRALID, DISSOLVED $(\mu \mathrm{g} / \mathrm{L})$ & 4 & $<0.230$ & $<0.050$ & -. & -- & -- & -. & -- & -- \\
\hline 49304 & DACTHALMONO-ACID, DISSOLVED $(\mu \mathrm{g} / \mathrm{L})$ & 4 & $<0.017$ & $<0.017$ & -- & -- & -- & -- & -- & -- \\
\hline 38442 & DICAMBA，DISSOLVED $(\mu \mathrm{g} / \mathrm{L})$ & 4 & $<0.035$ & $<0.035$ & -- & -- & -- & -- & -- & -- \\
\hline 49303 & DICHLOBENIL, DISSOLVED $(\mu \mathrm{g} / \mathrm{L})$ & 4 & $<1.200$ & $<0.020$ & -- & -- & -- & -- & -- & -- \\
\hline 49302 & DICHLORPROP, DISSOLVED $(\mu \mathrm{g} / \mathrm{L})$ & 4 & $<0.032$ & $<0.032$ & -- & -- & -- & -- & -- & -- \\
\hline 49301 & DINOSEB, DISSOLVED $(\mu \mathrm{g} / \mathrm{L})$ & 4 & $<0.035$ & $<0.035$ & -- & $\ldots$ & -- & -- & -- & -- \\
\hline 49300 & DIURON, DISSOLVED $(\mu \mathrm{g} / \mathrm{L})$ & 4 & $<0.020$ & $<0.020$ & -- & -- & -- & - - & -- & -. \\
\hline 49299 & 4, 6-DINITRO OCRESOL，DISSOLVED $(\mu \mathrm{g} / \mathrm{L})$ & 4 & $<0.420$ & $<0.035$ & -- & -- & -- & -- & -- & -- \\
\hline 49298 & ESFENVALERATE， DISSOLVED $(\mu \mathrm{g} / \mathrm{L})$ & 3 & $<0.019$ & $<0.019$ & -- & -- & -- & -- & -- & -- \\
\hline 49297 & FENURON, DISSOLVED $(\mu \mathrm{g} / \mathrm{L})$ & 4 & $<0.013$ & $<0.013$ & -- & -- & -- & -- & -- & -- \\
\hline 38811 & FLUOMETURON, DISSOLVED $(\mu \mathrm{g} / \mathrm{L})$ & 4 & $<0.035$ & $<0.035$ & - & - & - & -- & -- & -- \\
\hline 38478 & LINURON, DISSOLVED $(\mu \mathrm{g} / \mathrm{L})$ & 4 & $<0.018$ & $<0.018$ & - & -- & -- & -- & -- & -- \\
\hline 38482 & MCPA, DISSOLVED $(\mu \mathrm{g} / \mathrm{L})$ & 4 & $<0.170$ & $<0.050$ & -- & -- & -- & -- & -- & -- \\
\hline 38487 & MCPB, DISSOLVED $(\mu \mathrm{g} / \mathrm{L})$ & 4 & $<0.140$ & $<0.035$ & -- & -- & -- & -- & -- & -- \\
\hline 38501 & METHIOCARB, DISSOLVED $(\mu \mathrm{g} / \mathrm{L})$ & 4 & $<0.026$ & $<0.026$ & -- & -- & -- & -- & -- & -- \\
\hline 49296 & METHOMYL, DISSOLVED $(\mu \mathrm{g} / \mathrm{L})$ & 4 & $<0.017$ & $<0.017$ & -- & $\ldots$ & -- & - & -- & -- \\
\hline 49295 & 1 -NAPHTHOL, DISSOLVED $(\mu \mathrm{g} / \mathrm{L})$ & 3 & $<0.007$ & $<0.007$ & -- & -- & -- & -- & -- & -- \\
\hline 49294 & NEBURON， DISSOLVED $(\mu \mathrm{g} / \mathrm{L})$ & 4 & $<0.015$ & $<0.015$ & -- & - & -- & -- & -- & -- \\
\hline 49293 & NORFLURAZON， DISSOLVED $(\mu \mathrm{g} / \mathrm{L})$ & 4 & $<0.024$ & $<0.024$ & -- & -- & -- & -- & -- & -- \\
\hline 49292 & ORYZALIN, DISSOLVED $(\mu \mathrm{g} / L)$ & 4 & $<0.310$ & $<0.019$ & -- & -- & -- & -- & -- & -- \\
\hline 38866 & OXAMYL，DISSOLVED $(\mu \mathrm{g} / \mathrm{L})$ & 4 & $<0.018$ & $<0.018$ & -- & -- & -- & -- & -- & $\rightarrow$ \\
\hline 49291 & PICLORAM, DISSOLVED $(\mu \mathrm{g} / \mathrm{L})$ & 4 & $<0.050$ & $<0.050$ & -- & -- & -- & -- & -- & -- \\
\hline 49236 & PROPHAM, DISSOLVED $(\mu \mathrm{g} / \mathrm{L})$ & 4 & $<0.035$ & $<0.035$ & -- & -- & -- & -- & -- & -- \\
\hline 38538 & PROPOXUR, DISSOLVED $(\mu \mathrm{g} / \mathrm{L})$ & 4 & $<0.035$ & $<0.035$ & -- & -- & -- & -. & -- & -- \\
\hline 39762 & SILVEX, DISSOLVED $(\mu \mathrm{g} / \mathrm{L})$ & 4 & $<0.021$ & $<0.021$ & -- & -- & -- & -- & -- & -- \\
\hline 49235 & TRICLOPYR, DISSOLVED $(\mu \mathrm{g} / \mathrm{L})$ & 4 & $<0.250$ & $<0.050$ & $\ldots$ & -- & -- & -- & -- & - \\
\hline & & ORGANIC & COMPOUND & -ORGANONI & ROGEN, T & TAL & & & & \\
\hline 39057 & PROMETRYNE, TOTAL $(\mu \mathrm{g} / \mathrm{L})$ & 1 & $<0.100$ & -- & -- & -- & $\cdots$ & $\overline{--}$ & $\cdots$ & -- \\
\hline 39056 & PROMETONE， TOTAL $(\mu \mathrm{g} / L)$ & 1 & $<0.200$ & -- & -- & -- & -- & -- & -- & -- \\
\hline 39054 & SIMETRYNE, TOTAL $(\mu \mathrm{g} / \mathrm{L})$ & 1 & $<0.100$ & -- & - & -- & -- & -- & -- & -- \\
\hline 81757 & CYANAZINE, TOTAL $(\mu \mathrm{g} / \mathrm{L})$ & 1 & $<0.200$ & -- & -- & -- & - & -- & -- & -- \\
\hline 77825 & ALACHLOR，TOTAL $(\mu \mathrm{g} / \mathrm{L})$ & 1 & $<0.100$ & -- & -- & -- & -- & -- & -- & -- \\
\hline 82611 & METRIBUZIN, TOTAL $(\mu \mathrm{g} / \mathrm{L})$ & 1 & 0.300 & -- & -- & -- & -- & - & -- & -- \\
\hline 30311 & TERBACIL, TOTAL $(\mu \mathrm{g} / \mathrm{L})$ & 1 & $<0.200$ & -. & -- & -- & -- & -- & -. & -. \\
\hline 30245 & CARBOXIN, TOTAL $(\mu \mathrm{g} / \mathrm{L})$ & 1 & $<0.200$ & -- & $-\infty$ & -- & +- & -- & - & -- \\
\hline
\end{tabular}

NOTE: Multiple detection limits during the period of record may result in different values flagged with a "<."

* Value is estimated by using a log-probability regression to predict the values of data below the detection limit. 
Table 71. Statistical summary of water-quality data at site 39 (CSW05), June 1994 through September 1998-Continued

\begin{tabular}{|c|c|c|c|c|c|c|c|c|c|c|}
\hline \multirow{2}{*}{$\begin{array}{l}\text { PARAM- } \\
\text { ETER } \\
\text { CODE }\end{array}$} & \multirow{2}{*}{ PROPERTY OR CONSTITUENT } & \multicolumn{4}{|c|}{ DESCRIPTIVE STATISTICS } & \multicolumn{5}{|c|}{$\begin{array}{c}\text { PERCENT OF SAMPLES IN WHICH VALUES WERE LESS THAN OR } \\
\text { EQUAL TOTHOSE SHIOWN }\end{array}$} \\
\hline & & $\begin{array}{l}\text { SAMPLE } \\
\text { SIZE }\end{array}$ & MAXIMUM & MINIMUM & MEAN & $95 \%$ & $75 \%$ & $\begin{array}{c}50 \% \\
\text { (medlan) }\end{array}$ & $25 \%$ & $5 \%$ \\
\hline 30264 & HEXAZINONE, TOTAL $(\mu \mathrm{g} / \mathrm{L})$ & 1 & $<0.200$ & -- & -- & -- & -- & -- & $-\overline{-}$ & -- \\
\hline 30235 & BUTACHLOR, TOTAL $(\mu \mathrm{g} / \mathrm{L})$ & 1 & $<0.100$ & -- & -- & -- & -- & -- & -- & -- \\
\hline 30236 & BUTYLATE, TOTAL $(\mu \mathrm{g} / \mathrm{L})$ & 1 & $<0.100$ & -- & -- & - & -- & -- & -- & -- \\
\hline 75981 & DE-ETHYLATRAZINE, TOTAL $(\mu \mathrm{g} / \mathrm{L})$ & 1 & $<0.200$ & -- & -- & -- & -- & -- & -- & -- \\
\hline 39630 & ATRAZINE, TOTAL $(\mu \mathrm{g} / \mathrm{L})$ & 1 & 0.200 & -- & -- & -. & -- & -- & -- & -. \\
\hline 39055 & SIMAZINE, TOTAL $(\mu \mathrm{g} / \mathrm{L})$ & 1 & $<0.100$ & -- & -- & -- & -- & -- & -- & -- \\
\hline 39024 & PROPAZINE， TOTAL $(\mu \mathrm{g} / \mathrm{L})$ & 1 & $<0.100$ & -- & -- & -. & -- & -- & -- & -- \\
\hline 82184 & AMETRYNE, TOTAL $(\mu \mathrm{g} / \mathrm{L})$ & 1 & $<0.100$ & -- & -- & -- & -. & -- & -- & -- \\
\hline 39030 & TRIFLURALIN, TOTAL $(\mu \mathrm{g} / \mathrm{L})$ & 1 & $<0.100$ & -- & -- & -- & -- & -- & -- & -- \\
\hline 82612 & METOLACHLOR, TOTAL $(\mu \mathrm{g} / \mathrm{L})$ & 1 & $<0.200$ & -- & -- & -- & -- & -- & -- & -- \\
\hline 30234 & BROMACIL，TOTAL $(\mu \mathrm{g} / \mathrm{L})$ & 1 & $<0.200$ & -- & -- & -- & -- & -- & -- & -- \\
\hline 30255 & DIPHENAMID, TOTAL $(\mu \mathrm{g} / \mathrm{L})$ & 1 & $<0.100$ & -. & -- & -- & -. & -- & -- & -- \\
\hline 30324 & VERNOLATE, TOTAL $(\mu \mathrm{g} / \mathrm{L})$ & 1 & $<0.100$ & -- & -- & -- & -- & -- & -- & -- \\
\hline 30254 & CYCLOATE, TOTAL $(\mu \mathrm{g} / \mathrm{L})$ & 1 & $<0.100$ & -- & -- & -- & -- & -- & -- & -- \\
\hline 30295 & PROPACHLOR, TOTAL $(\mu \mathrm{g} / \mathrm{L})$ & 1 & $<0.100$ & -- & -- & -- & $\cdots$ & -- & -- & -- \\
\hline 75980 & DE-ISOPROPYLATRAZIN，TOTAL $(\mu \mathrm{g} / \mathrm{L})$ & 1 & $<0.200$ & -- & -- & -- & -- & -- & -- & -- \\
\hline \multicolumn{11}{|c|}{ ORGANIC COMPOUNDS-HERBICIDES, TOTAL } \\
\hline 39730 & $2,4-\mathrm{D}$, TOTAL $(\mu \mathrm{g} / \mathrm{L})$ & 1 & $<0.010$ & -- & -- & -- & -- & -- & $\ldots$ & -- \\
\hline 39760 & SILVEX, TOTAL $(\mu \mathrm{g} / \mathrm{L})$ & 1 & $<0.010$ & -- & -- & -- & -- & -- & -- & -- \\
\hline 39720 & PICLORAM，TOTAL $(\mu \mathrm{g} / \mathrm{L})$ & 1 & $<0.010$ & -- & -- & -- & -- & -- & -- & -- \\
\hline 39740 & $2,4,5-\mathrm{T}$, TOTAL $(\mu \mathrm{g} / \mathrm{L})$ & 1 & $<0.010$ & -- & -- & -- & -- & -- & -. & -- \\
\hline 82183 & $2,4-\mathrm{DP}$, TOTAL $(\mu \mathrm{g} / \mathrm{L})$ & 1 & $<0.010$ & -- & -- & -- & -- & -- & -- & -- \\
\hline 82052 & DICAMBA， TOTAL $(\mu \mathrm{g} / \mathrm{L})$ & 1 & $<0.010$ & -- & -- & -- & -- & -- & $=$ & -- \\
\hline \multicolumn{11}{|c|}{ ORGANIC COMPOUNDS-CARBAMATE PESTICIDES, TOTAL } \\
\hline 39750 & SEVIN, TOTAL $(\mu \bar{g} / \mathrm{L})$ & 1 & $<0.500$ & -- & -- & -- & $=-$ & -- & -- & -- \\
\hline 39051 & METHOMYL，TOTAL $(\mu \mathrm{g} / \mathrm{L})$ & 1 & $<0.500$ & -- & -- & -- & -- & -- & -- & -- \\
\hline 82619 & ALDICARD, TOTAL $(\mu \mathrm{g} / \mathrm{L})$ & 1 & $<0.500$ & -- & -- & -- & -- & -- & -- & -- \\
\hline 30296 & PROPOXUR, TOTAL $(\mu \mathrm{g} / \mathrm{L})$ & 1 & $<0.500$ & -- & -- & -- & -- & -- & -- & -- \\
\hline 39052 & PROPHAM, TOTAL $(\mu \mathrm{g} / \mathrm{L})$ & 1 & $<0.500$ & -- & -- & -- & -- & -- & -- & -- \\
\hline 82615 & CARBOFURAN，TOTAL $(\mu \mathrm{g} / \mathrm{L})$ & 1 & $<0.500$ & -- & -- & -- & -- & -- & -- & -- \\
\hline 77441 & 1 -NAPHTHOL，TOTAL $(\mu \mathrm{g} / \mathrm{L})$ & 1 & $<0.500$ & -- & -- & -- & -- & -- & -- & -- \\
\hline 30282 & METHIOCARB，TOTAL $(\mu \mathrm{g} / \mathrm{L})$ & 1 & $<0.500$ & -. & -- & -- & -- & -- & -- & -- \\
\hline
\end{tabular}

NOTE: Multiple detection limits during the period of record may result in different values flagged with a "<."

* Value is estimated by using a log-probability regression to predict the values of data below the detection limit. 
Table 72. Statistical summary of water-quality data at site 40 (CSW03), July 1994 through September 1998

\begin{tabular}{|c|c|c|c|c|c|c|c|c|c|c|}
\hline \multirow{2}{*}{$\begin{array}{l}\text { PARAM- } \\
\text { ETER } \\
\text { CODE }\end{array}$} & \multirow{2}{*}{ PROPERTY OR CONSTITUENT } & \multicolumn{4}{|c|}{ DESCRIPTIVE STATISTICS } & \multicolumn{5}{|c|}{$\begin{array}{l}\text { PERCENT OF SAMPLES IN WHICH VALUES WERE LESS THAN OR } \\
\text { EQUAL TO THOSE SHOWN }\end{array}$} \\
\hline & & $\begin{array}{l}\text { SAMPLE } \\
\text { SIZE }\end{array}$ & MAXIMUM & MINIMUM & MEAN & $95 \%$ & $75 \%$ & $\begin{array}{c}50 \% \\
\text { (medlan) }\end{array}$ & $25 \%$ & $5 \%$ \\
\hline \multicolumn{11}{|c|}{ PHYSICAL AND CHEMICAL PROPERTIES } \\
\hline 00061 & INSTANTANEOUS DISCHARGE $\left(\mathrm{ft}^{3} / \mathrm{s}\right)$ & 79 & 21.000 & 0.000 & 0.703 & 2.600 & 0.340 & 0.110 & 0.030 & 0.010 \\
\hline 00010 & WATER TEMPERATURE $\left({ }^{\circ} \mathrm{C}\right)$ & 79 & 28.000 & 2.000 & 15.196 & 26.000 & 21.500 & 15.500 & 8.500 & 3.300 \\
\hline 90095 & SPECIFIC CONDUCTANCE, LAB ( $\mu \mathrm{S} / \mathrm{cm}$ at $25^{\circ} \mathrm{C}$ ) & 52 & 309.000 & 19.000 & 67.231 & 180.300 & 69.750 & 53.500 & 40.000 & 27.300 \\
\hline 00095 & $\begin{array}{l}\text { SPECIFIC CONDUCTANCE, FIELD }(\mu \mathrm{S} / \mathrm{cm} \text { at } 25 \\
\left.{ }^{\circ} \mathrm{C}\right)\end{array}$ & 67 & 317.000 & 13.000 & 63.985 & 211.200 & 64.000 & 47.000 & 37.000 & 24.000 \\
\hline 00403 & pH, LAB (STANDARD pH UNITS) & 52 & 7.600 & 5.900 & 6.681 & 7.470 & 7.000 & 6.750 & 6.225 & 6.000 \\
\hline 00400 & pH，FIELD (STANDARD pH UNITS) & 67 & 7.500 & 6.100 & 6.763 & 7.300 & 7.100 & 6.700 & 6.500 & 6.200 \\
\hline 90410 & ALKALINITY, LAB (mg/L as $\left.\mathrm{CaCO}_{3}\right)$ & 52 & 120.000 & 1.000 & 16.090 & 60.450 & 17.750 & 11.000 & 6.225 & 2.520 \\
\hline 80154 & SUSPENDED SEDIMENT $(\mathrm{mg} / \mathrm{L})$ & 52 & 5530.000 & 14.000 & 381.615 & 1888.999 & 312.000 & 97.000 & 46.750 & 17.300 \\
\hline 00530 & $\begin{array}{l}\text { RESIDUE ON EVAPORATION AT } 105^{\circ} \mathrm{C} \text {, SUSPENDED } \\
(\mathrm{mg} / \mathrm{L})\end{array}$ & 30 & 1620.000 & 8.000 & 134.300 & 928.101 & 154.000 & 45.500 & 22.000 & 8.550 \\
\hline 00535 & RESIDUE VOLATILE， SUSPENDED (mg/L) & 49 & 586.000 & 1.000 & 55.633 & 327.000 & 57.500 & 22.000 & 9.500 & 3.000 \\
\hline 70300 & $\begin{array}{l}\text { DISSOLVED SOLIDS, RESIDUE AT } 180^{\circ} \mathrm{C} \text {, } \\
\text { SUSPENDED }(\mathrm{mg} / \mathrm{L})\end{array}$ & 52 & 192.000 & 11.000 & 52.673 & 133.100 & 61.250 & 47.000 & 26.000 & 14.000 \\
\hline 00310 & 5 DAY BIOCHEMICAL OXYGEN DEMAND (mg/L) & 39 & 43.000 & 1.400 & 10.464 & 37.000 & 10.000 & 7.100 & 5.900 & 2.000 \\
\hline 00340 & CHEMICAL OXYGEN DEMAND (mg/L) & 52 & 480.000 & 18.000 & 76.346 & 215.000 & 96.750 & 53.000 & 30.000 & 20.650 \\
\hline \multicolumn{11}{|c|}{ NUTRIENTS, TOTAL AND DISSOLVED } \\
\hline 00625 & $\begin{array}{l}\text { NITROGEN AMMONIA + ORGANIC, } \\
\text { TOTAL (mg/L as N) }\end{array}$ & 54 & 6.600 & 0.360 & 1.802 & 4.725 & $2 . \overline{125}$ & 1.450 & 1.000 & 0.565 \\
\hline 00631 & $\mathrm{NO}_{2}+\mathrm{NO}_{3}$, DISSOLVED $(\mathrm{mg} / \mathrm{L}$ as $\mathrm{N})$ & 54 & 2.700 & 0.150 & 0.730 & 2.263 & 0.850 & 0.540 & 0.393 & 0.175 \\
\hline 00608 & NITROGEN AMMONIA, DISSOLVED (mg/L as N) & 54 & 4.500 & 0.015 & 0.425 & 1.825 & 0.452 & 0.200 & 0.078 & 0.017 \\
\hline 00605 & NITROGEN ORGANIC, TOTAL (mg/L as N) & 54 & 3.800 & 0.280 & 1.372 & 3.200 & 1.750 & 1.100 & 0.875 & 0.410 \\
\hline 00600 & NITROGEN, TOTAL (mg/L as $\mathrm{N})$ & 54 & 8.000 & 0.800 & 2.537 & 5.425 & 3.250 & 2.000 & 1.475 & 0.870 \\
\hline 00665 & PHOSPHORUS, TOTAL (mg/L as P) & 54 & 2.120 & 0.060 & 0.435 & 1.412 & 0.532 & 0.335 & 0.195 & 0.120 \\
\hline 00671 & PHOSPHORUS ORTHO, DISSOLVED (mg/L as P) & 54 & 0.790 & 0.010 & 0.157 & 0.530 & 0.172 & 0.100 & 0.060 & 0.023 \\
\hline & & & OIL AND & REASE, TOT & & & & & & \\
\hline 00556 & OIL AND GREASE, TOTAL (mg/L) & 7 & 6.000 & $<1.000$ & -- & 6.000 & 4.000 & 3.000 & $<1.000$ & $<1.000$ \\
\hline & & & ORGANIC & ARBON, TOT & TAL & & & & & \\
\hline 00680 & CARBON ORGANIC， TOTAL $(\mathrm{mg} / \mathrm{L})$ & 33 & 76.000 & 9.300 & 18.839 & 55.700 & 20.000 & 14.000 & 12.500 & 9.370 \\
\hline & & & & IFORM & & & & & & \\
\hline 31679 & FECAL STREPTOCOCCI (COlonies per $100 \mathrm{~mL}$ ) & 24 & 640000.000 & 2100.000 & $83683 . \overline{336}$ & 560000.000 & 66750.000 & 32000.000 & 9425.000 & 2500.000 \\
\hline 31616 & FECAL COLIFORM (Colonies per $100 \mathrm{~mL}$ ) & 24 & 590000.000 & 810.000 & 76225.414 & 522500.000 & 81500.000 & 26000.000 & 5600.000 & 957.500 \\
\hline & & ORG & ANIC COMPOI & DS-PESTIC & IDES, TOTAL & & & & & \\
\hline 39330 & ALDRIN, TOTAL $(\mu \mathrm{g} / \mathrm{L})$ & 4 & $<0.010$ & $<0.010$ & -- & -- & - & -- & -- & -- \\
\hline 39340 & LINDANE， TOTAL $(\mu \mathrm{g} / \mathrm{L})$ & 4 & 0.010 & $<0.010$ & -- & -- & -- & -- & -- & -- \\
\hline 39350 & CHLORDANE, TOTAL $(\mu \mathrm{g} / \mathrm{L})$ & 4 & 0.320 & $<0.100$ & -- & -- & -- & -- & -- & -- \\
\hline 39370 & DDT, TOTAL $(\mu \mathrm{g} / \mathrm{L})$ & 4 & $<0.010$ & $<0.010$ & -- & -- & -- & -- & -- & -. \\
\hline 39365 & DDE, TOTAL $(\mu \mathrm{g} / L)$ & 4 & $<0.010$ & $<0.010$ & -- & -- & -- & -- & -- & -- \\
\hline 39360 & DDD, TOTAL $(\mu \mathrm{g} / \mathrm{L})$ & 4 & $<0.010$ & $<0.010$ & -- & -- & -- & -- & -- & -- \\
\hline 39380 & DIELDRIN, TOTAL $(\mu \mathrm{g} / \mathrm{L})$ & 4 & 0.010 & $<0.010$ & -- & -- & - & -- & -- & -- \\
\hline 39388 & ENDOSULFAN, TOTAL $(\mu \mathrm{g} / L)$ & 4 & $<0.010$ & $<0.010$ & -- & -- & - & -- & -- & -- \\
\hline 39390 & ENDRIN, TOTAL $(\mu \mathrm{g} / \mathrm{L})$ & 4 & $<0.010$ & $<0.010$ & -- & -- & -- & -- & -- & - \\
\hline 39410 & HEPTACHLOR， TOTAL $(\mu \mathrm{g} / \mathrm{L})$ & 4 & $<0.010$ & $<0.010$ & - & - & -- & -- & -- & -- \\
\hline 39420 & HEPTACHLOR EPOXIDE, TOTAL $(\mu \mathrm{g} / \mathrm{L})$ & 4 & 0.010 & $<0.010$ & -- & -- & -- & -- & -- & -- \\
\hline 39516 & PCB， TOTAL $(\mu \mathrm{g} / \mathrm{L})$ & 4 & 0.160 & $<0.100$ & -- & -. & -- & -- & -- & - \\
\hline 39400 & TOXAPHENE, TOTAL $(\mu \mathrm{g} / \mathrm{L})$ & 4 & $<1.000$ & $<1.000$ & -- & -- & -- & -- & -- & -- \\
\hline 39034 & PERTHANE, TOTAL $(\mu \mathrm{g} / \mathrm{L})$ & 4 & $<0.100$ & $<0.100$ & -- & -- & -- & -- & - & -- \\
\hline 39570 & DIAZINON, TOTAL $(\mu \mathrm{g} / \mathrm{L})$ & 4 & 0.010 & $<0.010$ & -- & -. & -- & -- & -- & -. \\
\hline 39398 & ETHION, TOTAL $(\mu \mathrm{g} / \mathrm{L})$ & 4 & $<0.010$ & $<0.010$ & -- & -- & -- & - & -- & - \\
\hline 39530 & MALATHION, TOTAL $(\mu g / L)$ & 4 & 0.010 & $<0.010$ & -- & - & -- & -- & -- & -- \\
\hline 39600 & METHYL PARATHION，TOTAL $(\mu \mathrm{g} / \mathrm{L})$ & 4 & 0.010 & $<0.010$ & -- & -- & -. & -- & -- & -- \\
\hline 39540 & PARATHION, TOTAL $(\mu g / L)$ & 4 & $<0.010$ & $<0.010$ & -- & -- & -- & -- & -- & -- \\
\hline 39786 & TRITHION, TOTAL $(\mu \mathrm{g} / \mathrm{L})$ & 4 & $<0.010$ & $<0.010$ & -- & -- & -- & - & -- & -- \\
\hline 39250 & PCN, TOTAL $(\mu \mathrm{g} / \mathrm{L})$ & 4 & $<0.100$ & $<0.100$ & -- & -- & -- & - & $\ldots$ & -- \\
\hline 39480 & METHOXYCHLOR, TOTAL $(\mu \mathrm{g} / \mathrm{L})$ & 4 & $<0.010$ & $<0.010$ & -- & -- & -- & -- & -- & -- \\
\hline 39755 & MIREX, TOTAL $(\mu \mathrm{g} / \mathrm{L})$ & 4 & $<0.010$ & $<0.010$ & -- & -- & -- & -- & -- & -- \\
\hline 39011 & DISYSTON, TOTAL $(\mu \mathrm{g} / \mathrm{L})$ & 4 & $<0.010$ & $<0.010$ & -- & - & - & -- & -- & -- \\
\hline 39023 & PHORATE, TOTAL $(\mu \mathrm{g} / \mathrm{L})$ & 4 & $<0.010$ & $<0.010$ & -- & -- & -- & -- & -- & -- \\
\hline 38932 & CHLORPYRIFOS, TOTAL $(\mu \mathrm{g} / \mathrm{L})$ & 4 & 0.060 & $<0.010$ & -- & -- & -- & -- & -- & -- \\
\hline 39040 & DEF, TOTAL $(\mu \mathrm{g} / \mathrm{L})$ & 4 & $<0.010$ & $<0.010$ & - & -- & -- & - & -- & - \\
\hline 82614 & FONOFOS, TOTAL $(\mu \mathrm{g} / \mathrm{L})$ & 4 & $<0.010$ & $<0.010$ & - & -- & -- & -- & -- & -- \\
\hline & & & LATILE ORGA & COMPOUN & NDS, TOTAL & & & & & \\
\hline$\overline{34030}$ & BENZENE, TOTAL $(\mu \mathrm{g} / \mathrm{L})$ & 7 & $<1.000$ & $<0 . \overline{200}$ & $-\infty$ & -- & -- & -- & -- & -- \\
\hline 32104 & BROMOFORM, TOTAL $(\mu \mathrm{g} / \mathrm{L})$ & 7 & $<1.000$ & $<0.200$ & -- & -- & -- & -- & -- & -- \\
\hline 32102 & CARBON TETRACHLORIDE, TOTAL $(\mu \mathrm{g} / \mathrm{L})$ & 7 & $<1.000$ & $<0.200$ & -- & -- & -- & -- & -- & -- \\
\hline 34301 & CHLOROBENZENE, TOTAL $(\mu \mathrm{g} / \mathrm{L})$ & 7 & $<1.000$ & $<0.200$ & -- & $\ldots$ & -- & -- & -- & - \\
\hline 32105 & CHLORODIBROMOMETHANE, TOTAL $(\mu \mathrm{g} / \mathrm{L})$ & 7 & $<1.000$ & $<0.200$ & -- & -- & -- & -- & -- & -- \\
\hline 34311 & CHLOROETHANE, TOTAL $(\mu \mathrm{g} / \mathrm{L})$ & 7 & $<1.000$ & $<0.200$ & -- & -- & -- & -- & -- & $\sim-$ \\
\hline 32106 & CHLOROFORM, TOTAL $(\mu \mathrm{g} / \mathrm{L})$ & 7 & $<1.000$ & $<0.200$ & - & -- & -- & -- & -- & -- \\
\hline 34496 & 1,1 -DICHLOROETHANE, TOTAL $(\mu \mathrm{g} / \mathrm{L})$ & 7 & $<1.000$ & $<0.200$ & -- & -- & . - & -- & -- & -- \\
\hline 32103 & 1,2-DICHLOROETHANE，TOTAL $(\mu \mathrm{g} / \mathrm{L})$ & 7 & $<1.000$ & $<0.200$ & -- & -- & - & -- & -- & -- \\
\hline 34501 & 1,1-DICHLOROETHYLENE，TOTAL $(\mu \mathrm{g} / \mathrm{L})$ & 7 & $<1.000$ & $<0.200$ & -- & -. & -. & -- & -- & -. \\
\hline 34541 & 1,2 -DICHLOROPROPANE, TOTAL $(\mu \mathrm{g} / \mathrm{L})$ & 7 & $<1.000$ & $<0.200$ & -- & -- & -- & - & -- & -- \\
\hline 34371 & ETHYLBENZENE, TOTAL $(\mu \mathrm{g} / \mathrm{L})$ & 7 & $<1.000$ & $<0.200$ & -- & -- & - & -- & -- & -- \\
\hline 34413 & METHYL BROMIDE, TOTAL $(\mu \mathrm{g} / \mathrm{L})$ & 7 & $<1.000$ & $<0.200$ & -- & -- & - & -- & -- & -- \\
\hline 34423 & METHYLENE CHLORIDE, TOTAL $(\mu \mathrm{g} / \mathrm{L})$ & 7 & $<1.000$ & $<0.200$ & -- & -- & -- & - & - & -- \\
\hline 34516 & $1,1,2,2$-TETRACHLOROETHANE, TOTAL $(\mu \mathrm{g} / \mathrm{L})$ & 7 & $<1.000$ & $<0.200$ & -- & -- & $-\infty$ & -- & -- & -- \\
\hline
\end{tabular}

NOTE: Multiple detection limits during the period of record may result in different values flagged with a "<."

* Value is estimated by using a log-probability regression to predict the values of data below the detection limit. 
Table 72. Statistical summary of water-quality data at site 40 (CSW03), July 1994 through September 1998—Contir'xed

\begin{tabular}{|c|c|c|c|c|c|c|c|c|c|c|}
\hline \multirow{2}{*}{$\begin{array}{l}\text { PARAM- } \\
\text { ETER } \\
\text { CODE }\end{array}$} & \multirow{2}{*}{ PROPERTY OR CONSTITUENT } & & DESCRIPTII & STATISTICS & & PERCENT O & $\begin{array}{r}\text { SAMPLES II } \\
\text { EQUA }\end{array}$ & $\begin{array}{l}\text { WHICH VALL } \\
\text { TO THOSES }\end{array}$ & $\begin{array}{l}\text { S WERE LE } \\
\text { DWN }\end{array}$ & THAN OR \\
\hline & & $\begin{array}{c}\text { SAMPLE } \\
\text { SIZE }\end{array}$ & MAXIMUM & MINIMUM & MEAN & $95 \%$ & $75 \%$ & $\begin{array}{c}50 \% \\
\text { (medlan) }\end{array}$ & $25 \%$ & $5 \%$ \\
\hline $34 \overline{75}$ & TETRACHLOROETHYLENE，TOTAL $(\mu \mathrm{g} / \mathrm{L})$ & 7 & $<1.000$ & $<0.200$ & -- & -- & -- & -- & -- & -- \\
\hline 34010 & TOLUENE, TOTAL $(\mu \mathrm{g} / \mathrm{L})$ & 7 & 0.900 & $<0.200$ & -- & 0.900 & $<1.000$ & $<0.800$ & $<0.400$ & $<0.400$ \\
\hline 34546 & 1,2 -TRANSDICHLOROETHENE, TOTAL $(\mu \mathrm{g} / \mathrm{L})$ & 7 & $<1.000$ & $<0.200$ & -- & -- & $-\infty$ & -- & -- & - \\
\hline 34506 & $1,1,1$-TRICHLOROETHANE, TOTAL $(\mu \mathrm{g} / \mathrm{L})$ & 7 & $<1.000$ & $<0.200$ & -- & -- & -- & - & -- & - \\
\hline 34511 & $1,1,2$-TRICHLOROETHANE, TOTAL $(\mu \mathrm{g} / \mathrm{L})$ & 7 & $<1.000$ & $<0.200$ & -- & -- & -- & -- & -- & -- \\
\hline 39180 & TRICHLOROETHYLENE， TOTAL $(\mu \mathrm{g} / \mathrm{L})$ & 7 & $<1.000$ & $<0.200$ & -- & -- & -- & -- & - & -. \\
\hline 39175 & VINYL CHLORIDE, TOTAL $(\mu \mathrm{g} / \mathrm{L})$ & 7 & $<1.000$ & $<0.200$ & - & -- & -- & -- & -- & - \\
\hline 30217 & DIBROMOMETHANE, TOTAL $(\mu \mathrm{g} / \mathrm{L})$ & 7 & $<1.000$ & $<0.200$ & -- & -- & -- & -- & -- & -. \\
\hline 32101 & DICHLOROBROMOMETHANE, TOTAL $(\mu \mathrm{g} / \mathrm{L})$ & 7 & $<1.000$ & $<0.200$ & - & -- & -- & -- & -- & -- \\
\hline 34668 & DICHLORODIFLUOROMETHANE, TOTAL $(\mu \mathrm{g} / \mathrm{L})$ & 7 & $<1.000$ & $<0.200$ & -- & -- & -- & -- & -- & $\ldots$ \\
\hline 34488 & TRICHLOROFLUOROMETHANE，TOTAL $(\mu \mathrm{g} / \mathrm{L})$ & 7 & $<1.000$ & $<0.200$ & - & -- & -- & -- & -- & -- \\
\hline 77651 & 1,2 -DIBROMOETHANE， TOTAL $(\mu \mathrm{g} / \mathrm{L})$ & 7 & $<1.000$ & $<0.200$ & -- & -- & -- & -- & -- & -- \\
\hline 34418 & METHYLCHLORIDE, TOTAL $(\mu \mathrm{g} / \mathrm{L})$ & 7 & $<1.000$ & $<0.200$ & -- & -- & - - & -- & -- & -. \\
\hline 34704 & CIS 1,3 -DICHLOROPROPENE, TOTAL $(\mu \mathrm{g} / \mathrm{L})$ & 7 & $<1.000$ & $<0.200$ & -- & -- & -- & -- & -- & - \\
\hline 34699 & TRANS 1,3 -DICHLOROPROPENE，TOTAL $\langle\mu \mathrm{g} / \mathrm{L}\rangle$ & 7 & $<1.000$ & $<0.200$ & -- & -- & -- & -- & -- & $\ldots$ \\
\hline 77128 & STYRENE, TOTAL $(\mu \mathrm{g} / \mathrm{L})$ & 7 & $<1.000$ & $<0.200$ & -- & -- & -- & -- & -- & -. \\
\hline 81551 & XYLENE, TOTAL $(\mu \mathrm{g} / \mathrm{L})$ & 7 & $<1.000$ & $<0.200$ & -- & -- & -- & -- & -- & -- \\
\hline 82625 & DIBROMOCHLOROPROPANE, TOTAL $(\mu \mathrm{g} / \mathrm{L})$ & 7 & $<5.000$ & $<1.000$ & -- & -- & - & -- & -- & -- \\
\hline 77168 & 1,1 -DICHLOROPROPENE，TOTAL $(\mu \mathrm{g} / \mathrm{L})$ & 7 & $<1.000$ & $<0.200$ & -- & -- & -- & -- & -- & - \\
\hline 77170 & 2,2 -DICHLOROPROPANE, TOTAL $(\mu \mathrm{g} / \mathrm{L})$ & 7 & $<1.000$ & $<0.200$ & -- & -- & -- & -. & -- & -- \\
\hline 77173 & 1,3 -DICHLOROPROPANE, TOTAL $(\mu \mathrm{g} / \mathrm{L})$ & 7 & $<1.000$ & $<0.200$ & -- & -- & -- & -- & -- & -- \\
\hline 77275 & O-CHLOROTOLUENE, TOTAL $(\mu \mathrm{g} / \mathrm{L})$ & 7 & $<1.000$ & $<0.200$ & -- & -- & -- & -- & -- & -. \\
\hline 77277 & P-CHLOROTOLUENE，TOTAL $(\mu \mathrm{g} / \mathrm{L})$ & 7 & $<1.000$ & $<0.200$ & - & - & -- & -- & - & - \\
\hline 77443 & 123-TRICHLOROPROPANE, TOTAL $(\mu \mathrm{g} / \mathrm{L})$ & 7 & $<1.000$ & $<0.200$ & -- & -- & -- & -- & -- & -- \\
\hline 77562 & 1112 -TETRACHLOROETHANE, TOTAL $(\mu \mathrm{g} / \mathrm{L})$ & 7 & $<1.000$ & $<0.200$ & -- & -- & -- & -- & -- & -- \\
\hline 78032 & TERTBUTYL METHYL ETHER, TOTAL $(\mu \mathrm{g} / \mathrm{L})$ & 7 & $<1.000$ & $<0.200$ & -- & -- & -- & -- & -- & -- \\
\hline 77297 & BROMOCHLORO METHANE，TOTAL $(\mu \mathrm{g} / \mathrm{L})$ & 7 & $<1.000$ & $<0.200$ & -- & -- & -- & -- & -- & -- \\
\hline 77093 & CIS- 1,2 -DICHLOROETHENE, TOTAL $(\mu \mathrm{g} / \mathrm{L})$ & 7 & $<1.000$ & $<0.200$ & -- & -- & -- & -- & -- & -- \\
\hline 34576 & 2-CHLOROETHYL VINYL ETHER, TOTAL $\{\mu \mathrm{g} / \mathrm{L}\}$ & 3 & $<4.000$ & $<1.000$ & -- & -- & -- & -- & -- & -- \\
\hline 77223 & ISOPROPYL BENZENE, TOTAL $(\mu \mathrm{g} / \mathrm{L})$ & 7 & $<1.000$ & $<0.200$ & -- & -- & -- & -- & -- & -- \\
\hline 77224 & N-PROPYL BENZENE, TOTAL $(\mu \mathrm{g} / \mathrm{L})$ & 7 & $<1.000$ & $<0.200$ & -- & -- & -- & -- & -- & -- \\
\hline 77353 & TERTBUTYL BENZENE， TOTAL $(\mu \mathrm{g} / \mathrm{L})$ & 7 & $<1.000$ & $<0.200$ & -- & -- & -- & -- & -- & -- \\
\hline 77222 & PSEUDOCUMENE, TOTAL $(\mu \mathrm{g} / \mathrm{L})$ & 7 & $<1.000$ & $<0.200$ & -- & -- & -- & -- & -- & -- \\
\hline 77350 & SEC-BUTYL BENZENE，TOTAL $(\mu \mathrm{g} / \mathrm{L})$ & 7 & $<1.000$ & $<0.200$ & -- & -- & -- & -- & -- & - - \\
\hline 77356 & P-ISOPROPYL TOLUENE, TOTAL $(\mu \mathrm{g} / \mathrm{L})$ & 7 & $<1.000$ & $<0.200$ & -- & -- & -- & -- & -- & -- \\
\hline 77342 & N-BUTYL BENZENE，TOTAL $(\mu \mathrm{g} / \mathrm{L})$ & 7 & $<1.000$ & $<0.200$ & - - & -. & -- & -- & - - & -- \\
\hline 77613 & $1,2,3$-TRICHLOROBENZENE，TOTAL $(\mu \mathrm{g} / \mathrm{L})$ & 7 & $<1.000$ & $<0.200$ & -. & -- & -- & -- & -- & -- \\
\hline 77652 & FREON-113, TOTAL $(\mu \mathrm{g} / \mathrm{L})$ & 7 & $<1.000$ & $<0.200$ & -- & -- & -- &.- & -- & -- \\
\hline 77226 & MESITYLENE, TOTAL $(\mu \mathrm{g} / \mathrm{L})$ & 7 & $<1.000$ & $<0.200$ & -- & -- & -- & -- & -- & -- \\
\hline 81555 & BROMOBENZENE， TOTAL $(\mu \mathrm{g} / \mathrm{L})$ & 7 & $<1.000$ & $<0.200$ & -. & -- & -- & -- & -. & -. \\
\hline 34551 & $1,2,4$-TRICHLOROBENZENE，TOTAL $(\mu \mathrm{g} / \mathrm{L})$ & 7 & $<1.000$ & $<0.200$ & -- & -- & -- & -- & -- & -- \\
\hline 34536 & 1,2 -DICHLOROBENZENE， TOTAL $(\mu \mathrm{g} / \mathrm{L})$ & 7 & $<1.000$ & $<0.200$ & -- & -- & -- & -- & -- & -- \\
\hline 34566 & 1,3-DICHLOROBENZENE，TOTAL $(\mu \mathrm{g} / \mathrm{L})$ & 7 & $<1.000$ & $<0.200$ & -- & -- & -- & -- & -- & -- \\
\hline 34571 & 1,4 -DICHLOROBENZENE, TOTAL $(\mu \mathrm{g} / \mathrm{L})$ & 7 & $<1.000$ & $<0.200$ & - & -- & -- & -- & -- & -- \\
\hline 39702 & HEXACHLOROBUTADI ENE, TOTAL $(\mu \mathrm{g} / \mathrm{L})$ & 7 & $<1.000$ & $<0.200$ & - & -- & -- & -- & -- & - \\
\hline 34696 & NAPHTHALENE, TOTAL $(\mu \mathrm{g} / \mathrm{L})$ & 7 & $<1.000$ & $<0.200$ & -- & -- & -- & -- & -- & - \\
\hline & & MET & LS AND MINC & CONSTITUE & JTS, TOTAL & & & & & \\
\hline$\overline{01097}$ & ANTIMONY, TOTAL $(\mu \mathrm{g} / \mathrm{L}$ as Sb) & 39 & 7.000 & $<1.000$ & $0.642^{\star}$ & 5.000 & $<1.000$ & $<1.000$ & $<1.00 \overline{0}$ & $<\overline{1.000}$ \\
\hline 01002 & ARSENIC, TOTAL $(\mu \mathrm{g} / \mathrm{L}$ as AS $)$ & 39 & 4.000 & $<1.000$ & $1.273^{*}$ & 4.000 & 2.000 & 1.000 & $<1.000$ & $<1.000$ \\
\hline 01012 & BERYLLIUM, TOTAL $(\mu \mathrm{g} / \mathrm{L}$ as Be $)$ & 9 & $<10.000$ & $<10.000$ & -- & $<10.000$ & $<10.000$ & $<10.000$ & $<10.000$ & $<10.000$ \\
\hline 01027 & CADMIUM, TOTAL $(\mu \mathrm{g} / \mathrm{L}$ as Cd) & 9 & 1.000 & $<1.000$ & - & 1.000 & $<1.000$ & $<1.000$ & $<1.000$ & $<1.000$ \\
\hline 01034 & CHROMIUM, TOTAL $(\mu \mathrm{g} / \mathrm{L}$ as $\mathrm{Cr})$ & 39 & 29.000 & 2.000 & 9.462 & 27.000 & 12.000 & 7.000 & 4.000 & 2.000 \\
\hline 01042 & COPPER, TOTAL $(\mu \mathrm{g} / \mathrm{L}$ as $\mathrm{Cu})$ & 39 & 50.000 & 3.000 & 16.949 & 41.000 & 22.000 & 15.000 & 8.000 & 4.000 \\
\hline 01051 & LEAD, TOTAL $(\mu \mathrm{g} / \mathrm{L}$ as $\mathrm{Pb})$ & 39 & 160.000 & 3.000 & 21.974 & 87.000 & 27.000 & 13.000 & 6.000 & 3.000 \\
\hline 71900 & MERCURY, TOTAL $(\mu \mathrm{g} / \mathrm{L}$ as $\mathrm{Hg})$ & 9 & 0.100 & $<0.100$ & -- & 0.100 & $<0.100$ & $<0.100$ & $<0.100$ & $<0.100$ \\
\hline 01067 & NICKEL, TOTAL $(\mu \mathrm{g} / \mathrm{L}$ as Ni) & 39 & 23.000 & 1.000 & 6.821 & 16.000 & 9.000 & 7.000 & 4.000 & 2.000 \\
\hline 01147 & SELENIUM, TOTAL $(\mu \mathrm{g} / \mathrm{L}$ as $\mathrm{Se})$ & 9 & $<1.000$ & $<1.000$ & - & $<1.000$ & $<1.000$ & $<1.000$ & $<1.000$ & $<1.000$ \\
\hline 01077 & SILVER, TOTAL $(\mu \mathrm{g} / \mathrm{L}$ as Ag) & 9 & $<1.000$ & $<1.000$ & -- & $<1.000$ & $<1.000$ & $<1.000$ & $<1.000$ & $<1.000$ \\
\hline 01092 & ZINC, TOTAL $(\mu \mathrm{g} / \mathrm{L}$ as $\mathrm{zn})$ & 38 & 490.000 & 30.000 & 100.000 & 262.000 & 110.000 & 75.000 & 60.000 & 30.000 \\
\hline 00720 & CYANIDE, TOTAL (mg/L as $\mathrm{Cn}$ ) & 9 & 0.010 & $<0.010$ & - & 0.010 & $<0.010$ & $<0.010$ & $<0.010$ & $<0.010$ \\
\hline & & ORGAP & C COMPOUN & -PESTICID & S, DISSOLVE & & & & & \\
\hline 49260 & ACETOCHLOR, DISSOLVED $(\overline{\mu \mathrm{g} / \mathrm{L})}$ & 3 & $<0 . \overline{002}$ & $<0.002$ & -- & -- & $-\overline{-}$ & -- & -- & -- \\
\hline 46342 & ALACHLOR, DISSOLVED $(\mu \mathrm{g} / \mathrm{L})$ & 3 & 0.011 & $<0.002$ & -- & -- & -- & -- & -- & -- \\
\hline 04040 & DEETHYL ATRAZINE, DISSOLVED $(\mu \mathrm{g} / \mathrm{L})$ & 3 & 0.006 & $<0.002$ & -- & -- & -- & -- & -- & -- \\
\hline 39632 & ATRAZINE, DISSOLVED $(\mu \mathrm{g} / \mathrm{L})$ & 3 & 0.029 & $<0.001$ & -- & - & -- & -- & -- & - \\
\hline 82686 & METHYL AZINPHOS， DISSOLVED $(\mu \mathrm{g} / \mathrm{L})$ & 3 & $<0.001$ & $<0.001$ & -- & - & -- & -- & -- & -- \\
\hline 82673 & BENFLURALIN, DISSOLVED $(\mu \mathrm{g} / \mathrm{L})$ & 3 & 0.037 & $<0.002$ & -- & -. & -- & -- & -- & -. \\
\hline 04028 & BUTYLATE， DISSOLVED $(\mu \mathrm{g} / \mathrm{L})$ & 3 & $<0.002$ & $<0.002$ & -- & -. & -- & -- & -- & -- \\
\hline 82680 & CARBARYL， DISSOLVED $(\mu \mathrm{g} / \mathrm{L})$ & 3 & 0.787 & 0.133 & -- & -- & -- & -- & -- & -- \\
\hline 82674 & CARBOFURAN, DISSOLVED $(\mu \mathrm{g} / \mathrm{L})$ & 3 & $<0.003$ & $<0.003$ & -- & - & -- & $\ldots$ & -- & -- \\
\hline 38933 & CHLORPYRIFOS, DISSOLVED $(\mu \mathrm{g} / \mathrm{L})$ & 3 & 0.009 & $<0.004$ & -- & -- & -- & -- & -- & -- \\
\hline 04041 & CYANAZINE, DISSOLVED $(\mu \mathrm{g} / \mathrm{L})$ & 3 & $<0.004$ & $<0.004$ & -- & - & -- & -- & -- & -- \\
\hline 82682 & DCPA， DISSOLVED $(\mu \mathrm{g} / \mathrm{L})$ & 3 & $<0.002$ & $<0.002$ & -- & -- & -- & -- & -- & -- \\
\hline 34653 & P, P' DDE, DISSOLVED $(\mu \mathrm{g} / \mathrm{L})$ & 3 & $<0.006$ & $<0.006$ & -- & -. & -- & -- & -- & -- \\
\hline 39572 & DIAZINON, DISSOLVED $(\mu \mathrm{g} / \mathrm{L})$ & 3 & 0.012 & $<0.002$ & -- & -- & -- & -- & -- & -- \\
\hline 39381 & DIELDRIN， DISSOLVED $(\mu \mathrm{g} / \mathrm{L})$ & 3 & $<0.001$ & $<0.001$ & -- & -- & -- & -- & -- & -- \\
\hline 82660 & 2,6 -DIETHYL ANILINE, DISSOLVED $(\mu \mathrm{g} / \mathrm{L})$ & 3 & $<0.003$ & $<0.003$ & -- & -- & -- & -- & -- & -- \\
\hline 82677 & DISULFOTON， DISSOLVED $(\mu \mathrm{g} / \mathrm{L})$ & 3 & $<0.017$ & $<0.017$ & -- & -- & -- & - & -- & -- \\
\hline 82668 & EPTC, DISSOLVED $(\mu \mathrm{g} / \mathrm{L})$ & 3 & $<0.002$ & $<0.002$ & -- & - & -- & - & -- & -- \\
\hline 82663 & ETHALFLURALIN， DISSOLVED $(\mu \mathrm{g} / \mathrm{L})$ & 3 & $<0.004$ & $<0.004$ & -- & -- & -- & -- & -- & -- \\
\hline 82672 & ETHOPROP, DISSOLVED $(\mu \mathrm{g} / \mathrm{L})$ & 3 & $<0.003$ & $<0.003$ & -- & -. & -- & -- & -- & -- \\
\hline 04095 & FONOFOS, DISSOLVED $(\mu \mathrm{g} / \mathrm{L})$ & 3 & $<0.003$ & $<0.003$ & -- & -- & -. & -- & -. & -- \\
\hline
\end{tabular}

NOTE: Multiple detection limits during the period of record may result in different values flagged with a "<."

* Value is estimated by using a log-probability regression to predict the values of data below the detection limit. 
Table 72. Statistical summary of water-quality data at site 40 (CSW03), July 1994 through September 1998-Continued

\begin{tabular}{|c|c|c|c|c|c|c|c|c|c|c|}
\hline \multirow{2}{*}{$\begin{array}{c}\text { PARAM- } \\
\text { ETER } \\
\text { CODE }\end{array}$} & \multirow{2}{*}{ PROPERTY OR CONSTITUENT } & & DESCRIPTI & STATISTICS & & PERCEN & $\begin{array}{r}\text { AMPLE } \\
\text { EQ }\end{array}$ & $\begin{array}{l}\text { WHICH VAL } \\
\text { TO THOSE }\end{array}$ & WERE & HAN OR \\
\hline & & $\begin{array}{c}\text { SAMPLE } \\
\text { SIZE }\end{array}$ & MAXIMUM & MINIMUM & MEAN & $\mathbf{9 5} \%$ & $75 \%$ & $\begin{array}{c}50 \% \\
\text { (median) }\end{array}$ & $25 \%$ & $5 \%$ \\
\hline 34253 & ALPHA BHC, DISSOLVED $(\mu \mathrm{g} / \mathrm{L})$ & 3 & $<0.002$ & $<0.002$ & -- & -- & $-\overline{-}$ & $-\overline{-}$ & $-\cdots$ & -- \\
\hline 39341 & LINDANE, DISSOLVED $\{\mu \mathrm{g} / \mathrm{L}\rangle$ & 3 & 0.006 & $<0.004$ & -- & -- & -- & -- & - & -- \\
\hline 82666 & LINURON，DISSOLVED $(\mu \mathrm{g} / \mathrm{L})$ & 3 & $<0.002$ & $<0.002$ & -- & -- & -. & -- & $-\infty$ & -- \\
\hline 39532 & MALATHION，DISSOLVED $(\mu \mathrm{g} / \mathrm{L})$ & 3 & 0.022 & $<0.005$ & -- & - & -- & -- & -- & -- \\
\hline 82667 & METHYL PARATHION, DISSOLVED $(\mu \mathrm{g} / \mathrm{L})$ & 3 & 0.033 & $<0.006$ & -- & $\ldots$ & -- & -- & -. & - \\
\hline 39415 & METOLACHLOR, DISSOLVED $(\mu \mathrm{g} / \mathrm{L})$ & 3 & 0.038 & $<0.002$ & -- & -- & -- & -- & -- & -- \\
\hline 82630 & METRIBUZIN，DISSOLVED $(\mu \mathrm{g} / \mathrm{L})$ & 3 & $<0.004$ & $<0.004$ & -- & -- & -- & -- & -- & -- \\
\hline 82671 & MOLINATE, DISSOLVED $(\mu \mathrm{g} / \mathrm{L})$ & 3 & $<0.004$ & $<0.004$ & -- & -- & -- & -- & -- & -- \\
\hline 82684 & NAPROPAMIDE, DISSOLVED $(\mu \mathrm{g} / \mathrm{L})$ & 3 & $<0.003$ & $<0.003$ & -- & -- & -- & -- & -- & -- \\
\hline 39542 & PARATHION， DISSOLVED $(\mu \mathrm{g} / \mathrm{L})$ & 3 & $<0.004$ & $<0.004$ & -- & -- & -- & -- & -- & -- \\
\hline 82669 & PEBULATE， DISSOLVED $(\mu \mathrm{g} / \mathrm{L})$ & 3 & $<0.004$ & $<0.004$ & -- & -- & -- & -- & -- & -- \\
\hline 82683 & PENDIMETHALIN, DISSOLVED $(\mu \mathrm{g} / \mathrm{L})$ & 3 & $<0.004$ & $<0.004$ & -- & -- & -- & -- & -- & -- \\
\hline 82687 & PERMETHRIN， DISSOLVED $\left(\mu g / L_{1}\right)$ & 3 & $<0.005$ & $<0.005$ & -- & -- & -- & -- & -- & -- \\
\hline 82664 & PHORATE, DISSOLVED $(\mu \mathrm{g} / \mathrm{L})$ & 3 & $<0.002$ & $<0.002$ & -- & -- & -- & -- & -- & -- \\
\hline 82676 & PRONAMIDE, DISSOLVED $\{\mu \mathrm{g} / \mathrm{L}\}$ & 3 & $<0.003$ & $<0.003$ & -- & -- & -- & - & -- & -- \\
\hline 04037 & PROMETON, DISSOLVED $(\mu \mathrm{g} / \mathrm{L})$ & 3 & $<0.018$ & $<0.018$ & -- & -- & -- & -- & -- & -- \\
\hline 04024 & PROPACHLOR, DISSOLVED $(\mu \mathrm{g} / \mathrm{L})$ & 3 & $<0.007$ & $<0.007$ & -- & -- & -- & -- & -- & -- \\
\hline 82679 & PROPANIL， DISSOLVED $(\mu \mathrm{g} / \mathrm{L})$ & 3 & $<0.004$ & $<0.004$ & -- & -- & -- & -- & -- & -- \\
\hline 82685 & PROPARGITE，DISSOLVED $(\mu \mathrm{g} / \mathrm{L})$ & 3 & $<0.013$ & $<0.013$ & -- & -- & -- & -. & -- & -- \\
\hline 04035 & SIMAZINE，DISSOLVED $(\mu \mathrm{g} / \mathrm{L})$ & 3 & $<0.005$ & $<0.005$ & -- & -- & -- & -- & -- & -- \\
\hline 82681 & THIOBENCARB，DISSOLVED $(\mu \mathrm{g} / \mathrm{L})$ & 3 & $<0.002$ & $<0.002$ & -- & -- & -- & -- & -- & -- \\
\hline 82670 & TEBUTHIURON， DISSOLVED $(\mu \mathrm{g} / \mathrm{L})$ & 3 & 0.020 & $<0.010$ & -- & -- & -- & -- & -- & -- \\
\hline 82665 & TERBACIL， DISSOLVED $(\mu \mathrm{g} / \mathrm{L})$ & 3 & $<0.007$ & $<0.007$ & -- & -- & -- & -- & -- & -- \\
\hline 82675 & TERBUFOS，DISSOLVED $(\mu g / L)$ & 3 & $<0.013$ & $<0.013$ & -- & -- & -- & -- & -- & -- \\
\hline 82678 & TRIALLATE， DISSOLVED $(\mu \mathrm{g} / \mathrm{L})$ & 3 & $<0.001$ & $<0.001$ & -- & -- & -- & -- & -- & -- \\
\hline 82661 & TRIFLURALIN, DISSOLVED $(\mu \mathrm{g} / \mathrm{L})$ & 3 & 0.023 & $<0.002$ & -- & -- & -- & -- & -- & -- \\
\hline 39742 & $2,4,5-T$, DISSOLVED $(\mu \mathrm{g} / \mathrm{L})$ & 3 & $<0.035$ & $<0.035$ & -- & -- & -- & -- & -- & -- \\
\hline 39732 & $2,4-\mathrm{D}$, DISSOLVED $(\mu \mathrm{g} / \mathrm{L})$ & 3 & $<0.150$ & $<0.035$ & -- & -- & -- & -- & -- & -- \\
\hline 38746 & $2,4-\mathrm{DB}$, DISSOLVED $(\mu \mathrm{g} / \mathrm{L})$ & 3 & $<0.240$ & $<0.035$ & -- & -- & -- & -- & -- & -- \\
\hline 49315 & ACIFLUORFEN, DISSOLVED $(\mu \mathrm{g} / \mathrm{L})$ & 3 & $<0.035$ & $<0.035$ & -- & -- & -- & -- & -- & -- \\
\hline 49312 & ALDICARB， DISSOLVED $(\mu \mathrm{g} / \mathrm{L})$ & 3 & $<0.550$ & $<0.016$ & -- & -- & -- & -- & -- & -- \\
\hline 49313 & ALDICARB SULFONE, DISSOLVED $(\mu \mathrm{g} / \mathrm{L})$ & 3 & $<0.100$ & $<0.016$ & -- & -- & -- & -- & -- & -- \\
\hline 49314 & ALDICARB SULFOXIDE, DISSOLVED $\left(\mu \mathrm{g} / L_{1}\right)$ & 3 & $<0.021$ & $<0.021$ & -- & -- & -- & -- & -- & -- \\
\hline 38711 & BENTAZON, DISSOLVED $(\mu g / L)$ & 3 & $<0.014$ & $<0.014$ & -- & -- & -- & -- & -- & -- \\
\hline 04029 & BROMACIL, DISSOLVED $(\mu \mathrm{g} / \mathrm{L})$ & 3 & $<0.035$ & $<0.035$ & -- & -- & $-\cdots$ & -- & -- & -- \\
\hline 49311 & BROMOXYNIL, DISSOLVED $(\mu \mathrm{g} / \mathrm{L})$ & 3 & $<0.035$ & $<0.035$ & -- & -- & -- & -- & -- & -- \\
\hline 49310 & CARBARYL， DISSOLVED $(\mu \mathrm{g} / \mathrm{L})$ & 3 & 0.540 & $<0.008$ & -- & -. & -- & -- & -. & -- \\
\hline 49309 & CARBOFURAN, DISSOLVED $(\mu \mathrm{g} / \mathrm{L})$ & 3 & $<0.120$ & $<0.028$ & -- & -- & -- & -- & -- & -- \\
\hline 49308 & 3-HYDROXY-CARBOFURAN $(\mu \mathrm{g} / \mathrm{L})$ & 3 & $<0.014$ & $<0.014$ & -- & -- & -- & -- & -- & -- \\
\hline 49307 & AMIBEN, DISSOLVED $(\mu \mathrm{g} / \mathrm{L})$ & 3 & $<0.420$ & $<0.011$ & -- & -- & -- & -- & -- & -- \\
\hline 49306 & CHLOROTHALONIL， DISSOLVED $(\mu \mathrm{g} / \mathrm{L})$ & 3 & $<0.480$ & $<0.035$ & -- & -- & -- & -- & -- & -- \\
\hline 49305 & CLOPYRALID， DISSOLVED $(\mu \mathrm{g} / \mathrm{L})$ & 3 & $<0.230$ & $<0.050$ & -- & -- & -- & -- & -- & -- \\
\hline 49304 & DACTHALMONO-ACID, DISSOLVED $(\mu \mathrm{g} / \mathrm{L})$ & 3 & $<0.017$ & $<0.017$ & -- & -- & -- & -- & -- & -- \\
\hline 38442 & DICAMBA， DISSOLVED $(\mu \mathrm{g} / \mathrm{L})$ & 3 & $<0.035$ & $<0.035$ & -- & -- & - & -- & -- & -- \\
\hline 49303 & DICHLOBENIL， DISSOLVED $(\mu \mathrm{g} / \mathrm{L})$ & 2 & $<1.200$ & $<0.020$ & -- & -- & -- & -- & -- & -- \\
\hline 49302 & DICHLORPROP, DISSOLVED $(\mu \mathrm{g} / \mathrm{L})$ & 3 & 0.090 & $<0.032$ & -- & -- & -- & -- & -- & -- \\
\hline 49301 & DINOSEB， DISSOLVED $(\mu \mathrm{g} / \mathrm{L})$ & 3 & $<0.035$ & $<0.035$ & -- & -- & -- & -- & -- & -- \\
\hline 49300 & DIURON, DISSOLVED $(\mu \mathrm{g} / \mathrm{L})$ & 3 & $<2.470$ & $<0.020$ & -- & -- & -- & -- & -- & -- \\
\hline 49299 & 4, 6 -DINITRO OCRESOL, DISSOLVED $(\mu \mathrm{g} / \mathrm{L})$ & 3 & $<0.420$ & $<0.035$ & -- & -- & -- & -- & -- & -- \\
\hline 49298 & ESFENVALERATE， DISSOLVED $(\mu \mathrm{g} / \mathrm{L})$ & 2 & $<0.019$ & $<0.019$ & -- & -- & -- & -- & - & -- \\
\hline 49297 & FENURON，DISSOLVED $(\mu \mathrm{g} / \mathrm{L})$ & 3 & $<0.013$ & $<0.013$ & -- & -- & -- & -- & -- & -- \\
\hline 38811 & FLUOMETURON, DISSOLVED $(\mu \mathrm{g} / \mathrm{L})$ & 3 & $<0.035$ & $<0.035$ & -- & -- & -- & -- & -- & -- \\
\hline 38478 & LINURON，DISSOLVED $(\mu \mathrm{g} / \mathrm{L})$ & 3 & $<0.018$ & $<0.018$ & -- & -- & -- & - & - & -- \\
\hline 38482 & MCPA， DISSOLVED $(\mu \mathrm{g} / \mathrm{L})$ & 3 & $<0.170$ & $<0.050$ & -- & -- & -- & -- & -- & -- \\
\hline 38487 & MCPB， DISSOLVED $(\mu \mathrm{g} / \mathrm{L})$ & 3 & $<0.140$ & $<0.035$ & -- & -- & - & -- & -- & -- \\
\hline 38501 & METHIOCARB， DISSOLVED $(\mu \mathrm{g} / \mathrm{L})$ & 3 & $<0.026$ & $<0.026$ & -- & -- & -- & -- & -- & -- \\
\hline 49296 & METHOMYL, DISSOLVED $(\mu \mathrm{g} / \mathrm{L})$ & 3 & $<0.017$ & $<0.017$ & -- & -- & -- & -- & -- & -- \\
\hline 49295 & 1-NAPHTHOL， DISSOLVED $(\mu \mathrm{g} / \mathrm{L})$ & 2 & $<0.007$ & $<0.007$ & -- & -- & -- & -- & -- & -- \\
\hline 49294 & NEBURON， DISSOLVED $(\mu \mathrm{g} / \mathrm{L})$ & 3 & $<0.015$ & $<0.015$ & -- & -- & -- & -- & -- & -- \\
\hline 49293 & NORFLURAZON， DISSOLVED $(\mu \mathrm{g} / \mathrm{L})$ & 3 & $<0.024$ & $<0.024$ & -- & -- & -- & -- & -- & -- \\
\hline 49292 & ORYZALIN， DISSOLVED $(\mu \mathrm{g} / \mathrm{L})$ & 3 & $<0.310$ & $<0.019$ & -- & -- & -- & -- & -- & -- \\
\hline 38866 & OXAMYL，DISSOLVED $(\mu \mathrm{g} / \mathrm{L})$ & 3 & $<0.018$ & $<0.018$ & -- & -- & -- & -- & -- & -- \\
\hline 49291 & PICLORAM， DISSOLVED $(\mu \mathrm{g} / \mathrm{L})$ & 3 & $<0.050$ & $<0.050$ & -- & -- & -- & -- & $\ldots$ & -- \\
\hline 49236 & PROPHAM， DISSOLVED $(\mu \mathrm{g} / \mathrm{L})$ & 3 & $<0.035$ & $<0.035$ & -- & -- & -- & -- & -- & -- \\
\hline 38538 & PROPOXUR, DISSOLVED $(\mu \mathrm{g} / \mathrm{L})$ & 3 & $<0.035$ & $<0.035$ & -- & -- & -- & -- & -- & -- \\
\hline 39762 & SILVEX， DISSOLVED $(\mu \mathrm{g} / \mathrm{L})$ & 3 & $<0.021$ & $<0.021$ & -- & -- & -- & -- & -- & -- \\
\hline 49235 & TRICLOPYR，DISSOLVED $(\mu \mathrm{g} / \mathrm{L})$ & 3 & $<0.250$ & $<0.050$ & -- & -- & -- & -- & -- & -- \\
\hline & & ORGANI & COMPOUND & -ORGANONI & ROGEN, T & TAL & & & & \\
\hline 39057 & PROMETRYNE， TOTAL $(\mu \mathrm{g} / \mathrm{L})$ & 1 & 0.100 & -- & -- & -- & -- & -- & -- & -- \\
\hline 39056 & PROMETONE, TOTAL $(\mu \mathrm{g} / \mathrm{L})$ & 1 & $<0.200$ & -- & -- & -- & -- & -- & -- & $-\cdots$ \\
\hline 39054 & SIMETRYNE, TOTAL ( $\mu \mathrm{g} / \mathrm{L})$ & 1 & $<0.100$ & -- & -- & -- & -- & -- & -- & -- \\
\hline 81757 & CYANAZINE，TOTAL $(\mu \mathrm{g} / \mathrm{L})$ & 1 & $<0.200$ & -- & - & -- & -- & -- & -- & -- \\
\hline 77825 & ALACHLOR， TOTAL $(\mu g / L)$ & 1 & $<0.100$ & -- & -- & - & -- & -- & -- & -- \\
\hline 82611 & METRIBUZIN, TOTAL $(\mu \mathrm{g} / \mathrm{L})$ & 1 & $<0.100$ & -- & -- & -- & -- & -- & -- & -- \\
\hline 30311 & TERBACIL, TOTAL $(\mu \mathrm{g} / \mathrm{L})$ & 1 & $<0.200$ & - & -- & -- & -- & -- & -- & -- \\
\hline 30245 & CARBOXIN, TOTAL $(\mu \mathrm{g} / \mathrm{L})$ & 1 & $<0.200$ & -- & -- & -- & -- & -- & -- & -- \\
\hline 30264 & HEXAZINONE, TOTAL $(\mu \mathrm{g} / \mathrm{L})$ & 1 & $<0.200$ & -- & -- & -- & -- & -- & -- & -- \\
\hline 30235 & BUTACHLOR, TOTAL $(\mu \mathrm{g} / \mathrm{L})$ & 1 & $<0.100$ & -- & -- & -- & $\sim$ & -- & -- & -- \\
\hline 30236 & BUTYLATE， TOTAL $(\mu \mathrm{g} / \mathrm{L})$ & 1 & $<0.100$ & -. & -- & -- & -- & -- & -- & -- \\
\hline 75981 & DE-ETHYLATRAZINE， TOTAL $(\mu \mathrm{g} / \mathrm{L})$ & 1 & $<0.200$ & -- & -- & -- & -- & -- & -- & -- \\
\hline 39630 & ATRAZINE, TOTAL $(\mu \mathrm{g} / \mathrm{L})$ & 1 & $<0.100$ & -- & -- & -- & -- & -- & -- & -- \\
\hline 39055 & SIMAZINE, TOTAL $(\mu \mathrm{g} / \mathrm{L})$ & 1 & $<0.100$ & -- & -- & -- & -- & -- & -- & -- \\
\hline
\end{tabular}

NOTE: Multiple detection limits during the period of record may result in different values flagged with a "<."

* Value is estimated by using a log-probability regression to predict the values of data below the detection limit. 
Table 72. Statistical summary of water-quality data at site 40 (CSW03), July 1994 through September 1998-Continued

\begin{tabular}{|c|c|c|c|c|c|c|c|c|c|c|}
\hline \multirow{2}{*}{$\begin{array}{l}\text { PARAM- } \\
\text { ETER } \\
\text { CODE }\end{array}$} & \multirow{2}{*}{ PROPERTY OR CONSTITUENT } & \multicolumn{4}{|c|}{ DESCRIPTIVE STATISTICS } & \multicolumn{5}{|c|}{$\begin{array}{c}\text { PERCENT OF SAMPLES IN WHICH VALU SS WERE LESS THAN OR } \\
\text { EQUAL TO THOSE SH'OWN }\end{array}$} \\
\hline & & $\begin{array}{l}\text { SAMPLE } \\
\text { SIZE }\end{array}$ & MAXIMUM & MINIMUM & MEAN & $95 \%$ & $75 \%$ & $\begin{array}{c}50 \% \\
\text { (medlan) }\end{array}$ & $25 \%$ & $\mathbf{5} \%$ \\
\hline 39024 & PROPAZINE, TOTAL $(\mu \mathrm{g} / \mathrm{L})$ & 1 & $<0.100$ & -- & -- & -- & -- & -- & -- & -- \\
\hline 82184 & AMETRYNE, TOTAL $(\mu \mathrm{g} / \mathrm{L})$ & 1 & 0.100 & -- & -- & -- & -- & -- & -- & -- \\
\hline 39030 & TRIFLURALIN, TOTAL $(\mu \mathrm{g} / \mathrm{L})$ & 1 & $<0.100$ & -- & -- & -- & -- & -- & -- & -- \\
\hline 82612 & METOLACHLOR, TOTAL $(\mu \mathrm{g} / \mathrm{L})$ & 1 & $<0.200$ & -- & -- & -. & .- & -- & -- & -- \\
\hline 30234 & BROMACIL， TOTAL $(\mu \mathrm{g} / L)$ & 1 & $<0.200$ & - - & -- & -- & -- & -- & -. & -- \\
\hline 30255 & DIPHENAMID, TOTAL $(\mu \mathrm{g} / \mathrm{I})$ & 1 & $<0.100$ & -- & -- & -- & -- & -- & -- & -- \\
\hline 30324 & VERNOLATE, TOTAL $(\mu \mathrm{g} / \mathrm{L})$ & 1 & $<0.100$ & -- & -- & -- & -- & -- & -- & -- \\
\hline 30254 & CYCLOATE, TOTAL $(\mu \mathrm{g} / \mathrm{L})$ & 1 & $<0.100$ & -- & -- & - - & -- & -- & -- & -- \\
\hline 30295 & PROPACHLOR， TOTAL $(\mu \mathrm{g} / \mathrm{L})$ & 1 & $<0.100$ & -- & -- & -- & -- & -- & -- & -- \\
\hline 75980 & DE-ISOPROPYLATRAZIN, TOTAL $(\mu \mathrm{g} / \mathrm{L})$ & 1 & $<0.200$ & -- & -- & -- & -- & -- & -- & -- \\
\hline \multicolumn{11}{|c|}{ ORGANIC COMPOUNDS-HERBICIDES, TOTAL } \\
\hline 39730 & $2,4-\mathrm{D}$, TOTAL $(\mu \mathrm{g} / \mathrm{L})$ & 1 & $<0.010$ & -- & -- & -- & -- & -- & -- & -- \\
\hline 39760 & SILVEX, TOTAL $(\mu \mathrm{g} / \mathrm{L})$ & 1 & $<0.010$ & -- & -- & -- & -- & -- & -- & -- \\
\hline 39720 & PICLORAM, TOTAL $(\mu \mathrm{g} / \mathrm{L})$ & 1 & $<0.010$ & -- & -- & -- & -- & -- & -- & -- \\
\hline 39740 & $2,4,5-\mathrm{T}$, TOTAL $\langle\mu \mathrm{g} / \mathrm{L}\rangle$ & 1 & $<0.010$ & -- & -- & -- & -- & -- & -- & -- \\
\hline 82183 & $2,4-\mathrm{DP}$, TOTAL $(\mu \mathrm{g} / \mathrm{L})$ & 1 & $<0.010$ & -- & -- & $\therefore$ & -- & -- & -- & -- \\
\hline 82052 & DICAMBA, TOTAL $(\mu \mathrm{g} / \mathrm{L})$ & 1 & $<0.010$ & -- & -- & -- & -- & -- & -- & $\ldots$ \\
\hline \multicolumn{11}{|c|}{ ORGANIC COMPOUNDS-CARBAMATE PESTICIDES, TOTAL } \\
\hline 39750 & SEVIN, TOTAL $(\mu \mathrm{g} / \mathrm{L})$ & 1 & $<0.500$ & -- & -- & -- & -- & -- & -- & -- \\
\hline 39051 & METHOMYL， TOTAL $(\mu \mathrm{g} / \mathrm{L})$ & 1 & $<0.500$ & -- & -- & -- & -- & -- & -- & -- \\
\hline 82619 & ALDICARD, TOTAL $(\mu \mathrm{g} / \mathrm{L})$ & 1 & $<0.500$ & -- & -- & -- & -- & -- & -- & -- \\
\hline 30296 & PROPOXUR, TOTAL $(\mu \mathrm{g} / \mathrm{L})$ & 1 & $<0.500$ & -- & -- & -- & -- & -- & - & -- \\
\hline 39052 & PROPHAM， TOTAL $(\mu \mathrm{g} / \mathrm{L})$ & 1 & $<0.500$ & -- & -- & -- & -- & -- & -- & -- \\
\hline 82615 & CARBOFURAN, TOTAL $(\mu \mathrm{g} / \mathrm{L})$ & 1 & $<0.500$ & -- & -- & -- & -- & -- & -- & -- \\
\hline 77441 & 1 -NAPHTHOL, TOTAL $(\mu \mathrm{g} / \mathrm{L})$ & 1 & $<0.500$ & -- & -- & -- & -- & -- & -- & -- \\
\hline 30282 & METHIOCARB， TOTAL $(\mu \mathrm{g} / \mathrm{L})$ & 1 & $<0.500$ & -- & -- & -- & -- & -. & -- & -- \\
\hline
\end{tabular}

NOTE: Multiple detection limits during the period of record may result in different values flagged with a "<."

* Value is estimated by using a log-probability regression to predict the values of data below the detection limit. 
Table 73. Statistical summary of water-quality data at site 41 (CSW02), May 1994 through September 1998

\begin{tabular}{|c|c|c|c|c|c|c|c|c|c|c|}
\hline \multirow{2}{*}{$\begin{array}{c}\text { PARAM- } \\
\text { ETER } \\
\text { CODE }\end{array}$} & \multirow{2}{*}{ PROPERTY OR CONSTITUENT } & \multicolumn{4}{|c|}{ DESCRIPTIVE STATISTICS } & \multicolumn{5}{|c|}{$\begin{array}{l}\text { PERCENT OF SAMPLES IN WHICH VALUES WERE LESS THAN OR } \\
\text { EQUALTO THOSE SHOW'N }\end{array}$} \\
\hline & & $\begin{array}{l}\text { SAMPLE } \\
\text { SIZE }\end{array}$ & MAXIMUM & MINIMUM & MEAN & $95 \%$ & $75 \%$ & $\begin{array}{c}50 \% \\
\text { (medlan) }\end{array}$ & $25 \%$ & $\mathbf{5} \%$ \\
\hline \multicolumn{11}{|c|}{ PHYSICAL AND CHEMICAL PROPERTIES } \\
\hline$\overline{00061}$ & INSTANTANEOUS DISCHARGE $\left(\mathrm{ft}^{3} / \mathrm{s}\right)$ & 98 & 253.000 & 0.030 & 15.850 & 99.150 & 7.625 & 1.200 & 0.390 & 0.158 \\
\hline 00010 & WATER TEMPERATURE $\left({ }^{\circ} \mathrm{C}\right)$ & 90 & 28.000 & 5.100 & 16.477 & 26.545 & 20.125 & 16.500 & 13.250 & 6.870 \\
\hline 90095 & SPECIEIC CONDUCTANCE, LAB $\left(\mu \mathrm{S} / \mathrm{cm}\right.$ at $\left.25^{\circ} \mathrm{C}\right)$ & 64 & 265.000 & 18.000 & 75.875 & 166.500 & 131.500 & 49.500 & 30.000 & 20.500 \\
\hline 00095 & $\begin{array}{l}\text { SPECIFIC CONDUCTANCE, FIELD ( } \mu \mathrm{S} / \mathrm{cm} \text { at } 25 \\
\left.{ }^{\circ} \mathrm{C}\right)\end{array}$ & 79 & 252.000 & 16.000 & 73.418 & 165.000 & 130.000 & 44.000 & 25.000 & 18.000 \\
\hline 00403 & $\mathrm{pH}$, LAB (STANDARD pH UNITS) & 64 & 7.400 & 5.500 & 6.711 & 7.200 & 6.900 & 6.800 & 6.425 & 5.825 \\
\hline 00400 & $\mathrm{pH}$, EIELD (STANDARD pH UNITS) & 75 & 7.200 & 5.900 & 6.760 & 7.100 & 6.900 & 6.800 & 6.600 & 6.300 \\
\hline 90410 & ALKALINITY, LAB (mg/L as $\mathrm{CaCO}_{3}$ ) & 63 & 61.000 & 2.000 & 19.567 & 55.000 & 30.000 & 10.000 & 6.000 & 3.200 \\
\hline 80154 & SUSPENDED SEDIMENT $(\mathrm{mg} / \mathrm{L})$ & 67 & 9370.000 & 7.000 & 259.104 & 669.200 & 156.000 & 46.000 & 26.000 & 9.000 \\
\hline 00530 & $\begin{array}{l}\text { RESIDUE ON EVAPORATION AT } 105^{\circ} \mathrm{C} \text {, SUSPENDED } \\
(\mathrm{mg} / \mathrm{L})\end{array}$ & 39 & 9800.000 & 4.000 & 342.256 & 708.000 & 150.000 & 22.000 & 14.000 & 7.000 \\
\hline 00535 & RESIDUE VOLATILE, SUSPENDED (mg/L) & 62 & 1090.000 & $<1.000$ & $38.687^{*}$ & 123.000 & 30.000 & 7.000 & 3.000 & $<1.000$ \\
\hline 70300 & DISSOLVED SOLIDS, RESIDUE AT $180^{\circ} \mathrm{C}$ (mg/L) & 62 & 224.000 & 6.000 & 59.435 & 148.900 & 101.750 & 39.000 & 20.000 & 13.450 \\
\hline 00310 & 5 DAY BIOCHEMICAL OXYGEN DEMAND $(\mathrm{mg} / \mathrm{L})$ & 48 & 30.000 & $<2.000$ & $7.843 *$ & 20.000 & 9.200 & 5.800 & 3.700 & $<2.000$ \\
\hline 00340 & CHEMICAL OXYGEN DEMAND $(\mathrm{mg} / \mathrm{L})$ & 63 & 250.000 & $<5.000$ & 43.184 * & 120.000 & 47.000 & 30.000 & 20.000 & $<10.000$ \\
\hline & & & NUTRIENTS, T & AL AND DIS & SSOLVED & & & & & \\
\hline 00625 & $\begin{array}{l}\text { NITROGEN AMMONIA + ORGANIC, } \\
\text { TOTAL (mg/L as N) }\end{array}$ & 65 & 16.000 & 0.300 & 1.676 & 7.710 & 1.550 & 0.900 & 0.660 & $0 . \overline{319}$ \\
\hline 00631 & $\mathrm{NO}_{2}+\mathrm{NO}_{3}$, DISSOLVED $(\mathrm{mg} / \mathrm{L}$ as $\mathrm{N})$ & 65 & 1.800 & 0.100 & 0.633 & 1.518 & 1.090 & 0.410 & 0.240 & 0.119 \\
\hline 00608 & NITROGEN AMMONIA, DISSOLVED (mg/L as N) & 65 & 1.600 & 0.023 & 0.297 & 1.189 & 0.405 & 0.190 & 0.100 & 0.053 \\
\hline 00605 & NITROGEN ORGANIC, TOTAL (mg/L as N) & 65 & 16.000 & 0.160 & 1.387 & 6.810 & 1.250 & 0.720 & 0.435 & 0.203 \\
\hline 00600 & NITROGEN，TOTAL (mg/L as N) & 65 & 17.000 & 0.540 & 2.307 & 8.670 & 2.150 & 1.500 & 1.150 & 0.645 \\
\hline 00665 & PHOSPHORUS, TOTAL (mg/L as P) & 65 & 1.580 & 0.060 & 0.344 & 1.100 & 0.410 & 0.260 & 0.130 & 0.060 \\
\hline 00671 & PHOSPHORUS ORTHO, DISSOLVED (mg/L as P) & 65 & 0.980 & 0.010 & 0.158 & 0.688 & 0.175 & 0.100 & 0.040 & 0.015 \\
\hline & & & OLL AND & REASE, TOT & & & & & & \\
\hline 00556 & OIL AND GREASE, TOTAL (mg/L) & 20 & 5.000 & $<1.000$ & $1.739^{*}$ & 5.000 & 2.000 & $<1.000$ & $<1.000$ & $<1.000$ \\
\hline & & & ORGANIC & ARBON, TOO & TAL & & & & & \\
\hline 00680 & CARBON ORGANIC, TOTAL (mg/L) & 40 & 82.000 & 2.000 & 17.270 & 71.000 & 16.750 & 11.000 & 7.525 & 2.995 \\
\hline & & & & IFORM & & & & & & \\
\hline 31679 & FECAL STREPTOCOCCI (COlonies per $100 \mathrm{~mL}$ ) & 32 & 115000.000 & $50 . \overline{000}$ & $\overline{3} 1411.563$ & 108500.000 & 59000.000 & 16500.000 & 1875.000 & 349.000 \\
\hline 31616 & FECAL COLIFORM (Colonies per $100 \mathrm{~mL}$ ) & 32 & 420000.000 & 110.000 & 58085.938 & 342000.063 & 47000.000 & 25500.000 & 2325.000 & 214.000 \\
\hline & & ORC & FANIC COMPOI & DS—PESTIC & IDES, TOTAL & & & & & \\
\hline 39330 & ALDRIN, TOTAL $(\mu \mathrm{g} / \mathrm{L})$ & 6 & $<0.010$ & $<0.010$ & -- & $<0.010$ & $<0.010$ & $<0.010$ & $<0.010$ & $<0.010$ \\
\hline 39340 & LINDANE，TOTAL $(\mu \mathrm{g} / \mathrm{L})$ & 6 & 0.010 & $<0.010$ & -- & 0.010 & $<0.010$ & $<0.010$ & $<0.010$ & $<0.010$ \\
\hline 39350 & CHLORDANE, TOTAL $(\mu \mathrm{g} / \mathrm{L})$ & 6 & 0.100 & $<0.100$ & -- & 0.100 & 0.100 & $<0.100$ & $<0.100$ & $<0.100$ \\
\hline 39370 & DDT, TOTAL $(\mu \mathrm{g} / \mathrm{L})$ & 6 & 0.020 & $<0.010$ & -- & 0.020 & $<0.010$ & $<0.010$ & $<0.010$ & $<0.010$ \\
\hline 39365 & DDE, TOTAL $(\mu \mathrm{g} / \mathrm{L})$ & 6 & $<0.010$ & $<0.010$ & -- & $<0.010$ & $<0.010$ & $<0.010$ & $<0.010$ & $<0.010$ \\
\hline 39360 & DDD, TOTAL $(\mu \mathrm{g} / L)$ & 6 & $<0.010$ & $<0.010$ & -- & $<0.010$ & $<0.010$ & $<0.010$ & $<0.010$ & $<0.010$ \\
\hline 39380 & DIELDRIN, TOTAL $(\mu \mathrm{g} / \mathrm{L})$ & 6 & 0.020 & $<0.010$ & -- & 0.020 & 0.010 & $<0.010$ & $<0.010$ & $<0.010$ \\
\hline 39388 & ENDOSULFAN, TOTAL $(\mu \mathrm{g} / \mathrm{L})$ & 6 & $<0.010$ & $<0.010$ & -- & $<0.010$ & $<0.010$ & $<0.010$ & $<0.010$ & $<0.010$ \\
\hline 39390 & ENDRIN， TOTAL $(\mu \mathrm{g} / \mathrm{L})$ & 6 & $<0.010$ & $<0.010$ & -- & $<0.010$ & $<0.010$ & $<0.010$ & $<0.010$ & $<0.010$ \\
\hline 39410 & HEPTACHLOR, TOTAL $(\mu \mathrm{g} / \mathrm{L})$ & 6 & $<0.010$ & $<0.010$ & -- & $<0.010$ & $<0.010$ & $<0.010$ & $<0.010$ & $<0.010$ \\
\hline 39420 & HEPTACHLOR EPOXIDE， TOTAL $(\mu \mathrm{g} / \mathrm{L})$ & 5 & 0.010 & $<0.010$ & - & 0.010 & $<0.010$ & $<0.010$ & $<0.010$ & $<0.010$ \\
\hline 39516 & PCB, TOTAL $(\mu \mathrm{g} / L)$ & 6 & 0.120 & $<0.100$ & -- & 0.120 & $<0.200$ & $<0.100$ & $<0.100$ & $<0.100$ \\
\hline 39400 & TOXAPHENE, TOTAL $(\mu \mathrm{g} / \mathrm{L})$ & 6 & $<1.000$ & $<1.000$ & -- & $<1.000$ & $<1.000$ & $<1.000$ & $<1.000$ & $<1.000$ \\
\hline 39034 & PERTHANE, TOTAL $(\mu \mathrm{g} / \mathrm{L})$ & 6 & $<0.100$ & $<0.100$ & -- & $<0.100$ & $<0.100$ & $<0.100$ & $<0.100$ & $<0.100$ \\
\hline 39570 & DIAZINON, TOTAL $(\mu \mathrm{g} / \mathrm{L})$ & 6 & 0.300 & $<0.010$ & $\rightarrow$ & 0.300 & 0.130 & 0.010 & $<0.010$ & $<0.010$ \\
\hline 39398 & ETHION, TOTAL $(\mu \mathrm{g} / \mathrm{L})$ & 6 & $<0.010$ & $<0.010$ & - & $<0.010$ & $<0.010$ & $<0.010$ & $<0.010$ & $<0.010$ \\
\hline 39530 & MALATHION, TOTAL $(\mu \mathrm{g} / L)$ & 6 & 0.100 & $<0.010$ & -- & 0.100 & 0.030 & $<0.010$ & $<0.010$ & $<0.010$ \\
\hline 39600 & METHYL PARATHION, TOTAL $(\mu \mathrm{g} / \mathrm{L})$ & 6 & $<0.010$ & $<0.010$ & -- & $<0.010$ & $<0.010$ & $<0.010$ & $<0.010$ & $<0.010$ \\
\hline 39540 & PARATHION, TOTAL $(\mu \mathrm{g} / \mathrm{L})$ & 6 & $<0.010$ & $<0.010$ & -- & $<0.010$ & $<0.010$ & $<0.010$ & $<0.010$ & $<0.010$ \\
\hline 39786 & TRITHION， TOTAL $(\mu \mathrm{g} / \mathrm{L})$ & 6 & 0.010 & $<0.010$ & -- & 0.010 & $<0.010$ & $<0.010$ & $<0.010$ & $<0.010$ \\
\hline 39250 & PCN, TOTAL $(\mu \mathrm{g} / \mathrm{L})$ & 6 & $<0.100$ & $<0.100$ & -- & $<0.100$ & $<0.100$ & $<0.100$ & $<0.100$ & $<0.100$ \\
\hline 39480 & METHOXYCHLOR， TOTAL $(\mu \mathrm{g} / \mathrm{L})$ & 6 & $<0.010$ & $<0.010$ & -- & $<0.010$ & $<0.010$ & $<0.010$ & $<0.010$ & $<0.010$ \\
\hline 39755 & MIREX, TOTAL $(\mu \mathrm{g} / \mathrm{L})$ & 6 & $<0.010$ & $<0.010$ & -- & $<0.010$ & $<0.010$ & $<0.010$ & $<0.010$ & $<0.010$ \\
\hline 39011 & DISYSTON, TOTAL $(\mu \mathrm{g} / L)$ & 4 & $<0.010$ & $<0.010$ & -- & - & -- & $\ldots$ & $\ldots$ & -- \\
\hline 39023 & PHORATE, TOTAL $(\mu \mathrm{g} / \mathrm{L})$ & 6 & $<0.010$ & $<0.010$ & -- & $<0.010$ & $<0.010$ & $<0.010$ & $<0.010$ & $<0.010$ \\
\hline 38932 & CHLORPYRIFOS, TOTAL $(\mu \mathrm{g} / \mathrm{L})$ & 6 & 0.050 & $<0.010$ & -. & 0.050 & 0.010 & $<0.010$ & $<0.010$ & $<0.010$ \\
\hline 39040 & DEF, TOTAL $(\mu \mathrm{g} / L)$ & 6 & $<0.010$ & $<0.010$ & -- & $<0.010$ & $<0.010$ & $<0.010$ & $<0.010$ & $<0.010$ \\
\hline 82614 & FONOFOS, TOTAL $(\mu \mathrm{g} / \mathrm{L})$ & 6 & $<0.010$ & $<0.010$ & -- & $<0.010$ & $<0.010$ & $<0.010$ & $<0.010$ & $<0.010$ \\
\hline & & & OLATILE ORGA & C COMPOU & NDS, TOTAL & & & & & \\
\hline 34210 & ACROLEIN, TOTAL $(\mu \mathrm{g} / \mathrm{L})$ & 1 & $<20.000$ & -- & -- & -- & -- & -- & -- & -- \\
\hline 34215 & ACRYLONITRILE, TOTAL $(\mu \mathrm{g} / \mathrm{L})$ & 2 & $<20.000$ & $<5.000$ & -- & -- & -- & -- & -- & -- \\
\hline 34030 & BENZENE, TOTAL $(\mu \mathrm{g} / \mathrm{L})$ & 13 & $<2.000$ & $<0.200$ & -- & -- & -- & -- & -- & -- \\
\hline 32104 & BROMOFORM，TOTAL $(\mu \mathrm{g} / \mathrm{L})$ & 13 & $<2.000$ & $<0.200$ & -- & -- & -- & -- & - & -- \\
\hline 32102 & CARBON TETRACHLORIDE, TOTAL $(\mu \mathrm{g} / \mathrm{L})$ & 13 & $<2.000$ & $<0.200$ & $\ldots$ & -- & $\ldots$ & -- & -- & -- \\
\hline 34301 & CHLOROBENZENE, TOTAL $(\mu \mathrm{g} / L)$ & 13 & $<2.000$ & $<0.200$ & -- & -- & -- & -- & -- & -- \\
\hline 32105 & CHLORODIBROMOMETHANE， TOTAL $(\mu \mathrm{g} / \mathrm{L})$ & 13 & $<2.000$ & $<0.200$ & -- & -- & -- & -- & -- & -- \\
\hline 34311 & CHLOROETHANE, TOTAL $(\mu \mathrm{g} / \mathrm{L})$ & 13 & $<2.000$ & $<0.200$ & -- & - & - & -- & -- & -- \\
\hline 32106 & CHLOROFORM, TOTAL $(\mu \mathrm{g} / \mathrm{L})$ & 13 & $<2.000$ & $<0.200$ & -- & - & -- & -- & -- & -- \\
\hline 34496 & 1,1 -DICHLOROETHANE，TOTAL $(\mu \mathrm{g} / \mathrm{L})$ & 13 & $<2.000$ & $<0.200$ & -- & -- & -- & -- & -- & -- \\
\hline 32103 & 1,2-DICHLOROETHANE, TOTAL $(\mu \mathrm{g} / \mathrm{L})$ & 13 & $<2.000$ & $<0.200$ & -- & -- & -- & - & $\cdots$ & -- \\
\hline 34501 & 1,1 -DICHLOROETHYLENE, TOTAL $(\mu \mathrm{g} / \mathrm{L})$ & 13 & $<2.000$ & $<0.200$ & -- & -- & -- & -- & -- & -- \\
\hline 34541 & 1,2-DICHLOROPROPANE， TOTAL $(\mu \mathrm{g} / \mathrm{L})$ & 13 & $<2.000$ & $<0.200$ & -- & -- & -- & -- & -- & -- \\
\hline 34371 & ETHYLBENZENE， TOTAL $(\mu \mathrm{g} / \mathrm{L})$ & 13 & $<2.000$ & $<0.200$ & -- & -. & -- & -- & -- & -- \\
\hline 34413 & METHYL BROMIDE， TOTAL $(\mu \mathrm{g} / \mathrm{L})$ & 13 & $<2.000$ & $<0.200$ & -- & -- & -- & -- & -- & -- \\
\hline 34423 & METHYLENE CHLORIDE， TOTAL $(\mu \mathrm{g} / \mathrm{L})$ & 13 & $<2.000$ & $<0.200$ & -- & $\ldots$ & -- & -- & -- & -- \\
\hline 34516 & $1,1,2,2$-TETRACHLOROETHANE, TOTAL $(\mu \mathrm{g} / \mathrm{L})$ & 13 & $<2.000$ & $<0.200$ & - & -- & -- & -- & -- & -- \\
\hline
\end{tabular}

NOTE: Multiple detection limits during the period of record may result in different values flagged with a "<."

* Value is estimated by using a log-probability regression to predict the values of data below the detection limit. 
Table 73. Statistical summary of water-quality data at site 41 (CSW02), May 1994 through September 1998-Continued

\begin{tabular}{|c|c|c|c|c|c|c|c|c|c|c|}
\hline \multirow{2}{*}{$\begin{array}{c}\text { PARAM- } \\
\text { ETER } \\
\text { CODE }\end{array}$} & \multirow{2}{*}{ PROPERTY OR CONSTITUENT } & & DESCRIPTI & STATISTICS & & PERCENT O & $\begin{array}{r}\text { SAMPLES I } \\
\text { EQUA }\end{array}$ & $\begin{array}{l}\text { WHICH VALI } \\
\text { TO THOSE S }\end{array}$ & $\begin{array}{l}\text { SWERE LE } \\
\text { OWN }\end{array}$ & THAN OR \\
\hline & & $\begin{array}{c}\text { SAMPLE } \\
\text { SIZE }\end{array}$ & MAXIMUM & MINIMUM & MEAN & $95 \%$ & $75 \%$ & $\begin{array}{c}50 \% \\
\text { (median) }\end{array}$ & $25 \%$ & $5 \%$ \\
\hline 34475 & TETRACHLOROETHYLENE， TOTAL $(\mu \mathrm{g} / \mathrm{L})$ & 13 & 1.300 & $<0.400$ & $0.706^{*}$ & 1.300 & $0 . \overline{900}$ & 0.600 & $<0.400$ & $<0.400$ \\
\hline 34010 & TOLUENE, TOTAL $(\mu \mathrm{g} / \mathrm{L})$ & 13 & 8.000 & $<0.200$ & $1.255^{*}$ & 8.000 & 0.500 & 0.224 & $<2.000$ & $<0.400$ \\
\hline 34546 & 1,2 -TRANSDICHLOROETHENE, TOTAL $(\mu \mathrm{g} / \mathrm{L})$ & 13 & $<2.000$ & $<0.200$ & -- & -- & -- & -- & -- & - \\
\hline 34506 & $1,1,1$-TRICHLOROETHANE， TOTAL $(\mu \mathrm{g} / \mathrm{L})$ & 13 & $<2.000$ & $<0.200$ & -- & -- & -- & - - & - & -- \\
\hline 34511 & $1,1,2$-TRICHLOROETHANE，TOTAL $\{\mu \mathrm{g} / \mathrm{L}\}$ & 13 & $<2.000$ & $<0.200$ & -- & -- & -- & -- & -- & -- \\
\hline 39180 & TRICHLOROETHYLENE, TOTAL $(\mu \mathrm{g} / \mathrm{L})$ & 13 & $<2.000$ & $<0.200$ & -- & -- & - & -- & -- & -- \\
\hline 39175 & VINYL CHLORIDE, TOTAL $(\mu \mathrm{g} / \mathrm{L})$ & 13 & $<2.000$ & $<0.200$ & -- & -- & -- & -- & -- & -. \\
\hline 30217 & DIBROMOMETHANE， TOTAL $\{\mu \mathrm{g} / \mathrm{L}\rangle$ & 13 & $<2.000$ & $<0.200$ & -- & -- & -- & -- & -- & - \\
\hline 32101 & DICHLOROBROMOMETHANE, TOTAL $(\mu \mathrm{g} / \mathrm{L})$ & 13 & $<2.000$ & $<0.200$ & -. & - & -- & -- & -- & - \\
\hline 34668 & DICHLORODIFLUOROMETHANE, TOTAL ( $\mu \mathrm{g} / \mathrm{L})$ & 13 & $<2.000$ & $<0.200$ & -- & -- & -- & -- & -- & -- \\
\hline 34488 & TRICHLOROFLUOROMETHANE， TOTAL $(\mu \mathrm{g} / \mathrm{L})$ & 13 & $<2.000$ & $<0.200$ & -- & -- & -- & -- & -- & -- \\
\hline 77651 & 1,2 -DIBROMOETHANE, TOTAL $(\mu \mathrm{g} / \mathrm{L})$ & 13 & $<2.000$ & $<0.200$ & -- & -- & -- & - & -- & -- \\
\hline 34418 & METHYLCHLORIDE，TOTAL $(\mu \mathrm{g} / \mathrm{L})$ & 13 & $<2.000$ & $<0.200$ & -- & -- & -- & -- & -- & -- \\
\hline 34704 & CIS 1,3 -DICHLOROPROPENE, TOTAL $\{\mu \mathrm{g} / \mathrm{L}\}$ & 13 & $<2.000$ & $<0.200$ & -- & -- & -- & -- & -- & -- \\
\hline 34699 & TRANS 1,3 -DICHLOROPROPENE, TOTAL $(\mu \mathrm{g} / \mathrm{L})$ & 13 & $<2.000$ & $<0.200$ & -- & -- & -- & -- & -- & -- \\
\hline 77128 & STYRENE, TOTAL $(\mu \mathrm{g} / \mathrm{L})$ & 13 & $<2.000$ & $<0.200$ & -- & -- & - & -- & -- & -- \\
\hline 81551 & XYLENE, TOTAL $(\mu \mathrm{g} / \mathrm{L})$ & 13 & $<2.000$ & $<0.200$ & -- & -- & -- & -- & -- & -- \\
\hline 82625 & DIBROMOCHLOROPROPANE, TOTAL $(\mu \mathrm{g} / \mathrm{L})$ & 13 & $<10.000$ & $<1.000$ & -- & -- & -- & -- & -- & -- \\
\hline 77168 & 1,1 -DICHLOROPROPENE, TOTAL $\{\mu \mathrm{g} / \mathrm{L}\}$ & 13 & $<2.000$ & $<0.200$ & -- & -- & -- & -- & -- & -- \\
\hline 77170 & 2,2 -DICHLOROPROPANE，TOTAL $(\mu \mathrm{g} / \mathrm{L})$ & 13 & $<2.000$ & $<0.200$ & -- & - & -- & -- & -- & -- \\
\hline 77173 & 1,3-DICHLOROPROPANE, TOTAL $\{\mu \mathrm{g} / \mathrm{L}\rangle$ & 13 & $<2.000$ & $<0.200$ & -- & -- & -- & -- & -- & -- \\
\hline 77275 & O-CHLOROTOLUENE, TOTAL $(\mu \mathrm{g} / \mathrm{L})$ & 13 & $<2.000$ & $<0.200$ & -- & -- & -- & -- & -- & -- \\
\hline 77277 & P-CHLOROTOLUENE, TOTAL $(\mu \mathrm{g} / \mathrm{L})$ & 13 & $<2.000$ & $<0.200$ & -- & -- & - & - & -- & -- \\
\hline 77443 & 123-TRICHLOROPROPANE，TOTAL $(\mu \mathrm{g} / \mathrm{L})$ & 13 & $<2.000$ & $<0.200$ & -- & -- & -- & -- & -- & - \\
\hline 77562 & 1112-TETRACHLOROETHANE，TOTAL $(\mu \mathrm{g} / \mathrm{L})$ & 13 & $<2.000$ & $<0.200$ & -- & -- & -- & -- & -- & -- \\
\hline 78032 & TERTBUTYL METHYL ETHER, TOTAL $(\mu \mathrm{g} / \mathrm{L})$ & 13 & $<2.000$ & $<0.200$ & -- & -- & -- & -- & -- & -- \\
\hline 77297 & BROMOCHLORO METHANE, TOTAL $(\mu \mathrm{g} / \mathrm{L})$ & 13 & $<2.000$ & $<0.200$ & -- & -- & -- & -- & -- & -- \\
\hline 77093 & CIS-1,2-DICHLOROETHENE, TOTAL $(\mu \mathrm{g} / \mathrm{L})$ & 13 & $<2.000$ & $<0.200$ & -- & -- & -- & -- & -- & -- \\
\hline 34576 & 2 -CHLOROETHYL VINYL ETHER, TOTAL $\{\mu \mathrm{g} / L)$ & 8 & $<10.000$ & $<1.000$ & -- & -- & -- & -- & -- & $\ldots$ \\
\hline 77223 & ISOPROPYL BENZENE, TOTAL $\{\mu \mathrm{g} / \mathrm{L}\}$ & 13 & $<2.000$ & $<0.200$ & -- & -- & -- & -- & -- & -- \\
\hline 77224 & N-PROPYL BENZENE，TOTAL $(\mu \mathrm{g} / \mathrm{L})$ & 13 & 0.600 & $<0.200$ & -- & 0.600 & $<0.800$ & $<0.400$ & $<0.200$ & $<0.200$ \\
\hline 77353 & TERTBUTYL BENZENE, TOTAL $(\mu \mathrm{g} / \mathrm{L})$ & 13 & $<2.000$ & $<0.200$ & -- & -- & -- & -- & -- & -- \\
\hline 77222 & PSEUDOCUMENE, TOTAL $(\mu \mathrm{g} / \mathrm{L})$ & 13 & 6.800 & $<0.200$ & -- & 6.800 & $<0.800$ & $<0.400$ & $<0.200$ & $<0.200$ \\
\hline 77350 & SEC-BUTYL BENZENE, TOTAL $(\mu \mathrm{g} / \mathrm{L})$ & 13 & $<2.000$ & $<0.200$ & -- & -- & -- & -- & -- & -- \\
\hline 77356 & P-ISOPROPYL TOLUENE, TOTAL $(\mu \mathrm{g} / \mathrm{L})$ & 13 & $<2.000$ & $<0.200$ & -- & -- & -- & -- & -- & -- \\
\hline 77342 & N-BUTYL BENZENE, TOTAL $(\mu \mathrm{g} / \mathrm{L})$ & 13 & $<2.000$ & $<0.200$ & -- & -- & -- & -- & -- & $\rightarrow$ \\
\hline 77613 & $1,2,3$-TRICHLOROBENZENE, TOTAL $(\mu \mathrm{g} / \mathrm{L})$ & 13 & $<2.000$ & $<0.200$ & -- & -- & - & -- & -- & -- \\
\hline 77652 & FREON-113, TOTAL $(\mu \mathrm{g} / \mathrm{L})$ & 13 & $<2.000$ & $<0.200$ & -- & -- & -- & -- & -- & -- \\
\hline 77226 & MESITYLENE， TOTAL $(\mu \mathrm{g} / \mathrm{L})$ & 13 & 1.800 & $<0.200$ & -- & 1.800 & $<0.800$ & $<0.400$ & $<0.200$ & $<0.200$ \\
\hline 81555 & BROMOBENZENE， TOTAL $(\mu \mathrm{g} / \mathrm{L})$ & 13 & $<2.000$ & $<0.200$ & -- & -- & -- & -- & -- & -- \\
\hline 34551 & $1,2,4$-TRICHLOROBENZENE, TOTAL $(\mu \mathrm{g} / \mathrm{L})$ & 13 & $<2.000$ & $<0.200$ & -- & -- & -- & $\rightarrow$ & -- & -- \\
\hline 34536 & 1,2-DICHLOROBENZENE, TOTAL $(\mu \mathrm{g} / \mathrm{L})$ & 13 & $<2.000$ & $<0.200$ & -- & -- & -- & -- & -- & -- \\
\hline 34566 & 1,3-DICHLOROBENZENE, TOTAL $(\mu \mathrm{g} / \mathrm{L})$ & 13 & $<2.000$ & $<0.200$ & -- & -- & -- & -- & -- & -- \\
\hline 34571 & 1,4 -DICHLOROBENZENE, TOTAL $\{\mu \mathrm{g} / \mathrm{L}\rangle$ & 13 & $<2.000$ & $<0.200$ & -- & -- & -- & -- & -- & -- \\
\hline 39702 & HEXACHLOROBUTADIENE, TOTAL $(\mu \mathrm{g} / \mathrm{L})$ & 13 & $<2.000$ & $<0.200$ & -- & -- & -- & -- & -- & -- \\
\hline 34696 & NAPHTHALENE, TOTAL $(\mu \mathrm{g} / \mathrm{L})$ & 13 & $<2.000$ & $<0.200$ & -- & -- & -- & -- & -- & -- \\
\hline & & MET & ILS AND MIN & CONSTTTUI & NTS, TOTAL & & & & & \\
\hline 01097 & ANTIMONY, TOTAL $(\mu \mathrm{g} / \mathrm{L}$ as $\mathrm{Sb})$ & 43 & 5.000 & $<1.000$ & -- & 1.000 & $<1.000$ & $<1.000$ & $<1.000$ & $<1.000$ \\
\hline 01002 & ARSENIC, TOTAL $(\mu \mathrm{g} / \mathrm{L}$ as As $)$ & 43 & 17.000 & $<1.000$ & $0.844^{\star}$ & 2.000 & $<1.000$ & $<1.000$ & $<1.000$ & $<1.000$ \\
\hline 01012 & BERYLLIUM, TOTAL $(\mu \mathrm{g} / \mathrm{L}$ as Be) & 12 & $<10.000$ & $<10.000$ & -- & $<10.000$ & $<10.000$ & $<10.000$ & $<10.000$ & $<10.000$ \\
\hline 01027 & CADMIUM, TOTAL $(\mu \mathrm{g} / \mathrm{L}$ as $\mathrm{Cd})$ & 12 & $<1.000$ & $<1.000$ & -- & $<1.000$ & $<1.000$ & $<1.000$ & $<1.000$ & $<1.000$ \\
\hline 01034 & CHROMIUM, TOTAL $(\mu \mathrm{g} / \mathrm{L}$ as $\mathrm{Cr})$ & 43 & 190.000 & 1.000 & 10.721 & 25.200 & 9.000 & 4.000 & 3.000 & 1.000 \\
\hline 01042 & COPPER, TOTAL $(\mu \mathrm{g} / \mathrm{L}$ as $\mathrm{Cu})$ & 43 & 270.000 & $<1.000$ & $18.346 *$ & 37.000 & 18.000 & 10.000 & 5.000 & $<1.000$ \\
\hline 01051 & LEAD, TOTAL $(\mu \mathrm{g} / \mathrm{L}$ as $\mathrm{Pb})$ & 43 & 190.000 & 1.000 & 21.349 & 86.000 & 26.000 & 13.000 & 6.000 & 1.200 \\
\hline 71900 & MERCURY, TOTAL $(\mu \mathrm{g} / \mathrm{L}$ as $\mathrm{Hg})$ & 12 & 0.100 & $<0.100$ & -- & 0.100 & $<0.100$ & $<0.100$ & $<0.100$ & $<0.100$ \\
\hline 01067 & NICKEL, TOTAL $(\mu \mathrm{g} / \mathrm{L}$ as Ni) & 43 & 52.000 & $<1.000$ & $4.485^{\star}$ & 10.000 & 5.000 & 3.000 & 1.000 & $<1.000$ \\
\hline 01147 & SELENIUM, TOTAL $(\mu \mathrm{g} / \mathrm{L}$ as Se) & 12 & 1.000 & $<1.000$ & - & 1.000 & $<1.000$ & $<1.000$ & $<1.000$ & $<1.000$ \\
\hline 01077 & SILVER, TOTAL $(\mu \mathrm{g} / \mathrm{L}$ as $\mathrm{Ag})$ & 12 & $<1.000$ & $<1.000$ & -- & $<1.000$ & $<1.000$ & $<1.000$ & $<1.000$ & $<1.000$ \\
\hline 01092 & ZINC, TOTAL $(\mu \mathrm{g} / \mathrm{L}$ as $\mathrm{Zn})$ & 43 & 860.000 & 10.000 & 125.814 & 354.000 & 150.000 & 100.000 & 60.000 & 20.000 \\
\hline 00720 & CYANIDE, TOTAL (mg/L as $\mathrm{Cn}$ ) & 12 & $<0.010$ & $<0.010$ &.- & $<0.010$ & $<0.010$ & $<0.010$ & $<0.010$ & $<0.010$ \\
\hline & & ORGAI & IC COMPOUN & -PEESTICID & S, DISSOLVE & & & & & \\
\hline 49260 & ACETOCHLOR, DISSOLVED $(\mu \mathrm{g} / \overline{\mathrm{L}})$ & 4 & $<0 . \overline{002}$ & $<0.002$ & -- & -- & -- & -- & -- & -- \\
\hline 46342 & ALACHLOR， DISSOLVED $(\mu \mathrm{g} / \mathrm{L})$ & 5 & $<0.009$ & $<0.002$ & - & - & - & - & -- & -- \\
\hline 04040 & DEETHYL ATRAZINE, DISSOLVED $(\mu \mathrm{g} / \mathrm{L})$ & 5 & $<0.005$ & $<0.002$ & -- & -- & -- & -- & -- & -- \\
\hline 39632 & ATRAZINE，DISSOLVED $(\mu \mathrm{g} / \mathrm{L})$ & 5 & 0.054 & $<0.017$ & -- & -- & -- & -- & -- & -- \\
\hline 82686 & METHYL AZINPHOS, DISSOLVED $(\mu \mathrm{g} / \mathrm{L})$ & 5 & $<0.050$ & $<0.001$ & - & -- & -- & -- & -- & -- \\
\hline 82673 & BENFLURAIIN，DISSOLVED $(\mu \mathrm{g} / \mathrm{L})$ & 5 & 0.079 & $<0.002$ & -- & -- & -- & -- & -- & -- \\
\hline 04028 & BUTYLATE, DISSOLVED $\{\mu \mathrm{g} / \mathrm{L}\}$ & 5 & $<0.008$ & $<0.002$ & -- & -- & -- & -- & -- & -- \\
\hline 82680 & CARBARYL，DISSOLVED $(\mu \mathrm{g} / \mathrm{L})$ & 5 & 0.155 & $<0.003$ & -- & -- & -- & -- & -- & -- \\
\hline 82674 & CARBOFURAN, DISSOLVED $(\mu \mathrm{g} / \mathrm{L})$ & 5 & $<0.013$ & $<0.003$ & -- & -- & -- & -- & -- & -- \\
\hline 38933 & CHLORPYRIFOS, DISSOLVED $(\mu \mathrm{g} / \mathrm{L})$ & 5 & 0.047 & $<0.004$ & -- & -- & - & -- & -- & -- \\
\hline 04041 & CYANAZINE, DISSOLVED $(\mu \mathrm{g} / \mathrm{L})$ & 5 & $<0.013$ & $<0.004$ & -- & -- & -- & -- & -- & -- \\
\hline 82682 & DCPA， DISSOLVED $(\mu \mathrm{g} / L)$ & 5 & 0.002 & $<0.002$ & -- & $-\cdots$ & -- & -- & -- & - \\
\hline 34653 & $P, P^{\prime}$ DDE, DISSOLVED $(\mu \mathrm{g} / \mathrm{L})$ & 5 & $<0.010$ & $<0.006$ & -- & -- & -- & -- & -- & -- \\
\hline 39572 & DIAZINON，DISSOLVED $(\mu \mathrm{g} / \mathrm{L})$ & 5 & 0.348 & $<0.002$ & -- & -- & -- & -- & -- & -- \\
\hline 39381 & DIELDRIN，DISSOLVED $(\mu \mathrm{g} / \mathrm{L})$ & 5 & 0.014 & $<0.001$ & -- & -- & -- & -- & -- & $\rightarrow-$ \\
\hline 82660 & 2,6 -DIETHYL ANILINE, DISSOLVED $(\mu \mathrm{g} / \mathrm{L})$ & 5 & $<0.006$ & $<0.003$ & - & -- & - & - & -- & -- \\
\hline 82662 & DIMETHOATE， DISSOLVED $(\mu \mathrm{g} / \mathrm{L})$ & 1 & $<0.020$ & -- & -- & -- & -- & -- & -- & -- \\
\hline 82677 & DISULFOTON， DISSOLVED $(\mu \mathrm{g} / \mathrm{L})$ & 5 & $<0.060$ & $<0.017$ & -- & - & -- & -- & - & -- \\
\hline 82668 & EPTC， DISSOLVED $(\mu \mathrm{g} / L)$ & 5 & $<0.005$ & $<0.002$ & -- & -- & -- & -- & -- & -- \\
\hline 82663 & ETHALFLURALIN, DISSOLVED $(\mu \mathrm{g} / \mathrm{L})$ & 5 & $<0.013$ & $<0.004$ & -- & -- & -- & -- & -- & -- \\
\hline 82672 & ETHOPROP， DISSOLVED $(\mu \mathrm{g} / \mathrm{L})$ & 5 & $<0.012$ & $<0.003$ & -- & -- & -- & -- & -- & -- \\
\hline
\end{tabular}

NOTE: Multiple detection limits during the period of record may result in different values flagged with a "<."

* Value is estimated by using a log-probability regression to predict the values of data below the detection limit. 
Table 73. Statistical summary of water-quality data at site 41 (CSW02), May 1994 through September 1998-Continued

\begin{tabular}{|c|c|c|c|c|c|c|c|c|c|c|}
\hline \multirow{2}{*}{$\begin{array}{l}\text { PARAM- } \\
\text { ETER } \\
\text { CODE }\end{array}$} & \multirow{2}{*}{ PROPERTY OR CONSTITUENT } & & DESCRIPTI & STATISTICS & & PERCEN & $\begin{array}{l}\text { AMPLE } \\
\text { EC }\end{array}$ & $\begin{array}{l}\text { WHICH VAL } \\
\text { TO THOSE }\end{array}$ & $\begin{array}{l}\text { WERE } \\
\text { IIN }\end{array}$ & HAN OR \\
\hline & & $\begin{array}{c}\text { SAMPLE } \\
\text { SIZE }\end{array}$ & MAXIMUM & MINIMUM & MEAN & $95 \%$ & $75 \%$ & $\begin{array}{c}50 \% \\
\text { (median) }\end{array}$ & $25 \%$ & $5 \%$ \\
\hline 04095 & FONOFOS, DISSOLVED $(\mu \mathrm{g} / \mathrm{L})$ & 5 & $<0.008$ & $<0.003$ & - & -- & -- & -- & -- & -- \\
\hline 34253 & ALPHA BHC, DISSOLVED $(\mu \mathrm{g} / \mathrm{L})$ & 5 & $<0.007$ & $<0.002$ & -- & -- & -- & -- & -- & -- \\
\hline 39341 & LINDANE， DISSOLVED $(\mu \mathrm{g} / \mathrm{L})$ & 5 & $<0.011$ & $<0.004$ & -- & -- & -- & -- & -- & -- \\
\hline 82666 & LINURON， DISSOLVED $(\mu \mathrm{g} / \mathrm{L})$ & 5 & $<0.039$ & $<0.002$ & -- & -- & -- & -- & -- & -- \\
\hline 39532 & MALATHION， DISSOLVED $(\mu \mathrm{g} / \mathrm{L})$ & 5 & 0.110 & $<0.005$ & -- & -- & -- & -- & -- & -- \\
\hline 82667 & METHYL PARATHION, DISSOLVED $(\mu \mathrm{g} / \mathrm{L})$ & 5 & $<0.035$ & $<0.006$ & -- & -- & -- & -- & -- & -- \\
\hline 39415 & METOLACHLOR, DISSOLVED $(\mu \mathrm{g} / \mathrm{L})$ & 5 & 0.035 & $<0.002$ & -- & -- & -- & -- & -- & -- \\
\hline 82630 & METRIBUZIN, DISSOLVED $(\mu \mathrm{g} / \mathrm{L})$ & 5 & 0.073 & $<0.004$ & -- & -- & -- & -- & -- & -- \\
\hline 82671 & MOLINATE, DISSOLVED $(\mu \mathrm{g} / \mathrm{L})$ & 5 & $<0.007$ & $<0.004$ & -- & -- & -- & -- & -- & - \\
\hline 82684 & NAPROPAMIDE, DISSOLVED $(\mu \mathrm{g} / \mathrm{L})$ & 5 & $<0.010$ & $<0.003$ & -- & -- & -. & -- & -- & -- \\
\hline 39542 & PARATHION， DISSOLVED $(\mu \mathrm{g} / \mathrm{L})$ & 5 & $<0.022$ & $<0.004$ & -- & -- & -- & -- & -- & -- \\
\hline 82669 & PEBULATE, DISSOLVED $(\mu \mathrm{g} / \mathrm{L})$ & 5 & $<0.009$ & $<0.004$ & -- & -- & -- & -- & -- & -- \\
\hline 82683 & PENDIMETHALIN， DISSOLVED $(\mu \mathrm{g} / \mathrm{L})$ & 5 & 0.180 & $<0.018$ & -- & -- & -- & -- & -- & -- \\
\hline 82687 & PERMETHRIN， DISSOLVED $(\mu \mathrm{g} / \mathrm{L})$ & 5 & $<0.016$ & $<0.005$ & -- & -- & -- & -- & -- & -- \\
\hline 82664 & PHORATE, DISSOLVED $(\mu \mathrm{g} / \mathrm{L}\}$ & 5 & $<0.011$ & $<0.002$ & -- & -- & -- & -- & -- & -- \\
\hline 82676 & PRONAMIDE， DISSOLVED $(\mu \mathrm{g} / \mathrm{L})$ & 5 & $<0.009$ & $<0.003$ & -- & -- & -- & -- & -- & -- \\
\hline 04037 & PROMETON, DISSOLVED $(\mu \mathrm{g} / \mathrm{L})$ & 5 & $<0.018$ & $<0.008$ & -- & -- & -- & - & -- & -- \\
\hline 04024 & PROPACHLOR, DISSOLVED $(\mu \mathrm{g} / \mathrm{L})$ & 5 & $<0.015$ & $<0.007$ & -- & -- & -- & -- & -- & -- \\
\hline 82679 & PEOPANIL, DISSOLVED $(\mu \mathrm{g} / \mathrm{L})$ & 5 & $<0.016$ & $<0.004$ & -- & -- & -- & -- & -- & -- \\
\hline 82685 & PROPARGITE, DISSOLVED $(\mu \mathrm{g} / \mathrm{L})$ & 5 & $<0.013$ & $<0.008$ & -- & -- & - & -- & -- & -- \\
\hline 04035 & SIMAZINE， DISSOLVED $(\mu \mathrm{g} / \mathrm{L})$ & 5 & $<0.008$ & $<0.005$ & -- & -- & -- & -- & -- & -- \\
\hline 82681 & THIOBENCARB，DISSOLVED $(\mu \mathrm{g} / \mathrm{L})$ & 5 & $<0.008$ & $<0.002$ & -- & -- & - & -- & -- & -- \\
\hline 82670 & TEBUTHIURON，DISSOLVED $(\mu \mathrm{g} / \mathrm{L})$ & 5 & $<0.015$ & $<0.010$ & -- & -- & -- & -- & -- & -- \\
\hline 82665 & TERBACIL, DISSOLVED $(\mu \mathrm{g} / \mathrm{L})$ & 5 & $<0.030$ & $<0.007$ & -- & -- & -- & -- & -- & -- \\
\hline 82675 & TERBUFOS, DISSOLVED $(\mu \mathrm{g} / \mathrm{L})$ & 5 & $<0.013$ & $<0.012$ & -- & -- & -- & -- & -- & -- \\
\hline 82678 & TRIALLATE， DISSOLVED $(\mu \mathrm{g} / \mathrm{L})$ & 5 & $<0.008$ & $<0.001$ & -- & -- & -- & -- & -- & - \\
\hline 82661 & TRIFLURALIN， DISSOLVED $(\mu \mathrm{g} / \mathrm{L})$ & 5 & 0.015 & $<0.002$ & -- & -- & -- & -- & -- & -- \\
\hline 39742 & $2,4,5-\mathrm{T}$, DISSOLVED $(\mu \mathrm{g} / \mathrm{L})$ & 5 & $<0.050$ & $<0.035$ & -- & -- & -- & -- & -- & - \\
\hline 39732 & $2,4-\mathrm{D}$, DISSOLVED $(\mu \mathrm{g} / \mathrm{L})$ & 5 & 1.620 & $<0.035$ & -- & -- & -- & -- & -- & -- \\
\hline 38746 & $2,4-D B$, DISSOLVED $(\mu \mathrm{g} / \mathrm{L})$ & 5 & $<0.240$ & $<0.035$ & -- & -- & -- & -- & -- & -- \\
\hline 49315 & ACIFLUORFEN， DISSOLVED $(\mu \mathrm{g} / \mathrm{L})$ & 5 & $<0.050$ & $<0.035$ & -- & -- & -- & -- & -- & $\cdots$ \\
\hline 49312 & ALDICARE, DISSOLVED $(\mu \mathrm{g} / \mathrm{L})$ & 5 & $<0.550$ & $<0.016$ & -- & -- & -- & -- & -- & -- \\
\hline 49313 & ALDICARB SULFONE, DISSOLVED $(\mu \mathrm{g} / \mathrm{L})$ & 5 & $<0.100$ & $<0.016$ & -- & -- & -- & -- & -- & -- \\
\hline 49314 & ALDICARB SULFOXIDE, DISSOLVED $(\mu \mathrm{g} / \mathrm{L})$ & 5 & $<0.050$ & $<0.021$ & -- & -- & -- & -- & -- & -- \\
\hline 38711 & BENTAZON， DISSOLVED $(\mu \mathrm{g} / \mathrm{L})$ & 5 & $<0.050$ & $<0.014$ & -- & -- & -- & -- & -- & -- \\
\hline 04029 & BROMACIL, DISSOLVED $(\mu \mathrm{g} / \mathrm{L})$ & 5 & $<0.050$ & $<0.035$ & -- & -- & -- & -- & -- & -- \\
\hline 49311 & BROMOXYNIL, DISSOLVED $(\mu \mathrm{g} / \mathrm{L})$ & 5 & $<0.050$ & $<0.035$ & -- & -- & -- & -- & -- & -- \\
\hline 49310 & CARBARYL， DISSOLVED $(\mu \mathrm{g} / \mathrm{L})$ & 5 & $<0.050$ & $<0.008$ & -- & -- & -- & -- & -- & -- \\
\hline 49309 & CARBOFURAN, DISSOLVED $(\mu \mathrm{g} / \mathrm{L})$ & 5 & $<0.120$ & $<0.028$ & -- & -- & -- & -- & -- & -- \\
\hline 49308 & 3-HYDKOXY-CARBOFURAN $(\mu \mathrm{g} / \mathrm{L})$ & 5 & $<0.050$ & $<0.014$ & -- & -- & -- & -- & -- & -- \\
\hline 49307 & AMIBEN, DISSOLVED $(\mu \mathrm{g} / \mathrm{L})$ & 5 & $<0.420$ & $<0.011$ & -- & -- & -- & -- & -- & -- \\
\hline 49306 & CHLOROTHALONIL, DISSOLVED $(\mu \mathrm{g} / \mathrm{L})$ & 5 & $<0.480$ & $<0.035$ & -- & -- & -- & -- & -- & -- \\
\hline 49305 & CLOPYRALID, DISSOLVED $(\mu \mathrm{g} / \mathrm{L})$ & 5 & $<0.230$ & $<0.050$ & -- & -- & -- & -- & -- & -- \\
\hline 49304 & DACTHALMONO-ACID, DISSOLVED $(\mu \mathrm{g} / \mathrm{L})$ & 5 & $<0.050$ & $<0.017$ & -- & -- & -- & -- & -- & -- \\
\hline 38442 & DICAMBA， DISSOLVED $(\mu \mathrm{g} / \mathrm{L})$ & 5 & 0.070 & $<0.035$ & -- & -- & -- & -- & -- & -- \\
\hline 49303 & DICHLOBENIL, DISSOLVED $(\mu \mathrm{g} / \mathrm{L})$ & 5 & $<1.200$ & $<0.020$ & -- & -- & -- & -- & -- & -- \\
\hline 49302 & DICHLORPROP， DISSOLVED $(\mu \mathrm{g} / \mathrm{L})$ & 5 & 0.210 & $<0.032$ & -- & -- & -- & -- & -- & -- \\
\hline 49301 & DINOSEB， DISSOLVED $(\mu \mathrm{g} / \mathrm{L})$ & 5 & $<0.050$ & $<0.035$ & -- & -- & -- & -- & -- & -- \\
\hline 49300 & DIURON, DISSOLVED $(\mu \mathrm{g} / \mathrm{L})$ & 5 & $<21.700$ & $<0.020$ & -- & -- & -- & -- & -- & -- \\
\hline 49299 & 4,6-DINITRO OCRESOL， DISSOLVED $(\mu \mathrm{g} / \mathrm{L})$ & 5 & 0.050 & $<0.035$ & -- & -- & -- & -- & -- & -- \\
\hline 49298 & ESFENVALERATE， DISSOLVED $(\mu \mathrm{g} / \mathrm{L})$ & 4 & $<0.050$ & $<0.019$ & -- & -- & -- & -- & -- & -- \\
\hline 49297 & FENURON， DISSOLVED $(\mu \mathrm{g} / \mathrm{L})$ & 5 & $<0.050$ & $<0.013$ & -- & -- & -- & -- & -- & -- \\
\hline 38811 & FLUOMETURON， DISSOLVED $(\mu \mathrm{g} / \mathrm{L})$ & 5 & $<0.050$ & $<0.035$ & -- & -- & -- & -- & -- & -- \\
\hline 38478 & LINURON, DISSOLVED $(\mu \mathrm{g} / \mathrm{L})$ & 5 & $<0.050$ & $<0.018$ & -- & -- & -- & -- & -- & -- \\
\hline 38482 & MCPA, DISSOLVED $(\mu \mathrm{g} / \mathrm{L})$ & 5 & $<0.170$ & $<0.050$ & -- & - & -- & -- & -- & -- \\
\hline 38487 & MCPB， DISSOLVED $(\mu \mathrm{g} / \mathrm{L})$ & 5 & $<0.140$ & $<0.035$ & -- & -- & -- & -- & -- & -- \\
\hline 38501 & METHIOCARB， DISSOLVED $(\mu \mathrm{g} / \mathrm{L})$ & 5 & $<0.050$ & $<0.026$ & -- & -- & -- & -- & -- & -- \\
\hline 49296 & METHOMYL， DISSOLVED $(\mu \mathrm{g} / \mathrm{L})$ & 5 & $<0.050$ & $<0.017$ & -- & -- & -- & -- & -- & -- \\
\hline 49295 & 1-NAPHTHOL, DISSOLVED $(\mu \mathrm{g} / \mathrm{L})$ & 4 & $<0.050$ & $<0.007$ & -- & -- & -- & -- & -- & -- \\
\hline 49294 & NEBURON， DISSOLVED $(\mu g / L)$ & 5 & $<0.050$ & $<0.015$ & -- & -- & -- & -- & -- & -- \\
\hline 49293 & NORFLURAZON，DISSOLVED $(\mu \mathrm{g} / \mathrm{L})$ & 5 & $<0.050$ & $<0.024$ & -- & -- & -- & -- & -- & -- \\
\hline 49292 & ORYZALIN, DISSOLVED $(\mu \mathrm{g} / \mathrm{L})$ & 5 & $<0.310$ & $<0.019$ & -- & -- & -- & -- & -- & -- \\
\hline 38866 & OXAMYL， DISSOLVED $(\mu g / L)$ & 5 & $<0.050$ & $<0.018$ & -- & -- & -- & -- & -- & -- \\
\hline 49291 & PICLORAM, DISSOLVED $(\mu \mathrm{g} / \mathrm{L})$ & 5 & $<0.050$ & $<0.050$ & -- & -- & -- & -- & -- & -- \\
\hline 49236 & PROPHAM, DISSOLVED $(\mu \mathrm{g} / \mathrm{L})$ & 5 & $<0.050$ & $<0.035$ & -- & -- & -- & -- & -- & -- \\
\hline 38538 & PROPOXUR, DISSOLVED $(\mu \mathrm{g} / \mathrm{L})$ & 5 & $<0.050$ & $<0.035$ & -- & -- & -- & -- & -- & -- \\
\hline 39762 & SILVEX, DISSOLVED $(\mu \mathrm{g} / \mathrm{L})$ & 5 & $<0.050$ & $<0.021$ & -- & -- & -- & -- & -- & -- \\
\hline 49235 & TRICLOPYR， DISSOLVED $(\mu \mathrm{g} / \mathrm{L})$ & 5 & $<0.250$ & $<0.050$ & -- & -- & -- & -- & -- & -- \\
\hline & & ORGAN1 & COMPOUND & -ORGANON1 & OGEN, & TAL & & & & \\
\hline 39057 & PROMETRYNE， TOTAL $(\mu \mathrm{g} / \mathrm{L})$ & 1 & $<0.100$ & -- & -- & -- & -- & -- & -- & -- \\
\hline 39056 & PROMETONE, TOTAL $(\mu \mathrm{g} / \mathrm{L})$ & 1 & $<0.200$ & -- & -- & -- & -- & -- & -- & -- \\
\hline 39054 & SIMETRYNE, TOTAL $(\mu \mathrm{g} / \mathrm{L})$ & 1 & $<0.100$ & -- & -- & -- & -- & -- & -- & -- \\
\hline 81757 & CYANAZINE， TOTAL $(\mu g / L)$ & 1 & $<0.200$ & -- & -- & -- & -- & -- & -- & - \\
\hline 77825 & ALACHLOR, TOTAL $(\mu g / L)$ & 1 & $<0.100$ & -- & -- & -- & -- & -- & -- & -- \\
\hline 82611 & METRIBUZIN， TOTAL $(\mu \mathrm{g} / \mathrm{L})$ & 1 & $<0.100$ & -- & -- & -- & -- & -- & -- & -- \\
\hline 30311 & TERBACIL, TOTAL $(\mu \mathrm{g} / \mathrm{L})$ & 1 & $<0.200$ & -- & -- & -- & -- & -- & -- & -- \\
\hline 30245 & CARBOXIN, TOTAL $(\mu \mathrm{g} / \mathrm{L})$ & 1 & $<0.200$ & -- & -- & -- & -- & -- & -- & -- \\
\hline 30264 & HEXAZINONE， TOTAL $(\mu \mathrm{g} / \mathrm{L})$ & 1 & $<0.200$ & -- & -- & -- & -- & -- & -- & -- \\
\hline 30235 & BUTACHLOR, TOTAL $(\mu g / L)$ & 1 & $<0.100$ & -- & -- & -- & -- & -- & -- & -- \\
\hline 30236 & BUTYLATE, TOTAL $(\mu g / L)$ & 1 & $<0.100$ & -- & -- & -- & -- & -- & -- & -- \\
\hline 75981 & DE-ETHYLATRAZINE, TOTAL $(\mu \mathrm{g} / \mathrm{L})$ & 1 & $<0.200$ & -- & -- & -- & -- & -- & -- & -- \\
\hline 39630 & ATRAZINE, TOTAL $(\mu \mathrm{g} / \mathrm{L})$ & 1 & $<0.100$ & -- & -- & -- & -- & -- & -- & -- \\
\hline
\end{tabular}

NOTE: Multiple detection limits during the period of record may result in different values flagged with a "<."

* Value is estimated by using a log-probability regression to predict the values of data below the detection limit. 
Table 73. Statistical summary of water-quality data at site 41 (CSW02), May 1994 through September 1998-Continued

\begin{tabular}{|c|c|c|c|c|c|c|c|c|c|c|}
\hline \multirow{2}{*}{$\begin{array}{c}\text { PARAM- } \\
\text { ETER } \\
\text { CODE }\end{array}$} & \multirow{2}{*}{ PROPERTY OR CONSTITUENT } & \multicolumn{4}{|c|}{ DESCRIPTIVE STATISTICS } & \multicolumn{5}{|c|}{$\begin{array}{c}\text { PERCENT OF SAMPLES IN WHICH VALUIIS WERE LESS THAN OR } \\
\text { EQUAL TO THOSE SHOWN }\end{array}$} \\
\hline & & $\begin{array}{l}\text { SAMPLE } \\
\text { SIZE }\end{array}$ & MAXIMUM & MINIMUM & MEAN & $95 \%$ & $75 \%$ & $\begin{array}{c}\mathbf{5 0} \% \\
\text { (median) }\end{array}$ & $25 \%$ & $5 \%$ \\
\hline 39055 & SIMAZINE, TOTAL $(\mu \mathrm{g} / \mathrm{L})$ & 1 & $<0.100$ & -- & $\because$ & - & -- & -- & -- & -- \\
\hline 39024 & PROPAZINE，TOTAL $(\mu \mathrm{g} / \mathrm{L})$ & 1 & $<0.100$ & -- & -- & -- & -- & -- & -- & -- \\
\hline 82184 & ANETRYNE, TOTAL $(\mu \mathrm{g} / \mathrm{L}\rangle$ & 1 & $<0.100$ & -- & -- & -- & -- & -- & -- & -- \\
\hline 39030 & TRIFLURALIN, TOTAL $(\mu \mathrm{g} / \mathrm{L})$ & 1 & $<0.100$ & -- & -- & -- & -- & -- & -- & -- \\
\hline 82612 & METOLACHLOR, TOTAL $(\mu \mathrm{g} / \mathrm{L})$ & 1 & $<0.200$ & -- & -- & -- & -- & -- & -- & -- \\
\hline 30234 & BROMACIL, TOTAL $(\mu \mathrm{g} / \mathrm{L})$ & 1 & 0.300 & -- & -- & -- & -- & -- & -- & -- \\
\hline 30255 & DIPHENAMID, TOTAL $(\mu \mathrm{g} / \mathrm{L})$ & 1 & $<0.100$ & -- & - & -- & -- & -- & -- & -. \\
\hline 30324 & VERNOLATE, TOTAL $(\mu \mathrm{g} / L)$ & 1 & $<0.100$ & -- & -- & -- & -- & - & -- & -- \\
\hline 30254 & CYCLOATE, TOTAL $(\mu \mathrm{g} / \mathrm{L})$ & 1 & $<0.100$ & -- & -- & -. & -- & -- & -- & -- \\
\hline 30295 & PROPACHLOR, TOTAL $(\mu \mathrm{g} / \mathrm{L})$ & 1 & 0.100 & -- & -- & -- & -- & -- & -- & -- \\
\hline 75980 & DE-ISOPROPYLATRAZIN, TOTAL $(\mu \mathrm{g} / \mathrm{L})$ & 1 & $<0.200$ & -- & -- & -- & -- & $\ldots$ & -- & - \\
\hline \multicolumn{11}{|c|}{ ORGANIC COMPOUNDS-HERBICIDES, TOTAL } \\
\hline 39730 & $2,4-\mathrm{D}$, TOTAL $(\mu \mathrm{g} / \mathrm{L})$ & 1 & 2.400 & -- & $\therefore$ & -- & -- & -- & -- & -- \\
\hline 39760 & SILVEX, TOTAL $(\mu \mathrm{g} / L)$ & 1 & $<0.010$ & -- & -- & -- & -- & -- & -- & -- \\
\hline 39720 & PICLORAM, TOTAL $(\mu \mathrm{g} / \mathrm{L})$ & 1 & $<0.010$ & -- & -- & -- & -- & -- & -- & -- \\
\hline 39740 & $2,4,5-\mathrm{T}$, TOTAL $(\mu \mathrm{g} / \mathrm{L})$ & 1 & $<0.010$ & -- & -- & -- & -- & -- & -- & -- \\
\hline 82183 & $2,4-\mathrm{DP}$, TOTAL $(\mu \mathrm{g} / \mathrm{L})$ & 1 & $<0.010$ & -- & -- & -- & -- & -- & -- & -- \\
\hline 82052 & DICAMBA, TOTAL $(\mu \mathrm{g} / \mathrm{L})$ & 1 & 0.380 & -- & -- & -- & -- & -- & -- & -- \\
\hline \multicolumn{11}{|c|}{ ORGANIC COMPOUNDS-CARBAMATE PESTICIDES, TOTAL } \\
\hline 39750 & SEVIN, TOTAL $(\mu \mathrm{g} / \mathrm{L})$ & 1 & $<0.500$ & -- & -- & -- & -- & -- & -- & -- \\
\hline 39051 & METHOMYL, TOTAL $(\mu \mathrm{g} / \mathrm{L})$ & 1 & $<0.500$ & -- & -- & -- & -- & -- & -- & -- \\
\hline 82619 & ALDICARD, TOTAL $(\mu \mathrm{g} / \mathrm{L})$ & 1 & $<0.500$ & -- & -- & -- & $-\infty$ & - & -- & -- \\
\hline 30296 & PROPOXUR, TOTAL $(\mu \mathrm{g} / \mathrm{L})$ & 1 & $<0.500$ & -- & -- & -- & -- & -- & -- & -- \\
\hline 39052 & PROPHAM, TOTAL $(\mu \mathrm{g} / \mathrm{L})$ & 1 & $<0.500$ & -- & -- & -- & -- & -- & -- & -- \\
\hline 82615 & CARBOFURAN, TOTAL $(\mu \mathrm{g} / \mathrm{L})$ & 1 & $<0.500$ & -- & -- & -- & -- & $-\infty$ & -- & -- \\
\hline 77441 & 1 -NAPHTHOL, TOTAL $(\mu \mathrm{g} / \mathrm{L})$ & 1 & $<0.500$ & -- & -- & -- & -- & -- & -- & -- \\
\hline 30282 & METHIOCARB, TOTAL $(\mu \mathrm{g} / \mathrm{L})$ & 1 & $<0.500$ & -- & -- & -- & -- & -- & -- & -- \\
\hline
\end{tabular}

NOTE: Multiple detection limits during the period of record may result in different values flagged with a "<."

* Value is estimated by using a log-probability regression to predict the values of data below the detection limit. 
Table 74. Statistical summary of water-quality data at site 42 (CSW04), May 1994 through September 1998

\begin{tabular}{|c|c|c|c|c|c|c|c|c|c|c|}
\hline \multirow{2}{*}{$\begin{array}{l}\text { PARAM- } \\
\text { ETER } \\
\text { CODE }\end{array}$} & \multirow{2}{*}{ PROPERTY OR CONSTITUENT } & \multicolumn{4}{|c|}{ DESCRIPTIVE STATISTICS } & \multicolumn{5}{|c|}{$\begin{array}{l}\text { PERCENT OF SAMPLES IN WHICH VALUES WERE LESS THAN OR } \\
\text { EQUAL TO THOSE SHOINN }\end{array}$} \\
\hline & & $\begin{array}{l}\text { SAMPLE } \\
\text { SIZE }\end{array}$ & MAXIMUM & MINIMUM & MEAN & $95 \%$ & $75 \%$ & $\begin{array}{c}50 \% \\
\text { (medlan) }\end{array}$ & $25 \%$ & $5 \%$ \\
\hline \multicolumn{11}{|c|}{ PHYSICAL AND CHEMICAL PROPERTIES } \\
\hline 00061 & INSTANTANEOUS DISCHARGE $\left(\mathrm{ft}^{3} / \mathrm{s}\right)$ & 93 & 229.000 & 0.020 & 15.378 & 81.000 & 13.000 & $2 . \overline{000}$ & 0.700 & $\overline{0.114}$ \\
\hline 00010 & WATER TEMPERATURE $\left({ }^{\circ} \mathrm{C}\right)$ & 85 & 25.000 & 2.500 & 14.865 & 23.500 & 20.000 & 15.500 & 9.400 & 3.500 \\
\hline 90095 & SPECIFIC CONDUCTANCE, LAB ( $\mu$ S/Cm at $25^{\circ} \mathrm{C}$ ) & 56 & 550.000 & 26.000 & 117.018 & 417.650 & 138.250 & 70.500 & 45.500 & 29.550 \\
\hline 00095 & $\begin{array}{l}\text { SPECIFIC CONDUCTANCE, FIELD }(\mu \mathrm{S} / \mathrm{cm} \text { at } 25 \\
\left.{ }^{\circ} \mathrm{C}\right)\end{array}$ & 67 & 425.000 & 19.000 & 103.507 & 307.800 & 130.000 & 65.000 & 49.000 & 24.600 \\
\hline 00403 & pH, LAB (STANDARD pH UNITS) & 56 & 7.600 & 5.700 & 6.809 & 7.515 & 7.100 & 6.850 & 6.400 & 6.085 \\
\hline 00400 & pH, FIELD (STANDARD pH UNITS) & 67 & 7.700 & 6.200 & 6.931 & 7.300 & 7.000 & 6.900 & 6.800 & 6.540 \\
\hline 90410 & ALKALINITY, LAB (mg/L as $\mathrm{CaCO}_{3}$ ) & 56 & 133.000 & 6.000 & 26.973 & 117.550 & 26.750 & 14.000 & 9.475 & 6.000 \\
\hline 80154 & SUSPENDED SEDIMENT $(\mathrm{mg} / \mathrm{L})$ & 59 & 1500.000 & 4.000 & 262.237 & 1390.000 & 240.000 & 95.000 & 54.000 & 23.000 \\
\hline 00530 & $\begin{array}{l}\text { RESIDUE ON EVAPORATION AT } 105^{\circ} \mathrm{C} \text {, SUSPENDED } \\
(\mathrm{mg} / \mathrm{L})\end{array}$ & 31 & 776.000 & 1.000 & 135.290 & 713.600 & 158.000 & 62.000 & 25.000 & 5.800 \\
\hline 00535 & RESIDUE VOLATILE, SUSPENDED $(\mathrm{mg} / \mathrm{L})$ & 54 & 172.000 & 1.000 & 25.148 & 105.250 & 28.250 & 12.500 & 7.750 & 1.750 \\
\hline 70300 & DISSOLVED SOLIDS, RESIDUE AT $180^{\circ} \mathrm{C}(\mathrm{mg} / \mathrm{L})$ & 56 & 340.000 & 1.000 & 81.679 & 242.000 & 95.750 & 56.500 & 34.250 & 18.250 \\
\hline 00310 & 5 DAY BIOCHEMICAL OXYGEN DEMAND $(\mathrm{mg} / \mathrm{L})$ & 41 & 42.000 & 2.000 & 10.956 & 31.000 & 14.000 & 7.400 & 5.250 & 2.020 \\
\hline 00340 & CHEMICAL OXYGEN DEMAND (mg/L) & 56 & 220.000 & 5.000 & 54.446 & 171.500 & 71.750 & 38.500 & 29.250 & 13.500 \\
\hline & & & NUTRIENTS, $\mathrm{T}$ & TAL AND DIS & SOLVED & & & & & \\
\hline$\overline{00625}$ & $\begin{array}{l}\text { NITROGEN AMMONIA + ORGANIC, TOTAL } \\
(\mathrm{mg} / \mathrm{L} \text { as N })\end{array}$ & 57 & 11.000 & 0.400 & 2.041 & 6.420 & 2.150 & 1.400 & 1.000 & $0 . \overline{498}$ \\
\hline 00631 & $\mathrm{NO}_{2}+\mathrm{NO}_{3}$, DISSOLVED $(\mathrm{mg} / \mathrm{L}$ as $\mathrm{N})$ & 56 & 1.700 & 0.110 & 0.581 & 1.388 & 0.780 & 0.500 & 0.303 & 0.180 \\
\hline 00608 & NITROGEN AMMONIA, DISSOLVED (mg/L as N) & 57 & 5.200 & 0.015 & 0.447 & 1.730 & 0.450 & 0.220 & 0.120 & 0.020 \\
\hline 00605 & NITROGEN ORGANIC, TOTAL (mg/L as N) & 57 & 5.800 & 0.250 & 1.591 & 5.050 & 1.650 & 1.100 & 0.870 & 0.475 \\
\hline 00600 & NITROGEN, TOTAL (mg/L as N) & 57 & 12.000 & 0.790 & 2.609 & 8.020 & 2.800 & 2.000 & 1.300 & 0.924 \\
\hline 00665 & PHOSPHORUS, TOTAL (mg/L as P) & 57 & 4.600 & 0.060 & 0.534 & 1.370 & 0.595 & 0.380 & 0.210 & 0.078 \\
\hline 00671 & PHOSPHORUS ORTHO, DISSOLVED (mg/L as P) & 57 & 4.800 & 0.010 & 0.321 & 1.108 & 0.360 & 0.140 & 0.060 & 0.020 \\
\hline & & & OIL AND & REASE, TOT & & & & & & \\
\hline 00556 & OIL AND GREASE, TOTAL (mg/L) & 20 & 5.000 & $<1,000$ & $1.503 *$ & $3 . \overline{000}$ & 2.000 & 1.000 & $<1.000$ & $<1.000$ \\
\hline & & & ORGANIC & ARBON. TOI & TAL & & & & & \\
\hline 00680 & CARBON ORGANIC, TOTAL $(\mathrm{mg} / \mathrm{L})$ & 35 & 49.000 & 6.800 & 17.977 & 45.000 & 24.000 & 14.000 & 11.000 & 7.440 \\
\hline & & & & IFORM & & & & & & \\
\hline$\overline{31679}$ & FECAL STREPTOCOCCI (Colonies per $100 \mathrm{~mL}$ ) & 36 & 540000.000 & 2000.000 & 66763.891 & 336000.375 & 66250.000 & 21000.000 & 5100.000 & 2340.000 \\
\hline 31616 & FECAL COLIFORM (Colonies per $100 \mathrm{~mL}$ ) & 36 & 700000.000 & 580.000 & 61437.500 & 368500.594 & 71500.000 & 14500.000 & 2325.000 & 622.500 \\
\hline & & ORG & ANIC COMPOI & DS-PESTIC & IDES, TOTAL & & & & & \\
\hline$\overline{39330}$ & ALDRIN, TOTAI $(\mu \mathrm{g} / \mathrm{L})$ & 5 & 0.040 & $<0.010$ & -- & -- & -- & -- & -- & -- \\
\hline 39340 & IINDANE, TOTAL $(\mu \mathrm{g} / \mathrm{L})$ & 5 & 0.010 & $<0.010$ & -- & -- & -- & -- & -- & -- \\
\hline 39350 & CHLORDANE, TOTAL $(\mu \mathrm{g} / \mathrm{L})$ & 5 & $<0.100$ & $<0.100$ & -- & -- & -- & -- & -- & -- \\
\hline 39370 & DDT, TOTAL $(\mu \mathrm{g} / \mathrm{L})$ & 5 & $<0.010$ & $<0.010$ & -- & -- & -- & -- & -- & -- \\
\hline 39365 & DDE，TOTAL $(\mu \mathrm{g} / \mathrm{L})$ & 5 & $<0.010$ & $<0.010$ & -- & -- & -- & -- & -- & -- \\
\hline 39360 & $\mathrm{DDD}, \quad$ TOTAL $(\mu \mathrm{g} / \mathrm{L})$ & 5 & $<0.010$ & $<0.010$ & -- & -- & -- & -. & - - & -. \\
\hline 39380 & DIELDRIN， TOTAL $(\mu \mathrm{g} / \mathrm{L})$ & 5 & $<0.010$ & $<0.010$ & -- & -- & -- & -- & -- & -- \\
\hline 39388 & ENDOSULFAN, TOTAL $(\mu \mathrm{g} / \mathrm{L})$ & 5 & $<0.010$ & $<0.010$ & -- & -- & -- & -- & -- & -- \\
\hline 39390 & ENDRIN, TOTAL $(\mu \mathrm{g} / \mathrm{L})$ & 5 & $<0.010$ & $<0.010$ & -- & -- & -- & -- & -- & -- \\
\hline 39410 & HEPTACHLOR, TOTAL $(\mu \mathrm{g} / \mathrm{L})$ & 5 & $<0.010$ & $<0.010$ & -- & -- & -- & -- & -- & -- \\
\hline 39420 & HEPTACHLOR EPOXIDE, TOTAL $(\mu \mathrm{g} / \mathrm{L})$ & 5 & 0.010 & $<0.010$ & -- & -- & -- & -- & - & -- \\
\hline 39516 & PCB, TOTAL $(\mu \mathrm{g} / \mathrm{L})$ & 5 & $<0.300$ & $<0.100$ & -- & -- & - & -- & -- & -- \\
\hline 39400 & TOXAPHENE, TOTAL $(\mu \mathrm{g} / \mathrm{L})$ & 5 & $<1.000$ & $<1.000$ & -- & -- & -- & -- & -- & -- \\
\hline 39034 & PERTHANE, TOTAL $(\mu \mathrm{g} / \mathrm{L})$ & 5 & $<0.100$ & $<0.100$ & -- & -- & -- & -- & -- & -- \\
\hline 39570 & DIAZINON, TOTAL $(\mu \mathrm{g} / \mathrm{L})$ & 5 & 0.060 & 0.010 & -- & -- & -- & $\ldots$ & -- & -- \\
\hline 39398 & ETHION, TOTAL $(\mu \mathrm{g} / \mathrm{L})$ & 5 & $<0.010$ & $<0.010$ & - & -- & -- & -- & -- & -- \\
\hline 39530 & MALATHION, TOTAL $(\mu g / L)$ & 5 & 0.010 & $<0.010$ & -- & -- & -- & -- & -- & -- \\
\hline 39600 & METHYL PARATHION, TOTAL $(\mu \mathrm{g} / \mathrm{L})$ & 5 & 0.010 & $<0.010$ & -- & -- & -- & -- & -- & -- \\
\hline 39540 & PARATHION, TOTAL $(\mu \mathrm{g} / \mathrm{L})$ & 5 & $<0.010$ & $<0.010$ & -- & -- & -- & -- & -- & -- \\
\hline 39786 & TRITHION, TOTAL $(\mu \mathrm{g} / \mathrm{L})$ & 5 & $<0.010$ & $<0.010$ & -- & -- & -- & - & -- & -- \\
\hline 39250 & PCN, TOTAL $(\mu \mathrm{g} / \mathrm{L})$ & 5 & $<0.100$ & $<0.100$ & -- & -- & - & -- & -- & -- \\
\hline 39480 & METHOXYCHLOR, TOTAL $(\mu \mathrm{g} / \mathrm{L})$ & 5 & 0.010 & $<0.010$ & -- & -- & -- & -- & -- & -- \\
\hline 39755 & MIREX, TOTAL $(\mu \mathrm{g} / \mathrm{L})$ & 5 & $<0.010$ & $<0.010$ & -- & -- & -- & -- & -- & -- \\
\hline 39011 & DISYSTON, TOTAL $(\mu \mathrm{g} / \mathrm{L})$ & 5 & $<0.010$ & $<0.010$ & -- & -- & - & -- & -- & -- \\
\hline 39023 & PHORATE, TOTAL $(\mu \mathrm{g} / \mathrm{L})$ & 5 & $<0.010$ & $<0.010$ & -- & -- & -- & - & -- & -- \\
\hline 38932 & CHLORPYRIFOS, TOTAL $(\mu \mathrm{g} / \mathrm{L})$ & 5 & 0.020 & $<0.010$ & -- & -- & -- & -- & -- & -- \\
\hline 39040 & DEF， TOTAL $(\mu \mathrm{g} / \mathrm{L})$ & 5 & $<0.010$ & $<0.010$ & -- & -- & -- & -- & -- & -- \\
\hline 82614 & FONOFOS, TOTAL $(\mu \mathrm{g} / \mathrm{L})$ & 5 & $<0.010$ & $<0.010$ & -- & -- & -- & -- & -- & -- \\
\hline & & & LATILE ORGA & C COMPOUN & NDS, TOTAL & & & & & \\
\hline 34210 & ACROLEIN, TOTAL $(\mu \mathrm{g} / \mathrm{L})$ & 2 & $<20.000$ & $<20.000$ & -- & - & -- & -- & -- & -- \\
\hline 34215 & ACRYLONITRILE, TOTAL $(\mu \mathrm{g} / \mathrm{L})$ & 2 & $<20.000$ & $<20.000$ & -- & -- & -- & -- & -- & -- \\
\hline 34030 & BENZENE，TOTAL $(\mu \mathrm{g} / \mathrm{L})$ & 14 & $<8.000$ & $<0.200$ & -- & - & -- & -- & -- & -- \\
\hline 32104 & BROMOFORM, TOTAL $(\mu \mathrm{g} / \mathrm{L})$ & 14 & $<8.000$ & $<0.200$ & -- & -- & -- & -- & -- & - \\
\hline 32102 & CARBON TETRACHLORIDE, TOTAL $(\mu \mathrm{g} / \mathrm{L})$ & 14 & $<8.000$ & $<0.200$ & -- & $\ldots$ & -- & -- & -- & -- \\
\hline 34301 & CHLOROBENZENE, TOTAL $(\mu \mathrm{g} / \mathrm{L})$ & 14 & $<8.000$ & $<0.200$ & +- & -- & -- & -- & -- & -- \\
\hline 32105 & CHLORODIBROMOMETHANE, TOTAL $(\mu \mathrm{g} / \mathrm{L})$ & 14 & $<8.000$ & $<0.200$ & - & - & -- & -- & -- & - \\
\hline 34311 & CHLOROETHANE, TOTAL $(\mu \mathrm{g} / \mathrm{L})$ & 14 & $<8.000$ & $<0.200$ & -- & -- & -- & - & -- & -- \\
\hline 32106 & CHLOROFORM, TOTAL $(\mu \mathrm{g} / \mathrm{L})$ & 14 & $<8.000$ & $<0.200$ & -- & $\ldots$ & -- & -- & -- & -- \\
\hline 34496 & 1,1 -DICHLOROETHANE, TOTAL $(\mu \mathrm{g} / \mathrm{L})$ & 14 & $<8.000$ & $<0.200$ & -- & -- & -- & -- & -- & -- \\
\hline 32103 & 1,2 -DICHLOROETHANE, TOTAL $(\mu \mathrm{g} / \mathrm{L})$ & 14 & $<8.000$ & $<0.200$ & -- & -- & -- & -- & -- & -- \\
\hline 34501 & 1,1 -DICHLOROETHYLENE, TOTAL $(\mu \mathrm{g} / \mathrm{L})$ & 14 & $<8.000$ & $<0.200$ & -- & -- & -- & -- & -- & -- \\
\hline 34541 & 1,2 -DICHLOROPROPANE, TOTAL $(\mu \mathrm{g} / \mathrm{L})$ & 14 & $<8.000$ & $<0.200$ & -- & -. & -- & -- & -- & -- \\
\hline 34371 & ETHYLBENZENE, TOTAL $(\mu \mathrm{g} / \mathrm{L})$ & 14 & $<8.000$ & $<0.200$ & -- & - & -- & - & -- & - \\
\hline 34413 & METHYL BROMIDE, TOTAL $(\mu \mathrm{g} / \mathrm{L})$ & 14 & $<8.000$ & $<0.200$ & -- & -- & -- & - & -- & - \\
\hline 34423 & METHYLENE CHLORIDE, TOTAL $(\mu \mathrm{g} / \mathrm{L})$ & 14 & $<8.000$ & $<0.200$ & -- & - & -- & -- & -- & -- \\
\hline
\end{tabular}

NOTE: Multiple detection limits during the period of record may result in different values flagged with a "<."

* Value is estimated by using a log-probability regression to predict the values of data below the detection limit. 
Table 74. Statistical summary of water-quality data at site 42 (CSW04), May 1994 through September 1998-Continued

\begin{tabular}{|c|c|c|c|c|c|c|c|c|c|c|}
\hline \multirow{2}{*}{$\begin{array}{c}\text { PARAM- } \\
\text { ETER } \\
\text { CODE }\end{array}$} & \multirow{2}{*}{ PROPERTY OR CONSTITUENT } & & DESCRIPTI & STATISTICS & & PERCENT OI & $\begin{array}{r}\text { AMPLES II } \\
\text { EQUAI }\end{array}$ & $\begin{array}{l}\text { WHICH VALU } \\
\text { TO THOSE S }\end{array}$ & $\begin{array}{l}\text { WERE LE } \\
\text { WN }\end{array}$ & THAN OA \\
\hline & & $\begin{array}{l}\text { SAMPLE } \\
\text { SIZE }\end{array}$ & MAXIMUM & MINIMUM & MEAN & $95 \%$ & $75 \%$ & $\begin{array}{c}50 \% \\
\text { (median) }\end{array}$ & $25 \%$ & $5 \%$ \\
\hline 34516 & $1,1,2,2$-TETRACHLOROETHANE, TOTAL $(\mu \mathrm{g} / \mathrm{L})$ & 14 & $<8,000$ & $<0.200$ & -- & -- & -- & -- & -- & -+ \\
\hline 34475 & TETRACHLOROETHYLENE，TOTAL $(\mu \mathrm{g} / \mathrm{L})$ & 14 & $<8.000$ & $<0.200$ & -- & -- & -- & -- & -- & -- \\
\hline 34010 & TOLUENE, TOTAL $(\mu \mathrm{g} / \mathrm{L})$ & 14 & $<8.000$ & $<0.200$ & -- & -- & -- & -- & -- & -- \\
\hline 34546 & 1,2-TRANSDICHLOROETHENE, TOTAL $(\mu \mathrm{g} / \mathrm{L})$ & 14 & $<8.000$ & $<0.200$ & -- & -- & -- & -- & -- & -- \\
\hline 34506 & $1,1,1$-TRICHLOROETHANE， TOTAL $(\mu \mathrm{g} / \mathrm{L})$ & 14 & $<8.000$ & $<0.200$ & -- & -- & - & -- & -- & -- \\
\hline 34511 & $1,1,2$-TRICHLOROETHANE, TOTAL $(\mu \mathrm{g} / \mathrm{L})$ & 14 & $<8.000$ & $<0.200$ & -- & - &.- & - & - & -- \\
\hline 39180 & TRICHLOROETHYLENE， TOTAL $(\mu \mathrm{g} / \mathrm{L})$ & 14 & $<8.000$ & $<0.200$ & -- & -- & -- & -- & -- & -- \\
\hline 39175 & VINYL CHLORIDE, TOTAL $(\mu \mathrm{g} / \mathrm{L})$ & 14 & $<8.000$ & $<0.200$ & -- & -- & -- & -- & -- & -- \\
\hline 30217 & DIBROMOMETHANE, TOTAL $(\mu \mathrm{g} / \mathrm{L})$ & 14 & $<8.000$ & $<0.200$ & -- & -- & -- & -- & -- & -- \\
\hline 32101 & DICHLOROBROMOMETHANE， TOTAL $(\mu \mathrm{g} / \mathrm{L})$ & 14 & $<8.000$ & $<0.200$ & -- & -- & -- & -- & -- & -- \\
\hline 34668 & DICHLORODIFLUOROMETHANE，TOTAL $(\mu \mathrm{g} / \mathrm{L})$ & 14 & $<8.000$ & $<0.200$ & -- & -- & -- & -- & -- & -- \\
\hline 34488 & TRICHLOROFLUOROMETHANE， TOTAL $(\mu \mathrm{g} / \mathrm{L})$ & 14 & $<8.000$ & $<0.200$ & -- & -- & -- & -- & -- & -- \\
\hline 77651 & 1,2 -DIBROMOETHANE， TOTAL $(\mu \mathrm{g} / \mathrm{L})$ & 14 & $<8.000$ & $<0.200$ & -- & -- & - & -- & -- & -- \\
\hline 34418 & METHYLCHLORIDE, TOTAL $(\mu \mathrm{g} / \mathrm{L})$ & 14 & $<8.000$ & $<0.200$ & -- & -- & -- & -- & -- & -- \\
\hline 34704 & CIS 1,3 -DICHLOROPROPENE, TOTAL $(\mu \mathrm{g} / \mathrm{L})$ & 14 & $<8.000$ & $<0.200$ & -- & -- & -- & -- & -- & -- \\
\hline 34699 & TRANS 1,3 -DICHLOROPROPENE, TOTAL $(\mu \mathrm{g} / \mathrm{L})$ & 14 & $<8.000$ & $<0.200$ & -- & -- & -- & -- & -- & -- \\
\hline 77128 & STYRENE， TOTAL $(\mu \mathrm{g} / \mathrm{L})$ & 14 & $<8.000$ & $<0.200$ & -- & -- & -- & -- & -- & -- \\
\hline 81551 & XYLENE, TOTAL $(\mu \mathrm{g} / \mathrm{L})$ & 14 & $<8.000$ & $<0.200$ & -- & -+ & 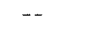 & -- & -- & -- \\
\hline 82625 & DIBROMOCHLOROPROPANE, TOTAL $(\mu \mathrm{g} / \mathrm{L})$ & 14 & $<40.000$ & $<1.000$ & -- & -- & -- & -- & -- & -- \\
\hline 77168 & 1,1 -DICHLOROPROPENE, TOTAL $(\mu \mathrm{g} / \mathrm{L})$ & 14 & $<8.000$ & $<0.200$ & -- & -- & - & -- & -- & -- \\
\hline 77170 & 2,2 -DICHLOROPROPANE, TOTAL $(\mu \mathrm{g} / \mathrm{L})$ & 14 & $<8.000$ & $<0.200$ & -- & -- & -- & -- & -- & -- \\
\hline 77173 & 1,3 -DICHLOROPROPANE, TOTAL $(\mu \mathrm{g} / \mathrm{L})$ & 14 & $<8.000$ & $<0.200$ & -- & -- & -- & -- & -- & -- \\
\hline 77275 & O-CHLOROTOLUENE, TOTAL $(\mu \mathrm{g} / \mathrm{L})$ & 14 & $<8.000$ & $<0.200$ & -- & -- & -- & -- & -- & -- \\
\hline 77277 & P-CHLOROTOLUENE， TOTAL $(\mu \mathrm{g} / \mathrm{L})$ & 14 & $<8.000$ & $<0.200$ & -- & -- & -- & -- & -- & -- \\
\hline 77443 & 123 -TRICHLOROPROPANE，TOTAL $(\mu \mathrm{g} / \mathrm{L})$ & 14 & $<8.000$ & $<0.200$ & -- & -- & -- & -- & -- & -- \\
\hline 77562 & 1112 -TETRACHLOROETHANE，TOTAL $(\mu \mathrm{g} / \mathrm{L})$ & 14 & $<8.000$ & $<0.200$ & -- & -- & -- & -- & -- & -- \\
\hline 78032 & TERTBUTYL METHYL ETHER, TOTAL $(\mu \mathrm{g} / \mathrm{L})$ & 14 & $<8.000$ & $<0.200$ & -- & -- & -- & -- & -- & -- \\
\hline 77297 & BROMOCHLORO METHANE, TOTAL $(\mu \mathrm{g} / \mathrm{L})$ & 14 & $<8.000$ & $<0.200$ & -- & -- & -- & -- & -- & -- \\
\hline 77093 & CIS-1,2-DICHLOROETHENE, TOTAL $(\mu \mathrm{g} / \mathrm{L})$ & 14 & $<8.000$ & $<0.200$ & -- & -- & -- & -- & -- & -- \\
\hline 34576 & 2 -CHLOROETHYL VINYL ETHER, TOTAL $(\mu \mathrm{g} / \mathrm{L})$ & 10 & $<40.000$ & $<1.000$ & -- & -- & -- & -- & -- & -- \\
\hline 77223 & ISOPROPYL BENZENE, TOTAL $(\mu \mathrm{g} / \mathrm{L})$ & 14 & $<8.000$ & $<0.200$ & -- & -- & -- & -- & -- & -- \\
\hline 77224 & N-PROPYL BENZENE, TOTAL $(\mu \mathrm{g} / \mathrm{L})$ & 14 & $<8.000$ & $<0.200$ & -- & -- & -- & -- & -- & - \\
\hline 77353 & TERTBUTYL BENZENE, TOTAL $(\mu \mathrm{g} / \mathrm{L})$ & 14 & $<8.000$ & $<0.200$ & -- & -- & -- & -- & -- & -- \\
\hline 77222 & PSEUDOCUMENE, TOTAL $(\mu \mathrm{g} / \mathrm{L})$ & 14 & $<8.000$ & $<0.200$ & -- & -- & -- & -- & -- & -- \\
\hline 77350 & SEC-BUTYL BENZENE, TOTAL $(\mu \mathrm{g} / \mathrm{L})$ & 14 & $<8.000$ & $<0.200$ & -- & -- & -- & -- & -- & -- \\
\hline 77356 & P-ISOPROPYL TOLUENE, TOTAL $(\mu \mathrm{g} / \mathrm{L})$ & 14 & $<8.000$ & $<0.200$ & -- & -- & -- & -- & -- & -- \\
\hline 77342 & N-BUTYL BENZENE, TOTAL $(\mu \mathrm{g} / \mathrm{L})$ & 14 & $<8.000$ & $<0.200$ & -- & -- & -- & -- & -- & -- \\
\hline 77613 & $1,2,3$-TRICHLOROBENZENE, TOTAL $(\mu \mathrm{g} / \mathrm{L})$ & 14 & $<8.000$ & $<0.200$ & -- & -- & -- & -- & -- & -- \\
\hline 77652 & FREON-113, TOTAL $(\mu \mathrm{g} / \mathrm{L})$ & 14 & $<8.000$ & $<0.200$ & -- & -- & -- & -- & -- & -- \\
\hline 77226 & MESITYLENE， TOTAL $(\mu \mathrm{g} / \mathrm{L})$ & 14 & $<8.000$ & $<0.200$ & -- & -- & -- & -- & -- & -- \\
\hline 81555 & BROMOBENZENE，TOTAL $(\mu \mathrm{g} / \mathrm{L})$ & 14 & $<8.000$ & $<0.200$ & -- & -- & -- & -- & -- & -- \\
\hline 34551 & $1,2,4$-TRICHLOROBENZENE, TOTAL $(\mu \mathrm{g} / \mathrm{L})$ & 14 & $<8.000$ & $<0.200$ & -- & -- & -- & -- & -- & -- \\
\hline 34536 & 1,2-DICHLOROBENZENE, TOTAL $(\mu \mathrm{g} / \mathrm{L})$ & 14 & $<8.000$ & $<0.200$ & -- & -- & -- & -- & -- & -- \\
\hline 34566 & 1,3-DICHLOROBENZENE, TOTAL $(\mu \mathrm{g} / \mathrm{L})$ & 14 & $<8.000$ & $<0.200$ & - & -- & -- & -- & -- & -- \\
\hline 34571 & 1,4-DICHLOROBENZENE, TOTAL $(\mu \mathrm{g} / \mathrm{L})$ & 14 & $<8.000$ & $<0.200$ & -- & -- & -- & -- & -- & -- \\
\hline 39702 & HEXACHLOROBUTADIENE, TOTAL $(\mu \mathrm{g} / \mathrm{L})$ & 14 & $<8.000$ & $<0.200$ & -- & -- & -- & -- & -- & -- \\
\hline 34696 & NAPHTHALENE，TOTAL $(\mu \mathrm{g} / \mathrm{L})$ & 14 & $<8.000$ & $<0.200$ & -- & -- & -- & -- & -- & -- \\
\hline & & MET & LS AND MIN & CONSTITUE & TS, TOTAL & & & & & \\
\hline 01097 & ANTIMONY, TOTAL $(\mu \mathrm{g} / \overline{\mathrm{L}}$ as Sb$)$ & 39 & 8.000 & $<1.000$ & $0.930^{*}$ & 5.000 & 1.000 & $<1.000$ & $<1.000$ & $<1.000$ \\
\hline 01002 & ARSENIC, TOTAL $(\mu \mathrm{g} / \mathrm{L}$ as AS $)$ & 39 & 28.000 & $<1.000$ & 1.878 * & 5.000 & 2.000 & 1.000 & $<1.000$ & $<1.000$ \\
\hline 01012 & BERYLLIUM, TOTAL ( $\mu \mathrm{g} / \mathrm{L}$ as Be) & 12 & $<10.000$ & $<10.000$ & -- & $<10.000$ & $<10.000$ & $<10.000$ & $<10.000$ & $<10.000$ \\
\hline 01027 & CADMIUM, TOTAL $(\mu \mathrm{g} / \mathrm{L}$ as $\mathrm{Cd})$ & 12 & 1.000 & $<1.000$ & -- & 1.000 & $<1.000$ & $<1.000$ & $<1.000$ & $<1.000$ \\
\hline 01034 & CHROMIUM, TOTAL $(\mu \mathrm{g} / \mathrm{L}$ as $\mathrm{Cr})$ & 39 & 68.000 & 1.000 & 12.077 & 35.000 & 17.000 & 7.000 & 3.000 & 2.000 \\
\hline 01042 & COPPER, TOTAL $(\mu \mathrm{g} / \mathrm{L}$ as $\mathrm{Cu})$ & 39 & 110.000 & 4.000 & 29.590 & 87.000 & 40.000 & 21.000 & 15.000 & 4.000 \\
\hline 01051 & LEAD, TOTAL $(\mu \mathrm{g} / \mathrm{L}$ as $\mathrm{Pb})$ & 39 & 130.000 & $<1.000$ & $18.291^{\star}$ & 73.000 & 19.000 & 11.000 & 8.000 & $<1.000$ \\
\hline 71900 & MERCURY, TOTAL $(\mu \mathrm{g} / \mathrm{L}$ as $\mathrm{Hg})$ & 12 & 0.110 & $<0.100$ & -- & 0.110 & $<0.100$ & $<0.100$ & $<0.100$ & $<0.100$ \\
\hline 01067 & NICKEL, TOTAL $(\mu \mathrm{g} / \mathrm{L}$ as $\mathrm{Ni})$ & 39 & 24.000 & $<1.000$ & $6.365^{\star}$ & 14.000 & 8.000 & 6.000 & 3.000 & $<1.000$ \\
\hline 01147 & SELENIUM, TOTAL $(\mu \mathrm{g} / \mathrm{L}$ as $\mathrm{Se})$ & 12 & $<1.000$ & $<1.000$ & -- & $<1.000$ & $<1.000$ & $<1.000$ & $<1.000$ & $<1.000$ \\
\hline 01077 & SILVER, TOTAL $(\mu \mathrm{g} / \mathrm{L}$ as $\mathrm{Ag})$ & 12 & $<1.000$ & $<1.000$ & -- & $<1.000$ & $<1.000$ & $<1.000$ & $<1.000$ & $<1.000$ \\
\hline 01092 & $\mathrm{ZINC}, \operatorname{TOTAL}(\mu \mathrm{g} / \mathrm{L}$ as $\mathrm{Zn})$ & 39 & 320.000 & 20.000 & 111.795 & 280.000 & 140.000 & 100.000 & 60.000 & 40.000 \\
\hline 00720 & CYANIDE, TOTAL (mg/L as Cn) & 12 & $<0.010$ & $<0.010$ & -- & $<0.010$ & $<0.010$ & $<0.010$ & $<0.010$ & $<0.010$ \\
\hline & & ORGA & C COMPOUN & -PESTICIDI & , DISSOLVF & & & & & \\
\hline 49260 & ACETOCHLOR， DISSOLVED $(\mu \mathrm{g} / \mathrm{L})$ & 2 & $<0.002$ & $<0.002$ & -- & -- & -- & -- & -- & -- \\
\hline 46342 & ALACHLOR, DISSOLVED $(\mu \mathrm{g} / \mathrm{L})$ & 3 & $<0.009$ & $<0.002$ & -- & -- & -- & -- & -- & -- \\
\hline 04040 & DEETHYL ATRAZINE, DISSOLVED $(\mu \mathrm{g} / \mathrm{L})$ & 3 & $<0.005$ & $<0.002$ & -- & -- & -- & -- & -- & -- \\
\hline 39632 & ATRAZINE, DISSOLVED $(\mu \mathrm{g} / \mathrm{L})$ & 3 & 0.019 & 0.011 & -- & -- & -- & -- & -- & -- \\
\hline 82686 & METHYL AZINPHOS, DISSOLVED $(\mu \mathrm{g} / \mathrm{L})$ & 3 & $<0.050$ & $<0.001$ & -- & -- & -- & -- & -- & -- \\
\hline 82673 & BENFLURALIN， DISSOLVED $(\mu \mathrm{g} / \mathrm{L})$ & 3 & $<0.013$ & $<0.002$ & -- & -- & -- & -- & -- & -- \\
\hline 04028 & BUTYLATE， DISSOLVED $(\mu \mathrm{g} / \mathrm{L})$ & 3 & $<0.008$ & $<0.002$ & -- & -- & -- & -- & -- & -- \\
\hline 82680 & CARBARYL，DISSOLVED $(\mu \mathrm{g} / \mathrm{L})$ & 3 & 0.221 & 0.016 & -- & -- & -- & -- & -- & -- \\
\hline 82674 & CARBOFURAN， DISSOLVED $(\mu \mathrm{g} / \mathrm{L})$ & 3 & $<0.013$ & $<0.003$ & -- & -- & -- & -- & -- & -- \\
\hline 38933 & CHLORPYRIFOS， DISSOLVED $(\mu \mathrm{g} / \mathrm{L})$ & 3 & $<0.008$ & $<0.004$ & -- & -- & -- & -- & -- & -- \\
\hline 04041 & CYANAZINE，DISSOLVED $(\mu \mathrm{g} / \mathrm{L})$ & 3 & $<0.013$ & $<0.004$ & -- & -- & -- & -- & -- & -- \\
\hline 82682 & DCPA， DISSOLVED $(\mu \mathrm{g} / \mathrm{L})$ & 3 & 0.004 & $<0.002$ & -- & -- & -- & -- & -- & - \\
\hline 34653 & $\mathrm{P}, \mathrm{P}^{\prime} \mathrm{DDE}, \mathrm{DISSOLVED}(\mu \mathrm{g} / \mathrm{L})$ & 3 & $<0.010$ & $<0.006$ & -- & -- & -- & -- & -- & -- \\
\hline 39572 & DIAZINON，DISSOLVED $(\mu \mathrm{g} / \mathrm{L})$ & 3 & 0.037 & $<0.002$ & -- & -- & -- & -- & -- & -- \\
\hline 39381 & DIELDRIN，DISSOLVED $(\mu \mathrm{g} / \mathrm{L})$ & 3 & $<0.008$ & $<0.001$ & -- & -- & -- & -- & -- & - \\
\hline 82660 & 2,6 -DIETHYL ANILINE, DISSOLVED $(\mu \mathrm{g} / \mathrm{L})$ & 3 & $<0.006$ & $<0.003$ & -- & -- & -- & -- & -- & -- \\
\hline 82662 & DIMETHOATE， DISSOLVED $(\mu \mathrm{g} / \mathrm{L})$ & 1 & $<0.020$ & -- & -- & -- & -- & -- & -- & -- \\
\hline 82677 & DISULFOTON， DISSOLVED $(\mu \mathrm{g} / \mathrm{L})$ & 3 & $<0.060$ & $<0.017$ & -- & -- & -- & -- & -- & -- \\
\hline 82668 & EPTC， DISSOLVED $(\mu \mathrm{g} / \mathrm{L})$ & 3 & $<0.005$ & $<0.002$ & -- & -- & - & -- & -- & -- \\
\hline 82663 & ETHALFLURALIN， DISSOLVED $(\mu \mathrm{g} / \mathrm{L})$ & 3 & $<0.013$ & $<0.004$ & -- & -- & -- & -- & -- & -- \\
\hline
\end{tabular}

NOTE: Multiple detection limits during the period of record may result in different values flagged with a "<."

* Value is estimated by using a log-probability regression to predict the values of data below the detection limit. 
Table 74. Statistical summary of water-quality data at site 42 (CSW04), May 1994 through September 1998-Continued

\begin{tabular}{|c|c|c|c|c|c|c|c|c|c|c|}
\hline \multirow{2}{*}{$\begin{array}{l}\text { PARAM- } \\
\text { ETER } \\
\text { CODE }\end{array}$} & \multirow{2}{*}{ PROPERTY OR CONSTITUENT } & & DESCRIPTI & STATISTICS & & PERCEN & $\begin{array}{l}\text { AMPLE } \\
\text { EC }\end{array}$ & $\begin{array}{l}\text { WHICH VAl } \\
\text { TO THOSE }\end{array}$ & $\begin{array}{l}\text { WERE } \\
\text { A'N }\end{array}$ & HAN OR \\
\hline & & $\begin{array}{c}\text { SAMPLE } \\
\text { SIZE }\end{array}$ & MAXIMUM & MINIMUM & MEAN & $95 \%$ & $75 \%$ & $\begin{array}{c}50 \% \\
\text { (medlen) }\end{array}$ & $25 \%$ & $5 \%$ \\
\hline 82672 & ETHOPROP, DISSOLVED $(\mu \mathrm{g} / \mathrm{L})$ & 3 & $<0.012$ & $<0.003$ & -- & -- & -- & +- & -- & -- \\
\hline 04095 & FONOFOS, DISSOLVED $(\mu \mathrm{g} / \mathrm{L})$ & 3 & $<0.008$ & $<0.003$ & -- & -- & -- & -- & -- & -- \\
\hline 34253 & ALPHA BHC, DISSOLVED $(\mu \mathrm{g} / \mathrm{L})$ & 3 & $<0.007$ & $<0.002$ & -- & -- & -- & -- & -- & -- \\
\hline 39341 & LINDANE，DISSOLVED $(\mu \mathrm{g} / \mathrm{L})$ & 3 & $<0.011$ & $<0.004$ & -- & - & -- & -- & -- & -- \\
\hline 82666 & LINURON, DISSOLVED $(\mu \mathrm{g} / \mathrm{L})$ & 3 & $<0.039$ & $<0.002$ & -- & -- & -- & -- & -- & -- \\
\hline 39532 & MALATHION, DISSOLVED $(\mu \mathrm{g} / \mathrm{L})$ & 3 & 0.029 & $<0.014$ & -- & -- & -- & -- & -- & -- \\
\hline 82667 & METHYL PARATHION, DISSOLVED $(\mu \mathrm{g} / \mathrm{L})$ & 3 & $<0.035$ & $<0.006$ & -- & -- & -- & -- & -- & -- \\
\hline 39415 & METOLACHLOR, DISSOLVED $(\mu \mathrm{g} / \mathrm{L})$ & 3 & 0.010 & $<0.002$ & -- & -- & -- & -- & +- & -- \\
\hline 82630 & METRIBUZIN， DISSOLVED $(\mu \mathrm{g} / \mathrm{L})$ & 3 & $<0.012$ & $<0.004$ & -- & -- & -- & -- & -- & -- \\
\hline 82671 & MOLINATE, DISSOLVED $(\mu \mathrm{g} / \mathrm{L})$ & 3 & $<0.007$ & $<0.004$ & -- & -- & -- & -- & -- & -- \\
\hline 82684 & NAPROPAMIDE， DISSOLVED $(\mu \mathrm{g} / \mathrm{L})$ & 3 & $<0.010$ & $<0.003$ & -- & -- & -- & -- & -- & -- \\
\hline 39542 & PARATHION， DISSOLVED $(\mu \mathrm{g} / \mathrm{L})$ & 3 & $<0.022$ & $<0.004$ & -- & -- & -- & -- & -- & -- \\
\hline 82669 & PEBULATE， DISSOLVED $(\mu \mathrm{g} / \mathrm{L})$ & 3 & $<0.009$ & $<0.004$ & -- & -- & -- & -- & -- & -- \\
\hline 82683 & PENDIMETHALIN，DISSOLVED $(\mu \mathrm{g} / \mathrm{L})$ & 3 & 0.210 & $<0.018$ & -- & -- & -- & -- & -- & -- \\
\hline 82687 & PERMETHRIN, DISSOLVED $(\mu \mathrm{g} / \mathrm{L})$ & 3 & $<0.016$ & $<0.005$ & -- & -- & -- & -- & -- & -- \\
\hline 82664 & PHORATE, DISSOLVED $(\mu \mathrm{g} / \mathrm{L})$ & 3 & $<0.011$ & $<0.002$ & -- & -- & -- & -- & -- & -- \\
\hline 82676 & PRONAMIDE， DISSOLVED $(\mu \mathrm{g} / \mathrm{L})$ & 3 & $<0.009$ & $<0.003$ & -- & -- & -- & -- & -- & -- \\
\hline 04037 & PROMETON, DISSOLVED $(\mu \mathrm{g} / \mathrm{L})$ & 3 & 0.027 & $<0.018$ & -- & -- & -- & -- & -- & -- \\
\hline 04024 & PROPACHLOR, DISSOLVED $(\mu \mathrm{g} / \mathrm{L})$ & 3 & $<0.015$ & $<0.007$ & -- & -- & -- & -- & -- & -- \\
\hline 82679 & PROPANIL， DISSOLVED $(\mu \mathrm{g} / \mathrm{L}\}$ & 3 & $<0.016$ & $<0.004$ & -- & $\ldots$ & -- & -- & -- & -- \\
\hline 82685 & PROPARGITE, DISSOLVED $(\mu \mathrm{g} / \mathrm{L})$ & 3 & $<0.013$ & $<0.008$ & -- & -- & -- & -- & -- & -- \\
\hline 04035 & SIMAZINE, DISSOLVED $(\mu \mathrm{g} / \mathrm{L})$ & 3 & $<0.008$ & $<0.005$ & -- & -- & -- & -- & -- & - \\
\hline 82681 & THIOBENCARB， DISSOLVED $(\mu \mathrm{g} / \mathrm{L})$ & 3 & $<0.008$ & $<0.002$ & -- & -- & -- & -- & -- & -- \\
\hline 82670 & TEBUTHIURON, DISSOLVED $(\mu \mathrm{g} / \mathrm{L})$ & 3 & 0.055 & $<0.010$ & -- & -- & -- & -- & -- & -- \\
\hline 82665 & TERBACIL, DISSOLVED $(\mu \mathrm{g} / \mathrm{L})$ & 3 & $<0.030$ & $<0.007$ & -- & -- & -- & -- & -- & -- \\
\hline 82675 & TERBUFOS, DISSOLVED $(\mu \mathrm{g} / \mathrm{L})$ & 3 & $<0.013$ & $<0.012$ & -- & -- & -- & -- & -- & -- \\
\hline 82678 & TRIALLATE，DISSOLVED $(\mu \mathrm{g} / \mathrm{L})$ & 3 & $<0.008$ & $<0.001$ & -- & -- & -- & -- & -- & -- \\
\hline 82661 & TRIFLURALIN， DISSOLVED $(\mu \mathrm{g} / \mathrm{L})$ & 3 & $<0.012$ & $<0.002$ & -- & -- & -- & -- & -- & -- \\
\hline 39742 & $2,4,5-T$, DISSOLVED $(\mu \mathrm{g} / \mathrm{L})$ & 4 & $<0.050$ & $<0.035$ & -- & -- & -- & -- & -- & -- \\
\hline 39732 & $2,4-D$, DISSOLVED $(\mu \mathrm{g} / \mathrm{L})$ & 4 & 1.180 & $<0.035$ & -- & -- & -- & -- & -- & -- \\
\hline 38746 & $2,4-D B$, DISSOLVED $(\mu \mathrm{g} / L)$ & 4 & $<0.240$ & $<0.035$ & -- & -- & -- & -- & -- & -- \\
\hline 49315 & ACIFLUORFEN, DISSOLVED $(\mu \mathrm{g} / \mathrm{L})$ & 4 & $<0.050$ & $<0.035$ & -- & -- & -- & + & -- & -- \\
\hline 49312 & ALDICARB, DISSOLVED $(\mu \mathrm{g} / \mathrm{L})$ & 4 & $<0.550$ & $<0.016$ & -- & -- & -- & -- & -- & -- \\
\hline 49313 & ALDICARB SULFONE, DISSOLVED $(\mu \mathrm{g} / \mathrm{L})$ & 4 & $<0.100$ & $<0.016$ & -- & -- & -- & -- & -- & -- \\
\hline 49314 & ALDICARB SULFOXIDE, DISSOLVED $(\mu \mathrm{g} / \mathrm{L})$ & 4 & $<0.050$ & $<0.021$ & -- & -- & -- & -- & -- & -- \\
\hline 38711 & BENTAZON， DISSOLVED $(\mu \mathrm{g} / \mathrm{L})$ & 4 & $<0.050$ & $<0.014$ & -- & -- & -- & -- & -- & -- \\
\hline 04029 & BROMACIL， DISSOLVED $(\mu \mathrm{g} / \mathrm{L})$ & 4 & $<0.050$ & $<0.035$ & -- & -- & -- & -- & -- & $\cdots$ \\
\hline 49311 & BROMOXYNIL, DISSOLVED $(\mu \mathrm{g} / \mathrm{L})$ & 4 & $<0.050$ & $<0.035$ & -- & -- & -- & -- & -- & -- \\
\hline 49310 & CARBARYL, DISSOLVED $(\mu \mathrm{g} / \mathrm{L})$ & 4 & $<0.050$ & $<0.008$ & -- & -- & -- & -- & -- & -- \\
\hline 49309 & CARBOFURAN, DISSOLVED $(\mu \mathrm{g} / \mathrm{L})$ & 4 & $<0.120$ & $<0.028$ & -- & -- & -- & -- & -- & -- \\
\hline 49308 & 3 -HYDROXY-CARBOFURAN $(\mu \mathrm{g} / \mathrm{L})$ & 4 & $<0.050$ & $<0.014$ & -- & -- & -- & -- & -- & -- \\
\hline 49307 & AMIBEN, DISSOLVED $(\mu \mathrm{g} / \mathrm{L})$ & 4 & $<0.420$ & $<0.011$ & -- & -- & $-\cdots$ & -- & -- & -- \\
\hline 49306 & CHLOROTHALONIL, DISSOLVED $(\mu \mathrm{g} / \mathrm{L})$ & 4 & $<0.480$ & $<0.035$ & -- & -- & -- & -- & -- & -- \\
\hline 49305 & CLOPYRALID， DISSOLVED $(\mu \mathrm{g} / \mathrm{L})$ & 4 & $<0.230$ & $<0.050$ & -- & -- & -- & -- & -- & -- \\
\hline 49304 & DACTHALMONO-ACID, DISSOLVED $(\mu \mathrm{g} / \mathrm{L})$ & 4 & $<0.050$ & $<0.017$ & -- & -- & -- & -- & -- & -- \\
\hline 38442 & DICAMBA， DISSOLVED $(\mu \mathrm{g} / \mathrm{L})$ & 4 & $<0.050$ & $<0.035$ & -- & -- & -- & -- & -- & -- \\
\hline 49303 & DICHLOBENIL，DISSOLVED $(\mu \mathrm{g} / \mathrm{L})$ & 4 & $<1.200$ & $<0.020$ & -- & -- & -- & - & -- & -- \\
\hline 49302 & DICHLORPROP， DISSOLVED $(\mu \mathrm{g} / \mathrm{L})$ & 4 & $<0.050$ & $<0.032$ & -- & -- & -- & -- & -- & -- \\
\hline 49301 & DINOSEB, DISSOLVED $(\mu \mathrm{g} / \mathrm{L})$ & 4 & $<0.050$ & $<0.035$ & -- & -- & -- & -- & -- & -- \\
\hline 49300 & DIURON, DISSOLVED $(\mu \mathrm{g} / \mathrm{L})$ & 4 & $<5.160$ & $<0.020$ & -- & -- & -- & +- & -- & -- \\
\hline 49299 & 4,6-DINITRO OCRESOL， DISSOLVED $(\mu \mathrm{g} / \mathrm{L})$ & 4 & $<0.420$ & $<0.035$ & -- & -- & -- & -- & -- & -- \\
\hline 49298 & ESFENVALERATE， DISSOLVED $(\mu \mathrm{g} / \mathrm{L})$ & 3 & $<0.050$ & $<0.019$ & -- & $-\cdots$ & -- & -- & -- & -- \\
\hline 49297 & FENURON, DISSOLVED $(\mu \mathrm{g} / \mathrm{L})$ & 4 & $<0.050$ & $<0.013$ & -- & -- & -- & -- & -- & -- \\
\hline 38811 & FLUOMETURON, DISSOLVED $(\mu \mathrm{g} / \mathrm{L})$ & 4 & $<0.050$ & $<0.035$ & -- & -- & -- & -- & -- & -- \\
\hline 38478 & LINURON, DISSOLVED $(\mu \mathrm{g} / \mathrm{L})$ & 4 & $<0.050$ & $<0.018$ & -- & -- & -- & -- & -- & -- \\
\hline 38482 & MCPA, DISSOLVED $(\mu \mathrm{g} / \mathrm{L})$ & 4 & $<0.170$ & $<0.050$ & -- & -- & -- & -- & -- & -- \\
\hline 38487 & MCPB， DISSOLVED $(\mu \mathrm{g} / \mathrm{L})$ & 4 & $<0.140$ & $<0.035$ & -- & -- & -- & -- & -- & -- \\
\hline 38501 & METHIOCARB， DISSOLVED $(\mu \mathrm{g} / \mathrm{L})$ & 4 & $<0.050$ & $<0.026$ & -- & -- & -- & -- & -- & -- \\
\hline 49296 & METHOMYL, DISSOLVED $(\mu \mathrm{g} / \mathrm{L})$ & 4 & $<0.050$ & $<0.017$ & -- & -- & -- & -- & -- & -- \\
\hline 49295 & 1-NAPHTHOL， DISSOLVED $(\mu \mathrm{g} / \mathrm{L})$ & 3 & $<0.050$ & $<0.007$ & -- & -- & -- & -- & -- & -- \\
\hline 49294 & NEBURON, DISSOLVED $(\mu \mathrm{g} / \mathrm{L})$ & 4 & $<0.050$ & $<0.015$ & -- & -- & -- & -- & - & -- \\
\hline 49293 & NORFLURAZON, DISSOLVED $(\mu \mathrm{g} / \mathrm{L})$ & 4 & $<0.050$ & $<0.024$ & -- & -- & -- & -- & -- & -- \\
\hline 49292 & ORYZALIN, DISSOLVED $(\mu \mathrm{g} / \mathrm{L})$ & 4 & $<0.310$ & $<0.019$ & -- & -- & -- & -- & -- & -- \\
\hline 38866 & OXAMYL, DISSOLVED $(\mu \mathrm{g} / \mathrm{L})$ & 4 & $<0.050$ & $<0.018$ & -- & -- & -- & -- & -- & -- \\
\hline 49291 & PICLORAM, DISSOLVED $(\mu \mathrm{g} / \mathrm{L})$ & 4 & $<0.050$ & $<0.050$ & -- & -- & -- & -- & -- & -- \\
\hline 49236 & PROPHAM， DISSOLVED $(\mu \mathrm{g} / \mathrm{L})$ & 4 & $<0.050$ & $<0.035$ & -- & -- & -- & -- & -- & -- \\
\hline 38538 & PROPOXUR, DISSOLVED $(\mu \mathrm{g} / \mathrm{L})$ & 4 & $<0.050$ & $<0.035$ & -- & -- & -- & -- & -- & -- \\
\hline 39762 & SILVEX, DISSOLVED $(\mu \mathrm{g} / \mathrm{L})$ & 4 & $<0.050$ & $<0.021$ & -- & -- & -- & -- & -- & - \\
\hline 49235 & TRICLOPYR, DISSOLVED $(\mu \mathrm{g} / \mathrm{L})$ & 4 & $<0.250$ & $<0.050$ & -- & -- & $\cdots$ & -- & -- & -- \\
\hline & & ORGANI & COMPOUND & ORGANONI & OGEN, 1 & TAL & & & & \\
\hline 39057 & PROMETRYNE, TOTAL $(\mu \mathrm{g} / \mathrm{L})$ & 1 & $<0.100$ & -- & -- & -- & -- & -- & -- & -- \\
\hline 39056 & PROMETONE, TOTAL $(\mu g / L)$ & 1 & $<0.200$ & + & -- & -- & -- & -- & -- & -- \\
\hline 39054 & SIMETRYNE, TOTAL $(\mu \mathrm{g} / \mathrm{L})$ & 1 & $<0.100$ & -- & -- & -- & -- & - & -- & -- \\
\hline 81757 & CYANAZINE, TOTAL $(\mu \mathrm{g} / \mathrm{L})$ & 1 & $<0.200$ & -- & -- & -- & -- & -- & -- & - \\
\hline 77825 & ALACHLOR, TOTAL $(\mu \mathrm{g} / \mathrm{L})$ & 1 & $<0.100$ & -- & -- & -- & -- & -- & -- & -- \\
\hline 82611 & METRIBUZIN, TOTAL $(\mu \mathrm{g} / \mathrm{L})$ & 1 & $<0.100$ & -- & -- & -- & -- & -- & -- & -- \\
\hline 30311 & TERBACIL, TOTAL $(\mu \mathrm{g} / \mathrm{L})$ & 1 & 0.300 & -- & -- & -- & -- & -- & -- & -- \\
\hline 30245 & CARBOXIN, TOTAL $(\mu \mathrm{g} / \mathrm{L})$ & 1 & $<0.200$ & -- & -- & -- & -- & -- & -- & -- \\
\hline 30264 & HEXAZINONE，TOTAL $(\mu \mathrm{g} / \mathrm{L})$ & 1 & $<0.200$ & -- & -- & -- & -. & -- & -- & -- \\
\hline 30235 & BUTACHLOR, TOTAL $(\mu \mathrm{g} / \mathrm{L})$ & 1 & $<0.100$ & -- & - & -- & -- & -- & -- & -- \\
\hline 30236 & BUTYLATE， TOTAL $(\mu \mathrm{g} / \mathrm{L})$ & 1 & $<0.100$ & -- & -- & -- & -- & -- & -- & -- \\
\hline 75981 & DE-ETHYLATRAZINE, TOTAL $(\mu \mathrm{g} / \mathrm{L})$ & 1 & $<0.200$ & -- & -- & -- & -- & -- & -- & -- \\
\hline
\end{tabular}

NOTE: Multiple detection limits during the period of record may result in different values flagged with a "<."

* Value is estimated by using a log-probability regression to predict the values of data below the detection limit. 
Table 74. Statistical summary of water-quality data at site 42 (CSW04), May 1994 through September 1998-Continued

\begin{tabular}{|c|c|c|c|c|c|c|c|c|c|c|}
\hline \multirow{2}{*}{$\begin{array}{l}\text { PARAM- } \\
\text { ETER } \\
\text { CODE }\end{array}$} & \multirow{2}{*}{ PROPERTY OR CONSTITUENT } & \multicolumn{4}{|c|}{ DESCRIPTIVE STATISTICS } & \multicolumn{5}{|c|}{$\begin{array}{l}\text { PERCENT OF SAMPLES IN WHICH VALUES WERE LESS THAN OR } \\
\text { EQUAL TO THOSE SHOWN }\end{array}$} \\
\hline & & $\begin{array}{l}\text { SAMPLE } \\
\text { SIZE }\end{array}$ & MAXIMUM & MINIMUM & MEAN & $95 \%$ & $75 \%$ & $\begin{array}{c}50 \% \\
\text { (medlan) }\end{array}$ & $25 \%$ & $5 \%$ \\
\hline 39630 & ATRAZINE, TOTAL $(\mu \mathrm{g} / \mathrm{L})$ & 1 & $<0.100$ & -- & -- & -- & -- & -- & -- & -- \\
\hline 39055 & SIMAZINE， TOTAL $(\mu \mathrm{g} / \mathrm{L})$ & 1 & $<0.100$ & -- & -- & -- & -- & -- & $-\cdots$ & -- \\
\hline 39024 & PROPAZINE, TOTAL $(\mu \mathrm{g} / \mathrm{L})$ & 1 & $<0.100$ & -- & -- & -- & -- & -- & -- & -- \\
\hline 82184 & AMETRYNE, TOTAL $(\mu \mathrm{g} / \mathrm{L})$ & 1 & $<0.100$ & - & -- & -- & -- & -- & -- & -- \\
\hline 39030 & TRIFLURALIN, TOTAL $(\mu \mathrm{g} / \mathrm{L})$ & 1 & $<0.100$ & -- & -- & -- & -- & -- & -- & -- \\
\hline 82612 & METOLACHLOR，TOTAL $(\mu \mathrm{g} / \mathrm{L})$ & 1 & $<0.200$ & -- & -. & -- & -- & -- & -- & -- \\
\hline 30234 & BROMACIL, TOTAL $(\mu \mathrm{g} / \mathrm{L})$ & 1 & $<0.200$ & -- & -- & -- & -- & -- & -- & -- \\
\hline 30255 & DIPHENAMID， TOTAL $(\mu \mathrm{g} / \mathrm{L})$ & 1 & $<0.100$ & -- & -- & -- & -- & -- & -- & -- \\
\hline 30324 & VERNOLATE, TOTAL $(\mu \mathrm{g} / \mathrm{L})$ & 1 & $<0.100$ & - & -- & -- & -- & -- & - - & -- \\
\hline 30254 & CYCLOATE, TOTAL $(\mu \mathrm{g} / \mathrm{L})$ & 1 & $<0.100$ & -- & - & -- & -- & -- & -- & -- \\
\hline 30295 & PROPACHLOR，TOTAL $(\mu \mathrm{g} / \mathrm{L})$ & 1 & $<0.100$ & -- & -- & -- & -- & -- & -- & -- \\
\hline 75980 & DE-ISOPROPYLATRAZIN， TOTAL $(\mu \mathrm{g} / \mathrm{L})$ & 1 & $<0.200$ & -- & -- & -- & -- & -- & -- & -- \\
\hline \multicolumn{11}{|c|}{ ORGANIC COMPOUNDS—HERBICIDES, TOTAL } \\
\hline 39730 & $2,4-\bar{D}$, TOTAL $(\mu \mathrm{g} / \mathrm{L})$ & 1 & 3.700 & -- & -- & -- & $-\cdots$ & -- & -- & -- \\
\hline 39760 & SILVEX, TOTAL $(\mu \mathrm{g} / \mathrm{L})$ & 1 & $<0.010$ & -- & -- & -- & -- & -- & -- & $\ldots$ \\
\hline 39720 & PICLORAM, TOTAL $(\mu \mathrm{g} / \mathrm{L})$ & 1 & $<0.010$ & -- & -- & -- & _- & -- & -- & - - \\
\hline 39740 & $2,4,5-\mathrm{T}$, TOTAL $(\mu \mathrm{g} / \mathrm{L})$ & 1 & $<0.010$ & -- & -- & -- & -- & -- & -- & -- \\
\hline 82183 & $2,4-\mathrm{DP}$, TOTAL $(\mu \mathrm{g} / \mathrm{L})$ & 1 & $<0.010$ & -- & -- & -- & -- & -- & -- & -- \\
\hline 82052 & DICAMBA, TOTAL $(\mu \mathrm{g} / \mathrm{L})$ & 1 & 0.650 & -- & $\ldots$ & -- & -- & -- & -- & -- \\
\hline \multicolumn{11}{|c|}{ ORGANIC COMPOUNDS_CARBAMATE PESTICIDES, TOTAL } \\
\hline 39750 & SEVIN, TOTAL $(\mu \mathrm{g} / \mathrm{L})$ & 1 & $<0.500$ & -- & -- & -- & -- & -- & $-m$ & -- \\
\hline 39051 & METHOMYL, TOTAL $(\mu \mathrm{g} / \mathrm{L})$ & 1 & $<0.500$ & -- & -- & -- & -- & -- & -- & -- \\
\hline 82619 & ALDICARD, TOTAL $(\mu \mathrm{g} / \mathrm{L}\}$ & 1 & $<0.500$ & -- & -- & -- & -- & -- & -- & -- \\
\hline 30296 & PROPOXUR, TOTAL $(\mu \mathrm{g} / \mathrm{L})$ & 1 & $<0.500$ & $\ldots$ & -- & - & -- & -- & -- & -- \\
\hline 39052 & PROPHAM, TOTAL $(\mu \mathrm{g} / \mathrm{L})$ & 1 & $<0.500$ & -- & -- & -- & -- & -- & -- & -- \\
\hline 82615 & CARBOFURAN, TOTAL $(\mu \mathrm{g} / \mathrm{L})$ & 1 & $<0.500$ & -- & -- & -- & -- & -- & -- &.- \\
\hline 77441 & 1-NAPHTHOL，TOTAL $(\mu \mathrm{g} / \mathrm{L})$ & 1 & $<0.500$ & -- & -- & -- & -- & - - & -- & -- \\
\hline 30282 & METHIOCARB， TOTAL $(\mu \mathrm{g} / \mathrm{L})$ & 1 & $<0.500$ & -- & -- & -- & -- & -- & -- & $\ldots$ \\
\hline
\end{tabular}

NOTE: Multiple detection limits during the period of record may result in different values flagged with a "<."

* Value is estimated by using a log-probability regression to predict the values of data below the detection limit. 
Table 75. Statistical summary of water-quality data at site 43 (CSW07), June 1994 through September 1998

\begin{tabular}{|c|c|c|c|c|c|c|c|c|c|c|}
\hline \multirow{2}{*}{$\begin{array}{l}\text { PARAM- } \\
\text { ETER } \\
\text { CODE }\end{array}$} & \multirow{2}{*}{ PROPERTY OR CONSTITUENT } & \multicolumn{4}{|c|}{ DESCRIPTIVE STATISTICS } & \multicolumn{5}{|c|}{$\begin{array}{l}\text { PERCENT OF SAMPLES IN WHICH VALUFS WERE LESS THAN OA } \\
\text { EQUAL TO THOSE SHNWN }\end{array}$} \\
\hline & & $\begin{array}{l}\text { SAMPLE } \\
\text { SIZE }\end{array}$ & MAXIMUM & MINIMUM & MEAN & $95 \%$ & $75 \%$ & $\begin{array}{c}50 \% \\
\text { (medlan) }\end{array}$ & $25 \%$ & $5 \%$ \\
\hline \multicolumn{11}{|c|}{ PHYSICAL AND CHEMICAL PROPERTIES } \\
\hline 00061 & INSTANTANEOUS DISCHARGE $\left(\mathrm{ft}^{3} / \mathrm{s}\right)$ & 85 & 344.000 & 0.030 & 12.183 & 36.000 & $1 \overline{1.000}$ & 2.700 & 0.950 & 0.193 \\
\hline 00010 & WATER TEMPERATURE $\left({ }^{\circ} \mathrm{C}\right)$ & 84 & 29.200 & 4.900 & 16.573 & 26.950 & 21.375 & 18.350 & 9.425 & 6.500 \\
\hline 90095 & SPECIFIC CONDUCTANCE, LAB ( $\mu \mathrm{S} / \mathrm{cm}$ at $\left.25{ }^{\circ} \mathrm{C}\right)$ & 59 & 1120.000 & 33.000 & 121.983 & 248.000 & 141.000 & 93.000 & 70.000 & 37.000 \\
\hline 00095 & $\begin{array}{l}\text { SPECIFIC CONDUCTANCE, FIELD ( } \mu \text { S/Cm at } 25 \\
{ }^{\circ} \mathrm{C} \text { ) }\end{array}$ & 79 & 1150.000 & 26.000 & 115.101 & 231.000 & 135.000 & 88.000 & 63.000 & 33.000 \\
\hline 00403 & pH, LAB (STANDARD pH UNITS) & 59 & 8.100 & 6.200 & 6.986 & 7.700 & 7.200 & 7.000 & 6.600 & 6.300 \\
\hline 00400 & pH, FIELD (STANDARD pH UNITS) & 76 & 7.900 & 6.600 & 7.042 & 7.415 & 7.200 & 7.000 & 6.900 & 6.700 \\
\hline 90410 & ALKALINITY, LAB (mg/L as $\mathrm{CaCO}_{3}$ ) & 58 & 145.000 & 8.000 & 30.069 & 87.100 & 36.750 & 22.000 & 16.000 & 10.950 \\
\hline 80154 & SUSPENDED SEDIMENT (mg/L) & 61 & 19400.000 & 42.000 & 2311.853 & 13180.005 & 2200.000 & 789.000 & 264.500 & 87.500 \\
\hline 00530 & $\begin{array}{l}\text { RESIDUE ON EVAPORATION AT } 105^{\circ} \mathrm{C} \text {, SUSPENDED } \\
(\mathrm{mg} / \mathrm{L})\end{array}$ & 30 & 23900.000 & 11.000 & 2173.933 & 16805.010 & 1797.500 & 648.500 & 110.000 & 14.300 \\
\hline 00535 & RESIDUE VOLATILE, SUSPENDED $(\mathrm{mg} / L)$ & 51 & 2200.000 & 1.000 & 188.569 & 1180.000 & 139.000 & 51.000 & 21.000 & 2.600 \\
\hline 70300 & DISSOLVED SOLIDS, RESIDUE AT $180^{\circ} \mathrm{C}(\mathrm{mg} / \mathrm{L})$ & 58 & 602.000 & 22.000 & 86.879 & 234.800 & 85.250 & 64.000 & 47.750 & 27.900 \\
\hline 00310 & 5 DAY BIOCHEMICAL OXYGEN DEMAND $(\mathrm{mg} / \mathrm{L})$ & 40 & 27.000 & $<2.000$ & $7.374 *$ & 19.000 & 8.800 & 5.500 & 3.100 & $<2.000$ \\
\hline 00340 & CHEMICAL OXYGEN DEMAND (mg/L) & 59 & 650.000 & 5.000 & 64.627 & 250.000 & 67.000 & 41.000 & 21.000 & 7.000 \\
\hline & & & NUTRIENTS, T & AL AND DIS & SOLVED & & & & & \\
\hline 00625 & $\begin{array}{l}\text { NITROGEN AMMONIA + ORGANIC, } \\
\text { TOTAL }(\mathrm{mg} / \mathrm{L} \text { as N) }\end{array}$ & 61 & 7.800 & 0.330 & 1.905 & 5.670 & 2.350 & 1.300 & 0.845 & 0.449 \\
\hline 00631 & $\mathrm{NO}_{2}+\mathrm{NO}_{3}$, DISSOLVED $(\mathrm{mg} / \mathrm{L}$ as $\mathrm{N})$ & 60 & 1.310 & 0.050 & 0.303 & 0.889 & 0.380 & 0.240 & 0.132 & 0.050 \\
\hline 00608 & NITROGEN AMMONIA, DISSOLVED (mg/L as N) & 60 & 1.100 & 0.015 & 0.197 & 0.884 & 0.230 & 0.120 & 0.050 & 0.017 \\
\hline 00605 & NITROGEN ORGANIC, TOTAL (mg/L as N) & 61 & 7.700 & 0.240 & 1.712 & 5.490 & 1.800 & 1.100 & 0.765 & 0.385 \\
\hline 00600 & NITROGEN, TOTAL (mg/L as N) & 61 & 8.300 & 0.450 & 2.204 & 5.940 & 3.000 & 1.500 & 1.000 & 0.622 \\
\hline 00665 & PHOSPHORUS, TOTAL (mg/L as P) & 61 & 33.500 & 0.110 & 3.312 & 14.560 & 2.935 & 1.000 & 0.520 & 0.194 \\
\hline 00671 & PHOSPHORUS ORTHO, DISSOLVED (mg/L as P) & 60 & 1.230 & $<0.010$ & $0.121 *$ & 0.620 & 0.090 & 0.050 & 0.020 & $<0.010$ \\
\hline & & & OIL AND & REASE, TOT & & & & & & \\
\hline 00556 & OIL AND GREASE, TOTAL (mg/L) & 15 & 4.000 & $<1.000$ & $1.451 *$ & $4 . \overline{000}$ & 2.000 & 1.000 & $<1.000$ & $<1.000$ \\
\hline & & & ORGANIC & ARBON, TOI & TAL & & & & & \\
\hline 00680 & CARBON ORGANIC, TOTAL $(\mathrm{mg} / \mathrm{L})$ & 34 & 56.000 & 4.400 & 17.035 & 35.750 & 21.250 & 16.000 & 11.375 & 6.650 \\
\hline & & & & IFORM & & & & & & \\
\hline 31679 & FECAL STREPTOCOCCI (Colonies per $100 \mathrm{~mL}$ ) & 26 & 70000.000 & 80.000 & 20948.076 & 69650.000 & 32000.000 & 10900.000 & 4125.000 & $145 . \overline{500}$ \\
\hline 31616 & FECAL COLIFORM (Colonies per $100 \mathrm{~mL}$ ) & 26 & 92000.0001 & 160.000 & 16888.846 & 77649.984 & 25750.000 & 11150.000 & 1200.000 & 167.000 \\
\hline & & ORC & GANIC COMPOI & DS-PESTIC & IIDES, TOTAL & & & & & \\
\hline 39330 & $\overline{\text { ALDRIN, TOTAL }(\mu \mathrm{g} / \mathrm{L})}$ & 4 & $<0.0 \overline{10}$ & $<0 . \overline{010}$ & -- & -- & -- & - & $\overline{--}$ & -- \\
\hline 39340 & LINDANE, TOTAL $(\mu \mathrm{g} / \mathrm{L})$ & 4 & $<0.010$ & $<0.010$ & -- & -- & -- & -- & -- & -- \\
\hline 39350 & CHLORDANE, TOTAL $(\mu \mathrm{g} / \mathrm{L})$ & 4 & $<0.100$ & $<0.100$ & -- & -- & -- & $\ldots$ & - & -- \\
\hline 39370 & DDT, TOTAL $(\mu \mathrm{g} / \mathrm{L})$ & 4 & $<0.010$ & $<0.010$ & -- & -- & -- & -- & - & -- \\
\hline 39365 & DDE, TOTAL $(\mu \mathrm{g} / \mathrm{L})$ & 4 & $<0.010$ & $<0.01 \mathrm{C}$ & -- & -- & -- & -- & -. & -- \\
\hline 39360 & DDD, TOTAL $(\mu \mathrm{g} / \mathrm{L})$ & 4 & $<0.010$ & $<0.010$ & -- & -- & -- & -- & -- & -- \\
\hline 39380 & DIELDRIN, TOTAL $(\mu \mathrm{g} / \mathrm{L})$ & 4 & $<0.020$ & $<0.010$ & -- & -- & -- & -- & -- & - \\
\hline 39388 & ENDOSULFAN, TOTAL $(\mu \mathrm{g} / \mathrm{L})$ & 4 & $<0.010$ & $<0.010$ & -- & -- & - & - & -- & -- \\
\hline 39390 & ENDRIN, TOTAL $(\mu \mathrm{g} / \mathrm{L})$ & 4 & $<0.010$ & $<0.010$ & -- & - & -- & -- & -- & $\rightarrow-$ \\
\hline 39410 & HEPTACHLOR, TOTAL $(\mu \mathrm{g} / \mathrm{L})$ & 4 & $<0.010$ & $<0.010$ & -- & -- & -- & -- & -- & -- \\
\hline 39420 & HEPTACHLOR EPOXIDE, TOTAL $(\mu \mathrm{g} / \mathrm{L})$ & 4 & $<0.010$ & $<0.010$ & -- & - & $\ldots$ & -- & -- & -- \\
\hline 39516 & $\mathrm{PCB}, \operatorname{TOTAL}(\mu \mathrm{g} / \mathrm{L})$ & 4 & $<0.400$ & $<0.100$ & -- & -- & -- & -- & -- & -- \\
\hline 39400 & TOXAPHENE, TOTAL $(\mu \mathrm{g} / \mathrm{L})$ & 4 & $<1.000$ & $<1.000$ & -- & -- & -- & - & -- & -- \\
\hline 39034 & PERTHANE, TOTAL $(\mu \mathrm{g} / \mathrm{L})$ & 4 & $<0.100$ & $<0.100$ & -- & -- & -- & -- & -- & - \\
\hline 39570 & DIAZINON， TOTAL $(\mu \mathrm{g} / \mathrm{L})$ & 4 & 0.080 & 0.010 & -- & -- & -- & $\ldots$ & - & -- \\
\hline 39398 & ETHION， TOTAL $(\mu \mathrm{g} / \mathrm{L})$ & 4 & $<0.010$ & $<0.010$ & -- & -- & -- & -- & -- & - \\
\hline 39530 & MALATHION, TOTAL $(\mu \mathrm{g} / L)$ & 4 & 0.020 & $<0.010$ & -- & - & -- & - & - & - \\
\hline 39600 & METHYL PARATHION, TOTAL $(\mu \mathrm{g} / \mathrm{L})$ & 4 & 0.010 & $<0.010$ & -- & -- & -- & -- & -- & -- \\
\hline 39540 & PARATHION, TOTAL $(\mu \mathrm{g} / \mathrm{L})$ & 4 & $<0.010$ & $<0.010$ & - & -- & - & - & -- & -- \\
\hline 39786 & TRITHION, TOTAL $(\mu \mathrm{g} / \mathrm{L})$ & 4 & $<0.010$ & $<0.010$ & -- & - & -- & - & - & -- \\
\hline 39250 & PCN, TOTAL $(\mu \mathrm{g} / \mathrm{L})$ & 4 & $<0.100$ & $<0.100$ & -- & -- & - & -- & -- & -- \\
\hline 39480 & METHOXYCHLOR, TOTAL $(\mu \mathrm{g} / \mathrm{L})$ & 4 & $<0.010$ & $<0.010$ & -- & -- & -- & -- & -- & -- \\
\hline 39755 & MIREX, TOTAL $(\mu \mathrm{g} / \mathrm{L})$ & 4 & $<0.010$ & $<0.010$ & -- & -- & -- & - - & $\ldots$ & -- \\
\hline 39011 & DISYSTON, TOTAL $(\mu \mathrm{g} / \mathrm{L})$ & 4 & $<0.010$ & $<0.010$ & -- & -- & -- & -- & -- & -- \\
\hline 39023 & PHORATE, TOTAL $(\mu \mathrm{g} / \mathrm{L})$ & 4 & $<0.010$ & $<0.010$ & -- & -- & -- & -- & -- & -- \\
\hline 38932 & CHLORPYRIFOS, TOTAL $(\mu \mathrm{g} / \mathrm{L})$ & 4 & 0.050 & $<0.010$ & -- & -- & -- & -- & -- & -- \\
\hline 39040 & DEF, TOTAL $(\mu \mathrm{g} / L)$ & 4 & $<0.010$ & $<0.010$ & -- & -- & -- & -- & - & -- \\
\hline 82614 & FONOFOS, TOTAL $(\mu \mathrm{g} / \mathrm{L})$ & 4 & $<0.010$ & $<0.010$ & -- & -- & -- & -- & -- & $\ldots$ \\
\hline & & & OLATILE ORGA & C COMPOU & NDS, TOTAL & & & & & \\
\hline 34210 & ACROLEIN, TOTAL $(\mu \mathrm{g} / \mathrm{L})$ & 1 & $<20.000$ & -- & -- & $\cdots$ & $\overline{--}$ & -- & -- & -- \\
\hline 34215 & ACRYLONITRILE, TOTAL $(\mu \mathrm{g} / \mathrm{L})$ & 2 & $<20.000$ & $<5.000$ & $\ldots$ & -- & -- & - & -- & -- \\
\hline 34030 & BENZENE，TOTAL $(\mu \mathrm{g} / \mathrm{L})$ & 10 & $<2.000$ & $<0.200$ & -- & -- & -- & -- & -- & -- \\
\hline 32104 & BROMOFORM, TOTAL $(\mu \mathrm{g} / \mathrm{L})$ & 10 & $<2.000$ & $<0.200$ & -- & -- & -- & -- & -- & -- \\
\hline 32102 & CARBON TETRACHLORIDE, TOTAL $(\mu \mathrm{g} / \mathrm{L})$ & 10 & $<2.000$ & $<0.200$ & -- & -- & -- & -- & - & -- \\
\hline 34301 & CHLOROBENZENE， TOTAL $(\mu \mathrm{g} / \mathrm{L})$ & 10 & $<2.000$ & $<0.200$ & -- & -- & -- & -- & -- & -- \\
\hline 32105 & CHLORODIBROMOMETHANE, TOTAL $(\mu \mathrm{g} / \mathrm{L})$ & 10 & $<2.000$ & $<0.200$ & -- & -- & -- & -- & $\ldots$ & -- \\
\hline 34311 & CHLOROETHANE, TOTAL $(\mu \mathrm{g} / \mathrm{L})$ & 10 & $<2.000$ & $<0.200$ & -- & -- & -- & -- & -- & -- \\
\hline 32106 & CHLOROFORM, TOTAL $(\mu \mathrm{g} / \mathrm{L})$ & 10 & $<2.000$ & $<0.200$ & -- & -- & -- & - & -- & -- \\
\hline 34496 & 1,1 -DICHLOROETHANE, TOTAL $(\mu \mathrm{g} / \mathrm{L})$ & 10 & $<2.000$ & $<0.200$ & -- & -- & -- & -- & -- & -- \\
\hline 32103 & 1, 2-DICHLOROETHANE, TOTAL $(\mu \mathrm{g} / \mathrm{L})$ & 10 & $<2.000$ & $<0.200$ & -- & - & -- & -- & -- & -- \\
\hline 34501 & 1,1 -DICHLOROETHYLENE, TOTAL $(\mu \mathrm{g} / \mathrm{L})$ & 10 & $<2.000$ & $<0.200$ & -- & -- & -- & - & -- & -- \\
\hline 34541 & 1,2 -DICHLOROPROPANE, TOTAL $(\mu \mathrm{g} / \mathrm{L})$ & 10 & $<2.000$ & $<0.200$ & -- & -- & -- & -- & -- & -- \\
\hline 34371 & ETHYLBENZENE, TOTAL $(\mu \mathrm{g} / \mathrm{L})$ & 10 & $<2.000$ & $<0.200$ & -- & -- & -- & -- & -- & -- \\
\hline 34413 & METHYL BROMIDE, TOTAL $(\mu \mathrm{g} / \mathrm{L})$ & 10 & $<2.000$ & $<0.200$ & -- & $\ldots$ & -- & - & $\ldots$ & -- \\
\hline 34423 & METHYLENE CHLORIDE, TOTAL $(\mu \mathrm{g} / \mathrm{L})$ & 10 & $<2.000$ & $<0.200$ & $\ldots$ & -- & -- & -- & -- & -- \\
\hline
\end{tabular}

NOTE: Multiple detection limits during the period of record may result in different values flagged with a "<."

* Value is estimated by using a log-probability regression to predict the values of data below the detection limit. 
Table 75. Statistical summary of water-quality data at site 43 (CSW07), June 1994 through September 1998-Contir'sed

\begin{tabular}{|c|c|c|c|c|c|c|c|c|c|c|}
\hline \multirow{2}{*}{$\begin{array}{l}\text { PARAM- } \\
\text { ETER } \\
\text { CODE }\end{array}$} & \multirow{2}{*}{ PROPERTY OR CONSTITUENT } & & DESCRIPTII & STATISTICS & & PERCENT O & $\begin{array}{r}\text { AMPLES } \\
\text { EQUA }\end{array}$ & $\begin{array}{l}\text { WHICHVALU } \\
\text { TO THOSE S }\end{array}$ & $\begin{array}{l}\text { SWERE LE } \\
\text { TNN }\end{array}$ & THAN OR \\
\hline & & $\begin{array}{l}\text { SAMPLE } \\
\text { SIZE }\end{array}$ & MAXIMUM & MINIMUM & MEAN & $95 \%$ & $75 \%$ & $\begin{array}{c}50 \% \\
\text { (median) }\end{array}$ & $25 \%$ & $5 \%$ \\
\hline 34516 & $1,1,2,2$-TETRACHLOROETHANE, TOTAL $(\mu \mathrm{g} / \mathrm{L})$ & 10 & $<2.000$ & $<0 . \overline{200}$ & -- & -- & -- & -- & -- & -- \\
\hline 34475 & TETRACHLOROETHYLENE， TOTAL $(\mu \mathrm{g} / \mathrm{L})$ & 10 & $<2.000$ & $<0.200$ & -- & -- & -- & -- & -- & -- \\
\hline 34010 & TOLUENE， TOTAL $(\mu \mathrm{g} / \mathrm{L})$ & 10 & $<2.000$ & $<0.200$ & - & -- & -- & -- & -- & -- \\
\hline 34546 & 1,2 -TRANSDICHLOROETHENE, TOTAL $(\mu \mathrm{g} / \mathrm{L})$ & 10 & $<2.000$ & $<0.200$ & -- & -- & -- & -- & -- & - \\
\hline 34506 & $1,1,1$-TRICHLOROETHANE, TOTAL $(\mu \mathrm{g} / \mathrm{L})$ & 10 & $<2.000$ & $<0.200$ & -- & -- & -- & -- & -- & -- \\
\hline 34511 & $1,1,2$-TRICHLOROETHANE, TOTAL $(\mu \mathrm{g} / \mathrm{L})$ & 10 & $<2.000$ & $<0.200$ & -- & -- & -- & -- & -- & -- \\
\hline 39180 & TRICHLOROETHYLENE， TOTAL $(\mu \mathrm{g} / \mathrm{L})$ & 10 & $<2.000$ & $<0.200$ & -- & -- & -- & -- & -- & -- \\
\hline 39175 & VINYL CHLORIDE, TOTAL $(\mu \mathrm{g} / \mathrm{L})$ & 10 & $<2.000$ & $<0.200$ & -- & -- & -- & - & -- & -- \\
\hline 30217 & DIBROMOMETHANE， TOTAL $(\mu \mathrm{g} / \mathrm{L})$ & 10 & $<2.000$ & $<0.200$ & -- & -- & -- & -- & -- & -- \\
\hline 32101 & DICHLOROBROMOMETHANE，TOTAL $(\mu \mathrm{g} / \mathrm{L})$ & 10 & $<2.000$ & $<0.200$ & -- & -- & -- & -- & -- & - \\
\hline 34668 & DICHLORODIFLUOROMETHANE, TOTAL $(\mu \mathrm{g} / \mathrm{L})$ & 10 & $<2.000$ & $<0.200$ & -- & -- & -- & $\rightarrow$ & -- & -- \\
\hline 34488 & TRICHLOROFLUOROMETHANE，TOTAL $(\mu \mathrm{g} / \mathrm{L})$ & 10 & $<2.000$ & $<0.200$ & -- & -- & -- & -- & -- & -- \\
\hline 77651 & 1,2-DIBROMOETHANE, TOTAL $(\mu \mathrm{g} / \mathrm{L})$ & 10 & $<2.000$ & $<0.200$ & -- & -. & -- & -- & -- & -- \\
\hline 34418 & METHYLCHLORIDE, TOTAL $(\mu \mathrm{g} / \mathrm{L})$ & 10 & $<2.000$ & $<0.200$ & -- & -- & -- & -- & -- & -- \\
\hline 34704 & CIS 1,3 -DICHLOROPROPENE, TOTAL $(\mu \mathrm{g} / \mathrm{L})$ & 10 & $<2.000$ & $<0.200$ & -- & -- & -- & -- & -- & -- \\
\hline 34699 & TRANS 1,3 -DICHLOROPROPENE, TOTAL $(\mu \mathrm{g} / \mathrm{L})$ & 10 & $<2.000$ & $<0.200$ & -- & -- & -- & -- & -- & -- \\
\hline 77128 & STYRENE， TOTAL $(\mu \mathrm{g} / \mathrm{L})$ & 10 & $<2.000$ & $<0.200$ & -- & -- & -- & -- & -- & - \\
\hline 81551 & XYLENE， TOTAL $(\mu \mathrm{g} / \mathrm{L})$ & 10 & $<2.000$ & $<0.200$ & -- & -- & -- & -- & -- & -- \\
\hline 82625 & DIBROMOCHLOROPROPANE， TOTAL $(\mu \mathrm{g} / \mathrm{L})$ & 10 & $<10.000$ & $<1.000$ & -- & -- & -- & -- & -- & -- \\
\hline 77168 & 1,1-DICHLOROPROPENE, TOTAL $(\mu \mathrm{g} / \mathrm{L})$ & 10 & $<2.000$ & $<0.200$ & -- & -- & -- & -- & -- & -- \\
\hline 77170 & 2,2 -DICHLOROPROPANE, TOTAL $(\mu \mathrm{g} / \mathrm{L})$ & 10 & $<2.000$ & $<0.200$ & -- & -- & -- & -- & -- & -- \\
\hline 77173 & 1.3-DICHLOROPROPANE， TOTAL $(\mu \mathrm{g} / \mathrm{L})$ & 10 & $<2.000$ & $<0.200$ & -- & -- & -- & -- & -- & -- \\
\hline 77275 & O-CHLOROTOLUENE, TOTAL $(\mu \mathrm{g} / \mathrm{L})$ & 10 & $<2.000$ & $<0.200$ & -- & -- & -- & -- & -- & -- \\
\hline 77277 & P-CHLOROTOLUENE, TOTAL $(\mu \mathrm{g} / \mathrm{L})$ & 10 & $<2.000$ & $<0.200$ & -- & -- & -- & -- & -- & -- \\
\hline 77443 & 123-TRICHLOROPROPANE，TOTAL $(\mu \mathrm{g} / \mathrm{L})$ & 10 & $<2.000$ & $<0.200$ & -- & -- & -- & -- & -- & -- \\
\hline 77562 & 1112-TETRACHLOROETHANE，TOTAL $(\mu \mathrm{g} / \mathrm{L})$ & 10 & $<2.000$ & $<0.200$ & -- & -- & -- & -- & -- & -- \\
\hline 78032 & TERTBUTYL METHYL ETHER, TOTAL $(\mu \mathrm{g} / \mathrm{L})$ & 10 & $<2.000$ & $<0.200$ & -- & -- & -- & -- & -- & -- \\
\hline 77297 & BROMOCHLORO METHANE，TOTAL $(\mu \mathrm{g} / \mathrm{L})$ & 10 & $<2.000$ & $<0.200$ & - & -- & -- & -- & -+ & -- \\
\hline 77093 & CIS- 1,2 -DICHLOROETHENE，TOTAL $(\mu \mathrm{g} / \mathrm{L})$ & 10 & $<2.000$ & $<0.200$ & - & -- & -- & -- & -- & -- \\
\hline 34576 & 2-CHLOROETHYL VINYL ETHER, TOTAL $(\mu \mathrm{g} / \mathrm{L})$ & 6 & $<2.000$ & $<1.000$ & -- & -- & -- & -- & -- & -- \\
\hline 77223 & ISOPROPYL BENZENE, TOTAL $(\mu \mathrm{g} / \mathrm{L})$ & 10 & $<2.000$ & $<0.200$ & -- & -- & -- & -- & -- & -- \\
\hline 77224 & N-PROPYL BENZENE, TOTAL $(\mu \mathrm{g} / \mathrm{L})$ & 10 & $<2.000$ & $<0.200$ & -- & -- & -- & -- & -- & -- \\
\hline 77353 & TERTBUTYL BENZENE，TOTAL $(\mu \mathrm{g} / \mathrm{L})$ & 10 & $<2.000$ & $<0,200$ & -- & -- & -- & -- & -- & -- \\
\hline 77222 & PSEUDOCUMENE, TOTAL $(\mu \mathrm{g} / \mathrm{L})$ & 10 & $<2.000$ & $<0.200$ & -- & -- & -- & -- & -- & -- \\
\hline 77350 & SEC-BUTYL BENZENE, TOTAL $(\mu \mathrm{g} / \mathrm{L})$ & 10 & $<2.000$ & $<0.200$ & - & -- & -- & -- & -- & -- \\
\hline 77356 & P-ISOPROPYL TOLUENE, TOTAL $(\mu \mathrm{g} / \mathrm{L})$ & 10 & $<2.000$ & $<0.200$ & -- & -- & -- & -- & -- & -- \\
\hline 77342 & N-BUTYL BENZENE, TOTAL $(\mu \mathrm{g} / \mathrm{L})$ & 10 & $<2.000$ & $<0.200$ & -- & -- & -- & -- & -- & -- \\
\hline 77613 & $1,2,3$-TRICHLOROBENZENE, TOTAL $(\mu \mathrm{g} / \mathrm{L})$ & 10 & $<2.000$ & $<0.200$ & -- & -- & -- & -- & -- & -- \\
\hline 77652 & FREON-113, TOTAL $\{\mu \mathrm{g} / \mathrm{L}\}$ & 10 & $<2.000$ & $<0.200$ & -- & -- & -- & -- & -- & -- \\
\hline 77226 & MESITYLENE， TOTAL $(\mu \mathrm{g} / \mathrm{L})$ & 10 & $<2.000$ & $<0.200$ & -- & -- & -- & -- & -- & - \\
\hline 81555 & BROMOBENZENE, TOTAL $(\mu \mathrm{g} / \mathrm{L})$ & 10 & $<2.000$ & $<0.200$ & -- & -- & -- & -- & -- & -- \\
\hline 34551 & $1,2,4$-TRICHLOROBENZENE, TOTAL $(\mu \mathrm{g} / \mathrm{L})$ & 10 & $<2.000$ & $<0.200$ & -- & -- & -- & -- & -- & -- \\
\hline 34536 & 1,2 -DICHLOROBENZENE， TOTAL $(\mu \mathrm{g} / \mathrm{L})$ & 10 & $<2.000$ & $<0.200$ & -- & -- & -- & -- & -- & -- \\
\hline 34566 & 1,3 -DICHLOROBENZENE, TOTAL $(\mu \mathrm{g} / \mathrm{L})$ & 10 & $<2.000$ & $<0.200$ & -- & -- & - & -- & -- & -- \\
\hline 34571 & 1,4 -DICHLOROBENZENE，TOTAL $(\mu \mathrm{g} / \mathrm{L})$ & 10 & $<2.000$ & $<0.200$ & -- & -- & -- & -- & -- & -- \\
\hline 39702 & HEXACHLOROBUTADIENE，TOTAI $(\mu \mathrm{g} / \mathrm{L})$ & 10 & $<2.000$ & $<0.200$ & -- & -- & -- & -- & -- & -- \\
\hline 34696 & NAPHTHALENE， TOTAL $(\mu \mathrm{g} / \mathrm{L})$ & 10 & $<2.000$ & $\leq 0.200$ & -- & -- & - & -- & -- & - \\
\hline & & ME] & ALS AND MIN & CONSTITUE & TS, TOTAL & & & & & \\
\hline 01097 & ANTIMONY, TOTAL $(\mu \mathrm{g} / \mathrm{L}$ as $\mathrm{Sb})$ & 43 & 20.000 & $<1.000$ & $2.280^{*}$ & 17.000 & $<5.000$ & $<1.000$ & $<1.000$ & $<1.000$ \\
\hline 01002 & ARSENIC, TOTAL $(\mu \mathrm{g} / \mathrm{L}$ aS AS $)$ & 43 & 59.000 & $<1.000$ & $11.469 *$ & 41.000 & 20.000 & 2.000 & $<1.000$ & $<1.000$ \\
\hline 01012 & BERYLLIUM, TOTAL ( $\mu \mathrm{g} / \mathrm{L}$ as Be) & 14 & 710.000 & $<10.000$ & -- & 710.000 & $<10.000$ & $<10.000$ & $<10.000$ & $<10.000$ \\
\hline 01027 & CADMIUM, TOTAL $(\mu \mathrm{g} / \mathrm{L}$ as $\mathrm{Cd})$ & 13 & $<1.000$ & $<1.000$ & -- & $<1.000$ & $<1.000$ & $<1.000$ & $<1.000$ & $<1.000$ \\
\hline 01034 & CHROMIUM, TOTAL $(\mu \mathrm{g} / \mathrm{L}$ as CI $)$ & 43 & 1100.000 & 6.000 & 170.116 & 744.000 & 160.000 & 62.000 & 27.000 & 7.600 \\
\hline 01042 & COPPER, TOTAL $(\mu \mathrm{g} / \mathrm{L}$ as $\mathrm{Cu})$ & 43 & 1200.000 & 3.000 & 163.279 & 798.000 & 240.000 & 44.000 & 14.000 & 3.800 \\
\hline 01051 & LEAD，TOTAL ( $(\mu \mathrm{g} / \mathrm{L}$ as $\mathrm{Pb})$ & 43 & 140.000 & 1.000 & 28.140 & 122.200 & 34.000 & 12.000 & 6.000 & 1.200 \\
\hline 71900 & MERCURY, TOTAL ( $\mu \mathrm{g} / \mathrm{L}$ as $\mathrm{Hg}$ ) & 42 & 0.700 & $<0.100$ & $0.160 *$ & 0.700 & 0.200 & $<0.100$ & $<0.100$ & $<0.100$ \\
\hline 01067 & NICKEL, TOTAL $(\mu \mathrm{g} / \mathrm{L}$ as Ni) & 43 & 1200.000 & 3.000 & 146.605 & 816.000 & 160.000 & 45.000 & 19.000 & 4.500 \\
\hline 01147 & SELENIUM, TOTAL ( $\mu \mathrm{g} / \mathrm{L}$ as $\mathrm{Se})$ & 13 & 2.000 & $<1.000$ & -- & 2.000 & $<5.000$ & $<1.000$ & $<1.000$ & $<1.000$ \\
\hline 01077 & SILVER, TOTAL $(\mu \mathrm{g} / \mathrm{L}$ as $\mathrm{Ag})$ & 13 & $<1.000$ & $<1.000$ & -- & $<1.000$ & $<1.000$ & $<1.000$ & $<1.000$ & $<1.000$ \\
\hline 01092 & ZINC, TOTAL $(\mu \mathrm{g} / \mathrm{L}$ as $\mathrm{zn})$ & 42 & 1800.000 & 20.000 & 267.381 & 822.500 & 337.500 & 120.000 & 57.500 & 30.000 \\
\hline 00720 & CYANIDE, TOTAL (mg/L as Cn) & 13 & $<0.010$ & $<0.010$ & -- & $<0.010$ & $<0.010$ & $<0.010$ & $<0.010$ & $<0.010$ \\
\hline & & ORGA & IC COMPOUN & -PESTICID & , DISSOLVE & & & & & \\
\hline 49260 & ACETOCHLOR, DISSOLVED $(\mu \mathrm{g} / \mathrm{L})$ & 3 & $<0.002$ & $<0.002$ & $\overline{--}$ & $-\overline{-}$ & -- & -- & -- & -- \\
\hline 46342 & ALACHLOR, DISSOLVED $(\mu \mathrm{g} / \mathrm{L})$ & 3 & 0.027 & $<0.002$ & -- & -- & -- & -- & -- & -- \\
\hline 04040 & DEETHYL ATRAZINE, DISSOLVED $(\mu \mathrm{g} / \mathrm{L})$ & 3 & $<0.002$ & $<0.002$ & -- & -- & -- & -- & -- & -- \\
\hline 39632 & ATRAZINE， DISSOLVED $(\mu \mathrm{g} / \mathrm{L})$ & 3 & 0.018 & $<0.001$ & -- & -- & -- & -- & -- & -- \\
\hline 82686 & METHYL AZINPHOS, DISSOLVED $(\mu \mathrm{g} / \mathrm{L})$ & 3 & $<0.001$ & $<0.001$ & -- & -- & -- & -- & -- & -- \\
\hline 82673 & BENFLURALIN, DISSOLVED $(\mu \mathrm{g} / \mathrm{L})$ & 3 & 0.011 & $<0.002$ & - & - & -- & -- & -- & -- \\
\hline 04028 & BUTYLATE， DISSOLVED $(\mu \mathrm{g} / \mathrm{L})$ & 3 & $<0.002$ & $<0.002$ & -- & -- & - & -- & -- & -- \\
\hline 82680 & CARBARYL，DISSOLVED $(\mu \mathrm{g} / \mathrm{L})$ & 3 & 0.310 & 0.045 & -- & -- & -- & -- & -- & -- \\
\hline 82674 & CARBOFURAN， DISSOLVED $(\mu \mathrm{g} / \mathrm{L})$ & 3 & $<0.003$ & $<0.003$ & -- & -- & - & -- & -- & -- \\
\hline 38933 & CHLORPYRIFOS， DISSOLVED $(\mu \mathrm{g} / \mathrm{L})$ & 3 & 0.032 & $<0.004$ & -- & -- & -- & -- & -- & -- \\
\hline 04041 & CYANAZINE， DISSOLVED $(\mu \mathrm{g} / \mathrm{L})$ & 3 & $<0.004$ & $<0.004$ & -- & -- & -- & -- & -- & -- \\
\hline 82682 & DCPA， DISSOLVED $(\mu \mathrm{g} / \mathrm{L})$ & 3 & 0.013 & $<0.002$ & -- & -- & - & -- & -- & -- \\
\hline 34653 & $\mathrm{P}, \mathrm{P}^{\prime}$ DDE, DISSOLVED $(\mu \mathrm{g} / \mathrm{L})$ & 3 & $<0.006$ & $<0.006$ & -- & -- & - & -- & -- & -- \\
\hline 39572 & DIAZINON, DISSOLVED $(\mu \mathrm{g} / \mathrm{L})$ & 3 & 0.110 & $<0.002$ & -- & -- & -- & -- & -- & -- \\
\hline 39381 & DIELDRIN, DISSOLVED $(\mu \mathrm{g} / \mathrm{L})$ & 3 & $<0.001$ & $<0.001$ & -- & -- & -- & -- & - & -- \\
\hline 82660 & 2,6 -DIETHYL ANILINE, DISSOLVED $(\mu \mathrm{g} / \mathrm{L})$ & 3 & $<0.003$ & $<0.003$ & -- & -- & -- & -- & $\cdots$ & -- \\
\hline 82677 & DISULFOTON, DISSOLVED $(\mu \mathrm{g} / \mathrm{L})$ & 3 & $<0.017$ & $<0.017$ & -- & -- & -- & -- & - & -- \\
\hline 82668 & EPTC, DISSOLVED $(\mu \mathrm{g} / \mathrm{L})$ & 3 & $<0.002$ & $<0.002$ & -- & -- & -- & -- & -- & -- \\
\hline 82663 & ETHALFLURALIN，DISSOLVED $(\mu \mathrm{g} / \mathrm{L})$ & 3 & $<0.004$ & $<0.004$ & -- & -- & -- & -- & -- & -- \\
\hline 82672 & ETHOPROP, DISSOLVED $(\mu \mathrm{g} / \mathrm{L})$ & 3 & $<0.003$ & $<0.003$ & -- & -- & -- & -- & -- & -- \\
\hline
\end{tabular}

NOTE: Multiple detection limits during the period of record may result in different values flagged with a "<."

* Value is estimated by using a log-probability regression to predict the values of data below the detection limit. 
Table 75. Statistical summary of water-quality data at site 43 (CSWO7), June 1994 through September 1998-Continued

\begin{tabular}{|c|c|c|c|c|c|c|c|c|c|c|}
\hline \multirow{2}{*}{$\begin{array}{l}\text { PARAM- } \\
\text { ETER } \\
\text { CODE }\end{array}$} & \multirow{2}{*}{ PROPERTY OR CONSTITUENT } & & DESCRIPTII & STATISTICS & & PERCEN & $\begin{array}{r}\text { AMPLE } \\
\text { EQ }\end{array}$ & $\begin{array}{l}\text { WHICHVAL } \\
\text { TO THOSE }\end{array}$ & $\begin{array}{l}\text { WERE } \\
\text { NN }\end{array}$ & HAN OR \\
\hline & & $\begin{array}{c}\text { SAMPLE } \\
\text { SIZE }\end{array}$ & MAXIMUM & MINIMUM & MEAN & $95 \%$ & $75 \%$ & $\begin{array}{c}\mathbf{5 0 \%} \\
\text { (medlan) }\end{array}$ & $25 \%$ & $5 \%$ \\
\hline 04095 & FONOFOS, DISSOLVED $(\mu \mathrm{g} / \mathrm{L})$ & 3 & $<0.003$ & $<0.003$ & -- & -- & -- & -- & $\cdots$ & $\overline{--}$ \\
\hline 34253 & ALPHA BHC, DISSOLVED $(\mu \mathrm{g} / \mathrm{L})$ & 3 & $<0.002$ & $<0.002$ & -- & -. & -- & -- & -- & -- \\
\hline 39341 & LINDANE, DISSOLVED $(\mu \mathrm{g} / \mathrm{L})$ & 3 & $<0.004$ & $<0.004$ & -- & - & -- & -- & -- & -- \\
\hline 82666 & LINURON, DISSOLVED $(\mu \mathrm{g} / \mathrm{L})$ & 3 & $<0.002$ & $<0.002$ & -- & -- & -- & -- & - & - \\
\hline 39532 & MALATHION, DISSOLVED $(\mu \mathrm{g} / \mathrm{L})$ & 3 & 0.029 & $<0.005$ & -- & -- & - & -- & -- & -- \\
\hline 82667 & METHYL PARATHION, DISSOLVED $(\mu \mathrm{g} / L)$ & 3 & $<0.006$ & $<0.006$ & -- & -- & -- & -- & -- & -- \\
\hline 39415 & METOLACHLOR, DISSOLVED $(\mu \mathrm{g} / \mathrm{L})$ & 3 & 0.038 & 0.018 & -- & -- & -- & -- & -- & -- \\
\hline 82630 & METRIBUZIN, DISSOLVED $(\mu \mathrm{g} / \mathrm{L})$ & 3 & $<0.030$ & $<0.004$ & -- & -- & - & -- & - & -- \\
\hline 82671 & MOLINATE, DISSOLVED $(\mu \mathrm{g} / \mathrm{L})$ & 3 & $<0.004$ & $<0.004$ & -- & -- & -- & -- & -- & -. \\
\hline 82684 & NAPROPAMIDE, DISSOLVED $(\mu \mathrm{g} / \mathrm{L})$ & 3 & $<0.003$ & $<0.003$ & -- & -- & -- & -- & -- & -- \\
\hline 39542 & PARATHION, DISSOLVED $(\mu \mathrm{g} / \mathrm{L})$ & 3 & $<0.004$ & $<0.004$ & -- & -- & -- & -- & -- & -- \\
\hline 82669 & PEBULATE, DISSOLVED $(\mu \mathrm{g} / \mathrm{L})$ & 3 & $<0.004$ & $<0.004$ & -- & -. & -- & -- & -- & -- \\
\hline 82683 & PENDIMETHALIN， DISSOLVED $(\mu \mathrm{g} / \mathrm{L})$ & 3 & 0.094 & $<0.004$ & -- & -- & - & -- & -- & -- \\
\hline 82687 & PERMETHRIN, DISSOLVED $(\mu \mathrm{g} / \mathrm{L})$ & 3 & $<0.005$ & $<0.005$ & -- & -- & -- & -- & -- & -- \\
\hline 82664 & PHORATE, DISSOLVED $(\mu \mathrm{g} / \mathrm{L})$ & 3 & $<0.002$ & $<0.002$ & -- & -- & -- & -- & -- & -- \\
\hline 82676 & PRONAMIDE， DISSOLVED $(\mu \mathrm{g} / \mathrm{L})$ & 3 & $<0.060$ & $<0.003$ & -- & -- & -- & -- & -- & - \\
\hline 04037 & PROMETON, DISSOLVED $(\mu \mathrm{g} / \mathrm{L})$ & 3 & $<0.018$ & $<0.018$ & -- & -- & -- & -- & -- & -- \\
\hline 04024 & PROPACHLOR, DISSOLVED $(\mu \mathrm{g} / \mathrm{L})$ & 3 & $<0.007$ & $<0.007$ & -- & -- & -- & -- & -- & -- \\
\hline 82679 & PROPANIL, DISSOLVED $(\mu \mathrm{g} / L)$ & 3 & $<0.004$ & $<0.004$ & -- & -- & -- & - & -- & -- \\
\hline 82685 & PROPARGITE, DISSOLVED $(\mu \mathrm{g} / \mathrm{L})$ & 3 & $<0.013$ & $<0.013$ & -- & - & -- & -- & -- & -- \\
\hline 04035 & SIMAZINE, DISSOLVED $(\mu \mathrm{g} / \mathrm{L})$ & 3 & $<0.030$ & $<0.005$ & -- & -- & -- & -- & -- & -- \\
\hline 82681 & THIOBENCARB， DISSOLVED $(\mu \mathrm{g} / \mathrm{L})$ & 3 & $<0.002$ & $<0.002$ & -- & -- & -- & -- & -- & -- \\
\hline 82670 & TEBUTHIURON，DISSOLVED $(\mu \mathrm{g} / \mathrm{L})$ & 3 & $<0.010$ & $<0.010$ & -- & -- & -- & -- & -- & -- \\
\hline 82665 & TERBACIL, DISSOLVED $(\mu \mathrm{g} / \mathrm{L})$ & 3 & $<0.007$ & $<0.007$ & -- & -- & -- & -- & - & - \\
\hline 82675 & TERBUFOS, DISSOLVED $(\mu \mathrm{g} / \mathrm{L})$ & 3 & $<0.013$ & $<0.013$ & -- & -- & -- & -- & -- & -- \\
\hline 82678 & TRIALLATE， DISSOLVED $(\mu \mathrm{g} / \mathrm{L})$ & 3 & $<0.001$ & $<0.001$ & -- & -- & -- & -- & -. & -- \\
\hline 82661 & TRIFLURALIN, DISSOLVED $(\boldsymbol{\mu} \mathrm{g} / \mathrm{L})$ & 3 & 0.016 & 0.005 & -- & -- & -- & -- & -- & - \\
\hline 39742 & $2,4,5-T$, DISSOLVED $(\mu \mathrm{g} / \mathrm{L})$ & 3 & $<0.035$ & $<0.035$ & -- & - & -- & -- & -- & -- \\
\hline 39732 & $2,4-\mathrm{D}$, DISSOLVED $(\mu \mathrm{g} / \mathrm{L})$ & 3 & 1.460 & $<0.035$ & -- & -- & -- & -- & -- & - \\
\hline 38746 & $2,4-\mathrm{DB}$, DISSOLVED $(\mu \mathrm{g} / \mathrm{L})$ & 3 & $<0.240$ & $<0.035$ & -- & -- & -- & -- & -- & -- \\
\hline 49315 & ACIFLUOREEN，DISSOLVED $(\mu \mathrm{g} / \mathrm{L})$ & 3 & $<0.035$ & $<0.035$ & -- & -- & -- & -- & -- & -- \\
\hline 49312 & ALDICARB, DISSOLVED $(\mu \mathrm{g} / \mathrm{L})$ & 3 & $<0.550$ & $<0.016$ & -- & -- & -- & -- & -- & -- \\
\hline 49313 & ALDICARB SULFONE, DISSOLVED $(\mu \mathrm{g} / \mathrm{L})$ & 3 & $<0.100$ & $<0.016$ & -- & -- & -- & -- & -- & - \\
\hline 49314 & ALDICARB SULFOXIDE, DISSOLVED $(\mu \mathrm{g} / \mathrm{L})$ & 3 & $<0.021$ & $<0.021$ & -- & -- & -- & -- & -- & -- \\
\hline 38711 & BENTAZON, DISSOLVED $(\mu \mathrm{g} / \mathrm{L})$ & 3 & $<0.014$ & $<0.014$ & - & -- & -- & -- & -- & -- \\
\hline 04029 & BROMACIL, DISSOLVED $(\mu \mathrm{g} / \mathrm{L})$ & 3 & $<0.035$ & $<0.035$ & -. & -- & -- & -- & -- & - - \\
\hline 49311 & BROMOXYNIL, DISSOLVED $(\mu \mathrm{g} / \mathrm{L})$ & 3 & $<0.035$ & $<0.035$ & $-\infty$ & -- & $\ldots$ & -- & -- & -- \\
\hline 49310 & CARBARYL，DISSOLVED $(\mu \mathrm{g} / \mathrm{L})$ & 3 & 0.150 & $<0.008$ & -- & -- & - & -- & -- & -- \\
\hline 49309 & CARBOFURAN, DISSOLVED $(\mu \mathrm{g} / \mathrm{L})$ & 3 & $<0.120$ & $<0.028$ & -- & -- & -- & -- & -- & -- \\
\hline 49308 & 3-HYDROXY-CARBOFURAN $(\mu \sigma / L)$ & 3 & $<0.014$ & $<0.014$ & - - & -- & -. & -- & -- & -- \\
\hline 49307 & AMIBEN, DISSOLVED $(\mu \mathrm{g} / \mathrm{L})$ & 3 & $<0.420$ & $<0.011$ & -- & -- & -- & -- & -- & -- \\
\hline 49306 & CHLOROTHALONIL， DISSOLVED $(\mu \mathrm{g} / \mathrm{L})$ & 3 & 0.800 & $<0.035$ & -- & -- & -- & -- & -- & -- \\
\hline 49305 & CLOPYRALID, DISSOLVED $(\mu \mathrm{g} / \mathrm{L})$ & 3 & $<0.230$ & $<0.050$ & -- & -- & -- & -- & -- & -- \\
\hline 49304 & DACTHALMONO-ACID, DISSOLVED $(\mu \mathrm{g} / \mathrm{L})$ & 3 & $<0.017$ & $<0.017$ & -- & -- & -- & -- & - & -- \\
\hline 38442 & DICAMBA, DISSOLVED $(\mu g / L)$ & 3 & $<0.035$ & $<0.035$ & -- & -- & -- & -- & -- & -- \\
\hline 49303 & DICHLOBENIL， DISSOLVED $(\mu \mathrm{g} / \mathrm{L})$ & 3 & $<1.200$ & $<0.020$ & -- & -- & -- & -- & -- & -- \\
\hline 49302 & DICHLORPROP， DISSOLVED $(\boldsymbol{\mu} \mathrm{g} / \mathrm{L})$ & 3 & $<0.032$ & $<0.032$ & -- & -- & - & -- & -- & -- \\
\hline 49301 & DINOSEB， DISSOLVED $(\mu \mathrm{g} / \mathrm{L})$ & 3 & $<0.035$ & $<0.035$ & -- & -- & -- & -- & -- & -- \\
\hline 49300 & DIURON, DISSOLVED $(\mu \mathrm{g} / \mathrm{L})$ & 3 & $<6.350$ & $<0.020$ & -- & -- & -- & -- & -- & -- \\
\hline 49299 & 4,6-DINITRO OCRESOL, DISSOLVED $(\mu \mathrm{g} / \mathrm{L})$ & 3 & $<0,420$ & $<0.035$ & -- & -- & -- & -- & -- & -- \\
\hline 49298 & ESFENVALERATE, DISSOLVED $(\mu \mathrm{g} / \mathrm{L})$ & 2 & $<0.019$ & $<0.019$ & -- & -- & -- & -- & -- & -- \\
\hline 49297 & FENURON, DISSOLVED $(\mu \mathrm{g} / \mathrm{L})$ & 3 & $<0.013$ & $<0.013$ & -- & -- & -- & -- & -- & -- \\
\hline 38811 & FLUOMETURON, DISSOLVED $(\mu \mathrm{g} / \mathrm{L})$ & 3 & $<0.035$ & $<0.035$ & -- & -- & -- & -- & -- & -- \\
\hline 38478 & LINURON， DISSOLVED $(\mu \mathrm{g} / \mathrm{L})$ & 3 & $<0.018$ & $<0.018$ & -- & -- & -- & -- & -- & -- \\
\hline 38482 & MCPA, DISSOLVED $(\mu \mathrm{g} / \mathrm{L})$ & 3 & $<0.170$ & $<0.050$ & -- & -- & - & -- & -- & -- \\
\hline 38487 & MCPB, DISSOLVED $(\mu \mathrm{g} / L)$ & 3 & $<0.140$ & $<0.035$ & -- & -- & -- & -- & -- & -- \\
\hline 38501 & METHIOCARB， DISSOLVED $(\mu \mathrm{g} / \mathrm{L})$ & 3 & $<0.026$ & $<0.026$ & -- & -- & -- & -- & -- & -- \\
\hline 49296 & METHOMYL, DISSOLVED $(\mu \mathrm{g} / \mathrm{L})$ & 3 & $<0.017$ & $<0.017$ & -- & -- & -- & -- & -- & -- \\
\hline 49295 & 1-NAPHTHOL, DISSOLVED $(\mu \mathrm{g} / \mathrm{L})$ & 2 & $<0.007$ & $<0.007$ & -- & -- & -- & -- & -- & -- \\
\hline 49294 & NEBURON， DISSOLVED $(\mu \mathrm{g} / \mathrm{L})$ & 3 & $<0.015$ & $<0.015$ & -- & -- & -- &.- & -- & -- \\
\hline 49293 & NORFLURAZON， DISSOLVED $(\mu \mathrm{g} / \mathrm{L})$ & 3 & $<0.024$ & $<0.024$ & -- & -- & -- & -- & -- & -- \\
\hline 49292 & ORYZALIN, DISSOLVED $(\mu \mathrm{g} / \mathrm{L})$ & 3 & $<0.310$ & $<0.019$ & -- & -- & -- & -- & -- & -- \\
\hline 38866 & OXAMYL，DISSOLVED $(\mu g / L)$ & 3 & $<0.018$ & $<0.018$ & - & -- & -- & - & -- & -- \\
\hline 49291 & PICLORAM, DISSOLVED $(\mu \mathrm{g} / \mathrm{L})$ & 3 & $<0.050$ & $<0.050$ & -- & -- & -- & -- & -- & -- \\
\hline 49236 & PROPHAM， DISSOLVED $(\mu \mathrm{g} / \mathrm{L})$ & 3 & $<0.035$ & $<0.035$ & -- & -- & -- & -- & -- & -- \\
\hline 38538 & PROPOXUR, DISSOLVED $(\mu \mathrm{g} / \mathrm{L})$ & 3 & $<0.035$ & $<0.035$ & -- & -- & -- & -- & -- & -- \\
\hline 39762 & SILVEX， DISSOLVED $(\mu \mathrm{g} / \mathrm{L})$ & 3 & $<0.021$ & $<0.021$ & -- & - & -- & -- & -- & -- \\
\hline 49235 & TRICLOPYR, DISSOLVED $(\mu \mathrm{g} / \mathrm{L})$ & 3 & $<0.250$ & $<0.050$ & -- & -- & -- & - & -- & -- \\
\hline & & ORGANI & COMPOUND & ORGANONI & OGEN, $T$ & CAL & & & & \\
\hline 39057 & PROMETRYNE， TOTAL $(\mu \mathrm{g} / \mathrm{L})$ & 1 & $<0.100$ & -- & -- & -- & -- & -- & -- & -- \\
\hline 39056 & PROMETONE, TOTAL $(\mu \mathrm{g} / \mathrm{L})$ & 1 & $<0.200$ & -- & -- & -- & -- & -- & -- & $\rightarrow-$ \\
\hline 39054 & SIMETRYNE, TOTAL $(\mu \mathrm{g} / \mathrm{L})$ & 1 & $<0.100$ & -- & -- & -- & -- & -- & -- & -- \\
\hline 81757 & CYANAZINE, TOTAL $(\mu g / L)$ & 1 & $<0.200$ & -- & -- & -- & -- & -- & -- & - \\
\hline 77825 & ALACHLOR, TOTAL $(\mu \mathrm{g} / \mathrm{L})$ & 1 & $<0.100$ & -- & -- & -- & -- & -- & -- & -- \\
\hline 82611 & METRIBUZIN, TOTAL $\left(\mu_{\mathrm{g} / \mathrm{L}}\right)$ & 1 & $<0.100$ & -- & -- & -- & -- & -- & -- & -- \\
\hline 30311 & TERBACIL, TOTAL $(\mu \mathrm{g} / \mathrm{L})$ & 1 & $<0.200$ & -- & -- & -- & -- & -- & -- & -- \\
\hline 30245 & CARBOXIN, TOTAL $(\mu \mathrm{g} / \mathrm{L})$ & 1 & $<0.200$ & -- & -- & -- & -- & -- & -- & -- \\
\hline 30264 & HEXAZINONE, TOTAL $(\mu \mathrm{g} / \mathrm{L})$ & 1 & $<0.200$ & -- & -- & -- & -- & -- & -- & -- \\
\hline 30235 & BUTACHLOR, TOTAL $(\mu \mathrm{g} / \mathrm{L})$ & 1 & $<0.100$ & -- & - & -- & -- & -- & -- & -- \\
\hline 30236 & BUTYLATE, TOTAL $(\mu \mathrm{g} / \mathrm{L})$ & 1 & 0.200 & - - & -- & -- & -- & -- & -- & -- \\
\hline 75981 & DE-ETHYLATRAZINE，TOTAL $(\mu \mathrm{g} / \mathrm{L})$ & 1 & $<0.200$ & -- & -- & -- & -- & -- & -- & -- \\
\hline 39630 & ATRAZINE, TOTAL $(\mu \mathrm{g} / \mathrm{L})$ & 1 & 0.200 & -- & -- & -- & -- & -- & -- & -- \\
\hline
\end{tabular}

NOTE: Multiple detection limits during the period of record may result in different values flagged with a "<."

* Value is estimated by using a log-probability regression to predict the values of data below the detection limit. 
Table 75. Statistical summary of water-quality data at site 43 (CSW07), June 1994 through September 1998-Continued

\begin{tabular}{|c|c|c|c|c|c|c|c|c|c|c|}
\hline \multirow{2}{*}{$\begin{array}{c}\text { PARAM- } \\
\text { ETER } \\
\text { CODE }\end{array}$} & \multirow{2}{*}{ PROPERTY OR CONSTITUENT } & \multicolumn{4}{|c|}{ DESCRIPTIVE STATISTICS } & \multicolumn{5}{|c|}{$\begin{array}{l}\text { PERCENT OF SAMPLES IN WHICH VALUES WERE LESS THAN OR } \\
\text { EQUAL TO THOSE SHIWWN }\end{array}$} \\
\hline & & $\begin{array}{c}\text { SAMPLE } \\
\text { SIZE }\end{array}$ & MAXIMUM & MINIMUM & MEAN & $95 \%$ & $75 \%$ & $\begin{array}{c}50 \% \\
\text { (median) }\end{array}$ & $25 \%$ & $5 \%$ \\
\hline 39055 & SIMAZINE, TOTAL $(\mu \mathrm{g} / \mathrm{L})$ & $\frac{2}{1}$ & 0.200 & -- & $=$ & -- & -- & -- & -- & -- \\
\hline 39024 & PROPAZINE, TOTAL $(\mu \mathrm{g} / \mathrm{L})$ & 1 & $<0.100$ & -- & -- & -- & -- & -- & -- & - \\
\hline 82184 & AMETRYNE， TOTAL $(\mu \mathrm{g} / \mathrm{L})$ & 1 & $<0.100$ & -- & -- & -- & -- & - & -- & -- \\
\hline 39030 & TRIFLURALIN, TOTAL $(\mu \mathrm{g} / \mathrm{L})$ & 1 & $<0.100$ & -- & -- & -- & - & $\ldots$ & -- & - \\
\hline 82612 & METOLACHLOR, TOTAL $(\mu \mathrm{g} / \mathrm{L})$ & 1 & $<0.200$ & -- & -- & - & -- & -- & -- & - \\
\hline 30234 & BROMACIL, TOTAL $(\mu \mathrm{g} / \mathrm{L})$ & 1 & 0.300 & -- & -- & -- & $\ldots$ & -- & -- & - \\
\hline 30255 & DIPHENAMID，TOTAL $(\mu \mathrm{g} / \mathrm{L})$ & 1 & $<0.100$ & -- & -- & -- & -- & -. & -- & - \\
\hline 30324 & VERNOLATE, TOTAL $(\mu \mathrm{g} / \mathrm{L})$ & 1 & $<0.100$ & -- & $\ldots$ & -- & -- & -- & -- & -- \\
\hline 30254 & CYCLOATE, TOTAL $(\mu \mathrm{g} / \mathrm{L})$ & 1 & $<0.100$ & -. & -- & -- & - & -- & -- & -- \\
\hline 30295 & PROPACHLOR, TOTAL $(\mu \mathrm{g} / \mathrm{L})$ & 1 & $<0.100$ & -- & - & -- & - & -- & -- & -- \\
\hline 75980 & DE-ISOPROPYLATRAZIN, TOTAL $(\mu \mathrm{g} / \mathrm{L})$ & 1 & $<0.200$ & - & -- & -- & -- & -- & - & -- \\
\hline \multicolumn{11}{|c|}{ ORGANIC COMPOUNDS-HERBICIDES, TOTAL } \\
\hline 39730 & $2, \overline{4-D}$, TOTAL $(\mu \mathrm{g} / \overline{\mathrm{L}})$ & 1 & 0.740 & -- & -- & -- & -- & -- & -- & - \\
\hline 39760 & SILVEX, TOTAL $(\mu \mathrm{g} / \mathrm{L})$ & 1 & $<0.010$ & -- & -- & -- & -- & -- & -- & -- \\
\hline 39720 & PICLORAM, TOTAL $(\mu \mathrm{g} / \mathrm{L})$ & 1 & $<0.010$ & -- & -- & -- & $\ldots$ & -- & -- & -- \\
\hline 39740 & $2,4,5-\mathrm{T}$, TOTAL $(\mu \mathrm{g} / \mathrm{L})$ & 1 & $<0.010$ & - & $\ldots$ & -- & $\ldots$ & -- & -- & -- \\
\hline 82183 & $2,4-\mathrm{DP}, \operatorname{TOTAL}(\mu \mathrm{g} / \mathrm{L})$ & 1 & $<0.010$ & -- & -- & -- & -- & -- & -- & - \\
\hline 82052 & DICAMBA, TOTAL $(\mu \mathrm{g} / \mathrm{L})$ & 1 & 0.170 & -- & -- & $\ldots$ & -- & -- & -- & - \\
\hline \multicolumn{11}{|c|}{ ORGANIC COMPOUNDS-CARBAMATE PESTICIDES, TOTAL } \\
\hline 39750 & SEVIN, TOTAL $(\mu \mathrm{g} / \mathrm{L})$ & 1 & $<0.500$ & -- & -- & -- & -- & -- & -- & -- \\
\hline 39051 & METHOMYL， TOTAL $(\mu \mathrm{g} / \mathrm{L})$ & 1 & $<0.500$ & -- & -- & $\ldots$ & $\ldots$ & -- & - & -- \\
\hline 82619 & ALDICARD, TOTAL $(\mu \mathrm{g} / \mathrm{L})$ & 1 & $<0.500$ & - & - & $\ldots$ & -- & -- & $\ldots$ & -- \\
\hline 30296 & PROPOXUR, TOTAL $(\mu \mathrm{g} / \mathrm{L})$ & 1 & $<0.500$ & -- & - & -- & - & -- & - & -- \\
\hline 39052 & PROPHAM, TOTAL $(\mu \mathrm{q} / \mathrm{L})$ & 1 & $<0.500$ & -. & .. & -. & -- & -- & .. & -- \\
\hline 82615 & CARBOFURAN, TOTAL $(\mu \mathrm{g} / \mathrm{L})$ & 1 & $<0.500$ & -- & - & -- & -- & -- & -. & -- \\
\hline 77441 & 1-NAPHTHOL, TOTAL $(\mu \mathrm{g} / \mathrm{L})$ & 1 & $<0.500$ & -- & -- & -- & -- & -. & -. & -- \\
\hline 30282 & METHIOCARB，TOTAL $(\mu \mathrm{g} / \mathrm{L})$ & 1 & $<0.500$ & -. & -. & -- & - & -. & -. & -- \\
\hline
\end{tabular}

NOTE: Multiple detection limits during the period of record may result in different values flagged with a "<."

* Value is estimated by using a log-probability regression to predict the values of data below the detection limit. 
Table 76. Water-quality data at site 37 (CSW06), July 1997 through September 1998

\begin{tabular}{|c|c|c|c|c|c|c|c|c|c|c|c|c|}
\hline DATE & TIME & $\begin{array}{l}\text { RAINFALL } \\
\text { ACCUM } \\
\text { (IN) } \\
(00045)\end{array}$ & $\begin{array}{l}\text { DISCHARGE, } \\
\text { INST. } \\
\text { CUBIC FEET } \\
\text { PEA } \\
\text { SECOND } \\
(00061) \\
\end{array}$ & $\begin{array}{l}\text { TEMPER- } \\
\text { ATURE } \\
\text { WATER } \\
\text { (DEG. C) } \\
(00010) \\
\end{array}$ & $\begin{array}{l}\text { SPECIFIC } \\
\text { CONDUC- } \\
\text { TANCE } \\
\text { LAB } \\
\text { (US/CM) } \\
(90095)\end{array}$ & $\begin{array}{l}\text { SPECIFIC } \\
\text { CONDUC- } \\
\text { TANCE } \\
\text { (US/CM) } \\
(00095 \\
\end{array}$ & $\begin{array}{c}\text { PH WATER } \\
\text { RAW } \\
\text { LAB } \\
\text { (STAN- } \\
\text { DARD } \\
\text { UNITS) } \\
\text { (00403) }\end{array}$ & $\begin{array}{c}\text { PH WATER } \\
\text { RAW } \\
\text { FIELD } \\
\text { (STAN- } \\
\text { DARD } \\
\text { UNITS) } \\
(00400)\end{array}$ & $\begin{array}{c}\text { ANC } \\
\text { UNFLTRD } \\
\text { TIT 4.5 } \\
\text { LAB } \\
\text { (MG/L AS } \\
\text { CACO3) } \\
(90410)\end{array}$ & $\begin{array}{l}\text { SEDIMENT, } \\
\text { SUS- } \\
\text { PENDED } \\
\text { (MG/L) } \\
(80154)\end{array}$ & $\begin{array}{l}\text { RESID JE } \\
\text { TOTA'. } \\
\text { AT } 1 \text { C5 } \\
\text { DEG. C, } \\
\text { SUS- } \\
\text { PEND =D } \\
(M G / 1-) \\
(00520)\end{array}$ & $\begin{array}{l}\text { RESIDUE } \\
\text { VOLATILE, } \\
\text { SUS- } \\
\text { PENDED } \\
\text { (MG/L) } \\
(00535)\end{array}$ \\
\hline \multicolumn{13}{|l|}{ JUL 1997} \\
\hline $\begin{array}{l}22 \ldots \\
\text { NOV }\end{array}$ & 2306 & 5.91 & 1.5 & 27.5 & 111 & 103 & 6.6 & 6.5 & 14 & 221 & $12^{?}$ & 36 \\
\hline $12 \ldots$ & 2005 & 1.20 & .02 & 11.0 & -- & 114 & -- & 7.2 & -- & -- & -- & -- \\
\hline $21 \ldots$ & 1158 & .73 & .81 & 8.0 & 83 & 80 & 6.8 & 6.7 & 12 & 63 & 57 & 14 \\
\hline $21 \ldots$ & 1352 & .73 & 7.3 & 9.0 & 55 & 51 & 6.8 & 6.7 & 6.0 & 149 & 140 & 22 \\
\hline $21 \ldots$ & 1447 & .73 & 1.7 & 9.0 & -- & 48 & -- & 6.5 & -- & -- & -- & -- \\
\hline $21 \ldots$ & 1504 & .73 & 1.2 & 9.0 & 53 & 49 & 6.8 & 6.6 & 6.0 & 36 & 2.5 & 5 \\
\hline \multicolumn{13}{|l|}{ JAN 1998} \\
\hline $22 \ldots$ & 2106 & .76 & .53 & 6.0 & 92 & 85 & 7.2 & 6.9 & 21 & 41 & 27 & 6 \\
\hline $22 \ldots$ & 2159 & .76 & 1.1 & 6.0 & 89 & 85 & 7.2 & 6.9 & 16 & 19 & 14 & 4 \\
\hline $23 \ldots$ & 0040 & .76 & 1.4 & 6.0 & 49 & 43 & 7.2 & 6.9 & 11 & 18 & 11 & 3 \\
\hline $23 \ldots$ & 0931 & .76 & .34 & 6.0 & 62 & 56 & 7.2 & 6.9 & 16 & 11 & 5 & 1 \\
\hline \multicolumn{13}{|l|}{ JUN } \\
\hline $10 \ldots$ & 0919 & .71 & 7.0 & -- & -- & 56 & -- & 6.7 & - & -- & -- & -- \\
\hline $10 \ldots$ & 0921 & .71 & 9.0 & - & 57 & 52 & 6.8 & 6.6 & 8.0 & 164 & 122 & 19 \\
\hline $10 \ldots$ & 0934 & .71 & 19 & -- & 46 & 41 & 6.7 & 6.6 & 6.0 & 138 & 119 & 22 \\
\hline $10 \ldots$ & 1001 & .71 & 4.1 & -- & -- & 41 & -- & 6.8 & -- & - & -- & -- \\
\hline $10 \ldots$ & 1016 & .71 & 1.8 & - & 45 & 40 & 7.0 & 6.6 & 7.0 & 21 & 9 & 5 \\
\hline DATE & $\begin{array}{c}\text { SOLIDS, } \\
\text { RESIDUE } \\
\text { AT } 180 \\
\text { DEG. C } \\
\text { DIS- } \\
\text { SOLVED } \\
\text { (MG/L) } \\
(70300) \\
\end{array}$ & $\begin{array}{c}\text { OXYGEN } \\
\text { DEMAND, } \\
\text { BIO- } \\
\text { CHEMICAL } \\
5 \text { DAY } \\
(\mathrm{MG} / \mathrm{L}) \\
(00310) \\
\end{array}$ & $\begin{array}{c}\text { OXYGEN } \\
\text { DEMAND, } \\
\text { CHEMICAL } \\
\text { (HIGHLEVEL) } \\
(M G / L) \\
(00340) \\
\end{array}$ & $\begin{array}{c}\text { NITROGEN, } \\
\text { AMMONIA + } \\
\text { ORGANIC } \\
\text { TOTAL } \\
\text { (MG/L AS N) } \\
(00625)\end{array}$ & $\begin{array}{c}\text { NITROGEN, } \\
\text { NO2+NO3 } \\
\text { DISSOLVED } \\
\text { (MG/ AS N) } \\
(00631) \\
\end{array}$ & $\begin{array}{c}\text { NITROGEN, } \\
\text { AMMONIA } \\
\text { DISSOLVED } \\
(M G / L \text { AS N) } \\
(00608) \\
\end{array}$ & $\begin{array}{l}\text { NITRO- } \\
\text { GEN, } \\
\text { ORGANIC } \\
\text { TOTAL } \\
\text { (MG/L } \\
\text { ASN) } \\
(00605)\end{array}$ & $\begin{array}{l}\text { NITROGEN, } \\
\text { TOTAL } \\
\text { (MG/LAS N) } \\
(00600)\end{array}$ & $\begin{array}{c}\text { PHOS- } \\
\text { PHORUS } \\
\text { TOTAL } \\
\text { (MG/ AS P) } \\
(00665) \\
\end{array}$ & $\begin{array}{c}\text { PHOS- } \\
\text { PHORUS } \\
\text { ORTHO, } \\
\text { DISSOLVED } \\
\text { (MG/L AS P) } \\
(00671) \\
\end{array}$ & $\begin{array}{c}\text { OILAND } \\
\text { GREASE, } \\
\text { TOTAL } \\
\text { RECOV. } \\
\text { GR,AVI- } \\
\text { METRIC } \\
\text { (MG/L) } \\
\text { (00556) } \\
\end{array}$ & \\
\hline \multicolumn{13}{|l|}{ JUL 1997} \\
\hline $\begin{array}{l}22 \ldots \\
\text { NOV }\end{array}$ & 117 & 23 & 99 & 3.2 & 0.450 & 0.430 & 2.8 & 3.7 & 1.90 & 1.18 & -- & \\
\hline $12 \ldots$ & -- & -- & -- & -- & -- & -- & -- & -- & -- & -- & 3 & \\
\hline $21 \ldots$ & 69 & - & 42 & .98 & .520 & .120 & .86 & 1.5 & .410 & .090 & -- & \\
\hline $21 \ldots$ & 46 & -- & 18 & 1.0 & .440 & .170 & .83 & 1.4 & .670 & .110 & -- & \\
\hline $21 \ldots$ & -- & -- & -- & -- & -- & -- & -- & -- & -- & -- & -- & \\
\hline $\begin{array}{c}21 \ldots \\
\text { JAN } 1998\end{array}$ & 45 & - & 16 & .63 & .370 & .140 & .49 & 1.0 & .350 & .110 & -- & \\
\hline $22 \ldots$ & 55 & 4.6 & 20 & .58 & .420 & .050 & .53 & 1.0 & .140 & .019 & -- & \\
\hline $22 \ldots$ & 53 & 5.1 & 18 & .49 & .440 & .017 & .47 & .93 & .110 & .013 & -- & \\
\hline $23 \ldots$ & 31 & 2.6 & 14 & .41 & .170 & $<.015$ & .41 & .58 & .080 & .200 & -- & \\
\hline $\begin{array}{l}23 \ldots \\
\text { JUN }\end{array}$ & 43 & $<2.0$ & 14 & .54 & .200 & $<.015$ & .54 & .74 & .100 & .027 & -- & \\
\hline $10 \ldots$ & -- & -- & -- & -- & -- & -- & -- & - & -- & -- & -- & \\
\hline $10 \ldots$ & 41 & 8.9 & 36 & 1.3 & .440 & .130 & 1.2 & 1.7 & .710 & .120 & 1 & \\
\hline $10 \ldots$ & 33 & 7.0 & 31 & 1.7 & .330 & .140 & 1.6 & 2.0 & .560 & .130 & -- & \\
\hline $10 \ldots$ & -- & -- & -- & -- & -- & -- & -- & -- & -- & -- & -- & \\
\hline $10 \ldots$ & 32 & 7.8 & 18 & .71 & .300 & .050 & .66 & 1.0 & .200 & .080 & -- & \\
\hline
\end{tabular}


Table 76. Water-quality data at site 37 (CSW06), July 1997 through September 1998-Continued

\begin{tabular}{|c|c|c|c|c|c|c|c|c|c|c|c|}
\hline DATE & $\begin{array}{c}\text { CARBON, } \\
\text { OR- } \\
\text { GANIC } \\
\text { TOTAL } \\
\text { (MG/L } \\
\text { AS C) } \\
(00680 \\
\end{array}$ & $\begin{array}{l}\text { STREPTO- } \\
\text { COCCI } \\
\text { FECAL, } \\
\text { (COLS. PER } \\
100 \mathrm{ML}) \\
(31679) \\
\end{array}$ & $\begin{array}{c}\text { COLIFORM, } \\
\text { FECAL, } \\
0.45 \\
\text { UM-MF } \\
\text { (COLS./ } \\
100 \mathrm{ML}) \\
(31616) \\
\end{array}$ & $\begin{array}{c}\text { ANTIMONY, } \\
\text { TOTAL } \\
\text { (UG/L AS SB) } \\
(01097) \\
\end{array}$ & $\begin{array}{c}\text { ARSENIC, } \\
\text { TOTAL } \\
\text { (UG/L AS AS) } \\
(01002) \\
\end{array}$ & $\begin{array}{l}\text { CHROMIUM, } \\
\text { TOTAL } \\
\text { RECOVER- } \\
\text { ABLE } \\
\text { (UG/L AS CR) } \\
(01034)\end{array}$ & $\begin{array}{c}\text { COPPER, } \\
\text { TOTAL } \\
\text { RECOVER- } \\
\text { ABLE } \\
\text { (UG/L } \\
\text { AS CU) } \\
(01042) \\
\end{array}$ & $\begin{array}{c}\text { LEAD, } \\
\text { TOTAL } \\
\text { RECOVER- } \\
\text { ABLE } \\
\text { (UG/L } \\
\text { AS PB) } \\
\text { (01051) }\end{array}$ & $\begin{array}{c}\text { MERCURY, } \\
\text { TOTAL } \\
\text { RECOVER- } \\
\text { ABLE } \\
\text { (UG/L AS HG) } \\
(71900)\end{array}$ & $\begin{array}{c}\text { NICKEL, } \\
\text { TOTAL } \\
\text { RECOVER- } \\
\text { ABLE } \\
\text { (UG/L AS NI) } \\
(01067) \\
\end{array}$ & $\begin{array}{c}\text { ZINC, } \\
\text { TOTAL } \\
\text { RECJVER- } \\
\text { ABLE } \\
\text { (UG/L AS ZN) } \\
(01092) \\
\end{array}$ \\
\hline \multicolumn{12}{|c|}{ JUL 1997} \\
\hline $22 \ldots$ & 32 & -- & -- & $<1$ & $<1$ & 11 & 23 & 11 & 0.20 & 32 & 140 \\
\hline \multicolumn{12}{|l|}{ NOV } \\
\hline $12 \ldots$ & - & $<100$ & K8800 & -- & -- & -- & -- & -- & -- & -- & -- \\
\hline $21 \ldots$ & 11 & -- & -- & $<1$ & $<1$ & 7 & 16 & 9 & $<.10$ & 11 & 80 \\
\hline $21 \ldots$ & 9.7 & -- & -- & $<1$ & $<1$ & 10 & 19 & 11 & $<.10$ & 14 & 90 \\
\hline $21 \ldots$ & -- & K1800 & 2900 & -- & -- & -- & -- & -- & -- & -- & -- \\
\hline $21 \ldots$ & -- & -- & -- & -- & -- & -- & - & -- & -- & -- & -- \\
\hline \multicolumn{12}{|c|}{ JAN 1998} \\
\hline $22 \ldots$ & 8.5 & -- & -- & $<1$ & $<1$ & $<1$ & 12 & 2 & $<.10$ & 6 & 70 \\
\hline $22 \ldots$ & 7.3 & - & -- & $<1$ & $<1$ & $<1$ & 5 & $<1$ & $<.10$ & 3 & 60 \\
\hline $23 \ldots$ & 5.9 & - & -- & $<1$ & 2 & 4 & 14 & 4 & .20 & 3 & 50 \\
\hline $23 \ldots$ & -- & 2300 & 540 & -- & -- & -- & -- & -- & -- & -- & -- \\
\hline \multicolumn{12}{|l|}{ JUN } \\
\hline $10 \ldots$ & -- & -- & -- & -- & -- & -- & -- & -- & -- & -- & -- \\
\hline $10 \ldots$ & 15 & 83000 & 43000 & $<1$ & 3 & 12 & 24 & 22 & $<.10$ & 12 & 130 \\
\hline $10 \ldots$ & 12 & 73000 & K77000 & $<1$ & 5 & 13 & 27 & 25 & .20 & 8 & 190 \\
\hline $10 \ldots$ & -- & 53000 & K15000 & - & -- & -- & -- & -- & -- & - & -- \\
\hline $10 \ldots$ & -- & 51000 & K66000 & -- & -- & -- & -- & -. & - & -- & -- \\
\hline
\end{tabular}

K Results based on colony count outside the acceptable range (nonideal colony count). 
Table 77. Water-quality data at site 39 (CSW05), July 1997 through September 1998

\begin{tabular}{|c|c|c|c|c|c|c|c|c|c|c|c|c|}
\hline DATE & TIME & $\begin{array}{c}\text { RAINFALL } \\
\text { ACCUM } \\
(\text { IN) } \\
(00045) \\
\end{array}$ & $\begin{array}{c}\text { DISCHARGE, } \\
\text { INST. } \\
\text { CUBIC FEET } \\
\text { PER } \\
\text { SECOND } \\
(00061) \\
\end{array}$ & $\begin{array}{l}\text { TEMPER- } \\
\text { ATURE } \\
\text { WATER } \\
\text { (DEG. C) } \\
\text { (00010) } \\
\end{array}$ & $\begin{array}{c}\text { SPECIFIC } \\
\text { CONDUC- } \\
\text { TANCE } \\
\text { LAB } \\
(\text { US/CM) } \\
(90095) \\
\end{array}$ & $\begin{array}{c}\text { SPECIFIC } \\
\text { CONDUC- } \\
\text { TANCE } \\
\text { (US/CM) } \\
(00095 \\
\end{array}$ & $\begin{array}{c}\text { PHWATER } \\
\text { RAW } \\
\text { LAB } \\
\text { (STAN- } \\
\text { DARD } \\
\text { UNITS) } \\
\text { (00403) } \\
\end{array}$ & $\begin{array}{l}\text { PH WATER } \\
\text { RAW } \\
\text { FIELD } \\
\text { (STAN- } \\
\text { DARD } \\
\text { UNITS) } \\
\text { (00400) } \\
\end{array}$ & $\begin{array}{c}\text { ANC } \\
\text { UNFLTRD } \\
\text { TIT } 4.5 \\
\text { LAB } \\
\text { (MG/L AS } \\
\text { CACO3) } \\
(90410) \\
\end{array}$ & $\begin{array}{l}\text { SEDIMENT, } \\
\text { SUS- } \\
\text { PENDED } \\
\text { (MG/L) } \\
(80154) \\
\end{array}$ & $\begin{array}{c}\text { RES DUE } \\
\text { TO'AL } \\
\text { AT } 105 \\
\text { DEG. C, } \\
\text { SUS- } \\
\text { PENDED } \\
\text { (MG/L) } \\
\text { (00530) } \\
\end{array}$ & $\begin{array}{c}\text { RESIDUE } \\
\text { VOLATILE, } \\
\text { SUS- } \\
\text { PENDED } \\
\text { (MG/L) } \\
(00535) \\
\end{array}$ \\
\hline \multicolumn{13}{|l|}{ JUL 1997} \\
\hline $22 \ldots$ & 2029 & 8.93 & 1.6 & 27.0 & 65 & 62 & 5.7 & 5.2 & 5.0 & 222 & 160 & 44 \\
\hline $23 \ldots$ & 0130 & 8.93 & 21 & 23.0 & 21 & 18 & 6.3 & 5.5 & 3.0 & 153 & 61 & 7 \\
\hline $23 \ldots$ & 0456 & 8.93 & .53 & 24.0 & 66 & 59 & 6.9 & 6.2 & 10 & 36 & 24 & 2 \\
\hline \multicolumn{13}{|l|}{ NOV } \\
\hline $12 \ldots$ & 1825 & 1.25 & .09 & 12.0 & -- & -- & -- & -- & - & -- & -- & -- \\
\hline $12 \ldots$ & 1910 & 1.25 & .09 & 11.5 & -- & 55 & -- & 7.3 & - & - & -- & -- \\
\hline $12 \ldots$ & 1935 & 1.25 & .07 & 11.5 & 55 & 51 & 7.0 & 6.7 & 11 & 49 & 58 & 10 \\
\hline $12 \ldots$ & 2146 & 1.25 & .19 & 10.5 & 43 & 40 & 7.0 & 6.8 & 9.0 & 58 & 6 & $<1$ \\
\hline $13 \ldots$ & 0030 & 1.25 & .09 & 10.5 & 53 & 48 & 7.1 & 6.9 & 12 & -- & 4 & $<1$ \\
\hline $13 \ldots$ & 1056 & 1.25 & .21 & 10.0 & -- & -- & -- & -- & - & -- & -- & -- \\
\hline \multicolumn{13}{|c|}{ JAN 1998} \\
\hline $22 \ldots$ & 1842 & .75 & .17 & 8.0 & 162 & 154 & 7.1 & 6.8 & 13 & 195 & 158 & 45 \\
\hline $22 \ldots$ & 1935 & .75 & .30 & 7.0 & 67 & 62 & 7.1 & 6.8 & 10 & 43 & 40 & 12 \\
\hline $23 \ldots$ & 0004 & .75 & .36 & 6.0 & 49 & 45 & 7.0 & 6.8 & 10 & 19 & 10 & 3 \\
\hline $23 \ldots$ & 1017 & .75 & .07 & 7.0 & 116 & 112 & 7.4 & 7.0 & 25 & 18 & 9 & 2 \\
\hline \multicolumn{13}{|l|}{ MAY } \\
\hline $20 \ldots$ & 0530 & .00 & .00 & 16.5 & 456 & 429 & 8.0 & 7.6 & 150 & 7 & 27 & 10 \\
\hline \multicolumn{13}{|l|}{ JUN } \\
\hline $10 \ldots$ & 0735 & .46 & .32 & 20.5 & 98 & 93 & 6.8 & 6.8 & 9.0 & 232 & 176 & 50 \\
\hline $10 \ldots$ & 0741 & .46 & .36 & 20.5 & -- & -- & -- & -- & -- & -- & -- & -- \\
\hline $10 \ldots$ & 0812 & .46 & .16 & 21.0 & -- & 47 & -- & 6.3 & - & -- & -- & -- \\
\hline $10 \ldots$ & 0837 & .46 & 1.0 & 21.5 & -- & 22 & -- & 6.8 & -- & -- & -- & -- \\
\hline $10 \ldots$ & 0840 & .46 & 1.6 & 21.5 & 27 & 21 & 6.7 & 7.1 & 5.0 & 130 & 116 & 28 \\
\hline $10 \ldots$ & 1028 & .46 & .16 & 22.0 & 61 & 54 & 7.0 & 6.8 & 11 & 24 & 14 & 6 \\
\hline DATE & $\begin{array}{l}\text { SOLIDS, } \\
\text { RESIDUE } \\
\text { AT } 180 \\
\text { DEG. C } \\
\text { DIS- } \\
\text { SOLVED } \\
(\mathrm{MG} / \mathrm{L}) \\
(70300) \\
\end{array}$ & $\begin{array}{c}\text { OXYGEN } \\
\text { DEMAND, } \\
\text { BIO- } \\
\text { CHEMICAL } \\
5 \text { DAY } \\
\text { (MG/L) } \\
(00310) \\
\end{array}$ & $\begin{array}{c}\text { OXYGEN } \\
\text { DEMAND, } \\
\text { CHEMICAL } \\
\text { (HIGHLEVEL) } \\
(\text { MG/L) } \\
(00340) \\
\end{array}$ & $\begin{array}{c}\text { NITROGEN, } \\
\text { AMMONIA + } \\
\text { ORGANIC } \\
\text { TOTAL } \\
\text { (MG/L AS N) } \\
(00625) \\
\end{array}$ & $\begin{array}{c}\text { NITROGEN, } \\
\text { NO2+NO3 } \\
\text { DISSOLVED } \\
\text { (MG/L AS N) } \\
(00631) \\
\end{array}$ & $\begin{array}{c}\text { NITROGEN, } \\
\text { AMMONIA } \\
\text { DISSOLVED } \\
\text { (MG/L AS N) } \\
(00608) \\
\end{array}$ & $\begin{array}{l}\text { NITRO- } \\
\text { GEN, } \\
\text { ORGANIC } \\
\text { TOTAL } \\
\text { (MG/L } \\
\text { AS N) } \\
(00605) \\
\end{array}$ & $\begin{array}{c}\text { NITROGEN, } \\
\text { TOTAL } \\
\text { (MG/LASN) } \\
(00600) \\
\end{array}$ & $\begin{array}{c}\text { PHOS- } \\
\text { PHORUS } \\
\text { TOTAL } \\
\text { (MG/L AS P) } \\
(00665)\end{array}$ & $\begin{array}{c}\text { PHOS- } \\
\text { PHORUS } \\
\text { ORTHO, } \\
\text { DISSOLVED } \\
\text { (MG/L AS P) } \\
(00671) \\
\end{array}$ & $\begin{array}{c}\text { OIL AND } \\
\text { GREASE, } \\
\text { TO־AL } \\
\text { REC.OV. } \\
\text { GRAVI- } \\
\text { METRIC } \\
\text { (MG/L) } \\
(00556) \\
\end{array}$ & \\
\hline \multicolumn{13}{|l|}{ JUL 1997} \\
\hline $22 \ldots$ & 63 & 23 & 140 & 2.6 & 0.890 & 0.690 & 1.9 & 3.5 & 0.390 & 0.100 & -- & \\
\hline $23 \ldots$ & 19 & 2.9 & 10 & .47 & .260 & .080 & .39 & .73 & .220 & .120 & -- & \\
\hline $23 \ldots$ & 64 & 4.9 & 7 & 1.0 & .680 & .130 & .87 & 1.7 & .270 & .200 & -- & \\
\hline \multicolumn{13}{|l|}{ NOV } \\
\hline $12 \ldots$ & -- & -- & -- & 1.3 & .530 & .220 & 1.1 & 1.8 & .440 & .190 & -- & \\
\hline $12 \ldots$ & -- & - & -- & -- & -- & -- & -- & - & -- & -- & 6 & \\
\hline $12 \ldots$ & 48 & 14 & 52 & .81 & .350 & .120 & .69 & 1.2 & .310 & .130 & -- & \\
\hline $12 \ldots$ & 36 & 10 & 36 & .73 & .240 & .040 & .69 & .97 & .240 & .080 & -- & \\
\hline $13 \ldots$ & 42 & 6.1 & 18 & .43 & .170 & $<.015$ & .43 & .60 & .120 & .050 & -- & \\
\hline $13 \ldots$ & - & -- & -- & -- & -- & -- & -- & -- & -- & -- & -- & \\
\hline \multicolumn{13}{|l|}{ JAN 1998} \\
\hline $22 \ldots$ & 102 & 18 & 64 & 1.8 & 1.56 & .420 & 1.4 & 3.4 & .750 & .180 & -- & \\
\hline $22 \ldots$ & 39 & 4.6 & 30 & .85 & .650 & .120 & .73 & 1.5 & .220 & .120 & -- & \\
\hline $23 \ldots$ & 29 & $<2.0$ & 13 & .43 & .350 & .080 & .35 & .78 & .140 & .070 & -- & \\
\hline $23 \ldots$ & 78 & $<2.0$ & 18 & .65 & .610 & .037 & .61 & 1.3 & .200 & .070 & -- & \\
\hline \multicolumn{13}{|l|}{ MAY } \\
\hline $20 \ldots$ & 291 & 4.3 & 7 & .69 & .900 & $<.015$ & .69 & 1.6 & .120 & .044 & 3 & \\
\hline \multicolumn{13}{|l|}{ JUN } \\
\hline $10 \ldots$ & 73 & 18 & 100 & 3.0 & 1.42 & .630 & 2.4 & 4.4 & .620 & .080 & -- & \\
\hline $10 \ldots$ & -- & -- & -- & -- & -- & -- & - & -- & -- & -- & -- & \\
\hline $10 \ldots$ & -- & -- & -- & -- & -- & -- & -- & -- & -- & -- & 4 & \\
\hline $10 \ldots$ & -- & -- & -- & -- & - & -- & -- & -- & -- & -- & -- & \\
\hline $10 \ldots$ & 14 & 6.5 & 38 & 1.2 & .270 & 1.20 & .00 & 1.5 & .340 & .050 & -- & \\
\hline $10 \ldots$ & 44 & 6.2 & 34 & 1.1 & .600 & .090 & 1.0 & 1.7 & .340 & .180 & -- & \\
\hline
\end{tabular}


Table 77. Water-quality data at site 39 (CSW05), July 1997 through September 1998-Continued

\begin{tabular}{|c|c|c|c|c|c|c|c|c|c|c|c|}
\hline DATE & $\begin{array}{c}\text { CARBON, } \\
\text { OR- } \\
\text { GANIC } \\
\text { TOTAL } \\
\text { (MG/L } \\
\text { AS C) } \\
(00680 \\
\end{array}$ & $\begin{array}{c}\text { STREPTO- } \\
\text { COCCI } \\
\text { FECAL, } \\
\text { (COLS. PER } \\
100 \mathrm{ML}) \\
(31679) \\
\end{array}$ & $\begin{array}{c}\text { COLIFORM, } \\
\text { FECAL, } \\
0.45 \\
\text { UM-MF } \\
\text { (COLS./ } \\
100 \mathrm{ML}) \\
(31616) \\
\end{array}$ & $\begin{array}{c}\text { ANTIMONY, } \\
\text { TOTAL } \\
\text { (UG/L AS SB) } \\
(01097) \\
\end{array}$ & $\begin{array}{c}\text { ARSENIC, } \\
\text { TOTAL } \\
\text { (UG/L AS AS) } \\
(01002) \\
\end{array}$ & $\begin{array}{c}\text { CHROMIUM, } \\
\text { TOTAL } \\
\text { RECOVER- } \\
\text { ABLE } \\
\text { (UG/L AS CR) } \\
(01034) \\
\end{array}$ & $\begin{array}{c}\text { COPPER, } \\
\text { TOTAL } \\
\text { RECOVER- } \\
\text { ABLE } \\
\text { (UG/L } \\
\text { AS CU) } \\
(01042) \\
\end{array}$ & $\begin{array}{c}\text { LEAD, } \\
\text { TOTAL } \\
\text { RECOVER- } \\
\text { ABLE } \\
\text { (UG/L } \\
\text { AS PB) } \\
(01051) \\
\end{array}$ & $\begin{array}{c}\text { MERCURY, } \\
\text { TOTAL } \\
\text { RECOVER- } \\
\text { ABLE } \\
\text { (UG/L AS HG) } \\
(71900) \\
\end{array}$ & $\begin{array}{c}\text { NICKEL, } \\
\text { TOTAL } \\
\text { RECOVER- } \\
\text { ABLE } \\
\text { (UG/L AS NI) } \\
(01067) \\
\end{array}$ & $\begin{array}{c}\text { ZINC } \\
\text { TCTAL } \\
\text { REC OVER- } \\
\text { ABLE } \\
\text { (UG/L AS ZN) } \\
(01092) \\
\end{array}$ \\
\hline \multicolumn{12}{|l|}{ JUL 1997} \\
\hline $22 \ldots$ & 41 & -- & -- & $<1$ & $<1$ & 15 & 24 & 33 & -- & 8 & 330 \\
\hline $23 \ldots$ & 4.2 & -- & -- & $<1$ & $<1$ & 9 & 9 & 7 & -- & 3 & 80 \\
\hline $\begin{array}{r}23 \ldots \\
\text { NOV }\end{array}$ & -- & -- & - & - & -- & -- & - & -- & -- & -- & -- \\
\hline $12 \ldots$ & -- & -- & -- & $<1$ & 2 & 15 & 27 & 31 & -- & 10 & 300 \\
\hline $12 \ldots$ & -- & K990 & 2000 & -- & -- & -- & -- & -. & -- & -- & -- \\
\hline $12 \ldots$ & 12 & -- & -- & $<1$ & $<1$ & 12 & 12 & 20 & -- & 6 & 200 \\
\hline $12 \ldots$ & 9.7 & - & -- & $<1$ & 2 & 10 & 14 & 13 & -- & 5 & 170 \\
\hline $13 \ldots$ & -- & -- & -- & -- & -- & - & - & -- & - & -- & -- \\
\hline $13 \ldots$ & -- & 6600 & 3900 & -. & -- & -- & -- & -. & - & -- & - \\
\hline JAN 1998 & & & & & & & & & & & \\
\hline $22 \ldots$ & 26 & -- & -- & $<1$ & $<1$ & 19 & 28 & 29 & -- & 10 & 270 \\
\hline $22 \ldots$ & 10 & - & -- & $<1$ & $<1$ & 6 & 12 & 10 & -. & $<1$ & 90 \\
\hline $23 \ldots$ & 5.3 & -- & -- & $<1$ & 3 & $<1$ & 7 & 4 & -- & $<1$ & 50 \\
\hline $23 \ldots$ & -- & 5800 & 1100 & -- & -- & -- & -- & - & - & - & - \\
\hline MAY & & & & & & & & & & & \\
\hline $\begin{array}{l}20 \ldots \\
\text { JUN }\end{array}$ & -- & K990 & K1900 & 1 & $<1$ & 7 & 16 & $<1$ & -- & 6 & 20 \\
\hline $10 \ldots$ & -- & -- & -- & 1 & 6 & 28 & 37 & 60 & -- & 12 & 430 \\
\hline $10 \ldots$ & - & - & -- & - & - & -- & -- & - & - & -- & -- \\
\hline $10 \ldots$ & 14 & 14000 & 28000 & -- & -- & -- & -- & - & - & -- & -- \\
\hline $10 \ldots$ & 10 & 20000 & K8000 & -- & -- & -- & -- & - & - & -- & -- \\
\hline $10 \ldots$ & -- & -- & -- & $<1$ & 3 & 65 & 18 & 21 & - & 4 & 120 \\
\hline $10 \ldots$ & -- & - & -- & -- & -- & -- & - & - & -- & -. & -- \\
\hline
\end{tabular}

K Results based on colony count outside the acceptable range (nonideal colony count). 
Table 78. Water-quality data at site 40 (CSW03), July 1997 through September 1998

\begin{tabular}{|c|c|c|c|c|c|c|c|c|c|c|c|c|}
\hline DATE & TIME & $\begin{array}{l}\text { RAINFALL } \\
\text { ACCUM } \\
\text { (IN) } \\
(00045)\end{array}$ & $\begin{array}{c}\text { DISCHARGE, } \\
\text { INST. } \\
\text { CUBIC FEET } \\
\text { PER } \\
\text { SECOND } \\
(00061)\end{array}$ & $\begin{array}{l}\text { TEMPER- } \\
\text { ATURE } \\
\text { WATER } \\
\text { (DEG. C) } \\
(00010) \\
\end{array}$ & $\begin{array}{c}\text { SPECIFIC } \\
\text { CONDUC- } \\
\text { TANCE } \\
\text { LAB } \\
(\text { US/CM) } \\
(90095) \\
\end{array}$ & $\begin{array}{l}\text { SPECIFIC } \\
\text { CONDUC- } \\
\text { TANCE } \\
\text { (US/CM) } \\
\text { (00095 }\end{array}$ & $\begin{array}{l}\text { PHWATER } \\
\text { RAW } \\
\text { LAB } \\
\text { (STAN- } \\
\text { DARD } \\
\text { UNITS) } \\
\text { (00403) } \\
\end{array}$ & $\begin{array}{l}\text { PH WATER } \\
\text { RAW } \\
\text { FIELD } \\
\text { (STAN- } \\
\text { DARD } \\
\text { UNITS) } \\
\text { (00400) } \\
\end{array}$ & $\begin{array}{c}\text { ANC } \\
\text { UNFLTRD } \\
\text { TIT 4.5 } \\
\text { LAB } \\
\text { (MG/L AS } \\
\text { CACO3) } \\
(90410) \\
\end{array}$ & $\begin{array}{l}\text { SEDIMENT, } \\
\text { SUS- } \\
\text { PENDED } \\
\text { (MG/L) } \\
\text { (80154) }\end{array}$ & $\begin{array}{c}\text { RESIDUE } \\
\text { TOTAL } \\
\text { AT } 105 \\
\text { DEG. C, } \\
\text { SUS- } \\
\text { PENDED } \\
\text { (MG/L) } \\
\text { (00530' }\end{array}$ & $\begin{array}{c}\text { RESIDUE } \\
\text { VOLATILE, } \\
\text { SUS- } \\
\text { PENDED } \\
(M G / L) \\
(00535)\end{array}$ \\
\hline \multicolumn{13}{|l|}{ JUL 1997} \\
\hline $22 \ldots$ & 2318 & 4.82 & 0.34 & 27.0 & 53 & 44 & 6.8 & 6.6 & 8.0 & 226 & 184 & 46 \\
\hline $23 \ldots$ & 0014 & 4.82 & .81 & 26.0 & 68 & 37 & 6.9 & 6.9 & 24 & 5530 & 1620 & 390 \\
\hline $23 \ldots$ & 0146 & 4.82 & 9.9 & 24.5 & -- & 25 & -- & 6.4 & -- & 2500 & -. & -- \\
\hline \multicolumn{13}{|l|}{ NOV } \\
\hline $21 \ldots$ & 1020 & .63 & .05 & 9.0 & 69 & 56 & 6.2 & 6.3 & 12 & 39 & 39 & 23 \\
\hline $21 \ldots$ & 1341 & .63 & .20 & 9.0 & -- & 44 & -- & 6.6 & -- & -- & -- & -- \\
\hline $21 \ldots$ & 1358 & .63 & .27 & 9.0 & 48 & 44 & 6.7 & 6.5 & 10 & -- & 48 & 21 \\
\hline $21 \ldots$ & 1438 & .63 & .03 & 9.5 & 43 & 39 & 6.8 & 6.7 & 9.0 & -- & 46 & 14 \\
\hline \multicolumn{13}{|c|}{ FEB 1998} \\
\hline $03 \ldots$ & 0814 & 1.79 & .02 & 7.5 & 63 & 56 & 7.2 & 6.9 & 10 & 58 & 49 & 15 \\
\hline $03 \ldots$ & 0850 & 1.79 & .03 & 8.0 & -- & 65 & -- & 7.1 & -- & -- & -- & -- \\
\hline $03 \ldots$ & 1604 & 1.79 & .07 & 8.5 & 47 & 41 & 7.0 & 7.1 & 12 & 178 & 172 & 49 \\
\hline $03 \ldots$ & 1656 & 1.79 & .27 & 8.0 & 35 & 26 & 6.9 & 6.9 & 10 & 285 & 256 & 73 \\
\hline $03 \ldots$ & 1814 & 1.79 & .59 & 7.0 & 39 & 32 & 7.0 & 6.6 & 11 & 245 & 107 & 26 \\
\hline $03 \ldots$ & 2249 & 1.79 & .04 & 7.5 & 104 & 97 & 7.4 & 7.0 & 37 & 16 & 14 & 4 \\
\hline $04 \ldots$ & 1002 & 1.79 & .05 & 7.0 & -- & 123 & -- & 7.1 & -- & -- & -- & -- \\
\hline \multicolumn{13}{|l|}{ JUN } \\
\hline $10 \ldots$ & 0806 & 1.48 & .15 & 21.5 & -- & 30 & -- & 6.7 & -- & -- & -- & -. \\
\hline $10 \ldots$ & 0837 & 1.48 & .76 & 21.5 & 33 & 24 & 6.7 & 6.6 & 5.0 & 399 & 227 & 49 \\
\hline $10 \ldots$ & 0940 & 1.48 & .04 & 21.5 & 54 & 47 & 6.9 & 6.7 & 12 & 32 & 12 & 6 \\
\hline DATE & $\begin{array}{c}\text { SOLIDS, } \\
\text { RESIDUE } \\
\text { AT } 180 \\
\text { DEG. C } \\
\text { DIS- } \\
\text { SOLVED } \\
\text { (MG/) } \\
(70300) \\
\end{array}$ & $\begin{array}{c}\text { OXYGEN } \\
\text { DEMAND, } \\
\text { BIO- } \\
\text { CHEMICAL } \\
5 \text { DAY } \\
(M G / L) \\
(00310) \\
\end{array}$ & $\begin{array}{c}\text { OXYGEN } \\
\text { DEMAND, } \\
\text { CHEMICAL } \\
\text { (HIGH LEVEL) } \\
(\text { MG/L) } \\
(00340) \\
\end{array}$ & $\begin{array}{c}\text { NITROGEN, } \\
\text { AMMONIA + } \\
\text { ORGANIC } \\
\text { TOTAL } \\
\text { (MG/L AS N) } \\
(00625)\end{array}$ & $\begin{array}{c}\text { NITROGEN, } \\
\text { NO2+NO3 } \\
\text { DISSOLVED } \\
(M G / L \text { AS N) } \\
(00631) \\
\end{array}$ & $\begin{array}{c}\text { NITROGEN, } \\
\text { AMMONIA } \\
\text { DISSOLVED } \\
(\text { MG } / \text { AS N) } \\
(00608)\end{array}$ & $\begin{array}{l}\text { NITRO- } \\
\text { GEN, } \\
\text { ORGANIC } \\
\text { TOTAL } \\
\text { (MG/L } \\
\text { AS N) } \\
(00605)\end{array}$ & $\begin{array}{c}\text { NITROGEN, } \\
\text { TOTAL } \\
\text { (MG/LASN) } \\
(00600)\end{array}$ & $\begin{array}{c}\text { PHOS- } \\
\text { PHORUS } \\
\text { TOTAL } \\
(M G / L \text { AS P) } \\
(00665) \\
\end{array}$ & $\begin{array}{c}\text { PHOS- } \\
\text { PHORUS } \\
\text { ORTHO, } \\
\text { DISSOLVED } \\
(\mathrm{MG} / \mathrm{L} \text { AS P) } \\
(00671) \\
\end{array}$ & $\begin{array}{c}\text { OIL AND } \\
\text { GREASE, } \\
\text { TOTAL- } \\
\text { RECO' } \\
\text { GRAV'- } \\
\text { METRIC } \\
\text { (MG/L) } \\
(00556) \\
\end{array}$ & \\
\hline \multicolumn{13}{|l|}{ JUL 1997} \\
\hline $22 \ldots$ & 48 & 8.1 & 74 & 1.9 & 1.02 & 0.320 & 1.6 & 2.9 & 0.560 & 0.230 & -- & \\
\hline $23 \ldots$ & 55 & 26 & 280 & 3.4 & .550 & .300 & 3.1 & 4.0 & 2.12 & .180 & -- & \\
\hline $23 \ldots$ & -- & -- & -- & 3.7 & .400 & .190 & 3.5 & 4.1 & 1.75 & .100 & -- & \\
\hline \multicolumn{13}{|l|}{ NOV } \\
\hline $21 \ldots$ & 70 & -- & 98 & 3.8 & .900 & .830 & 3.0 & 4.7 & .470 & .170 & - & \\
\hline $21 \ldots$ & -- & -- & -- & - & -- & -- & -- & -- & -- & -. & -- & \\
\hline $21 \ldots$ & 59 & -- & 74 & 1.5 & .490 & .400 & 1.1 & 2.0 & .320 & .100 & -- & \\
\hline $21 \ldots$ & 50 & -- & 48 & 1.5 & .340 & .370 & 1.1 & 1.8 & .330 & .100 & - & \\
\hline \multicolumn{13}{|c|}{ FEB 1998} \\
\hline $03 \ldots$ & 26 & 5.9 & 39 & .93 & .760 & .040 & .89 & 1.7 & .120 & .030 & - & \\
\hline $03 \ldots$ & -- & -- & -- & -- & -- & -- & -- & -- & -- & -- & 4 & \\
\hline $03 \ldots$ & 24 & 8.7 & 80 & 1.6 & .270 & .080 & 1.5 & 1.9 & .350 & .031 & -- & \\
\hline $03 \ldots$ & 11 & 6.0 & 22 & 1.8 & .150 & .060 & 1.7 & 2.0 & .440 & $<.010$ & -- & \\
\hline $03 \ldots$ & 18 & 3.9 & 43 & .97 & .160 & .110 & .86 & 1.1 & .350 & .024 & - & \\
\hline $03 \ldots$ & 56 & 2.0 & 23 & .36 & .450 & .080 & .28 & .81 & .150 & .029 & - & \\
\hline $04 \ldots$ & -- & -- & - & -- & -- & -- & -- & -- & -- & -- & -- & \\
\hline \multicolumn{13}{|l|}{ JUN } \\
\hline $10 \ldots$ & -- & -- & -- & -- & -- & -- & -- & -- & -- & -- & -- & \\
\hline $10 \ldots$ & 23 & 9.0 & 54 & 2.2 & .440 & .120 & 2.1 & 2.6 & .620 & .070 & -- & \\
\hline $10 \ldots$ & 41 & 7.0 & 28 & 1.2 & .780 & .070 & 1.1 & 2.0 & .260 & .120 & -- & \\
\hline
\end{tabular}


Table 78. Water-quality data at site 40 (CSW03), July 1997 through September 1998-Continued

\begin{tabular}{|c|c|c|c|c|c|c|c|c|c|c|c|}
\hline DATE & $\begin{array}{c}\text { CARBON, } \\
\text { OR- } \\
\text { GANIC } \\
\text { TOTAL } \\
\text { (MG/L } \\
\text { AS C) } \\
(00680 \\
\end{array}$ & $\begin{array}{c}\text { STREPTO- } \\
\text { COCCI } \\
\text { FECAL, } \\
\text { (COLS. PER } \\
100 \mathrm{ML}) \\
(31679) \\
\end{array}$ & $\begin{array}{c}\text { COLIFORM, } \\
\text { FECAL, } \\
0.45 \\
\text { UM-MF } \\
\text { (COLS./ } \\
100 \mathrm{ML}) \\
(31616) \\
\end{array}$ & $\begin{array}{c}\text { ANTIMONY, } \\
\text { TOTAL } \\
\text { (UG/L AS SB) } \\
\text { (01097) }\end{array}$ & $\begin{array}{c}\text { ARSENIC, } \\
\text { TOTAL } \\
\text { (UG/L AS AS) } \\
(01002) \\
\end{array}$ & $\begin{array}{c}\text { CHROMIUM, } \\
\text { TOTAL } \\
\text { RECOVER- } \\
\text { ABLE } \\
\text { (UG/L AS CR) } \\
(01034)\end{array}$ & $\begin{array}{c}\text { COPPER, } \\
\text { TOTAL } \\
\text { RECOVER- } \\
\text { ABLE } \\
\text { (UG/L } \\
\text { AS CU) } \\
(01042) \\
\end{array}$ & $\begin{array}{l}\text { LEAD, } \\
\text { TOTAL } \\
\text { RECOVER- } \\
\text { ABLE } \\
\text { (UG/L } \\
\text { AS PB) } \\
(01051) \\
\end{array}$ & $\begin{array}{c}\text { MERCURY, } \\
\text { TOTAL } \\
\text { RECOVER- } \\
\text { ABLE } \\
\text { (UG/L AS HG) } \\
(71900) \\
\end{array}$ & $\begin{array}{c}\text { NICKEL, } \\
\text { TOTAL } \\
\text { RECOVER- } \\
\text { ABLE } \\
\text { (UG/L AS NI) } \\
(01067)\end{array}$ & $\begin{array}{c}\text { ZINC, } \\
\text { TOTAL } \\
\text { REC.OVER- } \\
\text { ABLE } \\
\text { (UG/'-AS ZN) } \\
(01092)\end{array}$ \\
\hline \multicolumn{12}{|c|}{ ЛUL 1997} \\
\hline $22 \ldots$ & 14 & -- & -- & $<1$ & 1 & 22 & 18 & 15 & -- & 8 & 100 \\
\hline $23 \ldots$ & 28 & -- & - & $<1$ & 4 & 27 & 30 & 74 & -- & 16 & 250 \\
\hline $23 \ldots$ & -- & -- & - & $<1$ & 3 & 29 & 36 & 87 & - & 16 & 220 \\
\hline \multicolumn{12}{|l|}{ NOV } \\
\hline $21 \ldots$ & 18 & -- & - & $<1$ & $<1$ & 3 & 7 & 5 & -- & 9 & 70 \\
\hline $21 \ldots$ & -. & 4700 & KI400 & -- & -- & -- & -- & -- & -- & -- & - \\
\hline $21 \ldots$ & 13 & - & - & $<1$ & $<1$ & 12 & 6 & 6 & -- & 3 & 30 \\
\hline $21 \ldots$ & -- & -- & - & - & -- & -- & -- & -- & -- & -- & -- \\
\hline \multicolumn{12}{|c|}{ FEB 1998} \\
\hline $03 \ldots$ & 11 & -- & -- & $<1$ & $<1$ & 2 & 9 & 14 & -- & 2 & 60 \\
\hline $03 \ldots$ & -- & 29000 & 26000 & -- & -. & -- & -- & -- & -- & -- & -- \\
\hline $03 \ldots$ & 19 & -- & - & $<1$ & 1 & 8 & 21 & 27 & -- & 5 & 110 \\
\hline $03 \ldots$ & 20 & -- & -- & $<1$ & $<1$ & 12 & 27 & 29 & -- & 7 & 110 \\
\hline $03 \ldots$ & 14 & -- & -- & $<1$ & 2 & 7 & 15 & 18 & -- & 2 & 70 \\
\hline $03 \ldots$ & -- & -- & -- & -- & -- & -- & -- & -- & -- & -- & - \\
\hline $04 \ldots$ & -- & 31000 & 3000 & -- & -- & -- & -- & -- & -- & -- & -- \\
\hline \multicolumn{12}{|l|}{ JUN } \\
\hline $10 \ldots$ & -- & -- & -- & - & -- & - & $-\cdot$ & - & -- & -- & -- \\
\hline $10 \ldots$ & -- & -- & -- & $<1$ & 4 & 17 & 17 & 17 & -- & 8 & 70 \\
\hline $10 \ldots$ & -- & -- & -- & - & -- & -- & -- & -- & -- & -- & -- \\
\hline
\end{tabular}

K Results based on colony count outside the acceptable range (nonideal colony count). 
Table 79. Water-quality data at site 41 (CSW02), July 1997 through September 1998

\begin{tabular}{|c|c|c|c|c|c|c|c|c|c|c|c|c|}
\hline DATE & TIME & $\begin{array}{c}\text { RAINFALL } \\
\text { ACCUM } \\
(\text { IN }) \\
(00045)\end{array}$ & $\begin{array}{c}\text { DISCHARGE, } \\
\text { INST. } \\
\text { CUBIC FEET } \\
\text { PER } \\
\text { SECOND } \\
(00061)\end{array}$ & $\begin{array}{l}\text { TEMPER- } \\
\text { ATURE } \\
\text { WATER } \\
\text { (DEG. C) } \\
(00010)\end{array}$ & $\begin{array}{l}\text { SPECIFIC } \\
\text { CONDUC- } \\
\text { TANCE } \\
\text { LAB } \\
\text { (US/CM) } \\
(90095)\end{array}$ & $\begin{array}{l}\text { SPECIFIC } \\
\text { CONDUC- } \\
\text { TANCE } \\
\text { (US/CM) } \\
(00095\end{array}$ & $\begin{array}{l}\text { PH WATER } \\
\text { RAW } \\
\text { LAB } \\
\text { (STAN- } \\
\text { DARD } \\
\text { UNITS) } \\
\text { (00403) }\end{array}$ & $\begin{array}{c}\text { PH WATER } \\
\text { RAW } \\
\text { FIELD } \\
\text { (STAN- } \\
\text { DARD } \\
\text { UNITS) } \\
\text { (00400) }\end{array}$ & $\begin{array}{c}\text { ANC } \\
\text { UNFLTRD } \\
\text { TIT } 4.5 \\
\text { LAB } \\
\text { (MG/L AS } \\
\text { CACO3) } \\
(90410)\end{array}$ & $\begin{array}{l}\text { SEDIMENT. } \\
\text { SUS- } \\
\text { PENDED } \\
\text { (MG/L) } \\
(80154)\end{array}$ & $\begin{array}{l}\text { RESID JE } \\
\text { TOTA'. } \\
\text { AT 1C5 } \\
\text { DEG. C. } \\
\text { SUS. } \\
\text { PENDED } \\
\text { (MG'L) } \\
(0053))\end{array}$ & $\begin{array}{c}\text { RESIDUE } \\
\text { VOLATILE, } \\
\text { SUS- } \\
\text { PENDED } \\
\text { (MG/L) } \\
(00535)\end{array}$ \\
\hline \multicolumn{13}{|c|}{ JUL 1997} \\
\hline $22 \ldots$ & 2302 & 4.40 & 24 & 24.0 & 82 & 77 & 6.3 & 7.0 & 11 & 9370 & 9800 & 1090 \\
\hline $22 \ldots$ & 2326 & 4.40 & 27 & 28.0 & 22 & 18 & 6.4 & 7.1 & 3.0 & 797 & 708 & 88 \\
\hline $22 \ldots$ & 2340 & 4.40 & 50 & 27.0 & 18 & 17 & 6.5 & 7.0 & 4.0 & 365 & 282 & 48 \\
\hline $23 \ldots$ & 0453 & 4.40 & .12 & 26.5 & 62 & 42 & 6.7 & 6.8 & 6.0 & 28 & 21 & 3 \\
\hline $23 \ldots$ & 0756 & 4.40 & 253 & 24.0 & 30 & 29 & 6.7 & 7.2 & 6.0 & 392 & 178 & 30 \\
\hline \multicolumn{13}{|l|}{ NOV } \\
\hline $12 \ldots$ & 1824 & 1.12 & .36 & 14.0 & 165 & 159 & 7.2 & 6.8 & 49 & 26 & 46 & 6 \\
\hline $12 \ldots$ & 1834 & 1.12 & .30 & 14.0 & 155 & 155 & 7.1 & 7.1 & -- & -- & -- & -- \\
\hline $12 \ldots$ & 1958 & 1.12 & .40 & 14.0 & 69 & 67 & 6.9 & 7.1 & 12 & 31 & 23 & 2 \\
\hline $12 \ldots$ & 2224 & 1.12 & .76 & 13.5 & 49 & 48 & 6.9 & 7.0 & 5.0 & 13 & 10 & 1 \\
\hline $13 \ldots$ & 0032 & 1.12 & .21 & 12.0 & 35 & 31 & 7.0 & 6.8 & 8.0 & - & 27 & 3 \\
\hline $13 \ldots$ & 0822 & 1.12 & .21 & 11.0 & -- & - & -- & -- & -- & -- & -. & -- \\
\hline \multicolumn{13}{|c|}{ FEB 1998} \\
\hline $03 \ldots$ & 0346 & 1.23 & .60 & 11.0 & 117 & 112 & 7.0 & 6.9 & 29 & 9 & 15 & 4 \\
\hline $03 \ldots$ & 0840 & 1.23 & 1.1 & 9.5 & 69 & 62 & 6.9 & 6.9 & 12 & 37 & 39 & 12 \\
\hline $03 \ldots$ & 1438 & 1.23 & .22 & 9.0 & 48 & 43 & 7.0 & 7.0 & 11 & 24 & 16 & 6 \\
\hline $03 \ldots$ & 1659 & 1.23 & 7.0 & 7.5 & 28 & 22 & 6.9 & 7.0 & 7.0 & 157 & 150 & 39 \\
\hline $03 \ldots$ & 2249 & 1.23 & .46 & 8.5 & 85 & 80 & 7.1 & 6.9 & 20 & 10 & 8 & 2 \\
\hline $04 \ldots$ & 0738 & 1.23 & .44 & 9.0 & -- & 107 & -- & 6.8 & -- & -- & -- & -- \\
\hline \multicolumn{13}{|l|}{ MAY } \\
\hline $20 \ldots$ & 0845 & .00 & .09 & 17.0 & 167 & 152 & 6.9 & 7.0 & 55 & 11 & 8 & 3 \\
\hline \multicolumn{13}{|l|}{ JUN } \\
\hline $10 \ldots$ & 0756 & 1.53 & 81 & 18.0 & 90 & 78 & 7.0 & 6.7 & 26 & 686 & 218 & 55 \\
\hline $10 \ldots$ & 0757 & 1.53 & 140 & 19.0 & -- & 42 & -- & 6.8 & -- & -- & -- & -- \\
\hline $10 \ldots$ & 0800 & 1.53 & 150 & 21.0 & -- & 19 & -- & 6.7 & -- & -- & $\cdots$ & -- \\
\hline $10 \ldots$ & 0804 & 1.53 & 67 & 21.0 & -- & -- & -- & - & -- & -- & - & -- \\
\hline $10 \ldots$ & 0815 & 1.53 & 4.3 & 21.0 & -- & -- & -- & - & -- & -- & -. & -- \\
\hline $10 \ldots$ & 0913 & 1.53 & 83 & 20.5 & 26 & 21 & 6.7 & 6.9 & 5.0 & 297 & 104 & 24 \\
\hline $10 \ldots$ & 1023 & 1.53 & .84 & 21.0 & 79 & 73 & 6.9 & 6.8 & 17 & 61 & 22 & 8 \\
\hline DATE & $\begin{array}{c}\text { SOLIDS, } \\
\text { RESIDUE } \\
\text { AT } 180 \\
\text { DEG. C } \\
\text { DIS- } \\
\text { SOLVED } \\
(\mathrm{MG} / \mathrm{L}) \\
(70300) \\
\end{array}$ & $\begin{array}{c}\text { OXYGEN } \\
\text { DEMAND, } \\
\text { BIO- } \\
\text { CHEMICAL } \\
5 \text { DAY } \\
\text { (MG/L) } \\
(00310) \\
\end{array}$ & $\begin{array}{c}\text { OXYGEN } \\
\text { DEMAND, } \\
\text { CHEMICAL } \\
\text { (HIGH LEVEL) } \\
(\mathrm{MG} / \mathrm{L}) \\
(00340) \\
\end{array}$ & $\begin{array}{c}\text { NITROGEN, } \\
\text { AMMONIA + } \\
\text { ORGANIC } \\
\text { TOTAL } \\
\text { (MG/L AS N) } \\
(00625)\end{array}$ & $\begin{array}{c}\text { NITROGEN, } \\
\text { NO2+NO3 } \\
\text { DISSOLVED } \\
(\mathrm{MG} / \mathrm{L} \text { AS N) } \\
(00631)\end{array}$ & $\begin{array}{c}\text { NITROGEN, } \\
\text { AMMONIA } \\
\text { DISSOLVED } \\
(M G / L \text { AS N) } \\
(00608) \\
\end{array}$ & $\begin{array}{l}\text { NITRO- } \\
\text { GEN, } \\
\text { ORGANIC } \\
\text { TOTAL } \\
\text { (MG/L } \\
\text { AS N) } \\
(00605) \\
\end{array}$ & $\begin{array}{c}\text { NITROGEN, } \\
\text { TOTAL } \\
(\mathrm{MG} / \mathrm{LAS} \text { N) } \\
(00600)\end{array}$ & $\begin{array}{c}\text { PHOS- } \\
\text { PHORUS } \\
\text { TOTAL } \\
\text { (MG/ AS P) } \\
(00665)\end{array}$ & $\begin{array}{c}\text { PHOS- } \\
\text { PHORUS } \\
\text { ORTHO, } \\
\text { DISSOLVED } \\
\text { (MG/L AS P) } \\
(00671) \\
\end{array}$ & $\begin{array}{c}\text { OIL AN'D } \\
\text { GREASE, } \\
\text { TOTA': } \\
\text { RECO' } ' \\
\text { GRAVI- } \\
\text { METR'C } \\
\text { (MG/ ) } \\
\text { (00556) }\end{array}$ & \\
\hline \multicolumn{13}{|l|}{ JUL 1997} \\
\hline $22 \ldots$ & 77 & 30 & 68 & 2.7 & 1.08 & 0.930 & 1.8 & 3.8 & 1.35 & $<0.010$ & -- & \\
\hline $22 \ldots$ & 18 & 5.8 & 39 & 1.1 & .230 & .200 & .90 & 1.3 & .430 & .047 & -- & \\
\hline $22 \ldots$ & 16 & 5.1 & 31 & .85 & .220 & .130 & .72 & 1.1 & .290 & .090 & - & \\
\hline $23 \ldots$ & 38 & 5.6 & 18 & .76 & .600 & .260 & .50 & 1.4 & .070 & .070 & - & \\
\hline $23 \ldots$ & 30 & 3.7 & 34 & 1.2 & .530 & .120 & 1.1 & 1.7 & .410 & .160 & -- & \\
\hline \multicolumn{13}{|l|}{ NOV } \\
\hline $12 \ldots$ & 136 & 15 & 37 & 16 & 1.30 & .430 & 16 & 17 & .370 & .190 & - & \\
\hline $12 \ldots$ & -- & -- & -- & -- & -- & -- & -- & -- & -- & -- & 3 & \\
\hline $12 \ldots$ & 63 & 20 & 54 & 8.4 & .590 & .600 & 7.8 & 9.0 & .470 & .260 & - & \\
\hline $12 \ldots$ & 42 & 14 & 34 & 1.1 & .410 & .270 & .83 & 1.5 & .260 & .160 & -- & \\
\hline $13 \ldots$ & -- & 10 & 22 & .52 & .220 & .120 & .40 & .74 & .200 & .100 & -- & \\
\hline $13 \ldots$ & -- & -- & -- & -- & -- & -- & -- & -- & -- & -- & - & \\
\hline \multicolumn{13}{|c|}{ FEB 1998} \\
\hline $03 \ldots$ & 65 & 4.7 & 18 & 1.1 & 1.42 & .290 & .81 & 2.5 & .110 & .028 & - & \\
\hline $03 \ldots$ & 30 & 7.0 & 25 & .80 & .750 & .160 & .64 & 1.5 & .110 & .037 & 5 & \\
\hline $03 \ldots$ & 21 & 3.2 & 16 & .51 & .370 & .060 & .45 & .88 & .100 & .013 & -. & \\
\hline $03 \ldots$ & 13 & 5.4 & 37 & 1.2 & .110 & .080 & 1.1 & 1.3 & .340 & .029 & -- & \\
\hline $03 \ldots$ & 47 & 2.1 & 18 & .74 & 1.05 & .050 & .69 & 1.8 & .160 & .031 & - & \\
\hline $04 \ldots$ & -- & -- & -- & -- & -- & -- & -- & -- & -- & -- & - & \\
\hline \multicolumn{13}{|l|}{ MAY } \\
\hline $20 \ldots$ & \multirow{2}{*}{\multicolumn{6}{|c|}{ JUN }} & .33 & 1.5 & .080 & .025 & 2 & \\
\hline $10 \ldots$ & 62 & 18 & 88 & 4.3 & .730 & 330 & 4.0 & 5.0 & .760 & 050 & $\ldots$ & \\
\hline $10 \ldots$ & $\begin{array}{l}02 \\
--\end{array}$ & $\begin{array}{r}10 \\
--\end{array}$ & $\begin{array}{l}00 \\
--\end{array}$ & $\begin{array}{l}4.5 \\
--\end{array}$ & $-{ }_{-}^{.750}$ & ${ }_{--}^{.500}$ & $\begin{array}{l}4.0 \\
--\end{array}$ & $\begin{array}{l}5.0 \\
--\end{array}$ &.- & $-{ }_{-}^{.0 J 0}$ & 2 & \\
\hline $10 \ldots$ & - & -- & -- & -- & -- & -- & -- & -- & -- & -- & - & \\
\hline $10 \ldots$ & -- & -- & -- & -- & -- & -- & -- & -- & -- & -- & -- & \\
\hline $10 \ldots$ & -- & -- & -- & -- & -- & -- & -- & -- & -- & -- & - & \\
\hline $10 \ldots$ & 17 & 6.3 & 27 & 1.6 & .320 & .120 & 1.5 & 1.9 & .490 & .080 & -- & \\
\hline $10 \ldots$ & 59 & 7.3 & 30 & 1.4 & 1.12 & .070 & 1.3 & 2.5 & .400 & .180 & -- & \\
\hline
\end{tabular}


Table 79. Water-quality data at site 41 (CSW02), July 1997 through September 1998-Continued

\begin{tabular}{|c|c|c|c|c|c|c|c|c|c|c|c|}
\hline DATE & $\begin{array}{c}\text { CARBON, } \\
\text { OR- } \\
\text { GANIC } \\
\text { TOTAL } \\
\text { (MG/L } \\
\text { AS C) } \\
(00680 \\
\end{array}$ & $\begin{array}{c}\text { STREPTO- } \\
\text { COCCI } \\
\text { FECAL, } \\
\text { (COLS. PER } \\
100 \mathrm{ML}) \\
(31679) \\
\end{array}$ & $\begin{array}{c}\text { COLIFORM, } \\
\text { FECAL, } \\
0.45 \\
\text { UM-MF } \\
\text { (COLS } / \\
100 \mathrm{ML}) \\
(31616) \\
\end{array}$ & $\begin{array}{c}\text { ANTIMONY, } \\
\text { TOTAL } \\
\text { (UG/L AS SB) } \\
\text { (01097) }\end{array}$ & $\begin{array}{c}\text { ARSENIC, } \\
\text { TOTAL } \\
\text { (UG/ AS AS) } \\
(01002) \\
\end{array}$ & $\begin{array}{c}\text { CHROMIUM, } \\
\text { TOTAL } \\
\text { RECOVER- } \\
\text { ABLE } \\
\text { (UGL AS CR) } \\
(01034) \\
\end{array}$ & $\begin{array}{c}\text { COPPER, } \\
\text { TOTAL } \\
\text { RECOVER. } \\
\text { ABLE } \\
\text { (UG/L } \\
\text { AS CU) } \\
(01042) \\
\end{array}$ & $\begin{array}{c}\text { LEAD, } \\
\text { TOTAL } \\
\text { RECOVER- } \\
\text { ABLE } \\
\text { (UG/ } \\
\text { AS PB) } \\
(01051) \\
\end{array}$ & $\begin{array}{c}\text { MERCURY, } \\
\text { TOTAL } \\
\text { RECOVER- } \\
\text { ABLE } \\
\text { (UGL AS HG) } \\
\text { (71900) } \\
\end{array}$ & $\begin{array}{c}\text { NICKEL, } \\
\text { TOTAL } \\
\text { RECOVER- } \\
\text { ABLE } \\
\text { (UG/ AS NI) } \\
\text { (01067) }\end{array}$ & $\begin{array}{c}\text { ZINC, } \\
\text { TOTAL } \\
\text { RECOVER- } \\
\text { ABLE } \\
\text { (UG/ AS ZN) } \\
\text { (C1092) } \\
\end{array}$ \\
\hline \multicolumn{12}{|l|}{ JUL 1997} \\
\hline $22 \ldots$ & 38 & -- & -- & $<1$ & 17 & 190 & 270 & 190 & -- & 52 & 860 \\
\hline $22 \ldots$ & 10 & -- & -- & $<1$ & 2 & 19 & 22 & 32 & -- & 7 & 150 \\
\hline $22 \ldots$ & 11 & -- & -- & $<1$ & $<1$ & 15 & 18 & 11 & -- & 10 & 120 \\
\hline $23 \ldots$ & -- & -- & -- & -- & -- & -- & -- & -- & -- & -- & -- \\
\hline $23 \ldots$ & 14 & -- & -- & $<1$ & 1 & 12 & 16 & 24 & -- & 6 & 150 \\
\hline \multicolumn{12}{|l|}{ NOV } \\
\hline $12 \ldots$ & 9.9 & -- & -- & $<1$ & $<1$ & 5 & 5 & 7 & -- & 3 & 100 \\
\hline $12 \ldots$ & -- & 2100 & 29000 & -. & -- & -- & -- & -- & -- & -- & -- \\
\hline $12 \ldots$ & 12 & -- & - & $<1$ & $<1$ & 7 & 22 & 13 & -- & 5 & 140 \\
\hline $12 \ldots$ & 7.5 & -- & -- & $<1$ & $<1$ & 6 & 13 & 6 & -- & 3 & 100 \\
\hline $13 \ldots$ & -- & -- & -- & -- & -- & -- & -- & -- & -- & -- & -- \\
\hline $13 \ldots$ & -- & 7500 & 2300 & -- & -- & -- & -- & -- & -- & -- & -- \\
\hline \multicolumn{12}{|l|}{ FEB 1998} \\
\hline $03 \ldots$ & 6.0 & -. & -- & $<1$ & $<1$ & 4 & 14 & 8 & -- & 1 & 80 \\
\hline $03 \ldots$ & 8.5 & 22000 & 3000 & $<1$ & $<1$ & 2 & 28 & 15 & -- & 1 & 100 \\
\hline $03 \ldots$ & -- & -- & -- & -- & -- & -- & -- & -- & -- & -- & -- \\
\hline $03 \ldots$ & 17 & -- & -- & $<1$ & 1 & 8 & 19 & 26 & -- & 2 & 100 \\
\hline $03 \ldots$ & -- & -- & -- & -- & -- & -- & -- & -- & -. & -- & -- \\
\hline $04 \ldots$ & -- & 3100 & 2300 & -- & -- & -- & -- & -- & -- & -- & -- \\
\hline \multicolumn{12}{|l|}{ MAY } \\
\hline $20 \ldots$ & -- & K720 & K630 & $<1$ & $<1$ & 4 & 4 & 2 & -- & $<1$ & 70 \\
\hline \multicolumn{12}{|l|}{ JUN } \\
\hline $10 \ldots$ & -- & -- & -- & $<1$ & 6 & 22 & 37 & 47 & -- & 8 & 250 \\
\hline $10 \ldots$ & 33 & 75000 & K85000 & -- & -. & -- & -- & -- & -- & -- & -- \\
\hline $10 \ldots$ & -- & -- & -- & -- & -- & -- & -- & -- & -- & -- & -- \\
\hline $10 \ldots$ & -- & 99000 & K25000 & -- & -- & -- & -- & -- & -- & -- & -- \\
\hline $10 \ldots$ & -- & K115000 & 220000 & -. & -- & -- & -- & -- & -- & -- & -- \\
\hline $10 \ldots$ & -- & -- & -- & -- & -- & -- & -- & -- & -- & -- & -- \\
\hline $10 \ldots$ & -- & -- & -- & -- & -- & -- & -- & -- & -. & -- & -- \\
\hline
\end{tabular}

K Results based on colony count outside the acceptable range (nonideal colony count). 
Table 80. Water-quality data at site 42 (CSW04), July 1997 through September 1998

\begin{tabular}{|c|c|c|c|c|c|c|c|c|c|c|c|c|}
\hline DATE & TIME & $\begin{array}{l}\text { RAINFALL } \\
\text { ACCUM } \\
(\text { IN) } \\
(00045) \\
\end{array}$ & $\begin{array}{l}\text { DISCHARGE, } \\
\text { INST. } \\
\text { CUBIC FEET } \\
\text { PER } \\
\text { SECOND } \\
(00061) \\
\end{array}$ & $\begin{array}{l}\text { TEMPER- } \\
\text { ATURE } \\
\text { WATER } \\
\text { (DEG. C) } \\
(00010) \\
\end{array}$ & $\begin{array}{c}\text { SPECIFIC } \\
\text { CONDUC- } \\
\text { TANCE } \\
\text { LAB } \\
(\text { US/CM) } \\
(90095) \\
\end{array}$ & $\begin{array}{c}\text { SPECIFIC } \\
\text { CONDUC- } \\
\text { TANCE } \\
\text { (US/CM) } \\
\text { (00095 } \\
\end{array}$ & $\begin{array}{l}\text { PH WATER } \\
\text { RAW } \\
\text { LAB } \\
\text { (STAN- } \\
\text { DARD } \\
\text { UNITS) } \\
\text { (00403) } \\
\end{array}$ & $\begin{array}{l}\text { PHWATER } \\
\text { RAW } \\
\text { FIELD } \\
\text { (STAN- } \\
\text { DARD } \\
\text { UNITS) } \\
(00400) \\
\end{array}$ & $\begin{array}{c}\text { ANC } \\
\text { UNFLTRD } \\
\text { TIT } 4.5 \\
\text { LAB } \\
\text { (MG/L AS } \\
\text { CACO3) } \\
(90410) \\
\end{array}$ & $\begin{array}{l}\text { SEDIMENT, } \\
\text { SUS- } \\
\text { PENDED } \\
\text { (MG/L) } \\
(80154) \\
\end{array}$ & $\begin{array}{l}\text { RESIDUE } \\
\text { TOTAL } \\
\text { AT } 175 \\
\text { DEG C, } \\
\text { SUS- } \\
\text { PENL FD } \\
\text { (MG'L) } \\
\text { (005:30) } \\
\end{array}$ & $\begin{array}{c}\text { RESIDUE } \\
\text { VOLATILE, } \\
\text { SUS- } \\
\text { PENDED } \\
\text { (MG/L) } \\
(00535) \\
\end{array}$ \\
\hline \multicolumn{13}{|l|}{ JUL 1997} \\
\hline $23 \ldots$ & 0734 & 4.40 & 229 & 23.0 & 27 & 23 & 6.8 & 7.3 & 6.0 & 1070 & 672 & 90 \\
\hline \multicolumn{13}{|l|}{ NOV } \\
\hline $12 \ldots$ & 1828 & 1.12 & .36 & 10.5 & 213 & 207 & 7.4 & 7.0 & 47 & 48 & 45 & 8 \\
\hline $12 \ldots$ & 1904 & 1.12 & .56 & 11.0 & -- & 130 & -- & 7.1 & -- & -- & -- & -- \\
\hline $12 \ldots$ & 2302 & 1.12 & 1.3 & 10.5 & 57 & 55 & 7.1 & 6.9 & 12 & 54 & 42 & 7 \\
\hline $13 \ldots$ & 0036 & 1.12 & .70 & 10.5 & 59 & 55 & 7.1 & 7.0 & 13 & 23 & 13 & 2 \\
\hline $13 \ldots$ & 0928 & 1.12 & .54 & 9.5 & -- & -- & -- & - & -- & -- & -- & -- \\
\hline \multicolumn{13}{|l|}{ FEB 1998} \\
\hline $03 \ldots$ & 0802 & 1.23 & .56 & 7.5 & 111 & 106 & 7.3 & 7.0 & 27 & 27 & 25 & 8 \\
\hline $03 \ldots$ & 0816 & 1.23 & .83 & 7.5 & -- & 98 & -- & 7.2 & - & -- & -. & -- \\
\hline $03 \ldots$ & 0921 & 1.23 & 2.1 & 7.5 & 67 & 62 & 7.3 & 7.0 & 12 & 56 & 57 & 13 \\
\hline $03 \ldots$ & 1704 & 1.23 & 20 & 7.5 & 35 & 32 & 7.0 & 7.0 & 9.0 & 240 & 168 & 30 \\
\hline $03 \ldots$ & 2303 & 1.23 & 1.4 & 7.5 & 68 & 66 & 7.1 & 7.0 & 21 & 26 & 15 & 4 \\
\hline $04 \ldots$ & 0919 & 1.23 & 1.5 & 7.0 & -- & 62 & -- & 7.0 & - & -- & -- & -- \\
\hline \multicolumn{13}{|l|}{ MAY } \\
\hline $20 \ldots$ & 0815 & .00 & .05 & 19.5 & 308 & 283 & 7.5 & 7.3 & 115 & 4 & 9 & 2 \\
\hline \multicolumn{13}{|l|}{ JUN } \\
\hline $10 \ldots$ & 0758 & 1.53 & 50 & 20.0 & 34 & 27 & 6.7 & 6.7 & 6.0 & 1490 & $2 \Sigma 1$ & 44 \\
\hline $10 \ldots$ & 0802 & 1.53 & 75 & 20.5 & -- & 22 & -- & 6.8 & -- & -- & -- & -- \\
\hline $10 \ldots$ & 0824 & 1.53 & 16 & 20.0 & -- & 65 & -- & 6.9 & - & - & -- & -- \\
\hline $10 \ldots$ & 0837 & 1.53 & 100 & 20.0 & 50 & 44 & 6.6 & 6.6 & 6.0 & 1050 & 357 & 54 \\
\hline $10 \ldots$ & 0840 & 1.53 & 95 & 20.0 & -- & -- & -- & -- & - & -- & -- & -- \\
\hline $10 \ldots$ & 1101 & 1.53 & 1.1 & 21.0 & 104 & 99 & 7.0 & 6.9 & 16 & 49 & 18 & 8 \\
\hline DATE & $\begin{array}{c}\text { SOLIDS, } \\
\text { RESIDUE } \\
\text { AT } 180 \\
\text { DEG. C } \\
\text { DIS- } \\
\text { SOLVED } \\
\text { (MG/L) } \\
(70300) \\
\end{array}$ & $\begin{array}{c}\text { OXYGEN } \\
\text { DEMAND, } \\
\text { BIO- } \\
\text { CHEMICAL } \\
5 \text { DAY } \\
(\text { MG/L) } \\
(00310) \\
\end{array}$ & $\begin{array}{c}\text { OXYGEN } \\
\text { DEMAND, } \\
\text { CHEMICAL } \\
\text { (HIGH LEVEL) } \\
(\mathrm{MG} / \mathrm{L}) \\
(00340) \\
\end{array}$ & $\begin{array}{c}\text { NITROGEN, } \\
\text { AMMONIA + } \\
\text { ORGANIC } \\
\text { TOTAL } \\
\text { (MG/L AS N) } \\
(00625) \\
\end{array}$ & $\begin{array}{c}\text { NITROGEN, } \\
\text { NO2+NO3 } \\
\text { DISSOLVED } \\
\text { (MG/L AS N) } \\
(00631) \\
\end{array}$ & $\begin{array}{c}\text { NITROGEN, } \\
\text { AMMONIA } \\
\text { DISSOLVED } \\
\text { (MG/L AS N) } \\
(00608) \\
\end{array}$ & $\begin{array}{l}\text { NITRO- } \\
\text { GEN, } \\
\text { ORGANIC } \\
\text { TOTAL } \\
\text { (MG/L } \\
\text { AS N) } \\
(00605)\end{array}$ & $\begin{array}{c}\text { NITROGEN, } \\
\text { TOTAL } \\
\text { (MG/LASN) } \\
(00600)\end{array}$ & $\begin{array}{c}\text { PHOS- } \\
\text { PHORUS } \\
\text { TOTAL } \\
(\mathrm{MG} / \mathrm{L} \text { AS P) } \\
(00665)\end{array}$ & $\begin{array}{l}\text { PHOS- } \\
\text { PHORUS } \\
\text { ORTHO, } \\
\text { DISSOLVED } \\
\text { (MG/L AS P) } \\
\text { (00671) }\end{array}$ & $\begin{array}{c}\text { OILANID } \\
\text { GREA GE, } \\
\text { TOT''- } \\
\text { RECOV. } \\
\text { GRA'I- } \\
\text { METRIC } \\
\text { (MG'L) } \\
\text { (00556) }\end{array}$ & \\
\hline \multicolumn{13}{|l|}{ JUL 1997} \\
\hline $23 \ldots$ & 23 & 5.3 & 19 & 1.6 & 0.290 & 0.170 & 1.4 & 1.9 & 0.610 & 0.120 & -- & \\
\hline \multicolumn{13}{|l|}{ NOV } \\
\hline $12 \ldots$ & 172 & 31 & 98 & 3.0 & 1.34 & 1.00 & 2.0 & 4.3 & .490 & .280 & -- & \\
\hline $12 \ldots$ & -- & -- & -- & -- & -. & -- & -- & -- & -- & -- & 2 & \\
\hline $12 \ldots$ & 42 & 7.5 & 25 & .78 & .400 & .130 & .65 & 1.2 & .190 & .070 & -- & \\
\hline $13 \ldots$ & 47 & 6.8 & 18 & .50 & .370 & .070 & .43 & .87 & .170 & .080 & - & \\
\hline $13 \ldots$ & -- & -- & -- & -- & -- & -- & -- & -- & -- & -- & -- & \\
\hline \multicolumn{13}{|l|}{ FEB 1998} \\
\hline $03 \ldots$ & 53 & 6.3 & 31 & .81 & .840 & .170 & .64 & 1.6 & .090 & .040 & -- & \\
\hline $03 \ldots$ & -- & -- & -- & -- & -- & -- & -- & -- & -- & -- & 5 & \\
\hline $03 \ldots$ & 30 & 6.2 & 28 & 1.0 & .510 & .130 & .87 & 1.5 & .130 & .048 & - & \\
\hline $03 \ldots$ & 33 & 4.2 & 39 & 1.1 & .110 & .090 & 1.0 & 1.2 & .330 & .031 & -- & \\
\hline $03 \ldots$ & 54 & 2.9 & 28 & .92 & .290 & .100 & .82 & 1.2 & .210 & .044 & -- & \\
\hline $04 \ldots$ & -- & -- & -- & -- & -- & -- & -- & -- & -- & -- & - & \\
\hline \multicolumn{13}{|l|}{ MAY } \\
\hline $20 \ldots$ & 202 & 2.2 & $<5$ & .48 & .310 & $<.015$ & .48 & .79 & .090 & .023 & 2 & \\
\hline \multicolumn{13}{|l|}{ JUN } \\
\hline $10 \ldots$ & 19 & 12 & 46 & 2.4 & .470 & .280 & 2.1 & 2.9 & .750 & .050 & -- & \\
\hline $10 \ldots$ & -- & -- & -- & -- & -- & -- & -- & -- & -- & -- & - & \\
\hline $10 \ldots$ & -- & - & -- & -- & -- & -- & -- & -- & -- & -- & $<1$ & \\
\hline $10 \ldots$ & 35 & 7.5 & 40 & 2.6 & .540 & .340 & 2.3 & 3.1 & 1.29 & .620 & -- & \\
\hline $10 \ldots$ & -- & -- & -- & -- & -- & -- & -- & -- & - & -- & -- & \\
\hline $10 \ldots$ & 80 & 7.1 & 36 & 2.1 & .850 & .500 & 1.6 & 3.0 & 1.35 & .630 & -- & \\
\hline
\end{tabular}


Table 80. Water-quality data at site 42 (CSW04), July 1997 through September 1998-Continued

\begin{tabular}{|c|c|c|c|c|c|c|c|c|c|c|c|}
\hline DATE & $\begin{array}{c}\text { CARBON, } \\
\text { OR- } \\
\text { GANIC } \\
\text { TOTAL } \\
\text { (MG/ } \\
\text { AS C) } \\
\text { (00680 } \\
\end{array}$ & $\begin{array}{c}\text { STREPTO- } \\
\text { COCCI } \\
\text { FECAL, } \\
\text { (COLS. PER } \\
100 \mathrm{ML}) \\
(31679) \\
\end{array}$ & $\begin{array}{l}\text { COLIFORM, } \\
\text { FECAL, } \\
\text { O.45 } \\
\text { UM-MF } \\
\text { (COLSJ/ } \\
100 \mathrm{ML}) \\
(31616) \\
\end{array}$ & $\begin{array}{c}\text { ANTIMONY, } \\
\text { TOTAL } \\
\text { (UG/L AS SB) } \\
(01097)\end{array}$ & $\begin{array}{c}\text { ARSENIC, } \\
\text { TOTAL } \\
\text { (UG/L AS AS) } \\
(01002)\end{array}$ & $\begin{array}{c}\text { CHROMIUM, } \\
\text { TOTAL } \\
\text { RECOVER- } \\
\text { ABLE } \\
\text { (UG/L AS CA) } \\
(01034) \\
\end{array}$ & $\begin{array}{l}\text { COPPER, } \\
\text { TOTAL } \\
\text { AECOVER- } \\
\text { ABLE } \\
\text { (UG/L } \\
\text { AS CU) } \\
\text { (01042) }\end{array}$ & $\begin{array}{c}\text { LEAD, } \\
\text { TOTAL } \\
\text { RECOVER- } \\
\text { ABLE } \\
\text { (UG/L } \\
\text { AS PB) } \\
(01051)\end{array}$ & $\begin{array}{c}\text { MERCURY, } \\
\text { TOTAL } \\
\text { RECOVER- } \\
\text { ABLE } \\
\text { (UG/L AS HG) } \\
(71900)\end{array}$ & $\begin{array}{c}\text { NICKEL, } \\
\text { TOTAL } \\
\text { RECOVER- } \\
\text { ABLE } \\
\text { (UG/L AS NI) } \\
(01067)\end{array}$ & $\begin{array}{c}\text { ZINC, } \\
\text { TNTAL } \\
\text { REI COVER- } \\
\text { ABLE } \\
\text { (UG/L AS ZN) } \\
\text { (01092) }\end{array}$ \\
\hline \multicolumn{12}{|c|}{ JUL 1997} \\
\hline $23 \ldots$ & 16 & - & - & $<1$ & 2 & 26 & 40 & 20 & -- & 9 & 140 \\
\hline \multicolumn{12}{|l|}{ NOV } \\
\hline $12 \ldots$ & 24 & -- & -- & 3 & $<1$ & 7 & 19 & 9 & -- & 8 & 140 \\
\hline $12 \ldots$ & -- & 2000 & $\mathrm{~K} 110000$ & -- & -- & -- & -- & -- & -- & -- & -- \\
\hline $12 \ldots$ & 6.8 & -- & - & $<1$ & 3 & 7 & 26 & 11 & -- & 2 & 100 \\
\hline $13 \ldots$ & - & -- & -- & -- & -- & -- & -- & -- & -- & -- & -- \\
\hline $13 \ldots$ & -- & 7200 & K5000 & -- & -- & -- & -- & -- & -- & -- & -- \\
\hline \multicolumn{12}{|c|}{ FEB 1998} \\
\hline $03 \ldots$ & 8.8 & -- & -- & $<1$ & $<1$ & 1 & 18 & 10 & -- & 2 & 70 \\
\hline $03 \ldots$ & -- & K142000 & K580 & -- & - & -- & -- & - & -- & -- & -- \\
\hline $03 \ldots$ & 9.7 & - & -- & $<1$ & $<1$ & 4 & 40 & 13 & -- & 1 & 140 \\
\hline $03 \ldots$ & 14 & -- & -- & $<1$ & 2 & 14 & 36 & 19 & -- & 3 & 90 \\
\hline $03 \ldots$ & - & -- & -- & -- & - & -- & -- & -- & -- & -- & -- \\
\hline $04 \ldots$ & -- & 9800 & K810 & -- & -- & -- & -- & -- & -- & -- & -- \\
\hline \multicolumn{12}{|l|}{ MAY } \\
\hline $20 \ldots$ & - & 3400 & 2400 & 1 & $<1$ & 3 & 17 & $<1$ & -- & $<1$ & 20 \\
\hline \multicolumn{12}{|l|}{ JUN } \\
\hline $10 \ldots$ & -- & -- & -- & $<1$ & 5 & 31 & 39 & 130 & -- & 11 & 180 \\
\hline $10 \ldots$ & -- & -- & -- & -- & -- & -- & -- & -- & -- & -- & - \\
\hline $10 \ldots$ & 17 & 210000 & K73000 & -- & -- & -- & -- & - & -- & -- & -- \\
\hline $10 \ldots$ & -- & -- & -- & $<1$ & 28 & 35 & 59 & 16 & -- & 11 & 100 \\
\hline $10 \ldots$ & 19 & 100000 & К 67000 & -- & -- & -. & -- & -- & -- & -- & -. \\
\hline $10 \ldots$ & -- & -- & -- & -- & -- & -. & -- & -- & -- & -- & -- \\
\hline
\end{tabular}

K Results based on colony count outside the acceptable range (nonideal colony count). 
Table 81. Water-quality data at site 43 (CSW07), July 1997 through September 1998

\begin{tabular}{|c|c|c|c|c|c|c|c|c|c|c|c|c|}
\hline DATE & TIME & $\begin{array}{l}\text { RAINFALL } \\
\text { ACCUM } \\
(\text { IN) } \\
(00045)\end{array}$ & $\begin{array}{c}\text { DISCHARGE, } \\
\text { INST. } \\
\text { CUBIC FEET } \\
\text { PER } \\
\text { SECOND } \\
(00061)\end{array}$ & $\begin{array}{l}\text { TEMPER- } \\
\text { ATURE } \\
\text { WATER } \\
\text { (DEG. C) } \\
\text { (00010) }\end{array}$ & $\begin{array}{c}\text { SPECIFIC } \\
\text { CONDUC- } \\
\text { TANCE } \\
\text { LAB } \\
(\text { US/CM) } \\
(90095)\end{array}$ & $\begin{array}{l}\text { SPECIFIC } \\
\text { CONDUC- } \\
\text { TANCE } \\
\text { (US/CM) } \\
(00095\end{array}$ & $\begin{array}{l}\text { PH WATER } \\
\text { RAW } \\
\text { LAB } \\
\text { (STAN- } \\
\text { DARD } \\
\text { UNITS) } \\
\text { (00403) }\end{array}$ & $\begin{array}{l}\text { PHWATER } \\
\text { RAW } \\
\text { FIELD } \\
\text { (STAN- } \\
\text { DARD } \\
\text { UNITS) } \\
\text { (00400) }\end{array}$ & $\begin{array}{c}\text { ANC } \\
\text { UNFLTRD } \\
\text { TIT } 4.5 \\
\text { LAB } \\
\text { (MG/L AS } \\
\text { CACO3) } \\
(90410)\end{array}$ & $\begin{array}{l}\text { SEDIMENT, } \\
\text { SUS- } \\
\text { PENDED } \\
\text { (MG/L) } \\
(80154)\end{array}$ & $\begin{array}{l}\text { RESICIJE } \\
\text { TOT'' } \\
\text { AT } 175 \\
\text { DEG. C, } \\
\text { SUS- } \\
\text { PENEED } \\
\text { (MG'L) } \\
(00530)\end{array}$ & $\begin{array}{c}\text { RESIDUE } \\
\text { VOLATILE, } \\
\text { SUS- } \\
\text { PENDED } \\
\text { (MG/L) } \\
(00535)\end{array}$ \\
\hline \multicolumn{13}{|l|}{ JUL 1997} \\
\hline $23 \ldots$ & 0628 & 0.88 & 2.0 & 25.0 & 174 & 131 & 8.1 & 7.4 & 70 & 3680 & 3300 & 150 \\
\hline $23 \ldots$ & 0754 & .88 & 29 & 23.5 & 99 & 81 & 7.3 & 7.3 & 40 & 13500 & 11000 & 1160 \\
\hline \multicolumn{13}{|l|}{ NOV } \\
\hline $21 \ldots$ & 1058 & .64 & .27 & 8.5 & -. & 161 & - & 6.9 & -- & -- & -- & -- \\
\hline $21 \ldots$ & 1139 & .64 & 2.4 & 9.0 & -- & 63 & - & 7.2 & -- & -- & - & -- \\
\hline $21 \ldots$ & 1334 & .64 & 23 & 9.0 & 73 & 63 & 7.6 & 7.3 & 26 & 4160 & 2840 & 290 \\
\hline $21 \ldots$ & 1411 & .64 & 8.4 & 9.0 & -- & 68 & - & 6.8 & -. & -- & -- & -- \\
\hline $21 \ldots$ & 1504 & .64 & 3.6 & 9.5 & 79 & 74 & 7.1 & 7.0 & 20 & 848 & 627 & 77 \\
\hline \multicolumn{13}{|l|}{ JAN 1998} \\
\hline $22 \ldots$ & 1942 & .78 & 1.6 & 7.0 & 1120 & 1150 & 7.2 & 6.9 & 23 & 277 & 216 & 35 \\
\hline $22 \ldots$ & 2114 & .78 & 3.6 & 6.5 & 142 & 142 & 7.2 & 7.1 & 14 & 398 & 427 & 49 \\
\hline $23 \ldots$ & 0114 & .78 & 11 & 6.5 & 56 & 54 & 7.1 & 7.1 & 11 & 1130 & 677 & 71 \\
\hline $23 \ldots$ & 0738 & .78 & 1.9 & 7.0 & -- & 87 & - & 7.1 & -- & -. & -- & -- \\
\hline $23 \ldots$ & 0745 & .78 & 1.9 & 7.0 & 93 & 88 & 7.2 & 7.1 & 22 & 171 & 157 & 19 \\
\hline \multicolumn{13}{|l|}{ MAY } \\
\hline $20 \ldots$ & 0645 & .00 & .16 & 19.0 & 210 & 221 & 7.5 & 7.2 & 72 & 42 & 11 & 2 \\
\hline \multicolumn{13}{|l|}{ JUN } \\
\hline $10 \ldots$ & 0812 & 1.02 & 28 & 20.0 & -- & 63 & -- & 7.2 & -- & -- & -- & -- \\
\hline $10 \ldots$ & 0816 & 1.02 & 36 & 20.5 & 55 & 44 & 6.9 & 6.8 & -- & 8680 & -- & -- \\
\hline $10 \ldots$ & 0851 & 1.02 & 16 & 21.0 & -- & 67 & -- & 7.0 & -- & -- & -- & -- \\
\hline $10 \ldots$ & 0933 & 1.02 & 56 & 20.5 & 78 & 66 & 6.9 & 6.8 & 25 & 4280 & 5127 & 586 \\
\hline $10 \ldots$ & 1148 & 1.02 & 4.3 & 21.0 & 59 & 56 & 7.1 & 6.8 & 14 & 1950 & 1500 & 183 \\
\hline DATE & $\begin{array}{c}\text { SOLIDS, } \\
\text { RESIDUE } \\
\text { AT } 180 \\
\text { DEG. C } \\
\text { DIS- } \\
\text { SOLVED } \\
\text { (MGL) } \\
(70300)\end{array}$ & $\begin{array}{c}\text { OXYGEN } \\
\text { DEMAND, } \\
\text { BIO- } \\
\text { CHEMICAL } \\
5 \text { DAY } \\
(\text { MG/L) } \\
(00310)\end{array}$ & $\begin{array}{c}\text { OXYGEN } \\
\text { DEMAND, } \\
\text { CHEMICAL } \\
\text { (HIGHLEVEL) } \\
(\text { MG/L) } \\
(00340) \\
\end{array}$ & $\begin{array}{c}\text { NITROGEN, } \\
\text { AMMONIA + } \\
\text { ORGANIC } \\
\text { TOTAL } \\
\text { (MG/L AS N) } \\
(00625)\end{array}$ & $\begin{array}{c}\text { NITROGEN, } \\
\text { NO2+NO3 } \\
\text { DISSOLVED } \\
\text { (MG/L AS N) } \\
(00631)\end{array}$ & $\begin{array}{c}\text { NITROGEN, } \\
\text { AMMONIA } \\
\text { DISSOLVED } \\
\text { (MG/L AS N) } \\
(00608)\end{array}$ & $\begin{array}{l}\text { NITRO- } \\
\text { GEN, } \\
\text { ORGANIC } \\
\text { TOTAL } \\
\text { (MG/ } \\
\text { AS N) } \\
(00605)\end{array}$ & $\begin{array}{c}\text { NITROGEN, } \\
\text { TOTAL } \\
\text { (MG/L AS N) } \\
(00600)\end{array}$ & $\begin{array}{c}\text { PHOS- } \\
\text { PHORUS } \\
\text { TOTAL } \\
\text { (MG/L AS P) } \\
(00665)\end{array}$ & $\begin{array}{c}\text { PHOS- } \\
\text { PHORUS } \\
\text { ORTHO, } \\
\text { DISSOLVED } \\
\text { (MG/L AS P) } \\
(00671) \\
\end{array}$ & $\begin{array}{c}\text { OILAVD } \\
\text { GREA'E, } \\
\text { TOTFI- } \\
\text { RECOV. } \\
\text { GRA'/l- } \\
\text { METPIC } \\
\text { (MG/L) } \\
(00556) \\
\end{array}$ & \\
\hline \multicolumn{13}{|l|}{ JUL 1997} \\
\hline $23 \ldots$ & 98 & 19 & 180 & 2.6 & 0.540 & 0.900 & 1.7 & 3.1 & 9.70 & 0.011 & -. & \\
\hline $23 \ldots$ & 47 & 3.9 & $<5$ & 3.7 & .240 & 1.00 & 2.7 & 3.9 & 12.4 & $<.010$ & -- & \\
\hline \multicolumn{13}{|l|}{ NOV } \\
\hline $21 \ldots$ & -- & -- & .. & -- & -- & -- & -- & -- & -- & -- & 4 & \\
\hline $21 \ldots$ & -- & -- & 39 & 2.4 & .760 & .530 & 1.9 & 3.2 & 1.47 & .021 & -- & \\
\hline $21 \ldots$ & 48 & -- & 14 & 1.6 & .250 & .310 & 1.3 & 1.9 & 6.70 & .100 & -- & \\
\hline $21 \ldots$ & -- & -- & -- & -- & -- & -- & -- & -- & -- & -- &.- & \\
\hline $21 \ldots$ & 65 & -- & 31 & 1.1 & .330 & .140 & .96 & 1.4 & 1.58 & .100 & -- & \\
\hline \multicolumn{13}{|l|}{ JAN 1998} \\
\hline $22 \ldots$ & 602 & 9.6 & 42 & 1.6 & .860 & .340 & 1.3 & 2.5 & .360 & $<.010$ & -- & \\
\hline $22 \ldots$ & 73 & 3.6 & 18 & .77 & .370 & .060 & .71 & 1.1 & .540 & $<.010$ & -- & \\
\hline $23 \ldots$ & 31 & 2.5 & 18 & .80 & .140 & .027 & .77 & .94 & 1.56 & .012 & -- & \\
\hline $23 \ldots$ & -- & -- & -- & -- & -. & -- & -- & -- & -- & -- & -- & \\
\hline $23 \ldots$ & 56 & $<2.0$ & 10 & .59 & .190 & .032 & .56 & .78 & .340 & $<.010$ & -- & \\
\hline \multicolumn{13}{|l|}{ MAY } \\
\hline $20 \ldots$ & 126 & 2.9 & 7 & .53 & .420 & $<.015$ & .53 & .95 & .230 & $<.010$ & 2 & \\
\hline \multicolumn{13}{|l|}{ JUN } \\
\hline $10 \ldots$ & -- & -- & -- & -- & -- & -- & -- & -- & -- & -- & -- & \\
\hline $10 \ldots$ & 30 & -- & -- & 3.8 & .320 & .130 & 3.7 & 4.1 & 7.20 & .024 & -- & \\
\hline $10 \ldots$ & -- & -- & -- & -- & -- & -- & - & -- & -- & -- & 1 & \\
\hline $10 \ldots$ & 41 & 13 & 16 & 5.7 & .300 & .230 & 5.5 & 6.0 & 12.1 & .310 & -- & \\
\hline $10 \ldots$ & 31 & 5.9 & 8 & 1.4 & .240 & .070 & 1.3 & 1.6 & 2.16 & .021 & -- & \\
\hline
\end{tabular}


Table 81. Water-quality data at site 43 (CSW07), July 1997 through September 1998-Continued

\begin{tabular}{|c|c|c|c|c|c|c|c|c|c|c|c|}
\hline DATE & $\begin{array}{c}\text { CARBON, } \\
\text { OR- } \\
\text { GANIC } \\
\text { TOTAL } \\
\text { (MGL } \\
\text { AS C) } \\
\text { (00680 }\end{array}$ & $\begin{array}{l}\text { STREPTO- } \\
\text { COCCI } \\
\text { FECAL, } \\
\text { (COLS. PER } \\
100 \mathrm{ML}) \\
(31679)\end{array}$ & $\begin{array}{c}\text { COLIFORM, } \\
\text { FECAL, } \\
0.45 \\
\text { UM-MF } \\
\text { (COLS./ } \\
100 \mathrm{ML}) \\
(31616)\end{array}$ & $\begin{array}{c}\text { ANTIMONY, } \\
\text { TOTAL } \\
\text { (UG/L AS SB) } \\
\text { (01097) }\end{array}$ & $\begin{array}{c}\text { ARSENIC, } \\
\text { TOTAL } \\
\text { (UG/L AS AS) } \\
\text { (01002) }\end{array}$ & $\begin{array}{l}\text { CHROMIUM, } \\
\text { TOTAL } \\
\text { RECOVER- } \\
\text { ABLE } \\
\text { (UG/L AS CR) } \\
\text { (01034) }\end{array}$ & $\begin{array}{l}\text { COPPER } \\
\text { TOTAL } \\
\text { RECOVER- } \\
\text { ABLE } \\
\text { (UG/L } \\
\text { AS CU) } \\
\text { (01042) }\end{array}$ & $\begin{array}{c}\text { LEAD, } \\
\text { TOTAL } \\
\text { RECOVER- } \\
\text { ABLE } \\
\text { (UG/L } \\
\text { AS PB) } \\
(01051)\end{array}$ & $\begin{array}{c}\text { MERCURY, } \\
\text { TOTAL } \\
\text { RECOVER- } \\
\text { ABLE } \\
\text { (UG/L AS HG) } \\
\text { (71900) }\end{array}$ & $\begin{array}{c}\text { NICKEL. } \\
\text { TOTAL, } \\
\text { RECOVER- } \\
\text { ABLE } \\
\text { (UG/L AS NI) } \\
\text { (01067) }\end{array}$ & $\begin{array}{c}\text { ZINC, } \\
\text { TOTAL } \\
\text { RECOVER- } \\
\text { ABLE } \\
\text { (UG/L AS ZN) } \\
\text { (C1092) }\end{array}$ \\
\hline \multicolumn{12}{|l|}{ JUL 1997} \\
\hline $23 \ldots$ & 15 & -- & -- & $<1$ & 22 & 110 & 240 & 36 & 0.20 & 160 & 710 \\
\hline $23 \ldots$ & 9.5 & -- & -- & $<1$ & 34 & 330 & 500 & 83 & .70 & 410 & 830 \\
\hline \multicolumn{12}{|l|}{ NOV } \\
\hline $21 \ldots$ & -- & K270 & K360 & -- & -- & -- & -- & -- & -. & -- & -- \\
\hline $21 \ldots$ & -- & -- & -- & $<1$ & 9 & 77 & 100 & 28 & $<.10$ & 57 & 300 \\
\hline $21 \ldots$ & 8.5 & -- & -- & $<1$ & 23 & 150 & 270 & 30 & .10 & 73 & 310 \\
\hline $21 \ldots$ & -. & 4500 & K9300 & -- & -- & -- & -- & -- & - & -- & -- \\
\hline $21 \ldots$ & -- & 7100 & 9100 & -- & -. & -- & -- & -- & -- & -- & -. \\
\hline \multicolumn{12}{|l|}{ JAN 1998} \\
\hline $22 \ldots$ & 18 & -- & -- & 5 & 2 & 27 & 27 & 10 & .20 & 31 & 170 \\
\hline $22 \ldots$ & 8.3 & -- & -- & $<1$ & $<1$ & 29 & 39 & 7 & .10 & 23 & 90 \\
\hline $23 \ldots$ & 9.3 & -. & -- & $<1$ & $<1$ & 41 & 74 & 17 & $<10$ & 44 & 90 \\
\hline $23 \ldots$ & -- & 5200 & $\mathrm{~K} 1400$ & -- & -- & -- & -- & -- & -- & -- & -- \\
\hline $23 \ldots$ & - & -- & -- & - & -- & -. & -- & -- & -- & -- & -- \\
\hline \multicolumn{12}{|l|}{ MAY } \\
\hline $20 \ldots$ & -- & $\mathrm{K} 1300$ & K180 & 2 & $<1$ & 12 & 29 & $<1$ & $<.10$ & 3 & 30 \\
\hline \multicolumn{12}{|l|}{ JUN } \\
\hline $10 \ldots$ & -- & -- & -- & -- & -- & -. & -- & -- & -- & -- & -. \\
\hline $10 \ldots$ & -- & -- & - & $<1$ & 34 & 490 & 370 & 34 & .20 & 140 & 620 \\
\hline $10 \ldots$ & 26 & 45000 & 33000 & -- & -- & -. & -- & -- & -- & -- & -. \\
\hline $10 \ldots$ & 16 & 69000 & K19000 & $<1$ & 43 & 340 & 270 & 19 & .30 & 250 & 780 \\
\hline $10 \ldots$ & -- & -- & -- & -- & -- & -. & -- & -- & -- & -- & -- \\
\hline
\end{tabular}

K Results based on colony count outside the acceptable range (nonideal colony count). 
Table 82. Rainfall and streamflow characteristics for the monitored storms at site 37 (CSW06), July 1997 through September 1998

[Peak discharge for event may occur after storm duration ends. Sample types: A - Chemical, nutrients, and metals, B - Organic compounds, C - Volatile compounds, D - Total organic carbon, E - Oil and grease, F - Bacteria]

\begin{tabular}{|c|c|c|c|c|c|c|c|c|c|}
\hline $\begin{array}{l}\text { Date and time } \\
\text { storm began }\end{array}$ & $\begin{array}{l}\text { Storm } \\
\text { dura- } \\
\text { tion }^{a} \\
\text { (hours) }\end{array}$ & $\begin{array}{c}\text { Total } \\
\text { rainfallb }^{b} \\
\text { (inches) }\end{array}$ & $\begin{array}{l}\text { Time first/ } \\
\text { last samples } \\
\text { collected }\end{array}$ & $\begin{array}{l}\text { Total } \\
\text { rainfall } \\
\text { from } \\
\text { begin- } \\
\text { ning of } \\
\text { storm to } \\
\text { end of } \\
\text { sam- } \\
\text { pling }\end{array}$ & $\begin{array}{l}\text { Number } \\
\text { of dry } \\
\text { days } \\
\text { preceed- } \\
\text { ing } \\
\text { storm }\end{array}$ & $\begin{array}{l}\text { Number } \\
\text { of days } \\
\text { since } \\
\text { last } \\
\text { rainfall } \\
\text { of }>0.5 \\
\text { Inch }\end{array}$ & $\begin{array}{c}\text { Peak } \\
\text { dis- } \\
\text { charge } \\
\text { sampled } \\
\left(\mathrm{ft}^{3} / \mathrm{s}\right)\end{array}$ & $\begin{array}{l}\text { Peak } \\
\text { dis- } \\
\text { charge } \\
\text { during } \\
\text { the } \\
\text { storm } \\
\text { duration } \\
\left(\mathrm{ft}^{3} / \mathrm{s}\right)\end{array}$ & Szmple types \\
\hline $7 / 22 / 97$ at 2245 & 10 & 5.91 & $7 / 22 / 97$ at 2306 & .94 & $<1$ & $<1$ & 1.5 & d & $\mathrm{A}, \mathrm{D}$ \\
\hline $11 / 12 / 97$ at 1630 & 38 & 1.20 & $11 / 12 / 97$ at 2005 & .14 & 10 & 10 & .02 & 2.0 & $\mathrm{E}, \mathrm{F}$ \\
\hline $11 / 21 / 97$ at 0935 & 6 & .73 & $1158 / 1504$ & .72 & 6 & 7 & 7.3 & 7.6 & $\mathrm{~A}, \mathrm{D}, \mathrm{F}$ \\
\hline $1 / 22 / 98$ at 1835 & 19 & .76 & $\begin{array}{l}1 / 22 / 98 \text { at } 2106 \\
1 / 23 / 98 \text { at } 0931\end{array}$ & .73 & 2 & 2 & 1.4 & 2.2 & $A, D, F$ \\
\hline $6 / 10 / 98$ at 0750 & 2 & .71 & $0919 / 1016$ & .71 & 4 & 5 & 19 & 21 & $\mathrm{~A}, \mathrm{~B}, \mathrm{C}, \mathrm{D}, \mathrm{E}, \mathrm{F}$ \\
\hline
\end{tabular}

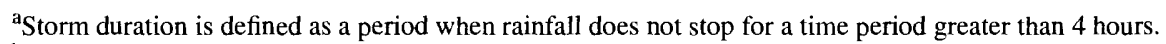

${ }^{b}$ Rainfall from site 37 (CRN28). Total rainfall is the amount of rain that occurred during the storm duration.

${ }^{\mathrm{c}}$ Number of dry days is defined as days that $\leq 0.10$ inch of rainfall occurred.

dot determined. 
Table 83. Rainfall and streamflow characteristics for the monitored storms at site 39 (CSW05), July 1937 through September 1998

[Peak discharge for event may occur after storm duration ends. Sample types: A - Chemical, nutrients, and metals, B - Organic compounds, C - Volatile compounds, D - Total organic carbon, E - Oil and grease, F - Bacteria]

\begin{tabular}{|c|c|c|c|c|c|c|c|c|c|}
\hline $\begin{array}{l}\text { Date and time } \\
\text { storm began }\end{array}$ & $\begin{array}{l}\text { Storm } \\
\text { dura- } \\
\text { tion }^{\mathrm{a}} \\
\text { (hours) }\end{array}$ & $\begin{array}{l}\text { Total } \\
\text { rainfalıb }^{b} \\
\text { (inches) }\end{array}$ & $\begin{array}{l}\text { Time first/ } \\
\text { last samples } \\
\text { collected }\end{array}$ & $\begin{array}{l}\text { Total } \\
\text { rainfall } \\
\text { from } \\
\text { begin- } \\
\text { ning of } \\
\text { storm to } \\
\text { end of } \\
\text { sam- } \\
\text { pling }\end{array}$ & $\begin{array}{l}\text { Number } \\
\text { of dry } \\
\text { days } \\
\text { preceed- } \\
\text { ing } \\
\text { storm c }\end{array}$ & $\begin{array}{c}\text { Number } \\
\text { of days } \\
\text { since } \\
\text { last } \\
\text { rainfall } \\
\text { of }>0.5 \\
\text { Inch }\end{array}$ & $\begin{array}{c}\text { Peak } \\
\text { dis- } \\
\text { charge } \\
\text { sampled } \\
\left(\mathrm{ft}^{3} / \mathrm{s}\right)\end{array}$ & $\begin{array}{c}\text { Peak } \\
\text { dis- } \\
\text { charge } \\
\text { during } \\
\text { the } \\
\text { storm } \\
\text { duration } \\
\left(\mathrm{ft}^{3} / \mathrm{s}\right)\end{array}$ & Sample types \\
\hline $7 / 22 / 97$ at 1545 & 18 & 8.93 & $\begin{array}{l}7 / 22 / 97 \text { at } 2029 \\
7 / 23 / 97 \text { at } 0456\end{array}$ & 6.91 & 12 & 24 & 21 & 27 & $A, D$ \\
\hline $11 / 12 / 97$ at 1700 & 33 & 1.25 & $\begin{array}{l}11 / 12 / 97 \text { at } 1825 \\
11 / 13 / 97 \text { at } 1056\end{array}$ & .61 & 10 & 10 & .21 & 1.6 & $\mathrm{~A}, \mathrm{D}, \mathrm{E}, \mathrm{F}$ \\
\hline $1 / 22 / 98$ at 1825 & 21 & .75 & $\begin{array}{l}1 / 22 / 98 \text { at } 1842 \\
1 / 23 / 98 \text { at } 1017\end{array}$ & .73 & 2 & 2 & .36 & 1.1 & A.D,F \\
\hline $6 / 10 / 98$ at 0610 & 4 & .46 & $0735 / 1028$ & .46 & 4 & $\geq 8$ & 1.6 & 2.3 & $F, \mathrm{~B}, \mathrm{C}, \mathrm{D}, \mathrm{E}, \mathrm{F}$ \\
\hline
\end{tabular}

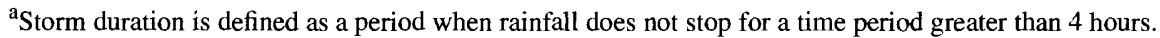

${ }^{b}$ Rainfall from site 15 (CRN03), except event of 7/22/97when rainfall from site 36 (CRN27) was used. Total rainfall is the amount of rain that occurred during the storm duration.

${ }^{c}$ Number of dry days is defined as days that $\leq 0.10$ inch of rainfall occurred. 
Table 84. Rainfall and streamflow characteristics for the monitored storms at site 40 (CSW03), July 1997 through September 1998

[Peak discharge for event may occur after storm duration ends. Sample types: A - Chemical, nutrients, and metals, B - Organic compounds, C - Volatile compounds, D - Total organic carbon, E - Oil and grease, F - Bacteria]

\begin{tabular}{|c|c|c|c|c|c|c|c|c|c|}
\hline $\begin{array}{l}\text { Date and time } \\
\text { storm began }\end{array}$ & $\begin{array}{l}\text { Storm } \\
\text { dura- } \\
\text { tion }^{\mathrm{a}} \\
\text { (hours) }\end{array}$ & $\begin{array}{c}\text { Total } \\
\text { rainfall } \\
\text { (inches) }\end{array}$ & $\begin{array}{l}\text { Time first/ } \\
\text { last samples } \\
\text { collected }\end{array}$ & $\begin{array}{l}\text { Total } \\
\text { rainfall } \\
\text { from } \\
\text { begin- } \\
\text { ning of } \\
\text { storm to } \\
\text { end of } \\
\text { sam- } \\
\text { pling }\end{array}$ & $\begin{array}{l}\text { Number } \\
\text { of dry } \\
\text { days } \\
\text { preceed- } \\
\text { ing } \\
\text { stormc }\end{array}$ & $\begin{array}{c}\text { Number } \\
\text { of days } \\
\text { since } \\
\text { last } \\
\text { rainfall } \\
\text { of }>0.5 \\
\text { inch }\end{array}$ & $\begin{array}{l}\text { Peak } \\
\text { dis- } \\
\text { charge } \\
\text { sampled } \\
\left(\mathrm{ft}^{3} / \mathrm{s}\right)\end{array}$ & $\begin{array}{l}\text { Peak } \\
\text { dis } \\
\text { charge } \\
\text { during } \\
\text { the } \\
\text { storm } \\
\text { duration } \\
\left(\mathrm{ft}^{3} / \mathrm{s}\right)\end{array}$ & Sample types \\
\hline $7 / 22 / 97$ at 2025 & 13 & 4.82 & $\begin{array}{l}7 / 22 / 97 \text { at } 2318 \\
7 / 23 / 97 \text { at } 0146\end{array}$ & 1.78 & 1 & 1 & 9.9 & $\geq 14$ & $A, D$ \\
\hline $11 / 21 / 97$ at 0940 & 5 & .63 & $1020 / 1438$ & .63 & 6 & 7 & .27 & .36 & $\mathrm{~A}, \mathrm{D}, \mathrm{F}$ \\
\hline $2 / 2 / 98$ at 2330 & 47 & 1.79 & $\begin{array}{l}2 / 3 / 98 \text { at } 0814 \\
2 / 4 / 98 \text { at } 1002\end{array}$ & 1.57 & 5 & 5 & .59 & .63 & $\mathrm{~A}, \mathrm{D}, \mathrm{E}, \mathrm{F}$ \\
\hline $6 / 10 / 98$ at 0725 & 3 & 1.48 & $0806 / 0940$ & 1.39 & 4 & 4 & .76 & 1.2 & $A, B$ \\
\hline
\end{tabular}

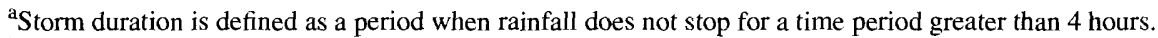

${ }^{b}$ Rainfall from site 29 (CRN20). Total rainfall is the amount of rain that occurred during the storm duration.

${ }^{\mathrm{c}}$ Number of dry days is defined as days that $\leq 0.10$ inch of rainfall occurred. 
Table 85. Rainfall and streamflow characteristics for the monitored storms at site 41 (CSW02), July 1 ( 77 through September 1998

[Peak discharge for event may occur after storm duration ends. Sample types: A - Chemical, nutrients, and metals, B - Organic compounds, C - Volatile compounds, D - Total organic carbon, E - Oil and grease, F - Bacteria]

\begin{tabular}{|c|c|c|c|c|c|c|c|c|c|}
\hline $\begin{array}{l}\text { Date and time } \\
\text { storm began }\end{array}$ & $\begin{array}{l}\text { Storm } \\
\text { dura- } \\
\text { tion }^{a} \\
\text { (hours) }\end{array}$ & $\begin{array}{c}\text { Total } \\
\text { rainfallb } \\
\text { (inches) }\end{array}$ & $\begin{array}{l}\text { Time first/ } \\
\text { last samples } \\
\text { collected }\end{array}$ & $\begin{array}{l}\text { Total } \\
\text { rainfall } \\
\text { from } \\
\text { begin- } \\
\text { ning of } \\
\text { storm to } \\
\text { end of } \\
\text { sam- } \\
\text { pling }\end{array}$ & $\begin{array}{l}\text { Number } \\
\text { of dry } \\
\text { days } \\
\text { preceed- } \\
\text { ing } \\
\text { stormc }\end{array}$ & $\begin{array}{l}\text { Number } \\
\text { of days } \\
\text { since } \\
\text { last } \\
\text { ralnfall } \\
\text { of }>0.5 \\
\text { inch }\end{array}$ & $\begin{array}{c}\text { Peak } \\
\text { dls- } \\
\text { charge } \\
\text { sampled } \\
\left(\mathrm{ft}^{3} / \mathrm{s}\right)\end{array}$ & $\begin{array}{l}\text { Peak } \\
\text { dis- } \\
\text { charge } \\
\text { during } \\
\text { the } \\
\text { storm } \\
\text { duration } \\
\left(\mathrm{ft}^{3} / \mathrm{s}\right)\end{array}$ & Sample types \\
\hline $7 / 22 / 97$ at 2245 & 11 & 4.40 & $\begin{array}{l}7 / 22 / 97 \text { at } 2302 \\
7 / 23 / 97 \text { at } 0756\end{array}$ & 3.52 & 16 & 16 & 253 & 268 & $\mathrm{~A}, \mathrm{D}$ \\
\hline $11 / 12 / 97$ at 1615 & 37 & 1.12 & $\begin{array}{l}11 / 12 / 97 \text { at } 1824 \\
11 / 13 / 97 \text { at } 0822\end{array}$ & .57 & 10 & 10 & .76 & 2.6 & $\mathrm{~A}, \mathrm{D}, \mathrm{E}, \mathrm{F}$ \\
\hline $2 / 2 / 98$ at 2225 & 36 & 1.23 & $\begin{array}{l}2 / 3 / 98 \text { at } 0346 \\
2 / 4 / 98 \text { at } 0738\end{array}$ & 1.18 & 5 & 5 & 7.0 & 8.3 & $\mathrm{~A}, \mathrm{D}, \mathrm{E}, \mathrm{F}$ \\
\hline $6 / 10 / 98$ at 0740 & 2 & 1.53 & $0756 / 1023$ & 1.53 & 4 & 5 & 150 & 172 & $\mathrm{~A}, \mathrm{~B}, \mathrm{C}, \mathrm{D}, \mathrm{E}, \mathrm{F}$ \\
\hline
\end{tabular}

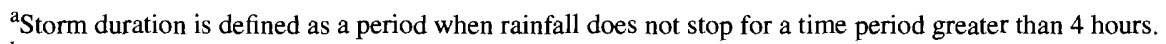

${ }^{\mathrm{b}}$ Rainfall from site 22 (CRN12). Total rainfall is the amount of rain that occurred during the storm duration.

${ }^{\mathrm{c}}$ Number of dry days is defined as days that $\leq 0.10$ inch of rainfall occurred. 
Table 86. Rainfall and streamflow characteristics for the monitored storms at site 42 (CSW04), July 1997 through September 1998

[Peak discharge for event may occur after storm duration ends. Sample types: A - Chemical, nutrients, and metals, B - Organic compounds, C - Volatile compounds, D - Total organic carbon, E - Oil and grease, F - Bacteria]

\begin{tabular}{|c|c|c|c|c|c|c|c|c|c|}
\hline $\begin{array}{l}\text { Date and time } \\
\text { storm began }\end{array}$ & $\begin{array}{l}\text { Storm } \\
\text { dura- } \\
\text { tion }^{a} \\
\text { (hours) }\end{array}$ & $\begin{array}{c}\text { Total } \\
\text { rainfall }^{\mathrm{b}} \\
\text { (inches) }^{\text {inches }}\end{array}$ & $\begin{array}{l}\text { Time first/ } \\
\text { last samples } \\
\text { collected }\end{array}$ & $\begin{array}{c}\text { Total } \\
\text { rainfall } \\
\text { from } \\
\text { begin- } \\
\text { ning of } \\
\text { storm to } \\
\text { end of } \\
\text { sam- } \\
\text { pling }\end{array}$ & $\begin{array}{l}\text { Number } \\
\text { of dry } \\
\text { days } \\
\text { preceed- } \\
\text { ing } \\
\text { stormc }\end{array}$ & $\begin{array}{l}\text { Number } \\
\text { of days } \\
\text { since } \\
\text { last } \\
\text { rainfall } \\
\text { of }>0.5 \\
\text { inch }\end{array}$ & $\begin{array}{c}\text { Peak } \\
\text { dls- } \\
\text { charge } \\
\text { sampled } \\
\left(\mathrm{ft}^{3} / \mathrm{s}\right)\end{array}$ & $\begin{array}{l}\text { Peak } \\
\text { dis- } \\
\text { charge } \\
\text { during } \\
\text { the } \\
\text { storm } \\
\text { duration } \\
\left(\mathrm{ft}^{3} / \mathrm{s}\right)\end{array}$ & Sample types \\
\hline $7 / 22 / 97$ at 2245 & 11 & 4.40 & $7 / 23 / 97$ at 0734 & 2.87 & 16 & 16 & 229 & 305 & A.D \\
\hline $11 / 12 / 97$ at 1615 & 37 & 1.12 & $\begin{array}{l}11 / 12 / 97 \text { at } 1828 \\
11 / 13 / 97 \text { at } 0928\end{array}$ & .58 & 10 & 10 & 1.3 & 19 & $\mathrm{~A}, \mathrm{D}, \mathrm{E}, \mathrm{F}$ \\
\hline $2 / 2 / 98$ at 2225 & 36 & 1.23 & $\begin{array}{l}2 / 3 / 98 \text { at } 0802 \\
2 / 4 / 98 \text { at } 0919\end{array}$ & 1.22 & 5 & 5 & 20 & 26 & $\mathrm{~A}, \mathrm{D}, \mathrm{E}, \mathrm{F}$ \\
\hline $6 / 10 / 98$ at 0740 & 2 & 1.53 & $0758 / 1101$ & 1.53 & 4 & 5 & 100 & 100 & $\mathrm{~A}, \mathrm{~B}, \mathrm{D}, \mathrm{E}, \mathrm{F}$ \\
\hline
\end{tabular}

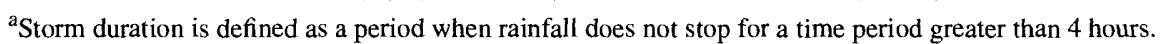

${ }^{b}$ Rainfall from site 22 (CRN12). Total rainfall is the amount of rain that occurred during the storm duration.

${ }^{\mathrm{c}}$ Number of dry days is defined as days that $\leq 0.10$ inch of rainfall occurred. 
Table 87. Rainfall and streamflow characteristics for the monitored storms at site 43 (CSW07), July 19 ? through September 1998

[Peak discharge for event may occur after storm duration ends. Sample types: A - Chemical, nutrients, and metals, B - Organic compounds, C - Volatile compounds, D - Total organic carbon, E - Oil and grease, F - Bacteria]

\begin{tabular}{|c|c|c|c|c|c|c|c|c|c|}
\hline $\begin{array}{l}\text { Date and time } \\
\text { storm began }\end{array}$ & $\begin{array}{c}\text { Storm } \\
\text { dura- } \\
\text { tion }^{a} \\
\text { (hours) }\end{array}$ & $\begin{array}{c}\text { Total } \\
\text { rainfall } \\
\text { (Inches) }\end{array}$ & $\begin{array}{l}\text { Time first/ } \\
\text { last samples } \\
\text { collected }\end{array}$ & $\begin{array}{c}\text { Total } \\
\text { rainfall } \\
\text { from } \\
\text { begin- } \\
\text { ning of } \\
\text { storm to } \\
\text { end of } \\
\text { sam- } \\
\text { pling }\end{array}$ & $\begin{array}{l}\text { Number } \\
\text { of dry } \\
\text { days } \\
\text { preceed- } \\
\text { ing } \\
\text { storm }\end{array}$ & $\begin{array}{c}\text { Number } \\
\text { of days } \\
\text { since } \\
\text { last } \\
\text { rainfall } \\
\text { of }>0.5 \\
\text { inch }\end{array}$ & $\begin{array}{c}\text { Peak } \\
\text { dls- } \\
\text { charge } \\
\text { sampled } \\
\left(\mathrm{ft}^{3} / \mathrm{s}\right)\end{array}$ & $\begin{array}{l}\text { Peak } \\
\text { dls- } \\
\text { charge } \\
\text { during } \\
\text { the } \\
\text { storm } \\
\text { duration } \\
\left(\mathrm{ft}^{3} / \mathrm{s}\right)\end{array}$ & Sample types \\
\hline $7 / 23 / 97$ at 0350 & 6 & .88 & $0628 / 0754$ & .79 & 6 & 25 & 29 & 42 & $\mathrm{~A}, \mathrm{D}$ \\
\hline $11 / 21 / 97$ at 0950 & 5 & .64 & $1058 / 1504$ & .64 & 6 & 7 & 23 & 24 & $\mathrm{~A}, \mathrm{D}, \mathrm{E}, \mathrm{F}$ \\
\hline $1 / 22 / 98$ at 1840 & 17 & .78 & $\begin{array}{l}1 / 22 / 98 \text { at } 1942 \\
1 / 23 / 98 \text { at } 0745\end{array}$ & .72 & 2 & 2 & 11 & 12 & $\mathrm{~A}, \mathrm{D}, \mathrm{F}$ \\
\hline $6 / 10 / 98$ at 0805 & 2 & 1.02 & $0812 / 1148$ & 1.02 & 3 & 5 & 56 & 66 & A,B,C,D,E,F \\
\hline
\end{tabular}

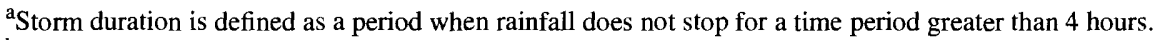

${ }^{b}$ Rainfall from site 19 (CRN08). Total rainfall is the amount of rain that occurred during the storm duration.

${ }^{\mathrm{c}}$ Number of dry days is defined as days that $\leq 0.10$ inch of rainfall occurred. 